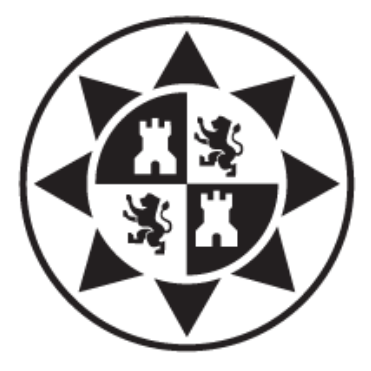

UNIVERSIDAD POLITÉCNICA DE CARTAGENA

Departamento de Tecnología Electrónica

\title{
CONTRIBUCIÓN A LAS REDES DE SENSORES INALÁMBRICAS. ESTUDIO E IMPLEMENTACIÓN DE SOLUCIONES HARDWARE PARA AGRICULTURA DE PRECISIÓN
}

Tesis Doctoral

D. Juan Antonio López Riquelme

Ingeniero en Automática y Electrónica Industrial

\section{Directores}

Dr. D. Juan Suardíaz Muro

Dr. D. Andrés Iborra García

Dr. D. Basil Al-Hadithi Abdul Qadir 
CONTRIBUCIÓN A LAS REDES DE SENSORES INALÁMBRICAS. ESTUDIO E IMPLEMENTACIÓN DE SOLUCIONES HARDWARE PARA AGRICULTURA DE PRECISIÓN.

Autor: Juan Antonio López Riquelme

Directores: Juan Suardíaz Muro

Andrés José Iborra García

Basil Al-Hadithi Abdul Qadir

\section{Contacto:}

Copyright 2011 @ Juan Antonio López Riquelme 


\title{
I N F O R M A:
}

Que la Tesis Doctoral titulada "CONTRIBUCIÓN A LAS REDES DE SENSORES INALÁMBRICAS. ESTUDIO E IMPLEMENTACIÓN DE SOLUCIONES HARDWARE PARA AGRICULTURA DE PRECISIÓN", ha sido realizada por D./Da . Juan Antonio López Riquelme, bajo la dirección y supervisión de D./Da . Juan Suardíaz Muro, Andrés Iborra García, Basil Al-Hadithi Abdul Qadir, y que la Comisión Académica ha dado su conformidad para que sea presentada ante la Comisión de Doctorado.

La rama de conocimiento por la que esta tesis ha sido desarrollada es:

\author{
Ciencias Básicas \\ Ciencias Sociales y Jurídicas \\ $x$ Ingeniería y Arquitectura
}

En Cartagena, a 12 de septiembre de 2011

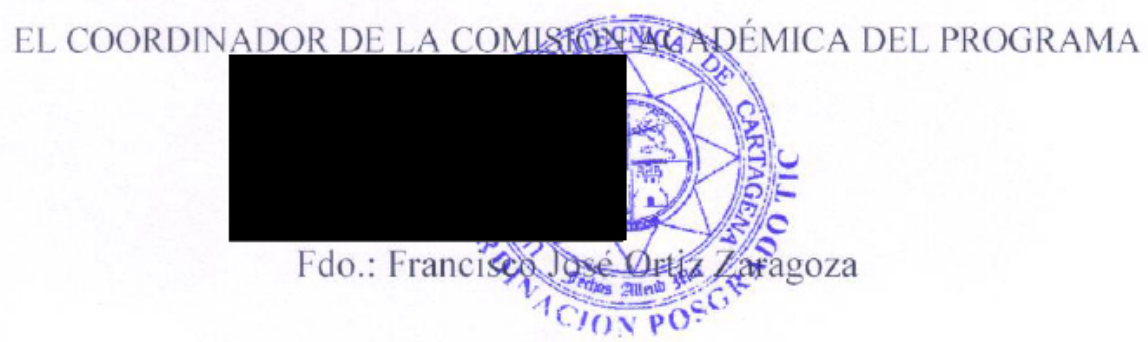

\section{Comisión de Doctorado}





\section{AUTORIZACIÓN DE LA PRESENTACIÓN DE LA TESIS DOCTORAL} POR EL DIRECTOR/A

D. Juan Suardíaz Muro, Profesor Doctor del Área de Tecnología Electrónica en el Departamento de Tecnología Electrónica

\section{A U T O R I Z A:}

La presentación de la Tesis Doctoral titulada "CONTRIBUCIÓN A LAS REDES DE SENSORES INALÁMBRICAS. ESTUDIO E IMPLEMENTACIÓN DE SOLUCIONES HARDWARE PARA AGRICULTURA DE PRECISIÓN", realizada por D. Juan Antonio López Riquelme, bajo mi dirección y supervisión, en el Departamento de Tecnología Electrónica, y que presenta para la obtención del grado de Doctor por la Universidad Politécnica de Cartagena.

En Cartagena, a 9 de septiembre de 2011

EL/LA DIRECTOR/A DE TESIS

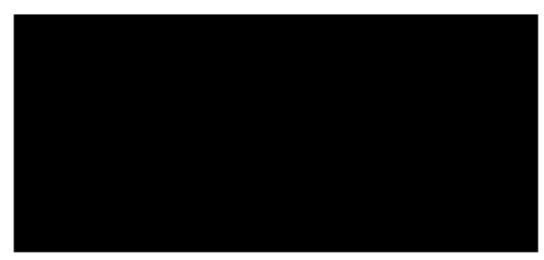

Fdo.: Juan Suardíaz Muro 



\section{AUTORIZACIÓN DE LA PRESENTACIÓN DE LA TESIS DOCTORAL} POR EL DIRECTOR/A

D. Andrés Iborra García, Profesor Doctor del Área de Tecnología Electrónica en el Departamento de Tecnología Electrónica

\section{A U T O R I Z A:}

La presentación de la Tesis Doctoral titulada "CONTRIBUCIÓN A LAS REDES DE SENSORES INALÁMBRICAS. ESTUDIO E IMPLEMENTACIÓN DE SOLUCIONES HARDWARE PARA AGRICULTURA DE PRECISIÓN", realizada por D. Juan Antonio López Riquelme, bajo mi dirección y supervisión, en el Departamento de Tecnología Electrónica, y que presenta para la obtención del grado de Doctor por la Universidad Politécnica de Cartagena.

En Cartagena, a 9 de septiembre de 2011

EL/LA DIRECTOR/A DE TESIS

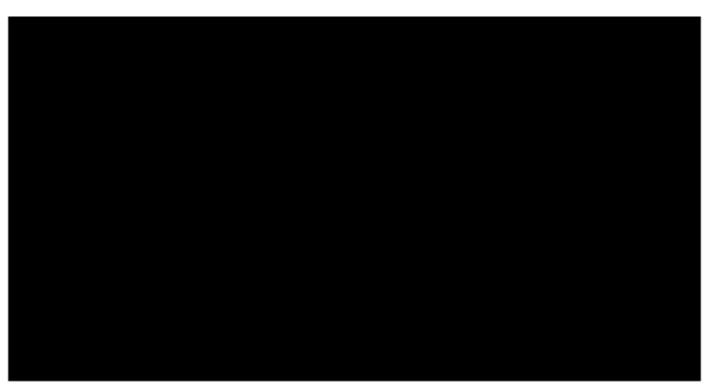

Fdo.: Andrés Iborra García 



\title{
AUTORIZACIÓN DE LA PRESENTACIÓN DE LA TESIS DOCTORAL
} POR EL DIRECTOR/A

D. Basil M. Al-Hadithi, Profesor Doctor del Área de Ingeniería de Sistemas y Automática en el Departamento de Electrónica, Automática e Informática Industrial

\begin{abstract}
A U T O R I Z A:
La presentación de la Tesis Doctoral titulada "CONTRIBUCIÓN A LAS REDES DE SENSORES INALÁMBRICAS. ESTUDIO E IMPLEMENTACIÓN DE SOLUCIONES HARDWARE PARA AGRICULTURA DE PRECISIÓN", realizada por D. Juan Antonio López Riquelme, bajo mi dirección y supervisión, en el Departamento de Tecnología Electrónica, y que presenta para la obtención del grado de Doctor por la Universidad Politécnica de Cartagena.
\end{abstract}

En Cartagena, a 9 de septiembre de 2011

EL/LA DIRECTOR/A DE TESIS

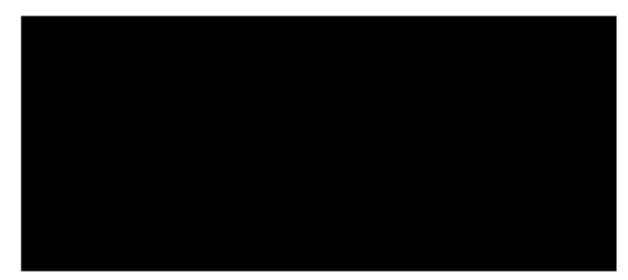

Fdo.: Basil M. Al-Hadithi 



\section{Agradecimientos}

Siempre es difícil recordar a todas las personas que han contribuido durante el largo camino que hay que recorrer para realizar una Tesis Doctoral. En primer lugar, quiero agradecer a mis directores Juan Suardíaz Muro, Andrés Iborra García y Basil Al-Hadithi Abdul Qadir su dedicación y apoyo durante este largo camino, especialmente a Juan Suardíaz que me ha guiado y aconsejado de una manera excelente desde que dirigió mi primer PFC en el año 2002.

Gracias a todos mis compañeros del laboratorio, Pencho Soto, Cristina Albaladejo, Roque Torres y Honorio Navarro. Especialmente, este trabajo no habría sido posible sin la inestimable ayuda de Pencho Soto, el cual me brindó una estrecha colaboración desde que empecé a trabajar en la línea de investigación de las redes de sensores inalámbricas. Tampoco puedo olvidar realizar un especial agradecimiento a Roque Torres, por sus sabios consejos, su amistad y los ratos agradables haciendo trabajos mecánicos en su taller.

Quiero agradecer a la Fundación Séneca de la Región de Murcia, su confianza por concederme la beca FPI que posibilitó que comenzara a desarrollar mis trabajos de investigación.

También quiero expresar mi agradecimiento a la empresa Edosoft Factory S.L., y especialmente a Juan Alberto Vera Gómez, por proporcionar la mayor parte de los fondos económicos necesarios para desarrollar los dispositivos descritos en la presente Tesis Doctoral.

Dar las gracias a los investigadores Joan García, Antonio Javier García y Felipe García, del grupo de investigación "Grupo de Ingeniería Telemática", por su colaboración en el desarrollo de las simulaciones con el entorno ns-2, así como en la comparación de los resultados del simulador con los obtenidos en el despliegue real.

Gracias a Jesús Rubio y a Raúl Borraz por su contribución en la implementación del GAIA Soil-Mote y del GAIA 2.

No puedo dejar de dar las gracias tampoco a los investigadores Raul Morais, Samuel Matos, Miguel Fernandes, Nuno Silva y Manuel Cabral, del grupo de investigación "Centro de Investigação e Tecnologias Agro-Ambientais e Biológicas", de la Universidad de Trás- 
os-Montes e Alto Douro por su cordial acogida y por su contribución a la ampliación de mis conocimientos sobre redes de sensores inalámbricas, durante el desarrollo de mi estancia predoctoral en Portugal.

También quiero darles especialmente las gracias a los investigadores, Rafael Domingo, Alejandro Pérez, Manuel García, María del Rosario Conesa, José María de la Rosa y Pablo Puerto, del grupo de investigación "Suelo-Agua-Planta", por proporcionarme los medios agronómicos necesarios para poder validar las soluciones hardware desarrolladas y por aportar su experiencia durante la elaboración de los requisitos que tenían que satisfacer los nodos sensores.

Agradecer a mis revisores más tenaces, Pedro Alcover y Senga Floyd, la mejora extraordinaria de la calidad de la lectura de este documento.

Este trabajo tampoco habría sido posible sin la inestimable ayuda, durante estos años, de todos los miembros del Dpto. de Tecnología Electrónica y del grupo de investigación "División de Sistemas e Ingeniería Electrónica".

No puedo olvidar, a la vez que me gustaría expresar mi más sincero agradecimiento a Pedro Sánchez y a Andrés Iborra, por su gran colaboración en la elaboración de las primeras publicaciones, con las que mejoré mi experiencia en esta dura tarea.

Y por último, gracias a Nieves por su gran apoyo durante estos últimos meses tan duros con las últimas pruebas realizadas y la preparación de este documento. 
A mis padres

y a todas las personas

que más quiero 



\section{Resumen}

En las regiones áridas y semiáridas, como el sureste español, la escasez de los recursos hídricos está causando importantes problemas de abastecimiento que afectan principalmente a la agricultura. Además, las predicciones sobre los efectos de cambio climático en estas regiones auguran un progresivo empeoramiento de la situación actual. Por tanto, uno de los objetivos prioritarios en el manejo y gestión de los recursos hídricos destinados al riego, debe ser el desarrollo e incorporación de nuevas tecnologías, así como de métodos que permitan conseguir una mayor eficiencia del uso del agua. Uno de los métodos más conocidos, y utilizados, para estimar, evaluar y entender las variaciones existentes en los cultivos es la agricultura de precisión. Este método se beneficia de numerosas tecnologías, entre las que se pueden citar los sistemas de posición global, las comunicaciones inalámbricas y los sistemas de instrumentación. Estas tecnologías permiten realizar tareas de monitorización de los cultivos almacenando los datos adquiridos, junto con las coordenadas geográficas del punto en el que se realizó la medida.

Las redes de sensores inalámbricas constituyen una tecnología emergente de adquisición de datos que recientemente está atrayendo gran interés gracias a sus posibilidades, siendo aplicadas en numerosos ámbitos científicos e industriales para la realización de estudios y control de procesos. El uso de comunicaciones inalámbricas permite que los dispositivos sensores que la forman sean emplazados, así como reubicados, fácilmente sobre el terreno.

Esta tesis se plantea con el objetivo global de estudiar, diseñar e implementar una arquitectura hardware, basada en redes de sensores inalámbricas, que pueda servir de herramienta a los ingenieros agrónomos para monitorizar, y así estimar, evaluar y entender las variaciones existentes en los cultivos, con objeto de determinar con mayor exactitud las necesidades de fertilizantes y riego, las fases de desarrollo y maduración de los productos, así como los puntos óptimos de siembra y recolección, que son los principales objetivos del método de la agricultura de precisión. 
Teniendo en cuenta las premisas de la arquitectura propuesta, durante el desarrollo de esta Tesis se han diseñado y construido diferentes prototipos de dispositivos inalámbricos (motes), los cuales han sido progresivamente depurados y mejorados a lo largo del transcurso de este trabajo, para poder ser validados en despliegues de redes de sensores, ubicadas en plantaciones agrícolas con diferentes cultivos. El resultado de este proceso ha concluido con el diseño de un dispositivo inalámbrico multi-entorno (MEWiN), con objeto de disminuir el número de elementos necesarios para configurar una red determinada y poder realizar tareas de monitorización mediante un único dispositivo.

Los resultados que se derivan del desarrollo de esta tesis pueden ser aprovechados en nuevos trabajos de investigación aplicada, no sólo en el ámbito de la agricultura de precisión, sino también en contextos más diversos, como la monitorización en entornos marinos, o la vigilancia preventiva de partículas nocivas en ambientes industriales. 


\section{Summary}

Water and food are the main resources that meet the needs of human beings. Agriculture provides most of the food but it also consumes most of the Earth's available fresh water.

In arid and semiarid regions, as Southern Spain, the water supply is an important factor that considerably affects agriculture. Development of innovative irrigation systems that efficiently use water is a high priority. In these regions, farmers must irrigate their crops efficiently; therefore, they must find new solutions and methods that improve the irrigation programming systems, taking into account not only the state of the soil and the plants but also information relating to the climate. All these data must be properly interpreted to decide the most suitable actions to carry out.

Precision Agriculture is a set of techniques that provide a suitable solution to these problems because it aims to optimize field-level management with regard to crop science by matching farming practices more closely to crop needs. Economic advantages are also obtained by boosting competitiveness through more efficient practices (e.g. better management of water usage and costs).

On the other hand, Information and Communication Technologies (ICT's) are used to acquire information about the crop needs. Precision Agriculture techniques use this information to achieve the proposed objectives, in particular, intensive monitoring of crops, data analysis, decision making and application of control actions. 
Sensor Networks is a technology to carry out the intensive monitoring of crops using both wired and wireless nets. Wired solutions have been used since the 90s, whereas Wireless Sensors Networks (WSNs) are more recent (from the year 2000). In Agriculture, WSN's are more suitable because costs are reduced and the final solution is more reliable, since wires can be damaged by farming machinery in the crop. The simple redeployment of the devices provides high network versatility, so it is also another advantage to consider. Furthermore, WSN's are proving to be a promising technology, and many WSN solutions are being successfully designed in several areas.

The interest aroused by WSN technology has prompted the appearance on the market of various hardware platforms (sensor nodes or motes) for the development of new applications (hospitals, unfriendly environments and mobile control applications, among others), and research works (e.g. energy-efficient optimization or target tracking). Worth noting among these platforms are MICAz, TelosB, IRIS, Imote2 and others. It should be noted that these motes, and others not mentioned here, are devices that normally include embedded low-cost sensors whose specifications (precision, resolution, drift, etc.) are not the same as those of the instruments that are normally required in Precision Agriculture applications. For instance, the TelosB that includes the temperature sensor Sensirion SHT11 has no protection level. Moreover the temperature accuracy of this sensor is very dependent on environmental conditions compared to the HMP41 Vaisala sensor, widely used in precision agriculture. Moreover, many of these motes have only been used in laboratory or research applications and are not robust enough for use in real agricultural environments. In fact, many theoretical references about WSN based applications in Precision Agriculture can be found in scientific literature, but very few real deployments had been carried out, due to the scarcity of resources and the high cost of technology in the agricultural sector. Therefore, cost reduction is necessary to guarantee the success of WSN based applications in Precision Agriculture.

Another important aspect of the motes is their capacity for connection to external instruments. There is a large group of external outdoor sensors used in the field of Precision Agriculture. Consequently, if motes are to be used in real agricultural applications, they first of all need to incorporate the necessary electronic interface to connect with external quality instruments. This feature together with robustness, autonomy and the possibility of connecting different types of instruments must be prioritized, as opposed to other functional characteristics less primordial, such as, wireless reprogramming, and the implementation of complex algorithms in the motes.

In this dissertation, an extensive review about commercial hardware solutions and motes prototypes developed by different research centers is described in detail. In addition, the state of the art shows how ICT's, such as Global Positioning Systems (GPS) and 
Geographic Information Systems (GIS), have been frequently used in Precision Agriculture. However WSN's are not yet popular. Therefore, the development of new low cost hardware devices (motes) is necessary because they can measure the state of the crops in real time and provide the essential support to Precision Agriculture. Furthermore, these devices must be reliable in both indoor and outdoor conditions. They must be designed using an open architecture to facilitate the connection between different sensors and actuators required by the farming application.

The main objective of this Thesis is the study and implementation of a hardware architecture based on WSN's and their application in Precision Agriculture, particularly in real-time crops monitoring. Specifically, using Precision Agriculture to get an optimal crops production involves carrying out the following stages: Monitoring crops by acquiring data from sensors, analyzing obtained data, making decisions, applying control actions.

This work only solves the problems related to the first stage. In order to achieve this aim, a general hardware architectures based on WSN's have been developed and validated.

First, it is proposed a general wireless sensor network architecture for monitoring horticultural crops that are distributed among small plots scattered at distances of up to 10 $\mathrm{km}$ from one another. The technology used for the real implementation of the architecture is based on the B-MAC (Berkeley Medium Access Control) medium access protocol to ensure a high degree of sensor node power autonomy. To resolve this issue, a series of specialized sensor nodes (Soil-Mote, Environmental-Mote and Water-Mote) have been developed, using header modules, along with a gateway to interconnect them with the farm central offices. Before starting device development, simulations were conducted to ensure that acceptable performance would be achieved with the selected technology in terms of node autonomy, achieved throughput and delays. To that end, it was necessary to implement the selected B-MAC protocol in the ns-2 (Network Simulator-2) simulation framework. The final system has been deployed on a real crop to check and validate the simulation results against experimental results.

Although the performance of this wireless sensor network is appropriate, having an only sensor node solving a specific problem is not suitable due to several reasons such as time-to-market and cost. Therefore, it is necessary to design a multifunctional sensor node whose hardware and software configuration can be changed. By selecting the right configuration, the device can operate as any of the specialized sensor nodes (Soil-Mote, Environmental-Mote, Water-Mote and Gateway). 
Before implementing the proposed node, a pre-commercial version of the final Soil-Mote (GAIA Soil-Mote) was carried out in order to provide experience about the hardware design of radiofrequency circuits using SMD components, necessary to deal with more complex designs.

The GAIA Soil-Mote is a new wireless sensor node for precision horticulture applications which allows the usage of precision agricultural instruments based on the SDI12 standard. Wireless communication is achieved with a transceiver compliant with the IEEE 802.15.4 standard. The GAIA Soil-Mote software implementation is based on TinyOS. A two-phase methodology was devised to validate the design of this sensor node. The first phase consisted of laboratory validation of the proposed hardware and software solution, including a study on power consumption and autonomy. The second phase consisted of implementing a monitoring application in a real broccoli (Brassica oleracea L. var Marathon) crop in Campo de Cartagena in south-east Spain. In this way the sensor node was validated in real operating conditions. This type of application was chosen because there is a large potential market for it in the farming sector, especially for the development of precision agriculture applications.

The experience achieved after designing the GAIA Soil-Mote has allowed to development and validation of a new sensor node commercial prototype. This new mote is a robust multifunctional device (called GAIA 2) that requires only a certification process. This is the main advantage obtained. It has been designed, implemented and tested in real farming applications. As mentioned before, this sensor node has been developed as a commercial product whose configuration can be changed using both hardware and software. GAIA 2 PCB consists of SMD components almost completely, even the short range radio module CC2420. Only the long range XStream radio modem is a commercial device. The mote provides several I/O interfaces both analogical and digital; therefore, it can be used with most of the sensors and actuators used in Precision Agriculture. Specifically, the mote has $0-2,5 \mathrm{~V}$ analogical inputs and 4-20mA, digital inputs/outputs, 0 $2,5 \mathrm{~V}$ analogical outputs and $\mathrm{I}^{2} \mathrm{C}$ and SDI-12 digital interfaces. The GAIA 2 board is protected by watertight casing designed ad-hoc. The design is expensive and therefore a disadvantage. Furthermore, the radio module XStream is also expensive, and this too, is a disadvantage.

In order to solve these drawbacks, a Multi-Environment Wireless sensor Node (called MEWiN) has also been designed, implemented and validated as a commercial product. This node is capable of carrying out monitoring and control tasks both in Precision Agriculture and other fields. MEWiN has been developed to reduce costs (due to the casing design and the use of the radio modem XStream), and to provide a temporary data storage system, very useful when the wireless communication fails. The mote can be 
used in a specific environment by designing a specific interface board. The necessary instrumentation can be connected to the sensor node MEWiN using this board. In particular, the main board MEWiN provides the following analogical and digital interfaces: 8/2 analogical inputs/outputs, 17 digital inputs/outputs and digital interfaces (SPI, 2 UARTs and $\mathrm{I}^{2} \mathrm{C}$ ). The interface boards SoilBoard and LVDTBoard have been developed as commercial products. They are used to connect soil sensors as Hydra Probe II and radial dendrometers to the main board.

In order to validate all the designed motes, several experiments in Precision Agriculture have been carried out. Specifically, the testing of the systems was done in two phases: the first in the laboratory, to validate the functional requirements of the developed devices, the networking solution and the motes' power management; the second on the farm, to assess the functional performance of the devices, such as range, robustness and flexibility. The result has been low cost, highly reliable and simple infrastructures for the collection of agronomical data over distributed areas in horticultural environments.

Specifically, the sensor nodes (Soil-Mote, Environmental-Mote and Water-Mote) based on header modules were successfully tested on a crop of ecological cabbage (Brassica oleracea).

The GAIA Soil-mote has been tested both in the laboratory and in a farm under real conditions. In particular, the laboratory tests were followed by the second phase of the experiment, consisting of another deployment. Two sensor networks were deployed in two plots of approximately 4 ha each. The plots were about 5.2 and $8.7 \mathrm{~km}$ respectively from the farm offices. Ten GAIA Soil-Motes, one Environmental-Mote and one Gateway were deployed on each of the plots. The Gateway was connected to an exterior $3 \mathrm{dBi}$ omnidirectional antenna placed on a post $5 \mathrm{~m}$ tall. This was to ensure direct line-of-sight between the antennas of the ground-level Motes and the Gateway. And again, to ensure line-of-sight between the Gateway and the offices, one $15 \mathrm{dBi}$ omnidirectional antenna was placed on the office roof ( $9 \mathrm{~m}$ high), and another identical antenna on the Gateway. Wireless communication between the rooftop antenna and the Base Station was achieved with a repeater. At the same time, a technology similar to that of the Gateway was used to develop a wireless node (Water-Mote) to monitor the quality of the water supplied to the crop. A monitoring application, running on the computer connected to the Base Station, was developed to control all the devices and keep a record of the information received. The trials were conducted on crops of Broccoli (Brassica oleracea L. var Marathon) covering an area of 4 ha each, located in Campo de Cartagena in south-east Spain.

The GAIA 2 also has been tested both in the laboratory and in a farm under real conditions. In particular, during the laboratory tests all output and input interfaces, short 
range communications and long range communications were validated. Also a real study was carried out in order to validate the correct autonomy of the device. After laboratory tests, a sensor network was deployed in a small crop of melons located at "Estación Experimental Agroalimentaria "Tomás Ferro", belonging to the Technical University of Cartagena. Eight GAIA 2 configured as Soil-Motes and one GAIA 2 configured as Gateway were deployed on the plot. Each GAIA 2 configured as Soil-Mote had connected two hydra probe II sensors. Three $3 \mathrm{dBi}$ omnidirectional antennas were used in the ground-level Motes and the Gateway to ensure direct line-of-sight. And again, to ensure line-of-sight between the Gateway and the offices (Base-Station), one $15 \mathrm{dBi}$ omnidirectional antenna was placed on the office roof $(8 \mathrm{~m}$ high), and $8 \mathrm{dBi}$ omnidirectional antenna on the Gateway. A monitoring application, running on the computer connected to the Base Station, was developed in LabView to control all the devices and keep a record of the information received.

Finally, the MEWiN sensor node has been validated in three experimental crops. One in an experimental crop of almond trees located at "Estación Experimental Agroalimentaria 'Tomás Ferro"'. The second deployment has been undertaken in a crop of olive trees in "Finca Experimental 'La Hampa" located at Coria del Río, Seville. The third deployment has been carried out in a grapes crop placed in Cieza, Murcia. Additionally, the node is also being validated in other kinds of applications such as oceanographic applications.

A general architecture based on wireless sensor networks has been proposed as one of the main contributions of this Thesis, derived from a previous study. The architecture allows us to carry out woody and horticultural crops monitoring and to perform the first stage needed to achieve the optimization of crop irrigation. Based on this architecture, different sensor nodes prototypes have been developed. These devices have been improved in order to reach a better performance and robustness. All the hardware solutions included in this architecture have been validated in real agricultural case studies. Finally, a multienvironmental device (MEWiN) has been developed in order to monitor agricultural crops using only one device. Moreover, MEWiN provides a temporary data storage system, very useful when the wireless communication fails.

New research lines will be considered after finishing this Thesis. Specifically, the proposed architecture will be improved in order to achieve new aims such as the irrigation control of the crop and the monitoring of other environments (oceanographic applications or environmental pollution in cities, among others). On the other hand, control algorithms based on Artificial Intelligence could be developed in order to increase the crop yield, in particular, when low reliable and low cost sensors are used. 


\section{Índice de Contenido}

$\begin{array}{lr}\text { Capítulo } 1 & 1\end{array}$

Introducción y Estructura de la Tesis. $\quad 1$

$\begin{array}{ll}1.1 \text { Motivación. } & 2\end{array}$

1.2 Marco de desarrollo de la Tesis. 4

$\begin{array}{ll}1.3 \text { Objetivos. } & 5\end{array}$

$\begin{array}{llr}1.4 & \text { Estructura de la Tesis. } & 6\end{array}$

Capítulo 2

El Papel de las Tecnologías de la Información y las Comunicaciones

$\begin{array}{ll}2.1 \text { Introducción. } & 12\end{array}$

$\begin{array}{ll}2.2 \text { Los sistemas agrícolas y la agricultura. } & 12\end{array}$

$\begin{array}{ll}2.3 \text { La toma de decisiones en la agricultura. } & 14\end{array}$

2.4 La variabilidad y la zona homogénea. 16

$\begin{array}{ll}2.4 .1 \text { La variabilidad espacial. } & 16\end{array}$

$\begin{array}{ll}\text { 2.4.2 La variabilidad temporal. } & 17\end{array}$

$\begin{array}{ll}\text { 2.4.3 La Zona Homogénea. } & 17\end{array}$

$\begin{array}{lll}2.5 & \text { La agricultura de precisión. } & 18\end{array}$

2.6 Tecnologías de la información y las comunicaciones en la agricultura de precisión. 23

2.7 Los sistemas de posicionamiento global y de navegación por satélites. 25

$\begin{array}{ll}\text { 2.7.1 Las correcciones diferenciales. } & 27\end{array}$

2.7.2 Aplicaciones del GPS en la Agricultura. 29

2.8 Los sistemas de información geográfica. 30

2.9 La Teledetección. $\quad 31$

2.10 Las redes de sensores. 33

2.11 Conclusiones.

$\begin{array}{lr}\text { Capítulo } 3 & 37\end{array}$

Las Redes de Sensores Inalámbricas. $\quad 37$

$\begin{array}{llr}3.1 & \text { Introducción. } & 38\end{array}$ 
3.2 Las redes de sensores inalámbricas.

3.3 Características de una red de sensores. $\quad 42$

3.4 Topologías de red y protocolos de comunicación. 46

3.4.1 El estándar IEEE 802.15.4. 46

$\begin{array}{ll}3.4 .2 \text { ZigBee. } & 50\end{array}$

3.4.3 B-MAC. 53

3.4.4 Otros estándares. 55

3.5 Estado de la técnica de las soluciones hardware. 57

3.5.1 Motes.

3.5.2 Soluciones comerciales.

3.5.2.1 Soluciones de propósito general. $\quad 59$

$\begin{array}{ll}\text { 3.5.2.2 Soluciones agrícolas. } & 61\end{array}$

3.5.2.3 El panorama nacional. $\quad 65$

$\begin{array}{lll}\text { 3.5.3 Prototipos desarrollados en centros de investigación. } & 67\end{array}$

3.6 Conclusiones. 75

$\begin{array}{ll}\text { Capítulo } 4 & 77\end{array}$

Diseño y Validación de una Arquitectura Basada en WSN para Cultivos Hortícolas.

$\begin{array}{lll}4.1 & \text { Introducción. } & 78\end{array}$

$\begin{array}{lll}4.2 & \text { Captura de especificaciones. } & 78\end{array}$

$\begin{array}{lll}\text { 4.2.1 Topología de la red. } & 78\end{array}$

4.2.2 Protocolo de comunicaciones. 80

4.2.3 Selección de sensores agronómicos. 81

4.2.3.1Sensores ambientales. $\quad 82$

4.2.3.2Sensores de suelo. $\quad 83$

4.2.3.3Sensores para monitorizar el estado hídrico de las plantas. $\quad 84$

4.2.4 Interfaces de entrada salida necesarias para los motes. 84

4.2.5 Principales componentes hardware de los motes desarrollados. 86

4.2.5.1 Transceptores de radio. 86

4.2.5.2 Antenas. $\quad 87$

4.2.5.3 Micro-controladores.

4.2.5.4 Sistemas de alimentación. $\quad 88$

4.3 Arquitectura de la red de sensores propuesta. $\quad 88$

4.4 Diseño e implementación de la red de sensores propuesta. 90

$\begin{array}{ll}\text { 4.4.1 Fase de simulación para el diseño de la red de sensores. } & 90\end{array}$

4.4.2 Diseño e implementación de los motes. 95

4.4.2.1 Soil-Mote. 96

$\begin{array}{ll}\text { 4.4.2.2 Environmental-Mote. } & 100\end{array}$

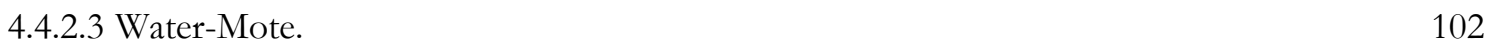

4.4.2.4 Gateway, Base-Station y Repeater. 104

4.4.3 Software de monitorización. 105

$\begin{array}{lll}4.5 & \text { Fase de validación. } & 106\end{array}$

$\begin{array}{ll}\text { 4.6 Resultados y discusión. } & 107\end{array}$

$\begin{array}{ll}\text { 4.6.1 Consumo de energía y autonomía. } & 107\end{array}$

$\begin{array}{ll}\text { 4.6.2 Throughput. } & 111\end{array}$

4.6.3 Delays. 111

4.6.4 Resultados experimentales en el cultivo. 112

$\begin{array}{lll}4.7 & \text { Conclusiones. } & 114\end{array}$ 
Capítulo 5

GAIA Soil-Mote: un Nodo Sensor para la Monitorización de Parámetros del Suelo Basado en el Estándar SDI-12.

5.1 Introducción.

5.2 Descripción del GAIA Soil-Mote. 120

5.2.1 Descripción del hardware. 121

$\begin{array}{ll}\text { 5.2.2 Descripción del software. } & 126\end{array}$

$\begin{array}{llr}5.3 & \text { Resultados experimentales. } & 126\end{array}$

$\begin{array}{ll}\text { 5.3.1 Experimentos de laboratorio. } & 127\end{array}$

$\begin{array}{llr}5.3 .2 & \text { Resultados agrícolas. } & 129\end{array}$

$\begin{array}{ll}5.4 \text { Conclusiones. } & 131\end{array}$

Capítulo 6

GAIA 2: un Nodo Sensor Multifuncional para Agricultura de Precisión. 133

$\begin{array}{ll}6.1 & 134\end{array}$

6.2 Descripción del GAIA 2. 135

6.2.1 Descripción del Hardware. 136

$\begin{array}{ll}\text { 6.2.2 Descripción del software. } & 142\end{array}$

$\begin{array}{ll}6.3 & \text { Resultados experimentales. } \\ 6.3 .145\end{array}$

6.3.1 Experimentos de laboratorio. 145

$\begin{array}{lll}\text { 6.3.2 Resultados agrícolas. } & 147\end{array}$

$\begin{array}{lr}6.4 \text { Conclusiones. } & 152\end{array}$

Capítulo $7 \quad 153$

MEWiN: Un Nodo Sensor Inalámbrico Multi-Entorno con una Arquitectura Abierta. 153

$\begin{array}{lll}7.1 & \text { Introducción. } & 154\end{array}$

$\begin{array}{llr}7.2 & \text { Descripción del sistema MEWiN. } & 155\end{array}$

\begin{tabular}{ll}
7.2 .1 & Requisitos funcionales. \\
\hline 7.2 .2 & 155
\end{tabular}

$\begin{array}{ll}\text { 7.2.2 Descripción del Hardware. } & 158\end{array}$

$\begin{array}{ll}\text { 7.2.2.1 MEWiN Mainboard. } & 158\end{array}$

$\begin{array}{lr}\text { 7.2.2.2 MEWiN Vegeboard. } & 161\end{array}$

7.2.2.3 MEWiN Routerboard. 163

7.2.2.4 Placas de Interfaz Comerciales MEWiN. 164

7.2.2.5 Carcasa, Antena y Batería. $\quad 164$

$\begin{array}{ll}\text { 7.2.3 Descripción del Software. } & 165\end{array}$

$\begin{array}{lll}7.3 & \text { Resultados experimentales. } & 167\end{array}$

$\begin{array}{ll}\text { 7.3.1 Experimentos de laboratorio. } & 167\end{array}$

$\begin{array}{llr}\text { 7.3.2 Resultados agrícolas. } & 169\end{array}$

$\begin{array}{llr}7.4 & \text { Conclusiones. } & 174\end{array}$

$\begin{array}{lr}\text { Chapter } 8 & 175\end{array}$

$\begin{array}{lr}\text { Conclusions and Future Work. } & 175\end{array}$

$\begin{array}{lll}8.1 \text { Conclusions. } & 176\end{array}$

$\begin{array}{ll}8.2 \text { Contributions. } & 179\end{array}$

8.3 Dissemination of the results. 181

$\begin{array}{llr}8.4 & \text { Future work. } & 182\end{array}$

$\begin{array}{lr}\text { Anexo I } & 185\end{array}$

Materiales, Herramientas y Métodos. 185 
I.1 Introducción.

I.2 Instrumentación agronómica.

I.2.1 Sensores medioambientales.

I.2.2 Sensores de suelo.

I.2.3 Sensores para monitorizar el estado hídrico de las plantas.

I.3 Principales componentes hardware de los motes.

I.3.1 Transceptor de radio.

I.3.2 Antenas.

I.3.3 Micro-controladores.

I.3.4 Baterías y módulos de alimentación seleccionados.

I.4 Herramientas.

208

I.4.1 Herramientas software.

I.4.1.1 Network Simulator 2.

I.4.1.2 TinyOS.

I.4.1.3 Bibliotecas software.

I.4.1.4 IAR Embedded Workbench para MSP430.

I.4.2 Herramientas hardware.

$\begin{array}{ll}\text { I.4.3 Herramientas para el despliegue. } & 220\end{array}$

$\begin{array}{lll}\text { I.5 Métodos. } & 221\end{array}$

$\begin{array}{ll}\text { I.5.1 Desarrollo de hardware para redes de sensores. } & 221\end{array}$

$\begin{array}{ll}\text { I.5.2 Desarrollo de software para redes de sensores. } & 223\end{array}$

\section{Anexo II}

Tramas de Datos Intercambiadas entre los Nodos Sensores Diseñados e Implementados durante la Fase de Validación de los Dispositivos.
II.1 Introducción.

II.2 Tramas de los nodos sensores basados en módulos de inserción.

II.3 Tramas utilizadas para validar el nodo sensor multi-entorno MEWiN.

\section{Anexo III}

Descripción del Software desarrollado para el GAIA Soil-Mote.

III.2 Descripción del software.

\section{Anexo IV}

Descripción del Software Desarrollado para un Nodo Sensor MEWiN con el Rol de ZigBee Router.

IV.2 Descripción del software. 


\section{Índice de Figuras}

Capítulo 2

Figura 2.1. Evolución del número de publicaciones obtenido mediante búsqueda con el término "precision agriculture" en el SciencieDirect. ............................................................................20

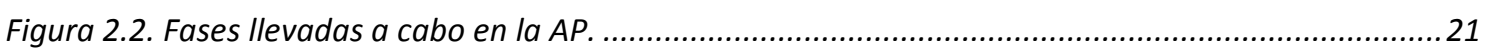

Figura 2.3. (a) Satélite NAVSTAR. (b) Constelación de los satélites del sistema GPS................................26

Figura 2.4. Determinación de la posición del receptor. ..........................................................................26

Figura 2.5. Corrección diferencial realizada con la ayuda de un satélite geoestacionario..........................28

Figura 2.6. Vehículos en los que se pueden instalar sensores para teledetección....................................32

Figura 2.7. (a) Funcionamiento de un sensor de tipo pasivo. (b) Teledetección por radar.........................32

Figura 2.8. Evolución del número de publicaciones obtenido mediante búsqueda con el

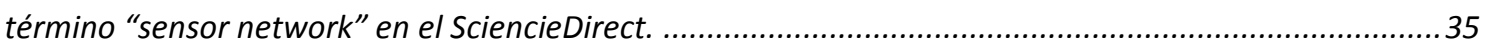

Figura 2.9. Evolución del número de publicaciones obtenido mediante búsqueda con el término "wireless sensor network" en el SciencieDirect...............................................................................35

Figura 2.10. Evolución del número de publicaciones obtenido mediante búsqueda cruzada de los términos "wireless sensor network" y "Precision Agriculture" en el SciencieDirect.

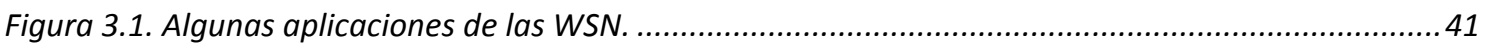

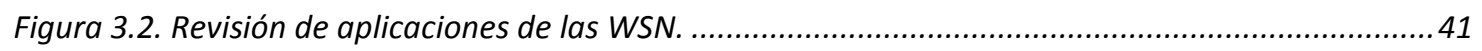

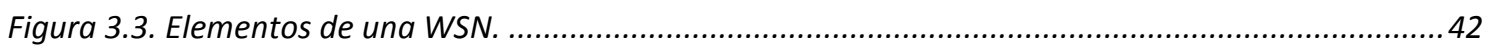

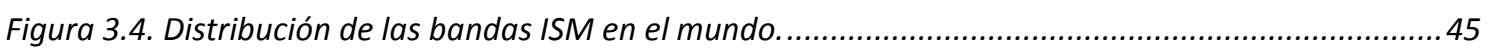

Figura 3.5. Modelo de referencia de la PHY [IEEE, 2006] ......................................................................47

Figura 3.6. Modelo de referencia de la subcapa MAC [IEEE, 2006] ............................................................48

Figura 3.7. Ejemplos de topologías en estrella y entre iguales (peer-to-peer) [IEEE, 2006]......................49

Figura 3.8. Arquitectura de capas funcional y la pila del protocolo ZigBee [ZigBee, 2007].......................51

Figura 3.9. Arquitectura de referencia de la capa de red [ZigBee, 2007] ...............................................51

Figura 3.10. Arquitectura de referencia de la capa de la subcapa APS [ZigBee, 2007]. ...........................52

Figura 3.11. Topologías de las redes ZigBee [Baronti, 2007] ............................................................5

Figura 3.12. Comunicación de dos estaciones mediante B-MAC........................................................5

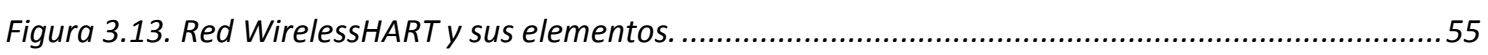


Figura 3.14. Red Wibree de sensores de deporte. ........................................................................5

Figura 3.15. Componentes de un mote...............................................................................................5

Figura 3.16. Familia de motes MICA. (a) MICA2. (b) MICA2dot. .............................................................59

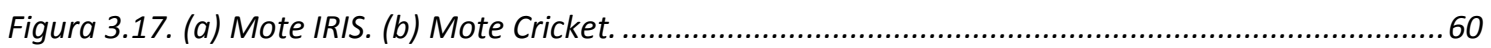

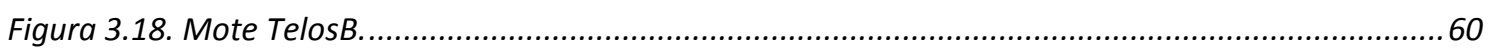

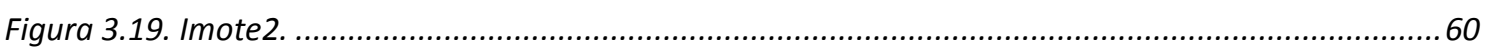

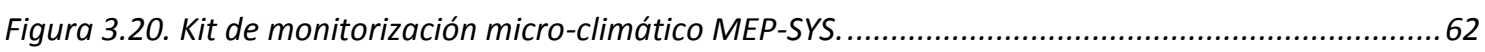

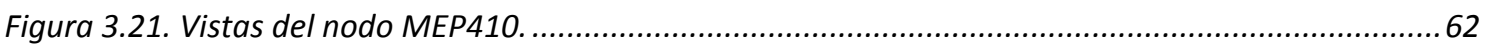

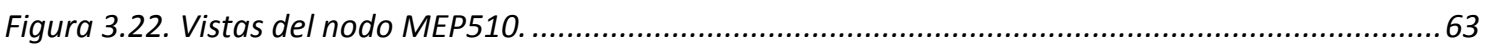

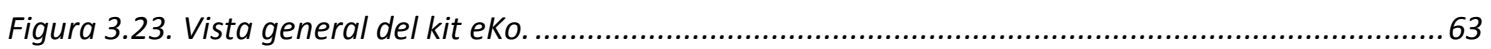

Figura 3.24. Sensores comercializados para el kit eko. .........................................................................64

Figura 3.25. Vista de la estación meteorológica conectada a un nodo del kit eKo. ..................................64

Figura 3.26. Vista del Waspmote y de la placa de sensores para agricultura de precisión.........................65

Figura 3.27. Nodo RFIX BAT-0901 comercializado por Balmart. ................................................................66

Figura 3.28. Vista y diagrama de bloques del MPWiNodeZ [Morais, 2008]............................................68

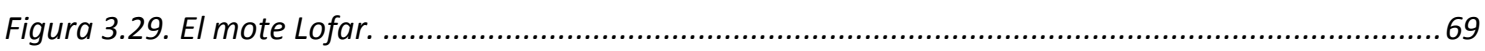

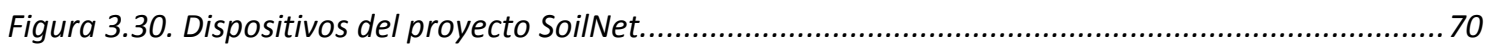

Figura 3.31. Arquitectura de la red de Anurag et. al. ....................................................................... 71

Figura 3.32. Vista de una SU y situación del despliegue realizado en el viñedo [Matese, 2009]. .............. 72

Figura 3.33. Fotografías de los prototipos primero y segundo de Vellidis et. al..................................... 73

Figura 3.34. Diversas vistas de la WSN implementada por Cano et. al. ................................................... 74

$\begin{array}{ll}\text { Capítulo } 4 & 77\end{array}$

Figura 4.1. Arquitectura propuesta para monitorizar cultivos hortícolas. ..............................................89

Figura 4.2. Comparativa de la evolución de la descarga de la batería entre B-MAC tradicional y B-MAC propuesto para los Soil-Motes (a) Check interval menor e igual a 1s. (b) Check interval igual a 5s. (c) Check interval mayor e igual a 10s................................................................................................93

Figura 4.3. Fotografía del Soil-Mote y diagrama de bloques de la arquitectura hardware. ......................96

Figura 4.4. Situación de los sensores del Soil-Mote...............................................................................97

Figura 4.5. Vista estática del diagrama de componentes nesC del Soil-Mote.........................................98

Figura 4.6. Fotografía del Soil-Mote desplegado en un cultivo de coles. ....................................................99

Figura 4.7. Fotografía del Environmental-Mote y diagrama de bloques de la arquitectura hardware. ...100

Figura 4.8. Vista del sensor utilizado en el Environmental-Mote y localización del mismo. ....................101

Figura 4.9. Vista estática del diagrama de componentes nesC del Environmental-Mote........................101

Figura 4.10. Fotografía del Environmental-Mote desplegado en la explotación agrícola Langmead...... 102

Figura 4.11. Vista del Water-Mote y diagrama de bloques de la arquitectura hardware. ......................102

Figura 4.12. Esquema del Water-Mote y vista del sensor EC250. .......................................................... 103

Figura 4.13. Vista estática del diagrama de componentes nesC del Water-Mote. ...................................104

Figura 4.14. Diferentes vistas del Gateway. ..................................................................................... 105

Figura 4.15. Vistas del software de monitorización. ............................................................................... 106

Figura 4.16. Consumos promedio de los diferentes dispositivos obtenidos con ns-2: (a) Gateway. (b) Soil-

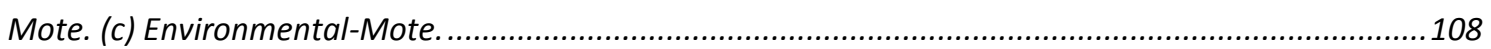

Figura 4.17. Consumo real del Soil-Mote en diferentes estados. ...........................................................109

Figura 4.18. Evolución del voltaje de la batería durante un estudio real. .................................................110

Figura 4.19. Tasa binaria de transmisión/recepción o throughput. (a) Subred Soil-Motes. (b) Subred

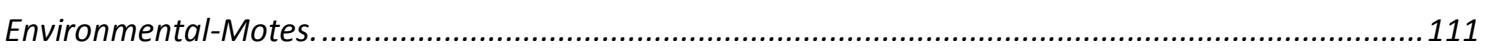

Figura 4.20. Delays obtenidos con el simulador y experimentalmente en la sub-red de los Soil-Motes. (a)

Delays del Gateway obtenidos con ns-2. (b) Delays del Soil-Mote obtenidos con ns-2. (c) Delays experimentales del Soil-Mote. 
Figura 4.21. Despliegue de los nodos sensores realizado en la explotación agrícola Langmead.............113

Figura 4.22. Humedad del suelo y temperatura del aire, monitorizada durante 10 semanas. ................114

$\begin{array}{lr}\text { Capítulo } 5 & 117\end{array}$

Figura 5.1. Vistas del GAIA Soil-Mote. (a) PCB. (b) Diagrama de bloques...............................................121

Figura 5.2. Layouts de las capas top y botton del diseño de referencia del módulo de inserción CC2420EM

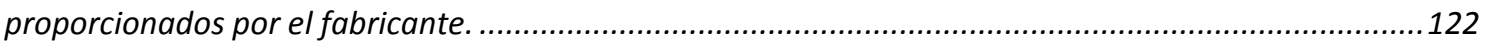

Figura 5.3. Esquemático de la interfaz SDI-12 ............................................................................ 123

Figura 5.4. Esquemático del cargador de la batería de LiPo. ..........................................................123

Figura 5.5. Esquemático del regulador de tensión de 2,5V .................................................................123

Figura 5.6. Esquemático del convertidor DC/DC que proporciona $12 \mathrm{~V}$ a los sensores. ............................124

Figura 5.7. Modelo 3D de la carcasa diseñada para el GSM. .................................................................. 124

Figura 5.8. Piezas fabricadas en resina semitransparente usando una impresora 3D........................... 125

Figura 5.9. Vistas del GAIA Soil-Mote. (a) Ubicación del dispositivo en el cultivo. (b) Esquema de montaje

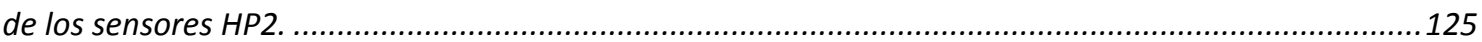

Figura 5.10. Vista estática de componentes nesC del GSM............................................................126

Figura 5.11. Arquitectura del sistema para llevar a cabo los experimentos de laboratorio.....................127

Figura 5.12. Diferentes estados de consumo del GSM: 'standby' $=0,25 \mathrm{~mA}$, 'communication module

wake-up' = 20mA, 'acquisiton' = 110mA y 'communication module transmitting' $=25 \mathrm{~mA}$...................128

Figura 5.13. Tensión de la batería del GSM durante el estudio realizado en el laboratorio.....................128

Figura 5.14. Descripción de la red desplegada para validar el GSM en un cultivo real. ..........................129

Figura 5.15. Porcentaje volumétrico del suelo monitorizado durante 12 semanas por un GSM. ............130

Capítulo $6 \quad 133$

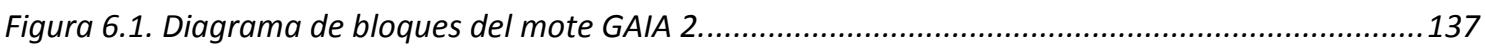

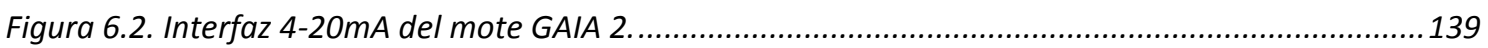

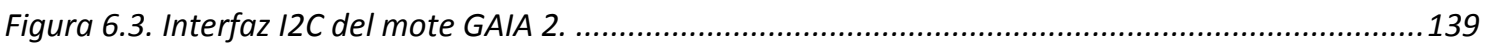

Figura 6.4. Interfaz entre el micro-controlador y el radio módem XStream..........................................139

Figura 6.5. PCB con componentes SMD y de 4 capas diseñada para el GAIA 2 .....................................140

Figura 6.6. Modelo 3D de la carcasa diseñada para el GAIA 2 con el software CAD SolidWorks.............140

Figura 6.7. Diferentes vistas de la carcasa fabricada para el GAIA 2. .................................................. 141

Figura 6.8. Fotografía de la segunda revisión de carcasa, fabricada con la PCB, ya instalada............... 141

Figura 6.9. Diagrama de componentes software para la funcionalidad HDM. .......................................142

Figura 6.10. Diagrama de componentes software para la funcionalidad GMM....................................144

Figura 6.11. Red desplegada en el laboratorio para validar el correcto funcionamiento del GAIA 2.......145

Figura 6.12. Diferentes estados de consumo del GAIA configurado como Environmental-Mote: 'standby'

$=0,03 \mathrm{~mA}$, 'receiving' $=22 \mathrm{~mA}$, 'acquisiton' $=0,5 \mathrm{~mA}$ y 'communication module transmitting' $=23 \mathrm{~mA} . .146$

Figura 6.13. Tensión de la batería del GSM durante el estudio realizado en el laboratorio.....................147

Figura 6.14. Despliegue realizado para validar el funcionamiento del GAIA 2 en un cultivo real. ...........147

Figura 6.15. Vista del Gateway que se instaló en la parcela. ...............................................................148

Figura 6.16. Fotografía del proceso de instalación de un nodo sensor. ...............................................148

Figura 6.17. Software de monitorización desarrollado con LabView. ...................................................149

Figura 6.18. Temperatura del suelo monitorizada, con uno de los sensores, durante 11 días.................150

Figura 6.19. Porcentaje volumétrico de humedad en el suelo obtenido de uno de los sensores..............150

Figura 6.20. Evolución de la tensión de la batería en el Gateway, registrada durante 11 días.................151

Figura 6.21. Evolución de la tensión de la batería registrada en un nodo sensor durante 25 días. ..........151

$\begin{array}{ll}\text { Capítulo } 7 & 153\end{array}$

Figura 7.1. Diagrama de bloques de la placa MEWiN Mainboard. .......................................................159 
Figura 7.2. Esquemático entre la batería y una ADC del micro-controlador. ........................................160

Figura 7.3. Esquemático de la interfaz SPI entre la tarjeta SD y el micro-controlador.............................160

Figura 7.4. Esquemático de la interfaz I2C entre el RTC y el micro-controlador...................................... 161

Figura 7.5. PCB de la placa base MEWiN Mainboard......................................................................... 161

Figura 7.6. Placa de interfaz MEWiN Vegeboard: (a) PCB. (b) Diagrama de bloques. ..............................162

Figura 7.7. Esquemático del convertidor DC/DC de la placa de interfaz Vegeboard................................162

Figura 7.8. Placa de interfaz MEWiN Routerboard: (a) Esquemático. (b) PCB.....................................163

Figura 7.9. Placas de interfaz MEWiN: (a) SoilBoard. (b) LVDTBoard. ..................................................164

Figura 7.10. Vista de la carcasa del nodo sensor MEWiN instalada en campo.....................................165

Figura 7.11. Diagrama simplificado del software de un nodo MEWiN con el rol de ZED.........................166

Figura 7.12. Red desplegada en el laboratorio para validar el correcto funcionamiento de la plataforma

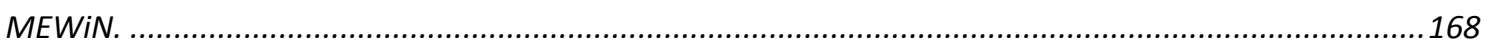

Figura 7.13. Despliegue para validar el funcionamiento de nodos MEWiN en un cultivo real.................170

Figura 7.14. Evolución del voltaje de la batería de un ZED durante 45 días.............................................170

Figura 7.15. Evolución del voltaje del panel solar y de la batería del ZR durante 7 días........................ 171

Figura 7.16. ZC instalado en el interior de la caseta próxima a la parcela de olivos.............................. 171

Figura 7.17. Uno de los dos ZED desplegados en la parcela de olivos....................................................172

Figura 7.18. Interfaz gráfica utilizada para validar el sistema MEWiN en cultivos reales. ......................172

Figura 7.19. Registro semanal de dos dendrómetros perimetrales de un nodo sensor...........................173

Figura 7.20. Vista de un nodo sensor desplegado en el parral de la explotación agrícola.......................174

$\begin{array}{lr}\text { Anexo I } & 185\end{array}$

Figura I.1. WSN que ha servido como caso de estudio. ................................................................. 186

Figura I.2. Estación climática de la ESEA y distribución de los distintos sensores. ....................................187

Figura I.3. Sensor de temperatura y humedad relativa del ambiente HMP45C de Vaisala...................... 188

Figura I.4. Piranómetro modelo CMP3 de Kipp \& Zonen instalado en la estación. .................................... 188

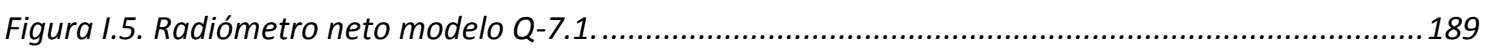

Figura I.6. Sensor FSS500 para registrar la pluviometría. ............................................................... 189

Figura I.7. Sensores de velocidad y dirección del viento..................................................................190

Figura I.8. Psicrómetro H301 de Vector Instruments.....................................................................190

Figura 1.9. Tensiómetro Jet Fill 2725ARL del Soilmoisture. ................................................................192

Figura I.10. Sensor digital Watermark junto con el medidor digital portátil..........................................192

Figura I.11. Diferentes vistas del equipo EnviroSCAN..................................................................... 193

Figura I.12. Sensor Hydra Probe II. ....................................................................................................... 194

Figura I.13. Vista del sensor IRTS-P y su instalación sobre un almendro en mástil. .................................195

Figura I.14. (a) Dendrómetro y portasensor instalados en tronco. (b) Representación gráfica de la

máxima contracción diaria (MCD) y tasa de crecimiento diaria de tronco (TCD) a partir de la variación del diámetro de tronco (VDT) en almendros. ........................................................................................ 196

Figura I.15. Comparativa de antenas direccionales, omnidireccionales y sectoriales.............................200

Figura I.16. Representación de la zona Fresnel con el transmisor y el receptor como focos....................201

Figura I.17. Ejemplo práctico de la zonas de Fresnel. ........................................................................202

Figura I.18. Antenas seleccionadas para los módulos de radio........................................................204

Figura I.19. Baterías seleccionadas para los nodos sensores objeto de estudio.....................................206

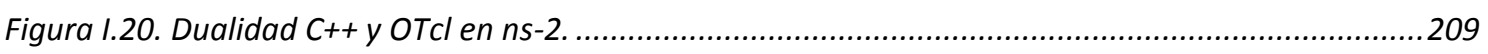

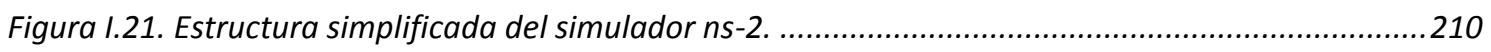

Figura I.22. Interfaz gráfica del simulador ns-2...................................................................................211

Figura I.23. Salida de Cygwin durante la compilación y descarga de una aplicación................................211

Figura I.24. Descripción gráfica de la aplicación BlinkAppC. .................................................................213

Figura I.25. Entorno de compilación y depuración IAR Embedded Workbench para MSP430. .................216 
Figura I.26. Vistas y detalle de componentes del mote TelosB

Figura I.27. Placa de desarrollo MSP430-P1611 y programador por interfaz JTAG MSP430-JTAG.........218

Figura I.28. Módulo de inserción MSP430-H1611 y kit de módulos de inserción CC2420EMK................218

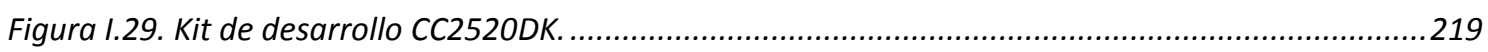

Figura I.30. Módulo de inserción CC2520-CC2591EM. ............................................................................219

Figura I.31. Adaptador de red 2400E y software SNA de Daintree Networks. ......................................221

Figura I.32. Selección de elementos para implementar un nodo sensor. .............................................223

$\begin{array}{ll}\text { Anexo III } & 237\end{array}$

Figura III.1. Vista estática de componentes nesC del GSM..............................................................238

Figura III.2. Pasos para realizar la lectura de un sensor HP2 .........................................................243

$\begin{array}{lr}\text { Anexo IV } & 251\end{array}$

Figura IV.1. Diagrama simplificado del software diseñado para el nodo sensor con el rol de ZR. ...........253 
CONTRIBUCIÓN A LAS REDES DE SENSORES INALÁMBRICAS. ESTUDIO E IMPLEMENTACIÓN DE SOluCiones Hardware Para Agricultura de PreCisión. 


\section{Índice de Tablas}

Capítulo 2

Tabla 2.1. Principales diferencias entre los ecosistemas naturales y los agrícolas.

$\begin{array}{lr}\text { Capítulo } 3 & 37\end{array}$

Tabla 3.1. Características principales de diferentes motes. ................................................................61

Tabla 3.2. Características principales de sistemas de monitorización comerciales....................................67

$\begin{array}{ll}\text { Capítulo } 4 & 77\end{array}$

Tabla 4.1. Características principales de los sensores ambientales seleccionados. ..................................83

Tabla 4.2. Características principales de los sensores de suelo seleccionados.........................................83

Tabla 4.3. Características principales de los sensores IRTS-P y DFg2.5. ...................................................84

Tabla 4.4. Resumen de las interfaces de las que debe estar dotado el mote a diseñar. ...........................84

Tabla 4.5. Diferentes estados de cada uno de los componentes principales de los motes y consumo asociado a cada uno de ellos.............................................................................................................91

Tabla 4.6. Descripción de las tramas intercambiadas entre el Gateway y los nodos sensores. .................94

Tabla 4.7. Consumo y autonomía de los diferentes dispositivos (i, intensidad; $t$, tiempo).......................109

Anexo I

Tabla I.1. Características principales de los sensores de la estación climática de la ESEA......................191

Tabla I.2. Características principales de los sensores de suelo de la ESEA. ...........................................193

Tabla I.3. Variables medidas por el sensor Hydra Probe II. .................................................................194

Tabla I.4. Características principales de los sensores IRTS-P y DFg2.5 ..............................................196

Tabla I.5. Características principales del módulo de radio CC2420 de Texas Instruments. ......................198

Tabla I.6. Características principales del módulo de radio CC2520 de Texas Instruments. ......................199

Tabla I.7. Características principales del micro-controlador MSP430F1611 de Texas Instruments. ........205

Tabla I.8. Características principales del micro-controlador MSP430F2618 de Texas Instruments. .........205 
Tabla II.1. Descripción de la trama enviada con la lectura de los sensores HP2 desde los Soil-Motes al Gateway.

Tabla II.2. Descripción de la trama enviada con la lectura del sensor SHT71 desde los Environmental-

Motes al Gateway.

Tabla II.3. Descripción de la trama enviada con la lectura del voltaje de la batería desde los

Environmental-Motes y los Soil-Motes al Gateway.

Tabla II.4. Descripción de la trama enviada desde el Gateway a los Soil-Motes y a los EnvironmentalMotes para configurar la frecuencia de lectura de los sensores.

Tabla II.5. Descripción de la trama enviada desde el Gateway al software de monitorización cuando el Gateway recibe una trama de datos de un Soil-Mote.

Tabla II.6. Descripción de la trama enviada desde el Gateway al software de monitorización cuando el Gateway recibe una trama de datos de un Environmental-Mote.

Tabla II.7. Descripción de la trama de datos del sensor EC250 enviada desde el Water-Mote al software de monitorización.

Tabla II.8. Descripción de la trama enviada desde el software de monitorización a un Gateway o al Water-Mote para modificar la frecuencia de lectura de los sensores.

Tabla II.9. Descripción de la trama enviada de la lectura de un sensor HP2 desde un ZED al ZC............233

Tabla II.10. Descripción de la trama enviada con la lectura del voltaje del panel solar del ZR al ZC. ......233 Tabla II.11. Descripción de la trama enviada desde el ZC a un nodo sensor (ZED ó ZR) para modificar la frecuencia de lectura de los sensores.

Tabla II.12. Trama que se envía a la interfaz gráfica de usuario al recibir una trama de datos de un ZED en el ZC.

Tabla II.13. Trama que se envía a la interfaz gráfica de usuario al recibir una trama de batería de un ZED o del ZR en el ZC.

Tabla II.14. Trama que se envía desde la interfaz gráfica de usuario para modificar la frecuencia de lectura de un nodo sensor de la red. 


\section{Índice de Listados}

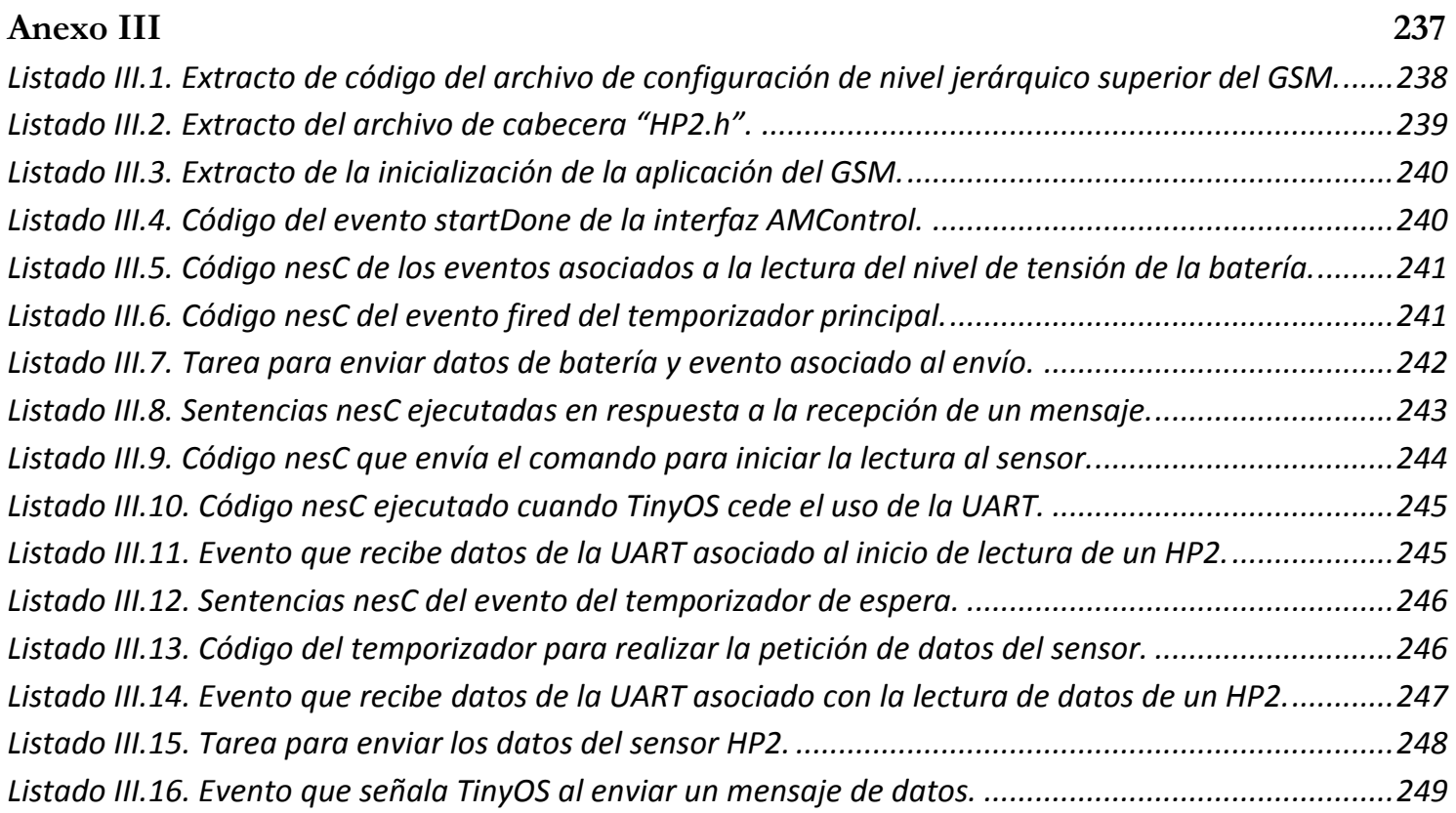

$\begin{array}{ll}\text { Anexo IV } & 251\end{array}$

Listado IV.1. Comandos de entrada y salida que recibe y envía el ZR con el coordinador.......................254

Listado IV.2. Extracto del método SampleApp_Init. ........................................................................25

Listado IV.3. Sentencias ejecutadas cuando el ZR se conecta con el coordinador.................................255

Listado IV.4. Sentencias del evento principal...................................................................................256

Listado IV.5. Sentencias de los eventos LEER_HORA_RTC, LEER_BATERIA y LEER_PANEL_SOLAR...........256

Listado IV.6. Sentencias del método para enviar los valores leídos de los ADCs. ..................................257

Listado IV.7. Sentencias de la máquina de estados implementada para la ejecución del evento CONTROL_PANEL_SOLAR. 
CONTRIBUCIÓN A LAS REDES DE SENSORES INALÁMBRICAS. ESTUDIO E IMPLEMENTACIÓN DE SOluCiones Hardware Para Agricultura de PreCisión.

$-x x i i-$ 


\section{Capítulo 1}

\section{Introducción y Estructura de la Tesis.}

\footnotetext{
$\Psi$ ste capitulo expone, en primer lugar, la motivación que ha llevado al desarrollo de este trabajo de tesis, junto con los objetivos que se persiguen. En segundo lugar, se presenta la estructura de esta memoria, describiendo de manera concisa el contenido.
} 


\subsection{Motivación.}

No hay duda de que los principales factores que garantizan el mantenimiento de la existencia humana y sostienen la vida en la tierra son el agua y la comida. La agricultura produce la gran mayoría de los alimentos, pero consume una gran proporción del agua dulce disponible en el planeta.

En las regiones áridas y semiáridas, como el sureste español, la creciente competitividad por los recursos hídricos está causando importantes problemas de abastecimiento que afectan principalmente a la agricultura. Además, las predicciones sobre los efectos del cambio climático en estas regiones auguran un progresivo empeoramiento de la situación actual [Bouwer, 2000]. Por tanto, uno de los objetivos prioritarios en el manejo y gestión de los recursos hídricos destinados al riego, debe ser el desarrollo e incorporación de nuevas tecnologías que permitan una mayor eficiencia del uso del agua.

Un claro ejemplo de región semiárida es la Región de Murcia, una de las zonas hortícolas más importantes de Europa localizada en el sureste de España. En términos climáticos, es una zona semiárida con una precipitación media anual de $400 \mathrm{~mm}$. A pesar de ésto, el 31\% del área total de cultivos (190.000ha) es de regadío, de las cuales unas 51.000ha están dedicadas a cultivos herbáceos y 91.000 ha a cultivos leñosos. Por ello, en esta región con recursos hídricos limitados es de suma importancia una gestión adecuada del agua de riego, debido a la importancia de la agricultura de regadío, a la escasa precipitación, al elevado coste del agua de riego y al empleo de aguas salinas.

Los agricultores deben maximizar la eficiencia de uso del agua de riego y, para este fin, aplicar estrategias y métodos de programación de riego más eficientes que los que aplican actualmente, basándose para ello en la información climática, el estado del suelo y el de las propias plantas; así como en una interpretación adecuada de esta información para la toma de decisión.

Esta gestión óptima del riego lleva a hablar de agricultura de precisión (AP en adelante) [Zhang, 2002], entendiéndolo como un método de estimar, evaluar y entender las variaciones existentes en los cultivos, con objeto de estimar con mayor exactitud las necesidades de fertilizantes y riego, las fases de desarrollo y maduración de los productos, los puntos óptimos de siembra y recolección, etc. La AP se beneficia de numerosas tecnologías, entre las que se pueden citar: los sistemas de posición global, los sistemas de información geográfica, la microelectrónica, las comunicaciones inalámbricas y los sistemas de instrumentación. En cuanto a las tecnologías inalámbricas, son muchas las que se han utilizado en la AP para implementar sensores inalámbricos con diferentes propósitos. Atendiendo a la distancia cubierta, es posible encontrar desde sencillos dispositivos basados 
en infrarrojos (IrDA) para distancias muy cortas, hasta sistemas de larga distancia basados en telefonía móvil, tales como GSM/GPRS y CDMA. En un punto intermedio se encontrarían las redes inalámbricas de área personal (WPAN, Wireless Personal Area Network) para distancias cortas, donde se encuentran tecnologías como Bluetooth y ZigBee; y las redes inalámbricas de área local (WLAN, Wireless Local Area Network) para distancias medias.

En el contexto de las WPAN se encuadran las Redes de Sensores Inalámbricas (WSN, Wireless Sensor Networks) [Akyildiz, 2002] como un tipo de red 'ad-hoc' autónoma y auto-organizada, constituida por unas decenas, cientos o miles de dispositivos o nodos inteligentes (motes); caracterizados por tener poca capacidad de procesamiento y una alimentación típica basada en baterías.

Las WSNs constituyen una tecnología emergente de adquisición de datos que recientemente está creando un gran interés gracias a sus posibilidades, siendo aplicadas en numerosos ámbitos científicos e industriales para la realización de estudios y control de procesos. El uso de comunicaciones inalámbricas permite que los dispositivos sensores que la forman sean emplazados fácilmente sobre el terreno. Esto, unido al bajo coste de algunos dispositivos, permite el despliegue de un gran número de dispositivos que actúan como nodos de la red.

Que las WSN sean una tecnología emergente es debido, en gran medida, a los recientes avances en los sistemas micro-electro-mecánicos (MEMS, Micro-Electro-Mechanical Systems), las comunicaciones inalámbricas y la electrónica digital. Estos avances han permitido el desarrollo de nodos sensores multifuncionales de bajo coste, baja potencia, pequeño tamaño y que permiten comunicarse inalámbricamente entre alcances cada vez mayores.

A pesar de que se han producido muchos avances en las distintas áreas tecnológicas relacionadas con las WSNs, pocos de ellos se han centrado en el diseño e implementación de arquitecturas hardware de bajo coste, especializadas para entornos agrícolas, que acerquen esta tecnología al agricultor que día a día trabaja en los cultivos.

Por otro lado, el interés que ha despertado el uso de esta tecnología ha dado lugar a la aparición de diferentes plataformas hardware (motes) para el desarrollo de aplicaciones y la investigación en diferentes campos relacionados con las WSN (topologías de redes, consumo de potencia, seguridad, estandarización, etc.). Entre ellas, cabe mencionar los TelosB, MicaZ, Mica2, Mica2Dot, etc. Estas plataformas existían cuando se comenzaron los trabajos de investigación de la presente tesis, en el año 2007. Cabe destacar que, tanto estos motes como otros no mencionados, son productos comerciales que incluyen sensores de bajo coste y cuyas especificaciones (precisión, resolución, derivas, etc.) se alejan de las de la instrumentación que se utiliza comúnmente en las aplicaciones de la AP. Por otro lado, 
muchos de estos motes se han utilizado en aplicaciones de laboratorio o de investigación, careciendo de la robustez necesaria para su aplicación en entornos reales. Por lo tanto, para que los motes puedan ser utilizados en aplicaciones reales es necesario, por un lado, que éstos incluyan la electrónica necesaria para la conexión de instrumentación externa de calidad y, por otro, que se mejore la robustez de los mismos para que puedan ser instalados en campo.

Esta tesis se plantea con el objetivo global de estudiar, diseñar e implementar una arquitectura hardware, basada en WSNs, que permita estimar, evaluar y entender las variaciones existentes en los cultivos, con objeto de estimar con mayor exactitud las necesidades de fertilizantes y riego, las fases de desarrollo y maduración de los productos, así como los puntos óptimos de siembra y recolección, que son los principales objetivos de la AP.

\subsection{Marco de desarrollo de la Tesis.}

La tesis que aquí se expone, surge por el interés común por las WSNs del grupo de investigación DSIE (División de Sistemas e Ingeniería Electrónica) [DSIE] de la Universidad Politécnica de Cartagena y de la PYME Edosoft Factory S.L. [EDOSOFT]. Concretamente se ha desarrollado como un trabajo de investigación del grupo DSIE. Este grupo de investigación desarrolla su labor de investigación, entre otras, en las siguientes áreas tecnológicas, dentro del ámbito de los sistemas reactivos:

- Sistemas de control y robótica para aplicaciones industriales.

- Robots de servicio.

- Sistemas de inspección visual automatizados.

- Tecnología electrónica para robótica y visión artificial.

- Automatización en viviendas y edificios (domótica).

- Redes de sensores inalámbricas.

El beneficio de la colaboración de la empresa Edosoft Factory S.L. es la comercialización de las soluciones hardware diseñadas en esta tesis, bajo el sobrenombre de la línea GAIA.

En esta tesis doctoral se llevan a cabo tareas de investigación y desarrollo en el área tecnológica de las WSNs. Concretamente, se aborda el estudio e implementación de arquitecturas hardware, basadas en WSNs, para AP. La línea de investigación de las redes de sensores inalámbricas fue abierta por el Dr. D. Fernando Losilla López que, en su tesis doctoral [Losilla, 2009], propuso la aplicación del Desarrollo del Software Dirigido por Modelos para diseñar software de forma automática para WSNs. 
El trabajo de tesis que se describe en esta memoria ha sido realizado en el marco de los proyectos de investigación RIMSI del Ministerio de Industria, Comercio y Turismo (ref. FIT-340000-2006-202), MOTE-FRUT (ref. 08850/PI/08) y SICORI (ref. 08754/PI/08) de la Fundación Séneca de la Región de Murcia, y EXPLORE de la Comisión Interministerial de Ciencia y Tecnología (TIN2009-08572).

\subsection{Objetivos.}

El objetivo final de la presente tesis es facilitar el uso de la tecnología de las WSN en el campo de la AP y, en concreto, estudiar e implementar soluciones hardware para llevar a cabo la tareas de monitorización y actuación requeridas en cultivos agrícolas de diversa especie (hortícolas, leñosos, frutícolas, etc.), así como optimizar el uso de los insumos en los cultivos. Para alcanzar dicho objetivo, se han cubierto las siguientes tareas de investigación y desarrollo:

- Analizar el estado del arte de las aplicaciones de WSN en el campo de la AP, prestando un principal interés en las soluciones hardware, sensores y actuadores empleados.

- Estudiar y proponer una arquitectura de red para llevar a cabo las tareas de monitorización y actuación en cultivos agrícolas mediante WSN.

- Analizar los protocolos de comunicación existentes y elegir el óptimo para una aplicación de AP.

- Determinar los tipos de nodos sensores adecuados a los requisitos del despliegue.

- Analizar los sensores y actuadores existentes en el mercado y escoger los adecuados para llevar a cabo las tareas de monitorización y control.

- Seleccionar la plataforma (radio y micro-controlador) hardware óptima que permita implementar una WSN con el protocolo de comunicaciones, los sensores y los actuadores seleccionados.

- Seleccionar un entorno y lenguaje de programación para el desarrollo software de las aplicaciones de los nodos.

- Definir los requisitos de diferentes tipos de nodos de acuerdo a la arquitectura diseñada anteriormente.

- Estudiar e implementar las soluciones hardware necesarias para monitorizar y controlar cultivos agrícolas.

- Implementar el software que dote de funcionalidad a los distintos tipos de nodos en el despliegue teniendo en cuenta el modo de funcionamiento en bajo consumo de los nodos sensores.

- Realizar pruebas de validación en laboratorio de las soluciones hardware implementadas (funcionamiento general y consumos de potencia). 
- Determinar el sistema de alimentación necesario para las soluciones hardware implementadas.

- Diseñar e implementar las carcasas que aseguren un correcto funcionamiento de los dispositivos en condiciones reales de trabajo.

- Seleccionar las infraestructuras necesarias (antenas, mástiles, cables, etc.) para desplegar los nodos sensores en cultivos agrícolas.

- Implementar una aplicación de usuario que permita la monitorización y actuación con los nodos sensores.

- Llevar a cabo la validación de las soluciones hardware implementas en condiciones reales de funcionamiento (consumo, correcto funcionamiento, pérdidas de paquetes, etc.

\subsection{Estructura de la Tesis.}

Esta memoria se ha dividido en un total de ocho capítulos. Además, el documento contiene cuatro anexos con información adicional. La estructura detallada de la tesis doctoral es la siguiente:

\section{Capítulo 1: Introducción y Estructura de la Tesis.}

Se trata del presente capítulo, donde se introducen los aspectos relacionados con la motivación y los objetivos perseguidos en el desarrollo de esta tesis. Asimismo, se presenta el marco de trabajo en el que se encuadra esta investigación y la estructura de esta tesis doctoral.

\section{Capítulo 2: E1 Papel de las Tecnologías de la Información y las Comunicaciones en} la Agricultura de Precisión.

En este capítulo, después de describir el concepto de la agricultura de precisión, se realiza un recorrido por las tecnologías de la información y las comunicaciones más empleadas en este campo. Finaliza con una breve presentación de una de las tecnologías más prometedoras dentro del campo de la AP y que es objeto de estudio de la presente tesis: las redes de sensores inalámbricas.

\section{Capítulo 3: Las Redes de Sensores Inalámbricas.}

El objetivo de este capítulo es hacer una revisión del estado de la técnica de las WSNs. Para ello, en primer lugar, se realiza una descripción general y, posteriormente, se describen con mayor detalle cada uno de los elementos involucrados en la configuración de una WSN. Además, al ser objetivos de esta tesis el estudiar e implementar soluciones 
hardware para entornos agronómicos, se revisa en la literatura científica el estado de las WSNs en el campo de la AP.

\section{Capítulo 4: Diseño y Validación de una Arquitectura Basada en WSN para Cultivos Hortícolas.}

En este capítulo se propone una arquitectura general, basada en WSNs, para llevar a cabo la monitorización de cultivos hortícolas y arbóreos. Para proponer dicha arquitectura de red, se comienza exponiendo las especificaciones que se han capturado, teniendo en cuenta las características de los cultivos a monitorizar y el tipo de sensores a utilizar. A partir de dichas especificaciones, se propone una arquitectura genérica de red, basada en el estándar de comunicaciones IEEE 802.15.4, y una serie de nodos diseñados ad-hoc para implementar la red. También se describen las simulaciones que se realizaron antes de desarrollar los dispositivos para confirmar que con la tecnología seleccionada se cumplen los requisitos en cuanto a autonomía, tasa binaría de envío/recepción de información y retrasos en las transmisiones. Posteriormente, se describen los dispositivos desarrollados y las dos fases de validación llevadas a cabo con los mismos, con objeto de contrastar las simulaciones realizadas con los datos obtenidos en un entorno real. El capítulo concluye que la alternativa de que cada nodo resuelva un problema concreto no es adecuada por las razones fundamentales de 'time-to-markę y los costes asociados. Además, a pesar de que los prototipos de laboratorio funcionaron correctamente durante la fase de validación realizada en un cultivo real, en agricultura los dispositivos tienen que ser muy robustos y económicos. Por ello, se planteó la alternativa de diseñar un único nodo multifuncional (GAIA 2), configurable tanto a nivel de hardware como de software, que permita reducir costes mediante un único proceso de certificación y que pueda desarrollar cualquiera de las funcionalidades descritas para los prototipos de laboratorio anteriores.

\section{Capítulo 5: GAIA Soil-Mote: un Nodo Sensor para la Monitorización de Parámetros del Suelo Basado en el Estándar SDI-12.}

Debido a la carencia de experiencia en el diseño electrónico con tecnología de montaje superficial de un nodo sensor con un circuito de radio frecuencia, llevar a cabo la implementación del GAIA 2 conllevaba un reto importante. Por ello, antes de abordar el diseño del GAIA 2, se planteó llevar a cabo un diseño menos ambicioso. Éste fue el diseño del GAIA Soil-Mote, que es la versión comercial, y por tanto, más robusta del prototipo de laboratorio Soil-Mote, descrito en el Capítulo 4. En primer lugar, se describen los requisitos funcionales contemplados para el diseño del dispositivo. Posteriormente, se describe la arquitectura hardware del dispositivo, así como los componentes software necesarios para implementar las especificaciones impuestas. Finalmente, se describen las pruebas llevadas a cabo, tanto en el laboratorio como en un cultivo real, con objeto de validar el diseño del nodo sensor. 


\section{Capítulo 6: GAIA 2: un Nodo Sensor Multifuncional para Agricultura de Precisión.}

Llegado este punto, ya se tienen los conocimientos necesarios para llevar a cabo el diseño e implementación de un nodo sensor multifuncional, el GAIA 2. Se trata de un diseño más ambicioso que el del GAIA Soil-Mote y que puede ser configurado para operar con la funcionalidad de cualquier nodo sensor de la arquitectura propuesta (Soil-Mote, Water-Mote y Environmental-Mote), o bien, como un elemento auxiliar de comunicaciones (Gateway). En primer lugar, se describen los requisitos funcionales tenidos en cuenta para el diseño del dispositivo. A continuación se describe la flexibilidad de la arquitectura hardware y las posibilidades software del GAIA 2 con objeto de demostrar la multifuncionalidad del nodo sensor. Finalmente, se describen las pruebas experimentales llevadas a cabo en el laboratorio y en un cultivo real con el prototipo comercial para validar el funcionamiento del mismo.

\section{Capítulo 7: MEWiN: Un Nodo Sensor Inalámbrico Multi-Entorno con una Arquitectura Abierta.}

Una vez desarrollado, se observó que el GAIA 2 presentaba varios inconvenientes: un elevado coste de la carcasa diseñada y del módulo comercial de comunicaciones de largo alcance XStream. Además, no dispone de un sistema para realizar el almacenamiento local de los datos adquiridos en previsión de un posible error de transmisión, presentando así un elevado riesgo de pérdida de los datos. Por ello, tras el diseño de este dispositivo se planteó el diseño del nodo sensor inalámbrico multi-entorno MEWiN, que permite eliminar estos inconvenientes y mejorar las prestaciones del GAIA 2. En este capítulo, se presenta el diseño, implementación y validación de dicho nodo, basado en una arquitectura abierta capaz de disminuir el número de elementos necesarios para configurar una red determinada y optimizar los costes de fabricación. En primer lugar se describen los requisitos funcionales para diseñar el dispositivo. También se presenta la arquitectura hardware y software de la placa base MEWiN Mainboard, así como el diseño hardware de las placas de interfaz realizadas para validar la arquitectura global en condiciones reales de funcionamiento (en un cultivo real). Finalmente, se presentan las pruebas experimentales realizadas sobre el dispositivo. Para ello se describen las pruebas realizadas en laboratorio, las pruebas de alcances y las llevadas a cabo en el despliegue realizado en un cultivo real. En cada una de ellas se presentan los resultados obtenidos.

\section{Capítulo 8: Conclusiones y Trabajos Futuros (Conclusions and Future Work).}

En este capítulo, que ha sido redactado en inglés con objeto de optar al suplemento europeo al título, se presentan las conclusiones obtenidas tras la realización de la presente tesis doctoral, así como los resultados que, hasta el momento, se han obtenido de ella. A 
continuación se enumeran las principales líneas de investigación que se abren a raíz de la realización de este trabajo de investigación y que permitirán su mejora y ampliación.

\section{Anexo I: Materiales, Herramientas y Métodos.}

A la hora de diseñar y desplegar una WSN es necesario escoger un conjunto de materiales, utilizar un conjunto de herramientas y seguir una serie de métodos para desarrollar la arquitectura necesaria. Este anexo recoge la experiencia acumulada durante el desarrollo de esta tesis en relación con estos aspectos. En primer lugar, se describen los principales elementos que se han contemplado para seleccionar la tecnología necesaria para desarrollar los principales componentes de las arquitecturas hardware propuestas. A continuación se describen las herramientas que han sido necesarias de utilizar tanto para el diseño y desarrollo del hardware/software, así como para el despliegue. Finalmente, se describen los métodos necesarios para llevar a cabo el diseño de la red.

\section{Anexo II: Tramas de Datos Intercambiadas entre los Nodos Sensores Diseñados e Implementados durante la Fase de Validación de los Dispositivos.}

En este anexo se describen los diferentes tipos de tramas intercambiadas por las WSNs desplegadas para validar los diferentes nodos sensores diseñados e implementados en la presente tesis doctoral. La descripción comienza presentando las tramas intercambiadas por los dispositivos de la WSN diseñada e implementada con módulos de inserción para el micro-controlador y el módulo de radio que fue descrita en el Capítulo 4. Finalmente, se describen las tramas utilizadas para validar el nodo sensor MEWiN, descrito en el Capítulo 7.

\section{Anexo III: Descripción del Software desarrollado para el GAIA Soil-Mote.}

Este anexo recoge en profundidad la descripción del software desarrollado para el GAIA Soil-Mote. La descripción comienza presentando la vista estática de componentes nesC del nodo sensor. A continuación, se describen brevemente cada uno de los componentes. Finalmente, se analiza en detalle el código fuente desarrollado del componente de tipo módulo que implementa la funcionalidad del nodo sensor.

\section{Anexo IV: Descripción del Software Desarrollado para un Nodo Sensor MEWiN con el Rol de ZigBee Router.}

En este anexo se describe en profundidad el software desarrollado para el nodo sensor multi-entorno MEWiN con el rol de ZigBee Router. La descripción comienza presentando un diagrama, que ilustra a grandes rasgos el funcionamiento del software del nodo sensor. Finalmente, se describe en detalle el código fuente desarrollado que implementa la funcionalidad del nodo sensor. 
CONTRIBUCIÓN A LAS REDES DE SENSORES INALÁMBRICAS. ESTUDIO E IMPLEMENTACIÓN DE SOluCiones Hardware Para Agricultura de PreCisión. 


\section{Capítulo 2}

\section{E1 Papel de las Tecnologías de la Información y las Comunicaciones en la Agricultura de Precisión.}

$L$ a productividad de un cultivo es uno de los principios básicos para su desarrollo y explotación, con lo que su optimización constituye un objetivo prioritario. Dicha optimización se encuentra relacionada con la minimización de la energía que se transfiere entre los seres vivos, que constituye lo que se denomina como 'cadena trófica'. En los ecosistemas agrícolas, al contrario que en los naturales, la biodiversidad debe ser baja con objeto de reducir las pérdidas de energía a lo largo de su cadena trófica. Para conseguir este objetivo, el uso de las tecnologías de la información y las comunicaciones en la agricultura puede resultar beneficioso, surgiendo asi el concepto de la agricultura de precisión (AP). El presente capitulo, tras describir el concepto de la agricultura de precisión, realiza un recorrido por las tecnologias de la información y las comunicaciones más empleadas en este campo. Finaliza con una breve presentación de una de las tecnologias más prometedoras dentro del campo de la AP y que es objeto de estudio de la presente tesis: las redes de sensores inalámbricos (WSN, Wireless Sensor Networks). En el siguiente capitulo se profundiza sobre el estado de la técnica de las WSN en el marco de la AP. 


\subsection{Introducción.}

No hay duda de que los principales factores que garantizan el mantenimiento de la existencia humana y sostienen la vida en la tierra son el agua y la comida. La agricultura produce la gran mayoría de los alimentos, pero consume hasta un $70 \%$ del agua dulce disponible en el planeta. Además, en las regiones del planeta con rentas bajas, como muchas zonas de África, la agricultura no es sólo una fuente de nutrición, sino también la principal fuente de ingresos para más del 70\% de la población [Chibuzor, 2006]. En estas regiones, las condiciones de la tierra son críticamente bajas, fundamentalmente debido a: las prácticas agrícolas inadecuadas, la escasez de los recursos y la variabilidad climática reciente. Se estima que, con las tasas actuales de degradación de los suelos, África pierde unos cuatro billones de dólares anualmente y que el rendimiento de algunos cultivos se reduciría en un $17-30 \%$ antes de 2020 [Henao, 2006]. Por otro lado, debido al calentamiento global se espera que aumente las necesidades mundiales de riego en un $26 \%$ para mantener el ritmo actual de producción [Chibuzor, 2006]. Un gran desafío es, entonces, maximizar la productividad de la agricultura de riego y los recursos, manteniendo la calidad e integridad de los cultivos y el medio ambiente [Kabashi, 2008].

En este capítulo se describe la agricultura de precisión como disciplina que aúna metodologías agronómicas de la información y las comunicaciones y que, por tanto, necesitan de una definición previa como es, la toma de decisiones en la agricultura, así como los tipos de variabilidad en los cultivos; conceptos que permiten definir el concepto de zona homogénea, que más adelante se analizará.

Tras describir la agricultura de precisión, se presentarán las principales tecnologías de la información y las comunicaciones empleadas en las explotaciones agrícolas.

Al final del capítulo se presentarán las redes de sensores como una tecnología y metodología muy apropiada para el uso en estas disciplinas, lo que repercutirá en la necesidad de describirla en profundidad en un capítulo posterior.

\subsection{Los sistemas agrícolas y la agricultura.}

Un ecosistema se define como 'el conjunto de organismos y el ambiente que coinciden en el tiempo y el espacio'. Dentro de un ecosistema, los organismos se relacionan entre sí mediante flujos de energía [Odum, 1968] y la caracterización unidimensional de los flujos de energía en el ecosistema se denomina 'cadena trófica'. En concreto, la cadena trófica (del griego 'throphe', alimentación) es el proceso de transferencia de energía alimenticia a través de una serie de organismos, en el que cada uno se alimenta del precedente y es alimento del siguiente. También conocida como 'cadena alimenticia', es la corriente de energía y nutrientes 
que se establece entre las distintas especies de un ecosistema en relación con su nutrición. Durante millones de años, el hombre obtuvo su energía de diversas cadenas tróficas, cazando y recolectando alimentos al azar, hasta que hace unos 8-10.000 años inventó la agricultura.

La agricultura es un conjunto de intervenciones humanas que modifican (y/o explotan) los ecosistemas para maximizar la producción deseada y minimizar las pérdidas de energía a lo largo de las cadenas tróficas. Estas intervenciones permiten al hombre producir alimentos y otros productos, manejando y manipulando las cadenas tróficas en los ecosistemas para su propio beneficio. Surgen así los conceptos de ecosistema natural y agrícola, según haya o no intervención humana. La tabla 2.1 resume las principales diferencias entre ambos [Villalobos, 2002].

\begin{tabular}{|c|c|c|}
\hline & Ecosistema natural & Ecosistema agrícola \\
\hline Biodiversidad & Alta & Baja \\
\hline Cadena trófica & Larga & Corta \\
\hline Autonomía & Alta & Baja \\
\hline
\end{tabular}

Tabla 2.1. Principales diferencias entre los ecosistemas naturales y los agrícolas.

Uno de los principales objetivos en un ecosistema agrícola es el de reducir la pérdidas de energía entre las cadenas tróficas, en los que se intentan eliminar todas la transferencias indeseables (hacia parásitos, patógenos o plantas que compiten con el cultivo). Esto se traduce en que la cadena trófica sea corta y que, por tanto, la presencia de diferentes especies sea baja frente al ecosistema natural y se incremente la productividad del cultivo, la cual se define como la producción por unidad de recurso utilizado; estando habitualmente referida a la superficie de suelo cultivado, factor limitante primario de la agricultura. En relación con la autonomía, es relativamente baja porque depende de los aportes del hombre.

Para caracterizar los sistemas agrícolas se usa el término 'eficiencia'. Ésta define cocientes entre determinados insumos y la productividad del cultivo. El término 'insumo', equivalente en ocasiones al de materia prima, es usado mayormente en el campo de la producción agrícola y hace referencia a un bien consumible utilizado en el proceso de producción de otro bien.

Los sistemas agrícolas se pueden clasificar en función de diversos criterios. Una primera clasificación viene dada por el tipo de cadena trófica. Atendiendo a este criterio, se pueden encontrar cadenas tróficas cortas, en las que los cultivos son consumidos directamente por el hombre y cadenas tróficas mayores, como, por ejemplo, cuando los pastos son consumidos por el ganado y este último por el hombre. Cabe destacar que la 
eficiencia energética disminuye conforme aumenta el número de niveles de la cadena trófica.

Otra alternativa a la hora de clasificar los sistemas agrícolas es en los de subsistencia y los de agricultura intensiva. Los de subsistencia se caracterizan por cultivar diversos tipos de especies, emplear mucha mano de obra y bajo nivel de nutrientes. Por ello, los rendimientos son bajos pero estables. Por otro lado, la agricultura intensiva se caracteriza por una baja diversidad, lo que conduce a un mayor rendimiento potencial. Además, se emplea más maquinaría y se usan más fertilizantes que en el caso anterior, la cual se traduce en conseguir una elevada productividad pero con baja eficiencia energética.

Por otro lado, la competitividad presente en el sector agrario ha llevado a la intensificación de la agricultura en muchos países de la Unión Europea. Esto a su vez ha llevado a la producción de excedentes en los principales cultivos y a episodios de contaminación ambiental por un uso excesivo de insumos como fertilizantes $y$ fitosanitarios. Surgieron así sistemas agrícolas alternativos, tales como la agricultura biológica o ecológica, también llamada 'agricultura orgánica' [Guzmán, 2009], que se caracterizan por no utilizar fertilizantes minerales y fitosanitarios de síntesis. Finalmente, además de los tipos de sistemas agrícolas citados anteriormente, cabe destacar la 'agricultura sostenible'. Ésta se caracteriza por practicar una agricultura más respetuosa con el medio ambiente y que asegura la calidad y sanidad de los alimentos.

\subsection{La toma de decisiones en la agricultura.}

Actualmente, la toma de decisiones en la agricultura resulta ser una tarea más compleja que en el pasado, ya que el agricultor debe tener muchos factores en cuenta, tales como la protección de los recursos naturales, las emisiones de gases de efecto invernadero, y no sólo y exclusivamente la productividad del cultivo. No obstante, existen nuevas herramientas de las que el agricultor puede hacer uso, entre las que caben destacar las tecnologías de la información y las comunicaciones.

La actividad agrícola se caracteriza por la incertidumbre propia de un sistema que depende de la climatología, que es altamente variable, y de otros factores bióticos relativamente imprevisibles como son las plagas, enfermedades, etc. Además, los precios de los productos agrícolas, del agua y de los insumos pueden desviarse sustancialmente respecto de los precios esperados por el agricultor, lo que dificulta aún más el proceso de toma de decisiones. Esto se debe a decisiones que no están en manos de los agricultores, sino en los políticos, que estudian estos asuntos en reuniones periódicas. En estas reuniones, tanto a nivel nacional como internacional, a partir de los datos de producción de cada país se realizan las correspondientes estimaciones de exportaciones e importaciones a 
realizar dentro de cada país, y se fijan, por tanto los precios por kilogramo que recibirán los agricultores al vender sus productos. Al establecer los precios, resulta evidente que los grandes empresarios del sector alimentario tengan gran influencia en su determinación. En resumen, los agricultores, y en general el sector primario, se enfrenta día a día con grandes dificultades.

La tendencia histórica general en los sistemas agrícolas del mundo ha sido desarrollar prácticas de manejo que reduzcan el riesgo, esto es, que aseguren unos beneficios suficientes en los años más adversos, aunque no permitan los beneficios máximos en los años más favorables. Cabe destacar que la tendencia a reducir el riesgo se traduce, en parte, en una lenta adopción de las nuevas tecnologías en el sector.

Según Fountas [Fountas, 2006], las decisiones en una explotación agrícola se pueden clasificar en operacionales, tácticas y estratégicas. Como el término indica, las operacionales se realizar a lo largo de la campaña agrícola (por ejemplo, fechas del riego, cantidades de fertilizante, fecha de aplicación de un insecticida). Por otro lado, las decisiones tácticas se llevan a cabo una sola vez por cada campaña agrícola (elección del cultivo y de la fecha de siembra, densidad de la siembra). Finalmente, las decisiones estratégicas afectan a un conjunto de campañas agrícolas (por ejemplo, inversión en maquinaría y mejoras de las infraestructura).

Para tomar decisiones, el agricultor necesita conocer cómo responden los cultivos a las prácticas agrícolas y cuál es el estado del cultivo y del suelo a lo largo de la campaña. El agricultor no está solo en esta tarea, sino que existen diferentes fuentes de información, más o menos accesibles, a las que puede acceder con un mayor o menor coste. Resulta evidente que la principal fuente de información es la propia experiencia del agricultor, que es la base tradicional de la actividad agrícola y puede ser la mejor fuente de información en sistemas agrícolas que varían poco con el tiempo. Otra fuente de información es la comercial. En los últimos años el sector primario está apostando por la investigación y participa muy activamente en la transferencia de tecnología al sector agrario. La investigación, experimentación y transferencia de tecnología es otra fuente de información que recibe el agricultor. La investigación y la experimentación son las únicas vías para producir nuevos conocimientos sobre el manejo de los sistemas agrícolas y existen numerosas universidades y centros de investigación que se dedican a esta tarea, y que realizan sus labores en estaciones experimentales [MOTE-FRUT, 2008][ESEA]. Finalmente, se encuentran los modelos de simulación de cultivos, los paquetes de ayuda a la toma de decisiones y los sistemas expertos. Los modelos de simulación de cultivos son representaciones lógicas y matemáticas que incluyen aspectos del suelo, del clima y del cultivo, de manera que permiten evaluar el comportamiento del cultivo ante determinadas situaciones. Los paquetes de ayuda incluyen modelos de simulaciones y otras utilidades que 
facilitan al agricultor con la tarea de la toma de decisiones. Un ejemplo sería el paquete DSSAT (Decision Support System for Agrotechnology) [Jones, 2003] desarrollado por la Universidad de Hawaii con la colaboración de investigadores de todo el mundo. Un sistema experto es equivalente a tener un experto humano, al que el agricultor le formula preguntas de manera interactiva. El sistema experto puede responder a estas preguntas a partir de su base de datos, una serie de reglas de decisión y un módulo de autoaprendizaje. Como ejemplo, se puede citar al sistema experto de manejo de algodón Comax, el cual incluye el modelo de simulación Gossym [Comax, 1990].

\subsection{La variabilidad y la zona homogénea.}

En las explotaciones agrícolas, el suelo de los terrenos dedicado a cultivo no es homogéneo y, por tanto, hay que distribuir los insumos en las parcelas de manera heterogénea. De hecho, la variabilidad es una característica que afecta a los parámetros del terreno, el estado de las plantas, al de las malas hierbas, etc. [Basso, 2006].

La variabilidad del terreno depende de una componente espacial, una temporal, así como del tipo de cultivo y de las labores realizadas por el hombre.

\subsubsection{La variabilidad espacial.}

La variabilidad espacial [Gallardo, 2007] es el resultado de muchos factores que interactúan de modo continuo en un contexto espacio-temporal. Hay factores que afectan sobre grandes superficies y que, durante largos periodos de tiempo, son modificados por otros procesos que afectan de forma diferente a superficies más pequeñas o durante menos tiempo. Esta variabilidad o cambios en los parámetros se deben tanto a razones sistemáticas como causales.

La variabilidad sistemática consiste en un cambio gradual y bien definido, que puede ser interpretado como el resultado de factores o procesos que actúan a una escala mayor que la que se aprecia a simple vista.

Por otro lado, también hay diferencias que no pueden ser atribuidas a causas conocidas. A esta parte de la heterogeneidad global, que en el estado actual del conocimiento no tiene interpretación, se la considera casual.

A continuación se clasifican las causas principales de la variabilidad espacial:

- Fija o estática, que no se puede modificar.

- Relativamente estática, que puede variar de un año a otro, pero nunca en un lapso de tiempo inferior al del periodo del cultivo. 
- Variable o dinámica, que se puede cambiar durante el periodo del cultivo.

La variabilidad de los parámetros está relacionada con la variabilidad de la productividad. En general, la variabilidad de la producción se ve poco afectada por la variabilidad de las propiedades estáticas, con una mayor influencia de las dinámicas.

\subsubsection{La variabilidad temporal.}

Por naturaleza el suelo es heterogéneo y, por tanto, los factores que afectan a la productividad de un cultivo varían espacialmente y temporalmente [Cichota, 2006]. Que varíen temporalmente implica que el parámetro medido presentará diferentes valores en un mismo punto a lo largo del tiempo. Se pueden distinguir dos componentes de la variabilidad temporal:

- Variabilidad temporal encontrada dentro del mismo año. Se dice de parámetros que tienen un valor diferente a lo largo del año, en función de las condiciones meteorológicas y de la interacción con otros factores presentes; como, por ejemplo, la humedad que varía con las estaciones y en función del riego.

- Variabilidad temporal encontrada a lo largo de años diferentes. Se dice de un parámetro que varía a lo largo del tiempo independientemente de las variaciones sufridas durante el ciclo del cultivo. Un claro ejemplo sería la producción.

En resumen, el estudio de la variabilidad debe tener el objetivo de cuantificarla y descubrir los factores fundamentales que influyen en ella. De esta manera, se puede determinar el grado de estabilidad, que representa la cuantía con la que varía un parámetro estudiado en el espacio y el tiempo. Esta fase es de vital importancia para delimitar las zonas homogéneas.

\subsubsection{La zona homogénea.}

Una zona homogénea es aquella en la que el conjunto de factores que influyen en la productividad ha causado una producción diferente al de otras zonas contiguas.

Las recientes herramientas tecnológicas (receptores GPS, imágenes de satélites, fotografías aéreas, índice de vegetación, etc.) ayudan al usuario con la tarea de delimitar las zonas que requieren un tratamiento diferente de las contiguas. Esta delimitación debe hacerse teniendo en cuenta el número, el tamaño y la forma de las mismas.

El número de zonas homogéneas puede ser muy diferente y se debe de tener en cuenta que, cuanto mayor sea, más complejo resultará la gestión de la explotación agrícola. En la práctica, si la explotación está compuesta de parcelas con una extensión media de una 
hectárea, no es conveniente definir más de dos zonas homogéneas por parcela [Basso, 2006].

El tamaño de las zonas homogéneas está directamente relacionado con el tamaño de las maquinarías usadas en el cultivo, que debe tenerse muy en cuenta en el momento de delimitarlas.

Resulta evidente que la forma de las zonas homogéneas depende de la variabilidad observada en los parámetros seleccionados. No obstante, al igual que en el caso anterior, deben definirse formas geométricas que sean compatibles con las características de las máquinas que realizan las labores en el cultivo.

Los métodos para delimitar las zonas homogéneas varían mucho, tanto en la información utilizada, como en los métodos empleados para delimitar las zonas. Así, en la literatura científica se pueden encontrar, entre otros, ejemplos de una delimitación manual de polígonos a partir de los mapas de la cosecha y/o fotos del suelo virgen [Fleming, 2000], de las propiedades del suelo y del paisaje [Fraisse, 2001], o a partir de imágenes remotas [Ahn, 1999], etc.

A modo de ejemplo, Kitchen et al. [Kitchen, 2005] realizaron un estudio real con objeto de determinar las zonas homogéneas y estudiar la correspondencia con las obtenidas a partir de los datos de la cosecha, en los correspondientes mapas de la cosecha elaborados durante años. Para ello utilizaron datos de la conductividad eléctrica aparente del suelo y de elevación. Al final concluye con que con el método empleado se obtuvo una coincidencia entre el 60 y el 70\% con la delimitación realizada a partir de los mapas de la cosecha y, además, que ambos métodos deberán tenerse en cuenta a la hora de delimitar las zonas homogéneas.

\subsection{La agricultura de precisión.}

El término agricultura de precisión (AP en adelante) ha estado presente desde hace algún tiempo. En 1994, Blackmore et al. [Blackmore, 1994] la definieron como: "un sistema completo diseñado para optimizar la producción agricola cuidando, al mismo tiempo, la adaptación del suelo y gestionando los cultivos para adecuarlos a las condiciones únicas que se encuentran en cada campo, y teniendo siempre en mente la preservación del medio ambiente".

Por otro lado, el National Research Council (1997), define a ésta como: "métodos de gestión empleando tecnologías de la información para obtener datos de múltiples fuentes y tomar decisiones asociadas con la productividad del cultivo" [Adrian, 2005]. 
En la misma línea, Anurag et al. [Anurag, 2008] definen la AP como: "una combinación de la perspectiva de una nueva gestión usando las nuevas tecnologías de la información y las comunicaciones, con el fin de aumentar la productividad y reducir los costes en el funcionamiento de las explotaciones agricolas a gran escala comercial'.

Conocida también como Site-Specific Management, la AP garantiza una rápida respuesta frente a las condiciones climáticas adversas, mejor control de la calidad del producto y un coste más bajo.

De las tres definiciones anteriores, se observa que todos ellos mencionan como principal objetivo la optimización de la productividad del cultivo. Como se puede recordar del concepto de cadena trófica, dicha optimización implica minimizar las pérdidas de energía a lo largo de dicha cadena. No es de extrañar, entonces, un predominio de la automatización y el uso de las tecnologías de la información y las comunicaciones en la AP, con objeto de asegurar una mayor rapidez de respuesta ante cambios en el cultivo; logrando así minimizar el impacto en la cadena trófica y, por tanto, aumentar la productividad. La AP también hace que el uso de máquinas completamente automatizadas sea una posibilidad. Esta forma de agricultura altamente automatizada, requiere una monitorización intensiva de las condiciones climáticas a pie de cultivo y una rápida transmisión de los datos hasta un repositorio central. Habitualmente en el denominado 'servidor central', caracterizado por una potencia de cálculo elevada, se lleva a cabo la toma de decisiones y el control de maquinaria agrícola.

La AP comenzó a desarrollarse en EEUU en la década de 1990, gracias a los avances de las Tecnologías de la Información y las Comunicaciones (sistemas de localización de bajo coste, potentes ordenadores, los sistemas de información geográfica, etc.). En ese país nació un lema que simplifica su definición: "do the right thing at the right place at the right time [Lowenberg-DeBoer, 2000], que se puede traducir por "haz la labor de cultivo apropiada en el lugar exacto en el momento justo". Por lo tanto, el uso de la AP ofrecía la oportunidad de incrementar la producción, mejorar el uso de los recursos y reducir la posible contaminación provocada por prácticas agrícolas no racionales [Basso, 2006]. Sin embargo, los primeros en adoptarla no la encontraron rentable. Existían pocas aplicaciones y muy diversificadas. Además, la alta inversión inicial de equipos electrónicos de monitorización y comunicaciones sólo estaba al alcance de las grandes explotaciones agrícolas.

En relación con la evolución de la AP, la Figura 2.1 muestra la evolución del número de publicaciones científicas realizadas por año, así como las acumuladas. Los datos se han obtenido con la herramienta SciencieDirect, buscando el término "precision agriculture". Como se puede observar en la tendencia exponencial de la figura, el "despegue" de la AP comenzó en el año 2001. 


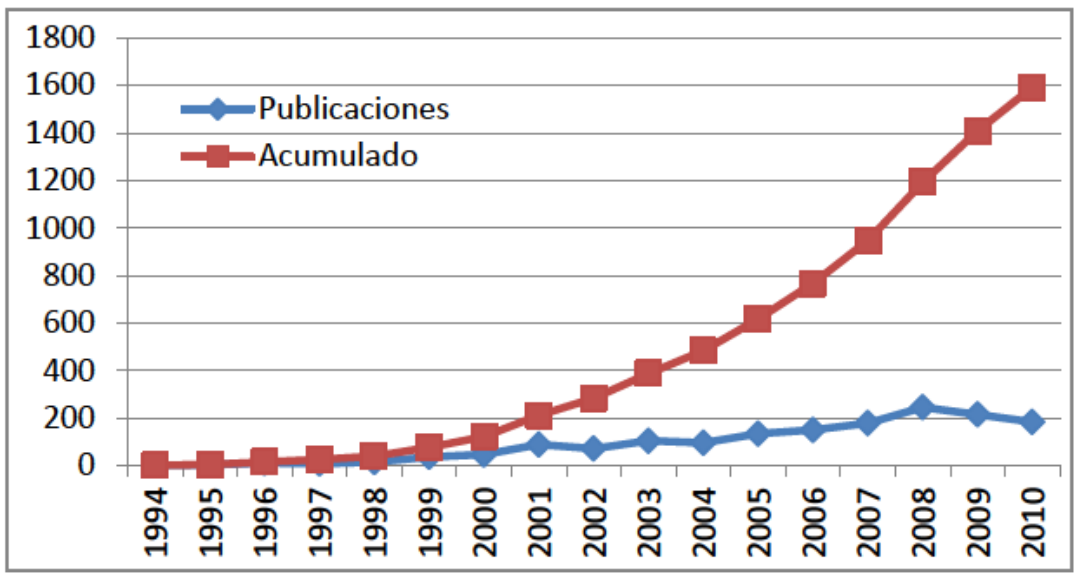

Figura 2.1. Evolución del número de publicaciones obtenido mediante búsqueda con el término "precision agriculture" en el SciencieDirect.

Para conseguir incrementar la producción, mejorar el uso de los recursos, reducir la posible contaminación provocada por prácticas agrícolas no racionales y que la AP resulte beneficiosa al mismo tiempo, se debe tener en cuenta que en los cultivos existe variabilidad y que hay que tratar cada zona homogénea de una manera distinta a las demás. La variabilidad se determina con diversos sensores, que son un eslabón del sistema completo:

- Sensorización de los parámetros agrícolas.

- Identificación de la posición de los sensores y la recolección de datos del cultivo.

- Transferencia de datos desde el cultivo hasta la estación de control para la toma de decisiones.

- Actuación y toma de decisiones en base a los datos obtenidos de los sensores.

Dentro del sistema completo se pueden identificar diferentes tecnologías involucradas, entre las que cabe resaltar las siguientes [Blackmore, 1994]: (a) sensores remotos, (b) sistemas de posicionamiento global y (c) sistemas de información geográfica [Panchard, 2006]. Estas tecnologías serán objeto de estudio de la siguiente sección.

A su vez, el agricultor debe llevar a cabo una serie de fases para poder llegar a la toma de decisiones de una zona homogénea concreta de la explotación agrícola. Estas fases (ver Figura 2.2) son las siguientes [Anurag, 2008]:

- Recolección de datos.

- Análisis de los datos.

- Toma de decisiones.

- Actuación. 


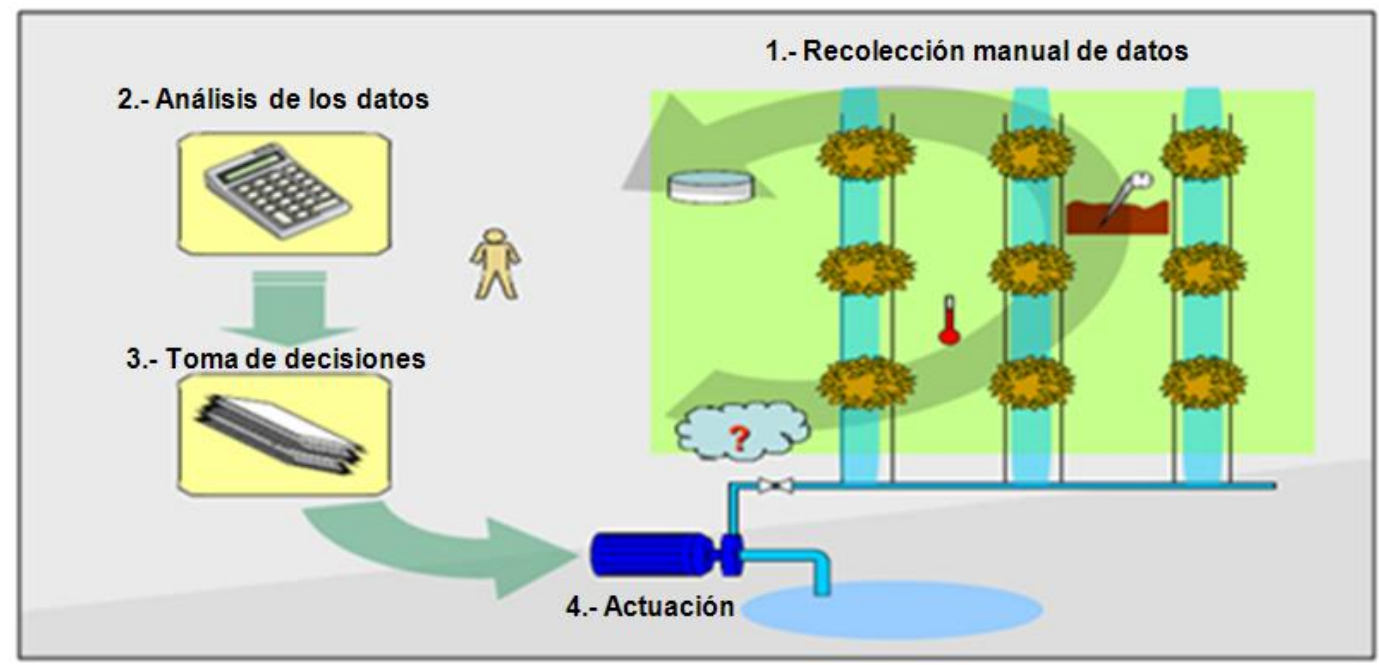

Figura 2.2. Fases llevadas a cabo en la AP.

La primera fase consiste en realizar la recogida de datos del cultivo, del ambiente, de las plantas, etc. Esta primera fase se puede realizar de forma manual o bien de manera automática, haciendo uso de una o varias determinadas tecnologías. La segunda fase consiste en analizar los datos para poder llevar a cabo la toma de decisiones en la tercera fase. Como se puede recordar, en la sección 2.3 se mencionaron los tipos de decisiones que se llevan a cabo en un sistema agrícola. La cuarta y última fase, es la encargada de cerrar el lazo del sistema. En esta fase se controlan los diferentes actuadores y/o maquinaría agrícola.

En relación con la primera fase, en la literatura se puede comprobar que existen diferentes parámetros de interés a la hora de monitorizar los cultivos. Por ejemplo, Hirafuji et al. [Hirafuji, 2004] desplegaron cinco dispositivos denominados 'Field-Monitoring Server' (FMS). Cada FMS tenía conectados diferentes sensores y, al final, usando todos los FMSs monitorizaban, entre otros, los siguientes parámetros: temperatura interior del dispositivo, humedad relativa y temperatura del ambiente, intensidad de la radiación solar, imágenes del cultivo con una cámara CCD, concentración de $\mathrm{CO}_{2}$, humedad de las hojas, densidad de flujo fotosintético de fotones, etc. En la misma línea, Matese et al. [Matese, 2009] realizaron una revisión de los parámetros monitorizados por diferentes autores en viñedos, y llegaron a la conclusión de que los trabajos presentaban importantes carencias a la hora de monitorizar de forma exhaustiva diferentes parámetros agro-meteorológicos, que serían de gran relevancia en la viticultura de precisión. Además, justificaron la necesidad de monitorizar los siguientes parámetros haciendo referencia a la literatura científica:

- El perfil de temperatura diario es uno de los factores ambientales más importantes para la calidad de la uva, especialmente en el mes antes de la maduración, y por lo tanto para el vino [Winkler, 1974]; 
- La temperatura de los racimos proporciona una importante información sobre el estado de maduración de los compuestos fenólicos de la uva [Castia, 1992];

- El potencial hídrico del suelo permite determinar el contenido de agua disponible en cualquiera de las fases fenológicas, a fin de proporcionar el riego en períodos de estrés hídrico [Sivilotti, 2005];

- Los datos de humedad de la hoja permiten una gestión precisa de los tratamientos de la viña, así como determinar qué micro-áreas con valores de humedad altos son más susceptibles a las enfermedades fúngicas [Johnson and Robinson, 2001];

- La radiación solar tiene una influencia importante sobre la biosíntesis de los compuestos fenólicos [Downey, 2006].

Una vez obtenidos los datos de los sensores, y antes de realizar la toma de decisiones, es necesario analizar los datos. En esta línea se pueden encontrar en la literatura científica gran cantidad de autores que analizan los datos de la producción [Zhang, 2002], así como investigadores que analizan los parámetros del suelo y del cultivo [Vrindts, 2003]. Una vez completadas las dos primeras fases, es posible realizar la toma de decisiones usando algunos de los paquetes de apoyo y/o sistemas expertos comentados en la sección 2.3. Destacar que los anteriores también incluyen herramientas para facilitar el análisis de los datos de la segunda fase. Finalmente, sólo resta traducir las decisiones a los actuadores (por ejemplo, válvulas de control del riego y de aplicación de fertilizante) y a la maquinaría agrícola automatizada o los operarios (laboreo del terreno, aplicación del fitosanitario, etc.).

Continuando con la relación entre la variabilidad del cultivo y la necesidad hacer uso de la AP, cabe destacar que el hecho de que exista una variabilidad no significa necesariamente que haya que aplicar tratamientos localizados.

Existen tres cuestiones clave que es necesario tener en cuenta antes de emplear la AP [Basso, 2006]: (a) determinar la variabilidad de las características del terreno y de las plantas, (b) análisis de la influencia de las variaciones anteriores en la cantidad y la calidad de la producción y (c) selección de la tecnología a emplear para tratar adecuadamente la variabilidad existente. Las respuestas a estas cuestiones permitirán decidir si la AP puede producir beneficios o no, sirviendo como indicador de si su uso es procedente o no.

En primer lugar, se debe determinar la variabilidad de las explotaciones, con objeto de poder encontrar las relaciones con la producción, en términos de cantidad y producción. Finalmente, hay que seleccionar cuidadosamente el sistema adecuado.

La monitorización de la producción es el punto de partida más lógico para introducir la AP. Si la producción no varía apreciablemente y el rendimiento es satisfactorio, el beneficio que pueda aportar la AP resultará mínimo. Por otro lado, producciones muy variables indican que las técnicas aplicadas hasta el momento no 
proporcionan las mejoras condiciones de cultivo en todos los puntos de la finca. En este último caso la práctica de la AP puede aportar beneficios, en el sentido de que minimiza las pérdidas de energía que pueda haber a lo largo de la cadena trófica.

\subsection{Tecnologías de la información y las comunicaciones en la agricultura de precisión.}

Los sistemas de producción agrícolas se han beneficiado de la incorporación de avances tecnológicos desarrollados inicialmente para otras industrias. La era industrial trajo la mecanización y fertilizantes sintetizados a la agricultura. La era tecnológica ofreció las ventajas de la aplicación de la ingeniería genética y la automatización. La era de la información trajo el potencial necesario para integrar los avances tecnológicos en la agricultura [Zhang, 2002].

Las tecnologías de monitorización permiten la identificación de plagas en los cultivos, la sequía o el incremento de la humedad, y pueden tener un efecto considerable con en el rendimiento agrícola. Disponer de dicha información en tiempo real, permite emplear actuadores que se pueden utilizar para controlar el riego, la fertirrigación y el control de plagas, con el fin de compensar las condiciones adversas presentes en el cultivo [Anurag, 2008].

El principal aporte del uso de las nuevas tecnologías en la agricultura es la generación de mapas del terreno, que representan gráficamente la variabilidad de parámetros de los cultivos, como por ejemplo, el rendimiento de la producción de los cultivos. La generación de mapas óptimos, así como de modelos de los campos agrícolas ha sido posible gracias a los sistemas remotos y de posicionamiento global. A su vez, el muestreo se realizaba generalmente a través de sensores electrónicos, tales como sondas de suelo y escáneres remotos ópticos localizados en satélites. La recopilación de esos datos en forma de bases de datos informáticas electrónicas dio lugar a los sistemas de información geográfica. Las tecnologías mencionadas, además de no tener características de tiempo real, conllevaban el uso de tecnologías costosas como el satélite de detección, e implicó el uso de mano de obra intensiva, ya que, en la mayoría de los casos, los mapas de los campos agrícolas se realizaban de forma manual.

En general, los agricultores son bastante reacios al uso de las tecnologías de la información y las comunicaciones (TIC en adelante), incluso para las aplicaciones que han demostrado beneficios económicos [Gelb, 1999][Parker, 1999]. Esta falta de interés se explica a menudo por factores tales como: bajos niveles de educación y una edad relativamente alta. Sin embargo, Ascough et al. [Ascough, 1999] encontraron complejas relaciones entre el uso de las TIC por los agricultores y su educación, edad y experiencia. Al 
parecer la principal razón para el uso de las TIC fue la falta de competencia agrícola, que hacía que la mejora en la productividad que el uso de las TIC pudiera suponer no les ofreciera ventaja frente a los costes asociados a su despliegue; mientras que los agricultores más experimentados no parecían encontrar beneficios significativos de las aplicaciones TIC disponibles, fundamentalmente debido al coste asociado a su implementación.

Kuhlmann [Kuhlmann, 1999] ofrece una explicación económica a la resistencia de los agricultores respecto al uso de las TIC: para éstos, la clave fundamental para la reducción de los costes del cultivo venía asociada más a una disminución en la profundidad de la producción (tamaño de los cultivos) y a la simplificación de las operaciones agrícolas con objeto de abaratar el coste final, antes que a afrontar un coste previo a través de un despliegue asociado a aplicaciones de las TIC dentro del cultivo. Por ejemplo, para un agricultor con esta mentalidad los costes de tratamientos fitosanitarios habituales son bajos, en comparación con el posible coste derivado del riesgo de las posibles pérdidas en caso de que el cultivo enferme, lo que hace que considere provechosa esa inversión. Sin embargo, no consideraban amortizable el coste asociado a un despliegue basado en TIC al no ser capaces de prever los beneficios que les podría reportar dicha inversión a largo plazo.

Öhlmer et al. [Öhlmer, 1998] encontraron en un estudio sobre la toma de decisiones que los agricultores no utilizaban el modelo de decisión lineal, que se implementaba normalmente en los sistemas de apoyo a las decisiones agrícolas, sino que utilizaban modelos de decisión no lineal, derivados de una continua actualización de los planes de actuación sobre el cultivo, basándose en su experiencia para llevar a cabo un análisis rápido y simple en cada instante que se requiriera una toma de decisiones, en contra de una previsión planificada de acciones destinadas a mejorar la productividad del cultivo y la aplicación gradual de acciones correctivas, frente a criterios pre-establecidos de mejora de productividad. Como consecuencia, las aplicaciones informáticas a disposición de los agricultores no se ajustaban a sus necesidades.

Uno de los efectos esperados de la Sociedad de la Información es que los intermediarios no sean necesarios y desaparezcan. La gente encuentra información, compra entradas y libros, y arregla los trámites bancarios por Internet. Ya no hay ninguna necesidad de tratar con personal cualificado que ofrezca físicamente estos servicios. En esta misma línea, se plantea si ocurrirá lo mismo con los servicios de extensión agrícola. Después de todo, el servicio de extensión recopila principalmente información de diversas fuentes y la redirecciona a medida, combinándola con la información del granjero; en principio se puede pensar que esto resulta más económico y rápido gestionándolo a través de Internet. Hoy en día, no es extraño disponer de un servidor web que ofrezca información sobre el estado de las plantas, principalmente sobre plagas y advertencia de posibles enfermedades, así como de pronósticos del tiempo [Jensen, 2000]. 
El uso de las TIC es beneficioso para la agricultura, no sólo a nivel de obtener información, sino también permitiendo acceso remoto a los cultivos con objeto de monitorizarlos y poder controlarlos en tiempo real.

En los últimos años, los avances en la detección y las comunicaciones así como la previsible reducción de los costes de implementación y funcionamiento de estos sistemas hacen prever que serán utilizados masivamente en la AP. En esta línea, se han desarrollado nuevas tecnologías inalámbricas con bajas necesidades de potencia y de capacidad de cómputo de datos, que pueden ser aplicables en la AP [Wang, 2006]. Esta tecnología, surgida gracias a los avances en los micro-sensores, la miniaturización de dispositivos, las comunicaciones inalámbricas y las redes ad-hoc se conocen como las redes de sensores inalámbricas (WSN, Wireless Sensor Networks).

Llegado a este punto, en los siguientes apartados se describirán algunos de los sistemas empleados para monitorizar en la AP desde largas distancias (satélites, teledetección) parámetros a gran escala de las explotaciones agrícolas de manera indirecta, así como sistemas a pie de campo, como por ejemplo, las redes de sensores [Stafford, 2000].

\subsection{Los sistemas de posicionamiento global y de navegación por satélites.}

Un sistema de posicionamiento es aquel que permite identificar y registrar la posición de un objeto o persona sobre la superficie terrestre o en la atmósfera. Los sistemas actualmente disponibles son los siguientes: sistema de posicionamiento global y navegación estadounidense (NAVSTAR-GPS), sistema ruso global de navegación por satélite (GLO-NASS) y servicio europeo geoestacionario de navegación (EG-NOS).

En el 78 se lanzó el primer satélite (Ver Figura 2.3a) del sistema NAVSTAR-GPS (Navigation System Time and Ranging Global Positioning System), más conocido de forma abreviada por las siglas GPS. El sistema consta de 24 satélites (ver Figura 2.3b), distribuidos en 6 órbitas localizadas a unos $20.180 \mathrm{~km}$ de la superficie terrestre. Esta distribución garantiza que cualquier punto situado en la superficie terrestre reciba en cualquier momento las ondas emitidas por, al menos, 4 satélites.

La literatura científica de este sistema es muy extensa y continua creciendo en publicaciones y congresos de diverso tipo. El sistema es la base para aplicar los tratamientos variables en los cultivos, empleados en pequeñas [Wang, 2001] y en extensas [Stafford, 1996] explotaciones agrícolas. 


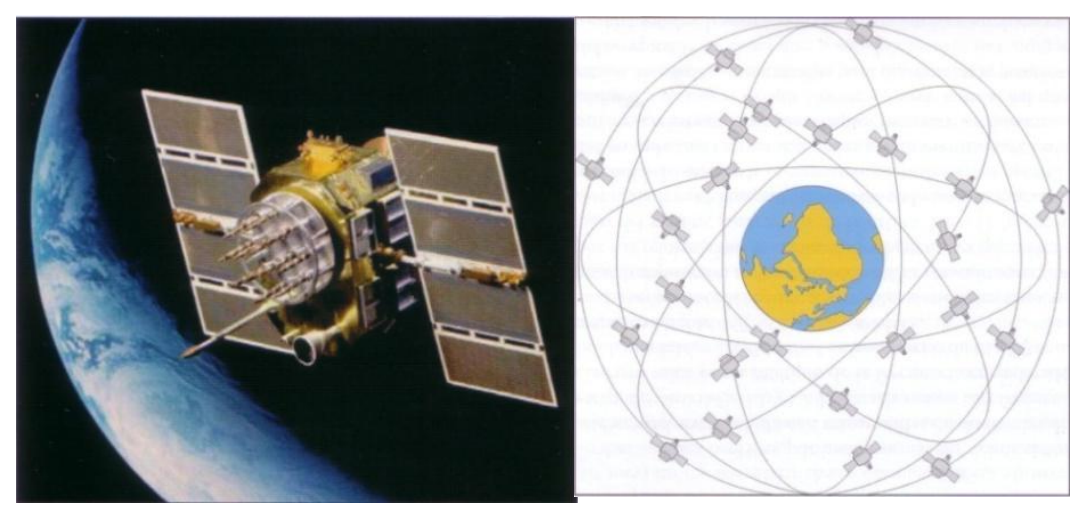

Figura 2.3. (a) Satélite NAVSTAR. (b) Constelación de los satélites del sistema GPS.

En relación con el sistema, los satélites están equipados con relojes atómicos alimentados por energía solar capaces de medir el tiempo con muchísima precisión. Esto debe ser así puesto que la hora marcada por los satélites es un dato básico para determinar la posición.

La trayectoria de las órbitas de los satélites puede sufrir variaciones debido a diferentes factores (asimetría del campo gravitatorio terrestre, radiaciones solares, etc.). Para corregir esta trayectoria, existe una red de estaciones de seguimiento en tierra que miden las desviaciones de la órbita real con la teórica y envían información a los satélites al pasar por la estación.

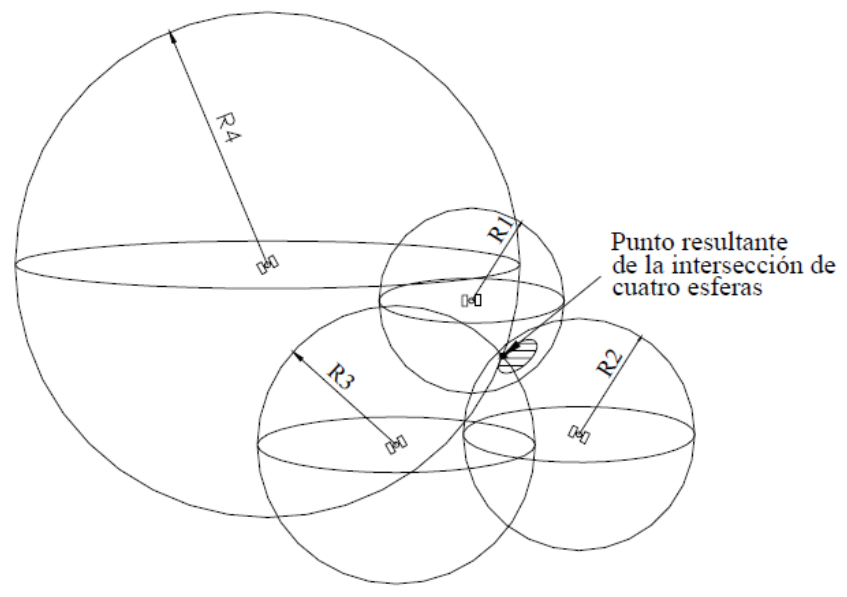

Figura 2.4. Determinación de la posición del receptor.

Los receptores GPS captan las señales enviadas por los satélites que, en cada momento, son visibles sobre el horizonte y las convierten en coordenadas del lugar donde está el receptor. Los satélites transmiten en las ondas de radio L1 y L2, de 1.575,42 y 1.227,60 MHz, respectivamente. Las señales contienen los siguientes datos: posición instantánea del satélite, tiempo marcado por sus relojes y varias características generales 
(identificación del satélite, parámetros de la órbita, etc.). Las señales tardan un determinado tiempo (retraso) del orden de centésimas de segundo en llegar del satélite al receptor. El retraso permite calcular la distancia entre el satélite y el receptor, y por tanto, el receptor se encontrará situado en una esfera con centro en el satélite y radio la distancia anterior (ver Figura 2.4). Este proceso se repite con 3 satélites más y la intersección de las 4 esferas determina el punto con la situación del receptor.

La versión rusa del sistema de posicionamiento y navegación por satélites se denomina por las siglas GLONASS (Global'naya Navigatsionnaya Sputnikovaya Sistema). Esta operativo desde el 24 de septiembre del 93, aunque no estuvo disponible para uso civil hasta el 96. Se lanzaron 24 satélites, de los cuales están disponibles actualmente 22. Los satélites están distribuidos en 3 órbitas situadas a $19.100 \mathrm{~km}$ de la superficie terrestre. Con la distribución comentada anteriormente se asegura que un receptor situado en la superficie terrestre reciba, al menos, las señales de 5 satélites. Cada satélite transmite las señales con una frecuencia propia comprendida en la banda L, determinada a partir de la frecuencias base $1.602 \mathrm{MHz}$ (L1) y $1.268 \mathrm{MHz}$ (L2) que se multiplican por una constante y por un coeficiente diferente para cada satélite.

EGNOS (European Geostationary Navigation Overlay Service) es la versión europea de los sistemas americanos WASS (Wide-Area Augmentation System) utilizados en Estados Unidos para la AP y ha sido desarrollada por la Agencia Espacial Europea. Consiste en la integración de los sistemas americanos GPS y ruso GLONASS. El sistema consta de 3 elementos: (1) una red de tres satélites geoestacionarios, (2) una red terrestre de estaciones de elaboración, distribuidas por el territorio europeo, que aplican procedimientos diferenciales diferente en cada zona particular de los datos relativos al retraso de las señales emitidas por los satélites del sistema GPS debido a la ionización de la atmósfera, y (3) red de estaciones centrales en tierra de recogida de los datos elaborados por los anteriores. El sistema empezó a emitir de formal operacional en julio de 2005 mostrando unas prestaciones excelentes en términos de precisión y disponibilidad.

El lector interesado puede encontrar más información sobre estos sistemas en [Wolfgang, 2000].

\subsubsection{Las correcciones diferenciales.}

A pesar de que la tecnología empleada en los sistemas GPS sea muy sofisticada y costosa, la determinación de la posición puede ser poco precisa debido a diversas causas, entre las que se pueden citar las siguientes: desfase entre la hora de los relojes del satélite y del receptor, desviaciones de las órbitas de los satélites, alteraciones producidas por la atmósfera terrestre, etc. A modo de comentario conviene resaltar que, hasta marzo de 2002, 
el Ministerio de Defensa de los EE. UU. introducía errores en las señales emitidas por los satélites por motivos de seguridad.

Mediante la corrección diferencial se mejora la precisión en el posicionamiento de un punto que, en el caso de no hacerse, con el sistema GPS podría generar errores de posición comprendidos entre 20 y 30 metros.

Para obtener la corrección es necesario emplear un segundo receptor, denominado estación base, situado en un punto fijo del que se conocen las coordenadas geográficas y la posición real respecto a los satélites [Cox, 2002].

La corrección diferencial puede realizarse en tiempo real y procesarse posteriormente, siendo más usado en la AP el primer método.

El sistema más habitual para realizar las correcciones diferenciales es el GPS diferencial (DGPS). Las estaciones base situadas en la tierra calculan el dato de corrección, lo transmiten a un satélite geoestacionario que, a su vez, realiza la transmisión a los receptores (ver Figura 2.5). Como se puede observar en la figura, la estación base (1) recibe las señales de los satélites GPS (2), efectúa la corrección de sus posiciones y las transmite al satélite geoestacionario (3). El receptor (4) recibe las señales GPS y la corrección diferencial. El sistema DGPS tiene la ventaja de conseguir una precisión en torno al metro, con el inconveniente de que el servicio lo gestionan empresas especializadas (OMNISTAR, LANDSTAR) a cambio del pago de un canon.

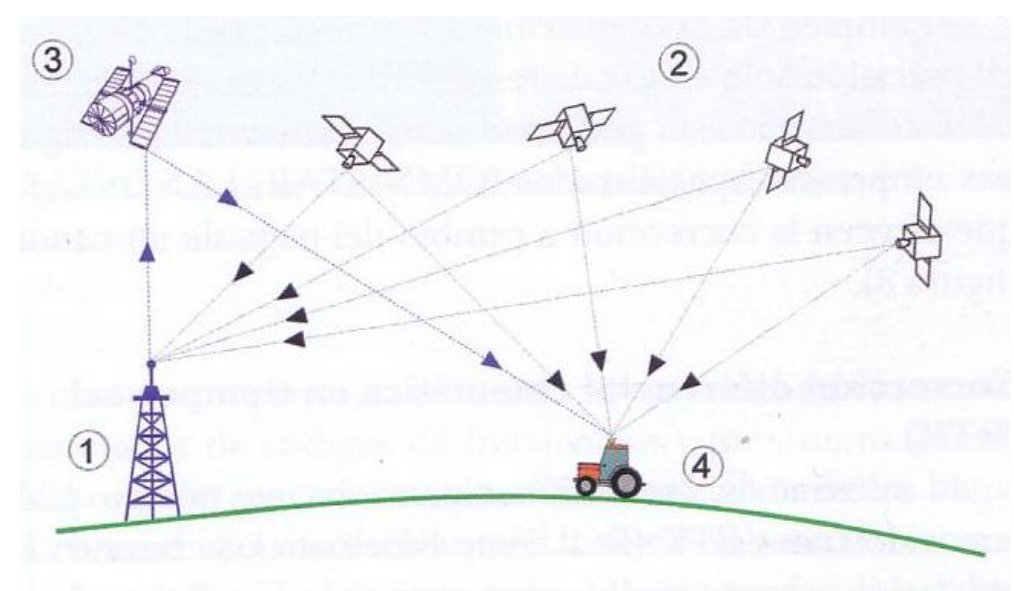

Figura 2.5. Corrección diferencial realizada con la ayuda de un satélite geoestacionario.

Otro sistema de corrección es el RTK (Real Time Kinematic). Al igual que con el DGPS existe una estación base, pero que, en este caso, envía la información de la corrección vía radio al receptor (situado a unos 3-5 km de las estación base). Usando este método, se consiguen precisiones del orden de pocos centímetros. 


\subsubsection{Aplicaciones del GPS en la Agricultura.}

Las aplicaciones de los sistemas de posicionamiento global y de navegación en la agricultura pueden ser muy diversas. No obstante, las más comunes que se pueden citar son las siguientes: determinación de los límites de la finca, guiado automático de maquinaría agrícola, asignar las coordenadas a las muestras tomadas con objeto de elaborar los mapas de producción u otra característica y determinar la actuación en cada punto. Un ejemplo de esto último, sería distribuir la dosis de fertilizantes y fitosanitarios en función de las coordenadas.

En la misma línea, los sistemas de navegación también resultan muy útiles en la agricultura. Estos sistemas son muy necesarios en explotaciones grandes a la hora de aplicar los tratamientos fitosanitarios y los fertilizantes. En estas tareas se emplea maquinaría agrícola pesada, y pequeños errores en la conducción pueden provocar que una franja del cultivo quede sin tratar (pérdidas de producción), que se aplique una sobredosis (perjuicios medioambientales y económicos), etc. Los sistemas de navegación también resultan de gran utilidad en casos concretos, como la aplicación de los herbicidas sistémicos que se deben aplicar durante el crespúsculo, así como en el guiado del vehículo cuando las condiciones atmosféricas son adversas. Por ello, no es de extrañar que numerosos investigadores trabajen en este campo.

Existen muchas publicaciones sobre la aplicación de la navegación por satélite en la agricultura. Un buena panorámica sobre este asunto está publicado en un Special Issue de la revista indexada Computers and Electronics in Agriculture [Auernhammer, 1994]. Otra referencia recomendada sobre el uso de estos sistemas en la agricultura se puede encontrar en la revisión (overview) a nivel mundial realizado por Zhang et. al [Zhang, 2002].

Por otro lado, tratando aplicaciones de los sistemas de navegación en la agricultura más concretas, Sun et. al [Sun, 2010] proponen un sistema automatizado de cultivo guiado por GPS con corrección RTK para conseguir un cultivo distribuido en hileras con el espacio optimizado. En la misma línea, Ge et. al [Ge, 2009] proponen a su vez un sistema para generar un mapa de producción de un cultivo de algodón, asociando las medidas de cantidad de producto recolectado con la posición geográfica obtenida mediante un sistema de localización GPS. Por su parte, Reyniers et. al [Reyniers, 2006] investigan un cultivo usando técnicas de AP con objeto de delimitar las zonas con diferente producción debido a la previa erosión del terreno. Para ello, unos de los métodos que llevaron a cabo fue la medida de la altitud y la pendiente del cultivo usando un sistema de posicionamiento global. 


\subsection{Los sistemas de información geográfica.}

Al aplicar los métodos de la AP en una explotación agrícola se recogen gran cantidad de datos del cultivo. A su vez, los datos están referidos a unas determinadas posiciones geográficas unívocas, gracias al uso de los sistemas de posicionamiento global. Por lo tanto, para que los datos recogidos sean de utilidad, es necesario realizar una representación gráfica adecuada, y es en este punto donde intervienen los sistemas de información geográfica (SIG en adelante).

Un SIG es un sistema formado por un equipo informático (ordenador, receptor GPS, etc.), programas, datos y personas especializadas que logran la representación y el análisis de los datos gráficos. Como resultado, se obtiene un mapa en formato digital sobre el que se sitúan elementos de los datos espaciales o atributos, que pueden estar clasificados por capas. Además, existe una base de datos asociada a los atributos con una descripción de los mismos. Al final, un SIG puede combinar datos geográficos con datos de otro tipo para generar mapas y esquemas, permitiendo al usuario recoger, gestionar e interpretar de forma planificada y sistemática la información relativa a lugares específicos.

Para obtener información específica de un SIG se puede emplear un método basado en una búsqueda de atributos o una búsqueda espacial. El primero, al tener cada atributo determinados criterios, resulta tan sencillo como recorrer la base de datos y localizar los atributos que satisfacen los criterios. Así, por ejemplo, una empresa agrícola puede localizar las parcelas (resaltarlas en el mapa digital) de su propiedad con una superficie entre 5 y 10 ha y una altitud entre 50 y $150 \mathrm{~m}$ sobre el nivel del mar. Por otro lado, la búsqueda espacial consiste en recabar información de los objetos gráficos seleccionados en el mapa.

Más información sobre los SIG, y en concreto, sobre lo que puede aportar esta tecnología en la AP, se puede encontrar en [Earl, 2000].

Mckinion et. al [Mckinion, 2001] realizan un análisis con el que llegan a la conclusión de que la AP en combinación con los modelos de simulación de los cultivos y los SIG pueden optimizar la producción de algodón, minimizando al mismo tiempo el agua de riego y los insumos de nitrógeno. Agostinho et. al [Agostinho, 2008] proponen un GIS con el fin de mejorar la evaluación de las explotaciones agrícolas de propiedad familiar que han adoptado ya sea los modelos de producción ecológicos o los de producción química. Ramos et. al [Ramos, 2007] desarrollan un sistema basado en tecnologías GPS y GIS para hacer un seguimiento del desplazamiento que sufre un cultivo de olivos debido a la erosión producida en el terreno con el paso del tiempo. 


\subsection{La Teledetección.}

La teledetección o 'remote sensing' en inglés, es el procedimiento que permite adquirir información del terreno y del cultivo a partir de las imágenes obtenidas por fotografías aéreas, desde satélites o por radiometría, utilizando una o más longitudes de onda reflejadas o emitidas por la superficie terrestre. Son, por lo tanto, métodos y técnicas de monitorización indirectos, en los que se emplean en la mayoría de los casos equipos con sensores de imagen situados en tierra o a bordo de aviones o satélites. Un ejemplo muy conocido de teledetección, son los datos obtenidos para predecir las condiciones meteorológicas a partir de los sensores instalados en los satélites.

Se emplea en la agricultura para obtener información sobre las características del terreno o sobre el estado sanitario del cultivo. Cabe destacar que es una herramienta muy poderosa para obtener datos en la AP, ya que permite obtener información de una manera relativamente rápida de grandes explotaciones agrícolas.

La teledetección unida con los SIG tiene múltiples aplicaciones en la agricultura, entre las que se pueden citar las siguientes: identificar las parcelas y los tipos de cultivo, valorar los daños por heladas, granizos, fuertes lluvias y otros efectos meteorológicos adversos para los cultivos, así como determinar el estado de la producción. Son, por lo tanto, métodos y técnicas para monitorizar cultivos a gran escala, y que no están pensados para pequeñas parcelas.

Para obtener información de los objetos se instalan sensores en diferentes vehículos especiales (ver Figura 2.6). Así, se pueden instalar sensores sobre vehículos terrestres a pie de cultivo, en aviones volando a diferentes altitudes, en vehículos espaciales y en satélites orbitando a muchos kilómetros del cultivo sobre el que se está aplicando la técnica de teledetección. Debido a los equipos de elevado coste involucrados en la teledetección, la utilización de ésta resulta ser costosa en la AP para los agricultores.

A su vez, en la teledetección se utilizan sensores con los que se obtiene la cantidad de energía y la longitud de onda de las radiaciones emitidas o reflejadas por los objetos. Estos sensores pueden ser de tipo activo o pasivo. Los de tipo pasivo aprovechan la energía del sol que incide sobre los objetos, y captan la radiación reflejada o emitida por éstos (ver Figura 2.7a). Su principal inconveniente son las interferencias producidas por la atmósfera. Por otro lado, los sensores activos se basan en emitir una radiación electromagmética que incide sobre la superficie terrestre y captar la energía reflejada con un sensor (ver Figura 2.7b). 


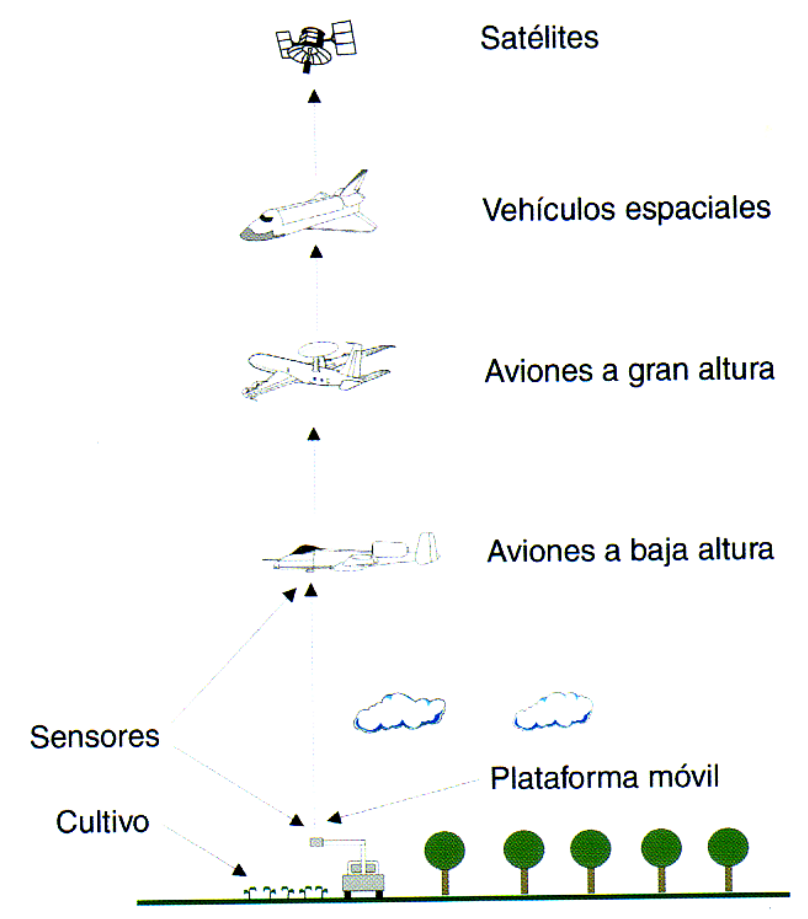

Figura 2.6. Vehículos en los que se pueden instalar sensores para teledetección.

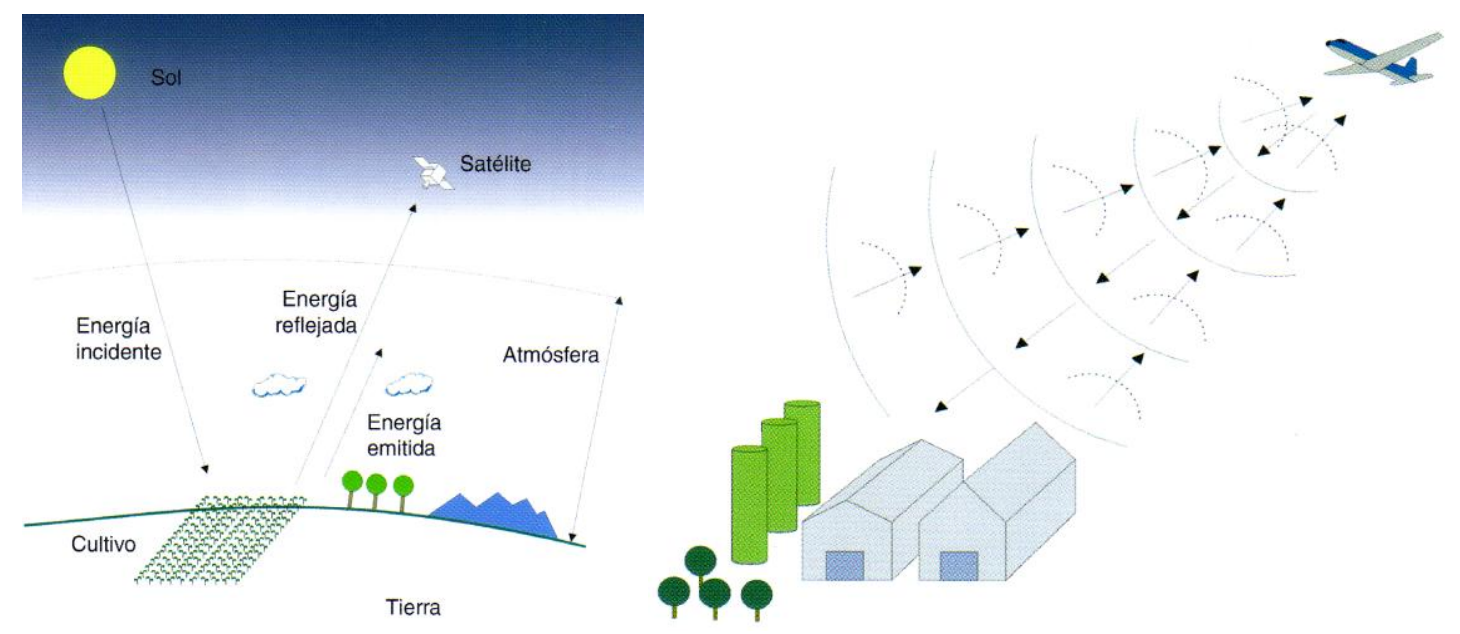

Figura 2.7. (a) Funcionamiento de un sensor de tipo pasivo. (b) Teledetección por radar.

Las tres variables que se pueden analizar en las explotaciones agrícolas a partir de las técnicas de teledetección son las siguientes: la cubierta vegetal, el suelo y el agua. La respuesta de la vegetación sobre la que incide la radiación electromagnética es útil para diferenciar las especies cultivadas en parcelas contiguas o, en el interior de una explotación, detectar los fenómenos de presencia de estrés en las plantas y deducir su vigor. La respuesta del suelo a la radiación electromagnética depende de sus propiedades orgánicas y físicas. También la cantidad de materia orgánica y su estado de descomposición influyen 
sobre la cantidad de energía absorbida y reflejada por el terreno. En definitiva, con la teledetección se puede obtener mucha información del suelo. Finalmente, como el agua absorbe por completo determinadas longitudes de onda del espectro electromagnético, es posible determinar las zonas con agua, en las que se encuentra infiltrada en el terreno, etc.

Yu et. al [Yu, 2010] utilizaron datos del sistema de teledetección MODIS (Terra Moderate-Resolution Imaging Spectroradiometer) y datos geoespaciales para estimar la productividad de la hierba y la capacidad de carga de ganado en la zona. Al final, concluyen que la integración de la teledetección y de los sistemas GIS, las condiciones espaciales y temporales de los pastos alpinos, la tendencia y las tasas de carga proyectadas podría facilitar la toma de decisiones. En el campo de la viticultura de precisión, Delenne et. al [Delenne, 2010] proponen una herramienta basada en la transformada rápida de Fourier, fácilmente integrable en un SIG, para detectar, delimitar y caracterizar viñedos. Ryu et. al [Ryu, 2009] proponen usar teledetección para proporcionar datos a un modelo de propósito con el que estimar el contenido de nitrógeno en el arroz durante en la etapa de iniciación de la panícula usando datos de tres años. Además, encontraron diferencias significativas en los datos de la vegetación, que fueron afectados por la absorción de nitrógeno del suelo según las condiciones climáticas.

\subsection{Las redes de sensores.}

En los apartados anteriores se han expuesto diferentes técnicas para obtener información sobre las explotaciones agrícolas de una forma remota y a gran escala. En muchas ocasiones, es necesario disponer además de medidas locales asociadas a determinadas variables que tengan impacto sobre la productividad del cultivo (temperatura ambiente, humedad relativa, porcentaje volumétrico y conductividad del suelo, etc.). Salvo en explotaciones de carácter experimental, la solución más habitual consiste en tomar medidas en puntos discretos mediante procedimientos manuales y, en el mejor de los casos, mediante la utilización de un número reducido de registradores de datos (data-loggers) situados en ubicaciones muy escogidas. En explotaciones experimentales de centros de investigación, se pueden encontrar despliegues más sofisticados que, en la mayoría de los casos, se llevan a cabo mediante una infraestructura de tipo cableado.

Las redes cableadas están basadas en buses que recorren las parcelas, y al que están conectados los diferentes sensores y/o actuadores. Además, suele ser habitual que los buses finalicen en un datalogger, con la función principal de almacenar los datos monitorizados del cultivo. Las redes cableadas tienen una problemática asociada importante con el cableado. Por un lado, hay que tener muy en cuenta los costes de los propios cables, de la mano de obra necesaria para su instalación en zanjas o líneas aéreas y los costes de mantenimiento por la rotura de los mismos, así como otros efectos eléctricos no deseados, 
entre los que se pueden citar el ruido eléctrico y los problemas de impedancias. Por este motivo, este tipo de tecnología es difícilmente exportable a explotaciones agrícolas reales.

Una alternativa prometedora a los sistemas cableados es el empleo de las redes de sensores inalámbricas (WSN, Wireless Sensor Networks). Con el uso de esta tecnología emergente se pretende monitorizar y gestionar en tiempo real las comunicaciones de varios dispositivos inalámbricos de bajo coste desplegados en el cultivo, lo que se traduce en reducir los tiempos de respuesta, minimizando las pérdidas de energía en la cadena trófica y, por tanto, mejorando así la productividad del cultivo.

Gracias al avance de la microelectrónica, hoy en día, se pueden diseñar y construir dispositivos inalámbricos lo suficientemente económicos para poder desplegarse en grandes redes malladas que ofrezcan una comunicación robusta gracias a la existencia de rutas redundantes [Akyildiz, 2005]. En el momento de iniciarse la presente tesis existían unos pocos dispositivos de carácter experimental para implementar redes de sensores en el laboratorio (TelosB, MicaZ, etc.), cuya utilización no era trasladable a entornos agrícolas reales. Estos dispositivos carecían de las interfaces eléctricas adecuadas para conectarse a instrumentación agrícola real y no incluían los elementos auxiliares (carcasa, sistema de alimentación, antena, etc.) necesarios que ofreciesen la robustez requerida para su operación en entornos agrícolas reales (hortícolas, viñedos, leñosos, etc.).

Uno de los objetivos de la presente tesis lo constituye el dar respuesta a esta carencia proporcionando una arquitectura robusta para trabajar en entornos agrícolas reales, que permitiera una implementación más rápida que la realizada mediante sistemas cableados. Los trabajos desarrollados se han llevado a cabo en un entorno en el que han trabajado intensamente diversos grupos de investigación: División de Sistemas e Ingeniería Electrónica (DSIE), Grupo de Investigación Suelo-Agua-Planta, Grupo de Investigación del Área de Ingeniería Telemática (AIT). Nuestro esfuerzo investigador, del grupo DSIE, se ha centrado en desarrollar una línea de investigación dedicada a abordar el estudio e implementación de plataformas hardware para entornos agrícolas.

Para estudiar la evolución de las tecnologías comentadas anteriormente a lo largo de los últimos años, se ha realizado una búsqueda en la literatura científica de los términos "Sensor Network", "Wireless Sensor Network". Además se ha realizado una búsqueda cruzada de los términos "Wireless Sensor Network" y "Precision Agriculture". Los resultados se muestran de la Figura 2.8 a la Figura 2.10. Se observa en dichas figuras que, tal y como se ha comentado anteriormente, las WSN son una tecnología emergente en la que cada vez más investigadores están confiando y obteniendo resultados satisfactorios. En consecuencia, en el siguiente capítulo se lleva a cabo un estudio de la técnica de las WSN, así como una revisión de las aplicaciones de las mismas en casos de estudio agrícolas. 


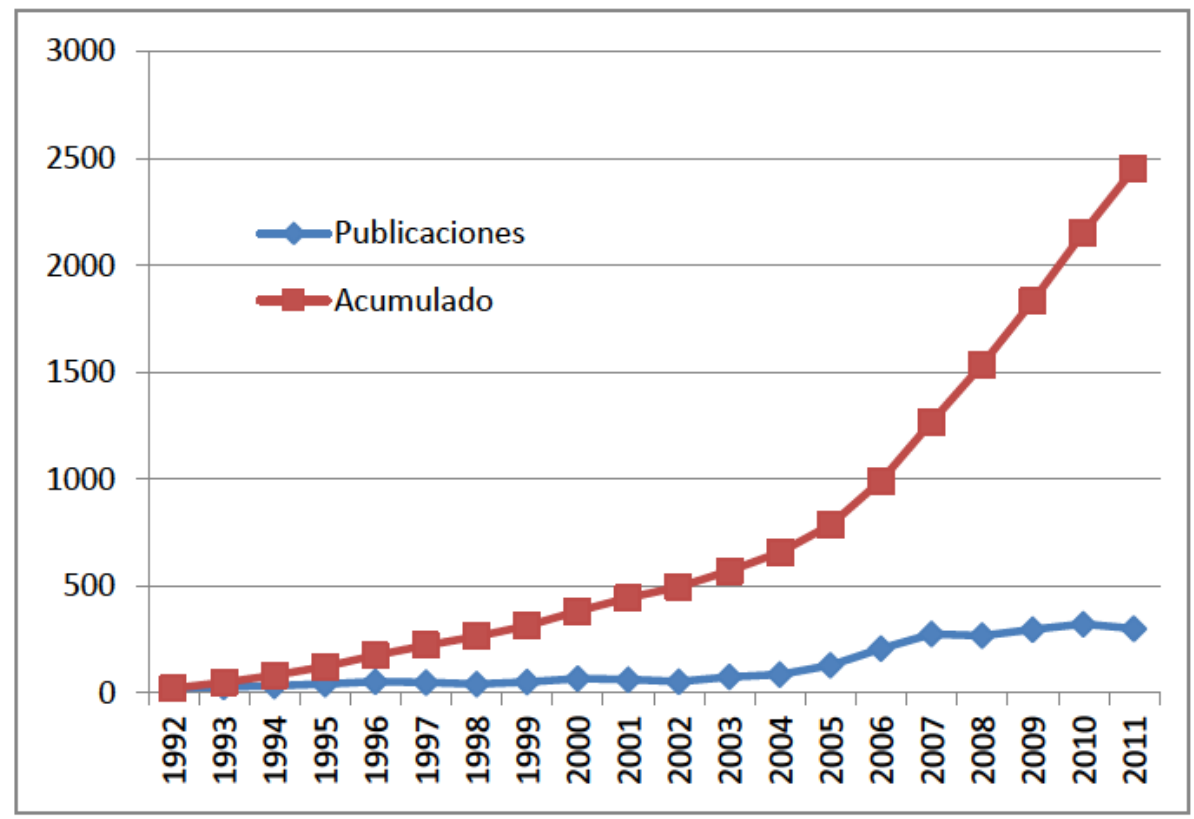

Figura 2.8. Evolución del número de publicaciones obtenido mediante búsqueda con el término "sensor network" en el SciencieDirect.

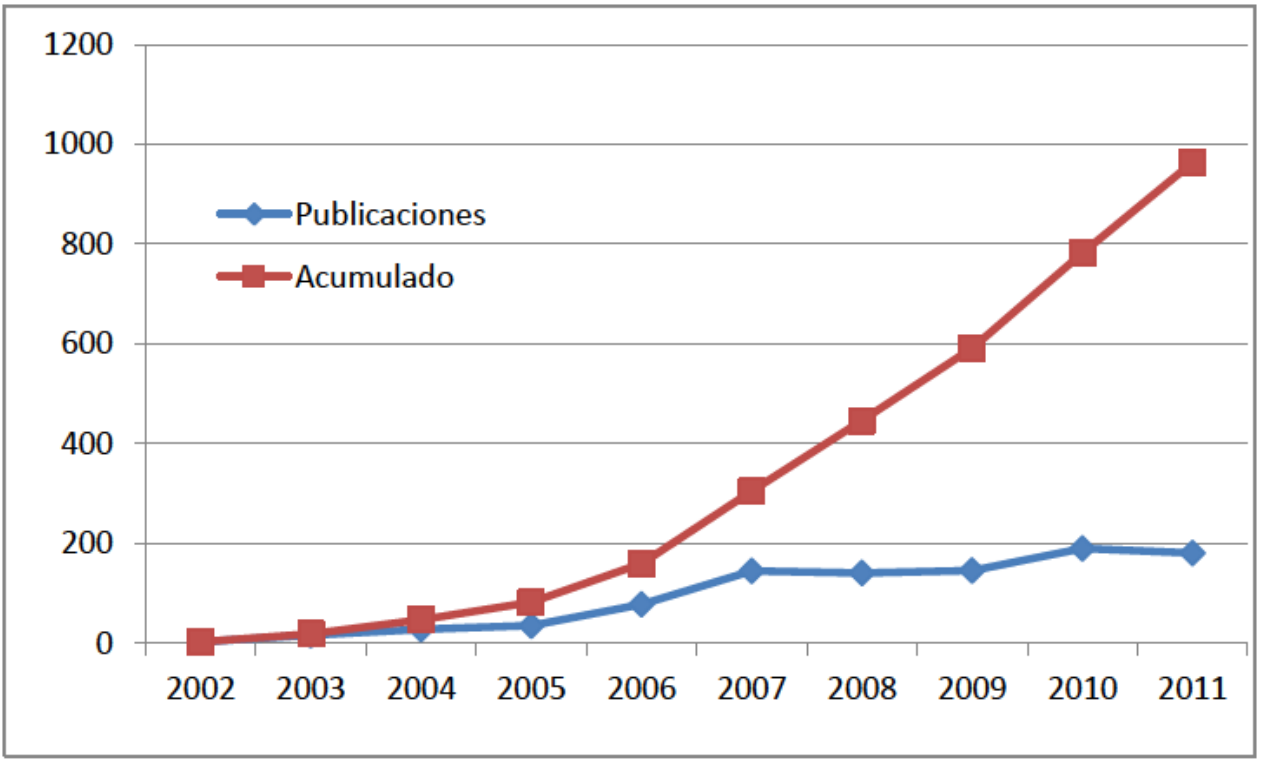

Figura 2.9. Evolución del número de publicaciones obtenido mediante búsqueda con el término "wireless sensor network" en el SciencieDirect. 


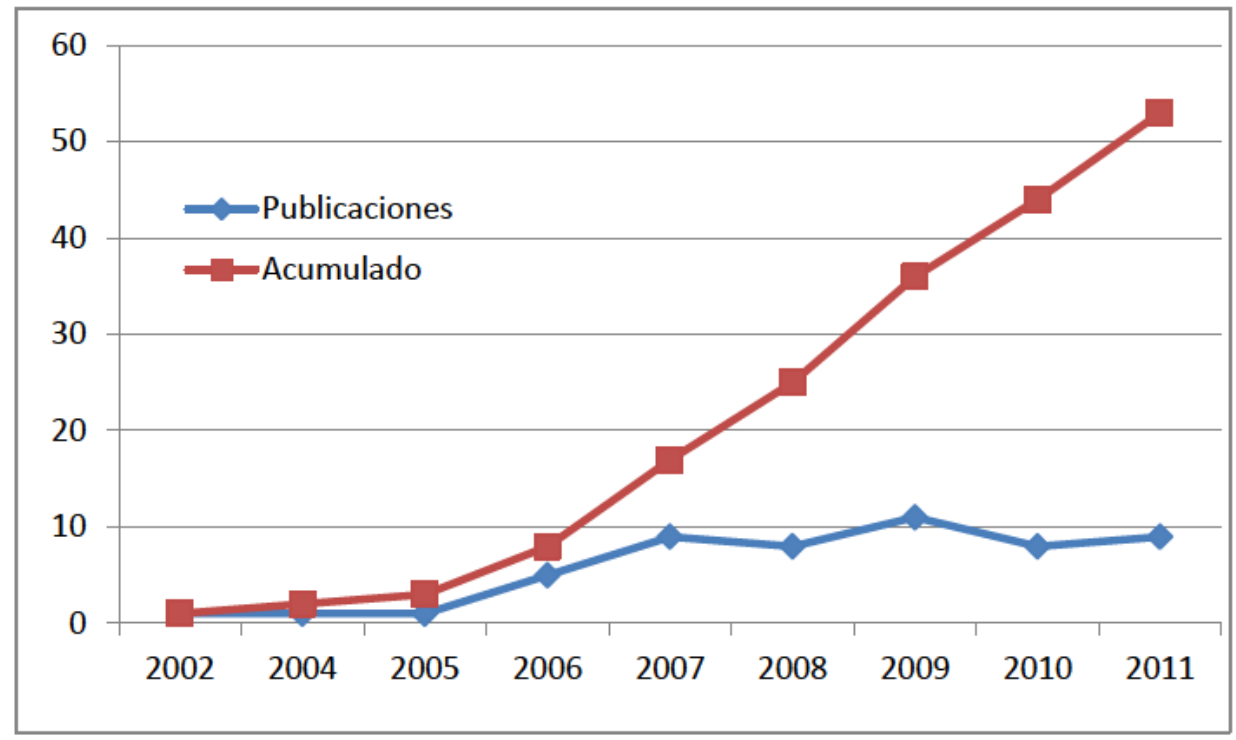

Figura 2.10. Evolución del número de publicaciones obtenido mediante búsqueda cruzada de los términos “wireless sensor network" y "Precision Agriculture" en el SciencieDirect.

\subsection{Conclusiones.}

La Agricultura de Precisión es una técnica que se caracteriza por realizar una monitorización intensiva de los cultivos. De esta forma, se pueden conocer las necesidades de una determinada zona del cultivo, aplicar los insumos necesarios, consiguiéndose así la optimización de la producción.

Para conocer las necesidades de las diferentes zonas de los cultivos y que se pueda aplicar la Agricultura de Precisión es necesario hacer uso de Tecnologías de la Información y las Comunicaciones. Estas tecnologías permiten realizar una monitorización intensiva y facilitan el análisis de los datos obtenidos.

Una tecnología que facilita la tarea de la monitorización intensiva son las redes de sensores, las cuales pueden ser cableadas e inalámbricas. Las redes de sensores cableadas se llevan usando desde el año 1990 y las inalámbricas desde, aproximadamente, el año 2000. De estos dos tipos, la tecnología más adecuada para su uso en agricultura es la inalámbrica por la reducción de costes y robustez del sistema que se consigue al eliminar el cableado.

En la presente tesis se estudiarán e implementarán soluciones hardware que constituyan una red de sensores inalámbrica con la que conseguir una de las primeras fases necesarias en la Agricultura de Precisión, la monitorización del cultivo. Por ello, en el siguiente capítulo se llevará a cabo un estudio de la técnica de las WSN, así como una revisión de las aplicaciones de las mismas en casos de estudio agrícolas. 


\section{Capítulo 3}

\section{Las Redes de Sensores Inalámbricas.}

$U$ na red de sensores inalámbrica es un conjunto de dispositivos electrónicos con tamaño reducido, elevada autonomía y baja capacidad de cómputo, que cooperan entre sí, vía radio, para llevar a cabo un objetivo común, como puede ser la monitorización de la bumedad del suelo de un cultivo. El objetivo de este capitulo es hacer una revisión del estado de la técnica de las redes de sensores inalámbricas. Para ello, en primer lugar se realizará una descripción general y, posteriormente, se describirán con mayor detalle cada uno de los elementos involucrados en la configuración de una red de sensores inalámbrica. Además, al ser objetivos de esta tesis el estudiar e implementar soluciones hardware para entornos agronómicos, se revisará en la literatura cientifica el estado de las redes de sensores inalámbricas en el campo de la agricultura de precisión. 


\subsection{Introducción.}

En el capítulo anterior, se han expuesto diferentes tecnologías de la información y las comunicaciones para obtener información sobre las explotaciones agrícolas de una forma remota y a gran escala. En muchas ocasiones, es necesario disponer además de medidas locales asociadas a determinadas variables que tengan impacto sobre la productividad del cultivo (temperatura ambiente, humedad relativa, porcentaje volumétrico y conductividad del suelo, etc.). En estos casos, tal y como se propone en esta tesis, una solución idónea consiste en el despliegue de una red de sensores a lo largo de la parcela a monitorizar. Por ello, en este capítulo se presentará una revisión del estado de la técnica de las redes de sensores inalámbricas y se detallarán características de la tecnología, tales como: las topologías de red, protocolos de comunicación y las soluciones hardware actuales, así como una revisión del estado de la cuestión en el ámbito de la agricultura.

\subsection{Las redes de sensores inalámbricas.}

La recolección de datos es una tarea indispensable en un gran número de aplicaciones. Ejemplos de ésto son las aplicaciones ambientales para el estudio de las condiciones de un terreno o las militares para control de equipamiento o tropas. En el pasado, tanto operadores humanos de forma manual, como equipos sensores conectados a una central mediante enlaces cableados eran los actores dedicados a la recogida de datos. Las dos opciones presentaban problemas: las mediciones realizadas por operadores humanos eran costosas en tiempo, lo que restringía la cantidad de ubicaciones en las que tomar medidas. Por otro lado, el uso de sensores cableados obligaba al despliegue de grandes cantidades de cable e impedía la toma de medidas en lugares de difícil acceso. Con los avances realizados en los campos de la electrónica y las comunicaciones inalámbricas se pudieron solventar estos problemas, abaratando el uso de dispositivos inalámbricos para adquisición de datos, lo que ha permitido el auge de las redes de sensores inalámbricas. Esta tecnología, reconocida en un informe del MIT (Massachusetts Institute of Technology) como una de las diez tecnologías emergentes que cambiarán el mundo [Huang, 2003], constituye una elección muy oportuna para los casos en los que se deben recoger datos de múltiples ubicaciones. Su uso permite el despliegue de un gran número de dispositivos sensores de bajo coste que forman una red inalámbrica robusta, escalable y adaptable a los cambios en el entorno o en su topología.

A pesar de su rápido desarrollo, actualmente las redes de sensores siguen siendo una tecnología emergente. Prueba de ello, es que en la lista de las 25 publicaciones más consultadas de junio a marzo de 2010 en el área de ingeniería del ScienceDirect, hay 3 publicaciones dedicadas a redes de sensores, y más concretamente del tipo revisión o 'survey'. 
Que las WSN sean una tecnología emergente, es debido en, gran medida, a los recientes avances en los sistemas micro-electro-mecánicos (MEMS, Micro-Electro-Mechanical Systems), las comunicaciones inalámbricas y la electrónica digital. Estos avances han permitido el desarrollo de nodos sensores multifuncionales de bajo coste, baja potencia, pequeño tamaño y que permiten comunicarse inalámbricamente dentro rangos cada vez mayores.

Una WSN se puede definir como: "un conjunto de dispositivos inalámbricos o motes que cooperan entre si para llevar a cabo un objetivo común, como puede ser la monitorización de la bumedad del suelo en una explotación agricola". Por este motivo, es posible considerar las WSN como sistemas distribuidos compuestos de dispositivos inalámbricos con baja capacidad de cómputo que solventan problemas sin requerir un equipo centralizado de procesamiento voluminoso y caro. En esta misma línea, Camilli et. al [Camilli, 2007] definen una WSN como: "redes de pequeños nodos sensores, con capacidad limitada de procesamiento, que incluyen los sensores y la electrónica especifica de acondicionamiento con los anteriores, y que se comunican dentro de pequeños alcances, normalmente usando una frecuencia de radio". Un concepto que va más allá de las WSN es el de las redes de sensores inalámbricas y actuadores (WSAN, Wireless Sensor and Actor Networks) [Akyildiz, 2004], que puede ser considerado como una extensión de las WSNs tradicionales. En este tipo de redes la función de los nodos sensores y actuadores es recoger datos del entorno y reaccionar adecuadamente a los eventos que se producen. La zona sensor-actuador define el área donde los nodos sensores y actuadores están distribuidos. La estación base central, conocida también como 'sumidero' (sink), monitoriza y coordina toda la actividad de la red. Cuando un nodo sensor observa un determinado fenómeno, transmite sus resultados a un nodo actuador cercano. El nodo actuador procesa toda la información recibida e inicia la respuesta apropiada o procesa y pasa la información al sumidero. Éste puede procesar la información recibida y consecuentemente enviar comandos adicionales a los nodos actuadores para obtener más información, o reaccionar, al evento detectado.

Los nodos actuadores son dispositivos ricos en recursos equipados con potentes procesadores, transceptores de radio de largo alcance, y equipados de sistemas de alimentación de mayor autonomía, y basados muchos de ellos en energías renovables. Los nodos actuadores también pueden tener capacidad de movimiento dentro del área de cobertura de los nodos sensores. En general, el número de nodos sensores sobrepasa al número de nodos actuadores en una cantidad considerable. Hay bastantes características que definen las WSANs [Stojmenovic, 2005]. Estas incluyen los requerimientos de tiempo real y la coordinación:

- Requerimientos de tiempo real: dependiendo de la aplicación, puede ser necesario que los nodos actuadores de una WSAN respondan dentro de unos plazos 
determinados a los eventos detectados. Por ejemplo, en una aplicación de monitorización de incendios, si se detecta un fuego, un conjunto de acciones tienen que ser llevadas a cabo lo más rápidamente posible.

- Coordinación: en una red de sensores inalámbrica, el proceso de recolección de los datos está coordinado por un dispositivo central, como una estación base o sumidero. En una WSAN, hay que llevar a cabo una coordinación en tres niveles: sensor-sensor, actuador-sensor y actuador-actuador. Los nodos sensores trasladan los eventos detectados a los nodos actuadores, que toman la acción adecuada. Esto puede incluir coordinar la respuesta de actividades con otros nodos actuadores, proporcionando instrucciones adicionales para los nodos sensores cercanos, o enviar de vuelta la información la información sensorizada del evento a la estación base central.

Las WSN están compuestas por numerosos nodos sensores, que están densamente desplegados en el interior de un fenómeno o muy cerca del caso de estudio a supervisar. Las posiciones de los nodos sensores no tienen por qué ser pre-determinadas. Esto permite realizar despliegues aleatorios en terrenos inaccesibles o en operaciones de emergencia. Por otra parte, esto implica que los protocolos y los algoritmos de la red deben poseer capacidades de auto-organización. Otra característica única de las redes de sensores es el esfuerzo conjunto que realizan los nodos sensores. Los nodos sensores están equipados con un elemento de cómputo, que en lugar de enviar todos los datos brutos de los sensores, tiene que procesarlos localmente mediante cálculos simples, con el fin de transmitir sólo la información necesaria y reducir las transmisiones dentro de la red [Akyildiz, 2002].

Las características descritas anteriormente, garantizan una amplia gama de aplicaciones para los nodos sensores o 'motes' que componen una WSN. Así, se pueden encontrar aplicaciones (ver Figura 3.1) de las WSN en hospitales [Handle, 2006], entornos hostiles [Song, 2008], aplicaciones de control remoto [Xia, 2007] y seguimiento de objetos [Wang, 2007], entre otras. Akyildiz et. Al [Akyildiz, 2002] prevén que, en el futuro, las redes inalámbricas serán una parte integral de nuestra vidas, más de lo que son actualmente los PCs.

La Figura 3.2 muestra una posible clasificación de las aplicaciones de las WSN [Yick, 2008]. Según ésta, es posible clasificarlas en dos categorías: monitorización (monitoring) y el seguimiento y control de objetos (tracking). Las aplicaciones de monitorización incluyen la monitorización de habitats interiores/exteriores, de pacientes hospitalarios, procesos industriales e infraestructuras y militares, entre otras. Las aplicaciones de seguimiento incluyen el seguimiento de objetos, animales, seres humanos y vehículos. 
Figura 3.1. Algunas aplicaciones de las WSN.

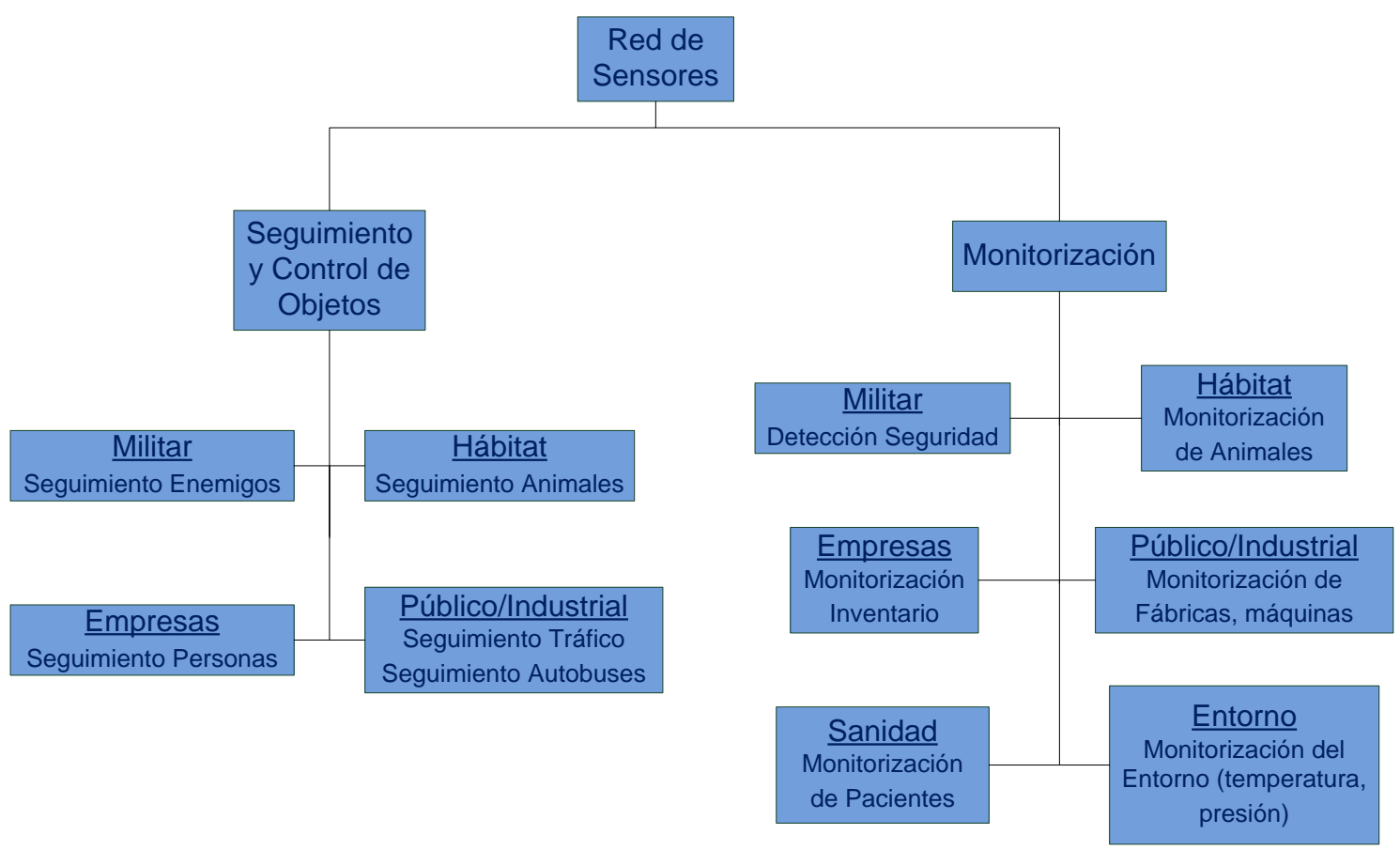

Figura 3.2. Revisión de aplicaciones de las WSN.

En relación con los elementos que componen una WSN (ver Figura 3.3), caben destacar: (1) los nodos sensores, (2) el nodo sumidero, y (3) la red inalámbrica propiamente dicha. A su vez, los nodos sensores están compuestos por un dispositivo de cómputo como un microcontrolador o un microprocesador, los sensores y/o actuadores correspondientes, elementos de almacenamiento temporal y permanente, un sistema de alimentación y un transceptor de radio. En cuanto a sus características, son dispositivos que procesan algoritmos sencillos, ya que requieren de una elevada autonomía. Por otro lado, el nodo sumidero hace de puente entre la red inalámbrica y el equipo que hace de servidor. Finalmente, hay que mencionar la red inalámbrica propiamente dicha está basada 
normalmente en un estándar de comunicaciones como el IEEE 802.15.4 [IEEE, 2006] o ZigBee [ZigBee, 2007; Baronti, 2007].

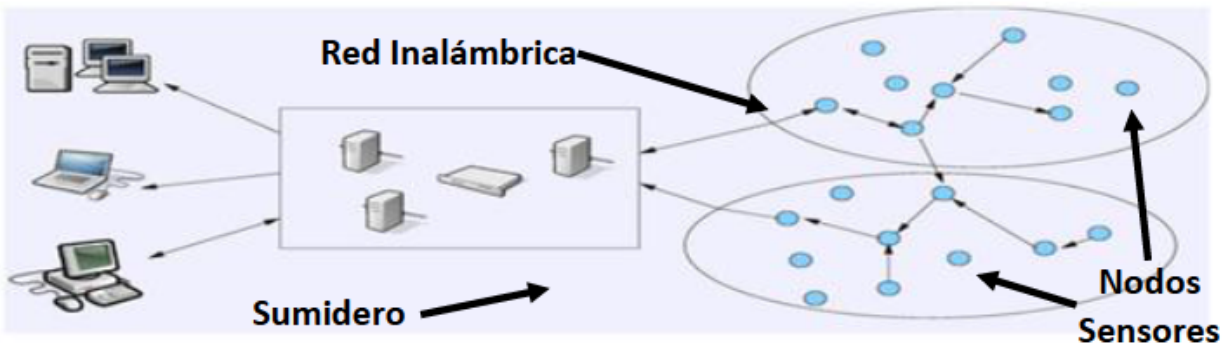

Figura 3.3. Elementos de una WSN.

Para finalizar este apartado, cabe señalar que, en el campo de las WSN, se están realizando trabajos de investigación principalmente en tres líneas diferentes: (1) topologías de red y los protocolos de comunicación, (2) implementación de nuevas soluciones hardware y (3) en proponer nuevas herramientas para diseñar WSN. En principio se podría pensar que estas tres líneas de investigación no tienen un denominador común. Sin embargo, el principal objetivo que se persigue con ellas es que los nodos sensores (motes) tengan un bajo consumo (o una elevada autonomía), que es una de las principales restricciones a la hora de diseñar una WSN. Otras restricciones importantes para diseñar una WSN son: el tamaño, la robustez y el coste de los motes. En las siguientes secciones se estudiará con más profundidad cada una de las líneas de investigación mencionadas anteriormente.

\subsection{Características de una red de sensores.}

Las WSN tienen una serie de características que hay que tener en cuenta en la fase de diseño. Entre ellas, se pueden mencionar las siguientes: tolerancia a fallos, escalabilidad, costes de producción, entorno de trabajo, topología de red, restricciones hardware, medio de transmisión y el consumo de potencia. Estos factores son importantes porque sirven como guía a la hora de diseñar un protocolo o un algoritmo para una red de sensores. Además, estos factores pueden ser usados para comparar diferentes sistemas [Akyildiz, 2002].

Tolerancia a fallos: los nodos sensores pueden dejar de funcionar por un fallo de alimentación, de la electrónica, daño en el dispositivo y las interferencias del entorno. Sin embargo, el fallo de uno o varios motes no debería provocar una pérdida de funcionamiento general en la WSN, sino que debería seguir funcionando. Esto es la fiabilidad (reliability) o la tolerancia a fallos. La tolerancia a fallos es la capacidad de mantener la operatibilidad de una WSN sin ninguna interrupción, a pesar de que uno o varios nodos no estén funcionando correctamente [Hoblos, 2000; Shen, 2001]. Resulta evidente que la tolerancia a fallos está 
condicionada por el entorno de trabajo. Así, no deberá ser tan crítica para una WSN que monitorice la temperatura y la humedad relativa en el interior de un edificio. Sin embargo, en aplicaciones militares a pie de batalla, la tolerancia a fallos tiene que ser elevada porque los datos de los sensores son críticos y los nodos pueden ser destruidos por acciones hostiles.

Densidad y Escalabilidad: el número de nodos sensores desplegados para el estudio de un fenómeno pueden ser teóricamente del orden de cientos o miles. Además, inicialmente se pueden desplegar unos cientos de nodos y meses después otros cientos. Por lo tanto, las WSN tienen que ser escalables. La densidad puede estar comprendida entre pocos y cientos nodos en una región, que puede ser menor de $10 \mathrm{~m}$ en diámetro [Cho, 2000]. La densidad de nodos depende de la aplicación, así, por ejemplo, para diagnóstico de máquinas la densidad puede ser de unos 300 motes en un espacio de $5 \mathrm{~m}^{2}$ y la densidad en el seguimiento y control de vehículos de unos 10 motes por región [Shih, 2001]. En aplicaciones de monitorización ambiental, el número de nodos sensores suele estar comprendido entre 25 y 100 por región o zona [Cerpa, 2000].

Costes de producción: una WSN consiste en un elevado número de nodos y por ello, es importante que el coste individual de cada nodo no sea muy elevado. Si el coste de la WSN es mayor que el necesario para desplegar soluciones tradicionales, el uso de una WSN pueda no estar justificado. Rabaey et. al [Rabaey, 2000] citan que para que las WSN sean factibles, el coste de un nodo debe ser mucho menor que $1 \$$. Pero hay que tener en cuenta que un mote no tiene solamente una unidad de sensado y procesamiento, sino que puede incluir entre otros elementos hardware específico de localización y generación de energía. En conclusión, se puede decir que el coste de un nodo sensor es un asunto complicado y que en ocasiones es difícil conseguir las funcionalidades necesarias para un precio inferior a $1 \$$. Por tanto, el coste dependerá de la aplicación.

Restricciones hardware: un nodo está constituido por cuatro unidades básicas: sensorización, procesamiento, transmisión y alimentación. Para algunos autores, la combinación de estas cuatro unidades debería tener el tamaño de una caja de cerillas [Intanagonwiwat, 2000]. El tamaño requerido podría ser menor que incluso $1 \mathrm{~cm}^{3}$ [Pottie, 2000], de manera que un nodo podría estar suspendido en el aire. Además del tamaño, hay otras características que deberían tenerse en cuenta. Según Kahn [Kahn, 1999], los nodos deberían: (1) tener un consumo de potencia relativamente bajo, (2) ser autónomos y funcionar sin supervisión y (3) tener capacidad de adaptación el medio ambiente. La unidad de alimentación es un elemento muy importante, ya que en algunas ocasiones los nodos son inaccesibles, y el tiempo de vida del nodo depende de la autonomía del sistema de alimentación. Además, la potencia es un recurso limitado debido a las restricciones de tamaño. Sin embargo, es posible extender el tiempo de vida del nodo mediante la obtención de energías alternativas [Rabaey, 2000a]. 
Topología de red: el difícil acceso a los nodos que funcionan de manera autónoma y sin supervisión, y que son propensos a sufrir fallos, hace que el mantenimiento de la topología sea una tarea complicada. Desplegar un elevado número de nodos con amplia densidad requiere manejar cuidadosamente el mantenimiento de la topología. Akyildiz et. al [Akyildiz, 2002] analizan el mantenimiento y cambio de la topología en tres fases: (1) fase previa al despliegue y la de despliegue, (2) la fase posterior al despliegue y (3) fase de redespliegue o de añadir nodos adicionales. En la primera fase, los nodos se pueden desplegar en masa o uno a uno. En relación con el método de despliegue, éstas pueden ser muy variadas. Se pueden lanzar desde un avión, con artillería o ser colocados físicamente por un humano. En esta fase es necesario establecer un plan de despliegue, con el fin de reducir los costes de la instalación y promover la auto-organización y la tolerancia a errores, entre otros factores. En relación con la segunda fase, es habitual que la topología de la red cambie debido a cambios en los nodos [Intanagonwiwat, 2000; Meguerdichian, 2001]. Entre los cambios posibles, se pueden citar los siguientes: cambio de posición, aparición de un nuevo obstáculo, la energía disponible y un malfuncionamiento de los nodos. La tercera fase hace referencia a que se pueden añadir nuevos nodos en la red y sustituir los nodos defectuosos. Esto obliga a que la red tenga capacidad de reorganización.

El entorno de trabajo: los nodos sensores se pueden desplegar en el interior de grandes máquinas, en el fondo de un océano, dentro de un tornado, en el campo de batalla junto a las líneas enemigas, en grandes invernaderos, en un lugar contaminado química o biológicamente, etc. Esta lista da una idea de las condiciones extremas que tienen que soportar algunas WSN y de la complejidad del diseño físico de las mismas asociada.

El medio de transmisión: los enlaces de los nodos sensores se pueden establecer por radio, infrarrojos o con tecnología óptica, estando la mayoría del hardware actual basado en circuitos de radio frecuencia (RF, Radio Frecuency). Sin embargo, se pueden encontrar diseños en los que se usa tecnología óptica y espejos para establecer las comunicaciones entre los nodos sensores [Kahn, 1999]. En los diseños RF, es muy importante seleccionar el medio de transmisión adecuado. Esto se traduce en escoger la frecuencia en la que transmitirán los dispositivos. Una opción es establecer los enlaces inalámbricos usando una de las bandas ISM (Industrial, Scientific and Medical), que no requieren licencia en la mayoría de países (ver Figura 3.4). Para las WSN es muy común encontrar aplicaciones en la banda ISM de 2,4GHz a nivel mundial, en la de $433 \mathrm{MHz}$ en América y usando la de $868 \mathrm{MHz}$ en Europa. Cabe destacar que, a menor frecuencia de trabajo, se irradian señales de mayor potencia, consiguiéndose alcances mayores entre nodos sensores. Sin embargo, conforme disminuye la frecuencia, la tasa de datos que se puede enviar por segundo (throughtput) es menor. Las WSN requieren un transceptor de tamaño reducido, bajo coste y muy bajo consumo energético. Según Porret et al [Porret, 2000], ciertas restricciones a nivel de hardware y la compensación entre la eficiencia de la 
antena y el consumo de potencia limitan la elección de una frecuencia de trabajo elevado. Ellos proponen el uso de la banda ISM de $433 \mathrm{MHz}$ en Europa y la banda ISM de $915 \mathrm{MHz}$ en América del Norte. Más información sobre el diseño de módulos de radio que operen en estas bandas se puede encontrar en [Favre, 1998; Melly, 1999].

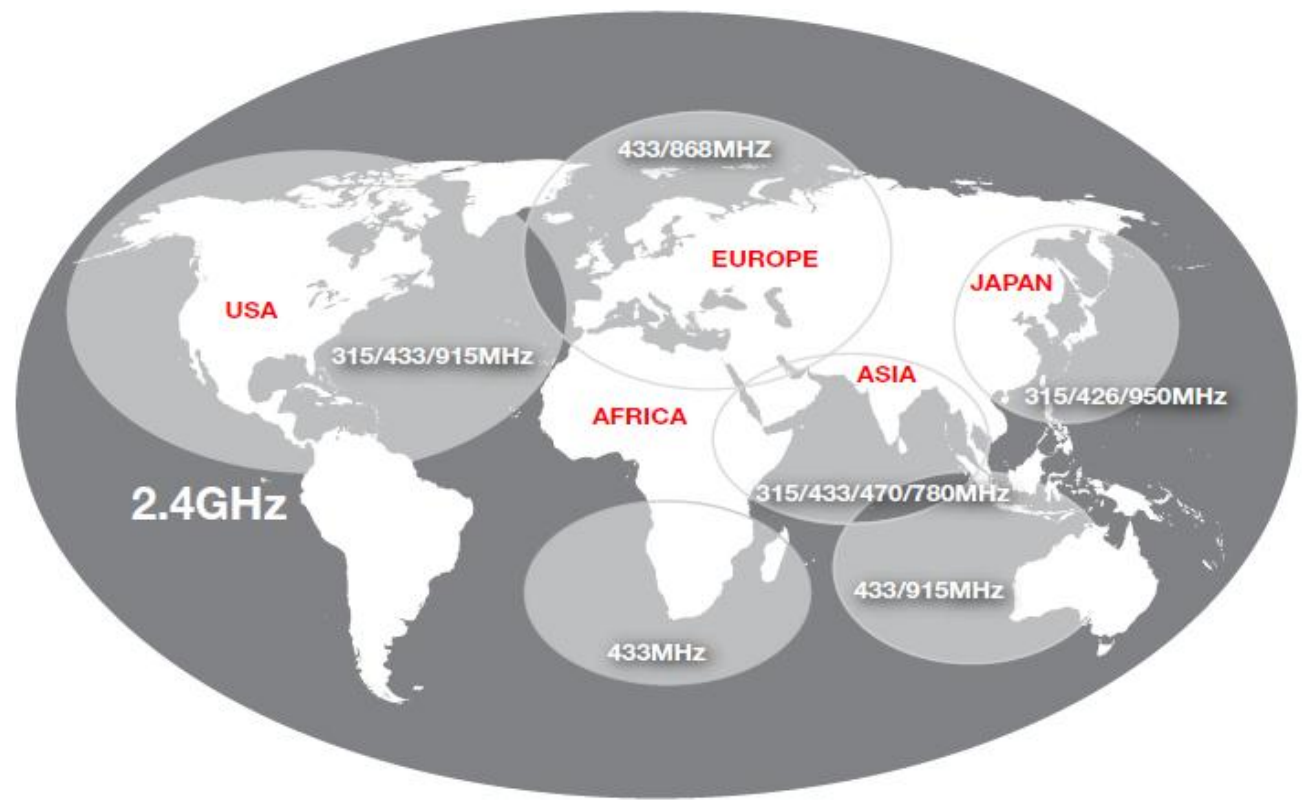

Figura 3.4. Distribución de las bandas ISM en el mundo.

El consumo energético: en otras redes 'ad-hoc' y móviles, el consumo energético ha sido un factor importante de diseño, pero no el más ponderado, simplemente porque los elementos de potencia pueden ser reemplazados por el usuario. Sin embargo, en las WSN el consumo energético es un factor muy importante, directamente relacionado con el tiempo de vida de la red. Por ejemplo, en una red 'ad-hoc' de tipo 'multihop', cada nodo realiza funciones de proporcionar datos y realizar el ruteado de mensajes en la red. En este caso, un mal funcionamiento de unos pocos nodos puede causar cambios significativos en la topología y que sea necesario reorganizar la red. Por ello, el consumo energético tiene una especial importancia. Éste puede ser debido a tres aspectos: sensorización, comunicaciones y procesamiento de datos. En el primer caso, dependerá mucho de los sensores y/o actuadores empleados y será particular para cada aplicación concreto. A pesar de ello, el mayor gasto energético se realiza con las comunicaciones, que engloban las transmisiones y las recepciones de datos. En esta línea, como las WSN se caracterizan por una baja de transmisión de información, los nodos sensores mantienen sus transceptores de radio en modo de bajo consumo la mayor parte del tiempo, y como el tamaño de los paquetes de datos es reducido, el consumo del encendido del módulo radio penaliza más que el tiempo que el transceptor está encendido. En [Shih, 2001] y [Porret, 2000] los autores presentan una formulación para obtener el consumo de potencia de la radio y discuten el diseño de un módulo de radio de pequeño tamaño, bajo coste y muy bajo consumo, respectivamente. Finalmente, como se ha comentado anteriormente, el consumo 
necesario en el procesamiento de datos es muy inferior al necesario en las comunicaciones. El ejemplo descrito en [Pottie, 2000] corrobora lo mencionado. En la misma línea, el consumo del procesamiento de datos dependerá del tipo de procesador y de la complejidad de los algoritmos a ejecutar. Por ello, para reducir el consumo en [Min, 1995; Pering, 1998] abogan por un escalado dinámico de la tensión de alimentación y la frecuencia en función de la tasa de procesamiento.

\subsection{Topologías de red y protocolos de comunicación.}

Una WSN debe funcionar de manera autónoma, aunque ocurran situaciones como el fallo de unos o varios nodos sensores o la adición de nuevos nodos en la red. Además, en determinadas aplicaciones, los motes se pueden encontrar desplegados en lugares de difícil acceso. Por ello, los nodos tienen que funcionar de manera autónoma y tener la máxima autonomía posible, que se traducirá en un tiempo de vida mayor en la WSN. En esta línea, es necesario utilizar topologías de red, así como estándares y protocolos de comunicación que garanticen lo anteriormente expuesto. Así, la mayoría de casos de estudio resueltos con WSN están basados en RF y siguen el estándar IEEE 802.15.4 [IEEE, 2006; Howitt, 2003; Gutierrez, 2001] y los protocolos de alto nivel definidos en la especificación ZigBee [Baronti, 2007], que están construidos sobre la subcapa MAC del estándar anterior.

\subsubsection{El estándar IEEE 802.15.4.}

El comité de nuevos estándares del IEEE (NesCom, IEEE New Standards Committee) estableció un grupo de trabajo en diciembre del año 2000, para comenzar el desarrollo de un estándar con baja tasa de transmisión de datos en redes inalámbricas de área personal. El grupo de trabajo 4 (TG4, Task Group 4) del IEEE 802.15 se encargaba de investigar una solución con baja velocidad de datos, con una duración de la batería de varios meses, o años, y de baja complejidad. A su vez la solución debía operar en una banda de frecuencia internacional y sin licencia. Las aplicaciones potenciales de comunicación del estándar serían los sensores, juguetes interactivos, tarjetas inteligentes, mandos a distancia, y en domótica. El TG4 se puso en estado de hibernación en la reunión de marzo 2004 después de formar un nuevo grupo de trabajo (TG4b). El nuevo TG4b completó su trabajo con la publicación de la revisión 2006 del estándar IEEE 802.15.4, que es compatible con la del 2003. Así, nuevos dispositivos 2006 pueden operar en una red 2003.

El estándar IEEE 802.15.4 define la capa física (PHY, Physical) y la especificación de la subcapa del control de acceso al medio (MAC, Medium Access Control) para dispositivos fijos, portátiles o móviles sin batería o con requerimientos de consumo para baterías 
limitadas que operen en redes inalámbricas personales de área local con tasas bajas de envío de datos (LR-WPAN, Low Rate-Wireless Personal Area Network). Las WPANs son usadas para intercambiar información sobre distancias relativamente cortas. A diferencia de las redes inalámbricas de área local (WLAN, Wireless Local Area Network), las conexiones realizadas a través de una WPAN requieren de infraestructuras muy sencillas e incluso de ninguna infraestructura. Esta característica permite implementar soluciones de pequeño tamaño, eficientes energéticamente y económicas para una amplia gama de dispositivos.

La capa física proporciona una interfaz entre la subcapa MAC y el canal de radio, a través del firmware y el hardware de RF. Conceptualmente la PHY (ver Figura 3.5) incluye una entidad de gestión denominada PLME (Physical Layer Management Entity). Esta entidad proporciona las interfaces de administración de servicios de la capa a través del cual las funciones de gestión de la capa pueden ser invocadas. La PLME también es responsable de mantener una base de datos de los objetos gestionados relacionados con la PHY. Esta base de datos se denomina la base de información PHY PIB (PIB, PAN Information Base). La PHY proporciona dos servicios: el servicio de datos PHY y el servicio de interfaz de gestión de la PHY, que está ligado con un servicio de acceso puntual (SAP, Sevice Access Point) de la entidad de gestión de la capa física (PLME, Physical Layer Management Entity), conocido por la siglas PLME-SAP. El servicio de datos de la PHY permite realizar transmisiones y recepciones de unidades de datos del protocolo PHY (MPDU, MAC Protocol Data Unit) a través del canal físico de radio. El PLME-SAP permite el transporte de comandos de gestión entre MLME (MAC Sublayer Management Entity) y la PLME. En definitiva, la PHY define las características físicas y funciones del enlace inalámbrico, entre las que se pueden citar las siguientes: bandas de frecuencia y número de canales de cada banda, la potencia de transmisión, la posibilidad de habilitar y deshabilitar el módulo de radio y la selección del canal. En relación con las bandas de frecuencia, el estándar define 16 canales en la banda disponible a nivel mundial de $2.450 \mathrm{MHz}, 30$ canales en la banda de $915 \mathrm{MHz}$ disponible en Norte América y 3 en la banda disponible en Europa de 868MHz.

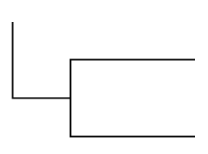

Figura 3.5. Modelo de referencia de la PHY [IEEE, 2006].

La subcapa MAC (ver Figura 3.6) proporciona dos servicios: el servicio de datos MAC y el servicio de gestión de la MAC, ligado con el servicio de acceso puntual de la 
entidad de gestión de la subcapa MAC (MLME-SAP, MAC Sublayer Management EntityService Access Point). El servicio de datos MAC permite la transmisión y recepción de MPDUs a través del servicio de datos PHY. Esta subcapa tiene, entre otras, las siguientes características: gestión de las balizas (beacons), acceso al canal, asociación, diasociación. Además, la subcapa MAC proporciona servicios para la aplicación de mecanismos de seguridad adecuados en las transmisiones de la aplicación. Los 'beacons' son tramas especiales de sincronización que, si están habilitadas, el coordinador de la red envía periódicamente a los nodos sensores, con el fin de tener la red sincronizada y evitar colisiones en las transmisiones.

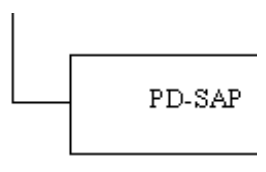

Figura 3.6. Modelo de referencia de la subcapa MAC [IEEE, 2006].

En relación con los dispositivos, hay dos tipos diferentes que pueden participar en una red basada el estándar IEEE 802.15.4: los dispositivos de funcionalidad completa (FFD, Full Function Device) y los de funcionalidad reducida (RFD, Reduced Function Device). El FFD puede operar en tres modos siendo el coordinador PAN (Personal Area Network), un coordinador o un dispositivo. Además, un FFD puede comunicarse con RFDs y otros FFDs, mientras que un RFD sólo puede establecer una comunicación con un dispositivo con el rol de FFD. Un RFD está pensado para aplicaciones que son extremadamente simples, como el interruptor de una luz o un sensor pasivo de infrarrojos; no necesitan envíar una gran cantidad de paquetes y sólo pueden estar asociados con un único FFD en un instante determinado. En consecuencia, el RFD puede ser implementado usando unos mínimos recursos y una memoria de reducida capacidad.

En función de los requerimientos de la aplicación, una IEEE 802.15.4 LR-WPAN puede estar configurada con dos topologías (ver Figura 3.7): la topología en estrella (star)y entre iguales (peer-to-peer). Con la topología en estrella la comunicación se establece entre los dispositivos y un único controlador central, denominado como el coordinador PAN. Típicamente, un dispositivo tiene una aplicación asociada y puede ser cualquiera, bien el punto de inicio, o bien el dispositivo final de la red de comunicación. Un coordinador PAN puede tener una aplicación específica, pero tiene la obligación de iniciar, liberar, o 
intercambiar los paquetes dentro de la red. El coordinador PAN es el coordinador principal de la PAN. Todos los dispositivos que operan en una red con cualquier topología deben tener direcciones únicas de 64-bits. Esta dirección se puede para realizar comunicaciones directas dentro de la PAN. También se pueden usar direcciones de 16 bits, que son asignadas a los dispositivos cuando se asocian con el coordinador y que se denotan con el término 'short address'. En relación con los requerimientos energéticos, el coordinador PAN tiene que estar siempre activo y generalmente no estará alimentado por baterías, salvo que haya un sistema de energía alternativa o no que la recargue. Al contrario, el resto de dispositivos de la PAN no tienen elevados consumos energéticos y, generalmente, estarán alimentados por baterías. Entre las aplicaciones que se pueden beneficiar de una topología en estrella, caben destacar las siguientes: automatización del hogar y la domótica, periféricos de un computador, juguetes y juegos, y la monitorización de la salud de pacientes.
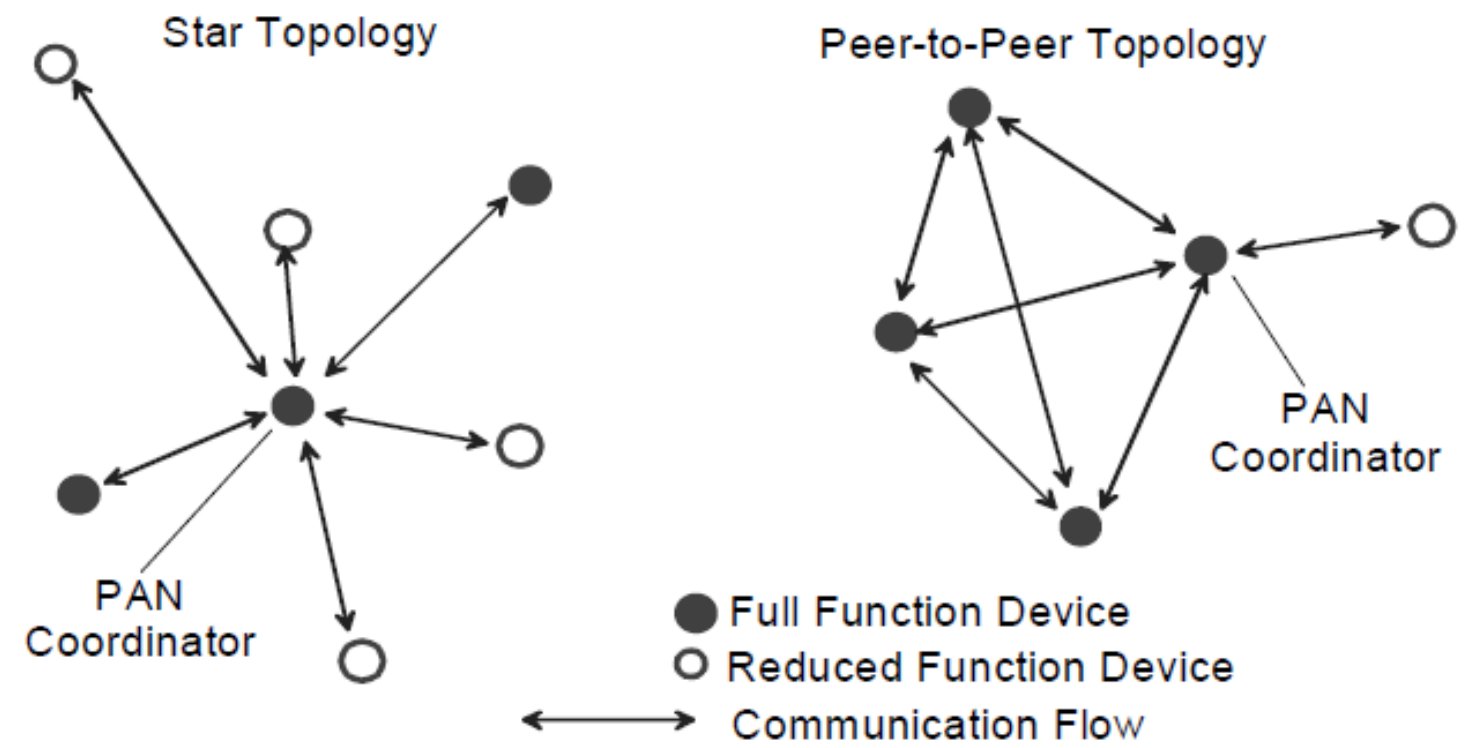

Figura 3.7. Ejemplos de topologías en estrella y entre iguales (peer-to-peer) [IEEE, 2006].

La topología peer-to-peer también tiene un coordinador PAN; sin embargo, se diferencia de la topología en estrella en que cualquier dispositivo puede intercambiar información con otro dispositivo, siempre y cuando esté dentro de su rango de alcance. Además, esta topología permite implementar redes más complejas, como una topología de tipo mesh. En este caso las aplicaciones que se pueden beneficiar de esta topología son las siguientes: control industrial y monitorización, WSNs, seguimiento de objetos, agricultura de precisión y la seguridad. Una red peer-to-peer puede ser ad hoc, auto-organizada y con capacidad de auto-reparación. También puede permitir intercambiar mensajes entre dos dispositivos a través de múltiples saltos ( $h o p s$ ) intermedios en otros nodos. Estas funciones pueden ser añadidas en capas superiores, pero no forman parte del estándar. Estas 
funciones han sido añadidas en el protocolo de comunicación de alto nivel ZigBee [Baronti, 2007].

\subsubsection{ZigBee.}

La ZigBee Alliance es una asociación de compañías trabajando en conjunto para desarrollar estándares (y productos) para redes inalámbricas de bajo consumo y coste y que sean también seguras. Probablemente, a lo largo de los próximos años la tecnología ZigBee será embebida en una gran cantidad de productos y aplicaciones a nivel mundial, como en el consumo de tipo comercial, industrial, entre otros [Baronti, 2007].

ZigBee define protocolos de comunicación de alto nivel construidos sobre la subcapa MAC del estándar IEEE 802.15.4 para LR-WPANs. ZigBee es simple, de bajo coste, y una tecnología inalámbrica de bajo consumo usada en aplicaciones empotradas. Los dispositivos ZigBee pueden formar redes malladas conectando entre ellos desde cientos hasta miles de nodos inalámbricos. Los dispositivos no requieren elevados consumos energéticos y pueden funcionar usando baterías durante varios años [Yick, 2008]. En general ZigBee define la especificación de la capa de red para topologías en estrella, árbol y peer-to-peer, y proporciona un marco de trabajo para la programación de las aplicaciones.

La versión 2004 del estándar ZigBee fue publicada en junio del año 2005. Esta versión fue revisada con la 2006, que fue publicada en diciembre de 2006. Y durante el último trimestre de 2007 finalizó la publicación de la versión 2007, que se puede encontrar referenciada como ZigBee Pro [ZigBee, 2008].

ZigBee estandariza las capas superiores (ver Figura 3.8) de la pila del protocolo. La capa de red (NWK, Network) está encargada de organizar y proporcionar rutado sobre una red de tipo multihop, al contrario que la capa de aplicación (APL, Application Layer) proporciona un marco de trabajo para el desarrollo de aplicaciones y comunicaciones distribuidas. La APL engloba el application framework, el ZigBee Device Object y la subcapa de aplicación (APS, Application Sub Layer). El application framework puede tener hasta 240 objetos de aplicación, esto es, módulos de aplicación definidos por el usuario que son parte de la aplicación ZigBee. El ZDO proporciona servicios que permiten descubrir unos APOs (Application Objects) de los otros, así como en organizarse en aplicaciones distribuidas. La APS ofrece una interfaz de datos y servicios de seguridad entre los APOs y el ZDO.

La capa de red (ver Figura 3.9) es necesaria para proporcionar la funcionalidad que asegure una correcta operación de la subcapa MAC del IEEE 802.15.4 y para proporcionar un conjunto de servicios de interfaz con la capa de aplicación. 


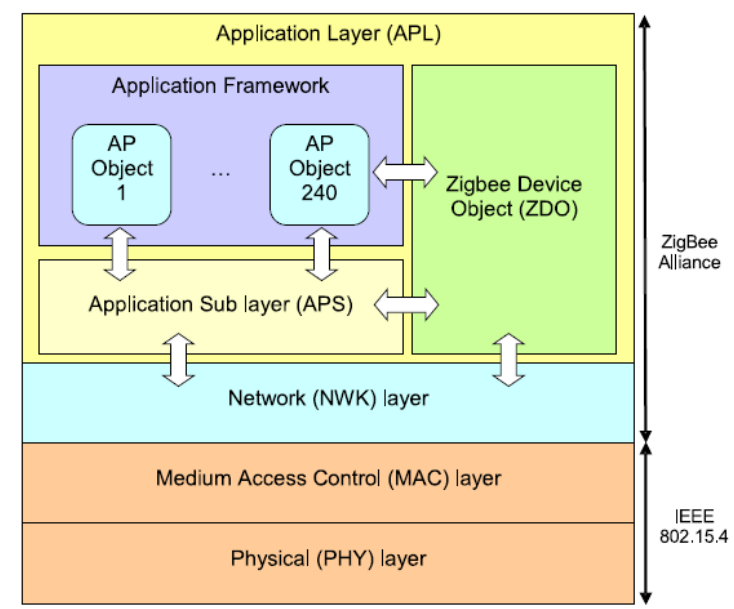

Figura 3.8. Arquitectura de capas funcional y la pila del protocolo ZigBee [ZigBee, 2007].

Para intercambiar información con la capa de aplicación, conceptualmente la capa de red incluye dos entidades de servicio que proporcionan la funcionalidad necesaria. Estas entidades de servicio son el servicio de datos y el servicio de gestión. La entidad de datos de la capa de red (NLDE, NWK Layer Data Entity) proporciona los servicios de transmisión de datos a través de su SAP asociado, el NLDE-SAP, y la entidad de gestión de la capa de red (NLME, NWK Layer Management Entity) proporciona el servicio de gestión por medio de su SAP asociado, el NLME-SAP. El NLME utiliza el NLDE para conseguir algunas de sus tareas de gestión y también manteniene una base de datos de los objetos manejados conocida como la network information base o por la siglas NIB [ZigBee, 2007].

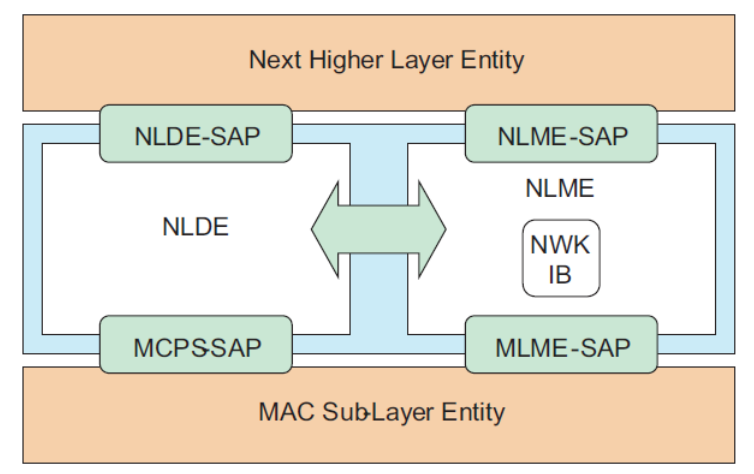

Figura 3.9. Arquitectura de referencia de la capa de red [ZigBee, 2007].

La capa de aplicación ZigBee está compuesta por la subcapa APS, el ZDO y los objetos aplicación definidos por el desarrollador. Las responsabilidades de la subcapa APS son mantener las tablas de binding, que es la habilidad de conectar dos dispositivos entre ellos basándose en sus servicios y sus necesidades, e intercambiar mensajes entre los dispositivos que tienen establecido el binding. Otra responsabilidad de la subcapa APS es el descubrimiento (discovery), que es la capacidad de determinar que otros dispositivos están funcionando en el espacio personal de operación del dispositivo. Las obligaciones del ZDO incluyen definir el rol del dispositivo dentro de la red, iniciar y/o responder a peticiones y 
establecimientos de binding y establecer una relación segura entre los dispositivos de la red. Los objetos aplicación implementados y definidos por el desarrollador implementan la aplicación en relación con las descripciones de la aplicación ZigBee definida.

Entrando más en detalles, la subcapa APS proporciona una interfaz entre la siguiente capa (NHLE, Next Higher Layer Entity) y la capa de red. Conceptualmente, la subcapa APS incluye una entidad de gestión llamada la entidad de gestión de la subcapa APS (APSME, APS Sub-layer Management Entity). Esta entidad proporciona las interfaces de servicio a través de las funciones de gestión que sean invocadas. El APSME es también responsable de mantener una base de datos de los objetos manejados pertenecientes a la subcapa APS. Esta base de datos se denota como la base de información de la subcapa APS (AIB, APS Information Base). La Figura 3.10 muestra las interfaces y los componentes de la subcapa APS.

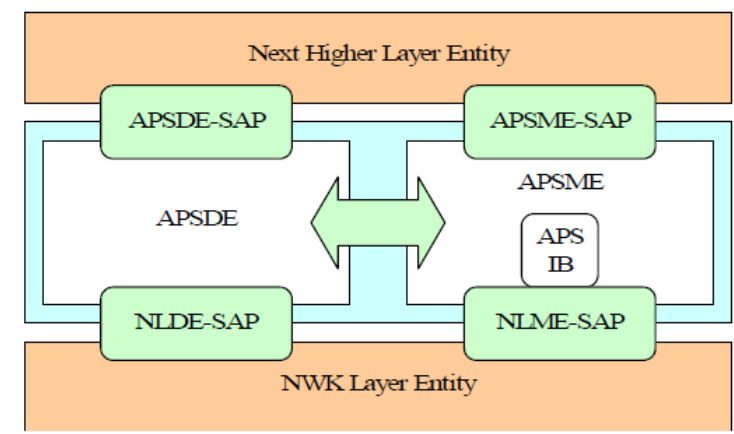

Figura 3.10. Arquitectura de referencia de la capa de la subcapa APS [ZigBee, 2007].

La subcapa APS proporciona dos servicios, accedidos por medio de dos SAPs. Estos son el servicio de datos APS, accedido a través del SAP de la entidad de datos de la subcapa APS (APSDE-SAP, APS Sublayer Data Entity SAP), y el servicio de gestión de la APS, accedido por medio del SAP de la entidad de gestión de la subcapa APS (APSMESAP, APS Sub-layer Management Entity SAP). Estos dos servicios proporcionan la interfaz entre el NHLE y la capa de red, a través del NLDE-SAP y, de forma limitada, con la interfaces NLME-SAP. La interfaz NLME-SAP entre la capa de red y la subcapa APS sólo soporta primitivas NLME-GET y NLME-SET; el resto de las primitivas NLME-SAP están disponibles sólo por medio del ZDO. Además de estas interfaces externas, también hay una interfaz implícita entre el APSME y el APSDE que permite que el primero use el servicio de datos de la APS.

En una red ZigBee hay involucrados tres tipos de dispositivos que define el estándar: el coordinador ZigBee (ZC, ZigBee Coordinator), el router ZigBee (ZR, ZigBee Router) y el dispositivo final ZigBee (ZED, ZigBee End Device). El ZED se corresponde con un RFD o un FFD definido por el estándar IEEE 802.15.4, el cual actúa como un 
dispositivo simple. Un ZR es un FFD con capacidad de realizar tareas de enrutado en la red. El ZC es un FFD que maneja la red completa y sólo puede haber uno en cada red.

Algunas de las funcionalidades de la capa de red son: enrutado multibop, descubrimiento y mantenimiento de rutas, seguridad y conexión/desconexión de la red, con la consecuente asignación de una short address (16-bits) a los nuevos dispositivos conectados. Además, la capa de red soporta topologías más complejas que las del estándar IEEE 802.15.4. Estas topologías son las siguientes (ver Figura 3.11g): estrella (star), árbol (tree) y malladas (mesh). En una topología en estrella, la red está controlada por un único dispositivo, el ZC. El ZC es el responsable de iniciar y mantener los dispositivos en la red. El resto de dispositivos, de tipo ZED, se comunican directamente con el ZC. En las topologías en árbol y malladas, el ZC tiene la responsabilidad de iniciar y configurar ciertos parámetros importantes de la red, pero la red puede extenderse haciendo uso de los ZRs. En redes en árbol, los ZRs intercambian datos y mensajes de control a lo largo de la red usando una estrategia de rutado jerárquica. Además, este tipo de redes pueden emplear comunicaciones con los beacon habilitados como describe el estándar IEEE. Las redes malladas permiten una comunicación peer-to-peer completa. Para ofrecer este soporte, se utiliza con ligeras modificacaciones el protocolo de rutado AODV (Ad-hoc On-demand Distance Vector) [Kwang, 2006]. Finalmente, cabe destacar que la revisión actual de ZigBee sólo describe redes intra-PAN, esto es, redes en las que las comunicaciones empiezan y terminar dentro de la misma red. Más información sobre ZigBee se puede encontrar en [ZigBee, 2007; Baronti, 2007].

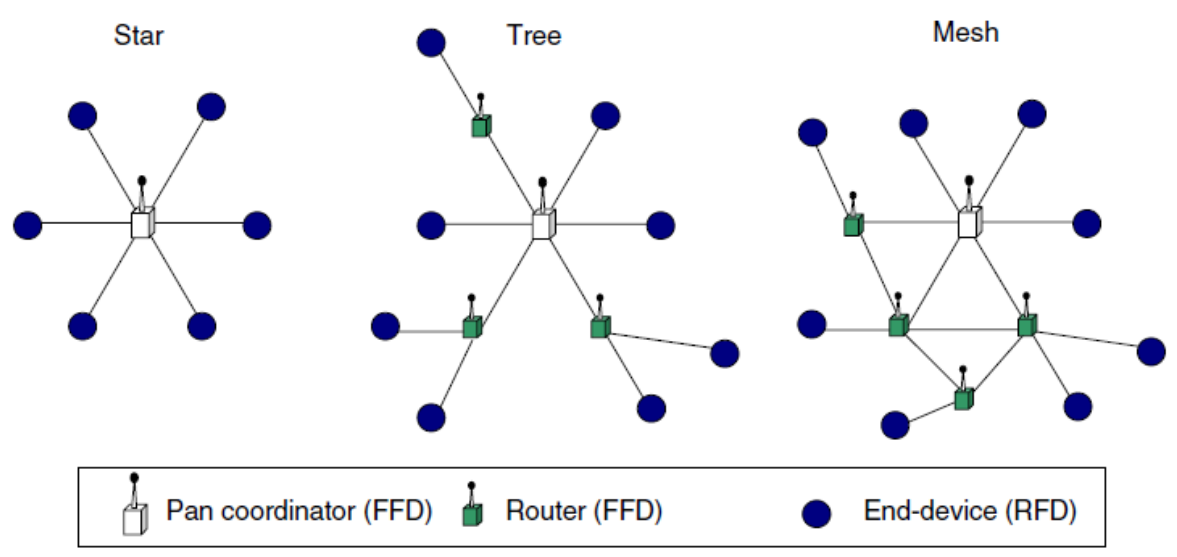

Figura 3.11. Topologías de las redes ZigBee [Baronti, 2007].

\subsubsection{B-MAC.}

Uno de los requisitos fundamentales que debe cumplir un dispositivo 802.15.4 es poseer un bajo consumo energético. B-MAC (Berkeley-Medium Access Contro) [Polastre, 2004] es un protocolo de acceso al medio que satisface este requisito añadiendo una serie de características que mejoran la gestión de la transmisión/recepción de tramas. B-MAC 
optimiza la gestión de las comunicaciones, de forma que los nodos conocen el máximo intervalo que deben permanecer despiertos para recibir la información. B-MAC permite una alta utilización del canal, un algoritmo que evita colisiones de tramas y una interfaz flexible de usuario. Además, B-MAC usa las funciones, primitivas y formatos de trama del estándar IEEE 802.15.4, pero usando B-MAC como protocolo de acceso al canal radio. Las tramas son unidades de información a nivel MAC, compuestas por una cabecera y un campo variable de datos del usuario con un tamaño máximo de 127 bytes.

B-MAC está basado en el muestreo periódico de ciclos de radio (períodos de tiempo) denominados Low Power Listening (LPL). Mediante la técnica LPL los nodos se despiertan periódicamente en intervalos denominados check intervals, activan la radio y sondean si hay actividad en el canal de comunicaciones. Este sondeo se realiza en función de la energía que recibe la interfaz radio del dispositivo mediante el mecanismo Clear Channel Assessment (CCA). El CCA permite distinguir tres posibles estados en función de si el canal se encuentra libre o no:

- Existe actividad y un nodo desea transmitir un mensaje. En este caso, el medio se encuentra ocupado y el nodo espera un período de tiempo (indicado por el usuario o definido por el propio protocolo) a efectos de poder enviar posteriormente la trama en otro check interval en donde se realiza el mismo proceso.

- No existe actividad y una estación desea transmitir una trama (caso mostrado en la Figura 3.12). El canal se encuentra libre y la estación emisora envía un preámbulo de longitud mínima el check interval antes de enviar la trama de información. La funcionalidad del preámbulo es ser detectado por la estación receptora, la cual sabrá que, una vez finalizado éste, la trama de datos se encuentra justo a continuación.

- Existe actividad y las tramas que se transmiten en el canal son recibidas por una estación. El nodo receptor despierta cada check interval, detecta el preámbulo y comienza su recepción. Una vez finalizado el preámbulo, la estación recibe la trama de datos propiamente dicha.

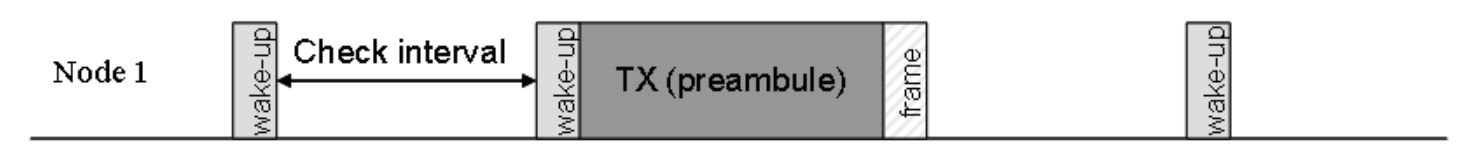

Node 2

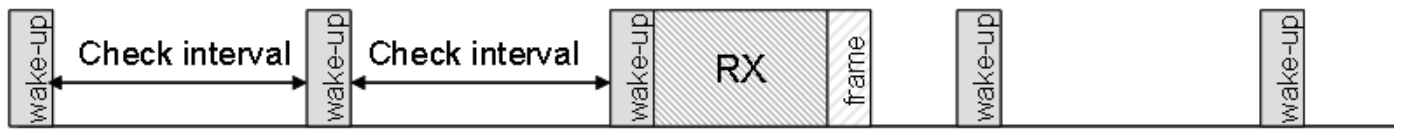

Figura 3.12. Comunicación de dos estaciones mediante B-MAC. 


\subsubsection{Otros estándares.}

El estándar WirelessHART [HART; HART, 2007] proporciona un protocolo de comunicación inalámbrica para aplicaciones de control de procesos e instrumentación. El estándar está basado en el IEEE 802.15.4 con una operación de bajo consumo en la banda de 2,4GHz. El WirelessHART es compatible con todos los productos existentes, herramientas y sistemas. Además, es fiable, seguro y eficiente en términos energéticos. Soporta redes malladas, salto de frecuencia y mensajes sincronizados. La red de comunicación es segura gracias a la encriptación, verificación, autentificación y a la gestión de la clave de la red. Las opciones de gestión energética permiten que los dispositivos inalámbricos sean más eficientes energéticamente. WirelessHART está diseñado para soportar topologías malladas, en estrella, así como combinaciones de las anteriores. Una red WirelessHART está compuesta de los dispositivos inalámbricos situados en campo, gateways, controladores de la automatización del proceso, las aplicaciones host y el gestor de la red (ver Figura 3.13). Los dispositivos inalámbricos de campo están conectados con el proceso o los equipos de la planta. Los gateways garantizan la comunicación entre los dispositivos inalámbricos de campo y las aplicaciones host. El controlador de la automatización del proceso actúa como un sencillo controlador para el proceso. El gestor de la red configura la red y organiza la comunicación entre los dispositivos. Además, se encargar del rutado y del tráfico de la red. El gestor de la red se puede integrar en un Gateway, en una aplicación 'host', o en un controlador del proceso. El estándar WirelessHART estuvo disponible para la industria en septiembre del 2007 y pronto estará disponible en productos comerciales.

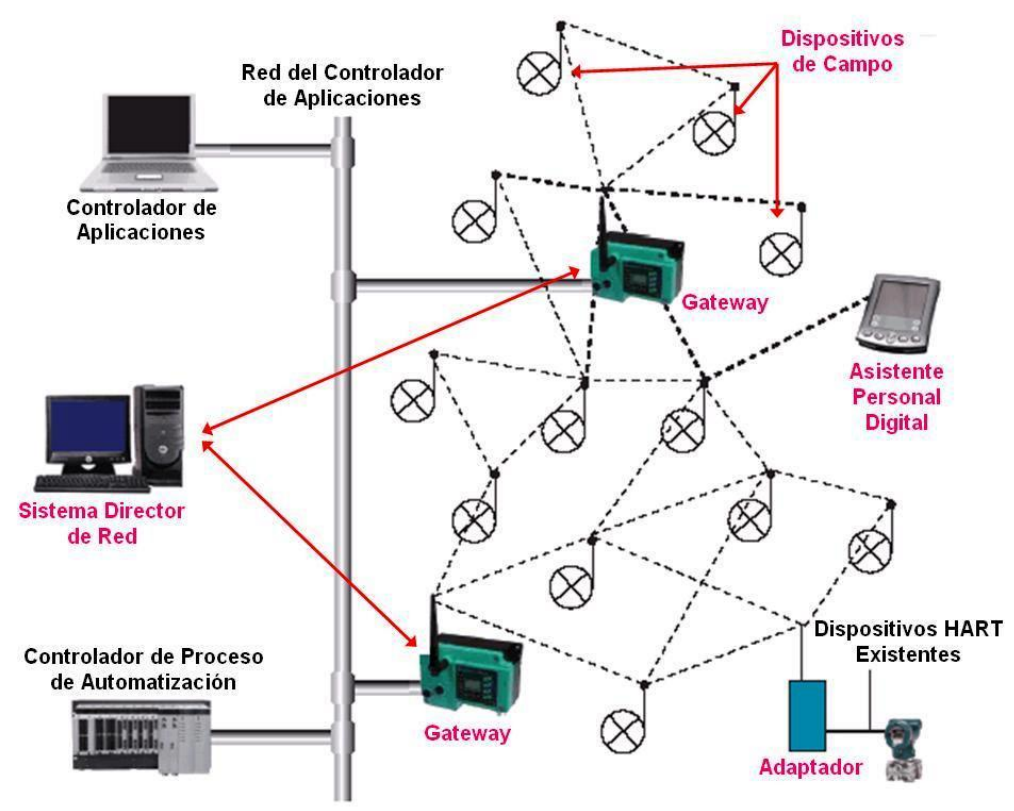

Figura 3.13. Red WirelessHART y sus elementos. 
El estándar ISA100.11a [ISA] está diseñado para monitorización inalámbrica y control de automatización de procesos con redes inalámbricas con baja velocidad de datos. Define la especificación para la capa OSI, seguridad y el sistema de gestión. El estándar tiene como objetivos un bajo consumo, la escalabilidad de la red, una infraestructura robusta y la interoperabilidad con otros dispositivos inalámbricos. La red sólo usa la banda de frecuencia de $2,4 \mathrm{GHz}$ y saltos de frecuencia para incrementar la fiabilidad de la red y minimizar las interferencias. En cuanto a las topologías, ofrece topologías malladas y en estrella. Además, el ISA100.11a proporciona una funcionalidad de seguridad simple, flexible y escalable.

El estándar 6LoWPAN (IPv6-based Low power Wireless Personal Area Networks) [6LoWPAN; Mulligan, 2007; Montenegro, 2007] proporciona el envío de paquetes IPv6 sobre una red basada en el IEEE 802.15.4. Los dispositivos de bajo consumo pueden comunicarse directamente con dispositivos IP usando protocolos IP. Usando 6LoWPAN, dispositivos de bajo consumo tienen todos los beneficios de una comunicación y una gestión IP. Este estándar proporciona una capa de adaptación, un nuevo formato de paquetes, así como gestión del direccionamiento. La capa de adaptación es usada porque el tamaño de los paquetes IPv6 son mucho mayores que el tamaño de trama del IEEE 802.15.4. La capa de adaptación lleva a cabo la funcionalidad para la compresión de la cabecera. Usando la compresión de la cabecera, se crean pequeños paquetes que encajan con el tamaño de trama del IEEE 802.15.4. El mecanismo de gestión de la dirección se encarga de la gestión de las direcciones de los dispositivos para garantizar la comunicación entre ellos. En definitiva, el estándar 6LoWPAN está pensado para aplicaciones en las que se utilizan dispositivos que tienen una baja tasa de envío de datos y que requieren comunicación con Internet.

El estándar IEEE 802.15.3 [IEEE 802.15.3, 2003] define una capa física y una capa de acceso al medio para WPANs con una elevada tasa de datos. Está diseñado para soportar transmisiones de audio y vídeo en tiempo real. El estándar define como la banda de operación la de 2,4GHz y tasas de envíos de datos desde 11 hasta 55Mbps. Para garantizar la calidad del servicio el estándar usa TDMA (Time Division Multiple Access). Soporta transferencias de datos síncronas y asíncronas y lleva a cabo las gestiones del consumo de potencia, la escalabilidad de la tasa de datos enviada y la frecuencia. El estándar se usa en dispositivos como: auriculares inalámbricos, equipos de vídeo portátil y conectividad inalámbrica para juegos, teléfonos inalámbricos, impresoras y televisores.

Wibree [Wibree] es una tecnología de comunicación diseñada para dispositivos de bajo consumo, comunicaciones dentro de pequeños alcances y bajo coste. Wibree permite la comunicación entre dispositivos provistos de pequeñas baterías y dispositivos Bluetooth. Entre los dispositivos provistos de pequeñas baterías caben mencionar los siguientes: 
relojes, teclados inalámbricos y los sensores de deporte (ver Figura 3.14) que están conectados con ordenadores personales o teléfonos móviles.

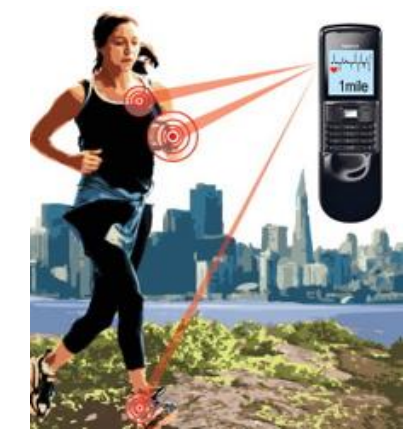

Figura 3.14. Red Wibree de sensores de deporte.

Wibree trabaja en la banda de $2,4 \mathrm{GHz}$, soporta una tasa de envío de datos de $1 \mathrm{Mbps}$ y la distancia de los enlaces puede estar comprendida entre 5 y $10 \mathrm{~m}$. Wibree está diseñado para trabajar con Bluetooth. La combinación de Bluetooth y Wibree consigue dispositivos más pequeños y más eficientes a nivel energético. Wibree se dio a conocer en octubre del 2006.

\subsection{Estado de la técnica de las soluciones hardware.}

El objetivo de esta sección es realizar un estado de la técnica de los nodos sensores o motes que se han desarrollado durante estos últimos años, alguno de ellos en paralelo con los trabajos desarrollados en esta tesis. Para ello, en primer lugar se describirán los componentes principales de un mote y, posteriormente, se repasarán brevemente las soluciones hardware que se han ido diseñando, describiendo tanto las desarrolladas en laboratorios a nivel de prototipos de investigación, como las disponibles comercialmente.

\subsubsection{Motes.}

Como ya se ha mencionado con anterioridad, los nodos sensores, más comúnmente conocidos como motes, son la pieza básica de una WSN. Se trata de dispositivos capaces de realizar medidas acerca de un determinado fenómeno y propagarlas vía radio. Sus principales características son su reducido tamaño, coste y consumo de potencia. Disponen de capacidad de procesado, comunicación inalámbrica así como de expansión a través de puertos de entrada/salida de su microprocesador.

El nombre de mote procede del inglés, donde su significado es mota de polvo. Esta denominación fue usada por primera vez en el Proyecto Smartdust [Warneke, 2001], concebido en 1998 con la intención de desarrollar nodos sensores de tamaño muy pequeño 
(en torno a $1 \mathrm{~mm}^{3}$ ) que en conjunto formarían un polvo inteligente (smart dust) que permitiría estudiar el entorno.

Un mote está formado por los subsistemas hardware que se muestran en la Figura 3.15 [Akyildiz, 2002]. Existen cuatro subsistemas que siempre se encuentran presentes en un mote. El primero de ellos, la unidad de procesado, se encarga de coordinar al resto de subsistemas, disparando las tareas de recogida de datos y comunicaciones con otros nodos, así como de procesar los datos tomados. La unidad de sensorización toma los datos del entorno a través de sus sensores y transforma las medidas analógicas tomadas en medidas digitales que el microprocesador puede manejar. El transceptor se encarga de las comunicaciones con el resto de nodos. Por último, nos encontramos con la unidad de alimentación que suministra energía al resto de subsistemas. Además, existen otros subsistemas que pueden existir opcionalmente en los motes como podrían ser movilizadores (si el mote necesita desplazarse), el subsistema de localización, o un generador de potencia (por ejemplo, un panel fotovoltaico).

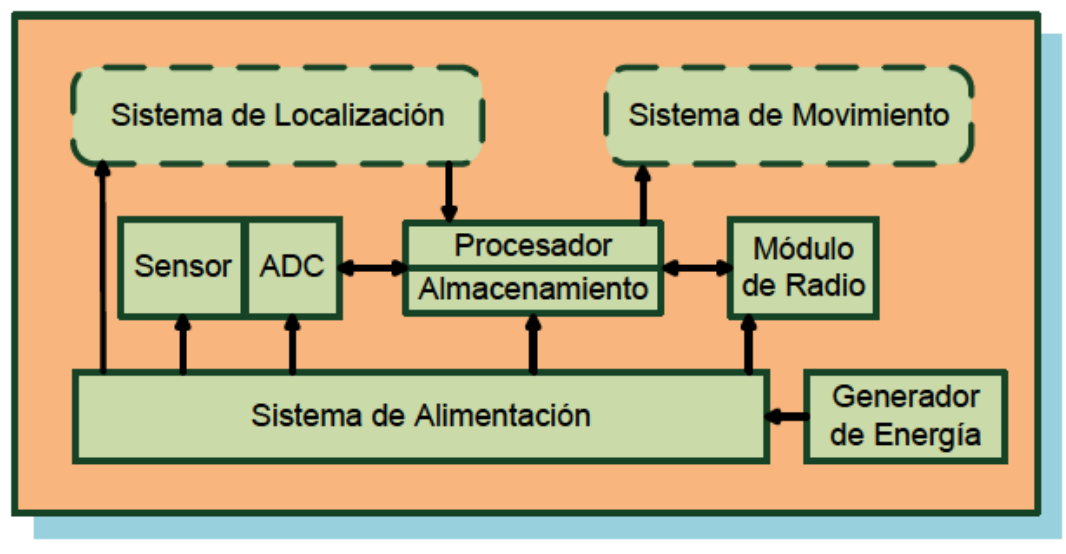

Figura 3.15. Componentes de un mote.

Los motes son dispositivos limitados en cuanto a sus prestaciones. Su CPU funciona a baja frecuencia, disponen de pocos kBytes de memoria RAM y tienen una fuente de alimentación que deben conservar durante el mayor tiempo posible. Debido a esto, las funciones que realizan los nodos se limitan a la captura y envío de datos, con algunas tareas de procesado destinadas a disminuir la cantidad de información que se transmite, o a obtener nueva información, ya sea a partir de las distintas medidas que se almacenan en un nodo, o a partir de un procesado distribuido en varios nodos. El almacenamiento en memoria no volátil está menos restringido, ya que disponen de una memoria flash del orden de cientos de kBytes, lo que permite el almacenamiento de gran cantidad de datos, que no tienen porqué ser inmediatamente transmitidos por la radio. Además, los motes cuentan con puertos de entrada/salida que permiten tanto el conexionado de subsistemas adicionales, como la comunicación con un PC o la programación del mote. 
En el capítulo 5 de [Mahgoub, 2006] se pueden encontrar ejemplos de motes que se han diseñado realizando una miniaturización con MEMS, y que, por tanto, son de muy pequeño tamaño. Uno de los dispositivos se ha desarrollado en la Universidad de Michigan por Mason et al. [Mason, 1998]. El dispositivo lleva embebidos sensores de temperatura, presión, humedad y vibración/posición, y tiene un tamaño de $10 \mathrm{~cm}^{3}$. Sin embargo, en algunos casos, dispositivos como el mencionado anteriormente no se puede utilizar en aplicaciones de Agricultura de Precisión, ya que requieren instrumentación específica de calidad. Con respecto al futuro de los dispositivos, se espera que se reduzcan significativamente los tamaños y costes de los motes, así como que se produzcan avances en los mecanismos de obtención de energía, en las capacidades de los motes que posibilitaran el éxito de las WSN y los actuadores inalámbricos.

\subsubsection{Soluciones comerciales.}

\subsubsection{Soluciones de propósito general.}

Durante estos últimos años, se han desarrollado motes que se pueden adquirir actualmente desde precios algo inferiores a los cien euros. Desde el año 2001, los motes más populares fueron los de la familia MICA de CrossBow (hoy conocida como MEMSIC), desarrollados en la Universidad de Berkeley [Berkeley] y que se empezaron a comercializar en ese año. En la Figura 3.16 se pueden ver dos motes de esta familia: los MICA2 (2002) [MEMSIC], ampliamente usados para investigación y los MICA2Dot (2002), más reducidos, y con el tamaño de $25 \mathrm{~mm}$ de diámetro. Otros motes de esta familia que salieron a la venta fueron el MICA (2001) y el MICAZ (2003).
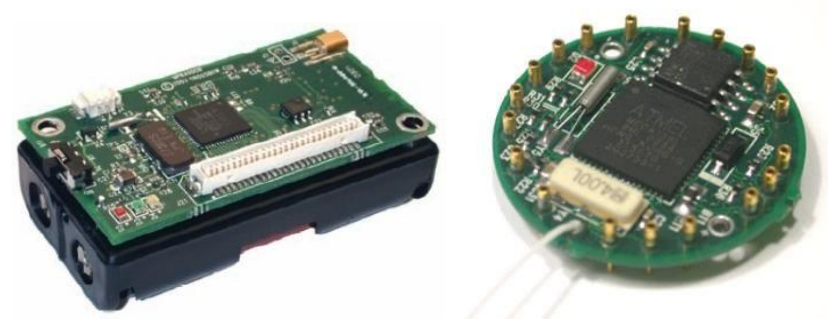

Figura 3.16. Familia de motes MICA. (a) MICA2. (b) MICA2dot.

Otros motes a destacar son los IRIS y el Cricket (también de Crossbow). El IRIS (ver Figura 3.17a) (2007) es un dispositivo con el que se pueden establecer aplicaciones de WSN de bajo consumo en la banda de frecuencia de 2,4GHz. El mote incrementa en cinco veces el alcance y en dos la capacidad de memoria, con respecto a las versiones previas de la familia MICA. El mote Cricket (ver Figura 3.17b) (2003) es la versión de localización del conocido MICA2. El dispositivo incluye todo el hardware de un MICA2, un receptor y un transmisor de ultrasonidos. 


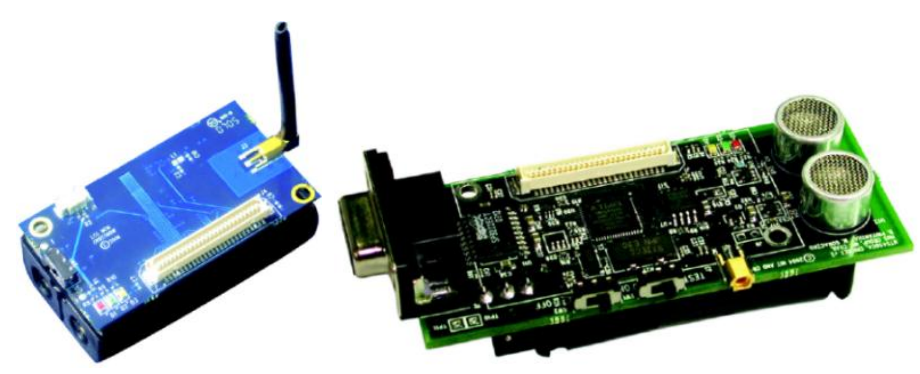

Figura 3.17. (a) Mote IRIS. (b) Mote Cricket.

Con posterioridad a los MICAs, aparecieron en el mercado los motes de la familia Telos, también desarrollados en la Universidad de Berkeley y comercializados por distintas empresas bajo dos marcas: TelosB [MEMSIC] (Crossbow, 2005) y Tmote Sky [Berkeley] (Universidad de Berkeley, 2004) (véase Figura 3.18). Estos motes tienen un coste más reducido y un menor consumo de potencia, en gran parte por soportar estándares de comunicación de bajo consumo, por lo que se están convirtiendo en los más usados actualmente a nivel de laboratorio.

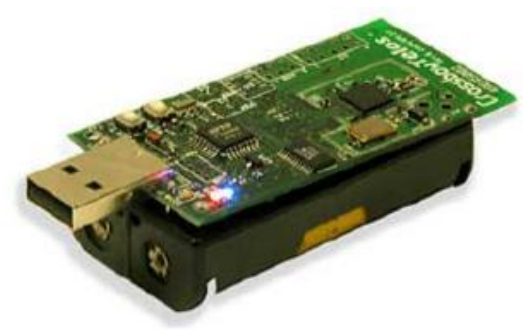

Figura 3.18. Mote TelosB.

A pesar de que los motes anteriores son los más conocidos, otros fabricantes no se han querido quedar atrás en el campo de las redes de sensores y han desarrollado sus propios motes. Así por ejemplo, el Imote2 (2007) (ver Figura 3.19), diseñado por Intel Corporation [Intel], ha aparecido hace pocos años con unas prestaciones más avanzadas que los anteriores y que su antecesor, el Imote, así como con unas dimensiones reducidas. Sin embargo, su precio también es notablemente superior.

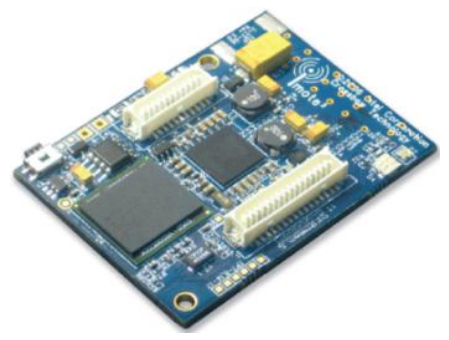

Figura 3.19. Imote2. 
La Tabla 3.1 resume las características principales de los motes descritos anteriormente.

\begin{tabular}{|c|c|c|c|c|c|c|c|}
\hline \multicolumn{2}{|c|}{ Mote } & MICA2 & MICA2Dot & IRIS & Cricket & TelosB & Imote2 \\
\hline \multirow{4}{*}{ MCU } & Chip & \multicolumn{2}{|c|}{ ATmega128L } & ATmega1281 & ATmega128L & Msp430f1611 & PXA271 \\
\hline & Tipo & \multicolumn{4}{|c|}{8 bits } & 16 bits & 32 bits \\
\hline & $\begin{array}{c}\text { Memoria } \\
\text { Programa }(K B)\end{array}$ & \multicolumn{4}{|c|}{128} & 48 & $\mathrm{~N} / \mathrm{A}$ \\
\hline & $R A M(K B)$ & & & 8 & 4 & 10 & 32768 \\
\hline \multirow{3}{*}{$\begin{array}{l}\text { Almacenamiento } \\
\text { Externo No Volátil }\end{array}$} & Chip & \multicolumn{2}{|c|}{ AT45DB014B } & \multirow{3}{*}{$\mathrm{N} / \mathrm{A}$} & AT45DB014B & M25P80 & $\mathrm{N} / \mathrm{A}$ \\
\hline & Conexión & \multicolumn{2}{|c|}{ SPI } & & SPI & SPI & $\mathrm{N} / \mathrm{A}$ \\
\hline & Tamaño (KB) & & & & 512 & 1024 & 32768 \\
\hline \multirow[b]{2}{*}{$\begin{array}{l}\text { Sistema de } \\
\text { Alimentación }\end{array}$} & Tipo & $2 \mathrm{xAA}$ & Coin Cell & \multicolumn{3}{|c|}{$2 \mathrm{xAA}$} & $3 \mathrm{xAAA}$ \\
\hline & $\begin{array}{c}\text { Capacidad } \\
\text { Típica } \\
(m A h)\end{array}$ & 2850 & 1000 & \multicolumn{3}{|c|}{2850} & 1100 \\
\hline \multirow{5}{*}{$\mathbf{R F}$} & Chip & \multicolumn{2}{|c|}{ CC1000 } & AT86RF230 & CC1000 & CC2420 & CC2420 \\
\hline & Frecuencia & \multicolumn{2}{|c|}{ 868/916, 433 ó $315 \mathrm{MHz}$} & $2,4 \mathrm{GHz}$ & $\begin{array}{c}\text { 868/916, } \\
433 \text { ó } 315 \\
\text { MHz }\end{array}$ & $2,4 \mathrm{GHz}$ & $2,4 \mathrm{GHz}$ \\
\hline & $\begin{array}{l}\text { datarate } \\
\text { (Kbps) }\end{array}$ & \multicolumn{2}{|c|}{38,4} & 250 & 38,4 & 250 & 250 \\
\hline & $\begin{array}{c}\text { Potencia } \\
\text { Transmisión } \\
\quad(d B m)\end{array}$ & \multicolumn{2}{|c|}{5} & 3 & 5 & 0 & 0 \\
\hline & $\begin{array}{c}\text { Alcance } \\
\text { Exterior }(m)\end{array}$ & 152,4 & $1524-304,8$ & $>300$ & 152,8 & $75-100$ & $\sim 30$ \\
\hline \multicolumn{2}{|c|}{ Año comercialización } & 2002 & 2002 & 2007 & 2003 & 2005 & 2007 \\
\hline
\end{tabular}

Tabla 3.1. Características principales de diferentes motes.

\subsubsection{Soluciones agrícolas.}

Tanto los motes presentados anteriormente, como otros no mencionados, son productos comerciales que incluyen sensores de bajo coste y cuyas especificaciones (precisión, resolución, derivas, etc.) se alejan de las de la instrumentación que habitualmente se utiliza en las aplicaciones de la agricultura de precisión. Por otro lado, muchos de estos motes se han utilizado en aplicaciones de laboratorio o de investigación, careciendo de la robustez necesaria para su aplicación en entornos agrícolas reales. Por lo tanto, para que los motes puedan ser utilizados en aplicaciones reales, es, por un lado, necesario que éstos incluyan la electrónica necesaria para la conexión de instrumentación externa de calidad y, 
por otro, que se mejore la robustez de los mismos, para que puedan ser instalados en campo.

Así, en el año 2005, la firma Crossbow comenzó a comercializar el kit de monitorización micro-ambiental MEP-SYS (Figura 3.20). Este estaba formado por seis nodos, de los cuales 4 eran del tipo "MEP510" y los otros 2 eran del tipo "MEP410". Disponía también de un nodo sumidero, tipo "MBR410". Se trata de una solución cerrada en la que los nodos incluyen sensores embebidos de bajo coste. No es, por tanto, un sistema versátil que se pueda usar en aplicaciones de Agricultura de Precisión que, como se ha ido comentando a lo largo de la tesis, en la mayoría de las ocasiones requieren de instrumentación externa de calidad.

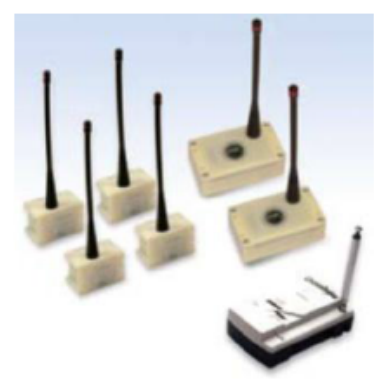

Figura 3.20. Kit de monitorización micro-climático MEP-SYS.

El nodo MEP410 (Figura 3.21) permitía monitorizar la temperatura y la humedad relativa del ambiente, la radiación (fotosintética y la total) y la presión barométrica. Externamente estaba compuesto por dos elementos: el nodo sensor y una caja estanca con las baterías $\left(2 \mathrm{xC}, 6.000 \mathrm{mAh}\right.$ ). Internamente englobaba 3 PCBs: un MICA2 ( $\mathrm{n}^{\circ} 2$ en la figura), que trabajaba en la banda de $433 \mathrm{MHz}$ y dos placas $\left(\mathrm{n}^{\circ} 1 \mathrm{y} \mathrm{n}^{\circ} 3\right.$ en la figura) de sensores, conectadas a la anterior a través del puerto de expansión.

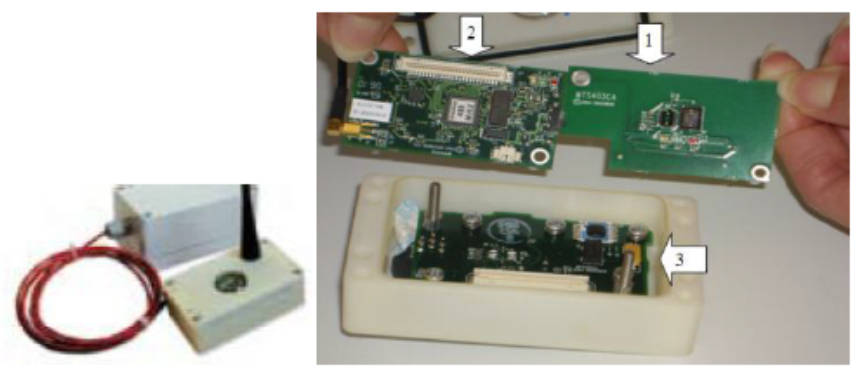

Figura 3.21. Vistas del nodo MEP410.

Por otro lado, el nodo MEP510 (ver Figura 3.22), a nivel de posibilidades de monitorización, era muy sencillo, ya que sólo permitía obtener periódicamente la temperatura y la humedad relativa del ambiente mediante el sensor de bajo coste SHT11 de Sensirion. A nivel externo constaba de un único dispositivo. En su interior incluía un 
MICA2dot, trabajando a una frecuencia de $433 \mathrm{MHz}$ ( $\mathrm{n}^{\circ} 2$ en la figura), la PCB con el sensor SHT11 y una batería de tipo A de 2/3.

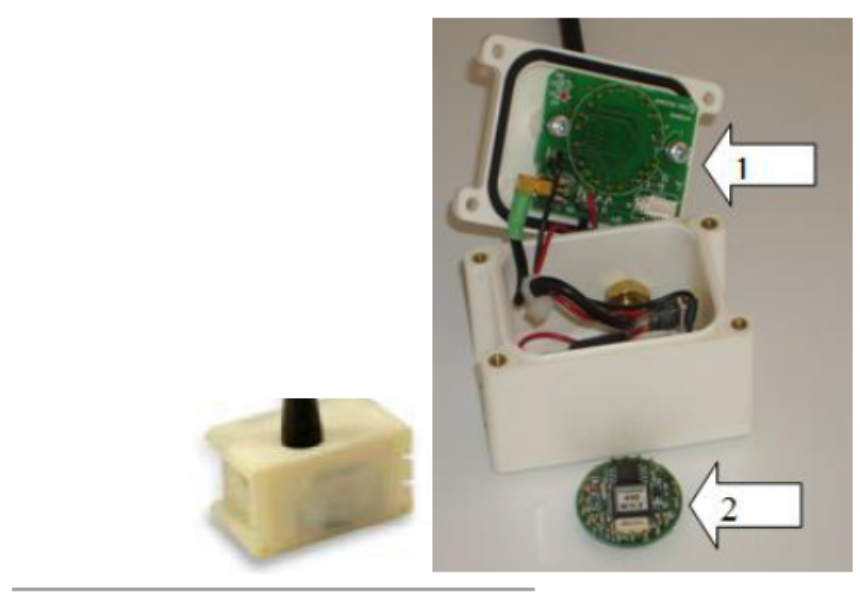

Figura 3.22. Vistas del nodo MEP510.

El sistema permitía monitorizar la temperatura, humedad relativa, radiación y la presión barométrica del ambiente. Además, debido a su carcasa de protección, se podían emplear tanto en cultivos a cielo abierto como en invernaderos, así como en otras aplicaciones exteriores/interiores.

En 2008, el mismo fabricante lanzó al mercado el kit eKo, que presentaba importantes mejoras en cuanto a robustez con respecto al producto anterior y que es, en la actualidad, su producto estrella en el ámbito de la agricultura. El fabricante lo define como un sistema inalámbrico de monitorización ambiental de exterior para aplicaciones de agricultura de precisión, microclima y estudios de conservación, investigación medioambiental y monitorización de cultivos. La Figura 3.23 muestra 4 nodos del kit, la estación base del sistema, que conectada con el eKo Gateway (esquina inferior derecha) permite tanto el almacenamiento local de los datos monitorizados, así como poder observar los datos monitorizados e interactuar con el sistema a través de Internet.

Figura 3.23. Vista general del kit eKo. 
Tanto los nodos sensores como la estación base se comercializan en dos versiones que operan en la banda de frecuencia de $2,4 \mathrm{GHz}$. La versión de corto ofrece coberturas de entre 152 y $457 \mathrm{~m}$ en línea de vista. La de largo alcance tiene coberturas de entre 610 y $3218 \mathrm{~m}$. Los nodos sensores incluyen un pequeño panel solar que recarga 3 baterías de tipo AA de NiMH. Además, los nodos disponen de 4 conectores externos en su base que permiten conectar hasta 4 sensores comercializados por el fabricante, así como la conexión de sensores ad hoc. La conexión de los sensores se realiza por el denominado bus plug-andplay ESB (Environmental Sensor Bus) desarrollado por el fabricante.

La Figura 3.24 muestra 3 de los 5 sensores que comercializa el fabricante para el kit $e K o$. De izquierda a derecha se tratan de un sensor de temperatura y humedad del suelo, una sonda de humedad relativa y temperatura del ambiente y un sensor de radiación solar.

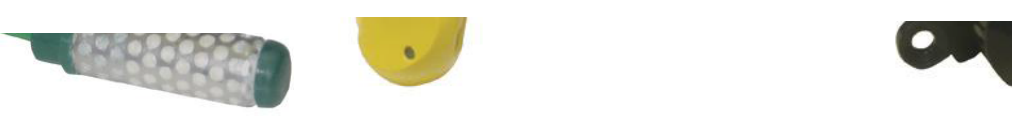

Figura 3.24. Sensores comercializados para el kit eKo.

Además de los sensores anteriores, al sistema también se puede conectar una estación meteorológica (ver Figura 3.25) que comercializa el fabricante. La estación incluye sensores de pluviometría, temperatura y humedad del ambiente, radiación solar, presión barométrica, velocidad y dirección del viento, y un anemómetro. Finalmente, cabe resaltar que el fabricante pone a disposición del usuario unas interfaces, las cuales permiten que el desarrollador pueda conectar algunos sensores propios.

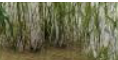

Figura 3.25. Vista de la estación meteorológica conectada a un nodo del kit eKo. 


\subsubsection{El panorama nacional.}

Libelium [Libelium], empresa spin off de la Universidad de Zaragoza, comercializa desde 2009/2010 un nodo sensor para agricultura de precisión. En concreto, el dispositivo permite controlar hasta catorce parámetros ambientales diferentes de cultivos en viñedos e invernaderos.

El elemento principal del sistema es el $W$ aspmote (ver Figura 3.26), que se trata de un nodo sensor que permite integrar varios sensores diferentes para monitorizar diversos tipos de entornos. Entre las aplicaciones que pueden llevarse a cabo con él destacan seguridad, medio ambiente, agricultura, control industrial, automatización, minería, energía y ganadería. Los datos adquiridos pueden ser enviados inalámbricamente a doce kilómetros de distancia usando el protocolo ZigBee o almacenados en el propio dispositivo. Además, la empresa comercializa diferentes placas de sensores que pueden ser conectadas al dispositivo. En la parte derecha de la Figura 3.26 se muestra la placa de sensores para agricultura de precisión. Con esta placa se pueden monitorizar hasta catorce parámetros medioambientales: temperatura y humedad relativa del aire, temperatura de suelo, humedad de suelo, humectación de hoja, presión atmosférica, radiación solar, diámetro de tronco/tallo/fruto, velocidad de viento, dirección de viento y precipitación.

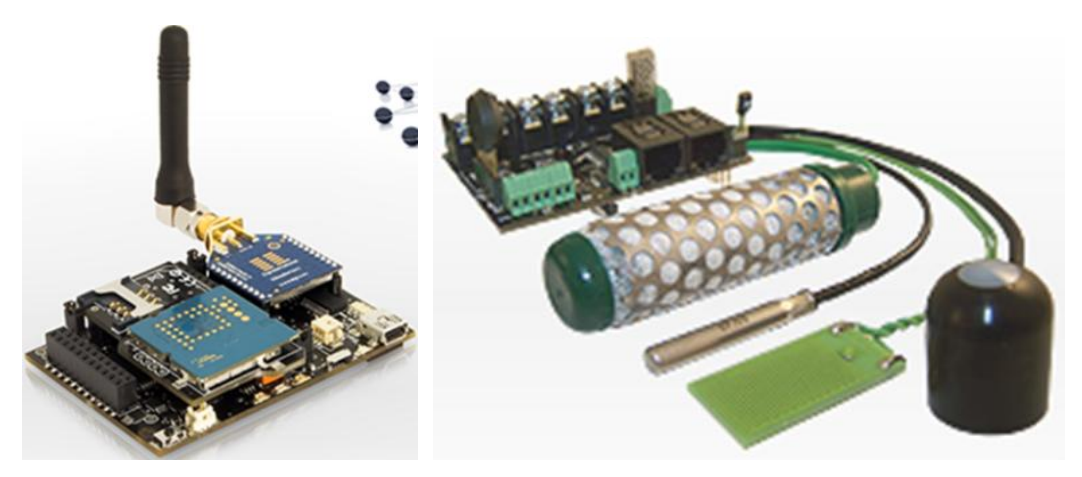

Figura 3.26. Vista del Waspmote y de la placa de sensores para agricultura de precisión.

Otra empresa nacional, spin off de la Universidad de Valencia, que comercializa nodos sensores es Balmart [Balmart]. En concreto, comercializar tres nodos sensores con características similares, y que difieren en el sistema de alimentación empleado.

El RFIX BAT-0901 es un nodo de telemedida por radiofrecuencia que opera en banda libre gratuita ISM a 2,4GHz y transmite los datos de hasta 4 sensores de variables medioambientales en paralelo. La Figura 3.27 muestra la caja estanca IP65 para instalaciones en exterior que integra todo el hardware del nodo. El dispositivo incluye cuatro entradas analógicas y cuatro E/S digitales configurables. Tres de las cuatro entradas analógicas tienen un rango fijo entre 0 y $3 \mathrm{~V}$, y la cuarta permite configurar el rango. En relación con 
las digitales, se pueden configurar de tipo $\mathrm{NO} / \mathrm{NC}$, para contar pulsos, 0-3V, y una de ellas puede ser configurada también entre 0 y $10 \mathrm{~V}$. Las comunicaciones están basadas en el estándar IEEE 802.15.4, con una potencia máxima de transmisión de $20 \mathrm{dBm}$ y 800m de alcance. El dispositivo trabaja con dos baterías tipo D de $13 \mathrm{Ah}$ de capacidad. El dato de la autonomía no aparece en las características técnicas del dispositivo. No obstante, se facilita que para una frecuencia de sensado y de transmisión de 2 y 10 minutos, respectivamente, el consumo sea de $1 \mathrm{~mA}$. Con este dato se puede estimar una autonomía de unos 540 días.

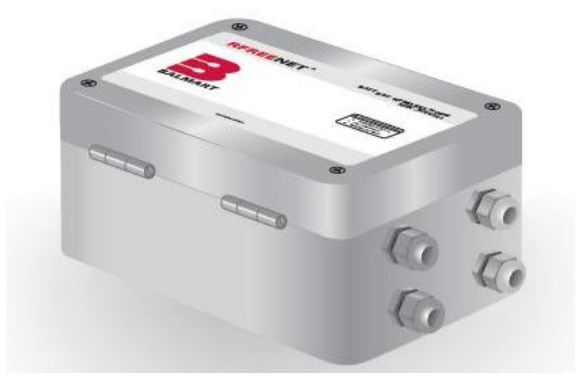

Figura 3.27. Nodo RFIX BAT-0901 comercializado por Balmart.

Otro dispositivo de la familia con las mismas características que el anterior es el RFIX AC-0901. La única diferencia es que éste se alimenta directamente desde la red eléctrica de $220 \mathrm{~V}$. Cabe destacar que también se puede alimentar con $24 \mathrm{~V}$ de corriente alterna y continua.

El último dispositivo de la familia es el RFIX SC-0901, que también se diferencia de los anteriores en el sistema de alimentación. En este caso está basado en 3 baterías recargables de NiMH de $2700 \mathrm{mAh}$ de capacidad y un panel solar de $3 \mathrm{~V}$ y $500 \mathrm{~mA}$. En este caso, con presencia de radiación solar la autonomía es ilimitada. En ausencia de radiación solar se puede estimar, a partir del consumo del dispositivo $(1 \mathrm{~mA})$ y la capacidad de las baterías, resultando un valor de 112 días.

En el año 2007, se comenzó una línea de colaboración con la empresa Edosoft Factory S.L., en el marco de la presente tesis doctoral, para desarrollar una línea de productos (GAIA) para aplicaciones de Agricultura de Precisión. Estos trabajos se desarrollaron en el marco de una serie de proyectos (RIMSI, SIRSIMA) financiados por el plan nacional de I+D+I. Como resultado de dicha colaboración, en el año 2009 se fabricó el prototipo pre-comercial del GAIA Soil-Mote (GSM), que se describirá en mayor detalle en el capítulo 5. A grandes rasgos, el GSM es un nodo sensor que proporciona una interfaz de tipo SDI-12, de manera que instrumentación de calidad muy empleada en la Agricultura de Precisión se conecta directamente a este dispositivo. 
Posteriormente, en el año 2010, se desarrolló el GAIA 2. Se trata de un prototipo multifuncional que dispone, en un único dispositivo, la funcionalidad necesaria en la mayoría de las aplicaciones (gateway, repetidor y nodo sensor). El GAIA 2 se caracteriza por disponer de diferentes interfaces de entrada y salidas, tanto analógicas como digitales, y por permitir la conexión de un radio modem de largo alcance comercial, que permite conseguir alcances de hasta $16 \mathrm{~km}$. El mote, que se describirá en mayor detalle en el capítulo 6, tiene la principal ventaja de proporcionar un único dispositivo comercial para aplicaciones de Agricultura de Precisión, requiriendo un único proceso de certificación (marcado CE).

La Tabla 3.2 resume las principales características de los nodos sensores comerciales descritos anteriormente comercializados por la corporación internacional MEMSIC y las empresas nacionales Libelium, Balmart y Edosoft.

\begin{tabular}{|c|c|c|c|c|}
\hline Dispositivo & Variables medidas & $\begin{array}{l}\text { Frecuencia } \\
\text { operación }\end{array}$ & $\begin{array}{l}\text { Alcance } \\
\text { máximo }\end{array}$ & $\begin{array}{l}\text { Autonomía } \\
\text { Máxima }\end{array}$ \\
\hline MEP-SYS & $\begin{array}{c}\text { Temperatura y humedad relativa del ambiente, radiación solar y } \\
\text { presión barométrica }\end{array}$ & $433 \mathrm{MHz}$ & $>150 \mathrm{~m}$ & 2 años \\
\hline eKo & $\begin{array}{c}\text { Temperatura y humedad del suelo, temperatura y humedad } \\
\text { relativa del ambiente, radiación solar, precipitación, presión } \\
\text { barométrica, velocidad y dirección del viento y humectación de } \\
\text { la hoja }\end{array}$ & $2,4 \mathrm{GHz}$ & $\begin{array}{c}150- \\
3200 \mathrm{~m}\end{array}$ & $\begin{array}{l}5 \text { años } 3 \text { meses } \\
\text { sin sol }\end{array}$ \\
\hline Waspmote & $\begin{array}{l}\text { Temperatura y humedad relativa del ambiente, temperatura y } \\
\text { humedad del suelo, humectación hoja, presión atmosférica, } \\
\text { radiación solar, diámetro del tronco/tallo/fruto, velocidad y } \\
\text { dirección del viento y precipitación }\end{array}$ & $\begin{array}{l}2400,900 \\
868 \mathrm{MHz}\end{array}$ & $\begin{array}{l}7,24 \\
40 \mathrm{~km}\end{array}$ & 1 año \\
\hline RFIX BAT-0901 & \multirow{3}{*}{ La empresa no comercializa sensores } & \multirow{3}{*}{$2400 \mathrm{MHz}$} & \multirow{3}{*}{$800 \mathrm{~m}$} & 540 días \\
\hline RFIX AC-0901 & & & & Ilimitada \\
\hline RFIX SC-0901 & & & & 112 días sin sol \\
\hline GAIA Soil-Mote & $\begin{array}{c}\text { Temperatura, porcentaje volumétrico y conductividad eléctrica } \\
\text { del suelo }\end{array}$ & $2400 \mathrm{MHz}$ & $100 \mathrm{~m}$ & 165 días \\
\hline GAIA 2 & $\begin{array}{c}\text { Temperatura, porcentaje volumétrico, conductividad eléctrica } \\
\text { del suelo, humedad relativa y temperatura del ambiente, } \\
\text { temperatura y conductividad eléctrica del agua en una balsa de } \\
\text { riego }\end{array}$ & $2400 \mathrm{MHz}$ & $\begin{array}{c}200 \mathrm{~m} / 16 \\
\mathrm{~km}\end{array}$ & $\begin{array}{c}521 \text { días } \\
\text { (batería } \\
\text { 12V/6,5Ah; } \\
\text { comunicación } \\
\text { de largo } \\
\text { alcance) }\end{array}$ \\
\hline
\end{tabular}

Tabla 3.2. Características principales de sistemas de monitorización comerciales.

\subsubsection{Prototipos desarrollados en centros de investigación.}

En la sección anterior se describieron soluciones comerciales tanto de motes sencillos como de sistemas listos para ser instalados y empezar a monitorizar. Pero no todos los desarrollos de motes para agricultura de precisión son comerciales. Existen prototipos que han sido desarrollados por investigadores, y que serán descritos en esta sección. 
Durante estos últimos años, se han implementado diversas aplicaciones de WSN en el campo de la agricultura. Así, se pueden encontrar diferentes referencias en la literatura. Camilli et al. [Camilli, 2007] demostraron la utilidad de una WSN en este campo a nivel de simulación. Muestran que una WSN puede validar una estimación del cultivo, construida sólo a partir de datos locales, con menos de un 3\% en pérdida de precisión en comparación con un enfoque centralizado. También muestran cómo utilizar la capacidad de comunicación y de procesamiento de una WSN para crear nuevos paradigmas en aplicaciones de agricultura de precisión, así como dilucidar algunas de las ventajas y los inconvenientes que se derivan de este enfoque distribuido de codificación.

Pierce et al. [Pierce, 2008] describieron el hardware y el software que desarrollaron, para redes de ámbito local y regional, así como sus implementaciones en dos aplicaciones de AP en el estado de Washington. La red regional está basada en el datlogger AWN200 (desarrollado por los autores) equipado con una radio operando en la banda de frecuencia de $900 \mathrm{MHz}$. La red de ámbito local está basada en el SS100 radio/logger desarrollado por los autores, que incluye una transceptor de radio de $900 \mathrm{MHz}$. Esta red está desplegada con una topología en estrella, en la que el nodo sumidero estratégicamente situado es el responsable de la sincronización de la red, la recogida de datos de las estaciones remotas y de retransmitir los datos recogidos hasta un nodo conectado a un ordenador portátil.

Morais et al. [Morais, 2008] desarrollaron el prototipo MPWiNodeZ (ver Figura 3.28), que está basado en el micro-controlador wireless de Jennic JN5121. El dispositivo incluye una batería de NiMH de $650 \mathrm{mAh}$ de capacidad, que se puede recargar desde 3 fuentes de energía alternativas (ver diagrama de bloques de la Figura 3.28) y dispone de 8 canales analógicos de 0 a 2,5V para la conexión de sensores. El MPWiNodeZ soporta ZigBee y, por tanto, se puede desplegar redes con topologías en estrella, árbol y malladas. Finalmente, cabe destacar que el dispositivo está pensado para monitorizar viñedos.

Figura 3.28. Vista y diagrama de bloques del MPWiNodeZ [Morais, 2008].

En California, Beckwith et. al [Beckwith, 2004; Beckwith, 2004a] diseñaron, desplegaron y analizaron los resultados de la implementación de una WSN de gran escala en un viñedo. Desplegaron 65 motes comerciales MICA2 en el área planificada, donde no se implementaron funcionalidades de descubrimiento de vecinos y un table driven protocol fue 
usando en lugar de una red auto-organizada. Los nodos, separados $15 \mathrm{~m}$ los unos de los otros, realizaban una lectura cada 5 minutos. El análisis de los datos permitió predecir el $\mathrm{pH}$, la acidez y el peso de las bayas. Usando WSN, pudieron determinar con 6 horas de antelación el inicio de las heladas cuando el productor del vino tendría que tomar medidas para hacer frente a un problema climático.

En Europa, el proyecto Lofar Agro [Baggio, 2005; Langendoen, 2006] consistía en un estudio de agricultura de precisión focalizado en el mantenimiento, a medida, de un cultivo. Esto implicaba monitorizar el suelo, las plantas y las condiciones ambientales en un cultivo, generalizando los resultados y proporcionando un sistema de soporte a las decisiones para los tratamientos, o tomar diferentes acciones, como la variación del fertilizante o aplicación de pesticidas. El sistema de soporte a las decisiones, obtenía información de una estación meteorológica y de la red inalámbrica. La información era utilizada para mapear la distribución de humedad y temperatura del suelo, que se usaba para determinar una estrategia efectiva para controlar las plagas. La WSN estaba constituida por 150 dispositivos denominados Lofar Mote (Figura 3.29), que eran similares a un mote MICA2. Los nodos realizaban una lectura de la humedad y la temperatura cada minuto y por motivos de eficiencia energética enviaban los datos cada 10 minutos. Para mejorar las comunicaciones, 30 de los 150 motes funcionaban como repetidores, y todos los dispositivos estaban instalados a $75 \mathrm{~cm}$ del suelo.

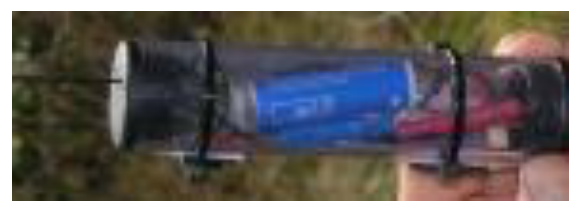

Figura 3.29. E1 mote Lofar.

La humedad del suelo juega un importante papel en la distribución del agua y de los flujos de energía, proporcionando humedad a la atmósfera para las precipitaciones, y controlando el patrón de recarga de las aguas subterráneas. La variabilidad de la humedad del suelo es impulsada por las precipitaciones espaciadas temporalmente y los patrones de radiación. A escala local, la cobertura del suelo, sus condiciones y su topografía actúan para redistribuir la humedad. A pesar de la importancia de la humedad del suelo, no ha sido medida todavía de forma operativa. En esta línea, el proyecto SoilNet [SoilNet, Bogena, 2007] impulsaba el desarrollo de redes de sensores de humedad del suelo para monitorizar los cambios del contenido de agua en el suelo a una elevada escala espacial y temporal. Las principales características del proyecto eran las siguientes:

- WSN basadas en ZigBee con topología mallada.

- Bajo consumo energético para tener una elevada autonomía.

- Dinámicas, redes escalables (hasta más de 1000 nodos). 
- Capacidad de reaccionar ante factores externos (cambiar la frecuencia de lectura).

- Diferentes configuraciones de nodos, adaptadas a las necesidades de medida.

- Almacenamiento de los datos en una base de datos (acceso fácil y variable).

La pequeña área de cuencas de Wüstebach (sobre 26,7ha) fue propuesta para ser monitorizada con la red de humedad del suelo SoilNet. La red estaba compuesta por 286 sub-nodos y 12 nodos con el rol de coordinador. En su totalidad, la red era gestionada por un servidor principal, que estaba conectado con las telecomunicaciones necesarias (por ejemplo, ADSL), para poder establecer transmisiones con el espacio de trabajo. La Figura 3.30 muestra de izquierda a derecha, una vista de los nodos sensores, del router y del servidor principal. Todos ellos estaban basados en un micro-controlador dotado de un enlace wireless.
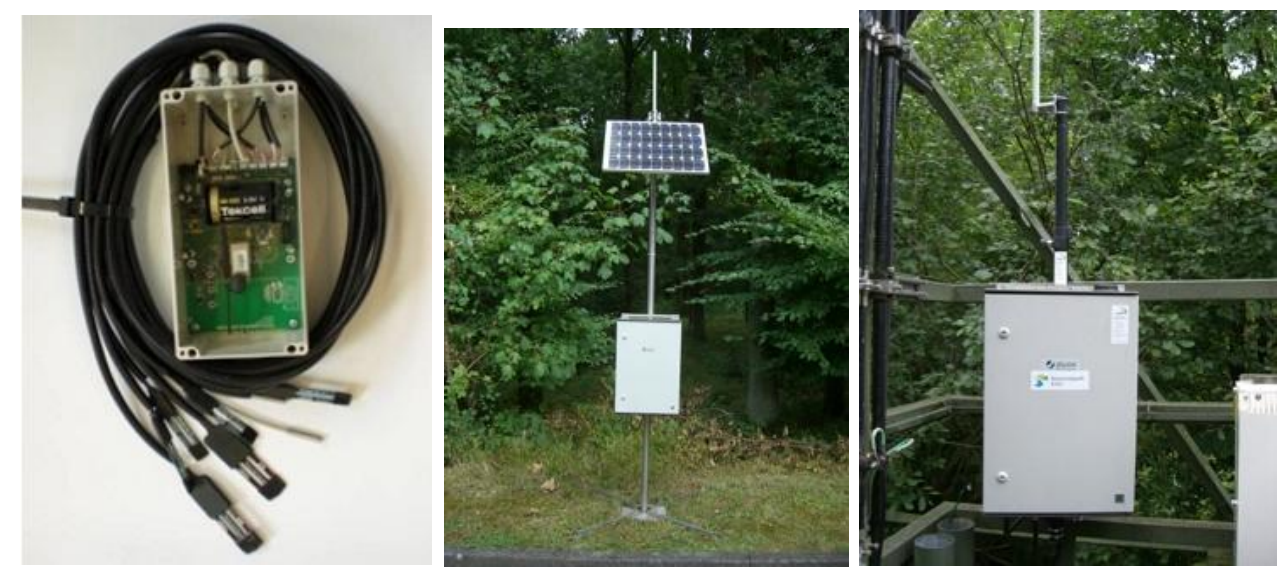

Figura 3.30. Dispositivos del proyecto SoilNet.

Como muchas de las condiciones que un agricultor puede querer monitorizar (por ejemplo, la presencia de un virus en una planta, el nivel de nutrientes en el suelo) se producen a nano-escala, y porque las superficies pueden ser alteradas a nano-escala obligadas de forma selectiva con proteínas biológicas particulares, los sensores con sensibilidad a nano-escala son particularmente importantes para obtener una medida del estado de los cultivos. En esta línea, el Departamento de Agricultura de Estados Unidos desarrolló un sistema completo inteligente para los cultivos (Smart Field System) que automáticamente detectaba, localizaba, informaba y dosificaba el agua, fertilizantes y pesticidas, es decir, que iba más allá de la detección y permitía conseguir una aplicación automática [AZoNanotechnology].

Anurag et. al [Anurag, 2008] diseñaron una WSN para monitorizar parámetros agrícolas (humedad, $\mathrm{pH}$, temperatura y conductividad del suelo) y, consecuentemente, controlar el riego y la fertirrigación que se llevaba a cabo en la agricultura de precisión. La 
red estaba basada en la tecnología IEEE 802.15.4. La salida de los sensores llegaba al microprocesador, que estaba basado en un dispositivo de radiofrecuencia como el CC2420 de Texas Instruments. Los datos, en formato digital, eran encapsulados en paquetes que se enviaban al repositorio central con la ayuda de los tres dispositivos diferentes que componían la red (ver Figura 3.31): Sensor, Router y Gateway.

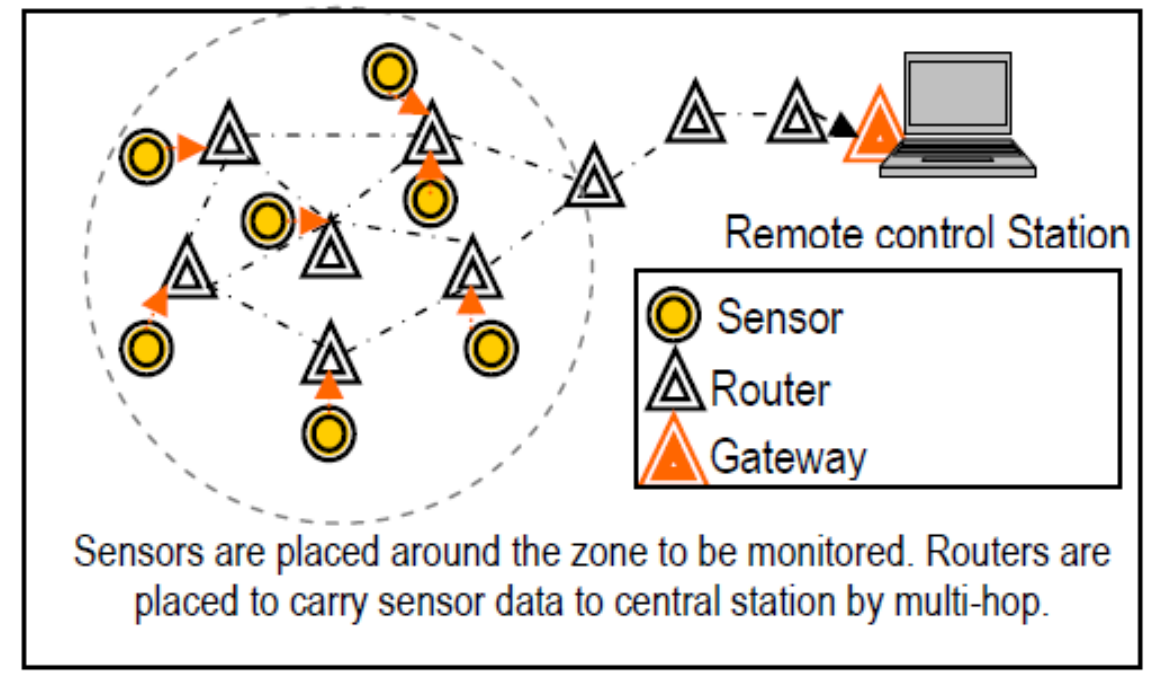

Figura 3.31. Arquitectura de la red de Anurag et. al.

Hirafuji et. al [Hirafuji, 2004] describían que para monitorizar el arroz en los campos de arroz son necesarias las comunicaciones inalámbricas de banda ancha, tecnologías para monitorizar imágenes de alta resolución y varios sensores. Por ello, para este tipo de aplicaciones proponían sistemas más potentes que los basados en una WSN. En concreto sugerían utilizar los denominados servidores de cultivo (FS, Field Server) y servidores de monitorización del cultivo (FMS, Field Monitoring Server), siendo este último un FS con capacidad de monitorización en tiempo real. El FMS estaría compuesto de un FS (servidor micro-web para control y adquisición de datos), un punto de acceso wireless LAN, una cámara inalámbrica y los sensores [Hirafuji, 2002; Fukatsu, 2003]. Estos equipos permitían una elevada velocidad de comunicación (54/11Mbps), pero tenían la principal desventaja del coste del equipo y las elevados consumos energéticos de estos equipos.

El sistema NAV (Network Avanzato per il Vigneto-Advanced Vineyard Network) era una WSN diseñada y desarrollada con el objetivo de monitorizar datos en tiempo real y obtener parámetros micro-meteorológicos en un viñedo [Matese, 2009]. El sistema incluía una estación base agro-meteorológica (MU, Master Unit) y un conjunto de nodos inalámbricos (SU, Slave Units), localizados en el viñedo. La MU era una estación de monitorización común, que estaba situada fuera del viñedo, en un punto representativo para recoger los datos agro-meteorológicos, y tenía la capacidad de manejar hasta 20 nodos dentro de un alcance máximo de 200m en línea de vista. Los elementos principales de la unidad eran un micro-controlador de 16 bits con 12 entradas analógicas y un módulo transmisor fabricado 
por AUREL (XTR9039), que operaba en la banda ISM de 433MHz. El sistema de alimentación incluía un panel solar fotovoltaico de $50 \mathrm{~W}$ y una batería de $12 \mathrm{~V}$ y $12 \mathrm{Ah}$. Las SUs estaban equipadas con diferentes sensores agro-meteorológicos (temperatura del aire, del suelo, de las hojas y de las uvas, velocidad del viento, etc.), y enviaban sus lecturas a la MU. Estaban compuestas por un microprocesador de 16 bits con varios canales analógicos de entrada, y del módulo de radio mencionado para la MU. En este caso, se usaba un panel fotovoltaico de $9 \mathrm{~W}$ y una batería de 4,5Ah y 6V. La Figura 3.32 muestra una imagen de una SU y del despliegue realizado.

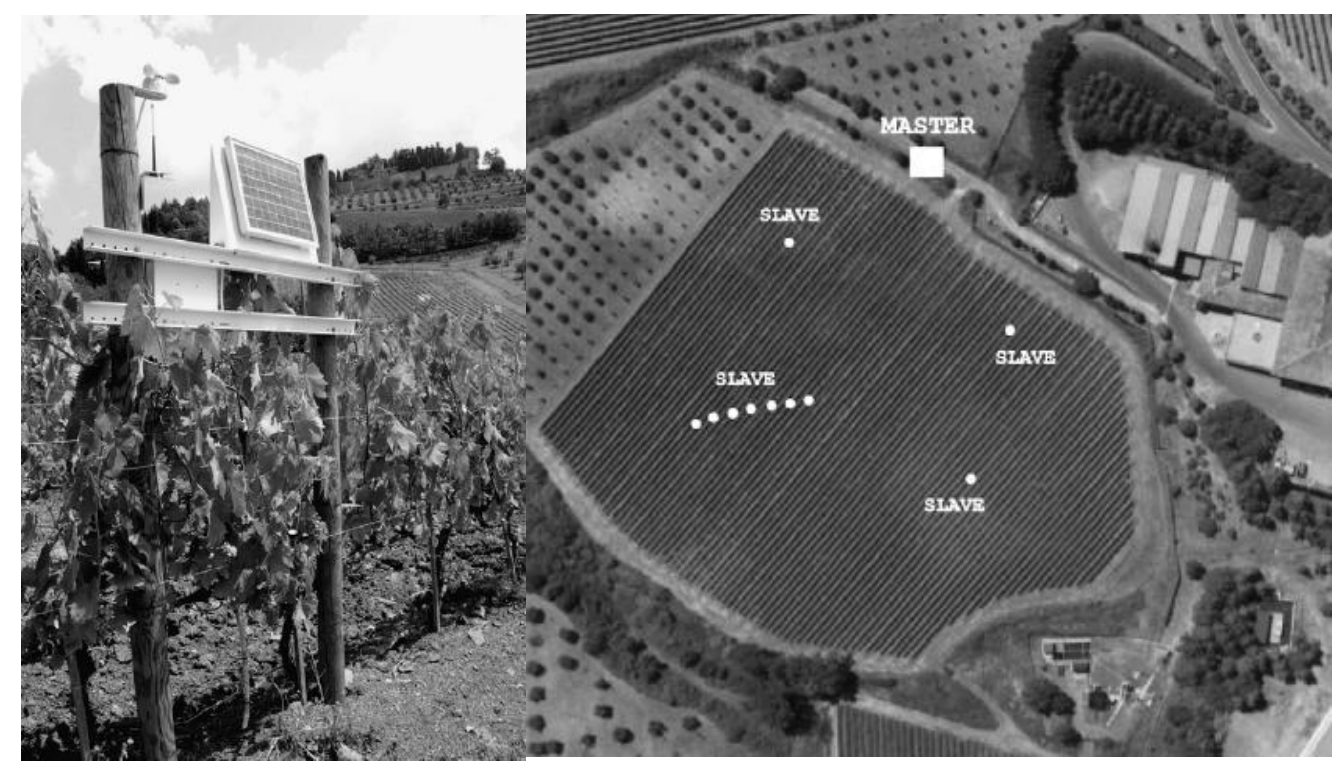

Figura 3.32. Vista de una SU y situación del despliegue realizado en el viñedo [Matese, 2009].

Allen [Allen, 2000] evaluó un sistema de gestión del riego que podía proporcionar información de la cantidad de agua en el suelo en tiempo real o cuasi tiempo real al operador del sistema del riego. Este sistema usaba dos tipos diferentes de registradores de datos (dataloggers) para obtener y almacenar datos de los sensores de suelo Watermark (Irrometer Co., Riverside, CA, USA). Los dataloggers estaban instalados en el cultivo, cerca de los sensores y cableados con los mismos. Sin embargo, este sistema no proporcionaba una solución inalámbrica porque requería que el operador se desplazara hasta los dataloggers para descargar los datos. Shock et. al [Shock, 1999] usaron un sistema similar, pero transmitiendo los datos desde los dataloggers hasta la central de recogida de datos vía radio. Este sistema permitía conectar hasta 16 sensores de suelo Watermark con un datalogger propietario. Pero, a menos que todos los sensores se situaran cerca del equipo, el sistema necesitaba un extenso cableado. El gasto de los dataloggers impedía desplegar una población densa de sensores en el campo. King et. al [King, 2000] y Wall y King [Wall, 2004] proponían utilizar la arquitectura de una red de sensores distribuidos que incluía los controladores para un sistema de riego variable. En los artículos se cita que, aunque este enfoque permitía distribuir un gran número de sensores, en este punto, seguía siendo un 
sistema teórico. Por otro lado, Hamrita y Hoffacker [Hamrita, 2005] exploraron la tecnología RFID como una solución para monitorización inalámbrica, en tiempo real, de las propiedades del suelo. A nivel de laboratorio, demostraron que la tecnología RFID es viable para la comunicación inalámbrica, en tiempo real, con un sensor de temperatura del suelo.

Vellidis et. al [Vellidis, 2008] desarrollaron y probaron un prototipo, con componentes disponibles en el mercado. Estaba basado en un array de sensores inteligentes, y medía la humedad y la temperatura del suelo para poder programar el riego en un cultivo de algodón. El array consistía en un receptor (situado en el centro), conectado a un ordenador portátil y múltiples nodos sensores de instalados en el campo. Los nodos de sensores estaban compuestos de los sensores (hasta 3 sensores de humedad del suelo Watermark ${ }^{\circledR}$ y cuatro termopares), una placa de circuito especialmente diseñada, y una etiqueta (tag) de identificación por radiofrecuencia que transmitía los datos al receptor. El array de sensores inteligentes ofrecía un potencial real para el seguimiento fiable del estado del suelo, que variaba espacialmente en los campos de un cultivo. El coste relativamente bajo del sistema (aproximadamente $2.400 \$$ para un sistema de 20 nodos sensores) permitía la instalación de un gran número de sensores de humedad del suelo, que permitían representar adecuadamente la variabilidad del suelo en las parcelas. La integración de los sensores con las tecnologías de riego proporcionaba un sistema de lazo cerrado de control del riego donde las entradas del array de sensores determinaban las fechas y las cantidades específicas de riego que se necesitaban aplicar en cada lugar. La comunicación entre los nodos sensores y el receptor se establecía usando las denominadas 'WhereNet tags activas RFID', que operaban en la banda de 2,4GHz, con un alcance de $800 \mathrm{~m}$ en línea de vista. La Figura 3.33 muestra una fotografía del primer prototipo instalado en campo y, en la parte derecha, una versión mejorada con la tag instalada por encima del cultivo, con objeto de mejorar la calidad y la fiabilidad de las comunicaciones.

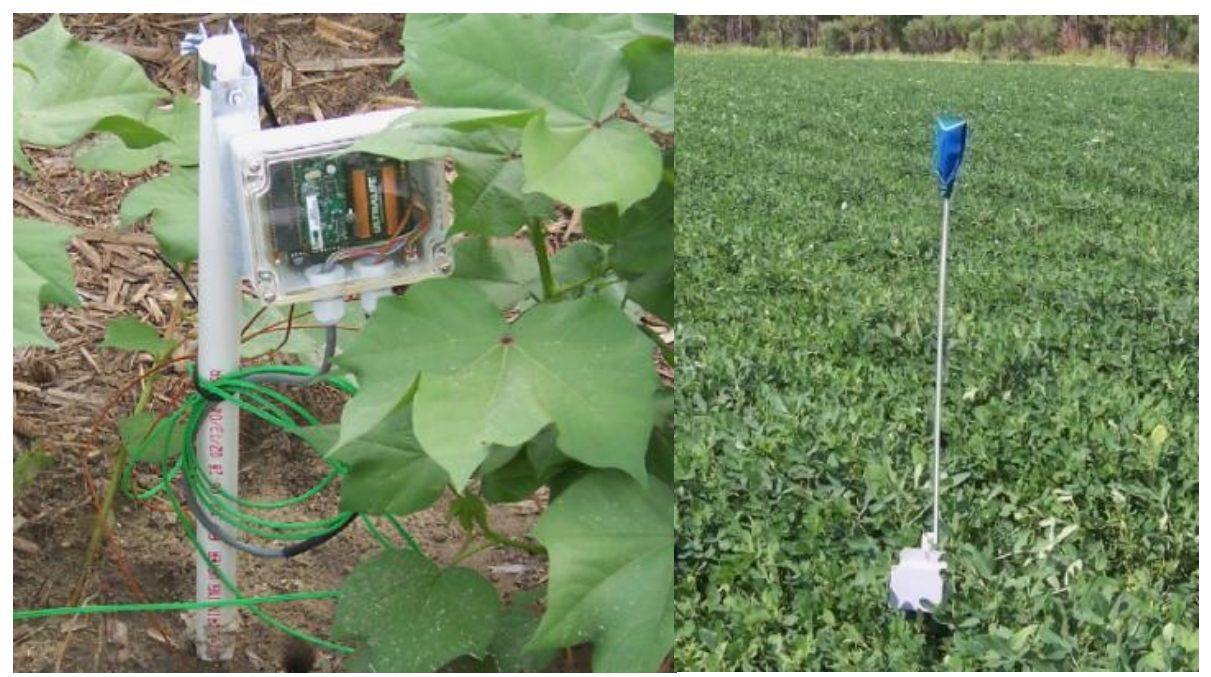

Figura 3.33. Fotografías de los prototipos primero y segundo de Vellidis et. al. 
Cano et. al [Cano, 2007] implementaron un sistema para monitorizar la humedad del suelo usando WSN en un área de $10 \times 10 \mathrm{~km}^{2}$. El despliegue englobaba 12 estaciones con la siguiente distribución: 5 en parcelas de vid, 3 en árboles frutales y 4 en zonas de arbustos. Los elementos principales de cada estación eran: un micro-controlador PIC16F876 de Microchip, la sonda capacitiva ThetaProbe ML2x, de Delta-T Devices, y el radio modem Wlink8S, comercializado por DMD, el cual operaba en la banda de $868 \mathrm{MHz}$. En algunos nodos, usaron antes omnidireccionales de 7,3dBi y, en otros, antenas direccionales Yagi de $12 \mathrm{dBi}$ (ver Figura 3.34). Con esta configuración, en un enlace de dos antenas omnidireccionales consiguieron un alcance de $4,7 \mathrm{~km}, \mathrm{y}$, entre una direccional y otra omnidireccional, el alcance fue de unos $8 \mathrm{~km}$.
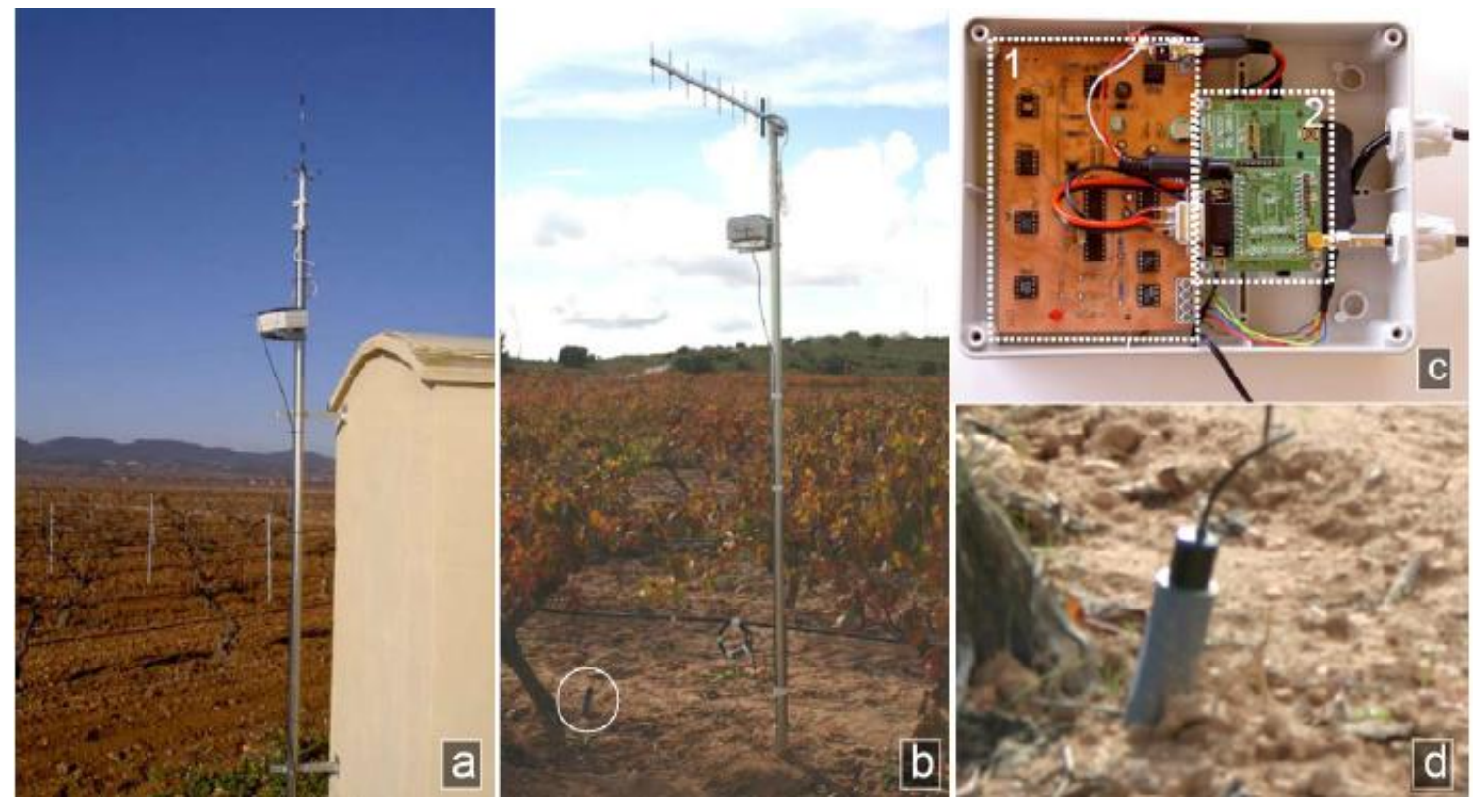

Figura 3.34. Diversas vistas de la WSN implementada por Cano et. al.

Conviene destacar que, debido a sus interesantes ventajas, la tecnología WSN ha sido progresivamente adoptada en una serie de proyectos de monitorización agrícolas posteriores [Konstantinos, 2007; Camilli, 2007; Cano, 2007]. Sin embargo, el enfoque ha sido principalmente en la agricultura de precisión en los países desarrollados, con la excepción de las recientes implementaciones de algunas regiones en desarrollo [Panchard, 2006]. A pesar de estas implementaciones, este campo de trabajo no ha alcanzado todavía la madurez con la mayoría de los proyectos, estando éstos o bien en fase de exploración, o en sus iteraciones iniciales, o en fases de evaluación. En otras palabras, todo el potencial de las WSNs en la agricultura, especialmente para la creación de entornos, todavía no ha sido explorado [Kabashi, 2008]. Es necesario resaltar también que Porter et al. [Porter, 2005] analizaron 52 publicaciones elegidas aleatoriamente en revistas de ecología para demostrar que las WSNs pueden cubrir el vacío existente, permitiendo a los investigadores muestrear a distancias y velocidades que no son actualmente posibles. 


\subsection{Conclusiones.}

La Agricultura de Precisión es una técnica que tiene como objetivo conocer de forma intensiva las características de una determinada zona del cultivo, con el fin de aplicar la dosis necesaria de insumos en esa zona y optimizar la producción.

Las redes de sensores inalámbricas están demostrando ser una tecnología prometedora, que se está aplicando con éxito en numerosas aplicaciones, incluidas en las aplicaciones de Agricultura de Precisión. Sin embargo, en este campo se pueden encontrar muchas referencias en la literatura científica a nivel de investigación, y pocos despliegues reales realizados en los últimos años. Esto se debe a que el sector agrícola es muy precario, y el precio de la tecnología está empezando a ser asequible para los agricultores, debido a los desarrollos hardware que se están llevando a cabo en los últimos 4 años.

El estado del técnica de las soluciones hardware comerciales y de prototipos de motes desarrollados en centros de investigación, justifica la falta de uso de las redes de sensores inalámbricas en la Agricultura de Precisión. Por ello, es necesario seguir diseñando nuevas plataformas hardware (motes) de bajo coste, que permitan conocer, en tiempo real, el estado de los cultivos y proporcionar el soporte necesario a la Agricultura de Precisión. Además, es necesario proporcionar en el mercado productos robustos, que puedan ser usados tanto en condiciones exteriores como interiores, con una arquitectura abierta que facilite la tarea de conexión de los sensores y actuadores comerciales requeridos. 
CONTRIBUCIÓN A LAS REDES DE SENSORES INALÁMBRICAS. ESTUDIO E IMPLEMENTACIÓN DE Soluciones Hardware para Agricultura de Precisión. 


\section{Capítulo 4}

\section{Diseño y Validación de una Arquitectura Basada en WSN para Cultivos Hortícolas.}

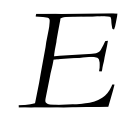

$n$ este capitulo se propone una red de sensores inalámbrica para poder monitorizar, en tiempo real, cultivos horticolas. Para dar solución al problema propuesto, se han capturado especificaciones teniendo en cuenta las características de los cultivos a monitorizar, y el tipo de sensores a utilizar. A partir de dichas especificaciones, se ha propuesto una arquitectura de red basada en el estándar de comunicaciones IEEE 802.15.4, y una serie de nodos que se han diseñado ad-hoc para implementar la red. Entre ellos, destacan una serie de nodos sensores especializados (Soil-Mote, Environmental-Mote y Water-Mote) y un conjunto de elementos auxiliares de comunicaciones (Gateway, Nodo-Sumidero y Estación Base). En un paso previo al desarrollo de los dispositivos, se realizaron las simulaciones necesarias para confirmar que las prestaciones obtenidas, en cuanto a autonomía, tasa binaría de envio/ recepción de información y retrasos en las transmisiones, fueran aceptables con la tecnología seleccionada. El capitulo, también describe los dispositivos desarrollados, y las dos fases de validación llevadas a cabo con los mismos, con objeto de contrastar los datos del entorno de simulación. En la primera fase, se confirmó en laboratorio el correcto funcionamiento de los dispositivos, y los resultados de consumo de los dispositivos obtenidos del simulador. En una segunda fase, se desplegó el sistema en un cultivo real de col picuda con el fin de contrastar los resultados de los retrasos obtenidos en las simulaciones con los resultados reales, así como para verificar un correcto funcionamiento de la red de sensores inalámbrica desarrollada. 


\subsection{Introducción.}

Los trabajos recogidos en este capítulo caen dentro del marco del proyecto de "Sistemas de Redes Inalámbricas de Micro Dispositivos para Monitorización Sensorial Inteligente", (RIMSI, del 12/2006-12/2007) [RIMSI]. Dicho proyecto estuvo subvencionado por el Ministerio de Industria, Comercio y Turismo y cofinanciado por el Fondo Europeo de Desarrollo Regional (FEDER). Dos eran los objetivos fundamentales de este proyecto. En primer lugar, la obtención de una guía de las mejores prácticas para la implementación de aplicaciones basadas en WSNs en los ámbitos de la agricultura y la construcción. En segundo lugar, pretendía proporcionar a sus participantes la tecnología necesaria para implantar soluciones tecnológicas en los dominios descritos, por lo que contaba con una parte innovadora considerable, ya que, en ese momento, existían muy pocas experiencias en España y Europa en el campo de las WSN. El consorcio estaba formado por los siguientes socios: Métodos y Tecnología de Sistemas y Procesos [MTSP], Edosoft Factory [EDOSOFT], Acciona Infraestructuras [Acciona] y Langmead [Langmead]. Durante el transcurso del proyecto, los socios desarrollaron componentes middleware reutilizables, protocolos de comunicación robustos, y obtuvieron experiencia en el diseño, desarrollo y aplicación real de las WSN, dentro de los campos de la construcción y la agricultura mediante el prototipado de soluciones ad-hoc.

Los socios del proyecto RIMSI, también participaron en el proyecto europeo de redes de sensores con acrónimo ESNA (European Sensor Network Architecture) [ESNA] y financiado por la ITEA (Information Technology for European Advancement).

Las tareas de Edosoft Factory eran, entre otras, llevar a cabo los estudios, pruebas e implementaciones necesarias para desarrollar un conjunto de motes con los que monitorizar en el ámbito agrícola, concretamente en cultivos hortícolas. Para llevar a cabo estas tareas, la empresa firmó un contrato Artículo 83 con investigadores del grupo de investigación DSIE (División de Sistemas e Ingeniería Electrónica) [DSIE], de la Universidad Politécnica de Cartagena, para desarrollar estos dispositivos en el marco de la presente Tesis Doctoral.

\subsection{Captura de especificaciones.}

\subsubsection{Topología de la red.}

En las WSNs, la eficiencia energética y la cobertura de amplias extensiones son requisitos esenciales en el desarrollo de aplicaciones destinadas a la agricultura de precisión [Akyildiz, 2002; Zhang, 2002]. Los dispositivos inalámbricos usados son autónomos, lo que significa que su fuente de alimentación es limitada, con una duración condicionada por los 
períodos agrícolas que se requieran monitorizar. Por otra parte, las amplias extensiones geográficas de terreno bajo observación requieren de una adecuada planificación para el despliegue de los dispositivos de red (topología de la red), y una selección de las tecnologías de comunicaciones más apropiadas. Esto constituye un punto crítico en el diseño, puesto que una selección adecuada permite enviar la información de, forma eficiente, a largas distancias.

Son varios los trabajos que profundizan en la forma de cumplir estos requisitos: en [Beckwith, 2004; Panchard, 2008; Katsalis, 2007] se proponen topologías malladas o en 'clúster' [IEEE, 2006] como solución para analizar amplias extensiones de terreno, donde los dispositivos inalámbricos además de realizar sus tareas de toma y envío de datos del entorno; también realizan labores de enrutamiento de la información. En estos trabajos, el consumo energético debido a esta doble funcionalidad (toma/envío de datos y enrutamiento) se obvia o queda en un segundo plano. Morais et al. [Morais, 2008] realiza un profundo estudio de los requisitos previos (eficiencia energética y cobertura), adoptando como solución una topología mallada que incorpora tres tipos de dispositivos: (1) dispositivos de toma y envío de datos sensoriales, con alimentación limitada la mayor parte de las veces, (2) dispositivos de enrutamiento, con alimentación ilimitada (recarga mediante paneles solares) y (3) gateways, que transmiten la información, vía GSM/GPRS, al usuario final o a Internet y que también disponen de alimentación independiente e ilimitada. Este sistema permite resolver los requisitos previamente mencionados, pero incrementando la dificultad de desarrollo, implantación y mantenimiento. En la referencia [Ayday, 2009], se implementa la monitorización de una parcela agraria con una red en topología en estrella, en donde las dimensiones de la parcela son apropiadas para una comunicación eficiente entre dispositivos de sensores inalámbricos comerciales y un gateway. En este trabajo, la captura de la información sensorial se realiza 'in-situ' de forma centralizada en el Gateway. Por tanto, no se dispone de un soporte físico que permita una comunicación a distancia entre la parcela a estudio y el usuario final.

En conclusión, la topología de la red es un factor de diseño crítico en una WSN, y que depende mucho de su despliegue en los cultivos a monitorizar. En el caso de la presente Tesis, el marco de trabajo se situaba en la zona agrícola conocida como "Campo de Cartagena"; localizado en el sureste de la Península Ibérica, formando una llanura que se extiende desde la Sierra de Carrasco hasta el Mar Mediterráneo. A lo largo de esta zona agrícola, predominan los cultivos hortícolas y sus explotaciones se caracterizan por estar distribuidas en parcelas de pequeña extensión (aproximadamente 4ha), y separadas entre sí por distancias no superiores a unos $10 \mathrm{~km}$, pudiendo cubrir extensiones de hasta $1.000 \mathrm{ha}$ (250 cultivos). 
Teniendo en cuenta estas circunstancias, se consideró que una topología en estrella era la más adecuada para monitorizar de forma distribuida las parcelas de explotaciones agrícolas con las características descritas anteriormente. Se optó por esta sencilla topología porque desplegando un nodo con el rol de coordinador en el centro de la parcela, y los nodos con el rol de dispositivo final, alrededor del anterior, era posible monitorizar adecuadamente las parcelas con una extensión media de 4ha descritas.

\subsubsection{Protocolo de comunicaciones.}

En el capítulo 3 se describieron un conjunto de protocolos de comunicación y las topologías de red asociadas con ellos. El objetivo de esta sección es seleccionar el protocolo de comunicación con el que garantizar las comunicaciones entre los nodos que componen la arquitectura de red objeto de estudio e implementación en esta Tesis. Esta selección permitió escoger el módulo o transceptor de radio utilizado en los prototipos finales.

Una WPAN (red inalámbrica de área personal) es la opción más adecuada para monitorizar un caso de estudio agronómico. A su vez, las WPANs disponen para implementar redes, bien de protocolos con baja tasa de envío de datos (IEEE 802.15.4), o bien de otros de envío intensivo de datos, pensados para transmitir audio y vídeo en tiempo real (IEEE 802.15.3). En los cultivos, las variables monitorizadas evolucionan despacio y, por ello, el estándar más adecuado para implementar las comunicaciones es el IEEE 802.15.4. La principal desventaja de éste es que sólo establece un nivel de comunicación dentro de la subcapa MAC y, por lo tanto, soporta topologías de red sencillas (en estrella o star y de pares o peer-to-peer). Sin embargo, tiene la gran ventaja de que el consumo de un dispositivo con el rol de dispositivo final es muy reducido, al no tener que realizar funciones de ruteado de paquetes procedentes de otros nodos.

En los últimos años, se han desarrollado otros protocolos de comunicación sobre la capa PHY y la subcapa MAC definidas por el IEEE 802.15.4, los cuales permiten gestionar las comunicaciones dentro la red inalámbricas con un nivel superior de abstracción. Entre ellos, cabe destacar ZigBee y 6LoWPAN. Como permiten gestionar las comunicaciones a un nivel mayor de abstracción, la cantidad de paquetes enviados por la red será mayor que la requerida al seguir el estándar IEEE 802.15.4 y, por el contrario, la autonomía de los nodos será menor. 6LoWPAN presenta un mayor nivel de abstracción que ZigBee, ya que permite un envío de paquetes IPv6 sobre una red basada en el IEEE 802.15.4.

En las redes WSN desplegadas en entornos agrícolas, los nodos se despliegan sobre el suelo y pueden recibir impactos, durante el desarrollo de las actividades habituales en el cultivo, por parte de los agricultores o de la maquinaría utilizada. Además, con el paso de 
las semanas, la vegetación del cultivo hortícola tendrá una mayor densidad y los nodos no serán fácilmente localizables y, por tanto, se incrementarán las dificultades asociadas a su mantenimiento. Entonces, es posible concluir que una arquitectura para monitorización agrícola debe caracterizarse por un bajo coste y una elevada autonomía de sus dispositivos. Así, a priori, la selección del estándar IEEE 802.15.4 sería la más adecuada. Sin embargo, en el capítulo 3 también se describió B-MAC [Polastre, 2004], que se trata de un protocolo de comunicaciones que permite establecer redes con la misma topología que el estándar IEEE 802.15.4, pero con consumo energético más reducido de los nodos que componen la arquitectura. Por ello, fue B-MAC el protocolo de comunicaciones seleccionado para intercambiar la información entre los nodos de la arquitectura.

\subsubsection{Selección de sensores agronómicos.}

En el año 2000, los investigadores de la Universidad Politécnica de Cartagena empezaron a implantar redes cableadas de sensores basadas en dataloggers en la Estación Experimental Agroalimentaria “Tomás Ferro" (ESEA en adelante) [ESEA]. Esta finca se encuentra ubicada frente al polígono industrial de La Palma, a $12 \mathrm{~km}$ de Cartagena, y pertenece a la Fundación Docente-Privada Tomás Ferro Navarro, constituida en 1985 con el fin de fomentar el desarrollo tecnológico de la agricultura en el campo de Cartagena.

La ESEA pone a disposición de los alumnos y del personal investigador de la UPCT modernos equipamientos agrícolas, como invernaderos experimentales de vidrio, diversa maquinaria agrícola, instalaciones automatizadas de riego, parcelas de regadío al aire libre, parcelas de cultivo ecológico, salas de investigación en tecnología e ingeniería de alimentos y salas de prácticas de mecanización agraria, entre otras. Alberga 13 laboratorios y una planta piloto. Cuenta, además, con una estación meteorológica automática, un lisímetro de pesada con drenaje forzado y un embalse de $10.000 \mathrm{~m}^{3}$. Contiene parcelas en las que se ha plantado diversas colecciones de cítricos, frutales y arbustos ornamentales, así como de otras destinadas a hortalizas, flor cortada y cereales. La superficie ocupada actualmente por la ESEA es de 10 hectáreas, ocupadas por el edificio administrativo y los laboratorios $\left(3.000 \mathrm{~m}^{2}\right)$, invernaderos $\left(2.500 \mathrm{~m}^{2}\right)$, umbráculos $\left(1.000 \mathrm{~m}^{2}\right)$ y terrenos de cultivo al aire libre. Dentro de la ESEA hay una red de sensores cableada, la cual se encuentra desplegada en una parcela de almendros (Prunus dulcis (Mill.) Webb, cv 'Marta' injertada sobre el portainjerto 'Mayor') [Nortes, 2008]. En esta parcela se aplican diferentes tratamientos a grupos de almendros seleccionados, y se monitoriza el estado del ambiente, del suelo y de las plantas mediante diferentes sensores, entre los que resaltan tensiómetros, dendrómetros, termoradiómetros y psicrómetros. Todos los sensores están conectados físicamente con los dataloggers, desde los que posteriormente se descarga, de manera local o remota, los datos almacenados procedentes de estos sensores. 
Esta experiencia en sensores agronómicos se ha utilizado en la presente Tesis para especificar el tipo de entradas/salidas necesarias en los nodos sensores, así como para definir otras características constructivas (tipo de micro-controlador, alimentación, etc.) de los mismos.

En el Anexo I de la presente tesis se describen, en detalle, las características técnicas de los sensores que se han considerado y, a continuación, las conclusiones a las que se han llegado.

\subsubsection{Sensores ambientales.}

Una WSN resulta de interés para controlar numerosas posiciones geográficas de la parcela de manera distribuida e independiente. Por ello, la conexión de sensores climáticos que sólo están presentes en una determinada posición (habitualmente en una estación agroclimática) no resulta de gran interés. No obstante, de los sensores descritos en el Anexo I, se considera importante que los nodos sensores soporten la conexión, tanto de una sonda comercial para la medida de temperatura y humedad relativa del ambiente, como de un psicrómetro especializado para la medida precisa de humedad relativa.

La mayoría de los micro-controladores disponibles en el mercado trabajan con tensiones de alimentación de 3,3V y sus entradas analógicas permiten digitalizar señales con valores comprendidos entre 0 y $2,5 \mathrm{~V}$. Ésta es una de las razones por las que se ha optado por utilizar como sensor de temperatura y humedad relativa del ambiente el sensor EE08 de E+E [EplusE]. Este sensor tienes características similares al HMP45C y un rango de salida de $0-2,5 \mathrm{~V}$, frente a los $0-1 \mathrm{~V}$ del sensor HMP45C utilizado en la estación agroclimática de la ESEA.

La temperatura y la humedad relativa del ambiente tienen una gran influencia en el estado del cultivo. Por ello, es importante poder obtener medidas fidedignas de estas variables. Esto se consigue con un psicrómetro y el sensor seleccionado es el H301. La humedad relativa se obtiene aplicando una serie de ecuaciones que tienen como entrada la temperatura de los bulbos seco y húmedo y un conjunto de constantes. Este sensor tiene la desventaja de requerir un mantenimiento periódico, el cual implica llenar cada semana el envase de agua destilada presente en éste, así como el sustituir periódicamente el cordón que humedece el sensor de la zona húmeda con el agua destilada de la botella. Además, el H301 debe tener activo de manera permanente un ventilador que trabaja con unos valores nominales de $12 \mathrm{~V}$ y $350 \mathrm{~mA}$. Por este motivo, será necesario diseñar un equipo de energía renovable para proporcionarle la alimentación necesaria cuando no se disponga de red eléctrica cerca del sensor. 
La Tabla 4.1 presenta las características principales de los dos sensores ambientales que incorporarán los nodos sensores de la arquitectura objeto de estudio e implementación.

\begin{tabular}{|c|c|c|c|c|}
\hline Sensor & Alimentación & Interfaz de salida & $\begin{array}{l}\text { Rango de medida } \\
\left(\mathrm{T}^{\mathrm{a}} / \mathrm{HR}\right)\end{array}$ & $\begin{array}{l}\text { Precisión } \\
\left(T^{\mathrm{a}} / \mathrm{HR}\right)\end{array}$ \\
\hline EE08 & $4,5-30 \mathrm{~V}$ & $\begin{array}{l}0-1 \mathrm{~V} / 0-2,5 \mathrm{~V} / 0-5 \mathrm{~V} / \\
0-10 \mathrm{~V} / \text { digital de } 2 \text { hilos }\end{array}$ & $-40-80^{\circ} \mathrm{C} / 0-100^{\circ} \%$ & $\pm 0,2^{\circ} \mathrm{C} / \pm 2^{2} \%$ \\
\hline H301 & $12 \mathrm{~V}, 350 \mathrm{~mA}$ & $\begin{array}{l}2 \text { RTDs con salida a } 4 \text { hilos Conexión } \\
\text { en puente de Wheastone }\end{array}$ & $-20-65^{\circ} \mathrm{C} / 0-100 \%$ & $\begin{array}{l}0,2^{\circ} \mathrm{C} / \text { relacionada } \\
\text { precisión de la } \mathrm{T}^{\mathrm{a}}\end{array}$ \\
\hline
\end{tabular}

Tabla 4.1. Características principales de los sensores ambientales seleccionados.

\subsubsection{Sensores de suelo.}

De los sensores descritos en el Anexo I, se consideró como solución óptima que los nodos de la arquitectura soporten la conexión de tensiómetros, de un Watermark y de sensores con prestaciones similares al sistema EnviroSCAN. La conexión con el Watermark es posible debido a las características típicas de los convertidores analógicos digitales (ADC) integrados en un micro-controlador, el cual trabaja con una tensión de alimentación de 3,3V. El acondicionamiento de señal con cada tensiómetro (lazo de corriente 4-20mA) no es complejo de diseñar y, una primera propuesta, consiste en una resistencia para convertir la corriente en tensión, junto con un amplificador operacional en la configuración de seguidor de tensión para adaptar las impedancias. El sensor con prestaciones similares al sistema EnviroSCAN seleccionado fue la sonda multi-paramétrica Hydra Probe II (HP2) de Stevens [Stevens]. El sensor HP2 se puede adquirir con dos interfaces de salida diferentes: SDI-12 [SDI12] y RS-485. De estas dos versiones, la que resulta más adecuada para aplicaciones con requerimientos de bajo consumo, es la de tipo SDI-12, ya que el consumo en standby es 10 veces menor que para la versión RS-485. Además, la versión SDI-12 también es más adecuada, a nivel de costes, ya que sólo son necesarios utilizar 3 hilos (alimentación, tierra y datos) de interconexión entre el sensor y el nodo sensor, frente a los 4 necesarios con la versión RS-485 (alimentación, tierra, com+ y com-).

Por lo tanto, los sensores de suelo que se pueden conectar a la arquitectura propuesta son: las sondas multi-paramétricas HP2, los tensiómetros Jet Fill 2725ARL y los sensores de tipo Watermark, cuyas principales características se resumen en la Tabla 4.2.

\begin{tabular}{|c|c|c|c|c|}
\hline Sensor & Alimentación & Interfaz de salida & Rango de medida & precisión \\
\hline HP2 & $9-20 \mathrm{~V}$ & $\begin{array}{l}\text { Digital de tipo } \\
\text { SDI-12 }\end{array}$ & $\begin{array}{l}\text { - Humedad: tanto por } 1 \\
\text { - Temperatura: }-10-65^{\circ} \mathrm{C} \\
\text { - Conductividad: } 0,01-1,5 \mathrm{~S} / \mathrm{m}\end{array}$ & $\begin{array}{l}\text { - Humedad: } \pm 0,03 \\
\text { - Temperatura: } \pm 0,1^{\circ} \mathrm{C} \\
\text { - Conductividad: } \pm 2 \% \text { ó } 0,005 \mathrm{~S} / \mathrm{m}\end{array}$ \\
\hline $\begin{array}{c}\text { Jet Fill } \\
\text { 2725ARL }\end{array}$ & $12-40 \mathrm{~V}$ & $\begin{array}{l}\text { Lazo de corriente } \\
\quad 4-20 \mathrm{~mA}\end{array}$ & $0-50 \mathrm{cb}$ & $\mathrm{N} / \mathrm{A}$ \\
\hline$\frac{\text { Watermark }}{200 S S-V}$ & $5 \mathrm{~V}$ & $0-2,8 \mathrm{~V}$ & $0-239 \mathrm{cb}$ & $\mathrm{N} / \mathrm{A}$ \\
\hline
\end{tabular}

Tabla 4.2. Características principales de los sensores de suelo seleccionados. 


\subsubsection{Sensores para monitorizar el estado hídrico de las plantas.}

El estado hídrico de las plantas es otra información muy importante del cultivo, que se puede determinar estimando la evapotranspiración de las plantas usando una instrumentación específica basada en dendrómetros y termoradiómetros. Los dendrómetros se utilizan para monitorizar la evolución del diámetro del tronco. El mismo sensor se puede utilizar para monitorizar la evolución del crecimiento del fruto, que también resulta de interés para estimar las necesidades hídricas de la plata monitorizada. Con el termoradiómetro se determina la temperatura de la superficie de la planta, que es una componente crucial de la transferencia de energía entre la planta y el ambiente.

Los sensores para monitorizar el estado de las plantas, escogidos para incorporar en los nodos de la arquitectura, son los siguientes: el termoradiómetro IRTS-P de Apogee [Apogee] y el tensiómetro basado en un sensor de desplazamiento variable y de respuesta lineal tipo LVDT (Linear Variable Differential Transformer) modelo DFg2.5 de Solartron [Solartron]. Más detalles sobre estos sensores se pueden encontrar en el Anexo I.

La Tabla 4.3 resume las principales características de los dos sensores, dedicados a monitorizar el estado hídrico de la plantas mencionados anteriormente.

\begin{tabular}{|c|c|c|c|c|}
\hline \multirow{2}{*}{ Sensor } & Alimentación & Interfaz de salida & Rango de medida & Precisión \\
\cline { 2 - 5 } IRTS-P & Pasivo & Salida de termopar tipo $\mathrm{K}$ & $-10-55^{\circ} \mathrm{C}$ & $\pm 0,5^{\circ} \mathrm{C}$ \\
\cline { 2 - 5 } & $10-24 \mathrm{Vcc}$ & $\begin{array}{c}750 \mathrm{mV} / \mathrm{mm} \operatorname{mon} 10 \mathrm{~V} \text { de } \\
\text { alimentación }( \pm 1,875 \mathrm{~V})\end{array}$ & $5 \mathrm{~mm}$ & $1-10 \mu \mathrm{m}$ \\
\hline
\end{tabular}

Tabla 4.3. Características principales de los sensores IRTS-P y DFg2.5.

\subsubsection{Interfaces de entrada salida necesarias para los motes.}

La Tabla 4.4 resume los sensores que deberán soportar los nodos de la arquitectura, basada en una WSN, objeto de estudio e implementación, detallando el sensor, la interfaz eléctrica necesaria para su lectura y la variable medida.

\begin{tabular}{|c|c|c|}
\hline Variable & Interfaz eléctrica & Sensor \\
\hline \multirow{2}{*}{ Temperatura y humedad relativa del ambiente } & $0-2,5 \mathrm{~V}$ & EE08 \\
\hline & RTD 4 hilos & H301 \\
\hline $\begin{array}{l}\text { Temperatura, porcentaje } \text { volumétrico } \\
\text { conductividad del suelo }\end{array}$ & SDI-12 & Hydra Probe II \\
\hline \multirow{2}{*}{ Potencial matricial. Depresión suelo } & $4-20 \mathrm{~mA}$ & Tensiómetro Jet Fill 2725ARL \\
\hline & $0-2,8 \mathrm{~V}$ & Watermark \\
\hline Temperatura superficie planta & Termopar tipo $\mathrm{K}$ & Apogee IRTS-P \\
\hline Variaciones diámetro tronco. Crecimiento fruto & $\pm 1,875 \mathrm{~V}$ & DFg2 5 \\
\hline
\end{tabular}

Tabla 4.4. Resumen de las interfaces de las que debe estar dotado el mote a diseñar. 
El objeto de esta sección es presentar las conclusiones más importantes sobre las interfaces eléctricas analógicas y digitales que deben aunar los nodos de la arquitectura, para que los sensores seleccionados en la sección anterior puedan ser conectados. Como se puede observar en la Tabla 4.4 todas las interfaces a describir son de tipo analógico, a excepción de la interfaz digital de tipo SDI-12 del sensor Hydra Probe II. Una descripción más detallada sobre estas interfaces se puede encontrar en el Anexo I.

La salida en lazo de corriente del tensiómetro se puede cambiar a valores de tensión comprendidos entre 0 y 2,4V utilizando una resistencia de $120 \Omega$. Además, la mayoría de micro-controladores que trabajan con tensiones de alimentación de 3,3V, proporcionan integrados en el mismo chip convertidores A/D con varios canales analógicos de entrada, capaces de muestrear señales analógicas entre $0 \mathrm{~V}$ y la tensión de referencia positiva configurada; siendo habitual disponer de márgenes de muestreo comprendidos en los rangos de tensión: [0-1,5V], [0-2,5V], y [0-3,3V]. Por ello, la electrónica de acondicionamiento entre el nodo sensor y los sensores EE08, el tensiómetro y el Watermark no merece una detallada descripción. En la misma línea, para acondicionar la salida del sensor DFg2.5 basta con convertir el rango de salida de $\pm 1,875 \mathrm{~V}$ al rango de 0 a $2,5 \mathrm{~V}$, o de 0 a 3,3V. Para ello se utilizan un conjunto de etapas basadas en amplificadores operacionales.

La salida del sensor Hydra Probe II tiene una interfaz de entrada/salida de tipo SDI12. El estándar SDI-12 define una interfaz de datos digital serie asíncrona, que hay que adaptarla con una interface de tipo UART (Universal Asynchronous Receiver-Transmitter) del micro-controlador. Esta electrónica se presentará cuando se describa el nodo que soporte la conexión de estos sensores.

El psicrómetro H301 dispone de 2 RTDs [Pérez, 2004] para proporcionar la medida de la temperatura de los bulbos seco y húmedo. RTD son las siglas de "Resistance Temperature Detector", las cuales designan a los sensores de temperatura de resistencia metálica, constituidos por metales, y caracterizados por poseer coeficientes térmicos positivos de variación de la resistencia eléctrica. Ello es el resultado de que al aumentar en los mismos la energía interna aumenta su resistividad.

Para acondicionar una RTD se utiliza un puente de Wheatstone y amplificadores operacionales. El acondicionamiento será estudiado y descrito en el nodo que soporte la conexión de este sensor.

La última interfaz a tratar es el acondicionamiento de las 2 salidas del termoradiómetro IRTS-P de Apogee, formada por termopares del tipo K. Un termopar [Pérez, 2004] es un sensor de temperatura constituido por dos metales diferentes, cuya característica principal es que se produce una tensión de pequeño valor, proporcional a la 
diferencia de temperaturas entre los puntos de unión de ambos metales. Además, la sensibilidad es del orden de algunos microvoltios por grado centígrado. Así pues, en el acondicionamiento de señal habrá que tener en cuenta dos aspectos básicos: (1) el circuito de acondicionamiento consistirá, básicamente, en un amplificador de tensión de buena calidad; (2) para determinar la temperatura objeto (unión caliente) de la medida será necesario conocer la temperatura de la otra unión (unión fría). La electrónica para conectar los termopares del sensor H301 con los ADCs del micro-controlador será estudiada al describir el nodo correspondiente.

\subsubsection{Principales componentes hardware de los motes desarrollados.}

En las siguientes secciones se especifican los componentes hardware, seleccionados para la implementación de la arquitectura de red de sensores objeto de la presente tesis. Además de los sensores ya descritos en las secciones previas, el mote deberá disponer también de los siguientes componentes hardware adicionales: módulos (transceptores) de radio, antenas, micro-controladores y sistemas de alimentación.

\subsubsection{Transceptores de radio.}

Para seleccionar el módulo de radio se tendrá en cuenta que tenga un bajo consumo, que soporte el protocolo de comunicaciones ZigBee (también soportará el estándar IEEE 802.15.4), que opere en la banda ISM de 2,4GHz y que sea compatible con el sistema operativo TinyOS [TinyOS]. Es importante que opere en la banda ISM de 2,4GHz porque esta banda tiene cobertura mundial. Además, los requisitos incluyen que sea compatible con TinyOS, porque se trata de un sistema operativo para WSN con extensa documentación, el cual es utilizado por numerosos investigadores en todo el mundo.

En este punto, se podrían describir las características principales de numerosos módulos de radio fabricados, entre otros, por Texas Instruments, Freescale y Atmel. Sin embargo, en la época de comienzo de los trabajos de investigación (año 2005), el más adecuado era el CC2420 [CC2420] de Texas Instruments porque tenía las mejores prestaciones y menores consumos que los productos de los competidores. Por ello, no es de extrañar que fuese el módulo de radio utilizado en varias plataformas hardware para WSN muy conocidas, como el TelosB y el Imote. Las características principales de este módulo de radio quedan recogidas en el Anexo I.

En el año 2007, Texas Instruments lanzó al mercado el módulo de radio CC2520 [CC2520]. Se trata de una mejora del chip previo CC2420, dotado con mayores prestaciones; el cual definieron como un transceptor $\mathrm{RF}$ de $2,4 \mathrm{GHz}$ de $2^{\mathrm{a}}$ generación para 
ZigBee y el IEEE 802.15.4. A grandes rasgos, el CC2520 es compatible con la nueva revisión del estándar IEEE 802.15.4 (versión 2006), y extiende algunas características del anterior, como la frecuencia de operación, la tensión de alimentación; incrementando también la potencia de transmisión hasta $+5 \mathrm{dBm}$, lo que se traduce en la posibilidad de conseguir alcances de hasta 400m entre dos estaciones, con sus antenas situadas en línea de vista. Las características técnicas de este transceptor de radio están recogidas en el Anexo I de la presente Tesis.

\subsubsection{Antenas.}

La antena es un elemento crítico en el diseño de una WSN. Dependiendo de la antena escogida, se podrán conseguir mayores o menores alcances entre los nodos sensores, y garantizar las comunicaciones inalámbricas bajo condiciones meteorológicas adversas.

Seleccionar una antena implica escoger las características de la antena, entre las que cabe destacar las siguientes [Balanis, 2005]: diagrama de radiación, ancho de banda, directividad y la ganancia. El ancho de banda estará definido por la frecuencia de trabajo de los nodos sensores.

El Anexo I recoge un estudio detallado justificando las dos antenas seleccionadas. En resumen, se utilizarán antenas omnidireccionales, de $2 \mathrm{dBi}$ y $3 \mathrm{dBi}$ de ganancia, respectivamente. El Anexo I también incluye la vista de las antenas seleccionadas para utilizar en los nodos de la arquitectura.

\subsubsection{Micro-controladores.}

En la sección que se especificó el módulo de radio, se hizo hincapié en que éste fuese compatible con el sistema operativo TinyOS. Al igual que con el transceptor de radio, también es necesario que el micro-controlador sea compatible con TinyOS. Así, los primeros nodos sensores estaban basados en el CC2420 y en el micro-controlador MSP430F1611 de Texas Instruments, elementos principales del ampliamente conocido mote TelosB. También se resaltó que los dispositivos debían tener el requisito de soportar ZigBee. En esta línea, posteriormente al CC2420, apareció en el mercado en CC2520, el cual, para que soportara la pila ZigBee desarrollada por Texas Instruments, tenía que estar conectado con el micro-controlador MSP430F2618 del mismo fabricante. En resumen, para seleccionar el micro-controlador es necesario conocer el módulo de radio escogido, y las bibliotecas software que deberá soportar la plataforma.

Las características técnicas de estos dos micro-controladores están recogidas en el Anexo I. Cabe destacar que los dos micro-controladores de la familia MSP430 tienen los recursos necesarios (interfaces de entrada y salida analógicas y digitales) para poder 
conectarles toda la instrumentación seleccionada anteriormente y son micro-controladores RISC de 16 bits con un consumo ultra-bajo.

\subsubsection{Sistemas de alimentación.}

Dado que, en la mayoría de los casos, no existe línea de red eléctrica cerca de la posición de instalación de un mote, un sistema de alimentación basado en baterías es el más adecuado para facilitar las tareas de despliegue y cambio de posición de un nodo.

En el Anexo I se describen las características que se deben tener en cuenta para seleccionar las baterías, y se menciona el método llevado a cabo para determinar la selección. Al final, se seleccionan las tres baterías que se usaron en las diferentes versiones de los nodos sensores de la arquitectura: 3 celdas AA recargables de $\mathrm{NiMH}$ de 1,2V y $2.700 \mathrm{mAh}$, celda de polímero de litio de $3,7 \mathrm{~V}$ y $2.000 \mathrm{mAh}$, y celda de Li-ION de 3,7V y $4.000 \mathrm{mAh}$.

Como las tensiones de los elementos principales de los motes tienen que estar comprendidas entre 2,1 y $3,6 \mathrm{~V}$, y todas las baterías mencionadas anteriormente ofrecen una tensión de unos 4,2V cuando están totalmente cargadas, son necesarios elementos adicionales para regular la tensión procedente de la batería. En concreto, la tensión se reguló a 2,5V con el regulador lineal de tensión LP38690DT-2.5 de National [National] y a 3V con el MCP1826S-3002E/DB de Microchip [Microchip].

La mayoría de los sensores seleccionados funcionan correctamente con una alimentación de $12 \mathrm{~V}$. Por ello, se utilizaron convertidores continua/continua para elevar la tensión de la batería hasta los 12V necesarios. En concreto, se utilizaron el MAX761ESA y el MAX1771, ambos de Maxim [Maxim].

Continuando con los sensores, la tensión de alimentación para acondicionar el H301 y alimentar los dendrómetros tiene que ser muy estable. Por ello, con objeto de alimentar los dendrómetros y el H301, la selección fue un circuito de referencia de tensión. En concreto, se escogió el MAX6176 de Maxim, que proporciona una tensión muy estable de $10 \mathrm{~V}$.

Una información más detallada sobre las baterías y el resto de elementos seleccionados se pueden encontrar en el Anexo I.

\subsection{Arquitectura de la red de sensores propuesta.}

Como ya se ha expuesto con anterioridad, el problema a resolver consiste en poder monitorizar remotamente el estado hídrico de los cultivos de una explotación agrícola especializada en productos hortícolas. Dicha explotación, puede estar constituida por un 
conjunto de parcelas, las cuales pueden encontrarse distribuidas alrededor de las oficinas centrales de la explotación, dentro de un radio no superior a unos $10 \mathrm{~km}$. La extensión media de las parcelas puede ser de, aproximadamente, unas 4ha. Esta distribución de los cultivos es la típica de los cultivos hortícolas situados en el sureste español. Con respecto a los parámetros que hay que monitorizar, es necesario obtener información del estado del suelo, del agua y del ambiente.

Con objeto de dar solución a este problema, se ha planteado una arquitectura general, la cual se muestra en la Figura 4.1.

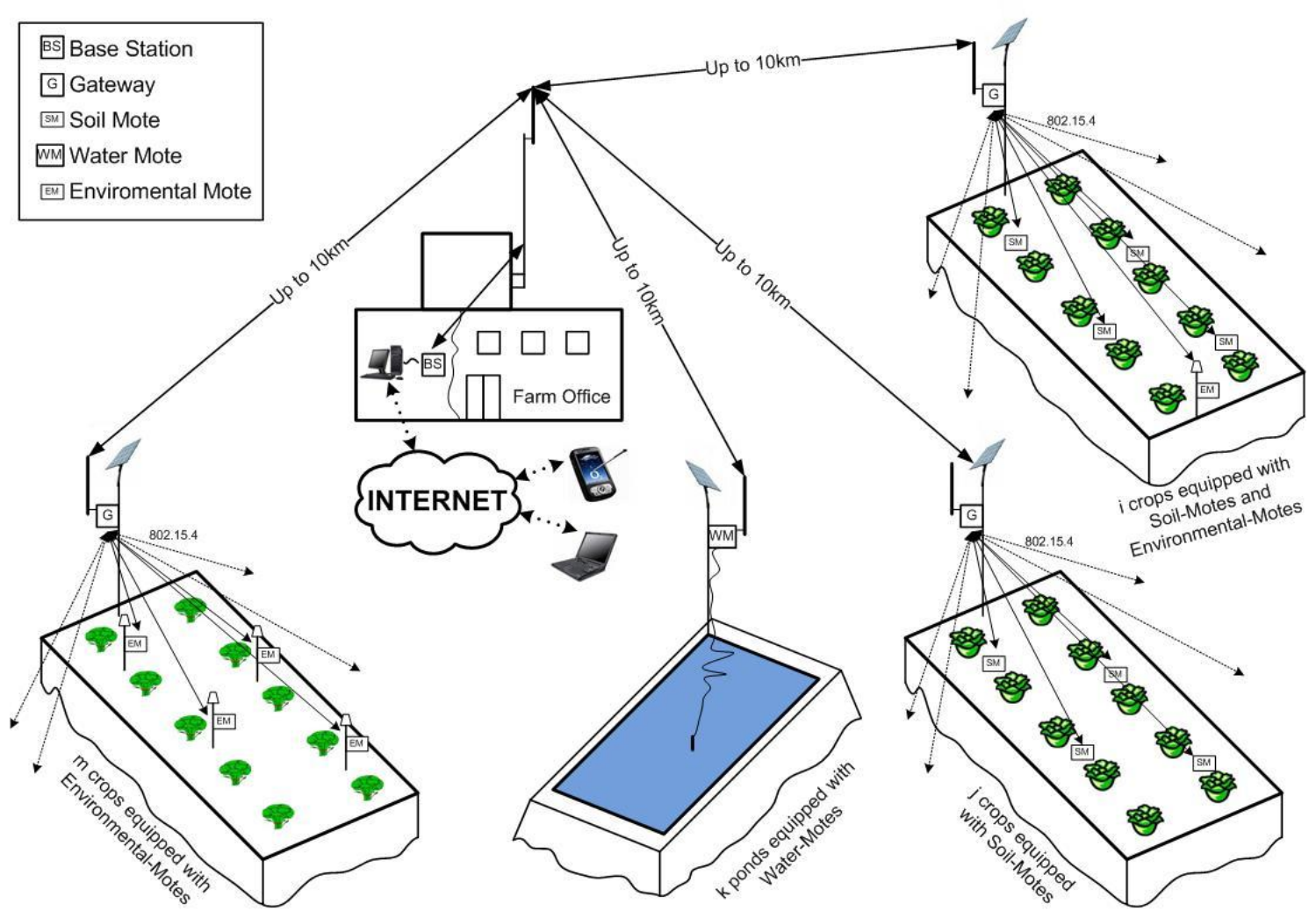

Figura 4.1. Arquitectura propuesta para monitorizar cultivos hortícolas.

Se ha considerado una explotación compuesta por varias parcelas y balsas de riego. El tamaño medio de una explotación agrícola del Campo de Cartagena es de unas 1.000ha, con un tamaño medio por parcela de 4 ha. Por ello, el número de balsas de riego y parcelas, en principio estará limitado y no será nunca superior a 250. Sin embargo, para ofrecer soporte a redes mayores, este número ha sido incrementado hasta 512. A su vez, en cada una de las parcelas se pueden desplegar diferentes redes que incluyen distintos motes dependiendo de los parámetros a monitorizar: características del suelo (Soil-Mote)-como el porcentaje volumétrico, conductividad, salinidad y temperatura; características del ambiente (Environmental-Mote) como la humedad relativa y la temperatura; o una mezcla de ambas (cultivos equipados con Soil-Mote y al menos un Environmental-Mote). Considerando el tamaño medio de las parcelas, el número máximo de nodos por parcela se ha limitado a unos 1.024 nodos. Con dicho número, se obtiene una densidad de sensores de, aproximadamente, 1 sensor por cada $40 \mathrm{~m}^{2}$. Esto supone una densidad más que suficiente 
para este tipo de aplicaciones, donde el número de sensores que, en realidad, se van a utilizar es muy inferior a este límite, debido principalmente al coste de la instrumentación necesaria. Por último, en las balsas de riego que abastecen a los cultivos, se colocarán nodos sensores (Water-Mote), para determinar la calidad del agua suministrada al cultivo a partir de su conductividad eléctrica. Como se puede observar, los Soil-Motes y los Environmental-Motes se comunican bidireccionalmente con el software de monitorización en la Base-Station (BS), a través del correspondiente Gateway. Se tratan por tanto de redes de sensores basadas en una topología en estrella, siendo el Gateway el coordinador de la subred. Con esta implementación, se pueden cubrir grandes áreas, incluso distribuidas, eliminando la necesidad de desarrollar e implementar topologías mesh/cluster, y con la consecuente reducción del consumo de energía de los dispositivos que realizan funciones de sensorización, ya que no tiene que realizar funciones de enrutamiento de mensajes de otros nodos.

\subsection{Diseño e implementación de la red de sensores propuesta.}

En la presente sección se presentan los trabajos realizados para el diseño e implementación de la red de sensores propuesta. Para ello, se mostrarán en primer lugar los trabajos de simulación realizados para la topología seleccionada y descrita en el apartado anterior de captura de especificaciones. Además, las simulaciones realizadas se basan en el empleo de los componentes tecnológicos también descritos y seleccionados en dicho apartado.

La tecnología se seleccionó con objeto de minimizar el consumo energético de los diferentes componentes de la red y garantizar que la autonomía de éstos fuera superior a la requerida por un ciclo agronómico hortícola (típicamente unas 10 semanas). Por su parte, las simulaciones permitieron determinar figuras de mérito de la red como son la autonomía, los retardos y la máxima tasa binaria alcanzada o 'throughput. Los datos obtenidos en las simulaciones fueron posteriormente corroborados mediante el despliegue real, en la fase de validación de resultados. Para ello, fue necesario desarrollar el conjunto de dispositivos que forman parte de la red. Dichos dispositivos son descritos en la sección 4.4.2.

\subsubsection{Fase de simulación para el diseño de la red de sensores.}

Para poder simular la arquitectura propuesta, se seleccionaron los componentes básicos (denominados Soil-Mote, Environmental-Mote, Water-Mote y Gateway) y un conjunto de dispositivos físicos (módulo de radio, micro-controlador y sensores) de una tecnología específica: módulo de radio Chipcon CC2420 (Texas Instruments, USA), microcontrolador MSP430F1611 (Texas Instruments, USA), sensor de temperatura y humedad relativa del ambiente SHT71 (Sensirion AG, Switzerland), y la sonda de suelo 
multiparamétrica Hydra Probe II (Stevens Water Monitoring Systems, USA). La combinación de los posibles estados de estos dispositivos físicos, determinaron los posibles estados de funcionamiento de un nodo sensor. Así, en el simulador se definieron los estados posibles de funcionamiento y el consumo estimado a partir de los datasheets de los dispositivos en cada estado. La Tabla 4.5 resume los datos de cada dispositivo físico que fueron necesarios para realizar una simulación fidedigna.

\begin{tabular}{|c|c|c|c|}
\hline \multirow{2}{*}{ Dispositivo } & \multicolumn{2}{|c|}{ Requisitos de consumo } & \multirow{2}{*}{$\begin{array}{l}\text { Tensión de } \\
\text { alimentación }\end{array}$} \\
\hline & Estado & Intensidad & \\
\hline \multirow{5}{*}{ CC2420 } & $\begin{array}{c}\text { Regulador Voltaje en } \\
\text { off (OFF) }\end{array}$ & $0,02 \mu \mathrm{A}$ & \multirow{5}{*}{$2,1-3,6 \mathrm{~V}$} \\
\hline & Desactivado & $20 \mu \mathrm{A}$ & \\
\hline & Inicial & $426 \mu \mathrm{A}$ & \\
\hline & Reciviendo & $18,8 \mathrm{~mA}$ & \\
\hline & Transmitiendo & $17,4 \mathrm{~mA}$ & \\
\hline \multirow{3}{*}{ MSP430F1611 } & Off & $0,2 \mu \mathrm{A}$ & \multirow{3}{*}{$1,8-3,6 \mathrm{~V}$} \\
\hline & Standby & $1,1 \mu \mathrm{A}$ & \\
\hline & Activo & $330 \mu \mathrm{A}$ & \\
\hline \multirow{2}{*}{ SHT71 } & Standby & $0,3 \mu \mathrm{A}$ & \multirow{2}{*}{$2,4-5,5 \mathrm{~V}$} \\
\hline & Midiendo & $0,5 \mathrm{~mA}$ & \\
\hline \multirow{2}{*}{$\begin{array}{l}\text { Hydra Probe II } \\
\text { (versión SDI-12) }\end{array}$} & Inactivo & $1 \mathrm{~mA}$ & \multirow{2}{*}{$12 \mathrm{~V}$} \\
\hline & Activo & $30 \mathrm{~mA}$ & \\
\hline
\end{tabular}

Tabla 4.5. Diferentes estados de cada uno de los componentes principales de los motes y consumo asociado a cada uno de ellos.

Otro aspecto relevante de la funcionalidad de la arquitectura, es la selección del protocolo de comunicación. El estándar que ha presentado el mayor desarrollo comercial en el campo de las WSNs es el IEEE 802.15.4. Es un protocolo óptimo para implementar las comunicaciones entre los nodos sensores y el Gateway, debido al bajo coste y consumo que ofrece. No obstante, en este trabajo se han incluido dos características más para reducir más el consumo. En primer lugar se seleccionó B-MAC (Berkeley-Medium Access Controh), que fue descrito en el Capítulo 3 como el protocolo de acceso al medio para gestionar las comunicaciones de múltiples sensores en el canal de radio. En segundo lugar, se propuso también un decremento aún mayor del consumo de los dispositivos inalámbricos, basado en el funcionamiento del Gateway (cuando éste no envíe datos, siempre estará escuchando el medio, ya que posee alimentación ilimitada) y consecuentemente, la modificación del protocolo B-MAC.

Antes de implementar los diferentes componentes, se simuló un caso de estudio con la tecnología y el protocolo de comunicación seleccionado. Las simulaciones se 
llevaron a cabo usando el entorno network simulator 2, conocido por ns-2 [Fall, 2010]. La selección de este simulador frente a otros (como, por ejemplo, OMNET ++ ) se debe a que ns-2 ya tiene implementados y validados los módulos correspondientes a redes inalámbricas de área personal (LR-WPAN)-802.15.4. El código fuente del simulador [Zheng, 2006] implementa las funciones, métodos y tramas del estándar 802.15.4 en sus diferentes modos de funcionamiento non-beacon enabled (sin sincronización entre nodos) y slotted beacon-enabled (con sincronización), siendo para ambos casos el mecanismo de acceso al medio CSMACA (Carrier Sense Multiple Access with Collision Avoidance). Este entorno de simulación también fue elegido porque numerosas referencias de la literatura científica [Camilli, 2007] lo identifican como uno de los entornos más adecuados para estudiar diferentes tecnologías, protocolos de comunicación y topologías de red usadas en aplicaciones del campo de la agricultura de precisión. Sin embargo, el simulador seleccionado no implementaba las condiciones de trabajo reales requeridas. En este caso, las comunicaciones están basadas en B-MAC, y no en el estándar 802.15.4, que sí está incorporado en el simulador. Por tanto, para simular la transferencia de información y los consumos energéticos sobre B-MAC, fue necesario modificar el simulador original. Para ello, se seleccionó un modo de comunicación non-beacon-enabled, no habiendo sincronización entre nodos, tal y como específica B-MAC. Asimismo, en el simulador se consideró el tipo de comunicación que se está realizando (Mote-Gateway o viceversa), con el objeto de representar el consumo real en los dispositivos inalámbricos. Finalmente, la simulación se realizó de modo que los motes y el Gateway enviarán sus tramas, con intervalos lo suficientemente distanciados para que no hubiese colisiones. Cuando se modificó la herramienta de simulación, los parámetros de interés elegidos para simular fueron el consumo energético, los retrasos y la tasa binaria de transmisión de datos. Debido a la experiencia y conocimiento del entorno de simulación por miembros del grupo de investigación Área de Ingeniería Telemática [AIT] de la Universidad Politécnica de Cartagena, las simulaciones se realizaron en colaboración con estos investigadores.

En este caso, la comunicación entre un nodo sensor y el Gateway se realizó teniendo en cuenta el requisito de que el Gateway se encuentra alimentado mediante un panel solar, por lo que se puede tener activo su módulo de radio de manera ininterrumpida. Mediante esta configuración, se puede mejorar la autonomía de los nodos sensores, ya que éstos no tendrán que enviar un preámbulo (una trama especial para sincronizar las estaciones de comunicación, cuyo tamaño mínimo en unidades de tiempo es el check interval), antes de enviar un mensaje al Gateway. Por otra parte, en la comunicación Gateway-nodo sensor, se hace necesario establecer el periodo de Check interval óptimo con el que despertarán periódicamente los nodos sensores (Soil-Motes y Environmental-Motes). Para determinar este parámetro se simularon las dos situaciones anteriores (B-MAC tradicional y nuestra propuesta, B-MAC con Gateway encendido permanentemente). Como se puede observar en la Figura 4.2, para un mismo check interval se consigue una mayor autonomía de los nodos 
sensores usando la alternativa propuesta que con B-MAC tradicional. En el caso de usar BMAC tradicional, en la figura también se aprecia que los intervalos de muestreo superiores a 10 s e inferiores a 1 s proporcionan los consumos más elevados. En el caso de intervalos de muestreo (sample intervals) superiores a 10s, el elevado consumo es debido al gran tamaño del preámbulo. Para un check interval inferior a $1 \mathrm{~s}$, el gasto de potencia es motivado por el alto número de intervalos de muestreo. Sin embargo, el B-MAC propuesto proporciona valores similares en este intervalo (1-10s), que se traduce en que el check interval diseñado presenta una baja dependencia sobre la energía consumida. Entonces, es posible seleccionar cualquier check interval en este rango de acuerdo con los requisitos de la aplicación. En este trabajo se seleccionó un check interval de 5s.

(a)

(b)

(c)

Figura 4.2. Comparativa de la evolución de la descarga de la batería entre B-MAC tradicional y BMAC propuesto para los Soil-Motes (a) Check interval menor e igual a 1s. (b) Check interval igual a 5s. (c) Check interval mayor e igual a $10 \mathrm{~s}$.

Determinado el check interval óptimo, se realizaron un conjunto de simulaciones para obtener la autonomía de los dispositivos, los retardos asociados a la transmisión de tramas (delays) y el throughput o tasa binaria de información recibida/transmitida por unidad de tiempo, para un determinado caso de estudio. La tasa de transferencia de datos indica la cantidad de bits por unidad de tiempo que están siendo transmitidos/recibidos por la red de sensores. Este parámetro no es crucial en este caso de estudio, ya que la cantidad de información transferida no es elevada; sin embargo, esta información combinada con los 
retardos es útil para determinar si se están produciendo pérdidas de paquetes en un instante determinado.

Para simular las características mencionadas, se definió un caso de estudio a partir de la arquitectura general mostrada en la Figura 4.1. Se consideraron 2 parcelas, una con 10 Soil-Motes conectados a 2 sensores Hydra Probe II cada uno de ellos, y otra con otros 10 Environmental-Motes conectados con un sensor SHT71. En los escenarios mencionados, los criterios de intercambio de información Gateway/motes son los siguientes:

- Los Soil-Motes/Environmental-Motes (nodos sensores) adquieren los datos de sus sensores y envían la información al Gateway cada 30 minutos. Los Soil-Motes envían dos tramas (una por cada sensor Hydra Probe II) con una demora entre ellas de 2s.

- Los nodos sensores envían información del estado de sus baterías cada hora.

- La red permitirá modificar la frecuencia de lectura de los sensores. Por ello, para considerar esta acción en los parámetros obtenidos con el simulador, se considera que el Gateway enviará cada 2000s información de control a los nodos sensores.

- Para todos los casos, el tamaño de la cabecera de la trama seleccionada fue el mínimo (9 bytes) según el estándar IEEE 802.15.4. Este tamaño mínimo disminuye la tasa de transmisión en la red, así como los consumos energéticos asociados.

- Se ha incluido una espera de 2 segundos cada vez que una estación emite una trama de información hasta que otra estación transmite. Este requisito permite que las estaciones no compitan por el medio inalámbrico, evitándose así colisiones, debido al reducido número de nodos sensores empleados en el caso de estudio.

La Tabla 4.6 detalla el payload (carga útil en bytes) asociado a cada uno de los mensajes anteriores. Una información más detallada sobre los campos de las tramas queda recogida en el Anexo II.

\begin{tabular}{|c|c|}
\hline Tipo de Mensaje & Payload (Bytes) \\
\hline Dato proporcionado por el Soil-Mote & 29 \\
\hline Dato proporcionado por el Environmental-Mote & 6 \\
\hline Estado de la batería & 4 \\
\hline Modificación de la frecuencia de lectura & 4 \\
\hline
\end{tabular}

Tabla 4.6. Descripción de las tramas intercambiadas entre el Gateway y los nodos sensores.

A la hora de realizar las simulaciones de los dos escenarios de trabajo, se configuró el entorno de simulación ns-2 con las topologías más cercanas a una situación real. Se definió una topología con el Gateway situado en el centro y 10 nodos sensores alrededor, siendo la distancia máxima entre los nodos sensores y el Gateway de 84m. En los dos 
escenarios se consideró que todos los nodos están situados a 1m de altura del suelo, con el fin de evitar problemas de interferencias.

Para simular este caso de estudio, se consideraron como entradas al simulador la topología de la red, el tráfico asociado a cada una de las redes y los parámetros intrínsecos a la tecnología seleccionada. La comunicación entre el Gateway y los nodos sensores se estableció con un schedule sin colisiones, pudiéndose establecer en dos sentidos: (1) comunicación desde el Gateway al mote y (2) comunicación desde el mote al Gateway.

- Comunicación desde el Gateway al mote. Los motes receptores despertarán cada check interval para comprobar si hay datos para ellos. Por su parte, el Gateway deberá enviar su información (preámbulo y los datos a transmitir al mote) durante todo un check interval. De este modo se asegura que el nodo receptor recibirá el paquete enviado por el Gateway.

- Comunicación desde el mote al Gateway. El sistema de alimentación, basado en panel solar, que se usará en el Gateway, permitirá tener el transceptor CC2420 siempre activo. La información de los sensores y del estado de la batería es enviada directamente al Gateway. En este caso, no se envía ningún tipo de preámbulo, ni existe un muestreo del canal, es decir, como se mencionó anteriormente, B-MAC no opera de forma convencional y sólo se encarga de la correcta recepción de la trama. El objetivo es reducir aún más los consumos de energía de los motes.

En el caso de la energía consumida, las simulaciones devuelven los consumos energéticos en todo momento y permiten estimar la autonomía de un dispositivo. Para ello, se implementó en el simulador ns-2 los niveles energéticos y transiciones de estados del transceptor CC2420 [Ramachandran, 2007]. La evaluación de consumos energéticos de los sensores Hydra Probe II y SHT71, y del micro-controlador MSP430F1611 supone también una aportación al código ns-2 original. Para ello, se modificó el código del simulador añadiendo las transiciones y estados de los sensores y del micro-controlador.

\subsubsection{Diseño e implementación de los motes.}

Los requisitos, en cuanto a distancia de cobertura de las redes (hasta $10 \mathrm{~km}$ ), junto con la no disponibilidad en el mercado de un mote adaptado al estándar SDI-12 de instrumentación agronómica justificó el desarrollo de los dispositivos que se describen en este trabajo. A la hora de diseñar estos dispositivos, los principales requisitos que se consideraron fueron los siguientes: (1) tecnología de radio robusta y alcance adecuado, (2) bajo coste y consumo de los dispositivos electrónicos empleados, (3) utilización de fuentes de energía de larga duración y (4) utilización de estándares de entrada/salida para poder conectar un amplio número de sensores externos. Estos requisitos se tuvieron en cuenta, 
previamente, para definir las entradas del simulador ns-2, con el que se realizó un estudio previo de la arquitectura propuesta. Para cada uno de los motes desarrollados se detallará la arquitectura hardware, los sensores empleados, la funcionalidad del mote y por último los componentes software desarrollados.

\subsubsection{Soil-Mote.}

La Figura 4.3 muestra una vista del Soil-Mote y el diagrama de bloques que describe la arquitectura hardware del dispositivo. Este mote consta de un micro-controlador, un transceptor de radio de corto alcance, una interfaz SDI-12, dos convertidores DC/DC y un conjunto de baterías para la alimentación. Todos los elementos están integrados dentro de una caja estanca con grado de protección IP67.

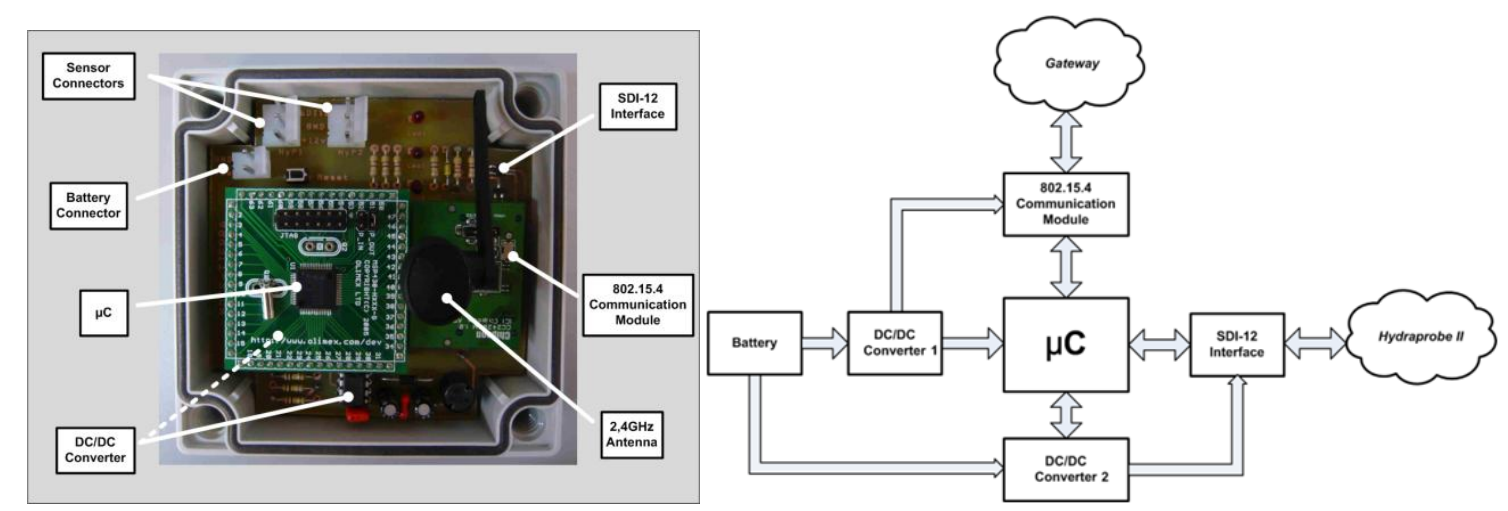

Figura 4.3. Fotografía del Soil-Mote y diagrama de bloques de la arquitectura hardware.

El dispositivo está compuesto por la tecnología mencionada en el sub-apartado anterior. El microcontrolador es el MSP430F1611 de Texas Instruments, y el lenguaje seleccionado para su programación ha sido el nesC [Gay, 2003] con bibliotecas de la versión 2 del sistema operativo TinyOS [Hill, 2000]. Este micro-controlador será el utilizado en el resto de motes. El módulo de radio de corto alcance se corresponde con el CC2420 de Texas Instruments. Este módulo de radio intercambia datos vía wireless, a 2,4 $\mathrm{GHz}$, y con un ancho de banda de $250 \mathrm{Kbps}$, con el Gateway y siguiendo el estándar IEEE 802.15.4. El Gateway sirve de pasarela entre la red de sensores y las oficinas de la explotación agrícola donde se encuentra instalada la aplicación de monitorización. El convertidor DC/DC 2, basado en el MAX761 de Maxim, se encarga de obtener la tensión adecuada (12V) para la interfaz SDI-12, a partir de la tensión de la batería. A su vez, el convertidor DC/DC 2 dispone de una salida que permite informar al micro de un nivel bajo de tensión en las baterías. Este nivel se configura en una entrada del convertidor, mediante un divisor de tensión resistivo. Otra característica muy importante a destacar, es que el convertidor se puede habilitar o deshabilitar reduciendo así el consumo de energía en periodos de tiempo en los que el mote no esté realizando ninguna lectura con los sensores. El sistema de alimentación está compuesto de tres baterías recargables tipo AA de NiMH 
de $2.700 \mathrm{mAh}$, conectadas con el convertidor DC/DC 1 encargado de estabilizar la tensión de las mismas a los 2,5V utilizados en los módulos principales (microcontrolador y módulo de radio). Como se presentará en un sección posterior, la duración de la batería es de unos 7 meses, tiempo más que suficiente para garantizar el funcionamiento durante una campaña agrícola hortícola habitual.

Cada mote está conectado a dos sensores Hydra Probe II (HP2 en adelante) de Stevens (véase Figura 4.4) a través de la interfaz SDI-12.

El Soil-Mote monitoriza cuatro parámetros: temperatura del suelo $\left(\mathrm{en}^{\circ} \mathrm{C}\right)$, salinidad del suelo (en gr/l de $\mathrm{ClNa}$ ), porcentaje volumétrico de humedad en el suelo (en tanto por 1) y conductividad de suelo con corrección de temperatura (en $S / m$ ), usando, para ello, los sensores HP2 con una frecuencia de muestreo, configurable por el usuario desde el PC receptor, comprendido en el intervalo desde 30 minutos hasta unos 10 días. Debido a que, habitualmente, se hace un muestreo cada hora, se optó por mandar los valores leídos en cada ciclo de lectura, consiguiendo con ello una disponibilidad de datos, en tiempo real, y un consumo razonable de energía. Además, el dispositivo monitoriza el nivel de las baterías y envía un mensaje cuando éstas alcanzan un nivel crítico.

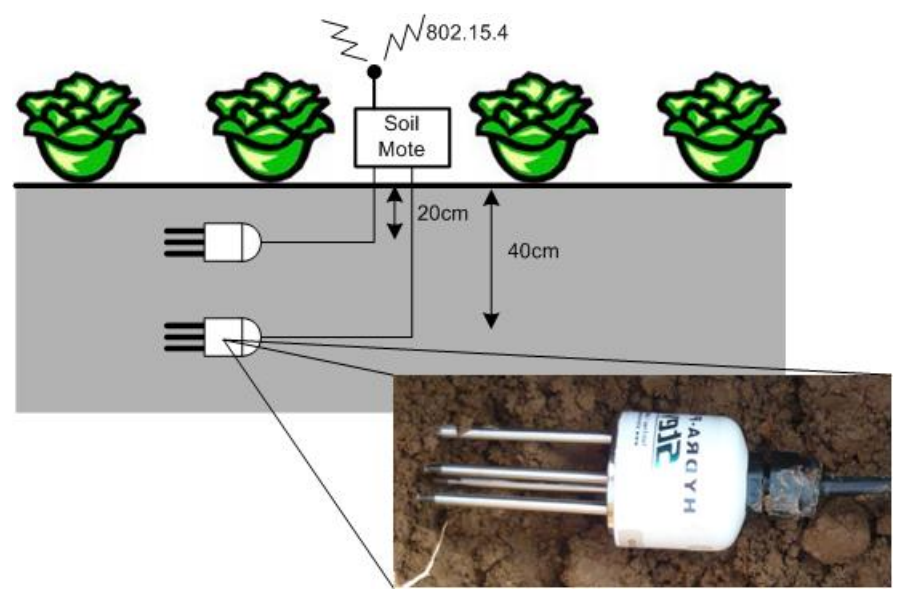

Figura 4.4. Situación de los sensores del Soil-Mote.

En relación con el software, TinyOS, es un sistema operativo específico del dominio de las WSNs, y es el estándar de facto de código abierto para el desarrollo de nuevas aplicaciones de WSNs, al tiempo que proporciona componentes reutilizables para garantizar comunicaciones fiables siguiendo el estándar IEEE 802.15.4.

En este caso, como se puede recordar del sub-apartado anterior, para la comunicación entre los Soil-Mote y el Gateway, se ha optado por el IEEE 802.15.4, pero utilizando B-MAC en lugar de MAC para acceder al medio. Este protocolo de comunicación está implementado en TinyOS. Además, puesto que el despliegue realizado en la explotación agrícola, junto con la cobertura ofrecida por los módulos de radio no 
hacen necesario utilizar topologías malladas o en árbol, la arquitectura propuesta está basada en una topología en estrella.

La Figura 4.5 muestra el diagrama de componentes utilizados en la implementación del mote usando el lenguaje nes $\mathrm{C}$, donde pueden observarse las distintas instancias de los componentes junto con las distintas interfaces suministrados al componente principal HP2C. Este componente, de tipo módulo, ha sido desarrollado para satisfacer la funcionalidad descrita anteriormente. El resto de componentes se reutilizan de la infraestructura que proporciona TinyOS para acceso a temporizadores, acceso a puertos de entrada/salida, módulo de radio, etc. Cada vez que se reutiliza un componente, se crea una o más instancias del mismo, distinguibles entre sí a través de un alias.

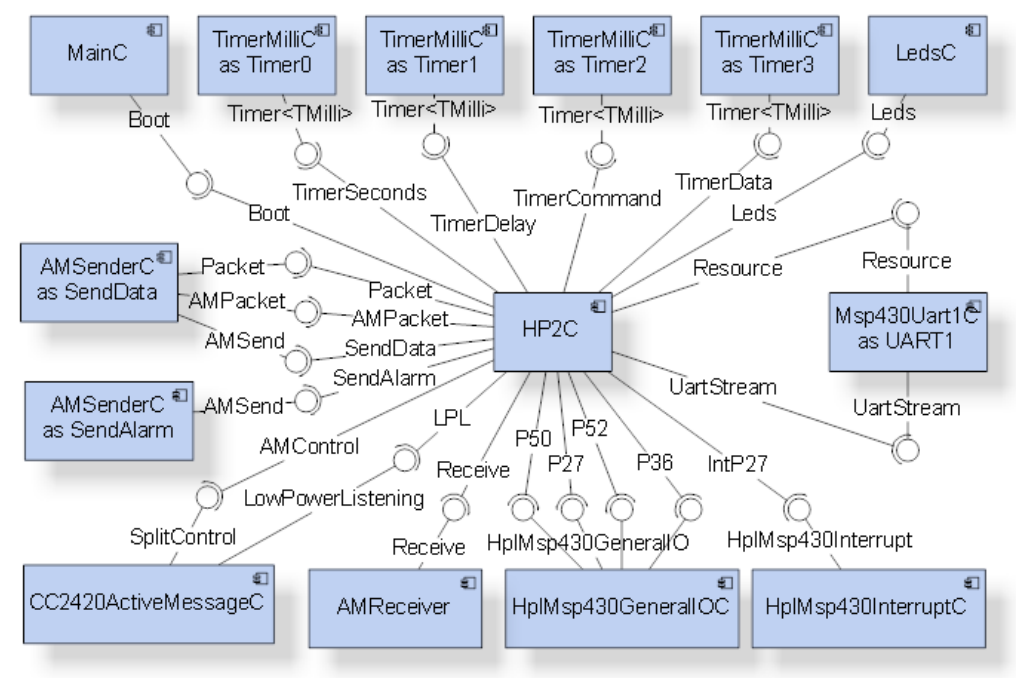

Figura 4.5. Vista estática del diagrama de componentes nesC del Soil-Mote.

El punto de entrada del programa lo proporciona el componente MainC, por medio de la interfaz predefinida Boot. Para el funcionamiento del programa, es necesario instanciar distintos componentes nesC:

- Componente TimerMilliC. Para leer los sensores HP2 es necesario enviar un primer comando de inicio de lectura (temporización fijada por Timer2), esperar la respuesta del sensor, la cual indica cuando los datos estarán disponibles. Pasado dicho tiempo (Timer1), se puede efectuar la medida de los datos enviando un nuevo comando de lectura (temporización fijada por Timer3). Como respuesta a dicho comando, el sensor envía las magnitudes que tiene configuradas para ser leídas y espera un tiempo (Timer0) para repetir el ciclo.

- Componente $A M S e n d e r C$. Este componente proporciona las interfaces necesarias para enviar mensajes vía radio al Mote-Gateway. Las instancias de este componente, llamadas SendData y SendAlarm, se utilizan para enviar los datos leídos y el aviso de nivel crítico en la batería, respectivamente. 
- Componente CC2420ActiveMessageC. La única instancia de este componente se utiliza para realizar las tareas de habilitación/deshabilitación del módulo de radio. Además, proporciona la funcionalidad necesaria para trabajar en modo bajo consumo.

- Componente AMReceiver. Esta instancia permite recoger los mensajes procedentes del Mote-Gateway, con la funcionalidad de modificar la frecuencia de muestreo de los motes.

- Componente HplMsp430GeneralIOC. Esta instancia ofrece el acceso a los puertos del microcontrolador, necesarios para controlar los sensores, y el convertidor DC/DC.

- Componente HplMsp430InterruptC. Esta instancia facilita el manejo de interrupciones desde el convertidor DC/DC 2, con el fin de tener constancia de cuándo la batería se encuentra por debajo del umbral establecido.

- Componente Msp430Uart1C. Con él se gestionan las comunicaciones con el sensor HP2, mediante el protocolo SDI-12.

- Componente LedsC. La instancia de este componente encapsula el hardware de los LEDs del mote y se emplea para la depuración de la aplicación y conocer el estado de ejecución de la misma.

El Anexo III describe el código fuente del componente de tipo módulo HP2C del GAIA Soil-Mote (versión comercial del Soil-Mote descrita en el capítulo 5). Como se ha descrito anteriormente, el módulo HP2C implementa la funcionalidad de este nodo sensor.

La Figura 4.6 muestra uno de los 10 Soil-Motes desplegados en una parcela de coles de 4ha de la explotación agrícola Langmead. Como se comentará más adelante, inicialmente se utilizaron antenas de tipo mono-polo, de 4,2dBi de ganancia, debido al crecimiento del cultivo, el cual originaba problemas de cobertura, posteriormente, fue necesario remplazarlas por antenas omnidireccionales, de $3 \mathrm{dBi}$ de ganancia.

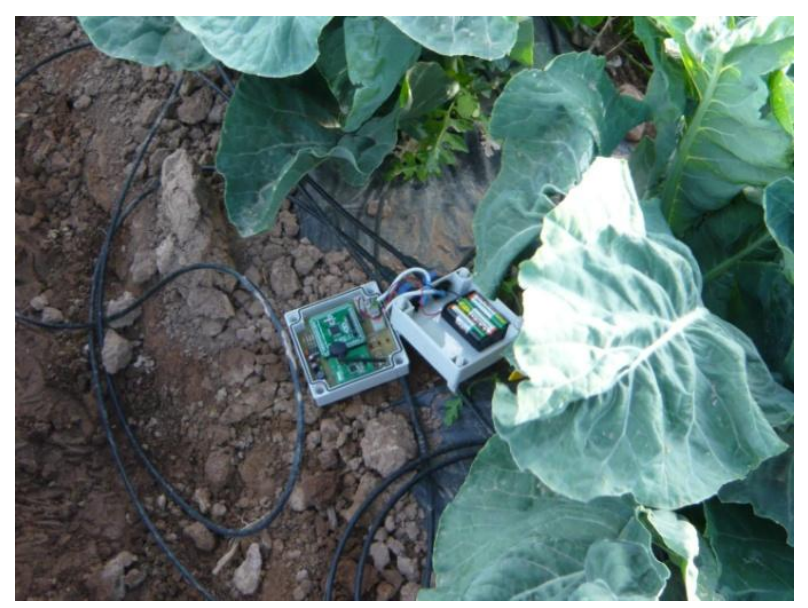

Figura 4.6. Fotografía del Soil-Mote desplegado en un cultivo de coles. 


\subsubsection{Environmental-Mote.}

Estos motes (ver Figura 4.7) son los encargados de registrar la temperatura y la humedad relativa del ambiente de un cultivo. Como se puede ver en la Figura 4.7, el mote tiene una arquitectura similar a la descrita anteriormente para el Soil-Mote, salvo la interfaz con la instrumentación externa.

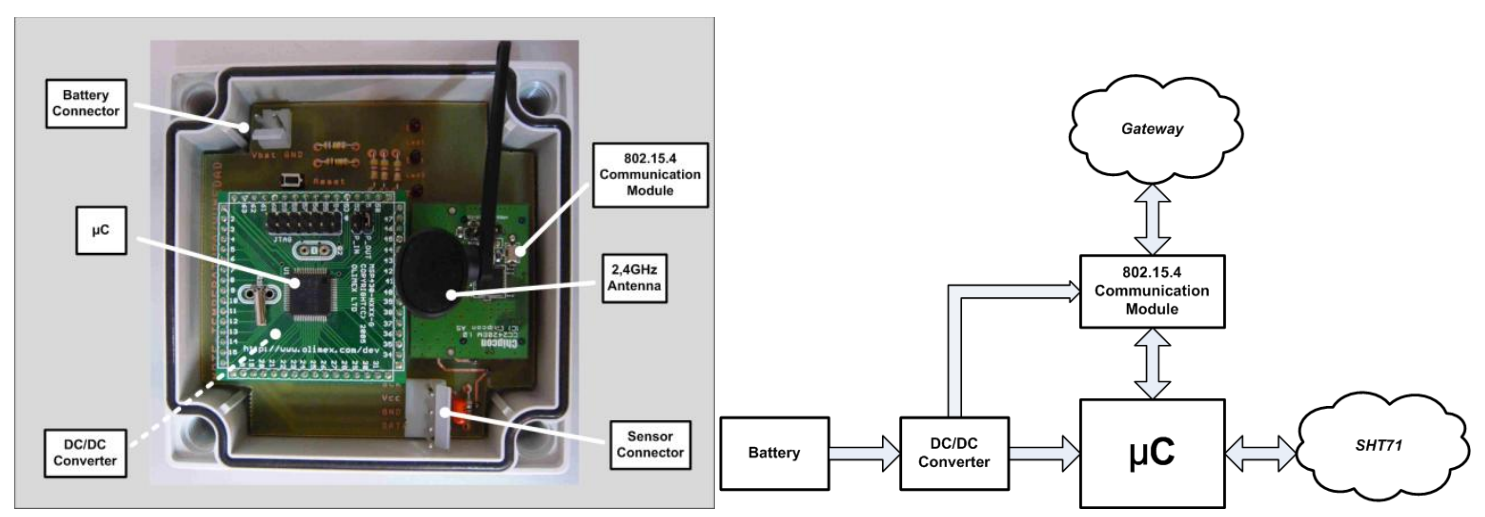

Figura 4.7. Fotografía del Environmental-Mote y diagrama de bloques de la arquitectura hardware.

Cada mote está conectado, a través de una interfaz de tipo $\mathrm{I}^{2} \mathrm{C}$, a un sensor SHT71 de Sensirion (véase Figura 4.8), el cual se encuentra dentro de un escudo de protección solar situado a un metro y medio del suelo. El sensor puede funcionar con la misma tensión de alimentación que el micro-controlador y el módulo de radio. Por ello, no es necesario un convertidor DC/DC que eleve el voltaje de la batería a 12V, como en el caso del Soil-Mote. Este sensor no está recogido en las especificaciones iniciales. Esto es así, porque se intentó reducir costes y evaluar un sensor de bajo coste (30\$), en lugar del sensor EE08 (unos $200 \$$ ), dentro del escudo de protección solar. Al final, el experimento con este sensor no resultó adecuado porque entraban insectos dentro del escudo de protección solar, que perturbaban la medida de éste.

Este tipo de motes efectúa lecturas de los citados parámetros con una frecuencia máxima configurable entre 30 minutos y 10 días.

La alimentación del dispositivo está basada, al igual que Soil-Mote, en una batería compuesta por 3 celdas de tipo AA de NiMH y $2.700 \mathrm{mAh}$ de capacidad. La tensión de la batería se estabiliza a 2,5V con un regulador lineal de tensión de bajo dropout. En este caso, no es necesario un segundo convertidor DC/DC, ya que el sensor SHT71 opera con una tensión de alimentación de 2,5V.

Para establecer el enlace inalámbrico con el Gateway utiliza una antena de tipo mono-polo, de 4,2dBi de ganancia. Al igual que con el Soil-Mote, también fue necesario reemplazar estas antenas por omnidireccionales, de $3 \mathrm{dBi}$ de ganancia. 


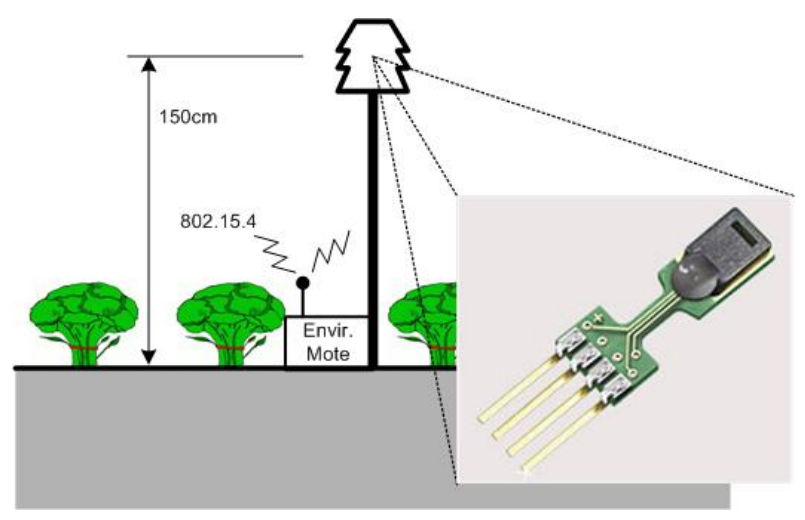

Figura 4.8. Vista del sensor utilizado en el Environmental-Mote y localización del mismo.

La programación de este mote ha sido igualmente llevada a cabo con bibliotecas de TinyOS. El componente SHT71C ha sido desarrollado para satisfacer la funcionalidad descrita. La Figura 4.9 muestra el diagrama de componentes utilizado en la implementación. Los componentes utilizados, que no han sido descritos en el mote anterior, son los siguientes:

- Componente SensirionSht11C. Este componente encapsula el acceso a los registros del sensor SHT71 mediante el protocolo $\mathrm{I}^{2} \mathrm{C}$.

- Componente Msp430ADCOC. Este componente permite muestrear el convertidor analógico/digital ADC0 del micro-controlador, con objeto de determinar cuándo se encuentra la batería por debajo de un umbral, enviándose una alerta de agotamiento de batería.

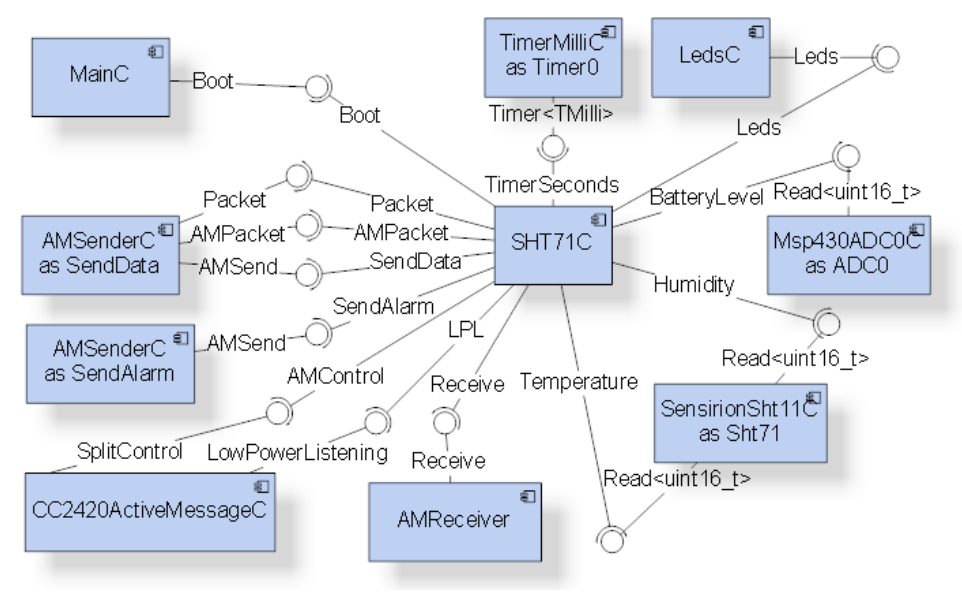

Figura 4.9. Vista estática del diagrama de componentes nesC del Environmental-Mote.

La Figura 4.10 muestra uno de los diez motes desplegados en la explotación agrícola, junto con el escudo de protección solar en el que se ubica el sensor SHT71. Los Environmental-Motes se desplegaron sobre el suelo y en un radio de unos cien metros alrededor del Gateway. 


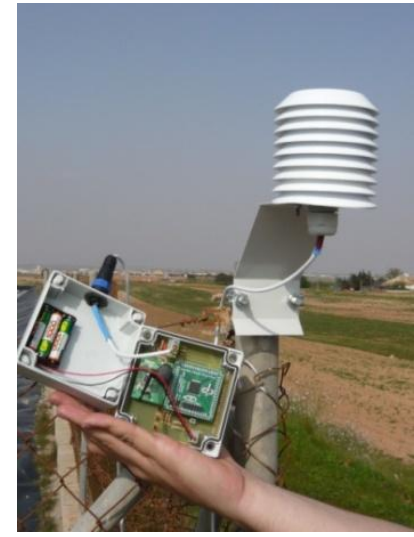

Figura 4.10. Fotografía del Environmental-Mote desplegado en la explotación agrícola Langmead.

\subsubsection{Water-Mote.}

El Water-Mote (ver Figura 4.11) es el encargado de medir la temperatura y conductividad eléctrica del agua, suministrada al cultivo en una balsa de riego de la explotación agrícola. En este caso, el mote se encuentra directamente conectado con las oficinas de la empresa a través del módulo de radio de largo alcance XStream X24-019PKIRA. Este módulo de radio proporciona alcances de hasta $16 \mathrm{~km}$ en línea de vista, y se conectó con una antena omnidireccional, de $8 \mathrm{dBi}$ de ganancia, instalada en un mástil, de $4 \mathrm{~m}$ de altura, al igual que la caja estanca con el resto de elementos. El resto de la arquitectura es muy similar a la de los motes anteriores (ver Figura 4.11).

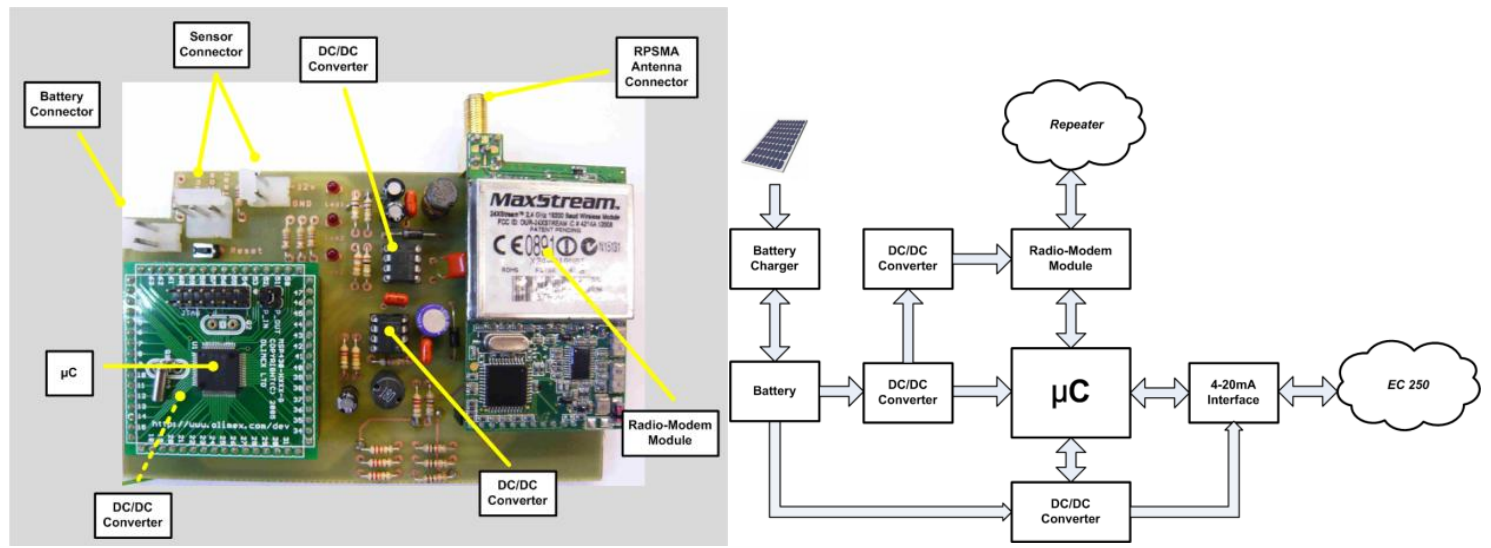

Figura 4.11. Vista del Water-Mote y diagrama de bloques de la arquitectura hardware.

El sensor utilizado es el EC250 de Stevens, que se encuentra sumergido dentro de la balsa (Figura 4.12). Este sensor no está recogido en las especificaciones iniciales y se utilizó para familiarizarse con este tipo de sensores y la información proporcionada. Las dos salidas del sensor (temperatura y la conductividad eléctrica) son proporcionadas en lazo de corriente 4-20mA, siendo leídas por los dos convertidores ADC0 y ADC1 del microcontrolador. Estos parámetros se adquieren con una frecuencia máxima parametrizable de 
2 lecturas/hora. El mote se alimenta mediante un panel solar modelo TPS 102/5 (12V, 5W), comercializado por Zodiac Solar, el cual mantiene cargada una batería de plomo-ácido de $12 \mathrm{~V}$ y $6,5 \mathrm{Ah}$. En este caso, la arquitectura hardware incluye 3 convertidores DC/DC, ya que los elementos principales del mote trabajan con 2,5V, el XStream con $5 \mathrm{~V}$ y el sensor EC250 requiere una tensión de alimentación de $12 \mathrm{~V}$.

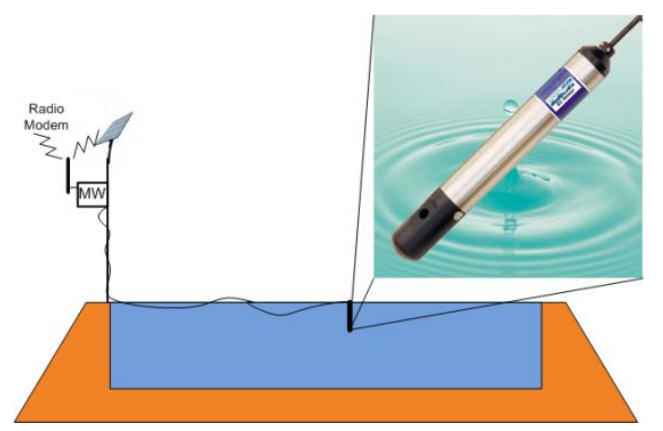

Figura 4.12. Esquema del Water-Mote y vista del sensor EC250.

El componente EC250C ha sido desarrollado igualmente en TinyOS para satisfacer la funcionalidad descrita. La Figura 4.13 muestra el diagrama de componentes utilizado en la implementación. Los componentes utilizados son los siguientes:

- Componente TimerMilliC. Cada vez que se tiene que leer del sensor EC250, se tiene que realizar un nuevo ciclo de lectura (instancia Timer) activándose el convertidor DC/DC que alimenta el sensor durante diez segundos. Pasado este tiempo (Timer1), se lanza la medida de los dos ADCs a los que están conectadas las salidas del sensor. Una vez se dispone de la lectura de los dos ADCs, se activa el módulo de radio y se espera un tiempo (Timer3) antes de enviar datos. En cada ciclo de lectura, una vez que se han mandado todos los datos (datos más alerta de batería si la hubiera), se mantiene activo el módulo de radio de largo alcance durante cinco segundos (Timer2) para que pueda escuchar un posible mensaje de cambio de la frecuencia de muestreo. Pasado este tiempo, el mote queda a la espera de un nuevo ciclo de lectura.

- Componentes Msp430ADC0C y Msp430ADC1C. Proporcionan el acceso a los convertidores analógicos digitales, a los que se encuentran conectados las salidas del sensor.

- Componente Msp430ADC2C. Esta instancia tiene conectada la tensión de batería, pasando antes por un divisor resistivo.

- Componente HplMsp430GeneralIOC. Ofrece el acceso a los puertos del microcontrolador, necesarios para controlar el convertidor DC/DC y el módulo de radio de largo alcance.

- Componente HplMsp430InterruptC. Genera un pulso de nivel bajo para indicar que se ha enviado un mensaje vía radio. 
- Componente PlatformSerialC. Permite gestionar la entrada/salida con la UART1 del microcontrolador, a la cual está conectado el módulo de radio de largo alcance.

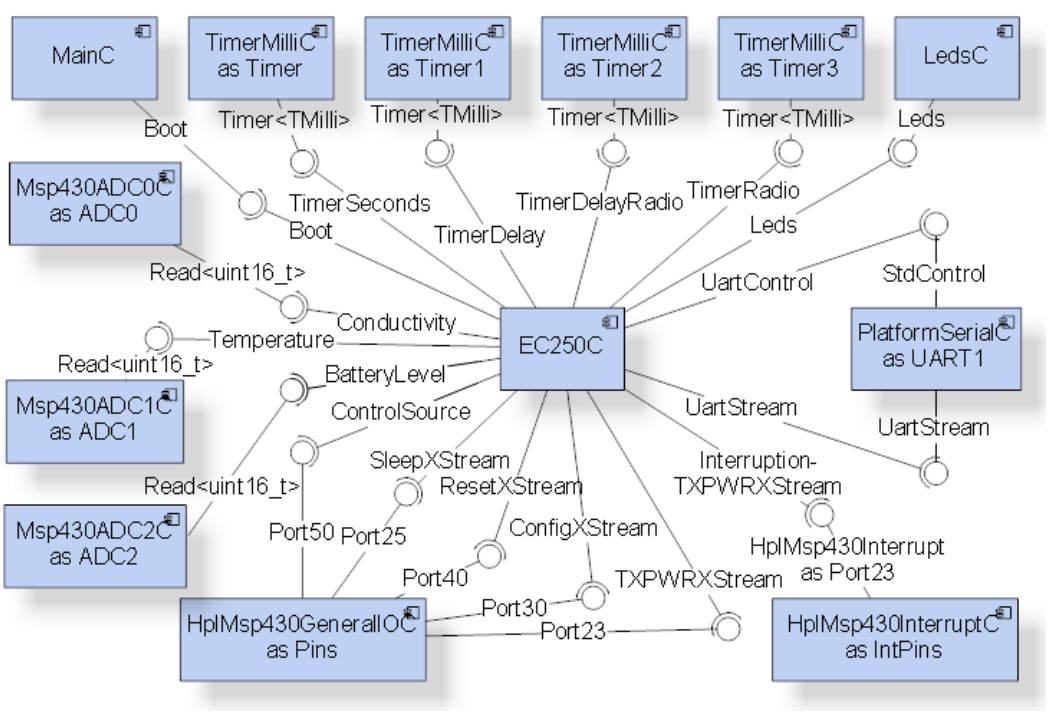

Figura 4.13. Vista estática del diagrama de componentes nesC del Water-Mote.

\subsubsection{Gateway, Base-Station y Repeater.}

La arquitectura propuesta (ver Figura 4.1) implica utilizar una infraestructura de dispositivos para interconectar las dos redes sensores y el sensor inalámbrico de este caso de estudio con las oficinas como la que se describe a continuación: (1) un Gateway por cada una de las redes de sensores, una de ellas basada en Environmental-Motes y la otra en SoilMotes, (2) un Repeater, ubicado en la azotea del edificio de las oficinas y (3) una Base-Station en el interior de las oficinas, conectada físicamente con el servidor de monitorización.

La Figura 4.14, apartados a y c, muestran la imagen detallada del Gateway, junto con el diagrama de bloques de la arquitectura hardware. El micro-controlador se comunica con los motes agronómicos a través de un módulo de radio de corto alcance y con el Repeater situado en las oficinas, usando un módulo de radio de largo alcance XStream. El tiempo de vida del sistema de alimentación es ilimitado, dado que se utiliza un sistema de baterías recargables mediante energía solar. La Figura 4.14d muestra el mote sobre su mástil en la explotación agrícola. La Figura 4.14b detalla los componentes nesC utilizados en la implementación.

La Base-Station recoge toda la información generada por la red de sensores, y se la transmite a la aplicación de monitorización instalada en el servidor. De igual modo, disemina a lo largo de la red de sensores cualquier orden proveniente de la aplicación software. Está basada en un dispositivo comercial XStream, constituido por un módulo de radio $(2,4 \mathrm{GHz})$ de largo alcance conectado a una antena omnidireccional, de $3 \mathrm{dBi}$ de ganancia, y una interfaz RS-232, utilizada en la conexión con el servidor. 


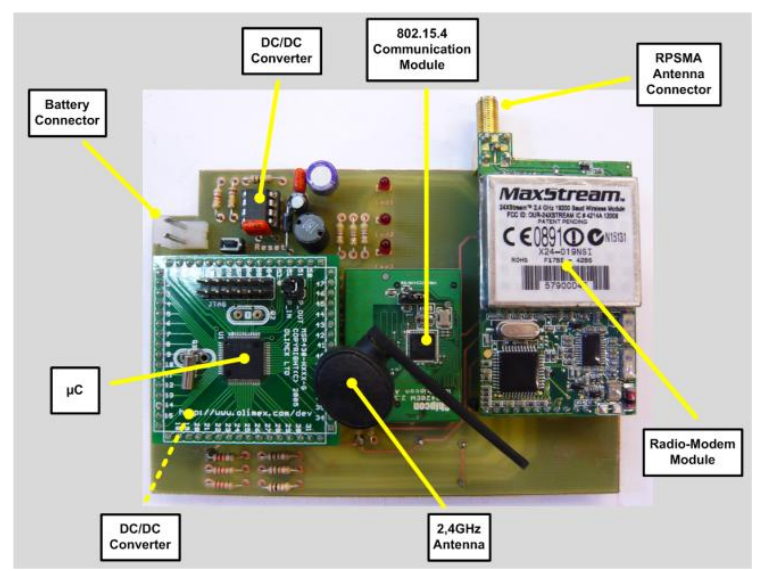

a) Imagen del Gateway.

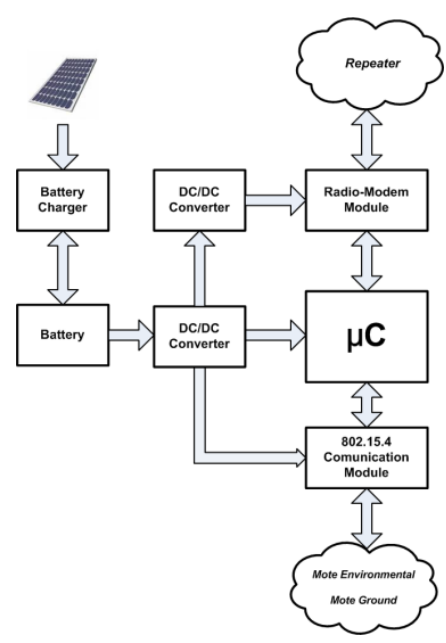

c) Arquitectura hardware.

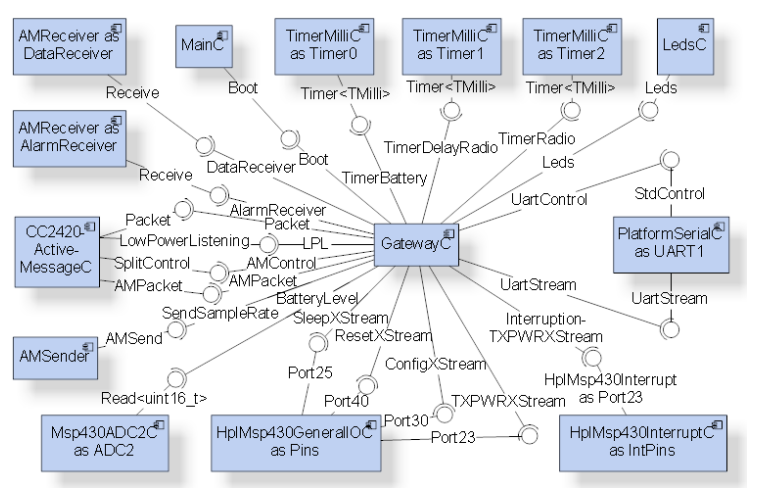

b) Diagrama de componentes nesC.

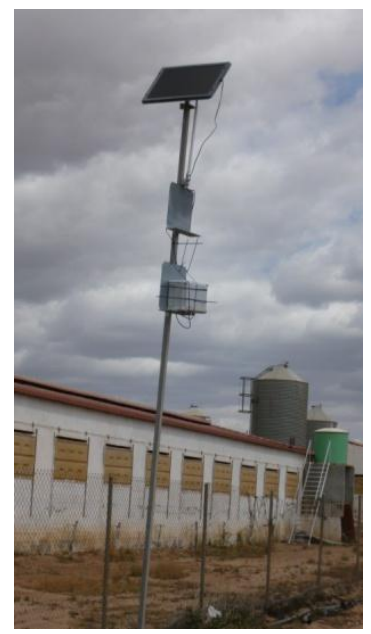

d) Imagen del dispositivo en campo.

Figura 4.14. Diferentes vistas del Gateway.

Con objeto de que la Base-Station, habitualmente situada en el interior de un edificio, tenga la cobertura suficiente con las redes desplegadas en la explotación agrícola a varios kilómetros, será necesario colocar la antena principal en el tejado de las oficinas (punto más alto). En este caso, la primera alternativa sería llevar el cable directamente desde la antena principal hasta la Base-Station, pero esto se traduciría en grandes pérdidas en las señales de transmisión y recepción. Por ello, se planteó incluir en la arquitectura el denominado Repeater, que se trata de un dispositivo igual a la Base-Station con una configuración software de repetidor de mensajes. Así, se pueden alcanzar coberturas de hasta $16 \mathrm{~km}$ en campo abierto y con visión directa entre la Base-Station y los Gateways de las diferentes subredes desplegadas en una explotación.

\subsubsection{Software de monitorización.}

En el marco del proyecto RIMSI, Edosoft Factory desarrolló un software de monitorización para WSNs desplegadas con la arquitectura general propuesta. La aplicación de monitorización (Figura 4.15) ha sido desarrollada utilizando el lenguaje de programación 
Java, con el entorno Eclipse y el sistema gestor de bases de datos relacional MySQL. En la parte derecha se visualiza la posición geográfica de los nodos usando la API de Google Maps. A la izquierda, se muestran los datos formateados en tablas de las diferentes subredes desplegadas. La parte inferior de las anteriores está dedicada a la realización de representaciones gráficas.

Figura 4.15. Vistas del software de monitorización.

\subsection{Fase de validación.}

La validación de los resultados de la simulación y del sistema se llevó a cabo en dos fases realizadas entre febrero y mayo de 2008. La primera de ellas, se realizó en el laboratorio y permitió validar los resultados relativos a consumos, que se obtuvieron con el entorno de simulación ns-2. Además, se desplegó una red con pocos dispositivos (4 SoilMotes y 4 Environmental-Motes, 2 Gateways y una Base-Station), con el fin de validar el correcto funcionamiento de los mismos en el laboratorio. El propósito de estas pruebas era determinar la autonomía de cada uno de los dispositivos desarrollados. Por ello, se llevó a cabo un estudio con objeto de determinar el consumo de potencia de todos los dispositivos. Además, se llevó a cabo un estudio real en laboratorio para validar los resultados obtenidos con el método citado anteriormente.

En una segunda fase, se llevó a cabo el despliegue de la WSN en la explotación agrícola real Langmead, con el fin de validar el correcto funcionamiento del sistema en condiciones reales y los resultados aportados por el simulador en cuanto a retrasos (delays) y el correcto funcionamiento de los dispositivos implementados. Langmead está situada en el Campo de Cartagena en la Región de Murcia, en el sureste de España. Esta zona es una de las más importantes áreas de producción hortícola de Europa. En relación con las características climáticas, es una zona semiárida con precipitaciones anuales de $400 \mathrm{~mm}$. A pesar de esto, $190.000 \mathrm{ha}$, el $31 \%$ del total de los cultivos que componen la zona, es de regadío. De esta área, 51.000 ha están destinadas a cultivos herbáceos y 91.000 a leñosos. 


\subsection{Resultados y discusión.}

En esta sección se presentan los resultados de consumo de energía, 'throughput' y 'delays', obtenidos con el entorno de simulación ns-2, así como los obtenidos tras la implementación de los diferentes dispositivos involucrados en la arquitectura general para monitorizar cultivos agrícolas. Además, sendos resultados se comparan y discuten en cada sub-apartado.

\subsubsection{Consumo de energía y autonomía.}

La Figura 4.16 muestra los consumos medios obtenidos en el Gateway (Figura 4.16a) y los producidos por la transmisión/recepción de la información de nodos sensores (Figura 4.16b y Figura 4.16c). Obsérvese que, como los sensores conectados a los Soil y Environmental Motes son diferentes, los consumos también lo son. El desglose de los consumos producidos es el siguiente:

- Consumos debidos a la transmisión/recepción de la información de los sensores y de la batería. Como muestra la Figura 4.16, los motes envían la información sensorial en períodos de $1.800 \mathrm{~s}$. El gasto energético en los Soil-Motes es muy superior a los Environmental-Motes (del orden de 5 veces mayor) debido al tipo de sensor utilizado (ver Tabla 4.5). En relación al envío de información del estado de las baterías, y debido a que los consumos energéticos del micro-controlador y del módulo de radio para ambos motes son idénticos, se observa un consumo medio similar del orden a $\operatorname{los} 21,5 \mathrm{~mA}$, durante la duración del envío de la trama. El Gateway recibe la información de los motes, y su consumo medio es de, aproximadamente, 21-22mA por trama recibida.

- Consumos debidos a la información de control. Cada 2.000s, el Gateway envía información de control a los motes, siendo su consumo medio de $22 \mathrm{~mA}$ durante la duración del envío ( $5 \mathrm{~s}$ incluyendo datos y preámbulo). Para los receptores, el consumo medio del transceiver es de $21 \mathrm{~mA}$. En el caso de consumos en períodos donde no se transmiten ni se reciben datos (período sleep), el Gateway es lógicamente el dispositivo que proporciona los mayores consumos, ya que es el único despierto $(20 \mathrm{~mA})$.

Finalmente, los consumos medios en los motes son los siguientes: Soil-Mote, 0,50mA y Environmental-Mote, 0,092mA. De este modo, si los motes estuvieran equipados con una batería de una capacidad de $2.700 \mathrm{mAh}$, se podría estimar una autonomía de aproximadamente 223 y 1.217 días, para el Soil-Mote y Environmental-Mote, respectivamente. Nótese que en la Figura 4.16 no se ha representado el pico de consumo energético asociado 
al encendido de la radio (cada 5s), debido a que impediría ver los consumos de los dispositivos en los diferentes estados.

Figura 4.16. Consumos promedio de los diferentes dispositivos obtenidos con ns-2: (a) Gateway. (b) Soil-Mote. (c) Environmental-Mote.

El objetivo de las primeras pruebas de laboratorio fue el determinar la autonomía de cada uno de los motes desarrollados. Para ello, se realizó un estudio de consumos de todos los dispositivos. A continuación, se detallará el estudio realizado para el Soil-Mote, siendo el proceso similar para el resto de dispositivos. El Soil-Mote presenta cinco estados de funcionamiento: reposo o standby, radio despertando, registro de los datos de los sensores, recepción de un mensaje y transmisión de los datos de los sensores y la batería. La Figura 4.17 presenta el consumo de corriente del mote en cada uno de dichos estados. Del mismo modo, en la Tabla 4.7 se recogen los valores de tiempo y consumo del mote para cada uno de estos estados, así como para el resto de los dispositivos. Cabe destacar que la Tabla 4.7 no incluye datos en el estado de recibir un mensaje, ya que es equivalente al estado de cuando se despierta el módulo de radio con una duración de 125ms. Para el caso del consumo promedio se consideró la situación más desfavorable, es decir, realizar una 


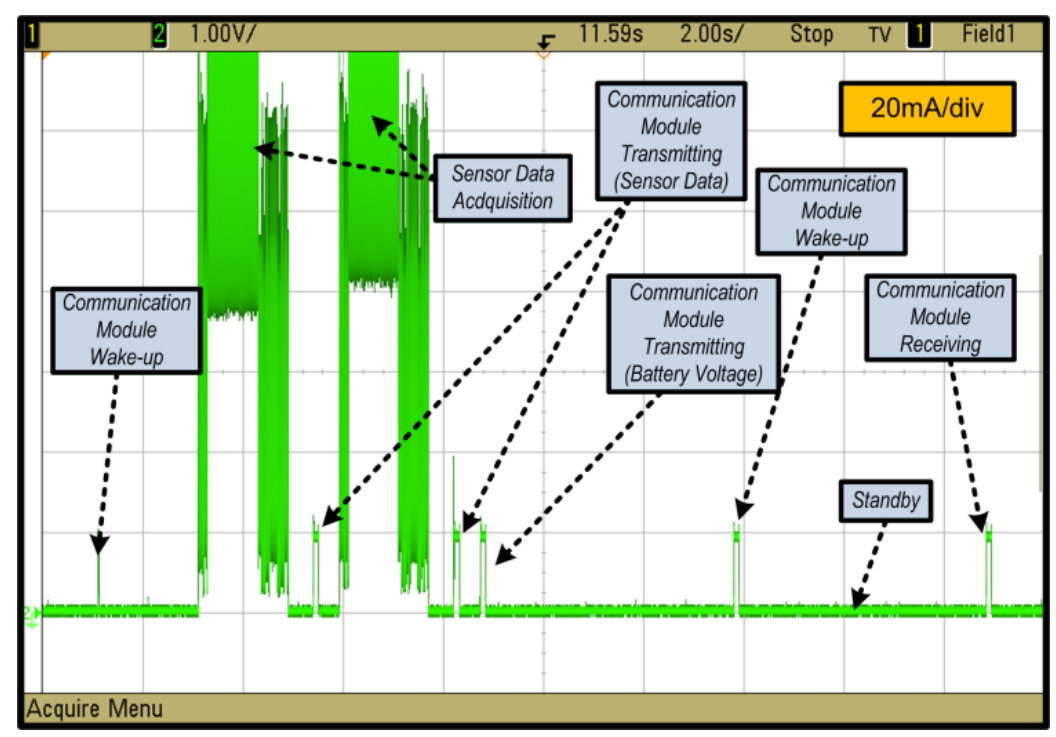

Figura 4.17. Consumo real del Soil-Mote en diferentes estados.

\begin{tabular}{|c|c|c|c|c|c|c|c|c|c|c|}
\hline & \multirow[b]{2}{*}{$\begin{array}{l}\text { Standby } \\
(\mathrm{mA})\end{array}$} & \multicolumn{2}{|c|}{ Despertando } & \multicolumn{2}{|c|}{ Leyendo Sensores } & \multicolumn{2}{|c|}{ Transmitiendo } & \multirow{2}{*}{$\begin{array}{c}\text { i } \\
\text { Promedio } \\
(\mathrm{mA})\end{array}$} & \multirow[b]{2}{*}{$\begin{array}{c}\text { Batería } \\
\text { (mAh) }\end{array}$} & \multirow[b]{2}{*}{$\begin{array}{c}\text { Autonomía } \\
\text { (días) }\end{array}$} \\
\hline & & $\begin{array}{c}\mathrm{i} \\
(\mathrm{mA})\end{array}$ & $t(m s)$ & $\begin{array}{c}\mathrm{i} \\
(\mathrm{mA})\end{array}$ & $\begin{array}{c}\mathrm{t} \\
(\mathrm{ms})\end{array}$ & $\begin{array}{c}\mathrm{i} \\
(\mathrm{mA})\end{array}$ & $\begin{array}{c}\mathrm{t} \\
(\mathrm{ms})\end{array}$ & & & \\
\hline Soil Mote & 0,25 & 20 & 15 & 110 & 1.800 & 25 & 125 & 0,5347 & 2.700 & 210 \\
\hline $\begin{array}{c}\text { Environmental } \\
\text { Mote }\end{array}$ & 0,03 & 20 & 15 & 0.5 & 350 & 25 & 125 & 0,093 & 2.700 & 1.204 \\
\hline Water Mote & 0,03 & 80 & 5.000 & 30 & 10.000 & 150 & 1.200 & 0,5188 & 6.500 & 521 \\
\hline $\begin{array}{c}\text { Gateway } \\
\text { (Soil Motes) }\end{array}$ & 20 & 80 & 5.000 & NA & NA & 150 & 1.200 & 26,4444 & 6.500 & 10 \\
\hline $\begin{array}{c}\text { Gateway } \\
\text { (Environmental } \\
\text { Motes) }\end{array}$ & 20 & 80 & 5.000 & NA & NA & 150 & 1.200 & 23,2222 & 6.500 & 11 \\
\hline
\end{tabular}

Tabla 4.7. Consumo y autonomía de los diferentes dispositivos (i, intensidad; $t$, tiempo).

adquisición y transmisión de los datos de los dos sensores cada 30 minutos. El objetivo final que se persiguió con este estudio, fue determinar la corriente media consumida por el mote, de forma que el valor resultante se pudiera relacionar directamente con el valor de la capacidad de las baterías, y poder determinar así la autonomía del dispositivo. La expresión de la corriente media de consumo del mote, se puede determinar a partir de la Ec. 4.1.

$\bar{I}_{\text {soil-mote }}=\bar{I}_{\text {stan dby }}+\bar{I}_{\text {despertan do }}+\bar{I}_{\text {acq }}+\bar{I}_{\text {trans }}+\bar{I}_{\text {receiv }}$

donde

$\bar{I}_{s \tan d b y}=0,25 m A$

$\bar{I}_{\text {despertan do }} \approx \frac{20 \mathrm{~mA} \cdot 15 \cdot 10^{-3}}{5}=0,06 \mathrm{~mA}$ 


$$
\begin{aligned}
& \bar{I}_{a c q} \approx 2 \cdot\left(\frac{110 m A \cdot 1800 \cdot 10^{-3}}{1800}\right)=0,22 m A \\
& \bar{I}_{\text {trans }} \approx 2 \cdot\left(\frac{25 m A \cdot 125 \cdot 10^{-3}}{1800}\right)=0,0035 m A \\
& \bar{I}_{\text {receiv }} \approx \frac{20 m A \cdot 125 \cdot 10^{-3}}{2000}=0,00125 m A
\end{aligned}
$$

La Ec. 4.2 indica el consumo de standby. En la Ec. 4.3 se expresa el valor medio del consumo del mote al activar el módulo de radio durante $15 \mathrm{~ms}$ y con un período de $5 \mathrm{~s}$. La Ec. 4.4 y la Ec. 4.5 se calculan de un modo similar, salvo que se multiplican por dos debido a que este es el número de sensores que lleva conectados cada mote. La Ec. 4.6 representa el consumo en la recepción de un mensaje (duración de 125ms). Para su cálculo, se ha mantenido la misma premisa que en la simulación de que, cada 2.000s, se recibe información de control del Gateway. Mediante la Ec. 4.1, se obtiene un valor de la corriente media de consumo del Soil-Mote de aproximadamente 0,5347mA. Como las baterías de este mote son de $2700 \mathrm{mAh}$, la autonomía estimada del mismo se corresponde con 210 días, tiempo que garantiza el funcionamiento correcto durante una campaña agrícola u hortícola habitual (10 semanas). El proceso para realizar el estudio del consumo del resto de dispositivos es el mismo que el empleado para el caso del Soil-Mote. Los resultados obtenidos se pueden consultar en la Tabla 4.7. Por lo tanto, los resultados mostrados en la Figura 4.17 y los valores obtenidos, validan los resultados de consumo de potencia obtenidos con el entorno de simulación (Figura 4.16).

Se realizó un estudio real en laboratorio, entre febrero y mayo de 2008, con los nodos sensores realizando una lectura de los sensores cada 30 minutos. Tras el estudio, el valor final de la batería era de, aproximadamente, 3,7V para el Soil-Mote y de 4,08V en el caso del Environmental-Mote (ver Figura 4.18). Por lo tanto, los resultados del entorno de simulación y los experimentales, muestran que la autonomía del Soil-Mote y del EnviromentalMote es mayor que la de un ciclo hortícola (típicamente 10 semanas).

Figura 4.18. Evolución del voltaje de la batería durante un estudio real. 


\subsubsection{Throughput.}

El throughput de los dispositivos con una optimización de consumos (es decir, tomando el check interval de 5s) se muestra en la Figura 4.19. La cantidad de datos enviados por el Gateway está relacionada con el propio funcionamiento del protocolo B-MAC y su operación de Low Power Listening (LPL). El Gateway envía un preámbulo de 5s antes de enviar los datos al mote seleccionado. La Figura 4.19 muestra cómo el throughput medio (para los 5 s) es de 238Kbps para el tamaño máximo de trama que, según el estándar 802.15.4, es de 127 bytes. Este valor es inferior a la tasa máxima de transmisión de estos dispositivos (250 Kbps), debido a los espacios temporales entre tramas MAC transmitidas (inter-frame space). En este caso, el espacio inter-frame se ha fijado en 6 bytes (denominado período SIFSShort Inter-frame Spacing en el estándar 802.15.4).
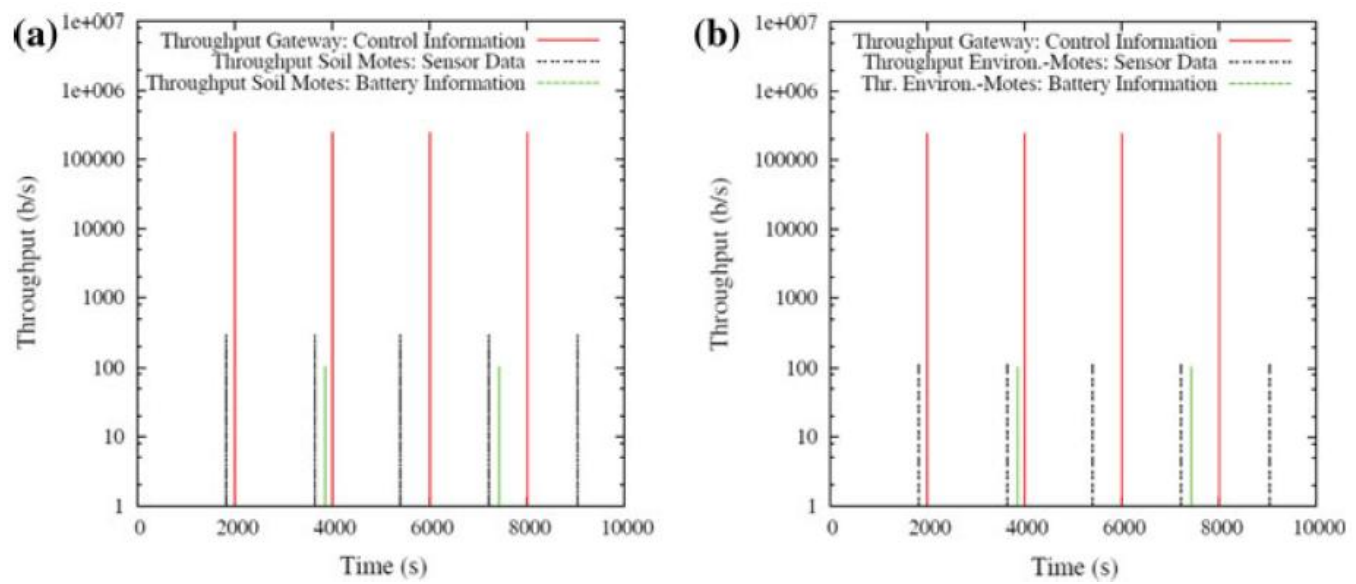

Figura 4.19. Tasa binaria de transmisión/recepción o throughput. (a) Subred Soil-Motes. (b) Subred Environmental-Motes.

La Figura 4.19 muestra el throughput de los motes. Éste depende del tipo de mote y del mensaje específico que se está transmitiendo, cuyo tamaño depende de la información obtenida de los sensores con los que se está monitorizando. Esto se traduce en diferentes throughputs para cada tipo de mote. Como se puede observar, el Gateway produce un tráfico mucho mayor que los nodos sensores (tramas de datos y voltaje de la batería).

\subsubsection{Delays.}

En la Figura 4.20a y la Figura 4.20b se muestran los retardos debidos a la subred de los Soil-Motes. Como se puede observar, los mayores retardos son los provocados por el Gateway (Figura 4.20a). El motivo se debe a que las tramas de control (compuestas de preámbulo y datos de control) van llenando las colas del dispositivo. Este proceso, y el propio funcionamiento del protocolo B-MAC, dan lugar a retardos en el envío de estas tramas a los dispositivos. 
En el caso de las tramas enviadas por los Soil-Motes, el retardo (Figura 4.20b) depende del protocolo de comunicaciones B-MAC y del procesamiento de los motes, desde que los datos son capturados por el sensor, hasta su transmisión. Los valores obtenidos son más reducidos en relación al Gateway debido a que éste no envía preámbulo, es decir, el SoilMote envía tramas de tamaño inferior a 127 bytes (menor tiempo de transmisión).

Los retardos de los Environmental-Motes, al igual que los Soil-Motes, son producidos por el funcionamiento del protocolo de acceso al medio y el procesamiento en los nodos, pero algo inferiores a éstos debido a la menor longitud del 'payload' enviado. Como se puede observar, los retrasos (delays) experimentales medidos en los dispositivos desarrollados (Figura 4.20c) son muy similares a los obtenidos con el entorno de simulación ns-2.

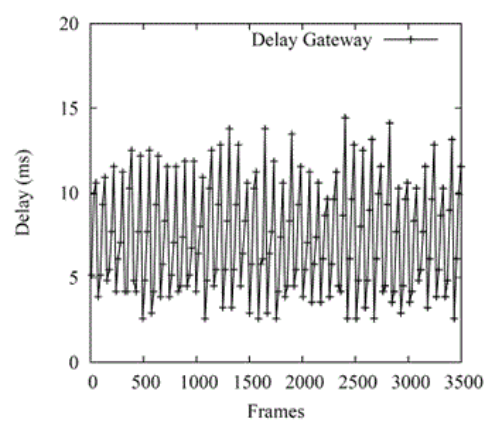

(a)

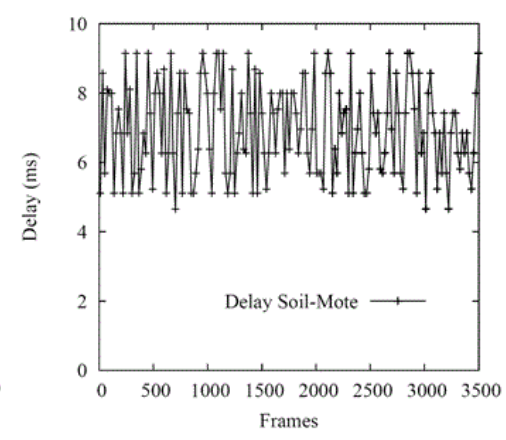

(b)

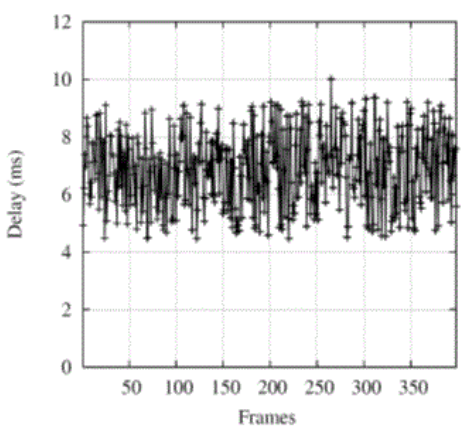

(c)

Figura 4.20. Delays obtenidos con el simulador y experimentalmente en la sub-red de los Soil-Motes.

(a) Delays del Gateway obtenidos con ns-2. (b) Delays del Soil-Mote obtenidos con ns2. (c) Delays experimentales del Soil-Mote.

\subsubsection{Resultados experimentales en el cultivo.}

Con objeto de validar los resultados de la simulación en cuanto a los retardos, así como el correcto funcionamiento de los dispositivos, se realizó un despliegue siguiendo la arquitectura propuesta en la Figura 4.1. Se desplegaron dos redes (Figura 4.21) de sensores en dos parcelas de aproximadamente 4ha cada una y separadas 5,2 y $8,7 \mathrm{~km}$, respectivamente, de las oficinas de la explotación agrícola. En una, se desplegaron 10 SoilMotes y un Gateway, y 10 Environmental-Motes y un Gateway en la otra. Al Gateway se conectaron dos antenas (omnidireccional de $3 \mathrm{dBi}$ y omnidireccional de $8 \mathrm{dBi}$ ) colocadas en un poste de $5 \mathrm{~m}$ de altura, lo que posibilitaba una visión directa entre las antenas de los nodos desplegados en el suelo y el Gateway. Además, para garantizar también una visión directa entre el Gateway y las oficinas, se colocó una antena omnidireccional, de 8dBi de ganancia, en la azotea de las oficinas ( $9 \mathrm{~m}$ de altura). La comunicación inalámbrica entre la antena del tejado y la Base-Station (XStream modem X24-019PKI-RA de Digi Inc., USA) se consiguió usando el mencionado repetidor (Repeater). Los Environmental-Motes utilizan una 
antena omnidireccional, de $3 \mathrm{dBi}$ de ganancia. Estos fueron desplegados a $1,5 \mathrm{~m}$ sobre el suelo. En el caso de la sub-red de los Soil-Motes, los nodos sensores se desplegaron sobre el suelo y estaban equipados con una antena omnidireccional, de $3 \mathrm{dBi}$ de ganancia. Al mismo tiempo, se utilizó una tecnología similar a la del Gateway para desarrollar un nodo inalámbrico (Water-Mote) para monitorizar la calidad del agua de una de las balsas de la explotación agrícola usando el sensor EC250. Por simplicidad, este dispositivo no se ha mostrado en la Figura 4.21.

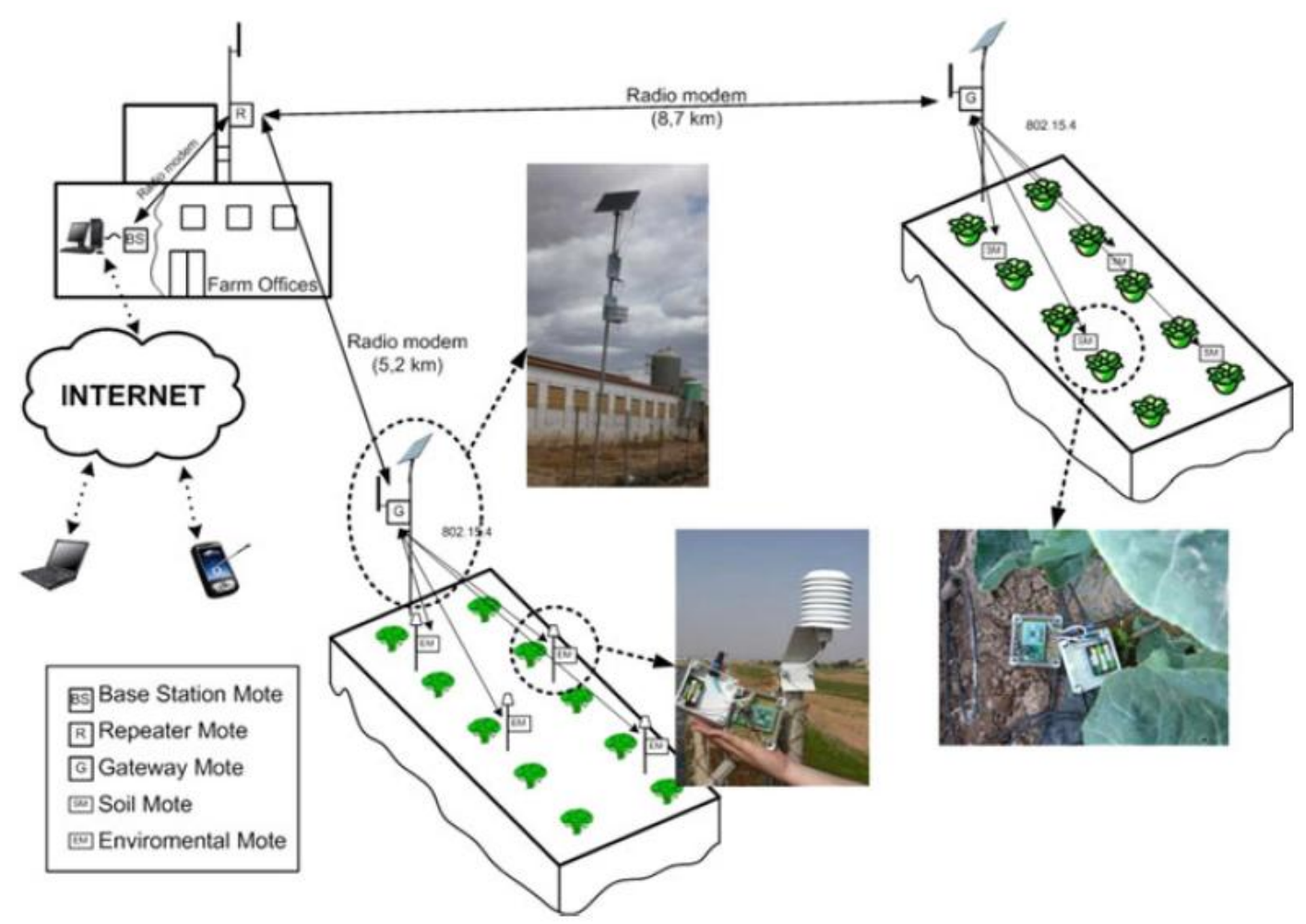

Figura 4.21. Despliegue de los nodos sensores realizado en la explotación agrícola Langmead.

Los nodos se desplegaron en la última semana de febrero de 2008, y los propietarios de la explotación agrícola comenzaron a obtener datos con las WSNs. Los sensores del Soil-Mote se colocaron a 200 y $400 \mathrm{~mm}$ de profundidad. Durante este periodo, se han producido precipitaciones por lluvia ( $80 \mathrm{~mm}$ acumulados), vientos moderadamente intensos de hasta $65 \mathrm{~km} / \mathrm{h}$ y temperaturas suaves (media de $15,2^{\circ} \mathrm{C}$ ), observándose un correcto funcionamiento de los nodos. Esto ofrece una garantía de robustez del despliegue para condiciones meteorológicas similares. La Figura 4.22 muestra los datos (porcentaje volumétrico del suelo y temperatura del aire) registrados durante un periodo de 10 semanas. El sensor Hydra Probe II proporciona medidas precisas de la humedad del suelo en unidades de fracción de agua por unidad de volumen (wfv o $\mathrm{m}^{3} \mathrm{~m}^{-3}$ ). La saturación completa (todos los espacios porosos del suelo llenos de agua) se produce, normalmente, entre 0,5 y 0,6 wfv, y es muy dependiente del suelo. Antes de enviar un mensaje del nodo sensor al Gateway, se configuran algunos intentos (10 para datos de los sensores y 5 para el 
voltaje de la batería) con un retraso de $100 \mathrm{~ms}$. Cuando los reintentos han llegado a su límite, se anota un error de transmisión en la memoria del mote. Estos valores se comprobaron cuando se realizaron los primeros ensayos y no se observaron errores de comunicación. Esto confirma que, bajo condiciones climáticas similares, la robustez del sistema está asegurada.
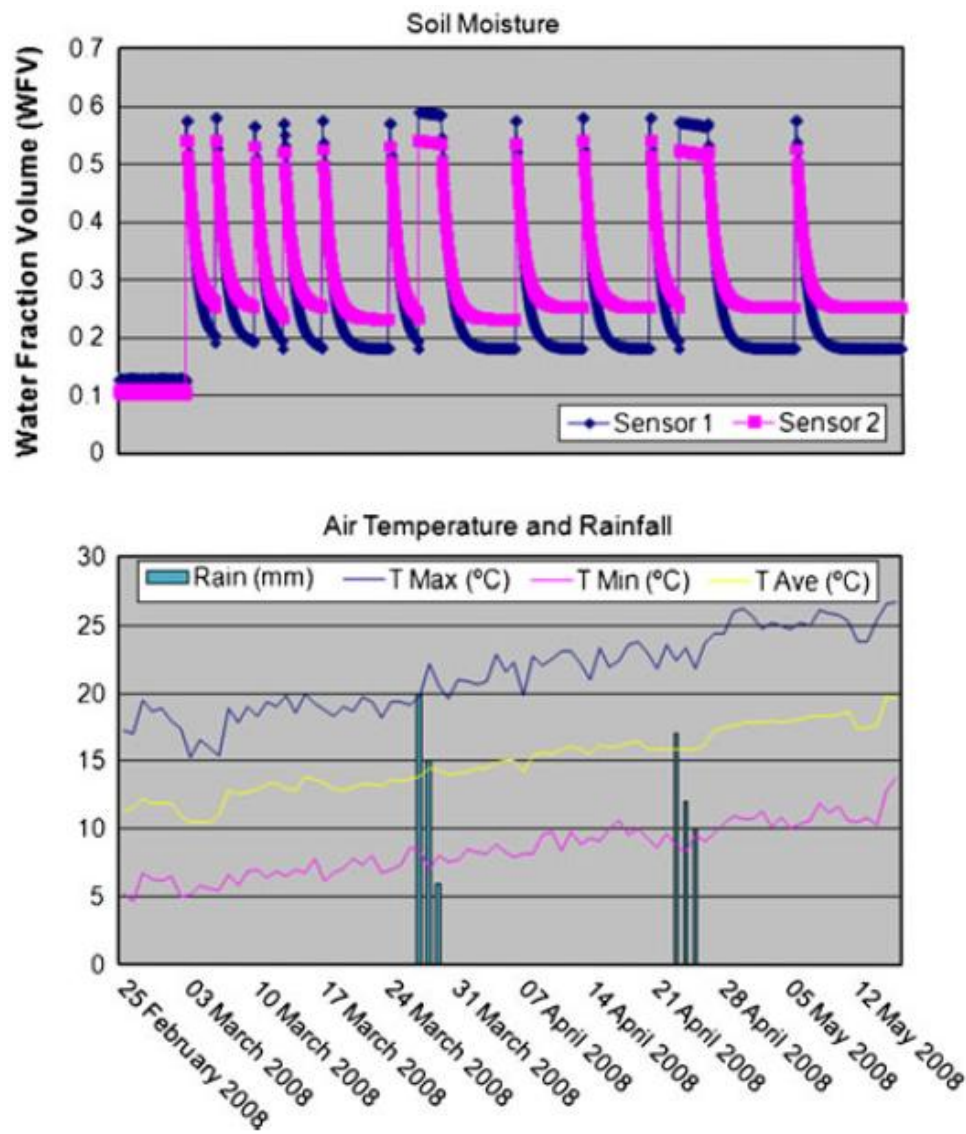

Figura 4.22. Humedad del suelo y temperatura del aire, monitorizada durante 10 semanas.

Antes de implantar esta tecnología, la empresa monitorizaba sus cultivos mediante un método tradicional. En la actualidad, con la tecnología desarrollada, es posible conocer las variables de los cultivos en tiempo real, de manera que se pueden estimar necesidades hídricas de los cultivos sin la necesidad de desplazarse hasta los mismos. Es, precisamente, en este último aspecto en el que se quiere seguir trabajando a corto plazo.

\subsection{Conclusiones.}

Se ha propuesto una arquitectura general, basada en una WSN, para llevar a cabo la monitorización en tiempo real y con medidas continuas en cultivos hortícolas. La arquitectura está compuesta por varios nodos (Soil-Mote, Environmental-Mote y Water-Mote) y una serie de elementos auxiliares (Gateway, Repeater y Base-Station) para interconectar los anteriores con las oficinas de la explotación agrícola. Todos los elementos citados 
anteriormente resuelven un problema concreto. La arquitectura ha demostrado que, utilizando una topología en estrella y el protocolo B-MAC con el módulo de radio del Gateway siempre activo, el consumo de los nodos sensores puede ser reducido y, por lo tanto, la vida útil de la red puede ser aún mayor.

A pesar de que el diseño de la arquitectura propuesta está optimizado para cultivos hortícolas, dicha arquitectura también puede ser utilizada en otro tipo de cultivos, como los leñosos.

La alternativa de que cada nodo resuelva un problema concreto, llevada a cabo en este trabajo, no es adecuada por razones fundamentales: el 'time-to-market' y los costes asociados. Surge, por tanto, la necesidad de culminar el desarrollo con el diseño de un único nodo multifuncional, el cual pueda ser configurable, tanto a nivel de hardware como de software. Surgió, así, la idea de desarrollar el nodo multifuncional GAIA 2, que será descrito en un capítulo posterior.

Debido a la carencia de experiencia en el diseño electrónico de un circuito de radio frecuencia con tecnología SMD, culminar en la implementación del GAIA 2 conllevaba un reto importante. Por ello, antes de abordar el diseño del GAIA 2, se planteo llevar a cabo un diseño menos ambicioso. Éste fue el diseño de la versión pre-comercial de Soil-Mote, que será descrita en el siguiente capítulo.

Los trabajos desarrollados en este capítulo han sido publicados en dos revistas internacionales listadas en el ISI [López, 2009a; López, 2011a], una revista nacional no listada en el ISI [López, 2008a], un congreso internacional con actas publicadas en Lecture Notes [López, 2009c] y un congreso nacional [López, 2008b]. 
CONTRIBUCIÓN A LAS REDES DE SENSORES INALÁMBRICAS. ESTUDIO E IMPLEMENTACIÓN DE Soluciones Hardware para Agricultura de Precisión. 


\section{Capítulo 5}

\section{GAIA Soil-Mote: un Nodo Sensor para la Monitorización de Parámetros del Suelo Basado en el Estándar SDI-12.}

$L$

a experiencia conseguida en el diseño, implementación y validación de una arquitectura basada en WSN para la monitorización de cultivos hortícolas, sentó las bases para llevar a cabo el diseño e implementación de versiones comerciales de nodos sensores o motes. En concreto, en este capitulo se describe el GAIA Soil-Mote, que se trata de una versión comercial del denominado Soil-Mote, descrito en el capitulo anterior. El GALA Soil-Mote permite utilizar instrumentación agricola de precisión basada en el estándar SDI-12. Sus principales elementos son el micro-controlador de 16 bits MSP430F1611 y el módulo de radio CC2420, que opera en la banda ISM de 2,4GHzy es compatible con el estándar IEEE 802.15.4. El diseño del software se llevó a cabo sobre el sistema operativo TinyOS, utilizando el lenguaje de programación nesC. Para validar el diseño de este nodo sensor, se ha planteado una metodología de dos fases. En una primera fase, se han validado en laboratorio las soluciones hardware y software propuestas, incluyendo un estudio sobre el consumo de potencia y autonomía del diseño logrado. En una segunda fase, se ha implementado una aplicación de monitorización de un cultivo real de Broccoli (Brassica oleracea L. var Marathon) en el Campo de Cartagena (37\%44'26'N, 1013'38'W) en el sureste de España. Ésta ha permitido la validación del nodo sensor, en condiciones reales de funcionamiento. 


\subsection{Introducción.}

Como se ha comentado en los capítulos anteriores, para gestionar con mayor eficiencia los cultivos agrícolas y sus recursos hídricos se puede hacer uso de la Agricultura de Precisión [Anurag, 2008], siendo las Redes de Sensores Inalámbricas (WSN, Wireless Sensor Networks) [Akyildiz, 2002] una de las tecnologías de la información y las comunicaciones con un futuro prometedor en esta área de estudio.

Por otro lado, el interés que ha despertado el uso de esta tecnología ha dado lugar a la aparición en el mercado de diferentes plataformas hardware (nodos sensores o motes), como los MICA2, los Imote2 y los TelosB [MEMSIC], ya descritos en el capítulo 3. Tanto estos motes como otros no mencionados aquí, son dispositivos que suelen incluir embebidos sensores de bajo coste, y cuyas especificaciones (precisión, resolución, derivas, entre otras) se alejan de las de la instrumentación que, habitualmente, es necesaria en las aplicaciones de la Agricultura de Precisión. Por otro lado, muchos de estos motes se han utilizado en aplicaciones de laboratorio o de investigación, careciendo de la robustez necesaria para su aplicación en entornos agronómicos reales. Por lo tanto, para que los motes puedan ser utilizados en aplicaciones agronómicas reales, por un lado, es necesario que éstos incluyan la electrónica necesaria para la conexión de instrumentación externa de calidad y, por otro, que se mejore la robustez de los mismos para que puedan ser instalados en campo.

Así, por ejemplo, hace ya algunos años, Crossbow (MEMSIC en la actualidad) [MEMSIC] comenzó a comercializar el kit de monitorización ambiental MEP-SYS, y posteriormente el kit eKo, ambos ya descritos en el capítulo 3. Al igual que Crossbow, hay algunos fabricantes que están proponiendo soluciones similares. En esta dirección, la empresa española Edosoft Factory S.L. se planteó hace un par de años el desarrollo de una línea de productos destinados para su uso en aplicaciones de Agricultura de Precisión. Para llevar a cabo estas tareas, la empresa se puso en contacto con el grupo de investigación División de Sistemas e Ingeniería Electrónica de la Universidad Politécnica de Cartagena, tal y como se expuso en el capítulo anterior. La conclusión de las reuniones mantenidas con la empresa fue desarrollar esta línea de productos en el marco de la presente Tesis Doctoral. En esta línea, se primarían características tales como la robustez, la autonomía y la posibilidad de conectar diferentes tipos de instrumentación, frente a otras características funcionales no tan prioritarias, como la reprogramación inalámbrica y la posibilidad de poder implementar algoritmos complejos en los motes.

Otra característica importante de los motes es la posibilidad que tienen de conexión con instrumentación externa. Existe en el mercado una amplia variedad de sensores, utilizados dentro del campo de la Agricultura de Precisión que proporcionan su salida mediante el protocolo SDI-12 [SDI12]. Por enumerar algunas soluciones comerciales 
basadas en este protocolo, Stevens [Stevens] comercializa sensores como el Hydra Probe II y el EC 1200. Otro distribuidor importante, como es Campbell Scientific [CampbellScientific], comercializa sensores como el CS245-L, CS408-L y el WINDSONIC4-L. En consecuencia, los motes necesitan incorporar la electrónica necesaria para conectar instrumentación de calidad, si se pretenden usar en aplicaciones reales de agricultura. Es por ello, que uno de los objetivos prioritarios ha sido incluir en los motes la posibilidad de conexión directa con sensores de tipo SDI-12. En este punto, cabe destacar que, en el momento de llevar a cabo el estudio e implementación del nodo descrito en este capítulo, no se encontró en la literatura científica un mote que proporcionase esta interfaz.

En el capítulo anterior, se presentó el diseño, implementación y validación de una WSN con la que llevar a cabo la monitorización de cultivos hortícolas. La arquitectura estaba compuesta por varios nodos sensores especializados, y una serie de elementos auxiliares de comunicación. Al final del capítulo, se llegó a la conclusión de que no era adecuado tener un nodo sensor que resuelva un problema concreto por razones de time-tomarket y de costes. Por ello, se planteó el diseño de un único nodo multifuncional, que pueda ser configurado, tanto a nivel de hardware como de software, para operar como cualquiera de los nodos sensores especializados

Este capítulo describe el nodo sensor denominado GAIA Soil-Mote (GSM en adelante), que proporciona una interfaz SDI-12 y dispone de una carcasa con un grado de protección IP67. Además, permite desplegar una gran cantidad de sensores en el cultivo y transferir inalámbricamente al sumidero de la red inalámbrica desplegada los datos adquiridos por éstos; todo ello gracias a que el diseño realizado permite que a cada GSM se le puedan conectar hasta 10 sensores Hydra Probe II (HP2 en adelante).

El diseño e implementación del GSM surge para resolver la carencia que se tenía en el diseño de circuitos de radio frecuencia con componentes SMD. Esto es así porque los nodos sensores especializados se diseñaron e implementaron con módulos de inserción. Es, por tanto, un diseño menos ambicioso que el de un nodo sensor multifuncional, con el que recabar experiencia en el diseño de circuitos de radio frecuencia y, en general, en todo el proceso necesario para diseñar e implementar un nodo sensor comercial.

Después de esta introducción, la siguiente sección detalla los requisitos funcionales y la descripción del hardware y software del dispositivo desarrollado para desplegar WSNs en horticultura de precisión. A continuación, en la sección 3, se describen las pruebas realizadas con el dispositivo. Las primeras, se llevaron a cabo en laboratorio determinando la autonomía del dispositivo, y verificando el correcto funcionamiento de una pequeña red desplegada. Posteriormente, se validó el dispositivo con el despliegue realizado en un cultivo real. Finalmente, la sección 4 detalla las conclusiones del trabajo realizado e introduce el nuevo dispositivo que será descrito en el próximo capítulo. 


\subsection{Descripción del GAIA Soil-Mote.}

A la hora de escoger los requisitos que debe satisfacer el diseño y construcción del nodo sensor, es posible que sea necesario llegar a un compromiso entre requisitos contrapuestos. En el diseño del GSM se primaron fundamentalmente aquellas decisiones arquitectónicas que favorecieron la robustez del sistema y también la interconexión de distintos tipos de sensores. Una vez fijadas estas premisas, junto con la compatibilidad requerida para las comunicaciones, se consideraron todos aquellos requisitos adicionales que pudieron hacer más idóneo y funcional el nodo sensor. El nuevo mote se desarrolló con el fin de cumplir las siguientes especificaciones:

1. El mote debe ser capaz de comunicarse con otros nodos sensores, por medio de un módulo de radio altamente fiable y compatible con el estándar IEEE 802.15.4 [IEEE, 2006].

2. Se pretende desarrollar un producto robusto para la monitorización de parámetros del suelo (temperatura, porcentaje volumétrico y conductividad eléctrica) en cultivos que pueden estar distribuidos en parcelas con una extensión media de 4ha, y separadas entre sí hasta unos $10 \mathrm{~km}$.

3. El mote debe proporcionar una interfaz SDI-12 para permitir la conexión al mote de cualquier sensor dotado de esta interfaz digital. En concreto, el software permitirá gestionar 2 sensores HP2. Además, con pequeñas variaciones en el software será posible usar otros sensores SDI-12, así como un mayor número de sensores del mismo tipo.

4. El diseño mecánico del dispositivo debe estar optimizado para ser utilizado en cultivos de tipo hortícola. Esto significa que el dispositivo debe poder instalarse a nivel del suelo y, por sus dimensiones, no debe ser necesario retirarlo cuando se utilice maquinaría agrícola pesada en el cultivo. Por ello, la carcasa del mote debe tener un grado de protección IP67 y la altura (incluyendo la antena) no debe superar los $20 \mathrm{~cm}$. Además, para evitar la substracción de los motes, éstos serán de un color discreto. Conviene destacar que, aunque el diseño debe estar optimizado para cultivos de tipo hortícola, los motes también deben poder ser utilizados en otro tipo de cultivos, como los frutícolas, los viñedos, etc.

5. El GSM soportará topologías en estrella, siendo un dispositivo de tipo Gateway el sumidero de la red.

6. Teniendo en cuenta que la antena del Gateway estará situada a $4 \mathrm{~m}$ de altura, y los GSMs se desplegarán sobre el suelo del cultivo, el alcance de las comunicaciones inalámbricas entre ambos será de, al menos, $100 \mathrm{~m}$.

7. El mote debe funcionar mediante baterías, debiéndose garantizar una autonomía mínima de 10 semanas, que es el tiempo habitual de duración de un ciclo agronómico hortícola. 
8. El desarrollo de aplicaciones software para el mote debe ser sencillo, con la ayuda de herramientas de programación y depuración estándar.

9. El mote debe disponer de un único conector externo, que tendrá un grado de protección IP67. Mediante este conector será posible programar y depurar el software del nodo, reiniciar el programa, cargar la batería y conectar los sensores externos.

10. El nodo sensor debe poder ser configurado inalámbricamente para realizar la lectura de los sensores con un período comprendido entre 30 minutos y 10 días. Además, el nodo enviará al software de monitorización la tensión de la batería cada hora.

En los siguientes apartados se describe el nodo sensor desarrollado, tanto a nivel hardware como software. Además, se mostrará una fotografía del dispositivo instalado en un cultivo real y un diagrama describiendo la situación del nodo sensor y de los sensores en el cultivo.

\subsubsection{Descripción del hardware.}

En la Figura 5.1 se puede ver el alto nivel de integración del nodo sensor que se ha desarrollado. La PCB (Figura 5.1a) tiene forma circular y $8 \mathrm{~cm}$ de diámetro. La Figura 5.1b muestra el diagrama de bloques del mote. Como se puede apreciar, la tarjeta del mote se ha desarrollado con un número mínimo de componentes. Esto se debe, en parte, al requisito de bajo consumo de potencia y, por otro lado, a la necesidad de reducir el tamaño del mote y los costes de fabricación.

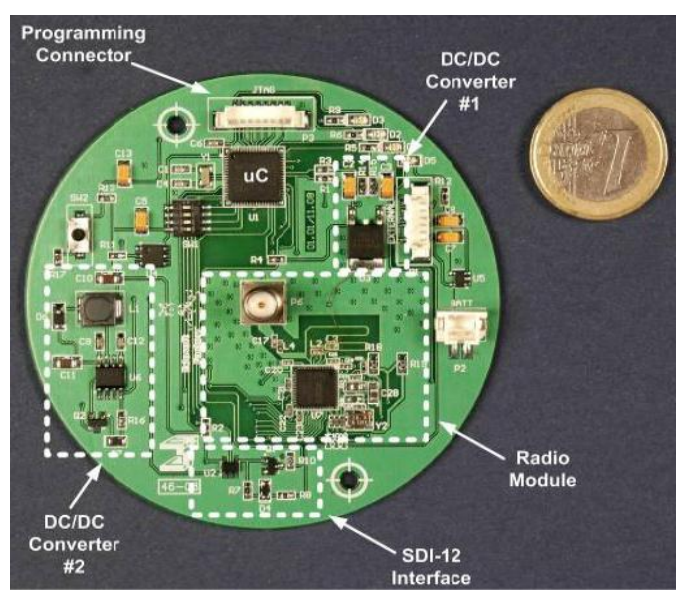

(a)

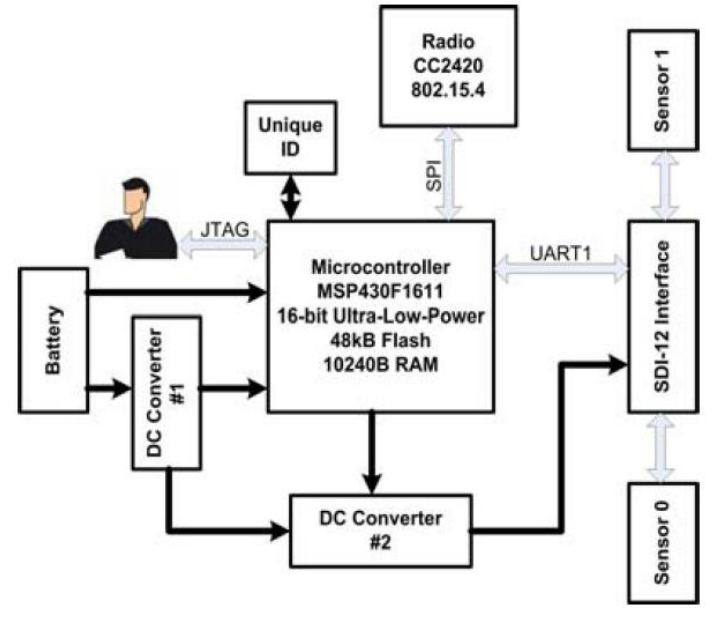

(b)

Figura 5.1. Vistas del GAIA Soil-Mote. (a) PCB. (b) Diagrama de bloques.

El elemento principal de la plataforma es el micro-controlador de bajo consumo MSP430F1611 de Texas Instruments. Su arquitectura, combinada con los 5 modos de bajo 
consumo [MSP430F1611] configurables, está optimizada para conseguir una elevada vida de la batería en aplicaciones portátiles. El dispositivo incluye una CPU, de tipo RISC, de 16 bits, y registros del mismo tamaño de palabra, que atribuyen al dispositivo una máxima eficiencia en el código. Su DCO (Digitally Controlled Oscillator) permite que el dispositivo pase de modos de bajo consumo a modo activo en menos de $6 \mu$ s. Este micro-controlador puede operar con frecuencias de reloj de hasta $8 \mathrm{MHz}$, y direccionar hasta $10 \mathrm{~KB}$ de memoria (mucho más de lo requerido en la práctica). En este caso, se usó un encapsulado de tipo QFN de 9,1x9,1 mm.

Las comunicaciones se gestionan con el módulo de radio CC2420 [CC2420] de Texas Instruments. Este módulo se incorporó en la PCB diseñada partiendo de la revisión A del diseño de referencia del módulo CC2420EM (ver Figura 5.2) proporcionado por el fabricante. Es un diseño de referencia de 2 capas que incluye los layouts de las diferentes capas requeridas a la hora de diseñar la PCB (top, botton, etc.). Para integrarlo, se han eliminado los 2 conectores SMD de los layouts y se han ruteado las pistas necesarias, que llegaban previamente a los conectores SMD, hasta las líneas del micro-controlador, y los puntos de VCC y GND. Las características principales del CC2420 son su bajo consumo y una operación eficiente, con soporte para el estándar IEEE 802.15.4. El dispositivo puede establecer las comunicaciones utilizando 16 canales en la banda libre ISM (IndustrialScientific-Medical) de 2,4GHz. El módulo de radio se ha conectado a la antena omnidireccional de exterior, de 2dBi de ganancia, descrita en el Anexo I.
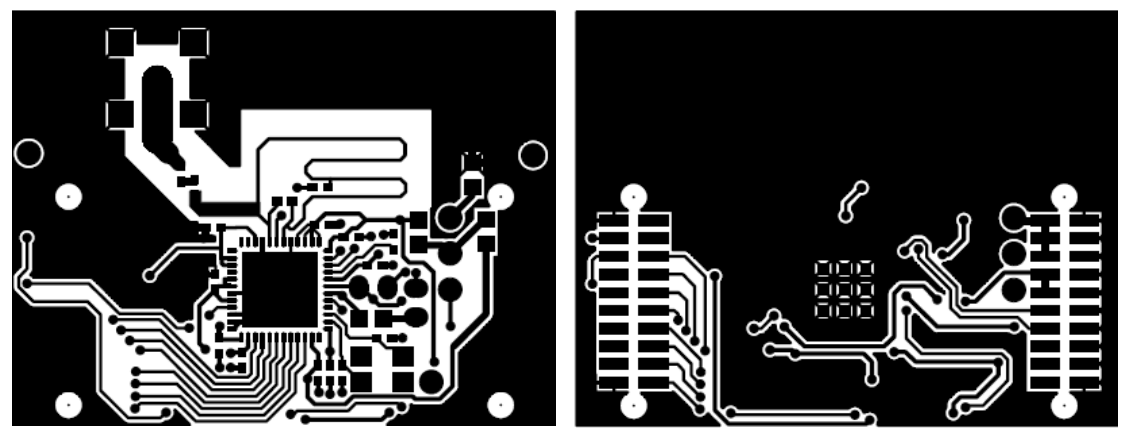

Figura 5.2. Layouts de las capas top y botton del diseño de referencia del módulo de inserción CC2420EM proporcionados por el fabricante.

Para poder conectar los sensores externos a la UART del micro-controlador, se ha incluido el módulo SDI-12 Interface. Esta interfaz es necesaria por dos motivos: (1) porque los niveles de tensión manejados por el micro-controlador y los sensores externos son diferentes y (2) porque, además, hay que interconectar los dos pines de la UART (Rx y Tx) con la única línea bidireccional de datos de los sensores externos. Todo esto se consigue mediante un buffer triestado, un transistor, un diodo Zener y algunos componentes analógicos, estableciéndose de este modo un bus SDI-12 en el mote (Figura 5.3). Cabe destacar, que en un bus SDI-12, se pueden conectar hasta 10 sensores con esta interfaz. 


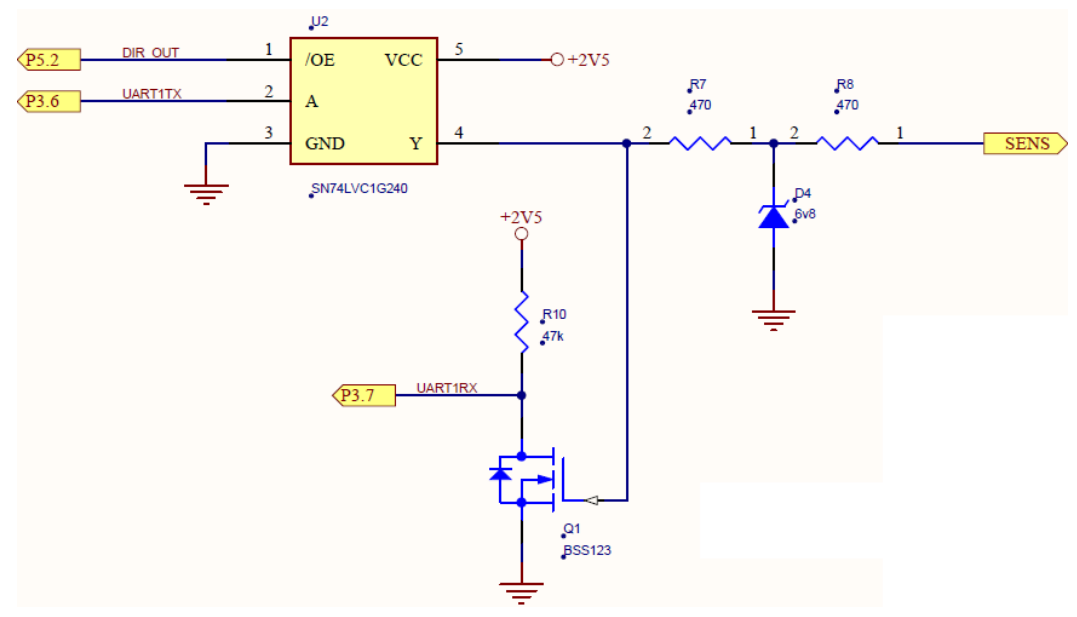

Figura 5.3. Esquemático de la interfaz SDI-12.

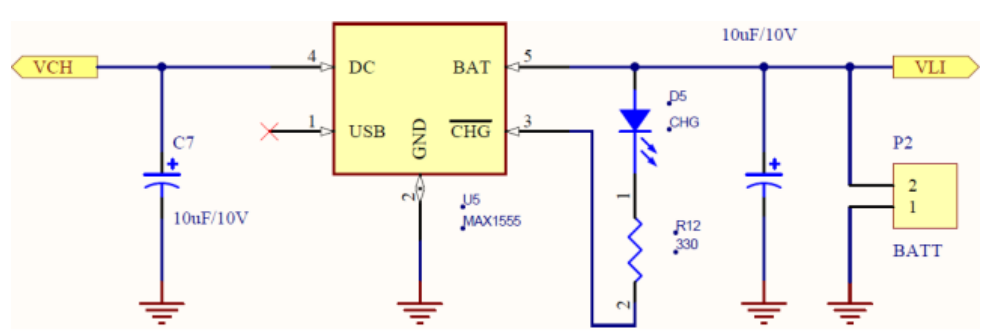

Figura 5.4. Esquemático del cargador de la batería de LiPo.

El mote se alimenta de una batería de polímero de litio (LiPo) de 3,7V y $2.000 \mathrm{mAh}$. El dispositivo integra en la PCB un cargador (Figura 5.4), basado en el circuito integrado MAX1555 de Maxim. Cuando la batería está cargada, la tensión de la misma es superior a 4V. Por ello, en el diagrama de bloques hay un convertidor DC/DC (\#1) de bajo consumo y bajo dropout (Figura 5.5), el cual regula la tensión de la batería a 2,5V. En concreto, se utiliza el LP38691DT-2.5 de National Semiconductor. La tensión anterior alimenta el micro-controlador, la radio y otros elementos del mote como la interfaz SDI-12 y los LEDs.

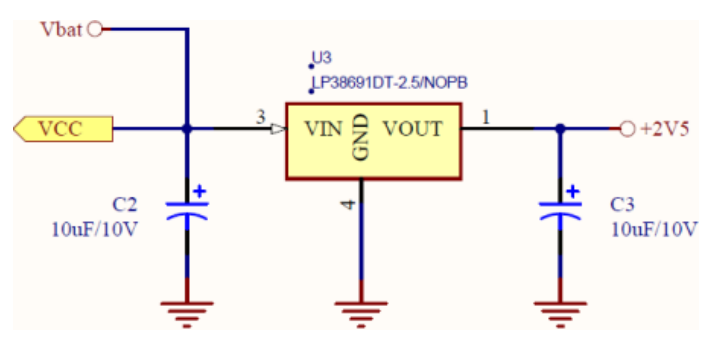

Figura 5.5. Esquemático del regulador de tensión de 2,5V.

Por otro lado, hay un segundo convertidor DC/DC (\#2) (ver Figura 5.6) que, a partir de la tensión de $2,5 \mathrm{~V}$, proporciona la salida de $12 \mathrm{~V}$ necesaria por los sensores externos. Éste está basado en el convertidor DC/DC de tipo elevador construido con el MAX761 de Maxim. Para un manejo eficiente de la energía, este convertidor es habilitado sólo durante el proceso de lectura de los sensores. Finalmente, el diagrama de bloques de la 
Figura 5.1b muestra que la batería está conectada con un ADC del micro-controlador con objeto de poder monitorizar el voltaje de la misma.

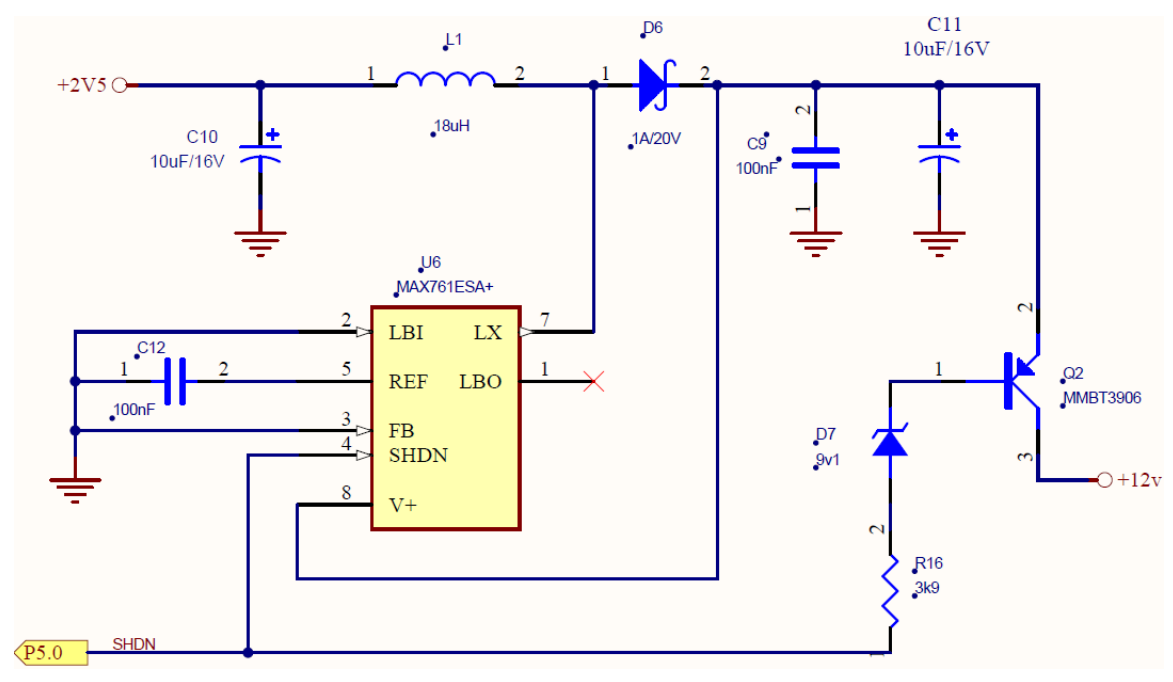

Figura 5.6. Esquemático del convertidor DC/DC que proporciona $12 \mathrm{~V}$ a los sensores.

La interfaz que proporciona el mote al usuario está constituida por 4 LEDs y un conector externo de 12 pines. Tres LEDs se usan para mostrar el estado del mote (enviando un mensaje, recibiendo un mensaje, etc.) y el cuarto es controlado por el cargador de la batería (Figura 5.4). El conector externo tiene la funcionalidad de reiniciar el mote, cargar la batería, programar y depurar el software, y conectar los sensores externos.

Una vez conocidas las dimensiones de la batería seleccionada y de la PCB diseñada y construida, y para que el GSM pueda utilizarse en cultivos reales, se diseñó una carcasa a medida con el software CAD SolidWorks. La Figura 5.7 muestra una vista del diseño realizado. Se puede observar que la carcasa está formada por dos piezas selladas mediante una junta tórica, y dos tornillos de $3 \mathrm{~mm}$ de paso. La antena está colocada con su junta tórica en la pieza superior. El conector de 12 pines IP67 está colocado en la pieza inferior. Además, en el interior del diseño se puede observar la PCB en color verde y la batería debajo de ésta.

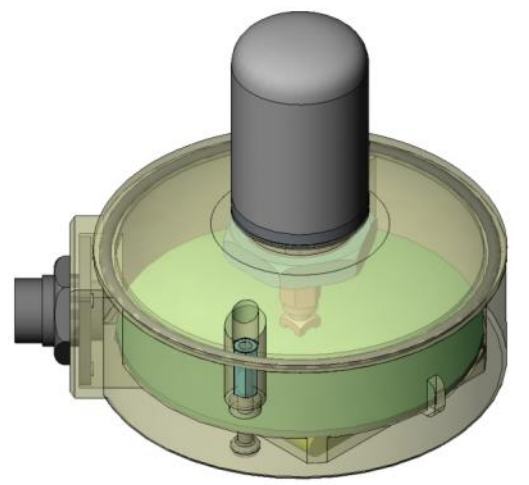

Figura 5.7. Modelo 3D de la carcasa diseñada para el GSM. 
El fichero de salida del software SolidWorks se envió al Centro Tecnológico del Metal, situado en el Polígono Industrial de Alcantarilla, en la Región de Murcia, con objeto de construir la carcasa (ver Figura 5.8) utilizando una impresora de inyección 3D. El material utilizado para fabricar la carcasa fue resina semitransparente. Se utilizó este material porque al ser semitransparente se podrían ver el estado de los LEDs de la PCB sin necesidad de incorporar una ventana en el diseño que redujese el grado de protección IP del conjunto.

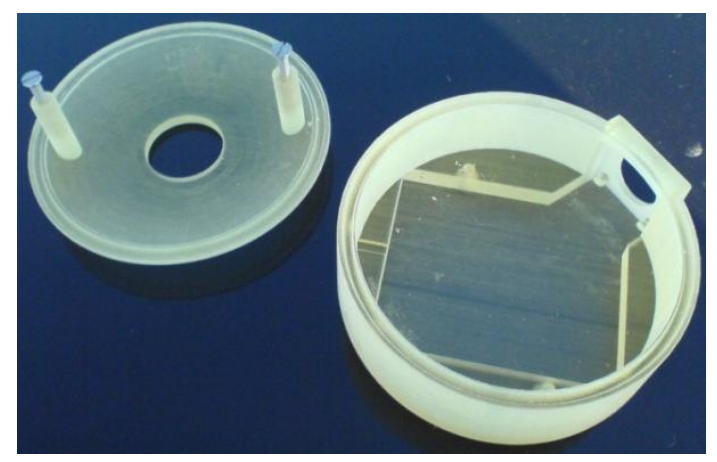

Figura 5.8. Piezas fabricadas en resina semitransparente usando una impresora 3D.

El conjunto (antena, conector externo, batería y PCB) se ha encerrado dentro de la carcasa, de $3 \mathrm{~mm}$ de sección, diseñada a medida para el GSM. La carcasa tiene un grado de protección IP67, al igual que el único conector de 12 pines que incluye. Cabe destacar que se usó un único conector externo para simplificar el diseño de la PCB. Mediante estas 12 conexiones, se puede cargar el firmware en el micro-controlador, reiniciar el programa del GSM, recargar la batería y conectar la instrumentación externa.

En la Figura 5.9a se ha representado la ubicación del mote en un cultivo hortícola. Como se puede ver en la Figura 5.9b, el nodo sensor está conectado a dos sensores HP2 de Stevens. Como se ha descrito anteriormente, los sensores HP2 se entierran a diferentes profundidades y permiten monitorizar los principales parámetros del suelo (temperatura, porcentaje volumétrico y conductividad eléctrica).

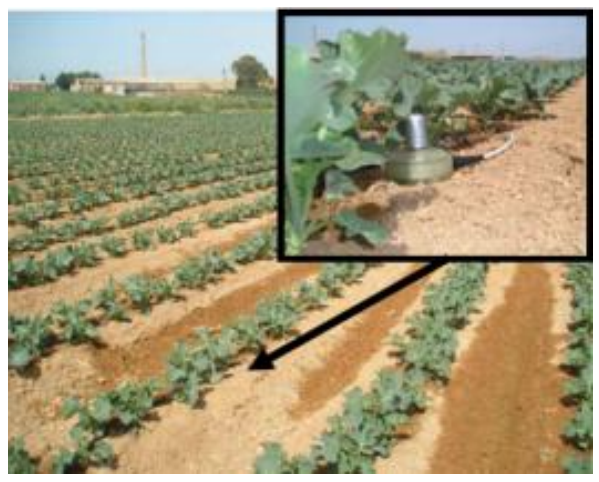

(a)

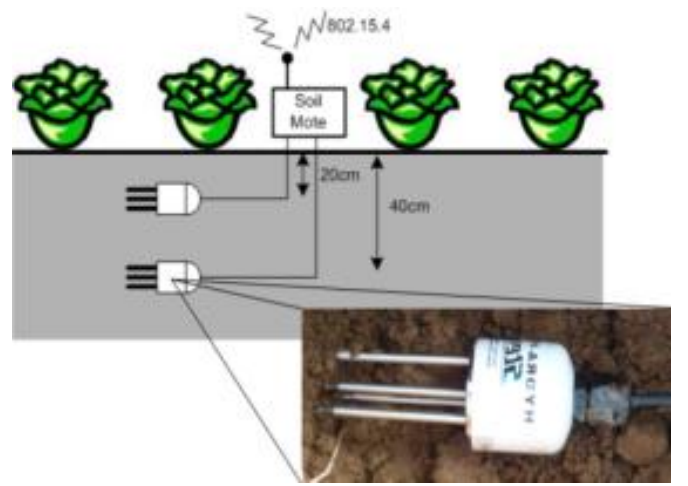

(b)

Figura 5.9. Vistas del GAIA Soil-Mote. (a) Ubicación del dispositivo en el cultivo. (b) Esquema de montaje de los sensores HP2. 


\subsubsection{Descripción del software.}

El GSM es la versión comercial del Soil-Mote descrito en el capítulo anterior. Por ello, el software del dispositivo se ha desarrollado también con componentes de la versión 2.0 del sistema operativo TinyOS [TinyOS] y el lenguaje de programación orientado a componentes asociado nesC [Gay, 2003]. El software se implementó partiendo de la versión realizada para el Soil-Mote.

El software desarrollado para el GSM tiene las funcionalidades principales de enviar la tensión de la batería cada hora, poder leer y enviar los datos adquiridos por medio de 1 hasta 10 sensores HP2, con una frecuencia configurable entre 30 minutos y aproximadamente 10 días; permitiendo, además, configurar remotamente la frecuencia de muestreo de estos sensores. Estas funcionalidades se resumen en la Figura 5.10, donde se muestra un diagrama con la vista estática de componentes nes $C$ del software desarrollado. Este diagrama se describió en el capítulo anterior. En ella, se muestra el componente de tipo módulo HP2C desarrollado, y los componentes instanciados del sistema operativo para conseguir esta funcionalidad.

La descripción detallada del código fuente nesC del componente de tipo módulo HP2C queda recogida en el Anexo III.

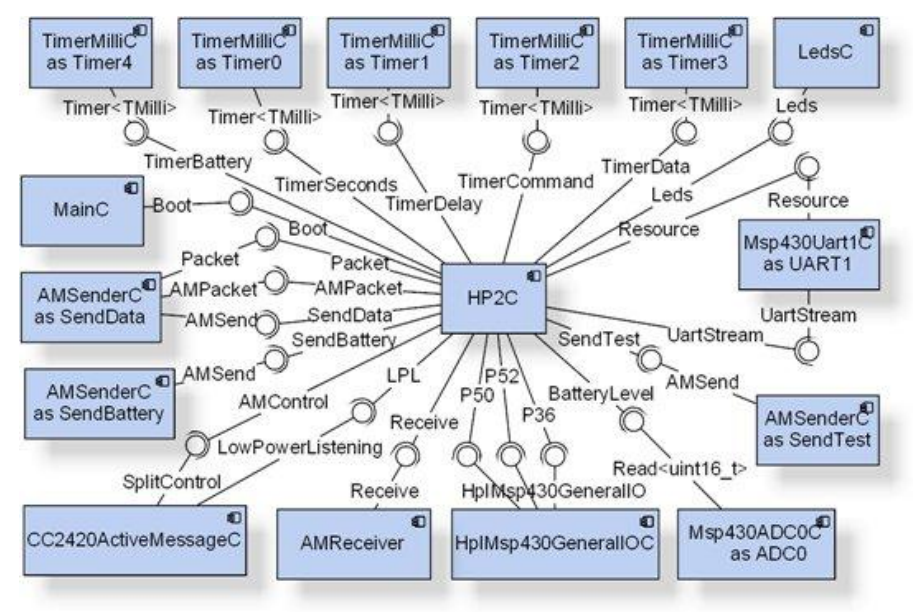

Figura 5.10. Vista estática de componentes nesC del GSM.

\subsection{Resultados experimentales.}

Con objeto de evaluar el correcto funcionamiento del mote GSM, se planteó una metodología basada en dos fases experimentales: pruebas en el laboratorio y pruebas sobre un cultivo real. El principal objetivo de la primera fase fue validar la arquitectura hardware propuesta y el software implementado. Inicialmente, se validó el hardware utilizando una versión del software que no usaba técnicas de bajo consumo. Posteriormente, cuando el 
dispositivo se validó correctamente, se revisó el software con objeto de incorporar los modos de bajo consumo. Así, fue posible conseguir que el dispositivos tuviese una autonomía necesaria para un ciclo hortícola (10 semanas). El objetivo de la segunda fase, que se llevó a cabo en un cultivo en condiciones reales, fue validar las condiciones funcionales del dispositivo desarrollado, como el alcance, la robustez y la flexibilidad.

\subsubsection{Experimentos de laboratorio.}

En la primera fase, se desplegó una red similar a la que se desplegaría en el cultivo real, pero con un número inferior de nodos (Figura 5.11). La red estaba formada por 4 nodos sensores GSM, que permitían monitorizar el estado del suelo de un cultivo. Adicionalmente, se añadió un Environmental-Mote para monitorizar los valores ambientales de temperatura y humedad del cultivo con la ayuda también de un sensor externo (Sensirion SHT71). Este nodo fue descrito en el capítulo anterior y su arquitectura difiere del GSM en el módulo interfaz. En este caso, la conexión con el sensor externo se hace a través de la interfaz $\mathrm{I}^{2} \mathrm{C}$. Como se puede ver en la Figura 5.11, los datos de los sensores son directamente transmitidos desde el nodo sensor al Gateway, el cual se encarga de transmitirlos a la Estación Base.

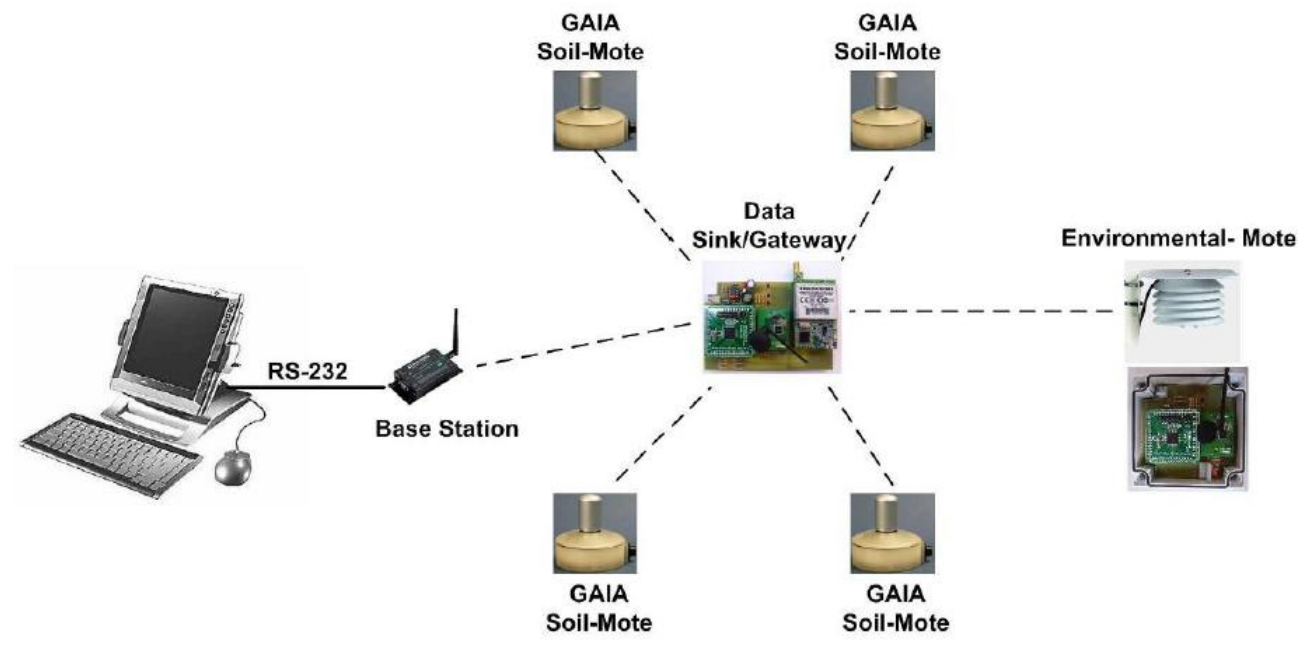

Figura 5.11. Arquitectura del sistema para llevar a cabo los experimentos de laboratorio.

Después de realizar la validación funcional del dispositivo desarrollado en la red mostrada en la Figura 5.11, los dispositivos se reprogramaron en modo de bajo consumo, por ser el modo más adecuado de operación para utilizar los dispositivos en un cultivo real. Antes de desplegar los dispositivos en un cultivo real, se llevó a cabo un estudio detallado del consumo del dispositivo con objeto de asegurar su autonomía durante un ciclo agronómico. Este estudio se realizó de la misma forma que el descrito para el Soil-Mote en el capítulo anterior. En este caso, se consideraron los cuatro siguientes estados funcionales: reposo o standy, radio activa esperando un mensaje o communication module wake-up, leyendo 
los sensores o sensor data acquisition y transmitiendo datos de batería o de los sensores (ver Figura 5.12). Para determinar el consumo promedio del GSM, se consideró el caso más desfavorable, es decir, realizar la lectura y transmisión de los datos de los dos sensores HP2 conectados con el GSM cada 30 minutos. Al final, se obtuvo una corriente promedio de $0,5034 \mathrm{~mA}$, con la que se puede estimar una autonomía de 165 días, teniendo en cuenta que la batería tiene una capacidad de $2.000 \mathrm{mAh}$. Cabe destacar que las intensidades del GSM en cada estado se han medido en la entrada, utilizando una tensión de $3 \mathrm{~V}$. De este modo, se explica que los 30mA del sensor HP2 en modo activo, con una tensión de alimentación de $12 \mathrm{~V}$, sean de $110 \mathrm{~mA}$ para una tensión de $3 \mathrm{~V}$. Además, en el estudio no se ha tenido en cuenta el estado de recepción de mensajes, al ser muy atípico en este tipo de redes.

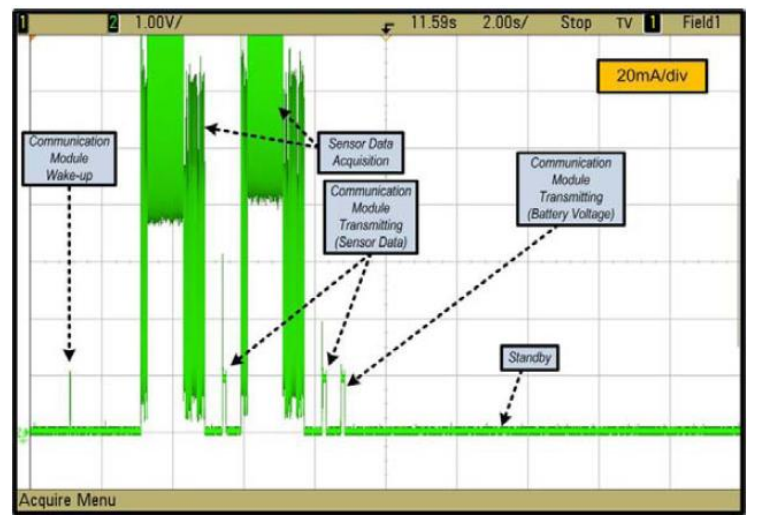

Figura 5.12. Diferentes estados de consumo del GSM: 'standby' $=0,25 \mathrm{~mA}$, 'communication module wake-up' $=20 \mathrm{~mA}$, 'acquisiton' $=110 \mathrm{~mA}$ y 'communication module transmitting' $=$ $25 \mathrm{~mA}$.

Para corroborar el resultado anterior, se realizó un estudio real en laboratorio (ver Figura 5.13) entre septiembre y diciembre de 2008, con el dispositivo realizando una lectura de los sensores cada 30 minutos. Tras el estudio, el valor final de la batería era de, aproximadamente, $3,8 \mathrm{~V}$; valor alejado de los $2,7 \mathrm{~V}$ que requiere la entrada del convertidor DC/DC principal para el correcto funcionamiento del sistema, y de los $3,4 \mathrm{~V}$ requeridos para recargar correctamente la batería.

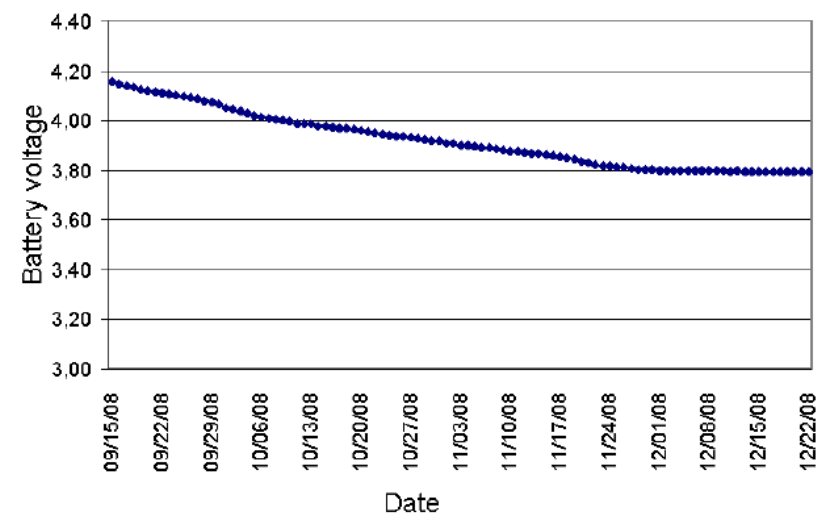

Figura 5.13. Tensión de la batería del GSM durante el estudio realizado en el laboratorio. 


\subsubsection{Resultados agrícolas.}

Después de realizar las pruebas de laboratorio, se inició la segunda fase del experimento realizando el despliegue mostrado en la Figura 5.14. Se han desplegado dos redes de sensores en dos parcelas de aproximadamente tha cada una de ellas. Las parcelas están alejadas de las oficinas de la explotación unos 5,2 y 8,7km, respectivamente. En cada una de dichas parcelas, se han desplegado 10 GAIA Soil-Motes, un Environmental-Mote y un Gateway. Éste último se ha conectado a una antena exterior omnidireccional, de $3 \mathrm{dBi}$ de ganancia, que se ha colocado en un poste de $5 \mathrm{~m}$ de altura. Con esto se asegura la visibilidad directa entre las antenas de los nodos sensores instalados a nivel de suelo y el Gateway. Del mismo modo, para garantizar la correcta comunicación entre el Gateway y las oficinas, se ha instalado una antena omnidireccional, de $15 \mathrm{dBi}$ de ganancia, en el tejado de las oficinas $(9 \mathrm{~m}$ de altura), y otra antena idéntica en cada Gateway. La comunicación entre la antena del tejado y la Estación Base se consigue inalámbricamente por medio de un repetidor. Por otro lado, con una tecnología similar al Gateway, también se ha desplegado un nodo inalámbrico (Water-Mote), para monitorizar la calidad del agua suministrada al cultivo con el sensor EC 250.

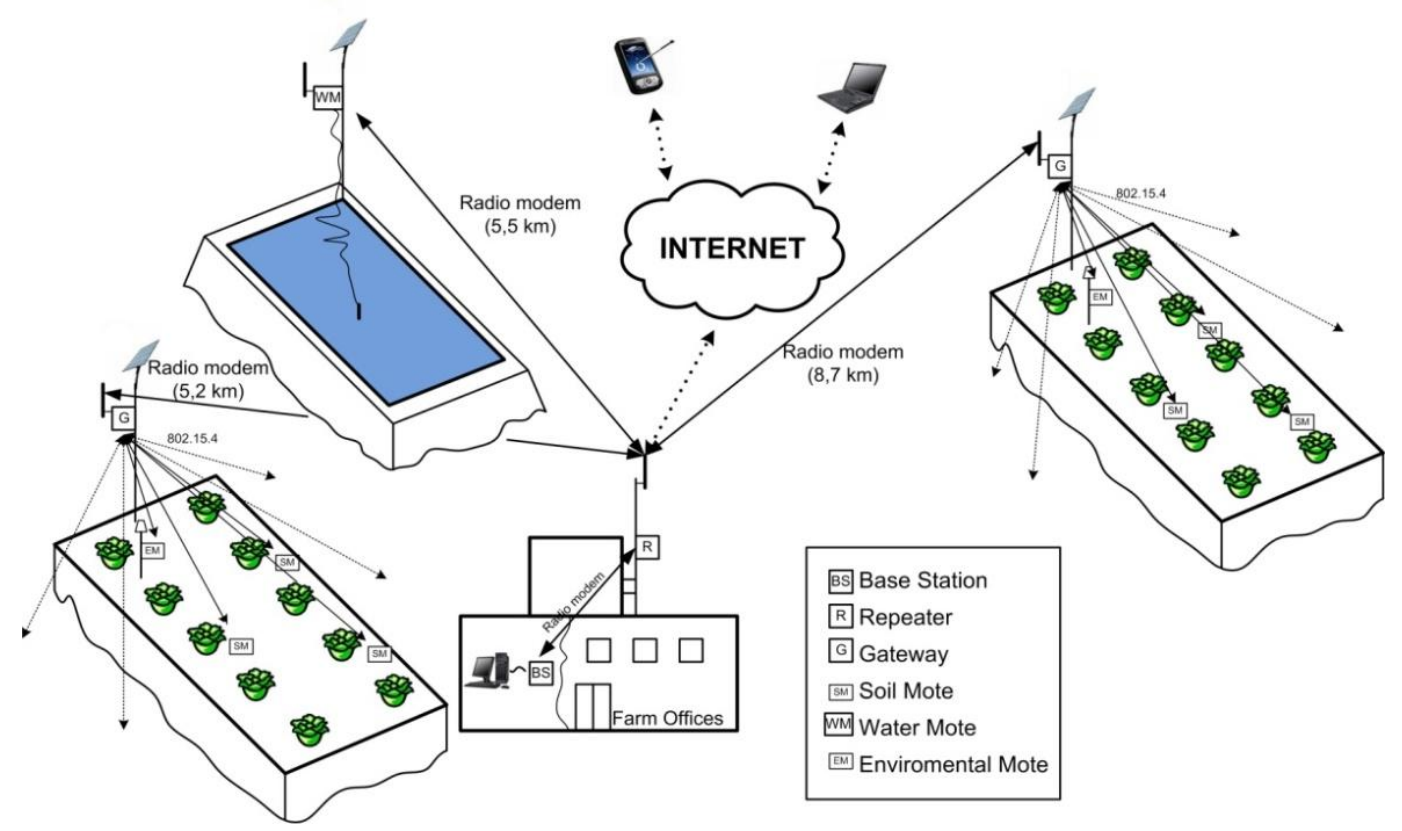

Figura 5.14. Descripción de la red desplegada para validar el GSM en un cultivo real.

Con objeto de visualizar y almacenar en una base de datos la información recibida de cada subred y del Water-Mote, se instaló en el servidor conectado con la Estación Base la aplicación de monitorización descrita en el capítulo anterior.

Las pruebas se llevaron a cabo en cultivos de Brócoli (Brassica oleracea L. var Marathon), de unas 4ha cada uno, localizados en el Campo de Cartagena (37\%44'26”N, 
1'13’38”W) en el sureste de España. Los cepellones se trasplantaron (con una densidad de 5 plantas $/ \mathrm{m}^{2}$ ) el 10 de noviembre de 2008 y la cosecha se recolectó 12 semanas después, en la segunda semana de febrero del 2009. Según datos facilitados por los expertos agronómicos, las características del suelo de los cultivos, a $40 \mathrm{~cm}$ de profundidad, fueron: textura clay loam, carbonatos totales 35,4 p.100, P(Olsen) 78,6ppm, K(Ac-NH4) 487ppm. Se instaló un sistema de riego por goteo entre cada dos hileras del cultivo, con emisores de $11 /$ h cada $20 \mathrm{~cm}$, y se aplicó fertilizante al cultivo utilizando fertirrigación. Los nodos se desplegaron en la segunda semana de noviembre, fecha desde la que los propietarios de la explotación agrícola comenzaron a monitorizar los cultivos mediante las WSNs. Los dos sensores HP2 de cada GSM se instalaron a 20 y $40 \mathrm{~cm}$ de profundidad, respectivamente. Durante este tiempo, la precipitación acumulada fue de $198 \mathrm{~mm}$, vientos moderadamente fuertes, de hasta $69 \mathrm{~km} / \mathrm{h}$, y temperaturas suaves (media de $11,5^{\circ} \mathrm{C}$ ). Los nodos funcionaron correctamente durante la fase de validación, llevada a cabo en los cultivos reales. Esto confirma que, bajo condiciones climáticas similares, la robustez del sistema está asegurada.

La Figura 5.15 muestra el porcentaje volumétrico de humedad en el suelo, registrado durante un periodo de 12 semanas por un GSM en unidades de fracción de agua por unidad de volumen ( $\mathrm{wfv} \mathrm{o} \mathrm{m}^{3} \mathrm{~m}^{-3}$ ). Esto es un porcentaje de agua en el suelo expresado de forma decimal. Así, por ejemplo, un contenido de agua de $0,2 \mathrm{wfv}$ indica que una muestra de suelo de 11 contiene $200 \mathrm{ml}$ de agua. Como se puede recordar del capítulo anterior, la saturación completa (todos los espacios porosos del suelo llenos de agua) se produce normalmente entre 0,5 y 0,6wfv y es muy dependiente del suelo.

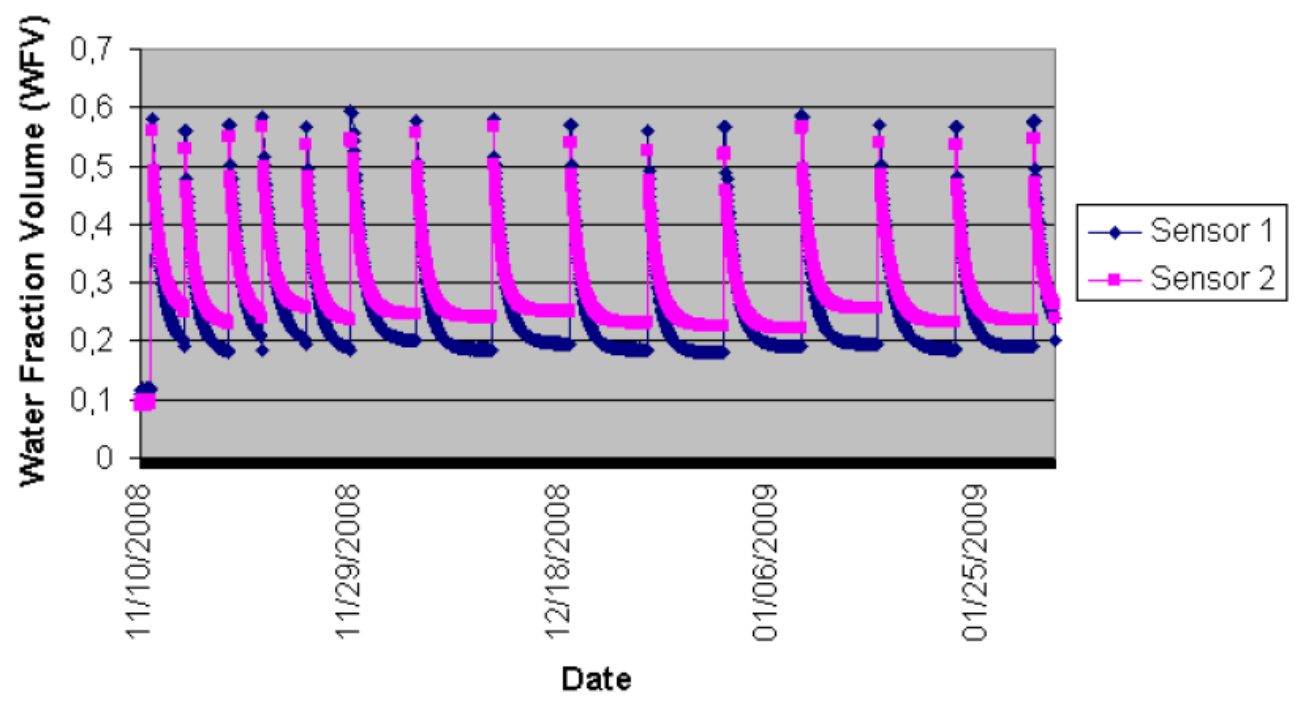

Figura 5.15. Porcentaje volumétrico del suelo monitorizado durante 12 semanas por un GSM.

Al igual que con el Soil-Mote, antes de enviar un mensaje del GSM al Gateway se configuraron distintos reintentos frente una no confirmación de la recepción por parte del dispositivo receptor (10 para datos de los sensores y 5 para el voltaje de la batería), todos 
con un retraso de 100ms; y se anotaban errores de comunicación si no se conseguía enviar un mensaje con los reintentos configurados. Estos valores se verificaron al final de los ensayos y no se observaron errores de este tipo. Además, se puso a prueba la autonomía de los dispositivos, que se han desarrollado en condiciones reales de operación. Las pruebas de campo también mostraron que los rangos especificados por los fabricantes eran correctos. Es importante señalar que, antes de desarrollar el GSM, se probaron diferentes antenas con un prototipo anterior. Por último, con las antenas seleccionadas (omnidireccionales, de 3dBi de ganancia) se lograron conseguir alcances entre los nodos sensores y el Gateway de $100 \mathrm{~m}$ bajo condiciones atmosféricas no favorables, como, por ejemplo, cuando hay una elevada humedad del ambiente $(>70 \%)$. Además, se comprobó que los enlaces de 8,7 y $5,2 \mathrm{~km}$ entre los Gateways y el repetidor en el tejado de oficina funcionaron correctamente.

Antes de implantar esta tecnología, la empresa monitorizaba sus cultivos mediante un método tradicional. En la actualidad, con la tecnología desarrollada, es posible conocer las variables de los cultivos en tiempo real, de manera que se pueden estimar necesidades hídricas de los cultivos sin la necesidad de desplazarse hasta los mismos. Es precisamente en este último aspecto en el que se quiere seguir trabajando a corto plazo.

\subsection{Conclusiones.}

Se ha diseñado y fabricado un nodo sensor con la robustez necesaria para su uso en aplicaciones hortícolas, y que permite medir los siguientes parámetros del suelo: temperatura, porcentaje volumétrico de humedad y conductividad eléctrica. El dispositivo proporciona una interfaz SDI-12 y el software del mismo se ha desarrollado utilizando la versión 2 del sistema operativo TinyOS. La versión actual del software desarrollado soporta la conexión de dos sensores HP2 de Stevens conectados a esta interfaz. Dicho software se ha realizado para que pueda ser fácilmente adaptado a cualquier otro tipo de sensor SDI12. Del mismo modo, el hardware del nodo desarrollado puede ser adaptado para soportar cualquier otro tipo de interfaces.

Como se comentó en la introducción, este dispositivo se ha diseñado e implementado con el propósito de conseguir experiencia en el diseño de una versión comercial de un nodo sensor.

Con el diseño y fabricación del GSM se ha obtenido experiencia en los pasos necesarios para fabricar un nodo sensor comercial, así como en el diseño de una PCB que integre un circuito de radio frecuencia con componentes SMD.

Llegado este punto, ya se tienen los conocimientos necesarios para llevar a cabo el diseño e implementación de un nodo sensor multifuncional. Es por tanto, un diseño más 
ambicioso que el del GSM y que podrá ser configurado tanto a nivel de hardware como de software para operar como un nodo sensor (Soil-Mote, Water-Mote y Environmental-Mote) o un elemento auxiliar de comunicaciones (Gateway).

Los trabajos desarrollados en este capítulo han sido publicados en una revista internacionales listadas en el ISI [López, 2009b], un congreso internacional [López, 2009d] y un congreso nacional [López, 2009e]. 


\section{Capítulo 6}

\section{GAIA 2: un Nodo Sensor Multifuncional para Agricultura de Precisión.}

\footnotetext{
$\Psi$ n el capitulo 5 se describió el diseño, implementación y validación del prototipo comercial de nodo sensor, que se desarrolló para obtener experiencia en el diseño de circuitos de radio frecuencia
} basados en componentes de montaje superficial. Esta tarea inicial, permitió llevar a cabo el estudio e implementación del prototipo comercial de nodo sensor multifuncional, que se planteó en las conclusiones del capitulo 4. Este dispositivo multifuncional, que se describe en el presente capitulo, se denominó GAIA 2. Es multifuncional porque es configurable tanto a nivel de hardware como de software. De este modo, mediante un único dispositivo, que requiere un único proceso de certificación, se puede configurar un Gateway, Water-Mote, Soil-Mote, etc.

En este capitulo se describe el hardware y las posibilidades software del GAIA 2, asi como las pruebas experimentales llevadas cabo con el prototipo comercial para validar el funcionamiento del mismo. 


\subsection{Introducción.}

En las zonas semiáridas del sureste español, como es el caso de la Región de Murcia, los recursos hídricos son un bien muy limitado y es necesario optimizar el uso de los mismos en los cultivos.

En el Capítulo 4 se presentó el diseño, implementación y validación de una arquitectura hardware basada en WSN con la que llevar a cabo la monitorización en tiempo real de cultivos hortícolas. La arquitectura está compuesta por varios prototipos de laboratorio (basados en módulos de inserción) de nodos sensores especializados y una serie de elementos auxiliares de comunicación. La principal conclusión de este capítulo fue que no era adecuado tener varios nodos sensores especializados por razones de costes y time-tomarket. Por ello, se planteó el diseño de un prototipo comercial de nodo multifuncional, que pudiese ser configurado tanto a nivel de hardware como de software, para operar como cualquiera de los nodos sensores especializados descritos en el Capítulo 4.

Antes de abordar el diseño e implementación del nodo multifuncional, se diseño el prototipo comercial de un nodo sensor menos complejo. Se trata del GAIA Soil-Mote, que fue descrito en el capítulo anterior. Antes del diseño del GAIA Soil-Mote, en el grupo de investigación no se tenía experiencia el diseño de circuitos de radio frecuencia con componentes de montaje superficial. Por ello, se planteó inicialmente el diseño de un dispositivo menos ambicioso, la versión comercial de Soil-Mote.

La experiencia conseguida con el diseño del prototipo comercial del Soil-Mote, permitió llevar a cabo el diseño, implementación y validación del prototipo comercial de nodo sensor multifuncional que se describe en este capítulo, y que recibe el nombre de GAIA 2. Éste se caracteriza por proporcionar diferentes interfaces de entrada y salida analógicas y digitales (0-2,5V , 4-20mA, SDI-12 e $\left.\mathrm{I}^{2} \mathrm{C}\right)$. La principal ventaja de este mote, es proporcionar un único dispositivo comercial para aplicaciones de agricultura de precisión, requiriendo de un único proceso de certificación (marcado CE). Además, sólo es necesario el diseño de una carcasa a medida, con la consecuente reducción de los gastos de desarrollo y producción.

Después de esta introducción, en la sección 2 se describen los requisitos funcionales con los que se diseñó el GAIA 2, así como el hardware y el software del dispositivo. En la sección 3 se presentan las pruebas experimentales realizadas sobre el dispositivo. Las primeras se llevaron a cabo en laboratorio, determinando la autonomía del GAIA 2 y verificando un correcto funcionamiento. Posteriormente, se validó el dispositivo con el despliegue realizado en un cultivo real. Finalmente, en la sección 4 se exponen las conclusiones y las observaciones finales. 


\subsection{Descripción del GAIA 2.}

A la hora de escoger los requisitos que debe satisfacer el diseño y construcción del nodo sensor es posible que sea necesario llegar a un compromiso entre requisitos contrapuestos. En el diseño del GAIA 2, al igual que en el diseño del GAIA Soil-Mote, se primaron fundamentalmente aquellas decisiones arquitectónicas que favorecieron la robustez del sistema y también la interconexión de distintos tipos de sensores. Una vez fijadas estas premisas, junto con la compatibilidad requerida para las comunicaciones, se consideraron todos aquellos requisitos adicionales que pudieron hacer más idóneo y funcional el nodo sensor. Los requisitos que se tuvieron en mente para el estudio e implementación del GAIA 2 se exponen en los siguientes párrafos.

El objetivo que se plantea es el desarrollo de un producto robusto y multifuncional (GAIA 2) para controlar cultivos que pueden estar distribuidos en diferentes parcelas, de una extensión media de 4ha, las cuales pueden estar separadas, entre sí y de las oficinas de la explotación agrícola, hasta unos $10 \mathrm{~km}$.

El dispositivo a desarrollar dispondrá de las siguientes interfaces de entrada y salida: 2 entradas/salidas (E/S) analógicas, $2 \mathrm{E} / \mathrm{S}$ digitales, 2 entradas analógicas con un rango máximo de 0 a 2,5V, 2 entradas analógicas de tipo 4-20mA, una interfaz $\mathrm{I}^{2} \mathrm{C}$ y otra de tipo SDI-12 [SDI12]. El dispositivo también dispondrá de una fuente de alimentación de 12V, basada en un convertidor DC/DC, destinado a proporcionar la alimentación a los diferentes sensores y/o actuadores conectados al mote.

Los elementos principales del mote serán el micro-controlador MSP430F1611 [MSP430F1611] y el transceptor de radio CC2420 [CC2420], ambos de Texas Instruments [TI], así como el módulo de radio de larga distancia XStream de Digi [Digi]. Los dos dispositivos de radio establecerán las comunicaciones en la banda ISM de 2,4GHz.

El dispositivo dispondrá de una PCB genérica, de manera que es posible comercializar series del motes configurables, tanto a nivel de hardware como de software, y poder competir en el mercado.

El diseño mecánico del dispositivo estará optimizado para ser utilizado en cultivos de tipo hortícola. Esto significa que el dispositivo estará instalado a nivel del suelo y, por sus dimensiones, no será necesario retirarlo cuando se realicen tareas con maquinaría agrícola pesada en el cultivo. Por ello, la carcasa del mote tendrá un grado de protección IP67 y la altura (incluyendo la antena) no superará los $20 \mathrm{~cm}$. Además, para evitar la substracción de los motes, éstos serán de un color discreto. Aunque el diseño esté optimizado para cultivos de tipo hortícola, también podrá ser utilizado en otro tipo diferentes de cultivos, como los frutícolas, viñedos, etc. 
Con respecto al conexionado con el exterior, la carcasa dispondrá de 4 conectores (uno de tipo prensaestopas y tres de conexión rápida), para poder conectar un panel solar, una batería externa y una antena externa, así como los correspondientes sensores y/o actuadores.

Como se describirá más adelante, una de las funcionalidades del mote será trabajar como nodo sensor con el módulo de radio CC2420. En este caso el dispositivo no dispondrá de panel solar y para garantizar la máxima autonomía posible, se comunicará punto-a-punto con el Gateway mediante tramas compatibles con el estándar IEEE 802.15.4 [IEEE, 2006]. La programación de los motes se llevará a cabo con el lenguaje de programación nesC [Gay, 2003], trabajando sobre el sistema operativo TinyOS [TinyOS]. Conviene recordar que este sistema operativo para sistemas empotrados se caracteriza por usar el algoritmo de acceso al medio B-MAC [Polastre, 2004], con objeto de optimizar los consumos. En el estándar IEEE 802.15.4, se define que el algoritmo de acceso al medio es MAC (Medium Access Control), de ahí que se hable de tramas compatibles con el estándar.

Estableciendo como restricción que la antena de corto alcance de un mote funcionando como Gateway estará a, no menos, de $5 \mathrm{~m}$ de altura por encima de los nodos desplegados. La cobertura mínima entre los nodos sensores y el Gateway será, al menos, de $100 \mathrm{~m}$.

El GAIA 2 usará una batería externa de NiMH y un panel solar cuando esté trabajando como Gateway. En el resto de casos, usará una batería de polímero de litio (LiPo), instalada en el interior del mote. Cuando se use esta última batería, se deberá garantizar una autonomía de al menos de 10 semanas, que es el tiempo habitual de duración de un ciclo agronómico hortícola.

En lo referido al software, la lectura periódica de los sensores, obtenida con dispositivos GAIA 2 configurados como nodos sensores (Soil-Mote y Environmental-Mote), será enviada al nodo sumidero a través del GAIA 2, configurado como Gateway. El periodo de lectura será configurable, desde el sumidero, con valore comprendidos desde 30 minutos hasta, aproximadamente, 10 días. El nodo sumidero también recibirá información sobre el estado de la batería cada hora. Además, para la fase de despliegue se establece que los motes recibirán mensajes de test del sumidero, que éstos deberán reenviar al anterior, con el fin de asegurar que existe una correcta comunicación entre ambos.

\subsubsection{Descripción del Hardware.}

En esta sección se describirá la arquitectura del prototipo desarrollado. En primer lugar, se presentará el hardware del GAIA 2, que incluye la PCB, la batería de polímero de litio, la antena y la carcasa diseñada para el dispositivo. Adicionalmente, como se explicará 
más adelante, puede incluir los siguientes elementos externos: batería, cargador de batería, antena y panel solar, además de los sensores y actuadores utilizados.

Como se ha comentado anteriormente, el GAIA 2 es un nodo sensor multifuncional, que es configurable tanto a nivel de hardware como de software. Esta configuración dual permite que el dispositivo funcione como nodo sensor de corto alcance, como Gateway y como nodo sensor de largo alcance. Si se recuerda la arquitectura de monitorización propuesta en el capítulo 4, un nodo sensor de corto alcance puede ser un Soil-Mote o un Environmental-Mote y un nodo de largo alcance es el Water-Mote. A continuación se describen estas tres funcionalidades.

En la Figura 6.1 se muestra el diagrama de bloques del GAIA 2. A la hora de diseñar el dispositivo, se consideraron las tres funcionalidades siguientes: High Density Mote (HDM), Long Distance Mote (LDM) y Gateway Mode Mote (GMM).

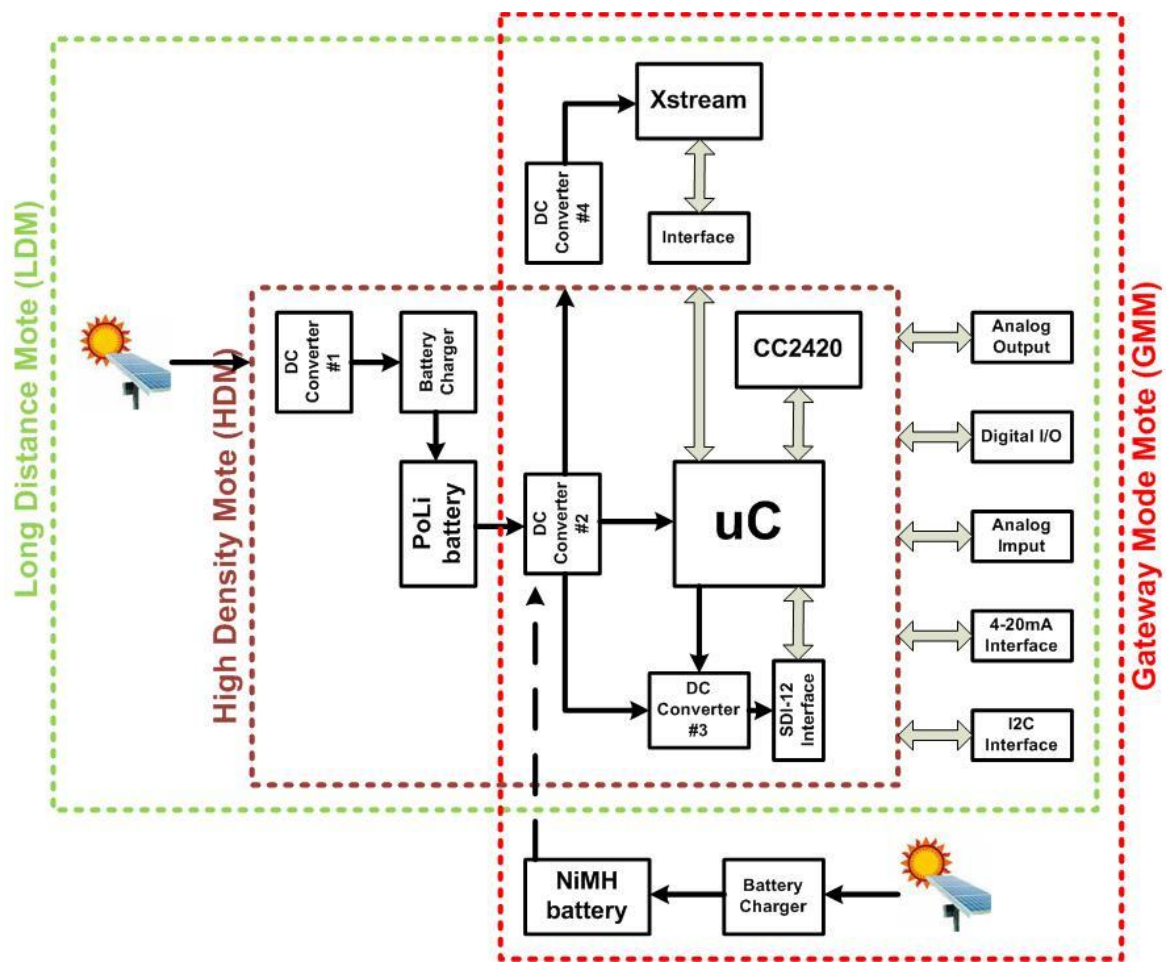

Figura 6.1. Diagrama de bloques del mote GAIA 2.

La funcionalidad HDM engloba a nodos sensores de corto alcance, como el GAIA Soil-Mote descrito en el capítulo anterior. En concreto, se utilizan los mismos elementos que en el GAIA Soil-Mote. Estos elementos eran los siguientes: batería de polímero de litio de $2.700 \mathrm{mAh}$ y 3,7V, micro-controlador MSP430F1611, módulo de radio CC2420, cargador de la batería de polímero de litio, 3 convertidores DC/DC y la electrónica de la interfaz SDI-12. La principal diferencia con el GAIA Soil-Mote es que el GAIA 2 proporciona más interfaces de entrada y salida analógicas y digitales. En concreto, además de la interfaz SDI12 que proporcionaba el GAIA Soil-Mote, dispone de 2 salidas analógicas (0-2,5V) que 
también pueden ser empleadas como entradas analógicas, $2 \mathrm{E} / \mathrm{S}$ digitales, 4 entradas analógicas (2 de 0-2,5V y 2 4-20mA) y de una interfaz $\mathrm{I}^{2} \mathrm{C}$.

La funcionalidad LDM engloba a nodos sensores de largo alcance como el $W$ aterMote. En este caso, en lugar del módulo de radio de corto alcance CC2420 se utiliza el radio-modem XStream, que trabaja con niveles de tensión de 5V. Por ello, es necesario incorporar otro convertidor DC/DC (DC Converter \#4) que proporciona los 5V de alimentación al dispositivo, así como de la electrónica de interfaz para las líneas de entrada y salida con el micro-controlador MSP430. Además, debido al uso de un módulo de radio de largo alcance que tiene un consumo elevado, es necesario usar una fuente de energía renovable para mantener cargada la batería. Esta fuente de energía será, generalmente, un panel solar (ver Figura 6.1). Finalmente, dependiendo de los sensores utilizados con el dispositivo, la batería de polímero de litio usada en la funcionalidad anterior puede que no proporcione la autonomía necesaria para el dispositivo. En este caso, se usará una batería externa compuesta de celdas de $\mathrm{NiMH}$, de 1,2V de tensión nominal y $6 \mathrm{Ah}$ de capacidad.

La última funcionalidad es la denominada como GMM. En este caso se trata de un dispositivo con la funcionalidad de Gateway, que puede estar dotado de interfaces de entrada y salida. Por ello, es necesario utilizar dos módulos de radio, el de corto y el de largo alcance. La otra principal diferencia con la funcionalidad LDM descrita anteriormente, recae en el sistema de alimentación. En este caso, se emplea una batería externa de NiMH de 4,8V y 6Ah. Además, debido a que los consumos requeridos por este dispositivo son mayores (el consumo medio es de unos $20 \mathrm{~mA}$ ), la batería se mantiene cargada mediante un panel solar, que se describirá en la sección dedicada a las pruebas experimentales.

Teniendo en cuenta las 3 funcionalidades descritas anteriormente, se diseñó una PCB genérica que garantiza la multifuncionalidad del GAIA 2. El dispositivo es multifuncional porque, en función de los componentes soldados durante el proceso de fabricación de la PCB, tendrá una u otra funcionalidad. Por tanto, el hardware del GAIA 2 se configura durante el proceso de fabricación. Esta característica, junto con el desarrollo de un software 'ad-hoc', culmina en un prototipo comercial de nodo sensor multifuncional.

Así, para fabricar una serie de Environmental-Motes, es necesario soldar lo siguientes elementos principales: DC Converter \#1, Battery Charger, DC Converter \#2, micro-controlador, CC2420 y I² C Interface. Además, será necesario realizar la soldadura de los componentes electrónicos pasivos y activos que requieren los elementos principales listados anteriormente. Ejemplo de estos son el oscilador y los dos condensadores de desacoplo, que requieren tanto el micro-controlador como el módulo de radio CC2420, así como las bobinas, condensadores, diodos y transistores que necesitan los convertidores DC/DC. 
El esquemático del GAIA 2 incluye todos los elementos presentes en el esquemático del GAIA Soil-Mote e incorpora elementos adicionales. Un módulo adicional es el de la interfaz 4-20mA (ver Figura 6.2). La interfaz convierte la entrada en lazo de corriente a un valor en tensión, que puede ser muestreado por el convertidor analógicodigital. Se han utilizado resistencias, con $120 \Omega$ de valor nominal y tolerancia del $1 \%$, para que el caso más desfavorable (resistencia de 121,2 $\Omega$ e intensidad de $20 \mathrm{~mA}$ ) no se supere el límite de 2,5V que puede muestrear el convertidor analógico-digital, cuando se utiliza la referencia interna de tensión de 2,5V.

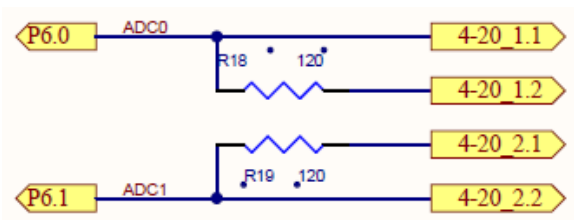

Figura 6.2. Interfaz 4-20mA del mote GAIA 2.

Otro módulo no presente en el GAIA Soil-Mote es la interfaz $\mathrm{I}^{2} \mathrm{C}$ (ver Figura 6.3). El micro-controlador implementa esta interfaz. Por ello, sólo hay que prolongar los pines de la interfaz del micro-controlador y colocar una resistencia de 'pull-up' en la línea de datos. Además, como los sensores que se pueden conectar con esta interfaz suelen tener un bajo consumo, se ha utilizado una línea digital del micro-controlador (P1.7) para proporcionar tensión de alimentación a estos durante el proceso de lectura.

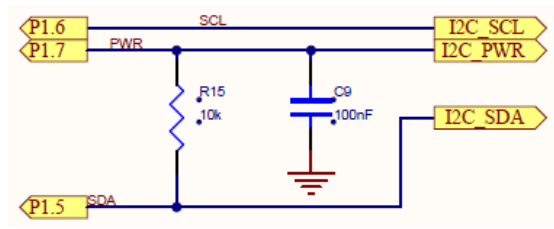

Figura 6.3. Interfaz $\mathrm{I}^{2} \mathrm{C}$ del mote GAIA 2.

El esquemático del GAIA 2 también incluye la interfaz con el radio modem XStream. En la Figura 6.4 se muestra uno de los tres adaptadores de nivel de tensión TXB0104 de Texas Instruments, que se utilizan para adaptar las líneas de 3,3V del microcontrolador con las de 5V del XStream.
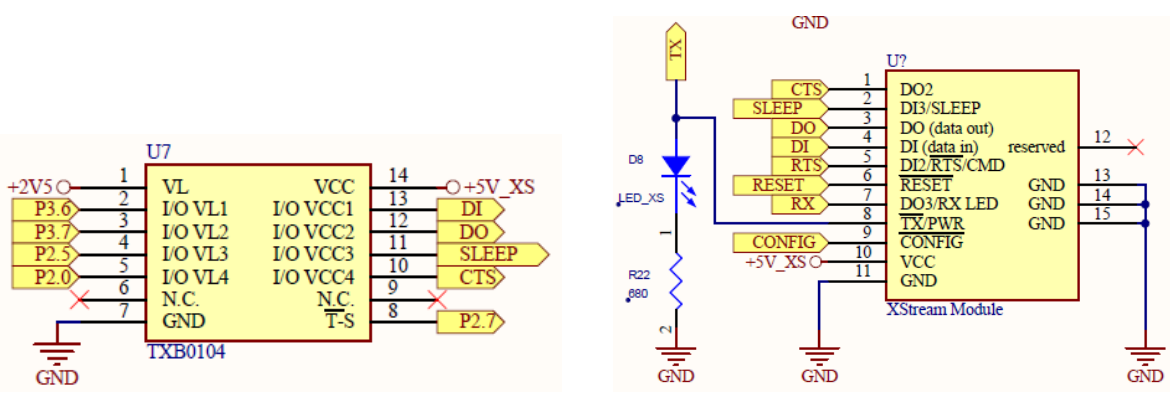

Figura 6.4. Interfaz entre el micro-controlador y el radio módem XStream. 
El esquemático del GAIA 2 también incluye un convertidor DC/DC, de tipo elevador, que proporciona los $5 \mathrm{~V}$ de alimentación que requiere el XStream. Este convertidor está diseñado con el MAX1555 de Maxim.

La Figura 6.5 muestra la PCB con componentes SMD y de 4 capas que se diseñó para soportar la multifuncionalidad del nodo sensor GAIA 2. Para su diseño, se utilizó, tal y como se hizo con el GAIA Soil-Mote, el layout del diseño de referencia del módulo de radio CC2420 proporcionado por el fabricante. En ella se han identificado los elementos principales, como la interfaz SDI-12, el módulo de radio de corto alcance, etc. Esta PCB dispone de todos los componentes soldados. Como se ha comentado anteriormente, dependiendo de la funcionalidad deseada, se soldarían sólo los componentes necesarios, optimizando los costes del producto final.

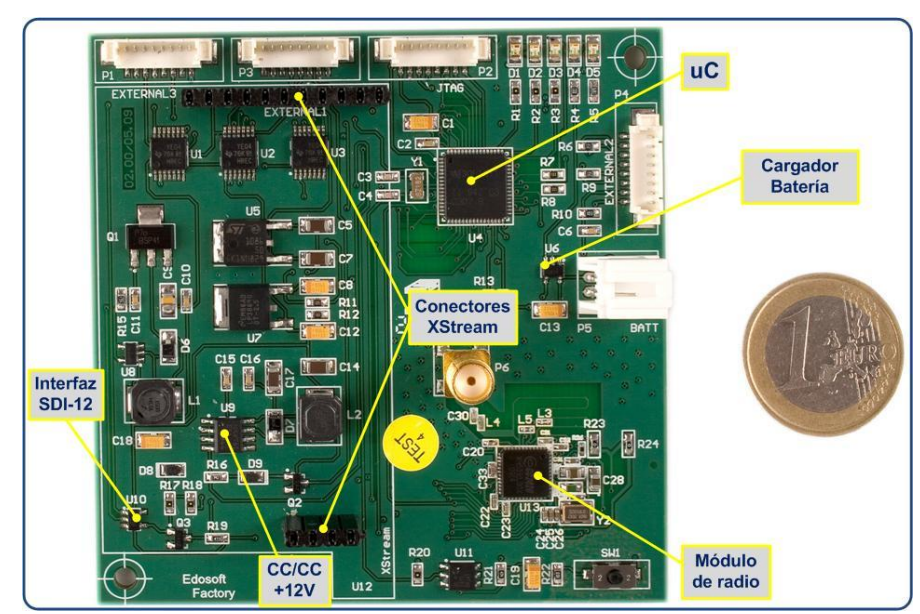

Figura 6.5. PCB con componentes SMD y de 4 capas diseñada para el GAIA 2.

Una vez diseñada y verificado el funcionamiento de la PCB, se diseñó la carcasa del mote utilizando el software CAD SolidWorks. La Figura 6.6 muestra que la carcasa incluye una funda para cubrir la antena omnidireccional de tipo rubber duck, de $3 \mathrm{dBi}$ de ganancia, conectada al CC2420. La carcasa dispone de una ventana lateral para visualizar el estado de los LEDS.

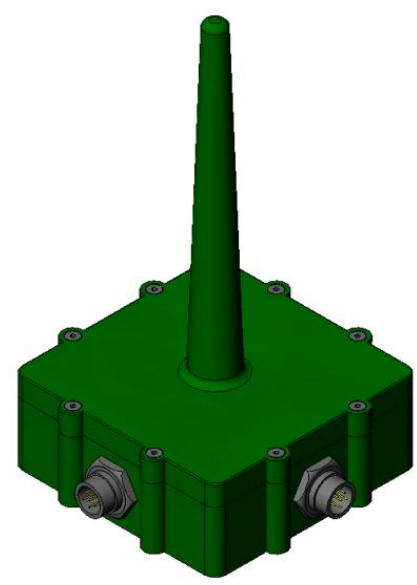

Figura 6.6. Modelo 3D de la carcasa diseñada para el GAIA 2 con el software CAD SolidWorks. 
La Figura 6.7 muestra varias vistas de la carcasa ya fabricada. La carcasa se fabricó con material ABSplus en el Servicio de Apoyo a la Investigación Tecnológica (SAIT) de la UPCT. Este centro dispone, entre otros, de una impresora 3D Dimension 1200es, que fue con la que se desarrollaron los prototipos de la carcasa. El precio de la carcasa es directamente proporcional a la cantidad de material utilizado y las horas de trabajo de la impresora. En concreto, cada unidad de esta carcasa tenía un coste de $150 €$.

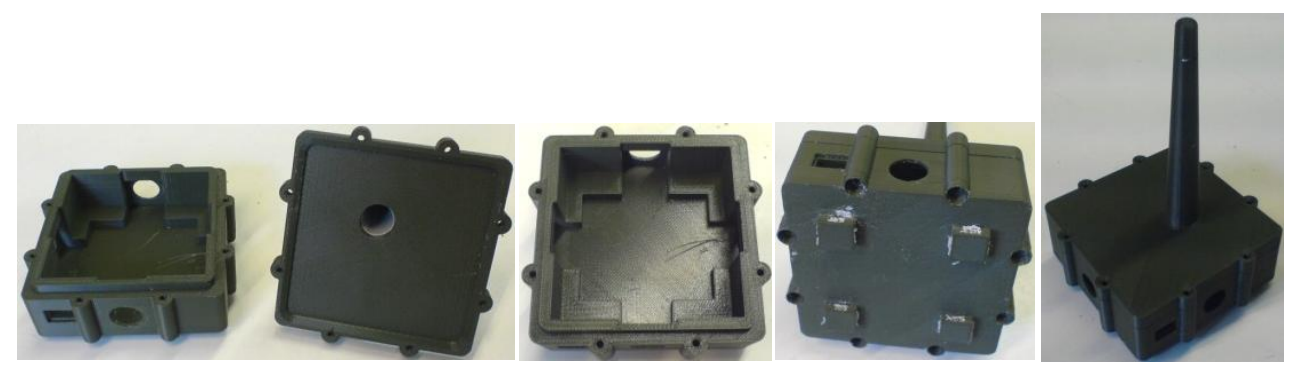

Figura 6.7. Diferentes vistas de la carcasa fabricada para el GAIA 2.

Para reducir los costes de la carcasa, se diseñó una segunda versión que utilizaba menos material y menos horas de trabajo de la impresora para su fabricación. Algunas de las modificaciones realizadas fueron las siguientes: utilizar sólo 4 tornillos situados en las esquinas, simplificar el alojamiento de la batería, y substituir la junta mecánica de la base con la tapa por una basada en junta tórica. Con estas modificaciones, se fabricó la carcasa mostrada en la Figura 6.8 con un coste de $100 €$. En dicha figura se pueden observar los conectores externos rápidos para la conexión de sensores, actuadores y alimentación externa, así como del conector de tipo prensaestopas utilizado en la conexión de una antena externa con en radio módem XStream. La batería de polímero de litio de 3,7V y $2.000 \mathrm{mAh}$ se encuentra alojada debajo de la PCB. Esta batería se usa en la funcionalidad HDM. El sistema de alimentación externo, que se utiliza para la funcionalidad GMM, se explicará en la sección de resultados experimentales. Finalmente, dependiendo de los sensores utilizados en la funcionalidad LDM, será adecuado utilizar la batería interna o la externa.

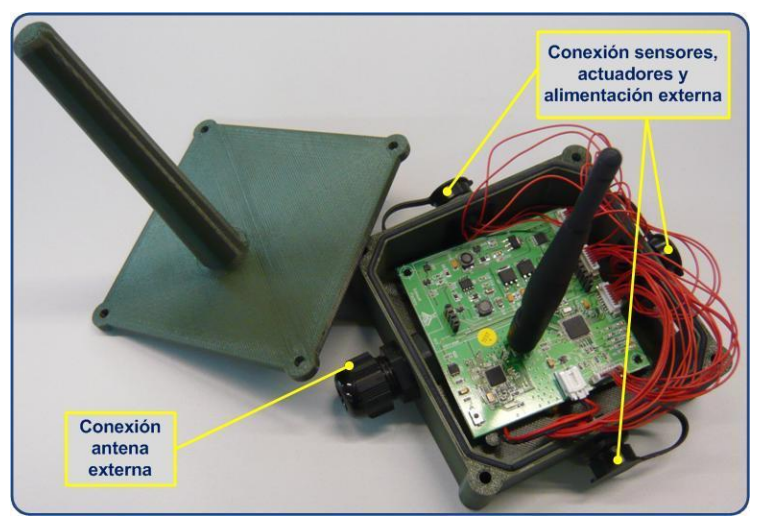

Figura 6.8. Fotografía de la segunda revisión de carcasa, fabricada con la PCB, ya instalada. 


\subsubsection{Descripción del software.}

El GAIA 2 tiene los mismos elementos principales (micro-controlador MSP430F1611 y módulo de radio CC2420) que el GAIA Soil-Mote. Por ello, el software del dispositivo también se ha desarrollado con la versión 2 del entorno para WSN TinyOS y el lenguaje de programación nesC asociado. Como se puede recordar, el conjunto TinyOS y nesC ofrecen un entorno de desarrollo de WSN con código abierto, orientado a componentes y dirigido por eventos. Una información más detallada sobre este tipo de programación se puede encontrar en [Gay, 2003].

En este sub-apartado se va a justificar que el GAIA 2 es dualmente configurable, es decir, a nivel de hardware y de software. Para ello, se describirá el hardware y el software necesario de dos ejemplos concretos: uno de funcionalidad HDM y otro de funcionalidad GMM, para obtener un GAIA Soil-Mote un Gateway, respectivamente.

Para comercializar un GAIA Soil-Mote a partir del GAIA 2, sería necesario soldar los módulos electrónicos, delimitados por la línea discontinua de la funcionalidad HDM, mostrados en la Figura 6.1. En concreto serían: los convertidores DC/DC \#1, \#2 y \#3, el cargador de la batería, la interfaz SDI-12, el micro-controlador y el módulo de radio CC2420. A nivel de software, serían necesarios los componentes mostrados en la Figura 6.9.

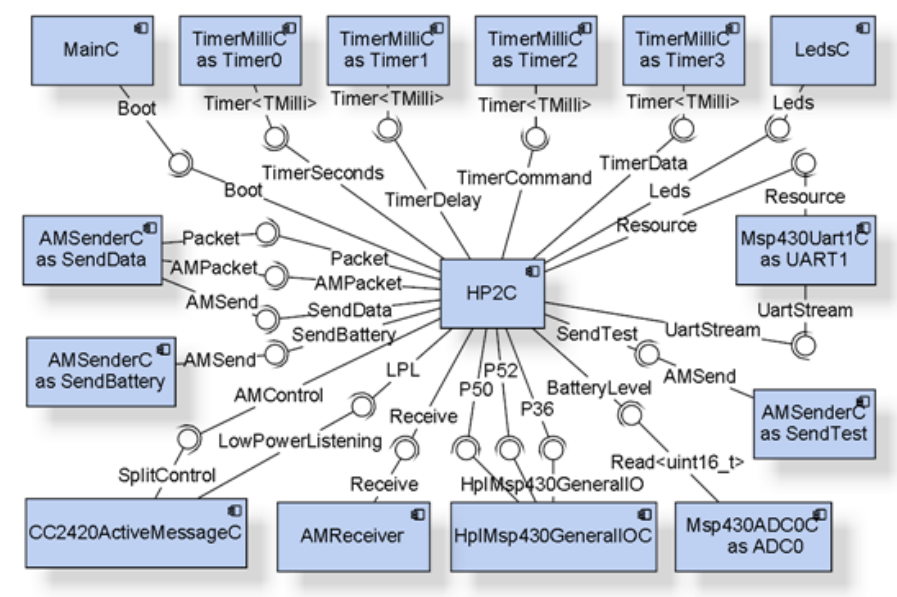

Figura 6.9. Diagrama de componentes software para la funcionalidad HDM.

En el componente de tipo módulo HP2C (ver Figura 6.9), es donde se ha escrito el código fuente nesC del para conseguir la funcionalidad de HDM propuesta. El resto de componentes, son primitivas del sistema operativo que se han instanciado.

El punto de entrada del programa lo proporciona el componente MainC por medio de la interfaz Boot. En concreto, TinyOS señala el evento boot() de la interfaz Boot cuando el sistema se ha inicializado correctamente. Es en esta parte del código donde el desarrollador inicializa la aplicación que está desarrollando. 
Para el funcionamiento del programa es necesario instanciar cuatro componentes de tipo TimerMilliC, que son necesarios para gestionar la temporización del programa, con el objetivo de poder saber cuándo hay que realizar una lectura (Timer0), mandar el comando para iniciar el proceso de lectura (Timer2), comenzar con el proceso de lectura de los datos (Timer3) y poder gestionar un retraso temporal desde que se inicia el proceso de lectura hasta que los datos están disponibles (Timer1).

En lo que ha comunicaciones se refiere, son necesarios tres componentes de tipo AMSenderC, uno de tipo CC2420ActiveMessageC y otro del tipo AMReceiver. Mediante la instancia SendData del componente $A M S$ ender $C$, se envían los datos recogidos de los sensores al nodo sumidero o estación base. Con la instancia SendBattery, se envía la información del nivel de batería cada hora. Finalmente, con la instancia SendTest, se hace el eco de un mensaje de prueba recibido desde el nodo sumidero, para facilitar la fase de despliegue de los nodos. Mediante el componente AMReceiver, se pueden recibir dos tipos de mensajes: mensajes de prueba y para cambiar la temporización de lectura de los sensores. El último componente, el CC2420ActiveMessageC, permite iniciar el módulo de radio y gestionar el periodo de encendido del mismo. De esta manera, se pueden optimizar los gastos energéticos debidos al transceptor de radio. TinyOS envía y recibe tramas compatibles con el estándar IEEE 802.15.4, usando para ello los componentes mencionados anteriormente. Las tramas son compatibles con el estándar porque, para optimizar el consumo, TinyOS usa el protocolo de acceso al medio B-MAC, y no MAC, que es el que está definido en el estándar IEEE 802.15.4.

El componente Msp430Uart1C permite gestionar todas las comunicaciones con el sensor Hydra Probe II. Este componente proporciona el acceso a una de las dos UARTs que tiene el micro-controlador.

Con el componente LedsC es posible controlar el encendido y apagado de los 3 LEDs que incluye el mote. Los LEDs se utilizan para indicar el estado en que se encuentra el programa, así como indicar que se ha producido un error.

El componente Msp430ADCOC permite muestrear el ADC0 del micro-controlador al que está conectada la batería, a través de un divisor de tensión resistivo. El intervalo de muestreo del ADC0 está configurado entre 0 y 2,5V, y la tensión de la batería, cuando está totalmente cargada, es de unos 4,2V. Por ello, es necesario el divisor de tensión.

Finalmente, es necesario un componente para gestionar una serie de pines del micro-controlador que se utilizan en la interfaz SDI-12. La línea P5.0 se utiliza para habilitar el convertidor DC/DC que proporciona los $12 \mathrm{~V}$ a los sensores durante el proceso de lectura. P5.2 es necesaria para controlar la lectura escritura en la interfaz SDI-12. El pin P3.6 se utiliza para habilitar los sensores Hydra Probe II, mediante el envío de un 'break'. 
A continuación, se describirá un ejemplo de la funcionalidad GMM (Gateway). En este caso, la PCB diseñada debería tener soldados los siguientes elementos: Convertidores DC/DC \#2 y \#4, MSP430, CC2420 y el XStream, así como la interfaz necesaria de acondicionamiento entre éste y el micro-controlador. Además, en este modo, para garantizar la autonomía necesaria por el dispositivo, son necesarios los siguientes elementos externos: batería externa de NiMH, cargador de batería y panel solar. Estos elementos se describirán en la sección de resultados experimentales. En relación con el software, la Figura 6.10 muestra los componentes necesarios para configurar un Gateway usando un dispositivo GAIA 2.

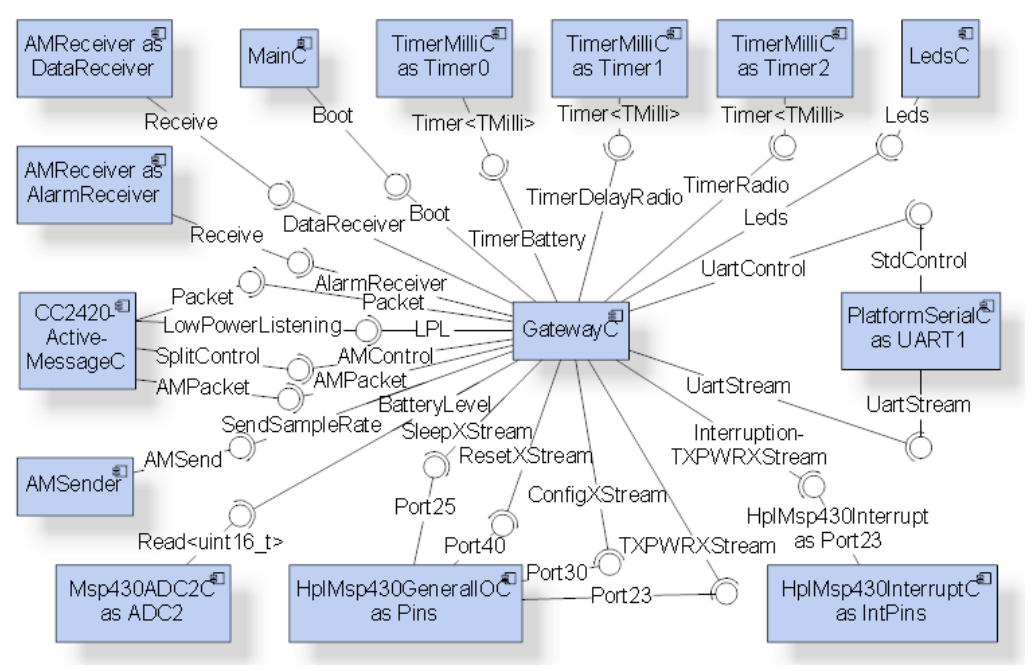

Figura 6.10. Diagrama de componentes software para la funcionalidad GMM.

En componente de tipo módulo GatewayC, es donde se ha escrito el código nesC de la aplicación. El resto son componentes instanciados de TinyOS.

Las comunicaciones de corto alcance (CC2420) se gestionan usando 4 componentes. Las instancias DataReceiver y AlarmReceiver se utilizan para recibir mensajes con datos de los sensores y del estado de la batería de los nodos sensores. El componente AMSender es utilizado para enviar los mensajes de cambio del tiempo de lectura de los sensores y de test a los motes remotos.

Las comunicaciones de largo alcance (XStream) se gestionan con la UART1 (componente PlatformSerialC) del MSP430 y una serie de pines del micro (componentes Pins y IntPins). P2.5 (sleep) se utiliza para que el XStream pase de modo de bajo consumo a modo activo y viceversa. P4.0 permite reiniciar el radio módem. P3.0 establece un valor fijo en el pin 'config' del XStream para que funcione correctamente. Finalmente, la línea P2.3 recibe interrupciones del XStream cuando se ha enviado correctamente un mensaje.

La instancia Timer0 del componente TimerMilliC es un temporizador periódico de una hora. Se utiliza para muestrear el valor de la batería (componente Msp430ADCOC). 


\subsection{Resultados experimentales.}

Para evaluar el correcto funcionamiento del GAIA 2, se planteó una metodología basada en dos fases experimentales: pruebas en el laboratorio y en un cultivo real. El principal objetivo de la primera fase, fue validar la arquitectura hardware propuesta y el software implementado. El objetivo de la segunda fase, que se llevó a cabo en un cultivo real, fue validar las condiciones funcionales del dispositivo desarrollado, como el alcance y la robustez.

\subsubsection{Experimentos de laboratorio.}

Sobre el mote GAIA 2, se realizaron en laboratorio una serie de pruebas para verificar la correcta funcionalidad y autonomía del dispositivo. En primer lugar, se validó la correcta funcionalidad de todas la interfaces de entrada y salida. Posteriormente, se realizaron pruebas con la radio de corto alcance, consiguiéndose alcances ligeramente superiores a $100 \mathrm{~m}$, usando antenas omnidireccionales de tipo rubber duck, de 3dBi de ganancia. También se realizaron pruebas de alcance con los XStream, consiguiendo una correcta comunicación entre dos GAIA 2 separados $10 \mathrm{~km}$. En esta prueba se utilizaron antenas externas omnidireccionales, de $15 \mathrm{dBi}$ de ganancia.

Para validar el correcto funcionamiento de un conjunto de GAIA operando al mismo tiempo, se realizo el despliegue en laboratorio de una red similar a la que se desplegaría en el cultivo real, pero con un número inferior de nodos (Figura 6.11).

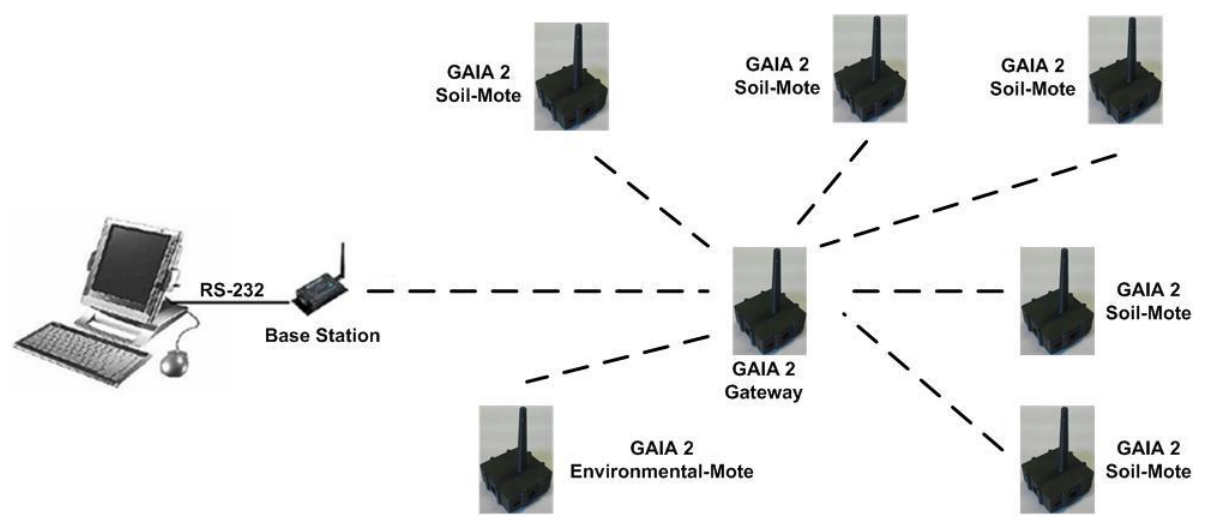

Figura 6.11. Red desplegada en el laboratorio para validar el correcto funcionamiento del GAIA 2.

La red estaba formada por 5 GAIA 2, configurados como Soil-Motes, para simular la monitorización del estado del suelo de un cultivo. Cada uno de los 5 nodos sensores tenía conectados 2 sensores Hydra Probe II. Además, se añadió un GAIA 2, configurado como Environmental-Mote, para monitorizar los valores ambientales de temperatura y humedad del 
cultivo con la ayuda también de un sensor externo (SHT71 de Sensirion). Este nodo fue descrito en el capítulo 4. Como se puede ver en la Figura 6.11, los datos de los sensores son directamente transmitidos desde los nodos sensores al GAIA 2 configurado como Gateway, el cual se encarga de transmitirlos al sumidero (Base Station).

Antes de desplegar los dispositivos en un cultivo real, se llevó a cabo un estudio detallado del consumo del dispositivo, con objeto de determinar la autonomía de un GAIA 2 con una configuración determinada. En este caso, se ha considerado un GAIA 2 configurado como Environmental-Mote. A nivel de hardware, un Environmental-Mote es un GAIA 2 con la funcionalidad HDM. En concreto, es necesaria una interfaz de tipo $\mathrm{I}^{2} \mathrm{C}$ y no son necesarios el convertidor DC/DC \#3 y la electrónica del la interfaz SDI-12.

La Figura 6.12 muestra la demanda de corriente del GAIA 2, con la funcionalidad de Environmental-Mote, en los diferentes estados. En este caso se consideraron los cuatro siguientes estados funcionales: reposo (standy), radio activa esperando un mensaje (communication module wake-up), leyendo el sensor (sensor data acquisition) y transmitiendo datos (communication module transmitting), este último utilizado para indicar que se transmiten datos tanto de la batería como del sensor. Para determinar el consumo promedio de este GAIA 2, se consideró el caso más desfavorable, es decir, realizar la lectura y transmisión de los datos del sensor SHT71 cada 30 minutos. Al final, realizando el mismo proceso que el descrito para el Soil-Mote en el capítulo 4, se obtuvo una corriente promedio de 0,093mA. A partir de este valor, y teniendo en cuenta la capacidad de la batería de $2.000 \mathrm{mAh}$, se puede estimar una autonomía de 896 días. Cabe destacar que las intensidades en cada estado se midieron en la entrada, utilizando una tensión de alimentación de 3V. Además, en el estudio no se ha tenido en cuenta el estado de recibir un mensaje, o communication module receiving, al ser muy atípico en este tipo de redes.

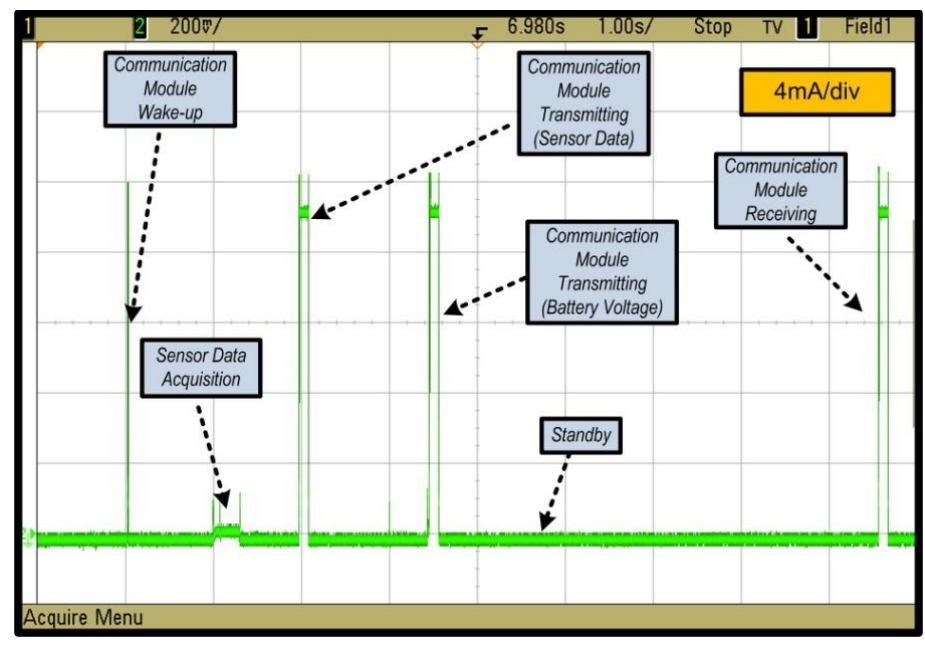

Figura 6.12. Diferentes estados de consumo del GAIA configurado como Environmental-Mote: 'standby' = 0,03 $\mathrm{mA}$, 'receiving' $=22 \mathrm{~mA}$, 'acquisiton' $=0,5 \mathrm{~mA}$ y 'communication module transmitting' $=23 \mathrm{~mA}$. 
Para corroborar el resultado anterior, se realizó un estudio real en laboratorio (Figura 6.13) de una duración de 75 días, con el dispositivo realizando una lectura del sensor cada 30 minutos. El valor inicial de la batería era de, aproximadamente, 4,14V. Tras el estudio, el valor final de la batería era de, aproximadamente, 4,08V, alejado del límite de descarga de la batería de $3,4 \mathrm{~V}$.

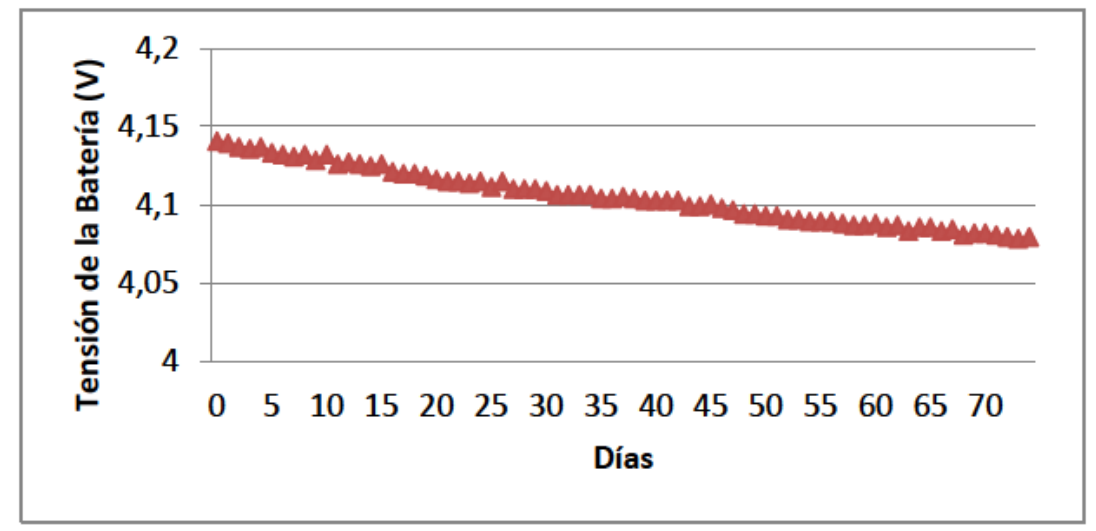

Figura 6.13. Tensión de la batería del GSM durante el estudio realizado en el laboratorio.

\subsubsection{Resultados agrícolas.}

Después de realizar las pruebas de laboratorio, se inició la segunda fase del experimento realizando el despliegue mostrado en la Figura 6.14. El despliegue se realizó en una zona de $375 \mathrm{~m}^{2}$ de una parcela de la Estación Experimental Agroalimentaria Tomás Ferro [ESEA]. En la parcela se plantaron melones, y se utilizó la WSN basada en el GAIA 2 para monitorizar el estado del cultivo durante todo el ciclo agrícola. Como se puede observar en la figura, se desplegaron 9 GAIA 2, teniendo uno de ellos la configuración de Gateway y el resto la de Soil-Mote. Cada uno de estos últimos tenían conectados 2 sensores Hydra Probe II, situados en un radio de $1 \mathrm{~m}$ de distancia con el mote. Todos los sensores se enterraron a $10 \mathrm{~cm}$ de profundidad.

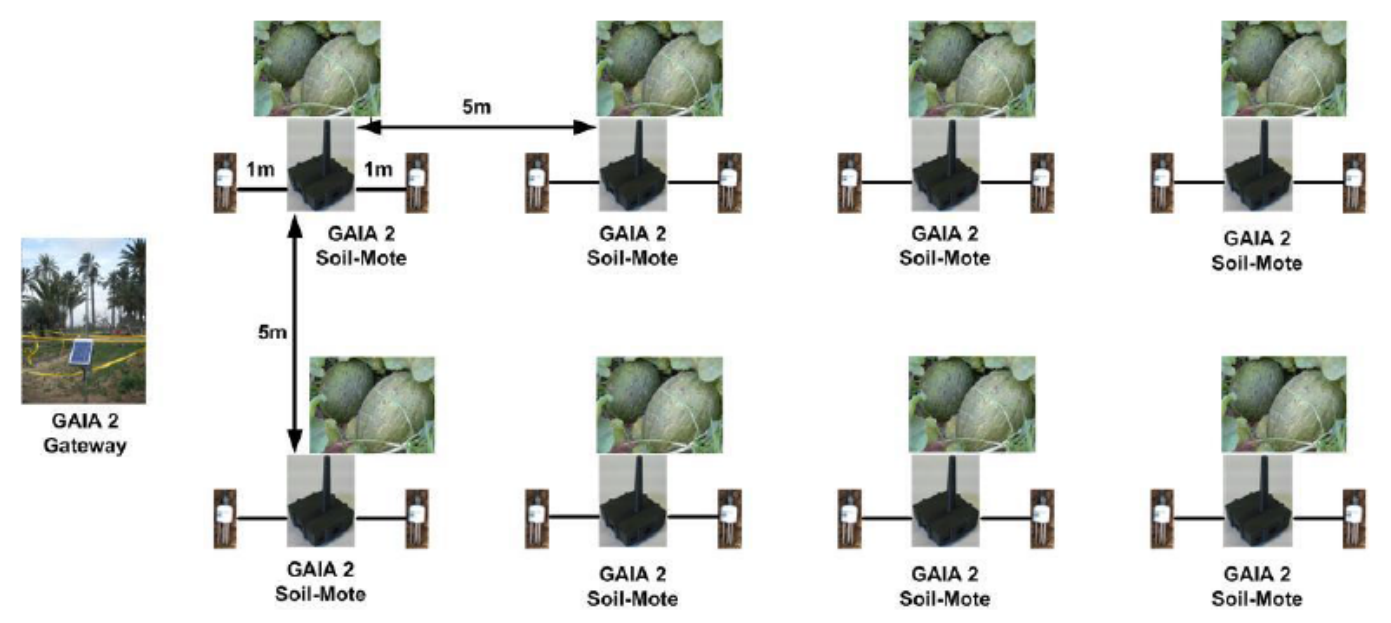

Figura 6.14. Despliegue realizado para validar el funcionamiento del GAIA 2 en un cultivo real. 
Los GAIA 2 configurados como Soil-Motes se comunicaban con el GAIA 2 configurado como Gateway, que hacía llegar los mensajes con la información de la batería, y de los sensores procedentes de los nodos sensores hasta el sumidero. Para ello, fue necesario equipar el Gateway (ver Figura 6.15) con una antena omnidireccional exterior, de $8 \mathrm{dBi}$ de ganancia (situada en el extremo superior del mástil de $3 \mathrm{~m}$ ), 4 celdas de NiMH tipo C (1,2V y 5Ah), un panel solar modelo SX310J (10W y 16,8V), comercializado por BP (British Petroleum) solar, y un cargador de baterías de NiMH, basado en el circuito integrado L6902D. Tanto el Gateway como los 8 nodos desplegados en el cultivo, disponían de una antena omnidireccional, de 3dBi de ganancia, para garantizar el funcionamiento de la red. Del mismo modo, para garantizar la correcta comunicación entre el Gateway y las oficinas se instaló una antena omnidireccional, de $15 \mathrm{dBi}$ de ganancia, en la fachada de las oficinas (8m de altura)

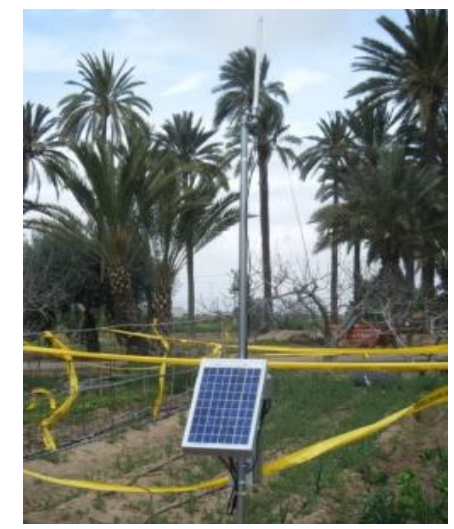

Figura 6.15. Vista del Gateway que se instaló en la parcela.

En la Figura 6.16 se muestra una fotografía tomada en el proceso de instalación de un nodo sensor en el cultivo. Como se puede observar, se utilizaron carcasas diferentes a la diseñada para el GAIA 2. Esta carcasa estaba basada en una caja comercial con grado de protección IP67, y un tubo para proteger la antena del mote. Fue necesario utilizar estas cajas porque, en el inicio del experimento, sólo se disponían de 3 carcasas diseñadas para el GAIA 2 (Figura 6.8).

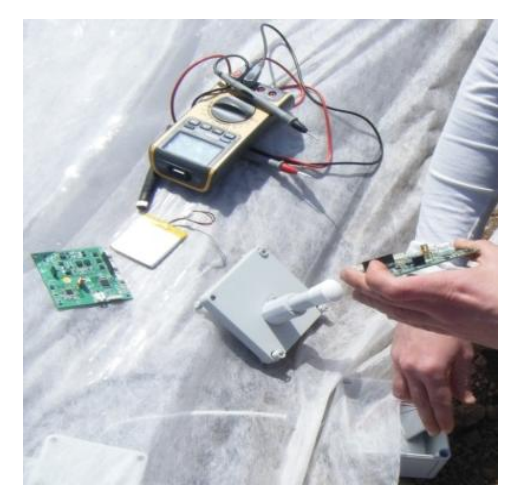

Figura 6.16. Fotografía del proceso de instalación de un nodo sensor. 
La infraestructura descrita anteriormente se utilizó para monitorizar el estado del cultivo desde que se plantaron las semillas (marzo 2010), hasta que se recolectó la cosecha (mayo 2010). En concreto, se monitorizó la temperatura, el porcentaje volumétrico y la conductividad eléctrica del suelo, con cada uno de los 16 sensores Hydra Probe II desplegados en el cultivo. Estos parámetros se monitorizaron cada 30 minutos.

En el interior de las oficinas se instaló el sumidero de la red de sensores, conectado por RS-232 con el servidor de monitorización. Tanto el servidor como el sumidero estaban alimentados de la red eléctrica. Además, ambos tenían conectado un SAI (Servicio de Alimentación ininterrumpida) para garantizar el correcto funcionamiento del sistema ante pequeños fallos en la red eléctrica.

Para comunicarse con la red de sensores, se desarrolló un software de monitorización (ver Figura 6.17) con LabView y la API de Google Maps. La interfaz gráfica tiene 3 vistas diferentes. La primera, permite ver gráficamente los datos recibidos de la WSN. La segunda, muestra el despliegue realizado sobre una vista de Google Maps, así como muestra los últimos datos recibidos por cada nodo sensor. La última vista se utiliza a modo de depuración. En esta vista se muestran los paquetes recibidos y los posibles errores en los paquetes recibidos, así como en el procesamiento de los mismos. Los datos recibidos se almacenan en un archivo con columnas para identificar los siguientes campos: subred, nodo, tipo de mensaje, sensor, temperatura, porcentaje volumétrico, conductividad eléctrica y tensión de la batería. En función del tipo de mensaje se completan determinadas columnas de la tabla. Así, por ejemplo, cuando llega una mensaje de batería, se almacena la subred y el nodo que envían el mensaje, el tipo de mensaje con el valor 20 y el valor de la batería recibido. El archivo generado se puedo abrir y utilizar con Excel, aunque, en este caso, se ha utilizado MatLab para representar gráficamente los datos monitorizados.

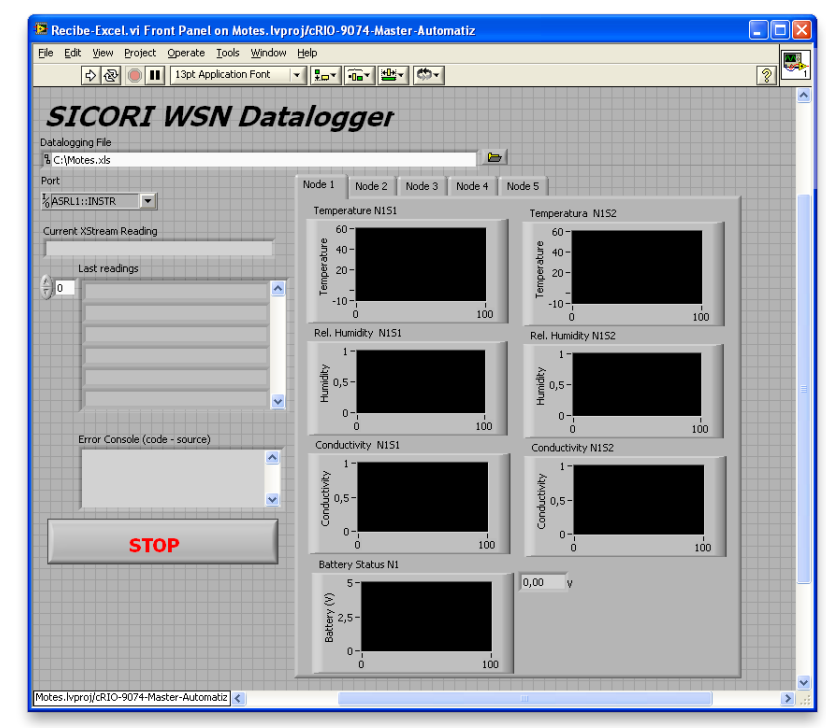

Figura 6.17. Software de monitorización desarrollado con LabView. 
La Figura 6.18 muestra un extracto, de aproximadamente 11 días, de la temperatura del suelo, monitorizada con uno de los sensores durante el experimento. Como se puede observar en la figura, la temperatura llega a un máximo cada día, y empieza a decrecer unas horas antes de la medianoche y durante la madrugada.

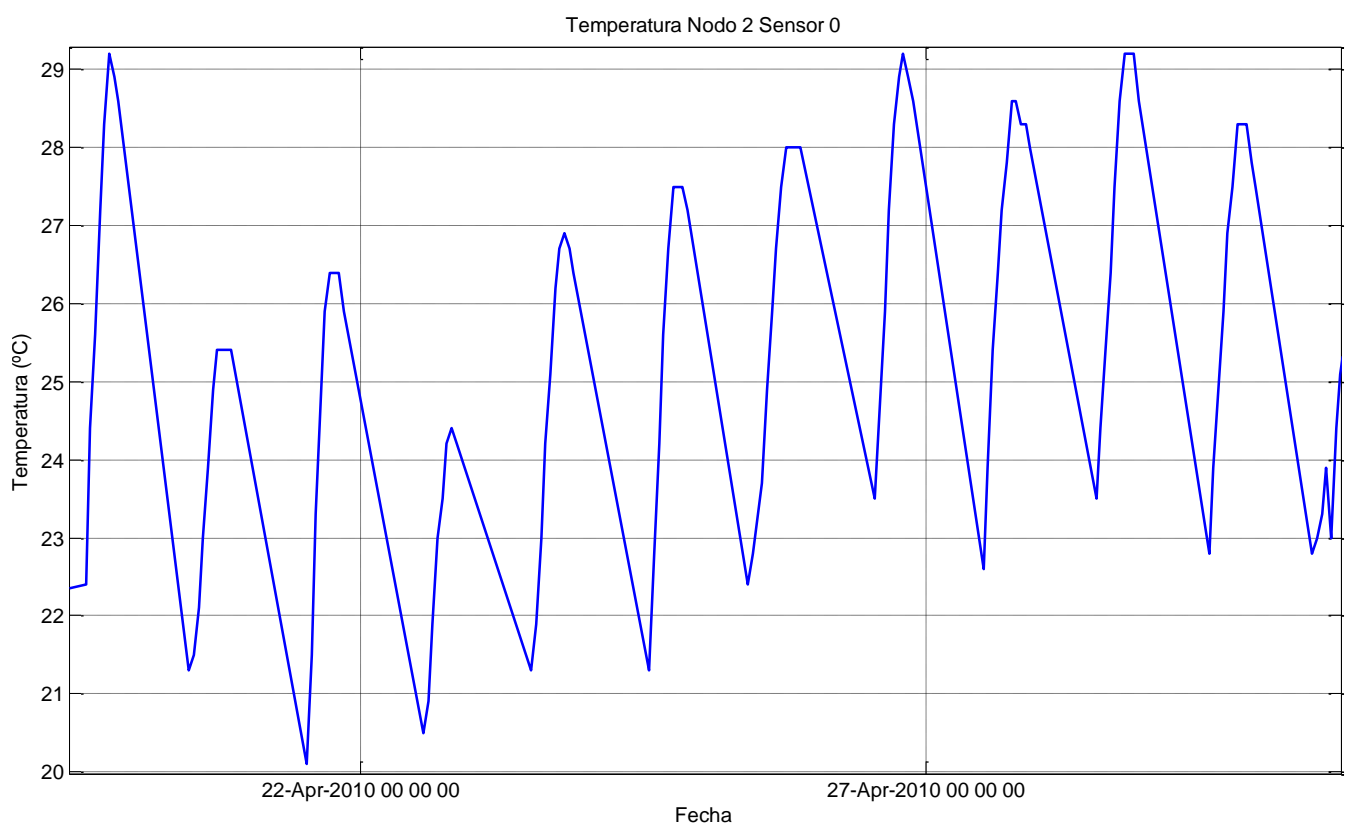

Figura 6.18. Temperatura del suelo monitorizada, con uno de los sensores, durante 11 días.

La Figura 6.19 muestra la evolución del porcentaje volumétrico de humedad en el suelo registrada, durante 11 días, en el cultivo. Los tres máximos indican los riegos realizados cada cinco días. La unidad 'wfv' se explicó en el Capitulo 4.

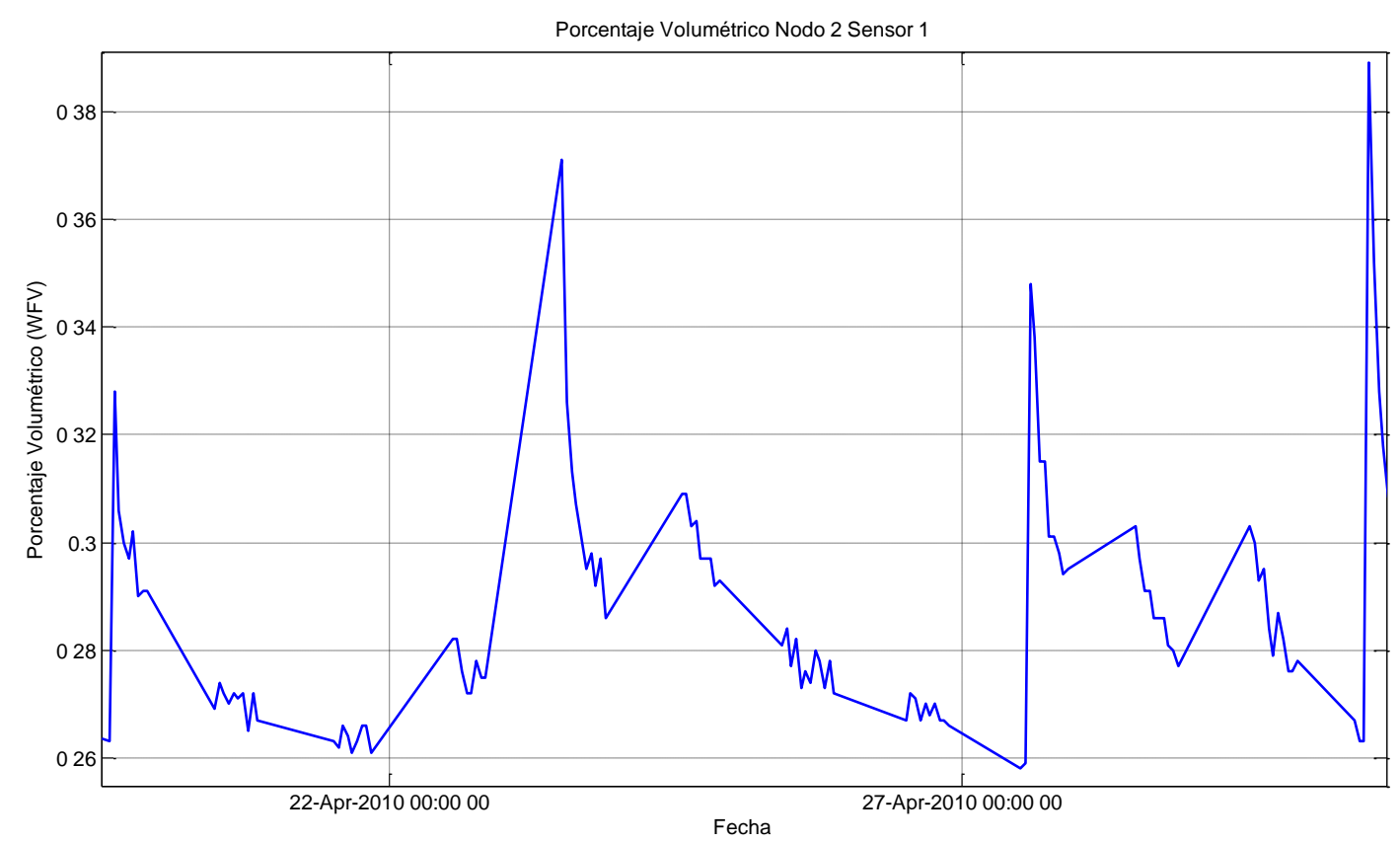

Figura 6.19. Porcentaje volumétrico de humedad en el suelo obtenido de uno de los sensores. 
Además de registrar los datos de los sensores, también se registró la tensión de la batería de los dispositivos desplegados durante todo el ciclo agronómico. La Figura 6.20 muestra la tensión de la batería, registrada en el Gateway durante las fechas indicadas. En ella, se puede observar la descarga producida por el consumo del dispositivo, así como la recarga, realizada en horas de sol con el panel solar, y el cargador de la batería de NiMH.

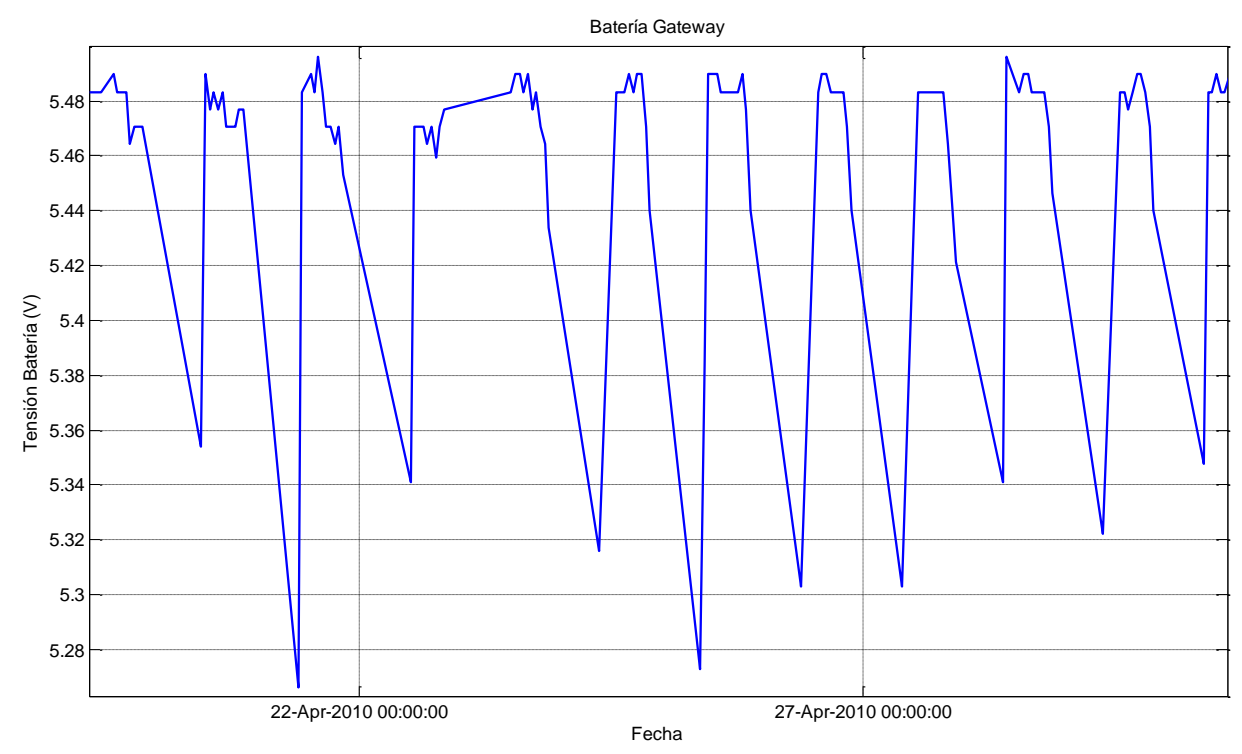

Figura 6.20. Evolución de la tensión de la batería en el Gateway, registrada durante 11 días.

La Figura 6.21 muestra la evolución de la descarga de la batería, monitorizada en un nodo sensor durante 25 días. En concreto, el nivel de la batería bajó 0,19V a lo largo de los 25 días que duró el experimento. Este resultado no se puede comparar con el obtenido en el laboratorio (Figura 6.13) con un GAIA 2 configurado con Environmental-Mote, ya que el consumo del sensor SHT71 es menor.

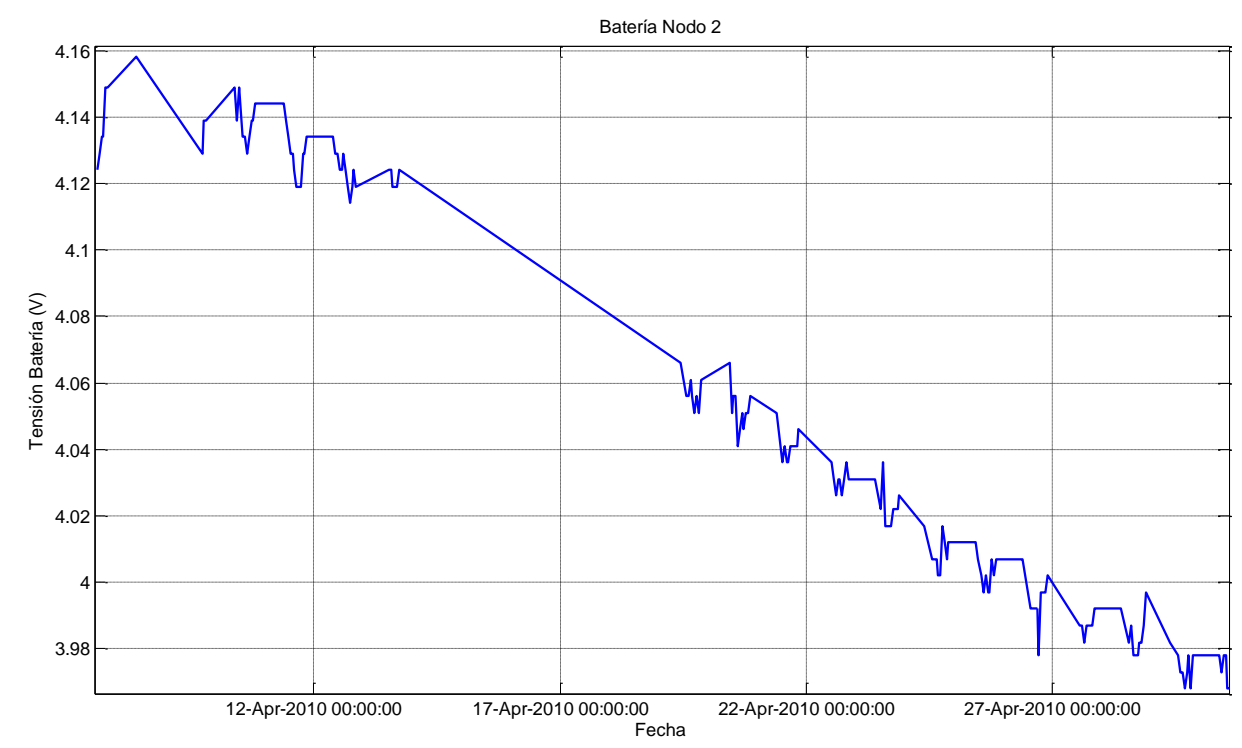

Figura 6.21. Evolución de la tensión de la batería registrada en un nodo sensor durante 25 días. 


\subsection{Conclusiones.}

Se ha llevado a cabo el diseño, implementación y validación de un nodo sensor, con una PCB constituida completamente con componentes SMD, incluso en el módulo de radio de corto alcance.

Se ha obtenido un mote multifuncional, a nivel de producto comercial, configurable tanto por hardware como por software, con la robustez necesaria para su uso en aplicaciones agrícolas reales. El mote dispone de varias interfaces de entrada y salida analógicas y digitales, compatibles con las de la mayoría de sensores y/o actuadores utilizados en agricultura de precisión. En concreto, dispone de entradas analógicas $0-2,5 \mathrm{~V}$ y 4-20mA, entradas/salidas digitales, salidas analógicas $0-2,5 \mathrm{~V}$ y las interfaces digitales $\mathrm{I}^{2} \mathrm{C}$ y SDI-12.

El principal inconveniente de este dispositivo, es el elevado coste de la carcasa diseñada. En la misma línea, el módulo comercial XStream, utilizado en una GAIA 2 configurado como Gateway en el sumidero, tiene un elevado coste (aproximadamente $90 €$ ). Otro inconveniente es que, al no disponer de un elemento de memoria temporal, no se almacenan en el propio nodo sensor los datos obtenidos de los sensores y, si ocurre un error en la transmisión inalámbrica, se pierden los datos. Por ello, tras el diseño de este dispositivo se planteó el diseño de un nodo sensor, el cual permita eliminar estos inconvenientes y mejorar las prestaciones del GAIA 2.

Los trabajos desarrollados en este capítulo han sido publicados en un congreso nacional [López, 2010], y está previsto publicar el GAIA 2 en revistas internacionales listadas en el ISI, así como en congresos internacionales. 


\title{
Capítulo 7
}

\section{MEWiN: Un Nodo Sensor Inalámbrico Multi-Entorno con una Arquitectura}

\author{
Abierta.
}

$\Psi$ Iproceso de monitorización de un entorno agronómico exige la utilización de redes compuestas por se presenta el diseño, implementación y validación un nodo sensor inalámbrico multi-entorno, basado en una arquitectura abierta, con objeto de disminuir el número de elementos necesarios para configurar una red determinada y optimizar los costes de fabricación. Dicho nodo, dispone de una placa base, con los componentes principales, sobre la que se pueden conectar las placas de interfaz para los diferentes sensores y/o actuadores necesarios. El dispositivo se ha diseñado en el marco de tres proyectos de investigación y, actualmente, se está validando en dos casos de estudio agronómicos, así como en uno oceanográfico.

En este capitulo se describe el hardware de la placa base del nodo y de las placas de interfar. También se describen el software del nodo sensor, las pruebas realizadas con el dispositivo, asi como los resultados obtenidos. 


\subsection{Introducción.}

En el capítulo anterior se describió el diseño, implementación y validación de un nodo sensor multifuncional GAIA 2, el cual se caracteriza por ser un diseño a nivel de producto comercial, configurable tanto por hardware como por software, con la robustez necesaria para su uso en aplicaciones agrícolas reales. La principal ventaja del mismo es tener un único dispositivo, que puede ser configurado como cualquier nodo requerido (nodo sensor de tierra, nodo sensor de ambiente, Gateway, etc.) en el despliegue de una red de sensores. De este modo, sólo es necesario realizar el proceso de certificación (marcado CE) de un único dispositivo. Sin embargo, el diseño presenta una serie de carencias que se discutieron en la sección de conclusiones del capítulo anterior.

El GAIA 2 dispone de una PCB cerrada, que proporciona un conjunto limitado de interfaces de entrada y salida analógicas y digitales, pudiendo ser usado con la mayoría de sensores y/o actuadores utilizados en la agricultura de precisión. Así, con el dispositivo se puede conectar directamente un tensiómetro con salida 4-20mA. Sin embargo, por ejemplo no se puede conectar directamente con el mote un termopar tipo $\mathrm{K}$, utilizados habitualmente en algunos termoradiómetros comerciales. En esta línea, para mejorar las prestaciones del GAIA 2 en un nuevo diseño, es más adecuado diseñar una PCB con una arquitectura abierta, constituida por una placa base a la que se puedan conectar diversas placas de interfaz diseñadas 'ad hoc' con la instrumentación necesaria.

El coste en el diseño de la carcasa del GAIA 2 fue muy elevado. Además, la fabricación de un número reducido de carcasas siempre tendrá un coste elevado por unidad. Por ello, es necesario abordar un nuevo diseño que utilice una caja estanca comercial como carcasa.

Otro elemento que eleva considerablemente el coste del GAIA 2, es el radio modem XStream, que es necesario cuando se utiliza el dispositivo como Gateway o nodo sensor de largo alcance. En concreto, el XStream tiene un coste aproximado de $90 €$. Este inconveniente se puede resolver en un nuevo diseño de PCB con la arquitectura abierta comentada anteriormente. Para conseguir esto, la placa base debe permitir la conexión de placas de interfaz 'ad hoc', así como de otros periféricos, como un segundo módulo de radio.

Las comunicaciones inalámbricas se ven afectadas por las condiciones atmosféricas $y$, en algunas ocasiones, no es posible realizar el correcto envío de un mensaje entre una estación emisora y otra transmisora. En este sentido, es muy útil que el nodo sensor incluya un elemento de memoria permanente que almacene, además de enviar inalámbricamente, las lecturas realizadas de los sensores. Además, con objeto de almacenar la lectura de los 
sensores junto con hora a la que se realizó, es necesario utilizar un elemento que proporcione la hora y la fecha, con la precisión adecuada, para el tratamiento posterior.

Por ello, una vez validado el GAIA 2 y vistos sus inconvenientes, se planteó el diseño de un nodo sensor inalámbrico multi-entorno con una arquitectura abierta. Se basa en una placa base que incluye los elementos principales necesarios en la mayoría de aplicaciones, así como de 2 conectores de expansión. Los conectores de expansión permiten la conexión de otras placas de interfaz para la instrumentación requerida y de otros periféricos, como un segundo módulo de radio. El sistema para conseguir esta arquitectura se ha denominado con el acrónimo MEWiN, que se extrae del inglés MultiEnvironmental Wireless Node. La placa base o principal se ha denominado MEWiN Mainboard.

El capítulo se estructura de la siguiente forma: la sección 2 describe los requisitos funcionales con los que se ha diseñado la placa base MEWiN Mainboard, el hardware y el software de la misma y las dos placas de interfaz realizadas para validar la arquitectura global en condiciones reales de funcionamiento (en un cultivo real). En la sección 3 se presentan las pruebas experimentales realizadas sobre el dispositivo. Para ello se describen las pruebas realizadas en laboratorio, las pruebas de alcances y las llevadas a cabo en el despliegue realizado en un cultivo real. En cada una de ellas se presentan los resultados obtenidos. Finalmente, en la sección 4 se exponen las conclusiones y las observaciones finales.

\subsection{Descripción del sistema MEWiN.}

En esta sección, en primer lugar, se describen los requisitos funcionales que se tuvieron en cuenta para llevar a cabo el diseño e implementación del sistema MEWiN. Una vez hecho esto, se describe el hardware de la placa base del sistema y las placas de interfaz diseñadas para validar el sistema en un cultivo real. Finalmente, se describirá el desarrollo del software que es necesario realizar en la placa base del sistema MEWiN.

\subsubsection{Requisitos funcionales.}

En este diseño, al igual que en el diseño del GAIA 2, se primaron, fundamentalmente, aquellas decisiones arquitectónicas que favorecieron la robustez del sistema y también la interconexión de distintos tipos de sensores. Una vez fijadas estas premisas, junto con la compatibilidad requerida para las comunicaciones, se consideraron todos aquellos requisitos adicionales que pudieron hacer más idóneo y funcional el sistema. Los requisitos que se tuvieron en cuenta para el estudio e implementación del sistema MEWiN se exponen en los siguientes párrafos. 
El objetivo que se plantea es el desarrollo de un nodo sensor inalámbrico multientorno, el cual permita llevar a cabo tareas de monitorización y actuación en casos de estudio agronómicos de diversa índole.

Se propone un diseño basado en una arquitectura abierta, que está compuesta por una placa base sobre la que se pueden conectar las placas de interfaz necesarias para la instrumentación utilizada en el caso de estudio. El diseño tiene en cuenta la posibilidad de conectar una placa de interfaz sobre otra ya conectada.

La placa base externaliza en dos conectores de expansión, de 16 pines cada uno, las siguientes interfaces de entrada y salida: 8 entradas analógicas $0-2,5 \mathrm{~V}, 2$ salidas analógicas 0 $2,5 \mathrm{~V}$, hasta 17 entradas/salidas digitales $0-3 \mathrm{~V}$ y las interfaces digitales de entrada/salida de tipo UART, SPI e I²C.

En los conectores de expansión se pueden conectar placas de interfaz para la instrumentación seleccionada, así como periféricos que se conecten mediante buses digitales como el SPI. Un ejemplo de periférico es el sensor de temperatura y humedad relativa del ambiente SHT71 de Sensirion. Este sensor fue descrito en el capítulo $4 \mathrm{y}$ requiere de una interfaz digital de tipo $\mathrm{I}^{2} \mathrm{C}$.

Los elementos principales del MEWiN son: el micro-controlador MSP430F2618 [MSP430F2618], el transceptor de radio CC2520 [CC2520] y el amplificador de radio CC2591 [CC2591], todos ellos de Texas Instruments [TI]. El CC2591 permite conseguir alcances mayores que el CC2520, ya que proporciona una menor sensibilidad y una mayor potencia de transmisión que el anterior.

La placa base debe disponer de los siguientes módulos principales: cargador de baterías de polímero de litio, slot para la conexión de una tarjea SD, un reloj de tipo real (RTC, Real Time Clock) y una interfaz RS-232. La PCB estará diseñada de forma que se puedan soldar de manera independiente los componentes mencionados anteriormente. Así se consigue fabricar placas base a medida para optimizar los costes y poder competir en el mercado.

El diseño mecánico del sistema estará optimizado para ser utilizado en cultivos de tipo hortícola. Esto significa que el dispositivo se podrá instalar a nivel del suelo, que el producto final tiene que ser discreto para evitar su substracción y que, por sus dimensiones, no será necesario retirarlo cuando se utilice maquinaría agrícola pesada en el cultivo. Por ello, la carcasa del mote tendrá un grado de protección IP67 y la altura (incluyendo la antena) no superará los $15 \mathrm{~cm}$. Aunque el diseño esté optimizado para cultivos de tipo hortícola, también podrá ser utilizado en otro tipo diferente de cultivos agronómicos, como los frutícolas, viñedos, etc. 
El nodo soportará los protocolos de comunicación definidos en la especificación ZigBee [ZigBee, 2007]. Para conseguir esto, la programación se llevará a cabo con la biblioteca Z-Stack [Z-Stack] que proporciona gratuitamente Texas Instruments. La programación con esta biblioteca permitirá desplegar redes de sensores con topologías sencillas (estrella) y topologías más complejas (árbol y malladas). Estas topologías, así como ZigBee fueron descritas en el capítulo 3.

En relación con los alcances, se establece como requisitos en el diseño del nodo sensor que el alcance sea de $2 \mathrm{~km}$ y $400 \mathrm{~m}$ en condiciones favorables y desfavorables del enlace, respectivamente. Condiciones favorables implica usar antenas omnidireccionales, de $8 \mathrm{dBi}$ de ganancia, que estén instaladas al menos a $2 \mathrm{~m}$ de altura de la superficie y que exista visión directa entre ellas. Por otro lado, utilizar una antena de $2 \mathrm{dBi}$ de ganancia en una de las estaciones, en la que no exista visión directa entre las antenas, o que uno de los nodos sensores esté instalado sobre el suelo se consideran como condiciones desfavorables para un enlace.

El nodo se alimentará con una batería Li-ION de 3,6V de tensión nominal. Con respecto a la capacidad, será de $4.000 \mathrm{mAh}$ en un dispositivo final y de $5.000 \mathrm{mAh}$ para un Router. Este último requiere una mayor capacidad, además de un panel para recargar la batería, porque su consumo medio, expresados en términos de intensidad, es de unos $30 \mathrm{~mA}$ y es necesario garantizar una autonomía de unos 3 días en ausencia de energía solar para recargar la batería. Con respecto a la autonomía de un dispositivo final, será de, al menos, 10 semanas, que es el tiempo habitual de duración de un ciclo agronómico hortícola.

La placa base del sistema MEWiN puede estar configurada para realizar las tareas de uno de los 3 tipos de nodos que intervienen en una red ZigBee. Estos son los siguientes: ZigBee Coordinator (ZC), ZigBee Router (ZR) y ZigBee End Device (ZED).

El software desarrollado para un ZED realizará la lectura de los sensores con la frecuencia configurada desde un software de monitorización. Esta frecuencia se podrá parametrizar remotamente desde 30 minutos hasta aproximadamente 10 días. Además de realizar la lectura de los sensores, el software diseñado deberá obtener la hora-fecha del RTC y almacenar los datos junto con el campo hora-fecha en la SD. De esta forma, el nodo sensor tendrá un registro de todas las lecturas realizadas. Esto es muy importante, ya que, en ocasiones, las comunicaciones inalámbricas no se realizan satisfactoriamente debido a las condiciones meteorológicas. Finalmente, el nodo realizará una lectura del nivel de la batería y lo enviará inalámbricamente cada hora. Este valor también se almacenará en la SD.

En el caso de tener sensores conectados, el software de un ZR será similar al del ZED. En todos los casos, el nodo realizará una lectura del nivel del voltaje de la batería 
cada hora. Este valor se enviará inalámbricamente y se almacenará en la SD cada hora. Además, en el caso de utilizar un panel solar fotovoltaico para recargar la batería, el software del nodo incluirá un algoritmo encargado de gestionar la recarga de la batería, así como de realizar lecturas periódicas de la voltaje del panel solar fotovoltaico y almacenar dicha información en la tarjeta de memoria SD del dispositivo.

Un ZC se encuentra, en la mayoría de los casos, conectado por un enlace físico con el servidor de la red. Por ello, el software desarrollado para un ZC deberá enviar los datos recibidos inalámbricamente de los sensores y de la tensión de la batería de cada nodo sensor hasta el software de monitorización con el formato establecido.

Las tramas intercambiadas por la red inalámbrica serán tramas crudas (raw). Así, por ejemplo, una trama para enviar la tensión de la batería contendrá 9 bytes de payload, con 2 bytes dedicados a la lectura del ADC de 12 bits y 7 bytes procedentes del valor devuelto por el RTC. El RTC devuelve los segundos, minutos, horas, día de la semana, día del mes, mes y año. Cada uno de estos valores se almacenan en 1 byte.

Por otro lado, las tramas en formato crudo recibidas en el coordinador serán transformadas en tramas en formato ASCII y, posteriormente, enviadas al servidor con el software de monitorización. El formato de estas tramas se describe en el Anexo II. Este formato es el mismo que se utiliza para almacenar las lecturas realizadas por los sensores en la SD. De este modo, un mismo software de monitorización puede procesar las lecturas recibidas de la red inalámbrica y de la tarjeta $\mathrm{SD}$ de un nodo sensor.

Para validar la arquitectura MEWiN se desarrollará una interfaz gráfica con el entorno $\mathrm{C}++$ Builder XE, comercializado por la empresa Embarcadero [Embarcadero]. Este software mostrará un log de las tramas recibidas de la red inalámbrica y las almacenará en archivos de texto para facilitar la representación de los datos en un entorno como MatLab. Además, tendrá los controles necesarios para modificar la frecuencia de lectura de los sensores de los nodos de la red.

\subsubsection{Descripción del hardware.}

En este apartado se describe la arquitectura del prototipo desarrollado. Se presenta la placa base y las placas de interfaz diseñadas para validar el sistema MEWiN. También se describirán el resto de elementos (batería, carcasa, etc.) que definen completamente la arquitectura.

\subsubsection{MEWiN Mainboard.}

La Figura 7.1 describe a nivel de diagrama de bloques la placa base MEWiN Mainboard desarrollada. En ella se puede observar que la unidad de procesamiento del 
sistema es el micro-controlador de bajo consumo MSP430F2618 de Texas Instruments. El micro-controlador está conectado por bus SPI con el módulo de radio CC2520 del mismo fabricante. Además, para conseguir mayores alcances que con el CC2520, éste se conecta con el range extender CC2591 del mismo fabricante. Con una infraestructura óptima de las antenas y usando sólo el CC2520, se pueden conseguir alcances entre dos estaciones de unos $400 \mathrm{~m}$. Este alcance se puede incrementar hasta $2 \mathrm{~km}$ usando el CC2591. Esto es así, ya que el CC2591 reduce la sensibilidad del CC2520 e incrementa la potencia de transmisión del CC2520.

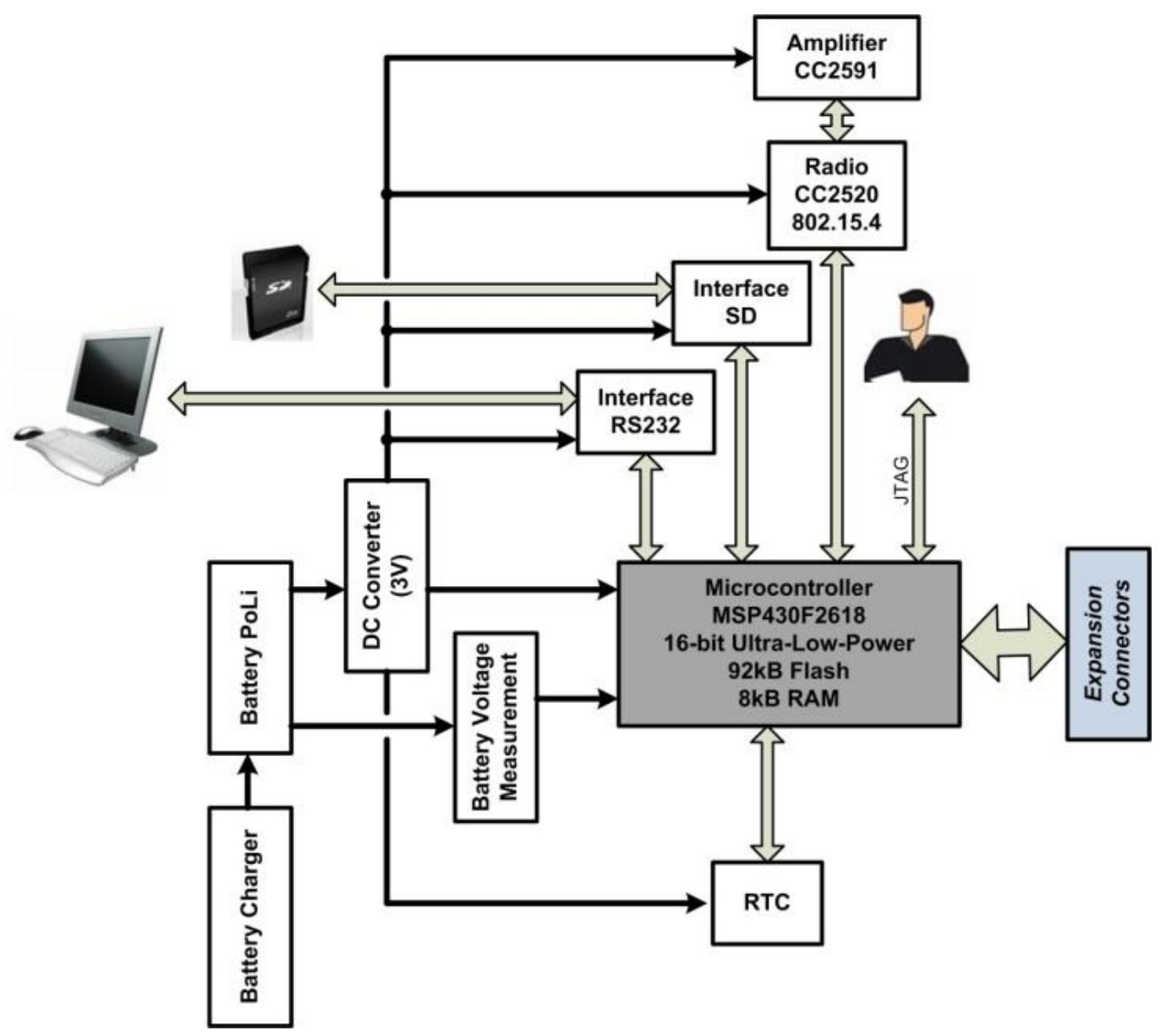

Figura 7.1. Diagrama de bloques de la placa MEWiN Mainboard.

Para alimentar el conjunto, se utiliza una batería de Li-ION, de 3,6V de tensión nominal. Con respecto a la capacidad de la batería, se utilizan celdas de $4.000 \mathrm{mAh}$ para un ZED y de $5.000 \mathrm{mAh}$ para un ZR. Más detalles sobre esta batería se pueden encontrar en el Anexo I. La batería se puede recargar mediante el cargador de baterías MAX1555 integrado en la PCB. En este diseño se utiliza el mismo esquemático para el cargador que el presentado en el Capítulo 5. La tensión de la batería se fija a 3V con un regulador de tensión lineal de bajo dropout MCP1826 y se monitoriza periódicamente mediante un ADC del micro-controlador usando un división resistivo y un amplificador operacional con la configuración de seguidor de tensión para adaptar las impedancias (ver Figura 7.2). 


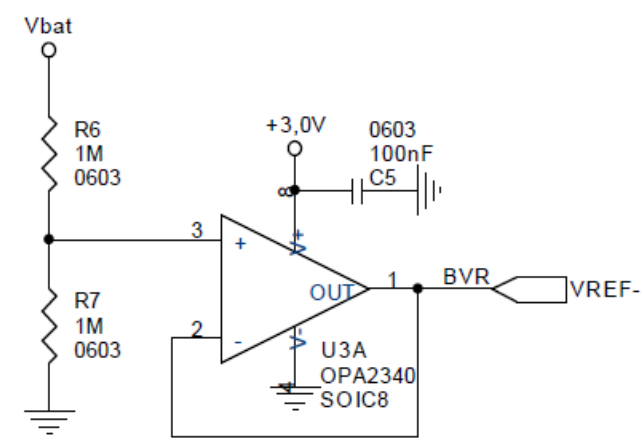

Figura 7.2. Esquemático entre la batería y una ADC del micro-controlador.

La Figura 7.3 muestra el esquemático de la interfaz entre el slot de la tarjeta SD y el micro-controlador. Esta interfaz se consigue mediante la conexión con pines de la USCI_B0 (Universal Serial Communication Interface) del micro-controlador. Esta USCI se puede configurar por software en modo SPI e $\mathrm{I}^{2} \mathrm{C}$, siendo necesario, en este caso, el modo SPI. La línea MOSI (Master Output Slave Input) es la línea de salida de datos del microcontrolador y de entrada a la SD. La línea MISO (Master Input Slave Output) tiene la función complementaria. La línea SCK es la señal de reloj de sincronización. Finalmente, también es necesaria la línea CS (Chip Select), que, en este caso, utiliza la P2.2.

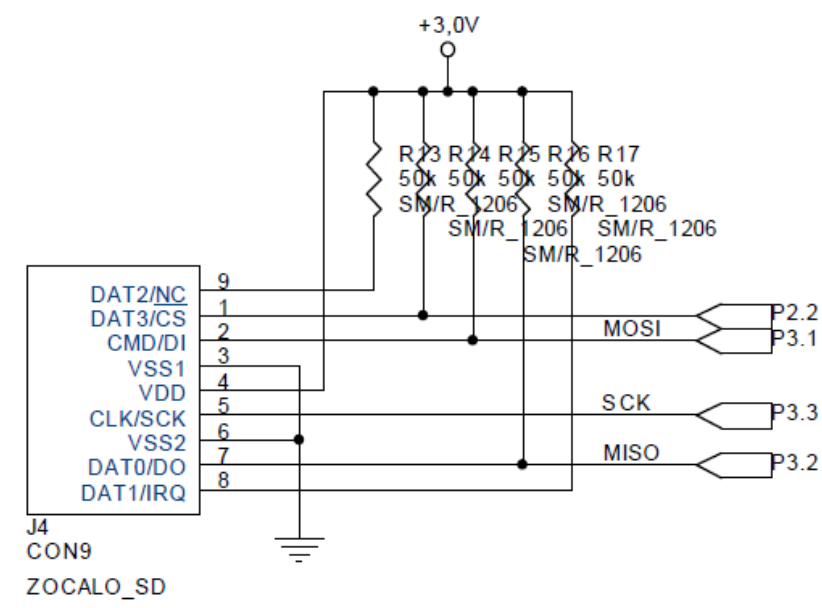

Figura 7.3. Esquemático de la interfaz SPI entre la tarjeta SD y el micro-controlador.

La USCI anterior también se utiliza en modo $\mathrm{I}^{2} \mathrm{C}$ para enviar y recibir tramas del RTC. En este caso se utilizan las líneas P3.1 y P3.2 (ver Figura 7.4), que se corresponden con la línea bidireccional de datos y la línea de sincronía de la interfaz $\mathrm{I}^{2} \mathrm{C}$, respectivamente. Además, como se puede observar en la Figura 7.4, el RTC tiene conectado un oscilador externo de $32 \mathrm{KHz}$ y una batería de back-up.

Las tareas de depuración y programación del dispositivo se realizan por interfaz JTAG. La interfaz RS-232 permite intercambiar datos con el nodo sensor. Además, se puede utilizar en tareas de depuración. 


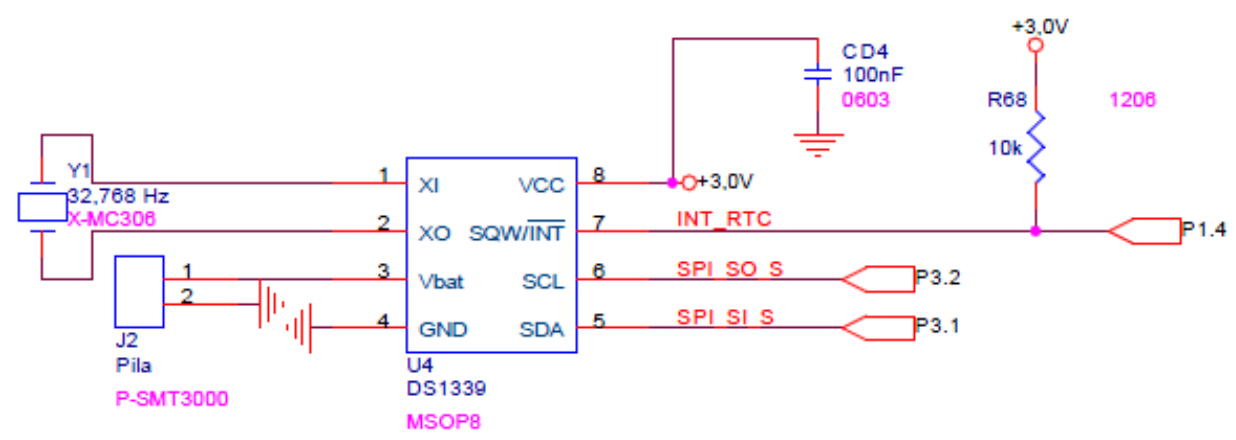

Figura 7.4. Esquemático de la interfaz $\mathrm{I}^{2} \mathrm{C}$ entre el RTC y el micro-controlador.

Como se ha descrito anteriormente, la placa base incluye un RTC y una tarjeta de memoria SD. Estos elementos proporcionan redundancia al sistema, ya que todos los mensajes de datos y nivel de batería que se envían por radio se almacenan también por seguridad en la tarjeta SD. Las tramas que se almacenan incluyen la fecha y la hora en la que se realizó la lectura de los sensores y/o la batería, que se obtienen del RTC.

La Figura 7.5 muestra la PCB con componentes SMD que se ha diseñado para la placa base MEWiN Mainboard. En ella, se pueden distinguir los elementos mencionados anteriormente, así como los 2 conectores de expansión de 16 pines para conectar las diferentes placas de interfaz con la instrumentación seleccionada, así como otros periféricos.

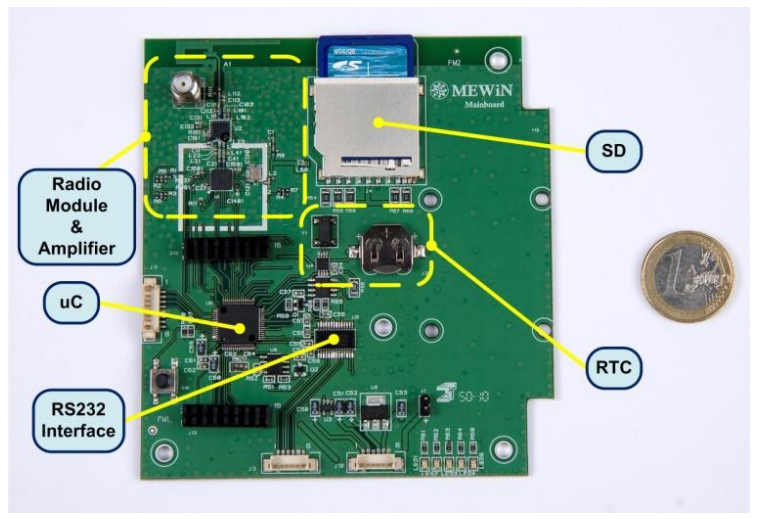

Figura 7.5. PCB de la placa base MEWiN Mainboard.

A continuación se describen las placas de interfaz que se han desarrollado para validad la placa base MEWiN Mainboard descrita anteriormente.

\subsubsection{MEWiN Vegeboard.}

Para validar un ZED se ha diseñado la placa de interfaz Vegeboard (ver Figura 7.6a), que permite la conexión de sensores Hydra Probe II (HP2) con la placa base MEWiN Mainboard. Este sensor ha sido descrito en el Anexo I. En la Figura 7.6b se muestra el diagrama de bloques de esta placa de interfaz. 


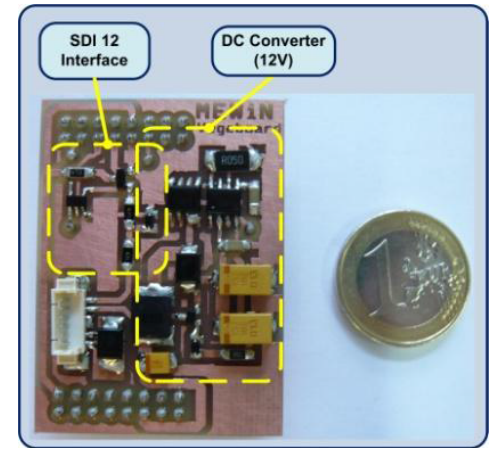

(a)

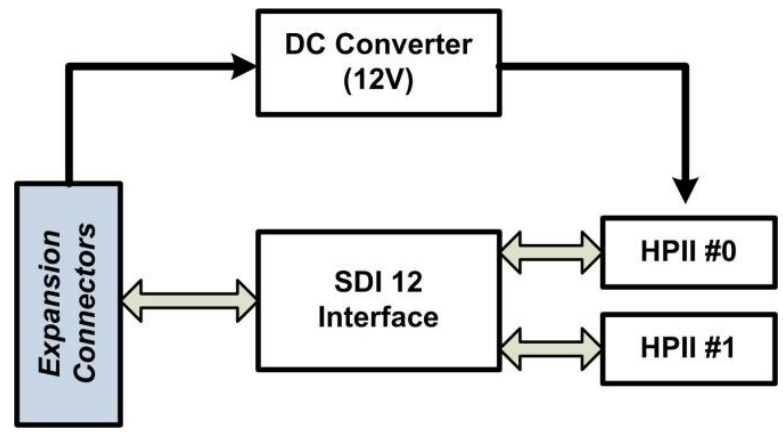

(b)

Figura 7.6. Placa de interfaz MEWiN Vegeboard: (a) PCB. (b) Diagrama de bloques.

Como se puede observar en la Figura 7.6b, el diagrama de bloques se ha particularizado para los 2 sensores HP2 que se han utilizado en las pruebas de validación de la plataforma. En relación con los esquemáticos asociados a los dos bloques mostrados en la Figura 7.6b, el esquemático de la interfaz SDI-12 es el mismo que ha sido descrito en el capítulo dedicado al GAIA Soil-Mote. Sin embargo, debido a que el fabricante (Maxim IC) dejó de comercializar el circuito integrado MAX761 utilizado en diseños previos, ha sido necesario volver a diseñar el convertidor conmutado DC/DC de tipo elevador, que proporciona los $12 \mathrm{~V}$ necesarios por los sensores a partir de la tensión estabilizada de la batería. El esquemático de este bloque, basado en el circuito integrado MAX1771 de Maxim IC, se muestra en la Figura 7.7. El transistor Q2 se ha añadido al diseño recomendado por el fabricante para asegurar que el consumo de los sensores es despreciable en periodos de no lectura.

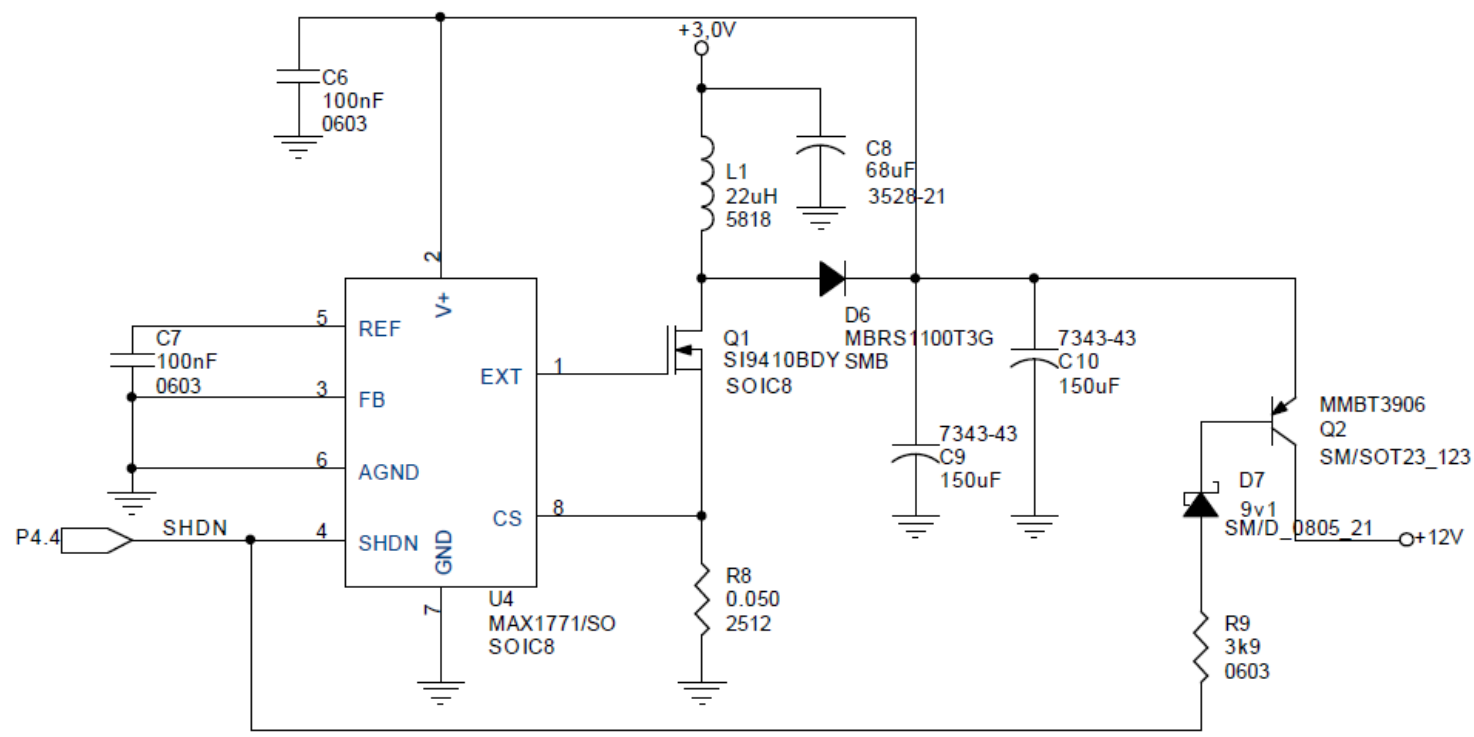

Figura 7.7. Esquemático del convertidor DC/DC de la placa de interfaz Vegeboard. 


\subsubsection{MEWiN Routerboard.}

Como se puede recordar del Capítulo 3, en una red de sensores que establece sus comunicaciones mediante ZigBee, un nodo con el rol de Router (ZR) resuelve las tareas de escalabilidad de la red e incrementa la robustez de la red de sensores desplegada. Lo primero se consigue porque el ZR permite comunicar un nodo remoto (ZED) con el ZC, que no se podría conectar directamente por limitaciones de alcance y, a nivel más general, porque ZigBee soporta topologías en árbol y malladas. Con respecto a la robustez de la red, se puede pensar en una red compuesta por un ZC, dos ZR y un ZED. Este último sólo se puede comunicar con el ZC a través de un ZR e inicialmente estableció la comunicación mediante el ZR número 1. Si este ZR fallara, el ZED podría establecer sus comunicaciones con el coordinador mediante el ZR número 2.

La placa de interfaz MEWiN Routerboard se ha diseñado para validar el funcionamiento de un ZR, constituido por la placa base MEWiN Mainboard y la mencionada anteriormente. En la Figura 7.8a y en la Figura 7.8b se muestra el esquemático y la PCB de la placa de interfaz que se ha diseñado para validar el funcionamiento de un nodo MEWiN con el rol de Router. El divisor resistivo de tres resistencias (divide por 9,72) permite monitorizar la tensión del panel solar con un convertidor analógico-digital (ADC3, pin P6.3) de rango 0-2,5V. La batería de Li-ION de 3,6V de tensión nominal y $5 \mathrm{Ah}$ de capacidad que se utiliza en un ZR, dispone de circuitos de protección de sobrecarga y sobredescarga. En ambos casos, cuando la batería está sobrecargada o sobredescargada, el circuito de protección desconecta la batería. En estos casos, a la entrada del regulador de tensión lineal, que está conectado a la salida de la batería, podrá llegar la tensión de cortocircuito del panel solar y se podría romper el nodo sensor. Para evitar esto, se controla la carga de la batería con el pin P1.7 del micro-controlador. Cuando este pin está a 3V, Q1 estará en saturación, el panel solar estará en cortocircuito y no se estará cargando la batería. Si el pin está a 0V, Q1 estará en corte y el panel solar estará cargando la batería.

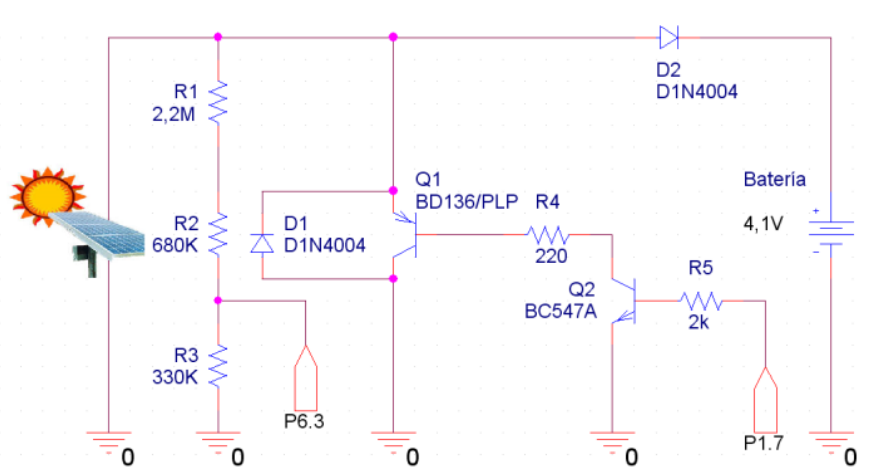

(a)

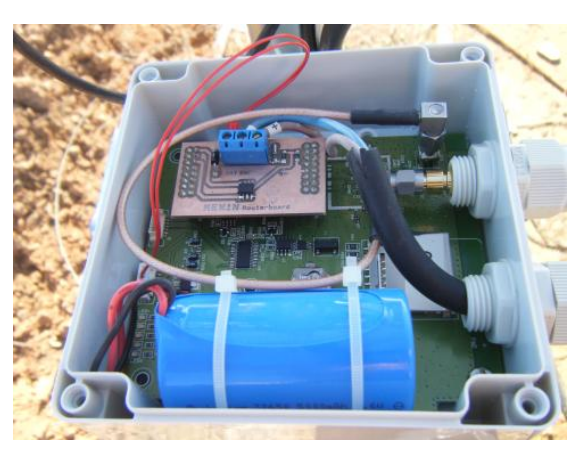

(b)

Figura 7.8. Placa de interfaz MEWiN Routerboard: (a) Esquemático. (b) PCB. 


\subsubsection{Placas de interfaz comerciales MEWiN.}

En paralelo con los trabajos de validación experimental del nodo MEWiN usando las placas de interfaz desarrolladas en laboratorio, se diseñaron y fabricaron dos placas de interfaz comerciales (ver Figura 7.9): MEWiN SoilBoard y MEWiN LVDTBoard.

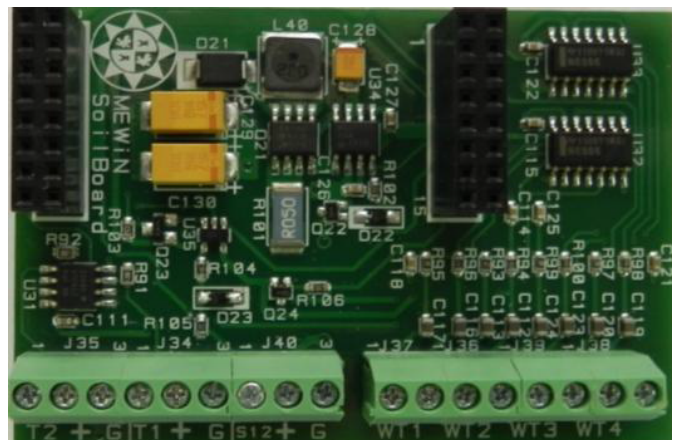

(a)

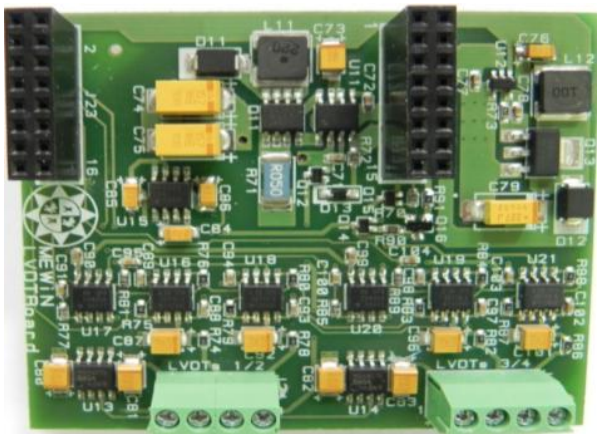

(b)

Figura 7.9. Placas de interfaz MEWiN: (a) SoilBoard. (b) LVDTBoard.

La PCB MEWiN SoilBoard es una placa de interfaz que permite la conexión directa de sensores de suelo con la placa base MEWiN. En concreto, observando las regletas de conexión de izquierda a derecha, permite la conexión de los siguientes sensores: 2 tensiómetros Irrometer con interfaz eléctrica de salida 4-20mA, un bus SDI-12, que permite la conexión de hasta 10 sensores con esta interfaz digital de salida, y 4 sensores de potencial hídrico del suelo $W$ atermark.

La placa de interfaz MEWiN LVDTBoard permite conectar, directamente con la placa base MEWiN, hasta 4 dendrómetros radiales, constituidos por un portasensor y el transductor de desplazamiento de precisión DfG2.5 de Solartron.

Las dos placas de interfaz disponen de conectores pasantes que permiten la conexión de múltiples placas de interfaz.

\subsubsection{Carcasa, antena y batería.}

En la Figura 7.10 se pueden observar el aspecto final de la carcasa comercial del nodo MEWiN. En este caso, para facilitar las pruebas iniciales y la correspondiente validación de la plataforma, así como reducir los costes, se ha utilizado como carcasa una caja estanca comercial con un grado de protección IP67. En el caso de un nodo sensor, se ha mecanizado la tapa superior para instalar la antena (ver sección 3.2 del Anexo I) externa omnidireccional, de $2 \mathrm{dBi}$ de ganancia. También se ha mecanizado una de las paredes laterales de la caja estanca para incorporar un prensaestopa de grado de protección IP67. En el caso de nodo Router que no tiene sensores conectados, la caja sólo es necesario mecanizarla en una de las paredes laterales para instalar dos prensaestopas. El primero se utilizar para introducir con estanqueidad la manguera del panel solar. El segundo hacer 
llegar al mote el cable SMA que proviene de la antena externa omnidireccional, de 8dBi de ganancia. Ambos dispositivos (nodo sensor y Router) incorporan en el interior de la caja una batería de Li-ION, de 3,6V (ver sección 3.4 del Anexo I) de tensión nominal, con una capacidad de $5 \mathrm{Ah}$ para un Router, y de $4 \mathrm{Ah}$ para un nodo sensor. Los elementos que no se han podido observar en la Figura 7.10 (antena del Router, conector rápido, etc.), se presentarán en la sección dedicada a los resultados experimentales.

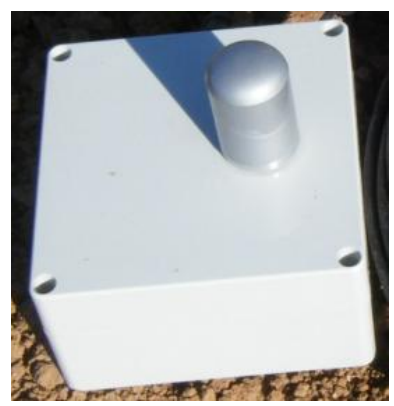

Figura 7.10. Vista de la carcasa del nodo sensor MEWiN instalada en campo.

\subsubsection{Descripción del software.}

El desarrollo de software de la plataforma MEWiN se ha llevado a cabo con la versión 2.3.0 de la biblioteca Z-Stack de Texas Instruments, detallada en mayor profundidad en el Anexo I. El desarrollo de aplicaciones con dicha biblioteca se realiza definiendo, al menos, una tarea a nivel de aplicación. Existen otras tareas de mayor prioridad, como la tarea de más bajo nivel encargada de la de gestión de control de acceso al medio (MAC, Medium Access Control). A nivel de aplicación final debe haber al menos una tarea.

Cada tarea tiene asociadas dos funciones. La primera, realiza la inicialización asociada a la tarea y se realiza una sola vez al iniciarse la ejecución. La segunda función es un manejador de eventos y se ejecuta periódicamente, comprobando si está pendiente el procesamiento de un determinado evento. En caso afirmativo, se procesa el evento y se desactiva el bit de la máscara correspondiente al evento procesado.

El desarrollador puede definir los eventos necesarios asociados a cada tarea para implementar el funcionamiento del software necesario. Estos eventos se pueden planificar para que se ejecuten pasado un determinado tiempo especificado.

Para validar la plataforma MEWiN se ha desplegado una red en la que interviene un coordinador (ZC, ZigBee Coordinator), un router (ZR, ZigBee Router) y varios nodos sensores (ZED, ZigBee End Device). Para ilustrar el flujo de diseño con la Z-Stack, se describirá, brevemente, el software desarrollado para el ZED (ver Figura 7.11). El software para este 
nodo sólo dispone de una tarea a nivel de aplicación. En esta tarea se planifican y ejecutan, principalmente, dos eventos: 'evento timer' y 'evento principal.

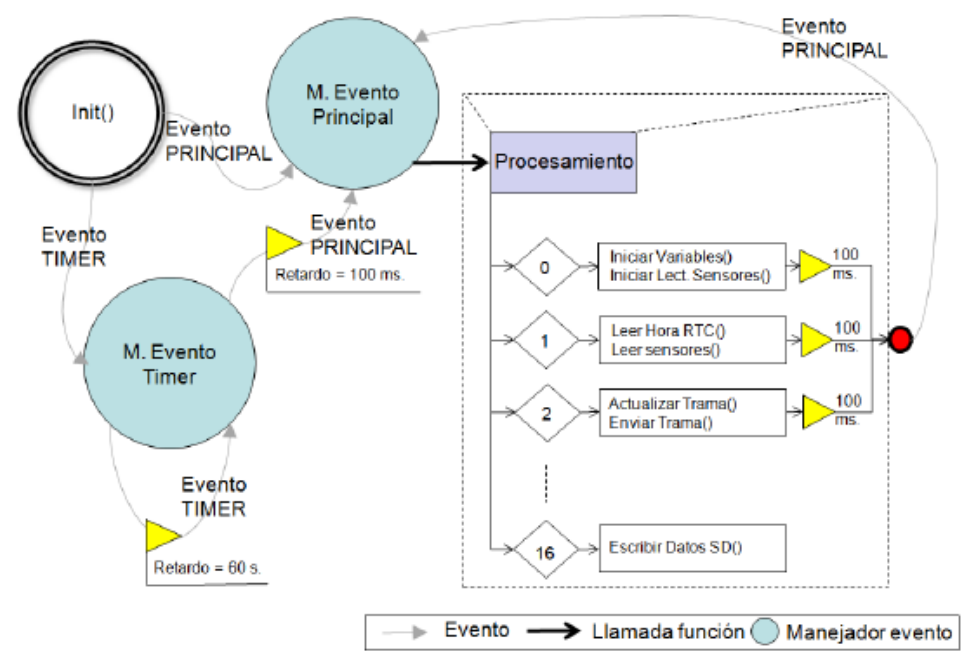

Figura 7.11. Diagrama simplificado del software de un nodo MEWiN con el rol de ZED.

El método Init) se ejecuta para realizar las inicializaciones necesarias antes de empezar la ejecución. En este método, se configuran las líneas de E/S necesarias, se inicializan las variables del temporizador software implementado, se planifica el 'evento timer' a los 60 s y el 'evento principal a los $100 \mathrm{~ms}$.

El 'evento principal implementa la máquina de estados necesaria para comenzar el proceso que engloba, entre otros procesamientos, la inicialización de las variables, la lectura de los sensores, el envío de la trama y actualizar el registro $(\log )$ implementado en la tarjeta de memoria SD con los nuevos datos leídos de los sensores. Esta máquina de estados se implementa con una función con una sentencia de tipo 'switch'. En cada 'case' del 'switcb' se incrementa la variable utilizada en la sentencia de control y se planifica de nuevo el "evento principal", de manera que, en la siguiente entrada al método 'procesamiento', se ejecuta el siguiente 'case' de la sentencia 'switch'.

Como se ha comentado anteriormente, el 'evento timer' se planifica por primera vez en el método Init). Dentro del manejador de dicho evento se planifica de nuevo el 'evento timer'. Además, se comprueba y se incrementa en su caso las variables (minutos y horas) del temporizador software implementado. Finalmente, se comprueba si los minutos y las horas coinciden con los minutos y las horas de lectura establecida. En este caso, se planifica el 'evento principal' con un retardo de $100 \mathrm{~ms}$.

Una mayor descripción sobre el desarrollo con la Z-Stack está recogida en el Anexo IV. Dicho anexo, describe el software desarrollado para un nodo con el rol de ZR. 


\subsection{Resultados experimentales.}

Para evaluar el correcto funcionamiento del nodo MEWiN, se planteó una metodología basada en dos fases experimentales: pruebas en el laboratorio y despliegue en un cultivo real. Además de validar los requisitos de alcance impuestos en el diseño del nodo sensor, el principal objetivo de la primera fase fue validar la arquitectura hardware propuesta y el software implementado. El objetivo de la segunda fase, que se llevó a cabo en un cultivo real, fue validar las condiciones funcionales del dispositivo desarrollado, en especial la robustez del mismo. El nodo también se está validando en un entorno oceanográfico, en el marco de otra tesis doctoral que se está realizando en el grupo de investigación DSIE [DSIE].

\subsubsection{Experimentos de laboratorio.}

Las pruebas de laboratorio realizadas sobre el nodo sensor MEWiN permitieron verificar la correcta funcionalidad y autonomía del dispositivo. En primer lugar, se diseñaron los componentes software, necesarios para validar la correcta operatividad de todas la interfaces de entrada y salida del nodo sensor. Posteriormente, se diseño el software necesario para realizar pruebas de PER (Packet Error Test) del dispositivo con diferentes antenas y distancias entre nodos. Para realizar estas pruebas se utilizaron dos dispositivos. Uno de ellos con el rol de ZC y otro con el rol de ZED. Las pruebas se llevaron a cabo configurando previamente un dispositivo como nodo receptor de paquetes y el otro como nodo emisor, y enviando 1000 tramas de datos desde la estación emisora hasta la estación receptora en, aproximadamente, 20s. De esta manera, se comprobó que existía una correcta comunicación bidireccional entre las dos estaciones.

Los resultados de las pruebas fueron el número de paquetes recibidos por la estación, el número de paquetes perdidos y el nivel de potencia de las señales recibidas (RSSI, Receive Signal Strength Indication). La primera prueba se realizó con antenas de $2 \mathrm{dBi}$ de ganancia, separando las estaciones aproximadamente $400 \mathrm{~m}$, y colocando los dispositivos a nivel del suelo. En este caso, los paquetes recibidos eran siempre superiores al 95\% de los paquetes enviados. La segunda prueba consistió en utilizar antenas externas omnidireccionales, de $8 \mathrm{dBi}$ de ganancia, instaladas a $2 \mathrm{~m}$ de la superficie con un mástil y separando los nodos sensores $2 \mathrm{~km}$. En este caso, el resultado de paquetes recibidos fue también siempre superior al 95\% de los paquetes enviados en todas las pruebas realizadas.

Para validar el correcto funcionamiento de un conjunto de nodos sensores MEWiN operando al mismo tiempo, se realizó el despliegue en laboratorio de una red similar a la que se desplegaría en el cultivo real, pero con un número inferior de nodos (Figura 7.12). La red estaba formada por 4 nodos sensores MEWiN. En concreto, un nodo con el rol ZC 
conectado por RS-232 con el servidor, un nodo con el rol de ZR y dos nodos sensores con el rol de ZED. Cada uno de los dos ZED tenía conectados dos sensores HP2, para simular la monitorización del estado del suelo de un cultivo durante las pruebas.

Para asegurar el uso del nodo router (ZR) durante las pruebas, se limito el número máximo de nodos conectados al coordinador (ZC) a dos, y se activó primero el ZR y, posteriormente, los dos nodos ZED. De este modo, sólo se podría conectar un ZED con el ZC y el otro ZED se tendría que conectar a la fuerza con el nodo ZR.

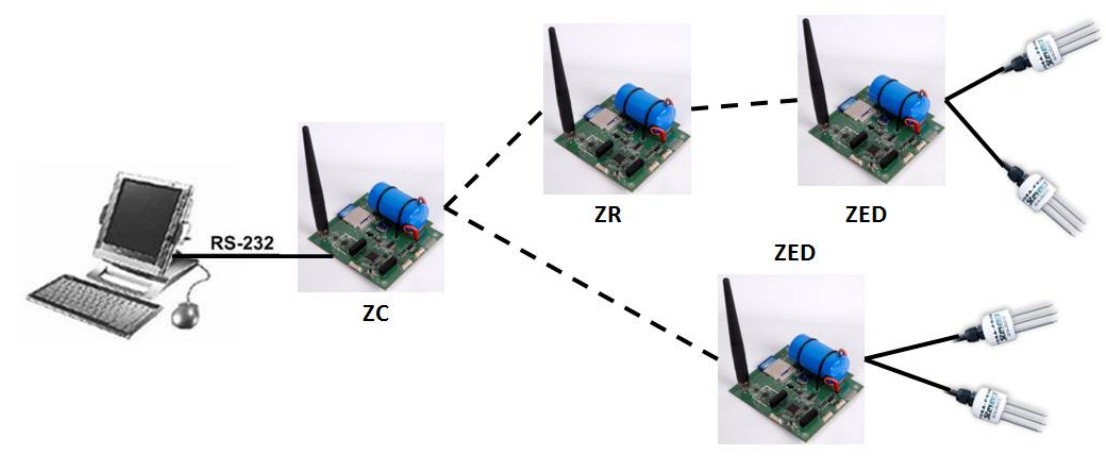

Figura 7.12. Red desplegada en el laboratorio para validar el correcto funcionamiento de la plataforma MEWiN.

Para validar el correcto funcionamiento de los nodos sensores MEWiN, se diseñó el software necesario para cada tipo de nodo, utilizando como base el ejemplo 'Sample App', de la biblioteca software Z-Stack.

En el caso del nodo coordinador, se declararon los posibles tipos de mensajes que se podían recibir (datos de los ZED, voltaje del panel del ZR y voltaje de la batería de todos los nodos), y en el método que se recibían estos mensajes, se formateaban adecuadamente, antes de ser enviados por la UART al software de monitorización. El formato con el que se envían los mensajes entre el nodo con el rol de ZC y el software de monitorización está recogido en el Anexo II.

En el nodo con el rol de ZR, se diseñó el software necesario para enviar el voltaje de la batería y del panel solar cada 30 minutos. Además, cada minuto se comprobaba el voltaje de la batería y del panel solar con objeto de, en función del estado anterior, activar o desactivar el cargador de la batería. Los componentes software de este nodo se describen en detalle en el Anexo IV.

Los nodos sensores con el rol de ZED son los que su software conllevó más tiempo implementar. Esto fue así porque hubo que diseñar el software de gestión de la interfaz digital SDI-12, con la que se configuran y reciben datos de los dos sensores HP2, que están conectados a cada nodo con el rol ZED. Se implementó un temporizador periódico para realizar una lectura de los dos sensores cada 30 minutos. Tras la lectura de cada sensor, se 
enviaba una trama por radio con la lectura realizada. Además, cada hora se medía el voltaje de la batería y se enviaba por radio. Además, el software se encargaba de almacenar en la tarjeta de memoria SD la información obtenida de los sensores y del voltaje medido en la batería.

Como se puede ver en la Figura 7.12, los datos enviados por uno de los dos ZED y por el nodo de tipo ZR se transmitían directamente hasta el software de monitorización a través del nodo con el rol de coordinador. Los datos enviados por el otro ZED llegan al nodo coordinador a través del nodo ZR. Este proceso de ruteo de paquetes lo realiza automáticamente la pila ZigBee y no requiere de una programación extra por parte del desarrollador.

En todos los nodos se realizaron las configuraciones necesarias para asegurar un bajo consumo medio, tanto en el nodo ZED como en los ZC o ZR. Para ello, en todos los nodos fue necesario configurar adecuadamente todo los pines digitales de entrada/salida del micro-controlador. Además, en el caso de un ZED se configuró el uso de un modo de bajo consumo con un periodo de activación del nodo de cada 10s para sondear si el nodo padre disponía de paquetes que habían sido enviados al nodo, cuando se encontraba en modo de bajo consumo.

\subsubsection{Resultados agrícolas.}

Después de realizar las pruebas de laboratorio, se inició la segunda fase del experimento, realizando el despliegue mostrado en la Figura 7.13. El despliegue se realizó en una parcela de almendros, con una extensión de unos $500 \mathrm{~m}^{2}$, situada en la Estación Experimental Agroalimentaria Tomás Ferro [ESEA]. En la parcela se utilizó la WSN basada en el nodo MEWiN para monitorizar el estado del suelo del cultivo durante unas 7 semanas. Como se puede observar en la Figura 7.13, se desplegaron 4 nodos MEWiN, con la composición de 1 nodo con el rol de coordinador, 1 nodo con el rol de router y 2 nodos con el rol de dispositivo final. Cada uno de estos dos últimos se desplegaron sobre el suelo y en el mismo tronco y tenían conectados 2 sensores HP2, situados en un radio de $2 \mathrm{~m}$ de distancia con el mote. Todos los sensores se enterraron a $20 \mathrm{~cm}$ de profundidad. El nodo coordinador se instaló en el interior de las oficinas junto con el servidor ejecutando el software de monitorización. El coordinador se conectó con una antena omnidireccional, de $8 \mathrm{dBi}$ de ganancia, para exteriores. Dicha antena se instaló en la fachada de las oficinas. En el nodo router se utilizó el mismo tipo de antena, y un panel solar fotovoltaico para recargar la batería.

La red anterior tenía la misma funcionalidad que la WSN de prueba que se desplegó en el laboratorio durante la fase 1. Los ZEDs enviaban, cada hora, tramas con la lectura de los sensores cada 30 minutos (configurable desde el software de monitorización) y del nivel 
de tensión de la batería. Además, el ZR enviaba, cada 30 minutos, el voltaje del panel solar y de la batería.

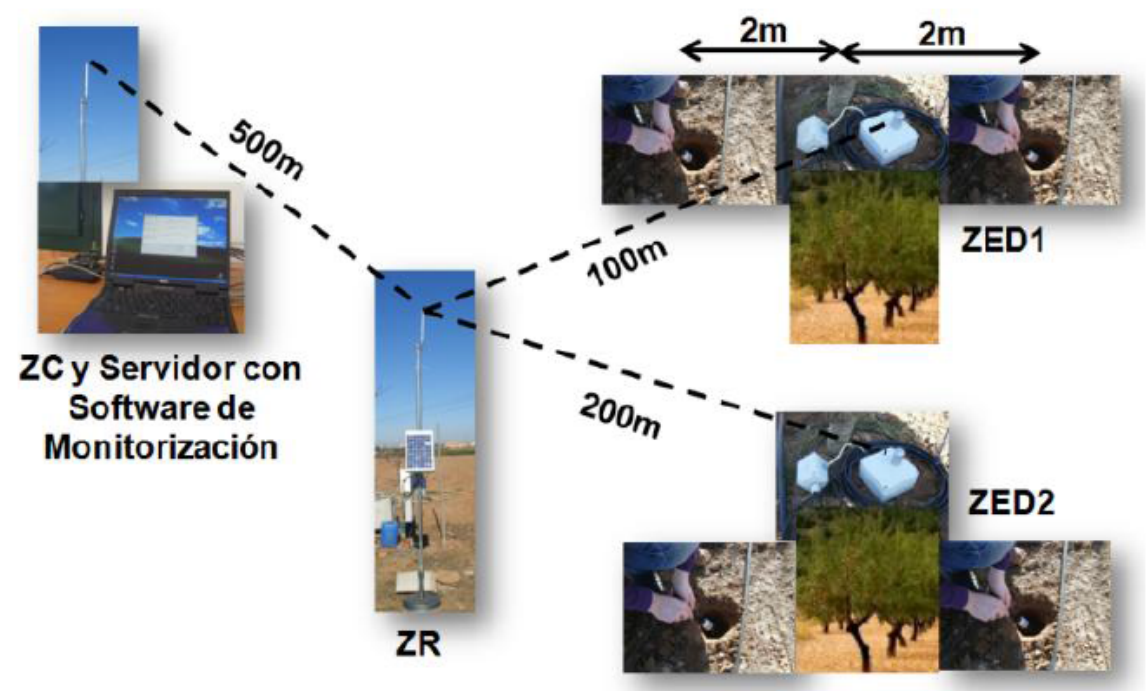

Figura 7.13. Despliegue para validar el funcionamiento de nodos MEWiN en un cultivo real.

Como resultados del despliegue realizado en la parcela de almendros, se presentan la Figura 7.14 y la Figura 7.15. La primera, muestra la evolución del voltaje de la batería del ZED2 durante 45 días. Tras siete semanas de funcionamiento, la batería tiene un voltaje de $3,7 \mathrm{~V}$, que está por encima del voltaje mínimo de 3,3V que requiere el regulador lineal de bajo dropout y de 3V de salida. La Figura 7.15 muestra la evolución del voltaje de la batería y del panel solar del nodo con el rol de ZR durante siete días. Como se puede observar, el cargador evita que la batería tenga un voltaje inferior a $4 \mathrm{~V}$.

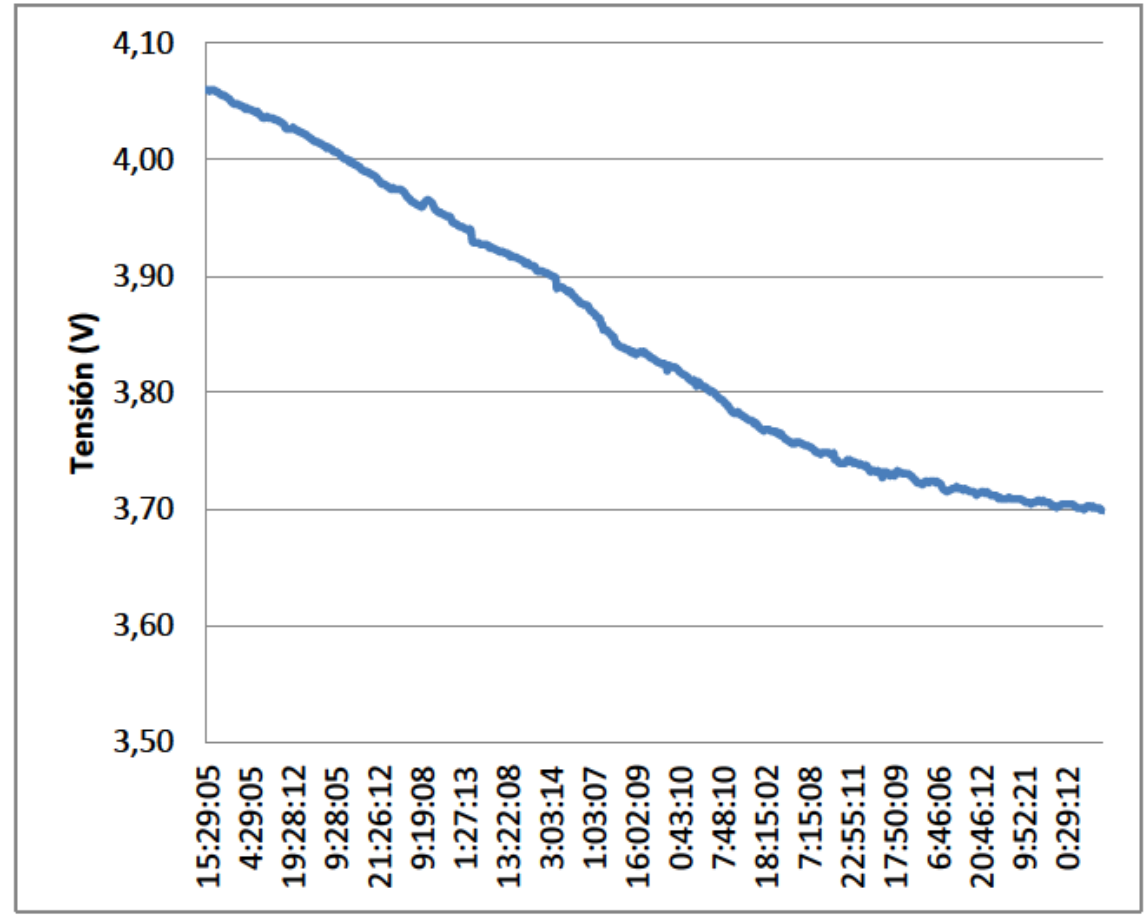

Figura 7.14. Evolución del voltaje de la batería de un ZED durante 45 días. 


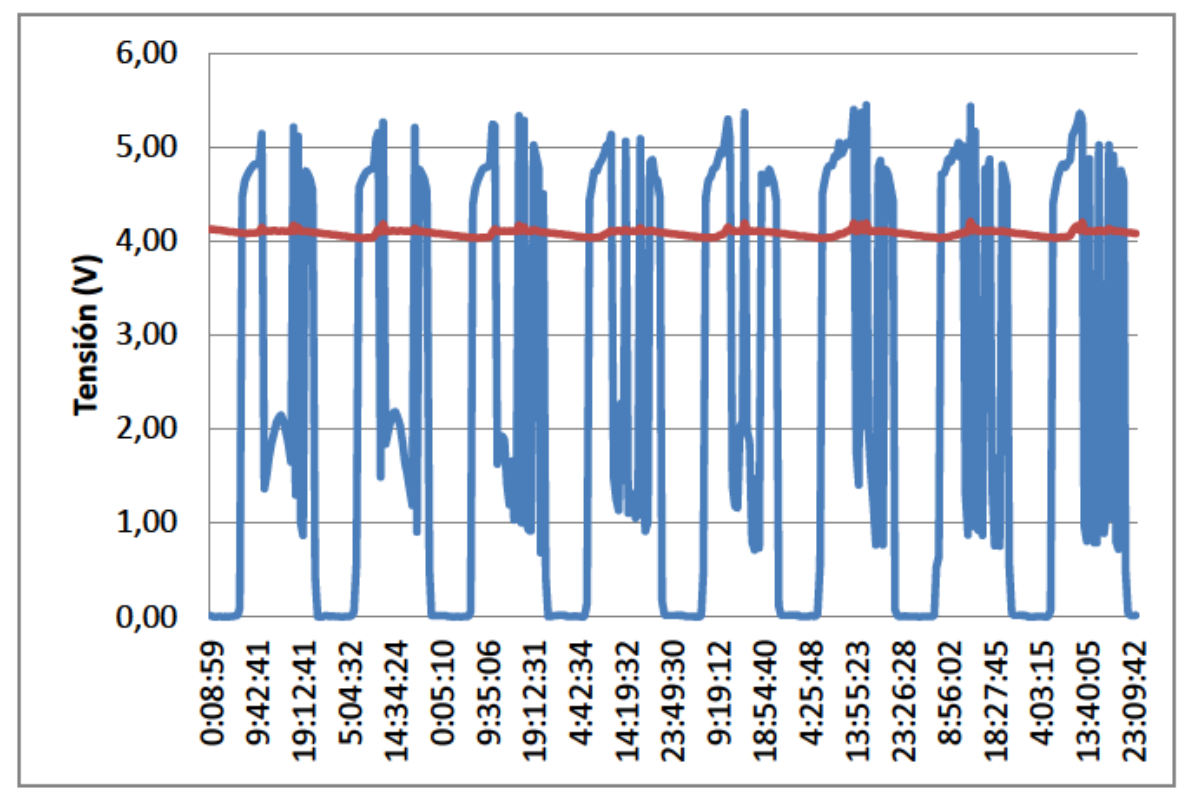

Figura 7.15. Evolución del voltaje del panel solar y de la batería del ZR durante 7 días.

Investigadores del CSIC, en concreto del "Instituto de Recursos Naturales y Agrobiología Sevilla" (IRNAS), mostraron interés por el sistema MEWiN. Por ello, en abril de 2011 se realizó un despliegue de un demostrador compuesto por 3 nodos sensores en "la Finca Experimental la Hampa", situada en Coria del Rio, Sevilla. En dicho emplazamiento, existen diferentes cultivos, entre ellos una parcela de olivos con una extensión de aproximadamente $4.000 \mathrm{~m}^{2}$. En dicha parcela, se realizó la instalación de un nodo (ver Figura 7.16) con el rol de ZC en una caseta próxima al cultivo. El dispositivo se instaló con una caja estanca en el interior de la caseta y se conectó por RS-232 con el servidor que ejecuta el software de monitorización. Además, el ZC se conectó con una antena omnidireccional de $8 \mathrm{dBi}$ de ganancia, colocada con un mástil a $5 \mathrm{~m}$ de altura, en la fachada de la caseta.

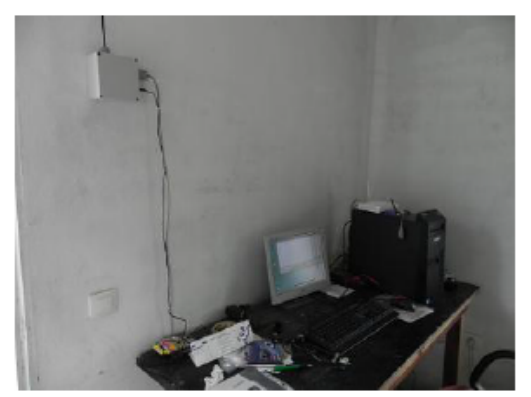

Figura 7.16. ZC instalado en el interior de la caseta próxima a la parcela de olivos.

En el cultivo se desplegaron 2 nodos sensores con el rol de ZED (ver Figura 7.17). Cada nodo sensor tiene conectados 2 dendrómetros de tipo perimetral. Como se puede observar en el detalle de la figura, el dendrómetro consta de un cable de acero que se mantiene tensado y permite variaciones con el uso de un muelle. Las variaciones del 
diámetro del tronco se traducen a valores eléctricos mediante galgas extensiométricas (situadas debajo de la resina roja).

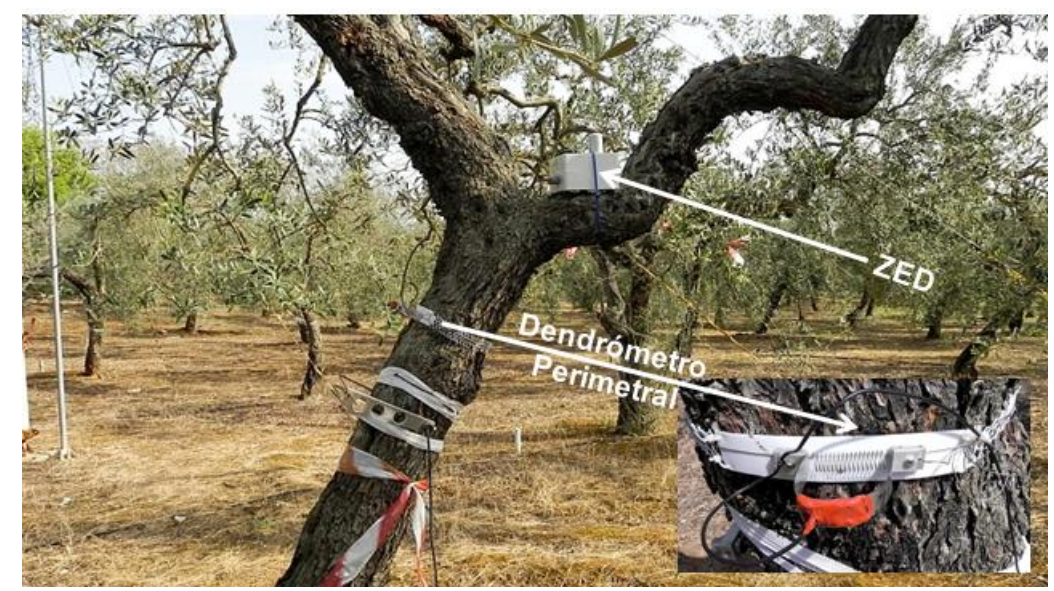

Figura 7.17. Uno de los dos ZED desplegados en la parcela de olivos.

Tras resolver unos problemas de estanqueidad y de alcances que se tuvieron con los nodos sensores durante el mes de abril, el sistema ha estado funcionando perfectamente hasta la actualidad. La Figura 7.18 muestra una captura de pantalla de la interfaz gráfica, que se implementó con $\mathrm{C}++$ Builder, de la empresa Embarcadero, en la que se reciben las tramas de los nodos sensores y se almacenan en los ficheros correspondientes. Como se puede observar en la figura, la interfaz gráfica muestra un registro $(\log )$ con las tramas recibidas, así como controles con el estado de las baterías y del número de tramas de datos y baterías recibidas. Además de visualizar gráficamente las tramas, el software las almacena en el fichero correspondiente (fichero de tramas de datos y de batería) para su posterior procesamiento y visualización. Finalmente, cabe mencionar que la misma interfaz gráfica se utilizó en las pruebas llevadas a cabo en laboratorio y en la parcela de almendros.

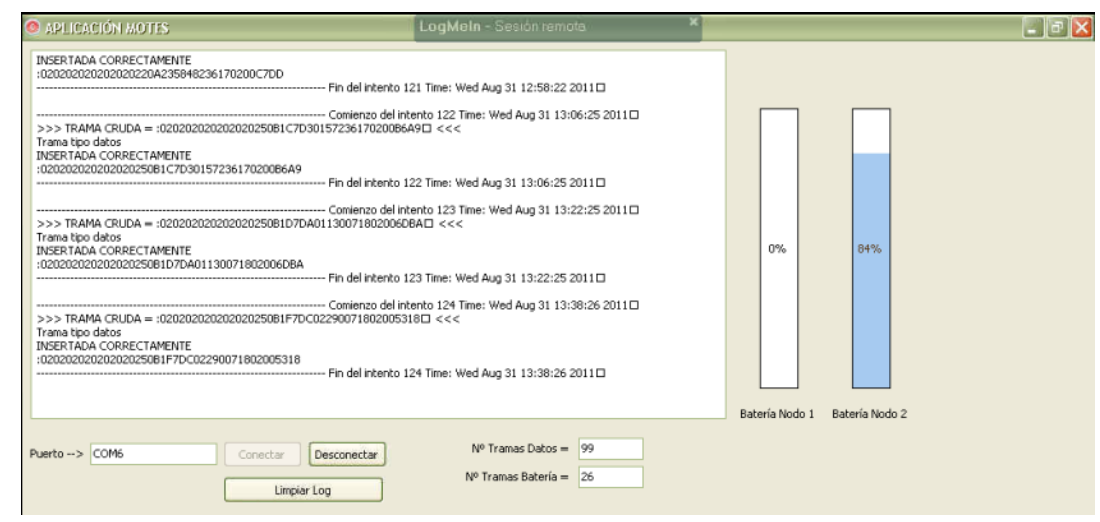

Figura 7.18. Interfaz gráfica utilizada para validar el sistema MEWiN en cultivos reales.

La Figura 7.19 muestra el registro realizado de los dos dendrómetros perimetrales de uno de los nodos sensores, durante una semana del mes de junio. Los valores están representados en unidades brutas del convertidor analógico-digital de 12 bits (entre $0 \mathrm{y}$ 
4.095). Como se comenta en el Anexo I, la gráfica permite determinar la máxima contracción diaria y la tasa de crecimiento diaria, parámetros que utilizan, posteriormente, los ingenieros agrónomos para estimar el estado y las necesidades hídricas de la planta.

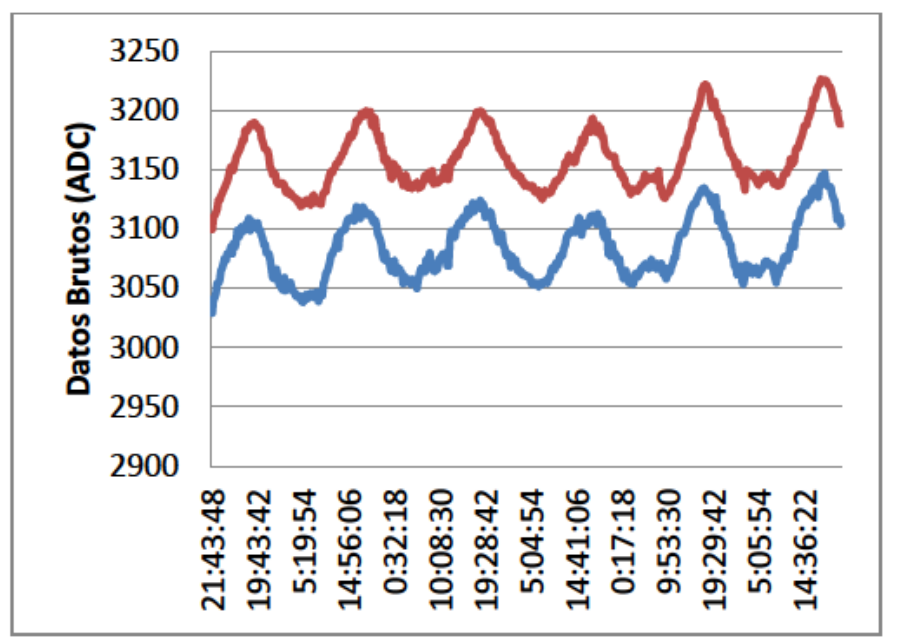

Figura 7.19. Registro semanal de dos dendrómetros perimetrales de un nodo sensor.

Además de los dos despliegues realizados en cultivos reales, en junio de 2011 se comenzó a realizar el despliegue de una WSN de 20 nodos sensores. Este despliegue se está realizando en un parral de 1 ha situado en Cieza (Región de Murcia). En dicho parral, los investigadores agronómicos están probando diferentes tratamientos de riego deficitario controlado, con objeto de proporcionar al gestor de la explotación agrícola una estrategia de riego con la que optimizar la productividad del cultivo con el mínimo uso de insumos, es decir, agua de riego entre otros.

Actualmente, se han desplegado 10 de los 20 nodos sensores que hay que instalar. En función del tipo de tratamiento, los nodos sensores tienen conectados diferentes sensores. Todos los tratamientos incorporan 2 dendrómetros radiales de tronco y, en el futuro, está previsto que incorporen 2 más para fruto. Otros sensores que incorporan todos los tratamientos, son al menos 2 sensores de potencial de tipo Watermark. Hay tratamientos que utilizan 4 sensores de tipo Watermark en lugar de 2. El último tipo de variable que se monitoriza en la mayoría de los tratamientos, es el porcentaje volumétrico de humedad en el suelo mediante el uso de 1 ó 2 sensores Enviroscan, de Sentek.

Para la fecha de defensa de la presente tesis, se espera que el despliegue esté terminado y se presentarán resultados del mismo durante la exposición.

La Figura 7.20 muestra una fotografía tomada de unos de los 10 nodos sensores instalados actualmente en el parral de Cieza. Dicha imagen muestra la situación del nodo sensor, de los 4 sensores de tipo Watermark y de los 2 dendrómetros radiales de tronco. En este nodo, dentro de la caja estanca superior, se encuentra alojada la placa base MEWiN y 
las placas de interfaz LVDTBoard y SoilBoard. La otra caja estanca tiene varios pasacables para conectar los sensores, y aloja el portabatería, de 3,7V y $4.000 \mathrm{mAh}$.

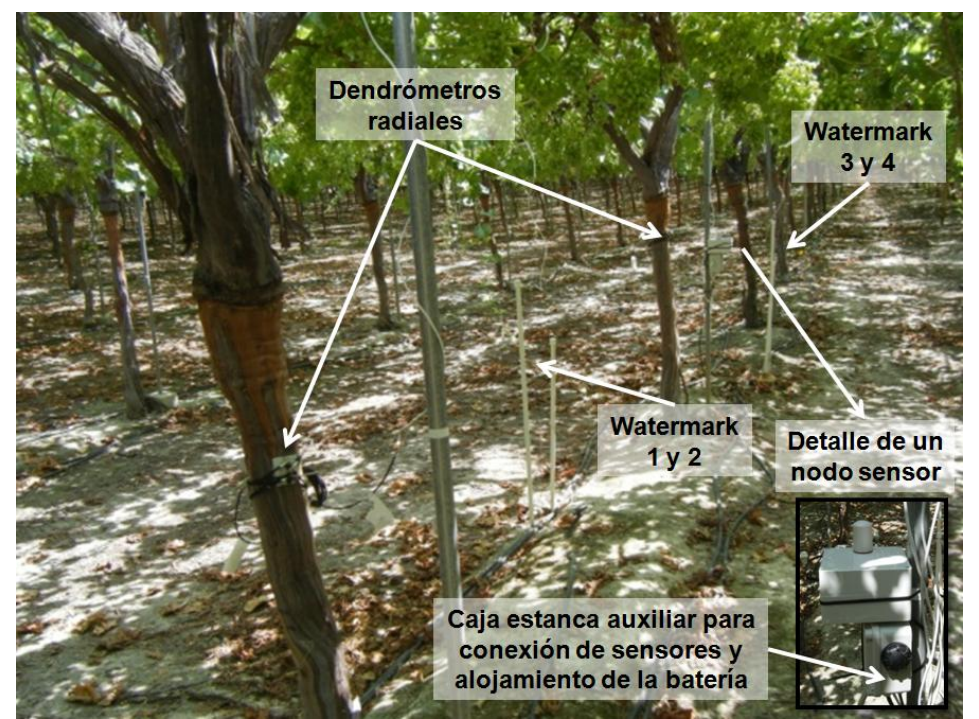

Figura 7.20. Vista de un nodo sensor desplegado en el parral de la explotación agrícola.

\subsection{Conclusiones.}

Se ha diseñado y desarrollado un nodo sensor inalámbrico multi-entorno, a nivel de producto comercial, que permite llevar a cabo tareas de monitorización y control en casos de estudio de diversa índole. El mote se puede utilizar en diferentes entornos de estudio, ya que la placa base externaliza un conjunto de interfaces de entrada y salida analógicas y digitales, que permiten utilizar el nodo con la instrumentación necesaria mediante el desarrollo de la placa de acondicionamiento necesaria. En concreto, la placa base MEWiN proporciona las siguientes interfaces analógicas y digitales de entrada y salida: 8/2 entradas/salidas analógicas, $17 \mathrm{E} / \mathrm{S}$ digitales e interfaces digitales de tipo SPI, 2 UARTs, e $\left.\mathrm{I}^{2} \mathrm{C}\right)$.

Se han diseñado e implementado las placas de interfaz SoilBoard y LVDTBoard, a nivel de producto comercial, que permiten conectar sensores de suelo y dendrómetros radiales con la placa base MEWiN.

El dispositivo se ha validado en 3 aplicaciones agrícolas reales, demostrando su robustez en condiciones reales de funcionamiento. El dispositivo también se está validando en un caso de estudio oceanográfico, en el marco de otra Tesis Doctoral que se está realizando en el grupo de investigación.

Los trabajos desarrollados en este capítulo han sido publicados en un congreso nacional [López, 2011b], y está previsto publicar el MEWiN en revistas internacionales listadas en el ISI, así como en congresos internacionales. 


\section{Chapter 8}

\section{Conclusions and Future Work.}

$\mathrm{R}$ esults and conclusions achieved in this dissertation are addressed in this chapter. The main research this work. 


\subsection{Conclusions.}

This PhD work has addressed the study and implementation of a hardware architecture, based on Wireless Sensor Networks (WSN) and their application in Precision Agriculture, specifically in real-time crops monitoring.

In arid and semiarid regions of Southern Spain, the water supply is an important problem that affects agriculture to a large extent. Development of innovative irrigation systems that efficiently use water is a priority. Therefore, farmers must find new solutions and methods that improve the irrigation programming systems, taking into account the state of both, the soil and the plants, and, as well, the climate information. All these data must be properly interpreted to decide the optimal actions to carry out. Precision Agriculture provides a suitable solution to address these problems.

Precision Agriculture aims to optimize field-level management with regard to crop science by matching farming practices more closely to crop needs. Economic advantages are obtained by boosting competitiveness through more efficient practices (e.g. better management of water usage and costs).

Information and Communication Technologies (ICT's) are used to acquire information about the crop needs. Precision Agriculture techniques use this information to achieve the proposed objectives, in particular, intensive monitoring of crops and data analysis.

Sensor Networks carry out the intensive monitoring of crops, by means of using wired or wireless nets. Wired solutions have been used since the 90s, whereas Wireless Sensor Networks are more recent (from the year 2000). In Agriculture, WSNs are more suitable because costs are reduced and the final solution is more robust, since wires can be damaged by farming machinery in the crop. The simple redeployment of the devices provides high network versatility, so it is also another advantage to consider.

Furthermore, WSNs are proving to be a promising technology, and many WSN solutions are being successfully applied into different fields, including Precision Agriculture applications. However, when the work described in this dissertation started to be developed in 2007, many theoretical references were found but very few real deployments had been carried out in Agriculture, due to the scarcity of resources and the high cost of technology in the agricultural sector. Cost reduction is necessary to guarantee the success of Precision Agriculture.

Therefore, developing new low cost hardware devices (motes) is necessary because they can measure the state of the crops in real time and provide the essential support to 
Precision Agriculture. Furthermore, these devices must be reliable in both indoor and outdoor conditions. They must be designed using an open architecture to facilitate the connection between different sensors and actuators required by the farming application.

This Thesis has focused on developing a hardware architecture that allows to improve some of the main drawbacks previously detailed. Specifically, according to the achieved results, the following particular conclusions can be enumerated:

- The proposed architecture has been tested in several crop monitoring real applications (woody and horticultural crops, among others). In order to validate this architecture, the following significant features have been taken into account: the suitability of the type of motes developed, the technology selected for the main components (micro-controller and radio module) of the motes, the sensors selected for a real-time monitoring of the crop, and the chosen communication protocol. The architecture proposed has the following advantages and disadvantages:

○ The use of a star topology and the B-MAC protocol, with the Gateway radio always powered on, makes possible that power consumption of the sensor nodes can be reduce, and the lifetime of the network increases.

- The cost of the motes case is low when a commercial waterproof case is used.

- The cost of a sensor node using radio and micro-controller header modules and the commercial radio modem XStream is very expensive.

o Time-to-market and cost are notable reasons why it is not suitable to use only a sensor node to solve a specific problem. Therefore, it is necessary to design a multifunctional sensor node, whose hardware and software configuration can be changed.

- It has been demonstrated that can be developed a robust multifunctional prototype (GAIA 2) of a commercial sensor node, whose configuration can be changed using both hardware and software, can be developed. Selecting the right configuration, the device can operate as any of specialized sensor nodes (SoilMote, Environmental-Mote, Water-Mote and Gateway) of the proposed architecture. The GAIA 2 device has the following advantages and disadvantages:

- The device requires only a certification process, in order to carry out its commercialization.

- The GAIA 2 provides several I/O interfaces both analogical and digital; therefore, it can be used with most of the sensors and actuators used in Precision Agriculture. Specifically, the mote has both $0-2.5 \mathrm{~V}$ and $4-20 \mathrm{~mA}$, analogical inputs, digital inputs/outputs, $0-2.5 \mathrm{~V}$ analogical outputs and $\mathrm{I}^{2} \mathrm{C}$ and SDI-12 digital interfaces. 
- The waterproof case for protecting the GAIA 2 board and the XStream commercial radio module used in a Gateway are expensive. In order to to reduce the mote cost, it is necessary to design a new device using alternative solutions for the case and the long range communications.

- It has been demonstrated that a Multi-Environment Wireless sensor Node (MEWiN) prototype can be designed, implemented and validated as a robust commercial product. This node is capable of carrying out monitoring and control tasks in both Precision Agriculture and other fields. MEWiN has been developed to reduce costs thanks to the casing design and the use of the radio modem XStream. The interface boards SoilBoard and LVDTBoard have been developed as commercial products, in order to validate the sensor node under real conditions. The MEWiN sensor node has been validated in three experimental crops. They are used to connect standard soil sensors as Hydra Probe II and radial dendrometers to the main board. The MEWiN device has the following advantages and disadvantages:

O Thanks to the open architecture of the MEWiN Mainboard design, the mote can be used in a specific environment by designing a specific interface board.

- MEWiN provides a temporary data storage system, based on a real-time clock and SD memory card, very useful when the wireless communication link fails or is lost.

○ The MEWiN Mainboard provides several analogical and digital I/O interfaces. Therefore, it can be used with most of the sensors and actuators typically used in Precision Agriculture. Specifically, the device provides the following analogical and digital interfaces: 8/2 analogical inputs/outputs, 17 digital inputs/outputs and digital interfaces (SPI, 2 UARTs and $\mathrm{I}^{2} \mathrm{C}$ ).

- The device has not included embedded sensors for monitoring the environmental conditions. The communication system will be improved by combining these sensors with the temporary data storage system already implemented.

- The MEWiN board is protected by a commercial and cheaper watertight casing whose mechanization is very laborious.

- The monitoring software implemented in this work is adequate to validate the hardware boards designed in this Thesis, but it is not yet suitable for commercial purposes.

- The MEWiN platform uses last generation chips (CC2520, CC2591 and MSP430F2618) from Texas Instruments, but it is not compatible with open source operating systems for embedded systems as TinyOS or Contiki. 
According to the results obtained in this Thesis, the following general conclusions can be enumerated:

- The cost of a fully functional sensor node is very expensive because in several applications the quality and the price of the individual standard sensors are excessive.

- The agricultural sector has many difficulties and the technology developed for this sector must be robust and low cost.

- Device autonomy is a critical issue and it is necessary to explore new renewable energy sources, in order to recharge the motes batteries.

$\circ$ The farmers demand products adapted to their needs and knowledge, and they usually prefer not to interact directly with the devices, that is, the provided technology should be transparent for the final users.

\subsection{Contributions.}

In this work, the main proposed goals, described in the chapter "Introduction", are accomplished. Next, the main contributions of this $\mathrm{PhD}$ work are enumerated:

- A study related to the existing WSN-based solutions applied to Precision Agriculture has been carried out, emphasizing both commercial hardware solutions and hardware architectures developed by research centres.

- An extensive review, regarding commercial agronomic instrumentation for water, soil and crops monitoring has been performed. From this study, a set of suitable sensors have been selected, in order to monitor the parameters required by the farming applications.

- A general and open architecture based on WSNs has been proposed, in order to solve the irrigation optimization problem. This architecture consists of several specialized nodes (Soil-Mote, Environmental-Mote and Water-Mote) and several auxiliary devices (Gateway, Repeater and Base-Station). Nodes are connected to the farm office using these devices. Each device was implemented, for the first time, from the MSP430F1611 microcontroller header modules and the CC2420 radio module. A star topology has been selected, together with the B-MAC protocol and the Gateway radio module always in active mode. This architecture has proved to be very efficient, since sensors nodes consumption can be reduced and the network's lifetime can be increased. As the system worked properly under real conditions, it was faced up the design of a more robust system. Consequently, the GAIA SoilMote was developed.

- A robust sensor node (called GAIA Soil-Mote) for horticultural applications has been designed, implemented and validated. This task provides experience about the 
design of hardware circuits that join a radiofrequency module and the SMD components, which are necessary to deal with more complex designs. This mote is the pre-commercial version of the final Soil-Mote. The following parameters can be measured using this device: soil temperature, soil moisture percentage and soil conductivity. The sensor provides a SDI-12 interface and the designed software has been implemented using the open source TinyOS operating system (version 2). The current version of the software supports the connection of two Hydra Probe II devices, from Stevens Inc. In addition, the software can be adapted to support any kind of SDI-12 sensor. At the same time, the hardware can be adapted to support any other kind of interface.

- A robust multifunctional mote (GAIA 2) for real farming applications has been designed, implemented and tested. This sensor node has been developed as a commercial product, whose configuration can be changed using both hardware and software. GAIA 2 PCB almost completely consists of SMD components, even the short range radio module CC2420. Only the long range XStream radio modem is a commercial device. The mote provides several I/O interfaces both analogical and digital, therefore, it can be used with most of the sensors and actuators used in Precision Agriculture. Specifically, the mote has $0-2.5 \mathrm{~V}$ analogical inputs and 4$20 \mathrm{~mA}$, digital inputs/outputs, $0-2.5 \mathrm{~V}$ analogical outputs and $\mathrm{I}^{2} \mathrm{C}$ and SDI-12 digital interfaces.

- Finally, a multi-environment wireless sensor node (MEWiN) has also been designed, implemented and validated as a commercial product. This node is capable of carrying out monitoring and control tasks in both Precision Agriculture and other fields. MEWiN has been developed to reduce costs (thanks to a new design of the previous case and the use of the radio modem XStream), and to provide a temporary data storage system, very useful when the wireless communication link fails. The mote can be used in a specific environment by designing a specific interface board. The necessary instrumentation can be connected to the sensor node MEWiN using this board. In particular, the main MEWiN board provides the following analogical and digital interfaces: 8/2 analogical inputs/outputs, 17 digital inputs/outputs and digital interfaces (SPI, 2 UARTs and I2C). At a first stage, the interface boards Vegeboard and RouterBoard were developed as laboratory prototypes, to validate the first MEWiN prototype. Next, the interface boards SoilBoard and LVDTBoard have been developed as commercial products. They are used to connect soil sensors and radial dendrometers to the main board. Several experiments in Precision Agriculture demonstrate that the wireless sensor node MEWiN is efficient and reliable. Currently, apart from the Precision Agriculture applications, the node is being validated in an oceanographic application by other researchers. 


\subsection{Dissemination of the results.}

The work described in this dissertation has generated several contributions in indexed international journals and international and local conferences. The ISI international journals are enumerated as follows:

- J.A. López, F. Soto, J. Suardiaz, A. Iborra, J. Longares, J.A.Vera. "Wireless Sensor Networks for precision horticulture in Southerm Spain". Internacional Journal of Computer and Electronics in Agriculture, volumen 68, páginas 25-35. 2009. FI 1.431, Q2.

- Juan A. López, F. Soto, P. Sánchez, A. Iborra, J. Suardíaz, J.A.Vera. “Development of a Sensor Node for Precision Horticulture”. Sensors, volumen 9, páginas 32403255. 2009. FI 1.771, Q1.

- J.A. López, A.J. García, F. Soto, A. Iborra, F. García, J. García. "Design and Validation of a Wireless Sensor Network Architecture for Precision Horticulture Applications". Precision Agriculture, volumen 12, páginas 280-295. 2011. FI 1.683, Q1.

- Emanuel Peres, Miguel A. Fernandes, Raul Morais, Carlos R. Cunha, Juan A. López, Samuel R. Matos, P.J.S.G. Ferreira, M.J.C.S. Reis. "An autonomous intelligent gateway infrastructure for in-field processing in precision viticulture". Internacional Journal of Computer and Electronics in Agriculture, volumen 78, páginas 176-187. 2011. FI 1.431, Q2.

- Cristina Albaladejo, Pedro Sánchez, Andrés Iborra, Fulgencio Soto, Juan A. López and Roque Torres. "Wireless Sensor Networks for Oceanographic Monitoring: A Systematic Review”. Sensors, volumen 10, páginas 6948-6968. 2009. FI 1.771, Q1.

The main non ISI journals are enumerated as follows:

- J. A. López Riquelme, F. Soto, J. Suardíaz, A. Iborra. "Red de Sensores Inalámbrica para Agricultura de Precisión”. Telecoforum 2008. ISSN: 1698-2924. Marzo 2009.

- Albaladejo Pérez, Cristina, López Riquelme, Juan Antonio, Navarro Hellín, Honorio, Rubio Aparicio, Jesús. "Sistema para monitorizar entornos marinos basado en redes sensores inalámbricas". IV Jornadas de Introducción a la Investigación de la UPCT. ISSN: 1888-8356. 2011.

Conferences published in Lecture Notes:

- Juan A. López, Fulgencio Soto, Andrés Iborra, Pedro Sánchez, Juan Suardíaz Muro. "Design and Implementation of a Wireless Sensor Network for Precision Horticulture". First International Conference on Sensor Applications, 
Experimentation, and Logistics (SENSAPPEAL 2009). Páginas 27-42. Atenas, Grecia.

Others international and national conferences:

- J.A. López, F. Soto, J. Suardíaz, P. Sánchez, A. Iborra, J.A. Vera. "Development of a Mote for Horticulture based on the SDI-12 standard". 35th Annual Conference of the IEEE Industrial Electronics Society (IECON 2009), Porto, Portugal.

- López Riquelme, J.A., Soto, F., Suardíaz, J., Iborra, A. "Desarrollo de una Red de Sensores para Agricultura de Precisión”. Seminario Anual de Automática, Electrónica Industrial e Instrumentación (SAAEI 2008), Cartagena, Murcia.

- J.A. López Riquelme, F. Soto, J. Suardíaz, A. Iborra. "GAIA: un Mote para la Monitorización de Parámetros del Suelo Basado en el Estándar SDI-12”. Seminario Anual de Automática, Electrónica Industrial e Instrumentación (SAAEI 2009), Leganés, Madrid.

- J.A. López Riquelme, F. Soto, C. Albaladejo, A. Iborra, J. Suardíaz. "GAIA 2: Un Mote Multifuncional para Agricultura de Precisión”. Seminario Anual de Automática, Electrónica Industrial e Instrumentación (SAAEI 2010), Bilbao, España.

- J.A. López Riquelme, F. Soto Valles, R. Torres Sánchez, C. Albaladejo Pérez, J. Suardíaz Muro, H. Navarro Hellín. "MEWiN: un nodo sensor inalámbrico multientorno. Validación en Agricultura de Precisión”. Seminario Anual de Automática, Electrónica Industrial e Instrumentación (SAAEI 2011), Badajoz, España.

The work presented in this dissertation has been supported by the firm Edosoft Factory S.L., by the research center Centro de Investigação e de Tecnologias AgroAmbientais e Biológicas of the Universidade de Trás-os-Montes e Alto Douro (Portugal), and the following research projects: RIMSI, Ministerio de Industria, Comercio y Turismo (ref. FIT-340000-2006-202), MOTE-FRUT (ref. 08850/PI/08), SICORI (ref. 08754/PI/08), Fundación Séneca de la Región de Murcia, and EXPLORE, Comisión Interministerial de Ciencia y Tecnología (TIN2009-08572).

\subsection{Future work.}

The use of Precision Agriculture to achieve optimal crop production involves to carry out the following stages:

1. Monitoring crops by acquiring data from sensors.

2. Analyzing obtained data.

3. Making decisions. 


\section{Acting.}

The first stage is carried out using the hardware architectures based on WSNs developed in this Thesis.

In order to carry out the acting stage, it is necessary to gather information about commercial actuators, select the most suitable devices and design the interface boards to connect the actuators to the boards implemented in this Thesis.

The second and third stages must also be tackled. New future works will address them together with the improvement of the hardware architectures developed in this work. In order to achieve these new goals, the following issues must be taken into account:

- From the information acquired during the monitoring step, it is possible to design a system capable of making decisions autonomously. Control Engineering theory is useful in solving this problem. The system can be controlled by using very simple PID controllers, or other more complex controllers, such as predictive, fuzzy or expert systems.

- The cost of a fully functional sensor node is very expensive, because in several applications the quality and the price of the sensors are excessive. Therefore, new cheaper sensors for precision agriculture should be designed.

- The farmers demand products adapted to their needs and knowledge, and they don't want to directly interact with the devices. For this reason, it is necessary to design and provide simple services to the farmers in order to manage the crop systems using only a smartphone, or any similar single device.

- The lifetime of the network is a critical issue and it is necessary to explore new renewable energy sources in order to charge the motes batteries. One way could be to extract energy from the waves available in the environment.

- The connectivity of the motes deployed in a crop is another important issue. The concept of "Internet of things" can make this task easy, since one of the aims of the systems based on ubiquitous computing is to connect heterogeneous wireless devices. In order to deal with this problem, it is necessary to use the IP addresses based on the IPv6 protocol and, therefore, the software of the devices must implement this communication protocol. TinyOS and Contiki can be extended to support the hardware architectures presented in this Thesis.

- The connectivity of the sensors and actuators with the motes also is another important issue. The IEEE 1451 Smart Transducer Interface Standard promotes the plug and play connection between the sensors and/or actuators with the devices. Then, the implementation of this standard on the MEWiN platform should be taken into account in the next future works. 
The hardware boards designed and implemented in this work have some deficiencies. Several future works are aimed at solving them:

- Currently, the MEWiN board is protected by a commercial watertight casing where the mechanization is very laborious. The design of a specific casing fulfilling certain requirements will be addressed.

- The monitoring software implemented in this work is functional enough to validate the hardware boards designed in this Thesis but it is not yet suitable for commercial purposes. The graphical interface of the application must take into account the needs of people who work in the farming sector. Therefore, it is necessary to improve the high level software architecture of the system.

- Device autonomy is a critical issue. Usage of renewable energy sources, such as hydro generators, to charge the motes batteries is a future goal to achieve.

- Wireless communications are very dependent on atmospheric conditions. The MEWiN board has a real-time clock and an SD memory card to locally store the monitoring information. However, the node does not include sensors on the casing to detect adverse environmental conditions. These sensors can be useful in making decisions about storing the data in the SD memory card without sending them via radio during adverse weather conditions. This improvement can reduce the device consumption and it will be also addressed in a future work. 


\section{Materiales, Herramientas y Métodos.}

$A$

la hora de diseñar y desplegar una red de sensores inalámbrica, es necesario escoger un conjunto de materiales, utilizar un conjunto de herramientas y seguir una serie de métodos para desarrollar la arquitectura necesaria. Este anexo recoge la experiencia que se ha acumulado durante el desarrollo de esta tesis en relación con estos aspectos. En primer lugar, se describen los principales elementos que se han contemplado para seleccionar la tecnología necesaria para desarrollar los principales componentes de la red de sensores propuesta. A continuación, se describen las herramientas que han sido necesarias de utilizar tanto para el diseño y desarrollo del hardware/software, así como para el despliegue. Finalmente, se describirán los métodos necesarios para el diseño de la red. 


\section{I.1 Introducción.}

Como se puede observar en la WSN (ver Figura l.1) que ha servido como caso de estudio para el desarrollo de la presente tesis, para llegar a desplegar una red de este tipo son necesarios un conjunto de elementos que, habitualmente, estarán presentes en la mayoría de ellas. Estos elementos son los siguientes: los nodos sensores o motes con las antenas apropiadas, la instrumentación necesaria para adquirir y acondicionar las señales asociadas al ámbito del problema, los gateways, los sumideros, los repetidores y los sistemas de alimentación.

En este anexo se recoge la experiencia que se ha acumulado durante la realización de la presente tesis en la selección de la instrumentación agrícola necesaria, así como en el diseño y desarrollo de los diferentes elementos que constituyen la red de sensores y el despliegue de la misma. Para ello, en primer lugar se revisa el estado actual de la instrumentación agronómica utilizada habitualmente en agricultura de precisión. A continuación, se presentan los principales componentes hardware que se han considerado en el diseño de los nodos sensores, gateways y elementos auxiliares necesarios para las comunicaciones. Posteriormente, también se recogen las principales herramientas que ha habido que utilizar tanto en el diseño hardware/software de los diferentes dispositivos, así como para su despliegue en campo. Finalmente, se recogen los métodos empleados en el diseño de la red de sensores propuesta.

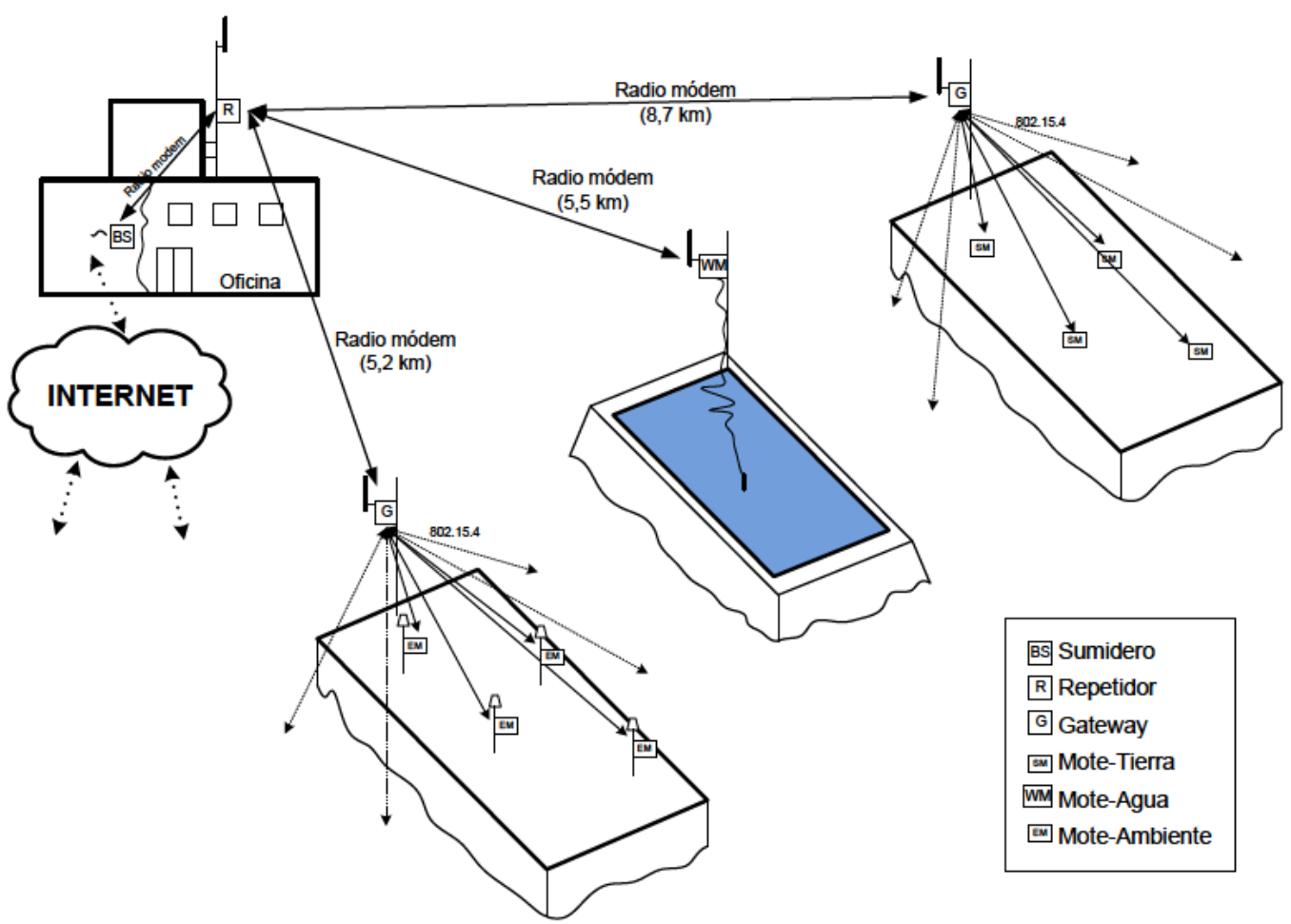

Figura I.1. WSN que ha servido como caso de estudio. 


\section{I.2 Instrumentación agronómica.}

Como se ha visto en el Capítulo 2, la agricultura de precisión en la fase de recolección de datos requiere el seguimiento de diferentes parámetros muy relacionados con el tipo de cultivo que se esté explotando. En consecuencia, se abre un amplio espectro de variables agronómicas a monitorizar y, por tanto, afecta a la elección de los sensores apropiados para realizar la adquisición de dichas variables. Cae fuera del ámbito de esta tesis hacer una recopilación de todo este abanico de sensores. En esta sección se presenta la instrumentación más habitual para la monitorización de las variables agronómicas críticas en cultivos similares a los enfocados en los casos de estudio analizados en esta tesis y llevados a cabo en su mayor parte en la Estación Experimental Agroalimentaria "Tomás Ferro" (ESEA en adelante). En todos ellos, es necesario obtener información del agua, del suelo, del ambiente y de las plantas usando instrumentación apropiada. Los datos muestreados son necesarios para que un sistema de decisión, humano o autónomo, llegue a responder a las preguntas de cuándo regar y cuánto riego aplicar al cultivo, además de poder predecir la presencia de una plaga, entre otras cosas.

\section{I.2.1 Sensores medioambientales.}

Para monitorizar las variables climáticas y medioambientales, en la ESEA se utiliza una estación meteorológica (ver Figura I.2) equipada con sensores para medir las siguientes variables: temperatura y humedad relativa del aire, radiación solar global, radiación neta, pluviometría, así como la velocidad y la dirección del viento.

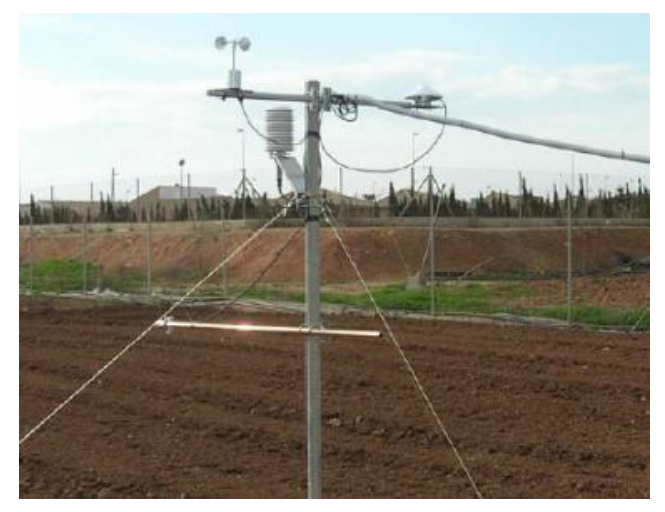

Figura I.2. Estación climática de la ESEA y distribución de los distintos sensores.

La temperatura y humedad relativa del aire se estima a partir de una sonda HMP45C de Vaisala [Vaisala], colocada a $2 \mathrm{~m}$ de altura del suelo. Esta sonda consta de dos sensores (Figura I.3): un sensor de temperatura ambiente Pt 1000 y otro de humedad relativa modelo HUMICAP 180. El primero está basado en una RTD (Resistance Temperature Detector) de platino y se fundamenta en la variación de la resistencia del metal conductor 
con la variación de la temperatura. El HUMICAP 180 consta de un polímero de plástico que tiende a absorber la humedad, y que cambia sus características eléctricas en función de la humedad ambiental. Además, la sonda HMP45C lleva como accesorio un protector, a fin de evitar el efecto de la radiación directa y difusa sobre el sensor de temperatura y, por tanto, los posibles efectos de histéresis que podrían producirse.

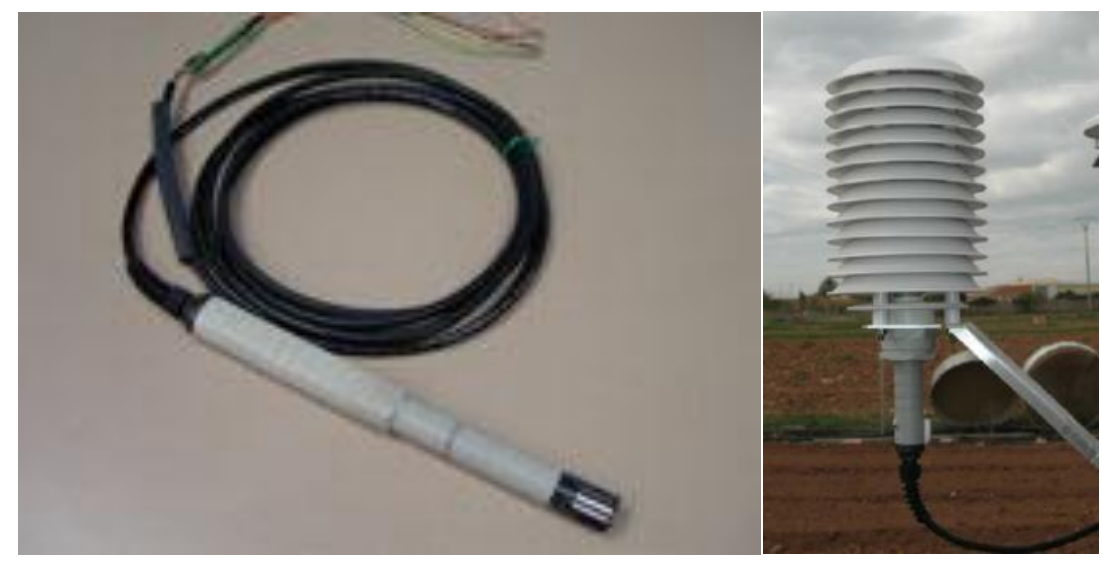

Figura I.3. Sensor de temperatura y humedad relativa del ambiente HMP45C de Vaisala.

La radiación solar global fue registrada mediante un piranómetro modelo CMP3 (Figura I.4), comercializado por Kipp \& Zonen [KippZonen]. La parte superior del sensor está formada por una fotocélula con un recubrimiento especial de silicona, asegurando una respuesta en la banda espectral entre 300 y $2800 \mathrm{~nm}$. El sensor pasivo proporciona una salida entre 0 y $20 \mathrm{mV}$, en función de la radiación recibida.

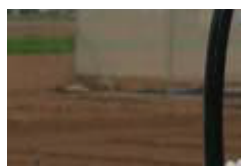

Figura I.4. Piranómetro modelo CMP3 de Kipp \& Zonen instalado en la estación.

La radiación neta fue registrada a partir de un radiómetro neto modelo Q-7.1 (ver Figura I.5), comercializado por Campbell Scientific [CampbellScientific]. Este sensor registra la suma algebraica de todas las longitudes de onda (componentes de onda corta y onda larga) entrantes y salientes. La radiación entrante se compone de la radiación solar directa y 
de la difusa más la radiación de onda larga de la bóveda celeste. La radiación saliente está compuesta por la radiación solar reflejada más la componente terrestre de onda larga, emitida por la superficie.

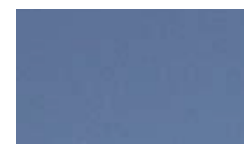

Figura I.5. Radiómetro neto modelo Q-7.1.

La precipitación fue registrada mediante un sensor FSS500 (Figura I.6), comercializado por Campbell Scientific. Éste consiste en un balancín que oscila sobre su eje, de forma que, cada vez que gira para verter el agua, cierra el circuito, hecho que es registrado por el sistema de adquisición de datos. Este fundamento se denomina la técnica de cazoletas basculantes, en la que el cierre del contacto de cada basculación puede ser registrado por la unidad de procesamiento. El sensor realiza medidas a partir de incrementos de $0.1 \mathrm{~mm}$. El diseño del sensor permite recoger en un área de $500 \mathrm{~cm}^{2}$ y ha sido diseñado con objeto de minimizar el efecto de que el flujo de aire disminuya la superficie de recogida. En concreto, el diseño se encuentra muy próximo al perfil "ideal" teórico que establece la oficina meteorológica británica.

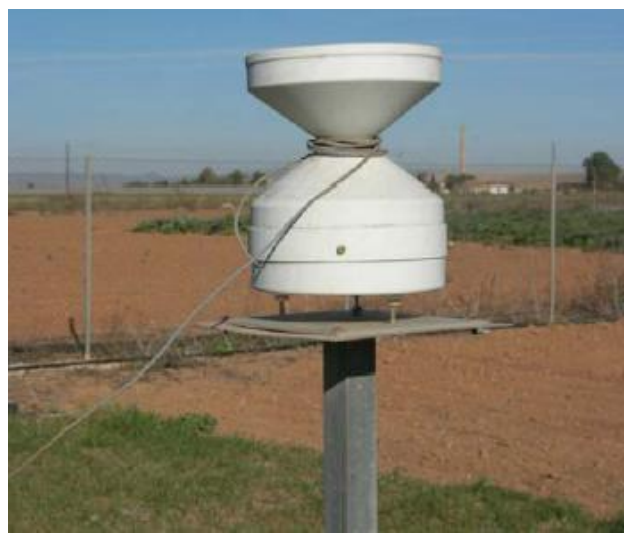

Figura I.6. Sensor FSS500 para registrar la pluviometría.

Para la determinación de la velocidad del viento se empleó un anemómetro de cazoletas modelo A100R fabricado por la empresa británica Vector Instuments [VectorInstruments]. El sensor se situó sobre la estación a 2 metros de altura (Figura I.7). Su 
fundamento se basa en un imán, el cual gira sobre el eje del molinete generando un campo variable, lo que provoca el cierre de un contacto, tipo lengüeta, para cada revolución del rotor. Este sensor no precisa de alimentación externa, ya que es alimentado por la propia energía eólica.

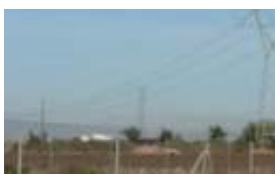

Figura I.7. Sensores de velocidad y dirección del viento.

La medida de la dirección del viento se realiza con una veleta modelo W200P, comercializada también por Campbell Scientific. Se trata de un potenciómetro circular de $1 \mathrm{k} \Omega$, en el que la dirección es función del valor resistivo del mismo (Figura 4.8). El rango de medida es de $0-360^{\circ}$, con una precisión de $\pm 2^{\circ}$.

Además de los sensores anteriores, en la parcela de almendros hay instalado un psicrómetro modelo H301 (Figura 1.8) de Vector Instruments. Se trata de un sensor de humedad aspirada usando el método de los bulbos seco y húmedo (dry and wet bulbs), que permite realizar una medida fiable de la humedad y la temperatura del ambiente. Los elementos sensores son dos resistencias de tipo RTD, con valor nominal de $100 \Omega$, una de ellas humedecida constantemente por agua destilada a través de un cordón. Finalmente, cabe destacar que se debe tener siempre activo un ventilador de $12 \mathrm{~V}$ y $350 \mathrm{~mA}$.

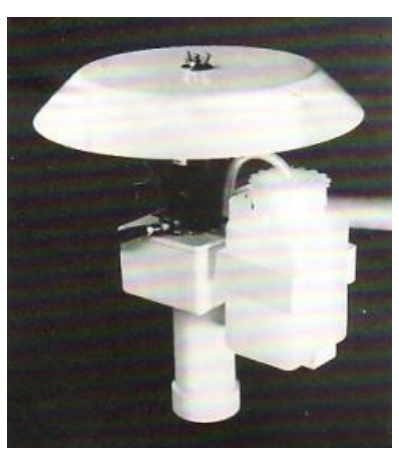

Figura I.8. Psicrómetro H301 de Vector Instruments. 
Las principales características de los sensores descritos anteriormente aparecen descritas en la Tabla I.1.

\begin{tabular}{|c|c|c|c|c|c|}
\hline $\begin{array}{l}\text { Variable } \\
\text { Climática }\end{array}$ & Rango de Señal & Tipo de Salida & Precisión & Sensor & $\begin{array}{c}\text { Tensión de } \\
\text { Alimentación }\end{array}$ \\
\hline Temperatura & $0-1 \mathrm{~V}$ & $-40-60^{\circ} \mathrm{C}$ & $\pm 2^{\circ} \mathrm{C}$ & Pt 1000 & $12 \mathrm{~V}$ \\
\hline $\begin{array}{l}\text { Humedad } \\
\text { Relativa }\end{array}$ & $0-1 \mathrm{~V}$ & $0-100 \%$ & $\pm 1 \%$ & HUMICAP 180 & $12 \mathrm{~V}$ \\
\hline Radiación Global & $0-20 \mathrm{mV}$ & $0-2000 \mathrm{Wm}^{-2}$ & $\pm 5 \%$ & CMP3 & Pasivo \\
\hline Radiación Neta & $-25-100 \mathrm{mV}$ & $-290-1160 \mathrm{Wm}^{-2}$ & $1 \%$ & Q-7 1 & Pasivo \\
\hline Lluvia & $\mathrm{N} / \mathrm{A}$ & $\begin{array}{c}\text { Cierre contacto } \\
\text { cada } 0,2 \mathrm{~mm}\end{array}$ & $0,2 \mathrm{~mm}$ & FSS500 & Pasivo \\
\hline $\begin{array}{l}\text { Velocidad del } \\
\text { Viento }\end{array}$ & $\begin{array}{l}\text { Cierre de contacto } \\
\text { por revolución }\end{array}$ & $0,2-75 \mathrm{~ms}^{-1}$ & $1 \% \pm 0,1 \mathrm{~ms}^{-1}$ & A100R & Pasivo \\
\hline $\begin{array}{l}\text { Dirección del } \\
\text { Viento }\end{array}$ & $\begin{array}{l}\text { Potenciómetro En } \\
\text { función del valor } \\
\text { de la alimentación }\end{array}$ & $0-360^{\circ}$ & $\pm 2^{\circ}$ & W200P & Máximo $20 \mathrm{~V}$ \\
\hline
\end{tabular}

Tabla I.1. Características principales de los sensores de la estación climática de la ESEA.

\section{I.2.2 Sensores de suelo.}

Para medir el estado hídrico del suelo en la ESEA se utilizan sensores para monitorizar el potencial matricial y el contenido de humedad en el suelo. Con los sensores de potencial matricial se realiza una medida indirecta de la cantidad de agua presente en el suelo a partir de la depresión o vacío existente. Básicamente, si las raíces de la planta están absorbiendo agua del suelo y el suelo está seco, el instrumento medirá una depresión que no estará presente cuando el suelo esté húmedo. Por otro lado, los sensores que miden el contenido de humedad en el suelo realizan una medida directa del porcentaje volumétrico de agua en la posición en la que está instalado el sensor.

Para medir el potencial matricial en la ESEA se usan baterías de tensiómetros con vacuómetro, tipo Bourdon, modelo R, comercializado por Irrometer [Irrometer] y sensores de matriz granular Watermark modelo 200SS, también comercializados por Irrometer. Los sensores están instalados a 30, 60 y $90 \mathrm{~cm}$ de profundidad y a $30 \mathrm{~cm}$ del emisor. Los tensiómetros, tienen un rango de medida de 0 a $100 \mathrm{cb}$, que se puede visualizar en un medidor analógico, de manera que $0 \mathrm{cb}$ indican que en el suelo no existe vacío o depresión y no es necesario activar el sistema de riego, y una lectura de 100cb representa una elevada presencia de vacío en el suelo, debida a la absorción de la planta en ausencia de agua. El 
sensor incluye un transductor electrónico modelo RSU con salida en lazo de corriente 4$20 \mathrm{~mA}(0-100 \mathrm{cb})$, que requiere de una tensión de alimentación comprendida entre 12 y $24 \mathrm{Vcc}$ para su correcto funcionamiento. Los tensiómetros tienen una punta porosa en contacto con el terreno, y es necesario rellenar la caña tensiométrica con agua o agua destilada y una solución que evita la formación de algas. Así, cuando el suelo está seco, el líquido del tensiómetro sale a través de la punta porosa y se va incrementando la presión. Cuando el terreno se riega, el tensiómetro absorbe líquido a través de la punta porosa y disminuye la presión. Por motivos de robustez, los tensiómetros anteriores fueron sustituidos por los Jet Fill modelo 2725ARL (Figura I.9) de Soilmoisture [Soilmoisture] y el transductor de corriente 5301-B.5, con características similares al anterior.

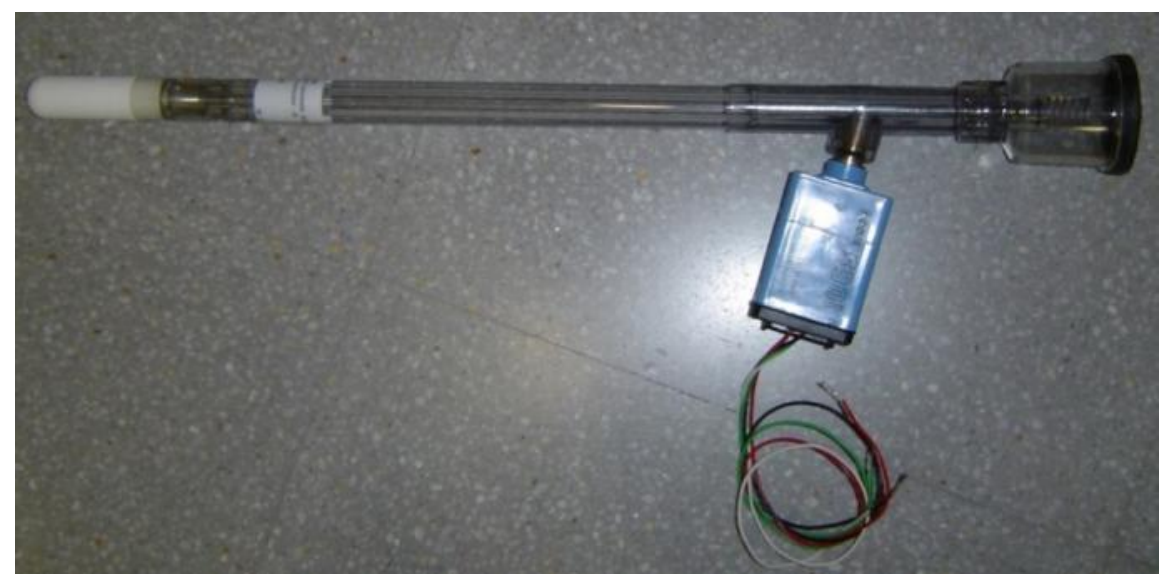

Figura I.9. Tensiómetro Jet Fill 2725ARL del Soilmoisture.

Los sensores de tipo Watermark (Figura I.10) son más económicos que los anteriores (aproximadamente, un coste 10 veces inferior). Su funcionamiento está basado una resistencia que cambia su valor debido a los cambios de tensión en el suelo con la variación del agua presente. El sensor tiene un rango de salida de 0-200cb y, al ser resistivo, requiere de la conexión en un puente o semipuente de Wheatstone para obtener las medidas. Cabe destacar que, actualmente, existe una versión que ofrece salida en tensión (modelo 200SS$\mathrm{V}$ ) en un rango de 0 a 2,8V (0-239cb), para lo que requiere de una excitación de $5 \mathrm{Vcc}$.

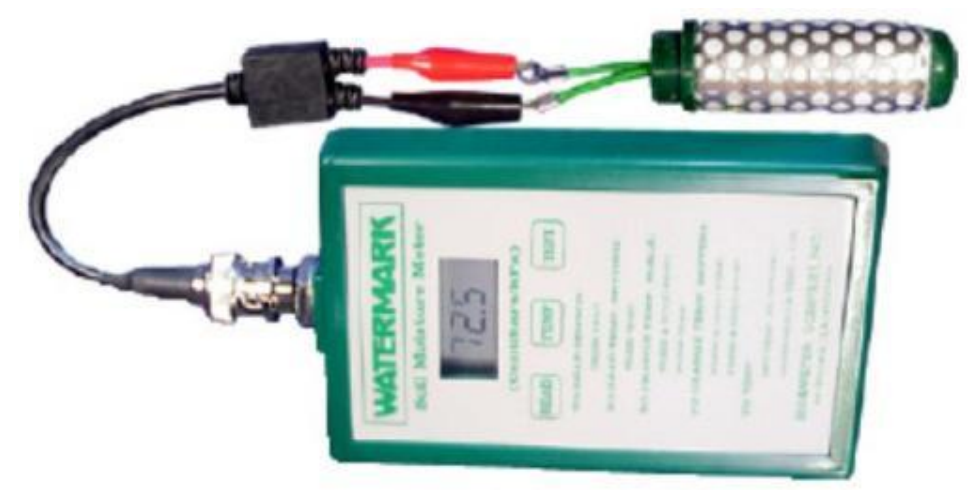

Figura I.10. Sensor digital Watermark junto con el medidor digital portátil. 
Para la medida del contenido de humedad en el suelo, los investigadores de la ESEA emplean sensores de reflectometría en el dominio de la frecuencia (FDR, Frequency Domain Reflectometry) y, en concreto, el equipo EnviroSCAN de Sentek [Sentek]. El sistema mide el porcentaje volumétrico de agua en el suelo y está disponible con diferentes interfaces de salida (Modbus RS-232 y RS-485, SDI-12, analógica en tensión, entre otros). Las versiones digitales tienen una resolución del 0,008\%, frente al 0,025\% de la versión analógica. Su principal característica es que se trata de un sistema modular que permite la conexión de hasta 16 elementos de medida, conectados en serie y separados a incrementos de $10 \mathrm{~cm}$. La Figura 1.11 muestra el sistema EnviroSCAN, instalado a pie de cultivo, mostrando en la imagen de la izquierda los sensores fuera de la guía.
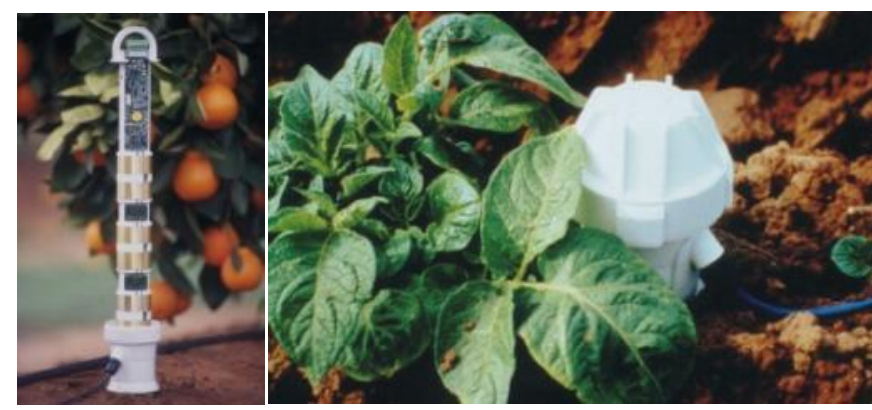

Figura I.11. Diferentes vistas del equipo EnviroSCAN.

La Tabla I.2 resume los principales parámetros de los sensores de suelo descritos anteriormente.

\begin{tabular}{|c|c|c|c|c|}
\hline Sensor & Alimentación & Interfaz Salida & Rango de Medida & Precisión \\
\hline $\begin{array}{l}\text { Tensiómetro } \\
\text { Irrometer R. } \\
\text { Transductor } \\
\text { RSU }\end{array}$ & $12-24 \mathrm{~V}$ & Lazo de corriente $4-20 \mathrm{~mA}$ & $0-100 \mathrm{cb}$ & N/A \\
\hline $\begin{array}{l}\text { Tensiómetro } \\
\text { Jet Fill } \\
\text { 2725ARL. } \\
\text { Transductor } \\
\text { 5301-B5 }\end{array}$ & $12-40 \mathrm{~V}$ & Lazo de corriente $4-20 \mathrm{~mA}$ & $0-50 \mathrm{cb}$ & $\mathrm{N} / \mathrm{A}$ \\
\hline $\begin{array}{l}\text { Watermark } \\
\text { 200SS-V }\end{array}$ & $5 \mathrm{~V}$ & $0-2,8 \mathrm{~V}$ & $0-239 \mathrm{cb}$ & $\mathrm{N} / \mathrm{A}$ \\
\hline EnviroSCAN & $\begin{array}{l}\text { 4,5-15, típica } \\
12 \mathrm{~V}\end{array}$ & $\begin{array}{c}\text { Tensión, SDI-12, Modbus RS- } \\
\text { 232, RS-485 }\end{array}$ & $0-100 \%$ & $\begin{array}{l}\text { Digital } 0,008 \% \\
\text { Analógico } 0,025 \%\end{array}$ \\
\hline
\end{tabular}

Tabla I.2. Características principales de los sensores de suelo de la ESEA.

El sistema de medida de porcentaje volumétrico (EnviroSCAN) tiene un coste elevado. Un sensor alternativo, con un coste menor, es la sonda multi-paramétrica Hydra Probe II (HP2) de Stevens [Stevens]. El sensor HP2 (Figura I.12) se puede adquirir con dos interfaces de salida diferentes: SDI-12 y RS485. De estas dos versiones, la que resulta más 
adecuada para aplicaciones con requerimientos de bajo consumo, es el de tipo SDI-12, ya que el consumo en standby es 10 veces menor que para la versión RS-485. Además, sólo son necesarios utilizar de 3 hilos (alimentación, tierra y datos) frente a los 4 necesarios con la versión RS485 (alimentación, tierra, com+ y com-).

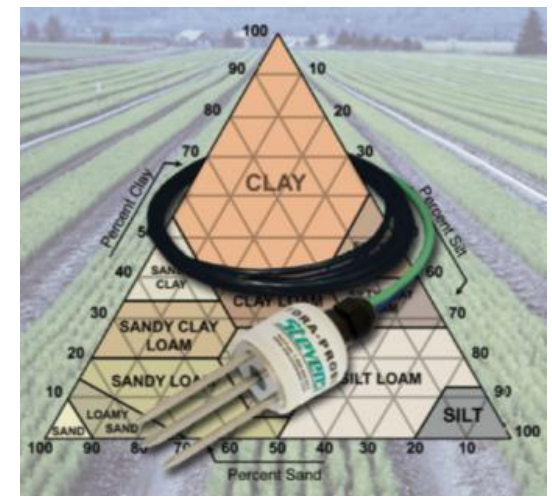

Figura I.12. Sensor Hydra Probe II.

Con el sensor se pueden obtener diversos parámetros del terreno, siendo los más importantes la temperatura, el porcentaje volumétrico, la conductividad y la salinidad del suelo. La calibración se realiza en el propio sensor y, por tanto, los datos son recibidos en unidades físicas de forma digital y en código ASCII. No obstante, para el usuario puede resultar interesante realizar una calibración propia y, por este motivo, es posible obtener la información de las variables directamente de los ADC, sin calibrar, en formato 'raw'.

Como en cualquier dispositivo SDI-12, la comunicación se realiza mediante el envío de comandos ASCII y la interpretación de la respuesta de los anteriores. Mediante estos comandos, se puede realizar la lectura de parámetros mencionados anteriormente, así como la configuración del tipo de suelo, constante del agua, tiempo de establecimiento y el conjunto de datos a medir.

Por lo tanto, con el HP2 se pueden obtener más datos del suelo que con el sistema EnviroSCAN y tiene un coste inferior.

El sensor requiere una tensión de alimentación comprendida entre 9 y $20 \mathrm{~V}$. La Tabla I.3 resume las características de las principales variables medidas por el sensor.

\begin{tabular}{|c|c|c|}
\hline Variable & Rango de Medida & Precisión \\
\hline $\begin{array}{l}\text { Porcentaje volumétrico } \\
\text { de humedad }\end{array}$ & $\begin{array}{l}\text { De completamente } \\
\text { seco a saturado }\end{array}$ & $\begin{array}{l} \pm 0,03 \text { fracción de agua } \\
\text { por volumen }\end{array}$ \\
\hline Temperatura & $-10-65^{\circ} \mathrm{C}$ & $\pm 0,1^{\circ} \mathrm{C}$ \\
\hline Conductividad & $0,01-1,5 \mathrm{~S} / \mathrm{m}$ & $\pm 20 \%$ ó $0,005 \mathrm{~S} / \mathrm{m}$ \\
\hline
\end{tabular}

Tabla I.3. Variables medidas por el sensor Hydra Probe II. 


\section{I.2.3 Sensores para monitorizar el estado hídrico de las plantas.}

Para la medida del estado hídrico de la plantas, en la ESEA se utilizan diferentes equipos portátiles, así como sensores de despliegue fijo. Al igual que en las secciones anteriores, en los nodos sensores interesa integrar los sensores de posición fija, entre los que cabe destacar los dendrómetros y los termoradiómetros. El objetivo que se persigue con estos sensores es estimar la evapotranspiración de las plantas. De esta manera se pueden conocer las necesidades hídricas de la planta, así como si está consumiendo nutrientes en ese momento.

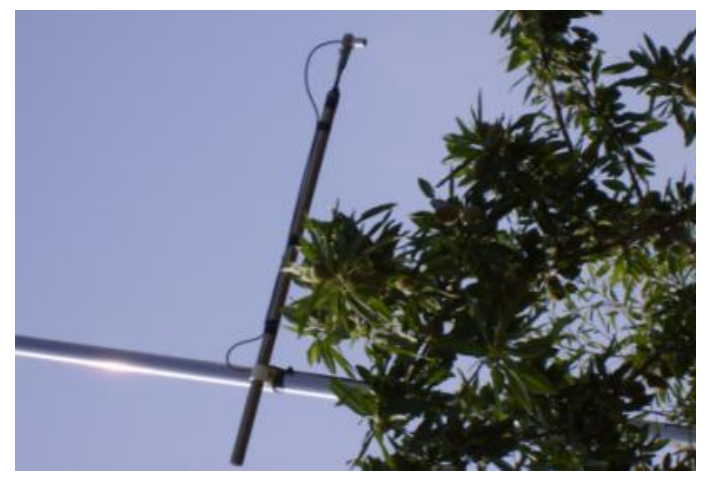

Figura I.13. Vista del sensor IRTS-P y su instalación sobre un almendro en mástil.

La medida de la temperatura de superficie de la planta es una componente crucial de la transferencia de energía. Una medida precisa del gradiente de temperatura de la hoja al ambiente es esencial para determinar la tasa de transpiración y la conductancia estomática en hojas individuales, así como en los doseles de las plantas. Generalmente, este gradiente es menor que $3^{\circ} \mathrm{C}$, lo que implica que la temperatura de la hoja debe ser medida con una precisión de unos $0,3^{\circ} \mathrm{C}$. Para conseguir esta precisión, el sensor IRTS-P (Figura I.13) de Apogee [Apogee] integra dos termopares de tipo K. El primer termopar ofrece la medida propiamente dicha y, el segundo, mide la temperatura del cuerpo del sensor, que se emplea para corregir la lectura de temperatura realizada por el sensor. La salida del sensor, al ser directamente el cableado con un termopar tipo $\mathrm{K}$, no requiere de una excitación eléctrica externa, pero sí de una electrónica de acondicionamiento compleja, ya que las tensiones de salida del termopar de tipo $\mathrm{K}$ son de unos pocos $\mathrm{mV}$.

Las variaciones de diámetro de tronco fueron medidas con sensores de desplazamiento variable y de respuesta lineal tipo LVDT (Linear Variable Differential Transformer), modelo DFg2.5, fabricados por Solartron [Solartron]. El sensor tiene un rango de medida lineal de 5mm, con una precisión de 1 a 10 micras (Figura I.14a). Estos sensores constan de un núcleo magnético, que se mueve impulsado por una aguja en contacto con el tronco. Dicho núcleo discurre por el interior de un cilindro, y el movimiento hace que la 
tensión que se induce del primario al secundario del transformador sea diferente en función de la posición. Además del LVDT, el sensor incluye la electrónica necesaria para que, tanto la tensión de excitación como la salida, sean de corriente continua.

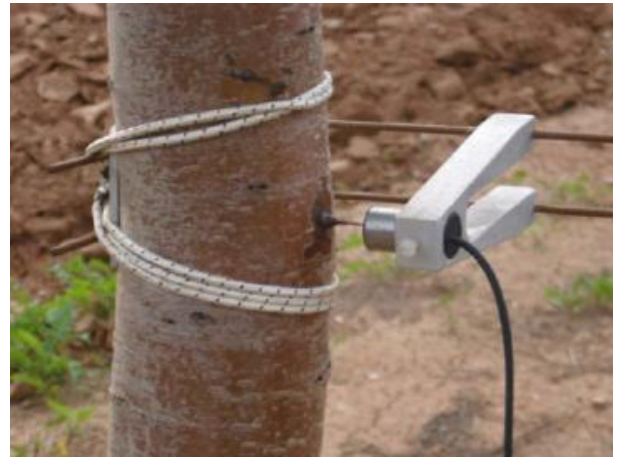

(a)

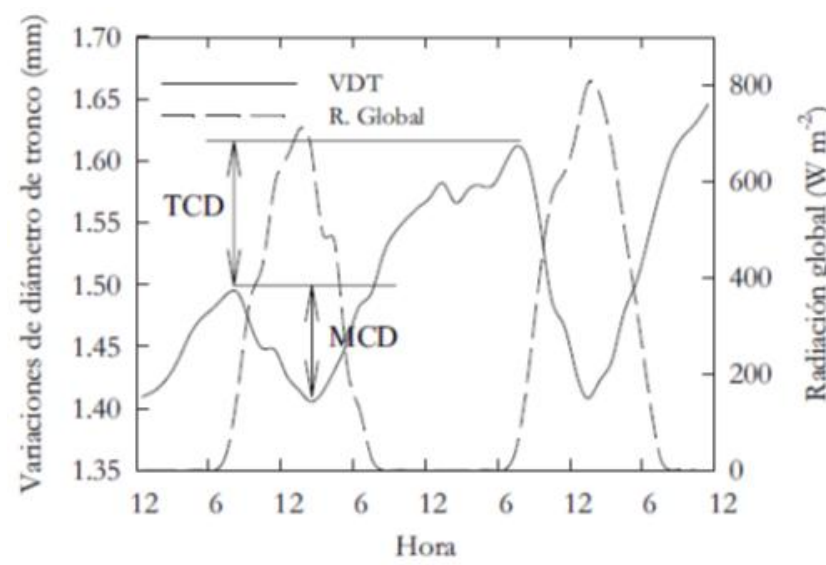

(b)

Figura I.14. (a) Dendrómetro y portasensor instalados en tronco. (b) Representación gráfica de la máxima contracción diaria (MCD) y tasa de crecimiento diaria de tronco (TCD) a partir de la variación del diámetro de tronco (VDT) en almendros.

A partir de las variaciones de diámetro de tronco se pueden determinar los parámetros indicadores del estado hídrico propuestos por Kozlowski [Kozlowski, 1967]: máxima contracción diaria de tronco $(\mathrm{MCD})$, tasa de crecimiento diaria de tronco (TCD) y crecimiento acumulado (CA). La MCD se obtiene como la diferencia entre el diámetro máximo alcanzado antes del alba y el mínimo a mediodía [Fereres, 2000]. La TCD se obtiene a partir de la diferencia entre el máximo diámetro de dos días consecutivos y la CA como la suma de los TCD en un intervalo de tiempo determinado (Figura I.14b). Finalmente, sobre el dendrómetro cabe destacar que, usando un portasensor para frutos, se puede monitorizar el crecimiento de los mismos.

La Tabla I.4 resume las principales características de los dos sensores utilizados para monitorizar el estado hídrico de la plantas, descritos anteriormente.

\begin{tabular}{|c|c|c|c|c|}
\hline Sensor & Alimentación & Interfaz Salida & Rango de Medida & Precisión \\
\hline IRTS-P & Pasivo & Salida de termopar tipo K & $-10-55^{\circ} \mathrm{C}$ & $\pm 0,5^{\circ} \mathrm{C}$ \\
\hline DFg2.5 & $10-24 \mathrm{Vcc}$ & $\begin{array}{l}750 \mathrm{mV} / \mathrm{mm} \text { con } 10 \mathrm{~V} \text { de } \\
\text { alimentación }( \pm 1,875 \mathrm{~V})\end{array}$ & $5 \mathrm{~mm}$ & $1-10 \mu \mathrm{m}$ \\
\hline
\end{tabular}

Tabla I.4. Características principales de los sensores IRTS-P y DFg2.5.

Existen otros métodos con los que se puede estimar la evapotranspiración de las plantas. Estos métodos son los siguientes: la técnica del pulso de calor [Huber, 1937] y el método del lisímetro [Allen, 1990]. El primero, consiste en realizar tres orificios en el 
tronco. En el del centro se coloca un calentador construido con un resistor. Aguas arriba y abajo del calentador se instalan baterías de termopares. El método consiste en aplicar un pulso de calor, que será recibido por la savia que circula por el árbol y, a partir de la lectura de los termopares, determinar la velocidad del flujo de savia; valor con el que es posible estimar la evapotranspiración de la planta. Por otro lado, el lisímetro es una báscula instalada en el propio cultivo con la planta a estudiar plantada en el interior del mismo. Las variaciones en la medida del peso del terreno en el que están esparcidas las raíces, realizadas con células de carga, permiten determinar el consumo de la planta.

\section{I.3 Principales componentes hardware de los motes.}

\section{I.3.1 Transceptor de radio.}

Cuando se comenzaron los trabajos de investigación existían numerosos entornos de desarrollo de software para WSN, entre los que caben destacar: TinyOS, Contiki, etc. Todos ellos ofrecían soporte para el mote TelosB, que era muy usado a nivel de investigación. Este mote fue desarrollado por un grupo de investigadores de la Universidad de Berkeley, los cuales fueron los principales impulsores de las WSN. Estos investigadores seleccionaron como elementos principales del mote el micro-controlador MSP430F1611 y el módulo de radio CC2420 [CC2420], ambos de Texas Instruments.

No es de extrañar que el TelosB y otros motes utilizaran el CC2420 como módulo de radio, teniendo en cuenta que era la mejor alternativa, en términos de costes y prestaciones ofrecidas. En concreto, algunos de los motes que lo han utilizado son los siguientes: MicaZ, TelosB, Imote, Imote2.

Por ello, con objeto de disponer de una plataforma que fuese compatible con los sistemas operativos o entornos de desarrollo de la fecha y que utilizase un módulo de radio con la mejor relación costes/prestaciones, se optó por iniciar los trabajos de investigación con una plataforma que utilizase el CC2420 como módulo de radio.

Las características principales del módulo de radio CC2420 quedan recogidas en la tabla I.8. Cabe destacar que la unidad $\mathrm{dBm}$ se define como el nivel de potencia en decibelios, en relación a un nivel de referencia de $1 \mathrm{~mW}$. Así, el valor en $\mathrm{dBm}$ en un punto para una potencia $\mathrm{P}$, viene dado por la siguiente ecuación:

$$
d B m=10 \cdot \log \left(\frac{P}{1 m W}\right)
$$




\begin{tabular}{|c|c|c|}
\hline \multicolumn{2}{|r|}{ Característica } & Descripción \\
\hline & General & $\begin{array}{l}\text { Transceptor RF de 2,4GHz compatible con el IEEE } 8021542003 \text { con } \\
\text { soporte MAC en un solo chip Requiere de pocos elementos externos } \\
\text { (cristal y algunos elementos pasivos) No precisa de filtros externos }\end{array}$ \\
\hline & Data rate (máximo) & $250 \mathrm{kbps}$ \\
\hline \multicolumn{2}{|r|}{ Frecuencia de trabajo } & $2400-2483,5 \mathrm{MHz}$ \\
\hline \multicolumn{2}{|c|}{ Potencia de transmisión } & Programable desde -25 hasta $0 \mathrm{dBm}$ en pasos $(0,-5,-10,-15,-25 \mathrm{dBm})$ \\
\hline \multicolumn{2}{|r|}{ Sensibilidad } & Alta Mínima de $-90 \mathrm{dBm}$ y típica de $-95 \mathrm{dBm}$ \\
\hline \multicolumn{2}{|r|}{ Interfaz } & Digital SPI de 4 hilos con la frecuencia de reloj de hasta $10 \mathrm{MHz}$ \\
\hline \multicolumn{2}{|r|}{ Tensión de trabajo } & $2,1-3,6 \mathrm{~V}$ \\
\hline \multirow{9}{*}{ Consumo } & Voltage regulator off (OFF) & $0,02-1 \mu \mathrm{A}$ \\
\hline & Power Down mode & $20 \mu \mathrm{A}$ \\
\hline & Idle mode & $426 \mu \mathrm{A}$ \\
\hline & Receive mode & $18,8 \mathrm{~mA}$ \\
\hline & transmit mode: $-25 \mathrm{dBm}$ & $8,5 \mathrm{~mA}$ \\
\hline & transmit mode: $-15 \mathrm{dBm}$ & $9,9 \mathrm{~mA}$ \\
\hline & transmit mode: $-10 \mathrm{dBm}$ & $11 \mathrm{~mA}$ \\
\hline & transmit mode: $-5 \mathrm{dBm}$ & $14 \mathrm{~mA}$ \\
\hline & transmit mode: $0 \mathrm{dBm}$ & $17,4 \mathrm{~mA}$ \\
\hline
\end{tabular}

Tabla I.5. Características principales del módulo de radio CC2420 de Texas Instruments.

Al utilizarse un nivel de referencia determinado ( $1 \mathrm{~mW})$, la medida en $\mathrm{dBm}$ constituye una verdadera medición de la potencia y no una simple relación de potencias, como en el caso de la medida en decibelios. Así, una lectura de $20 \mathrm{dBm}$ significa que la potencia medida es 100 veces mayor que $1 \mathrm{~mW}$ y por tanto igual a $100 \mathrm{~mW}$. En el caso del CC2420, la potencia de transmisión, con valores comprendidos entre -25 y 0dBm, equivale a $3,16 \mu \mathrm{W}$ y $1 \mathrm{~mW}$, respectivamente.

En el año 2007, Texas Instruments lanzó al mercado el módulo de radio CC2520 [CC2520]. Se trataba de un CC2420 con mayores prestaciones, y era definido como un transceptor $\mathrm{RF}$ de $2,4 \mathrm{GHz}$ de $2^{\mathrm{a}}$ generación para los protocolos de comunicación IEEE 802.15.4 y ZigBee. La Tabla I.6 resume sus principales características. 
A grandes rasgos, el CC2520 es compatible con la nueva versión del estándar IEEE 802.15.4, la versión 2006, y extiende algunas características del anterior, como la frecuencia de operación, la tensión de alimentación e incrementa la potencia de transmisión. Con el incremento de esta última característica, el fabricante proporciona con el CC2520 un alcance de $400 \mathrm{~m}$ en línea de vista, junto a una máxima potencia de transmisión $(+5 \mathrm{dBm})$.

\begin{tabular}{|c|c|c|}
\hline \multicolumn{2}{|r|}{ Característica } & Descripción \\
\hline & General & $\begin{array}{l}\text { Transceptor RF de } 2,4 \mathrm{GHz} \text { compatible con el IEEE } 8021542006 \text { y } \\
\text { ZigBee Sólo requiere de un cristal y algunos elementos pasivos externos } \\
\text { No requiere de filtros externos e incrementa el alcance del CC2420 }\end{array}$ \\
\hline & Data rate (máximo) & $250 \mathrm{kbps}$ \\
\hline & recuencia de trabajo & $2394-2507 \mathrm{MHz}$ \\
\hline & otencia de transmisión & Programable desde -18 hasta $5 \mathrm{dBm}$ \\
\hline & Sensibilidad & Entre -99 y $-95 \mathrm{dBm}$ \\
\hline & Interfaz & Digital SPI de 4 hilos con la frecuencia de reloj de hasta $8 \mathrm{MHz}$ \\
\hline & Tensión de trabajo & $1,8-3,8 \mathrm{~V}$ \\
\hline \multirow{7}{*}{ Consumo } & $\begin{array}{l}\text { LPM2: oscilador y regulador } \\
\text { en off. No guarda estado }\end{array}$ & $30 \mathrm{nA}-4,5 \mu \mathrm{A}$ \\
\hline & $\begin{array}{l}\text { LPM1: oscilador off y } \\
\text { regulador on. Guarda estado }\end{array}$ & $175-250 \mu \mathrm{A}$ \\
\hline & $\begin{array}{l}\text { Modo Activo: oscilador y } \\
\text { regulador en on }\end{array}$ & $1,6-1,9 \mu \mathrm{A}$ \\
\hline & Receive mode & $18,5 \mathrm{~mA}$ \\
\hline & transmit mode: $-\mathbf{- 1 8 d B m}$ & $16,2 \mathrm{~mA}$ \\
\hline & transmit mode: $0 \mathrm{dBm}$ & $25,8 \mathrm{~mA}$ \\
\hline & transmit mode: $\mathbf{5 d B m}$ & $33,6 \mathrm{~mA}$ \\
\hline
\end{tabular}

Tabla I.6. Características principales del módulo de radio CC2520 de Texas Instruments.

\section{I.3.2 Antenas.}

Las antenas son unos elementos críticos en el diseño de una WSN. Dependiendo de la antena escogida, se podrán conseguir mayores o menores alcances entre los nodos sensores y garantizar las comunicaciones inalámbricas bajo condiciones meteorológicas adversas.

Seleccionar una antena implica escoger las características de la antena, entre las que cabe destacar las siguientes [Balanis, 2005]: diagrama de radiación, ancho de banda, directividad y la ganancia. El ancho de banda estará definido por la frecuencia de trabajo de los nodos sensores. Un diagrama de radiación es la representación gráfica de las características de radiación de una antena, en función de la dirección (coordenadas en 
azimut y elevación). Lo más habitual, es representar la densidad de potencia radiada, aunque también se pueden encontrar diagramas de polarización o de fase. Atendiendo al diagrama de radiación, se puede hacer una clasificación general de los tipos de antena y se puede definir la directividad de la antena. La directividad indica la zona en la que irradia la antena y permiten clasificarlas en: antena isotrópica, antena directiva, antena bidireccional, antena omnidireccional, entre otras. La ganancia de una antena es la relación (cociente) de potencias por unidad de superficie, entre una antena dada y una antena isótropa que se toma como referencia, estando ambas alimentadas con la misma potencia. Se expresa en $\mathrm{dBi}$ o decibelio isótropo, que son decibelios con una i añadida para recordar que se trata de una ganancia con respecto a una antena isótropa. El fabricante también especifica la ganancia de la antena en $\mathrm{dBd}(\mathrm{dBi}=\mathrm{dBd}+2,15 \mathrm{~dB})$. En este caso, la antena de referencia es un dipolo de media onda.

Recordando la selección del transceptor de radio de la sección anterior, se escogerán antenas con un ancho de banda suficiente para englobar los 16 canales de la banda ISM de 2,4GHz.

A grandes rasgos, la directividad permite clasificar las antenas en: (1) direccionales o directivas, (2) omnidireccionales y (3) sectoriales. Las primeras, orientan la señal en una dirección muy determinada con un haz estrecho pero de largo alcance. Las antenas omnidireccionales orientan la señal en todas direcciones, con un haz amplio pero de corto alcance y menor que usando una antena direccional en la misma situación. Las antenas sectoriales son la mezcla de las antenas direccionales y las omnidireccionales. Éstas emiten un haz más amplio que una direccional, pero no tan amplio como una omnidireccional. El alcance de la antena sectorial es mayor que la omnidireccional, pero algo menor que con la direccional. En la práctica es habitual usar 3 antenas sectoriales de $120^{\circ}$ para conseguir una directividad omnidireccional con un mayor alcance. La Figura I.15 compara gráficamente el alcance y los grados del sector con los que irradian los 3 tipos de antenas mencionados.

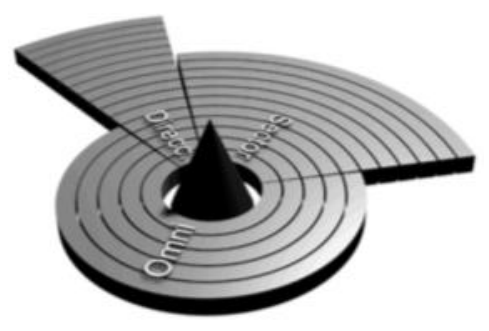

Figura I.15. Comparativa de antenas direccionales, omnidireccionales y sectoriales.

En una WSN de ámbito agrícola, los nodos sensores tendrán una posición fija, pero interesa que puedan ser instalados en otras posiciones sin realizar ajustes de orientación de las antenas y que, además, las comunicaciones sean robustas frente a movimientos del dispositivo, debido a que el agricultor lo pueda mover sin darse cuenta, al viento o a 
cualquier tipo de condición climática adversa. Por ello, la antena seleccionada para los nodos sensores instalados en campo es de tipo omnidireccional. No obstante, en un nodo Gateway (comunica red inalámbrica con las oficinas de la explotación agrícola) de posición fija será interesante estudiar la necesidad de emplear una antena direccional o sectorial en lugar de una omnidireccional.

Finalmente, para poder seleccionar la antena por completo, es necesario determinar la ganancia necesaria. Para ello, es necesario tratar las zonas de Fresnel [Bertoni, 2000] y la fórmula de transmisión de Friis [Friis, 1946; Balanis, 2005]. Las zonas de Fresnel son elipsoides de revolución sobre la línea entre el punto transmisor y el receptor, siendo los puntos del transmisor y del receptor los focos de la elipse (Figura I.16). Existen ' $n$ ' zonas de Fresnel, en las que las impares contribuyen a la recepción de la señal (o están en fase) y las pares contribuyen negativamente (o están en contrafase). Los desfases se producen por la reflexión de la señal, y el rayo directo que llega del transmisor al receptor se considera que tiene fase cero. En la primera zona Fresnel es en la que se transmite la mayor parte de la señal, y la presencia de obstáculos en ella es muy perjudicial para una correcta recepción de la señal, queda definida por el volumen de onda electromagnética, de modo que el desfase de las ondas en dicho volumen no supere los $180^{\circ}$.

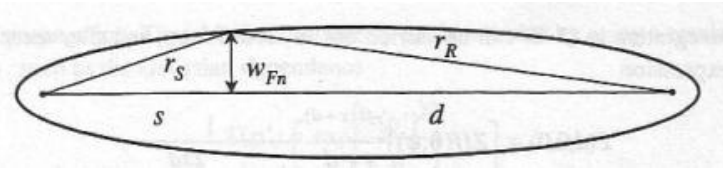

Figura I.16. Representación de la zona Fresnel con el transmisor y el receptor como focos.

Analíticamente, el radio de la zona Fresnel enésima, $\mathrm{W}_{\mathrm{Fn}}$, se puede determinar con la Ec. I.2:

$$
W_{F n}=\sqrt{n \cdot \frac{\lambda s d}{s+d}}
$$

A partir de la Ec. I.2 se puede obtener una expresión más útil en la práctica, o en unidades prácticas:

$$
R_{n}=548 \cdot \sqrt{n \cdot \frac{d_{1} \cdot d_{2}}{f \cdot d}}
$$

En la que, $\mathrm{R}_{\mathrm{n}}$ es el radio de la enésima zona Fresnel $(n)$, $\mathrm{f}$ es la frecuencia de la señal transmitida $(\mathrm{MHz}), \mathrm{d}_{1}$ es la distancia del transmisor al plano considerado $(\mathrm{km}), \mathrm{d}_{2}$ es la distancia del plano considerado al receptor $(\mathrm{km})$ y d es la distancia que separa al transmisor del receptor $\left(\mathrm{d}_{1}+\mathrm{d}_{2}\right)$, expresada en kilómetros. 
Llevado a la práctica, la utilidad de las zonas de Fresnel es la determinar la altura a la que se deben situar las antenas para asegurar la recepción de la señal en un enlace punto-apunto. La Figura 1.17 servirá de ejemplo para ilustrar este cálculo. En la figura, los 3 edificios tienen la misma altura, y el edificio central (obstáculo) impedirá la recepción de la señal si las antenas no están situadas a la altura necesaria (el edificio central no debe estar dentro de la primera zona Fresnel de radio $r_{1}$ ). Suponiendo que la distancia del transmisor al objeto $\left(\mathrm{d}_{1}\right)$ y que la distancia del receptor al objeto $\left(\mathrm{d}_{2}\right)$ son de $500 \mathrm{~m}$, y que la frecuencia de la señal transmitida es de $900 \mathrm{MHz}$, el radio de la primera zona Fresnel $(\mathrm{n}=1)$ se puede calcular usando la Ec. I.3:

$r_{1}=548 \cdot \sqrt{1 \cdot \frac{0,5 \cdot 0,5}{900 \cdot 1}}=9,13 m$

Ec. I.4

Por lo tanto, las antenas se deberían instalar a 9,13m sobre los tejados.

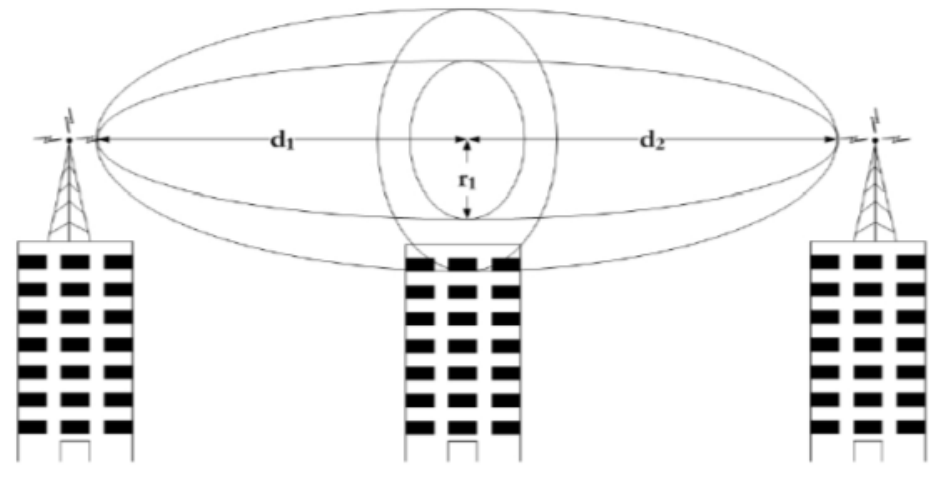

Figura I.17. Ejemplo práctico de la zonas de Fresnel.

Por otro lado, la fórmula de transmisión de Friis (Ec. I.5) relaciona las potencias recibida $\left(\mathrm{P}_{\mathrm{r}}\right)$ y transmitida $\left(\mathrm{P}_{t}\right)$ entre dos antenas que están separadas una distancia $\mathrm{R}$ en espacio libre. El término cuadrático entre paréntesis es llamado el factor de pérdidas en espacio libre, y tiene en cuenta las pérdidas debidas a la propagación esférica de la energía de la antena. Esta ecuación, permite dimensionar la ganancia necesaria que debe tener la antena de los motes objeto de estudio e implementación.

$\frac{P_{r}}{P_{t}}=\left(\frac{\lambda}{4 \pi \cdot R}\right)^{2} \cdot G_{0 t} \cdot G_{0 r}$

A continuación, se va a llevar a cabo un estudio para dimensionar la antena requerida para comunicar dos estaciones equipadas con el módulo de radio CC2420, que estén separadas $100 \mathrm{~m}$. El estudio se extenderá a dos estaciones equipadas con el transceptor de radio CC2520, separadas por una distancia de 400m. 
Para determinar la antena necesaria se van a considerar condiciones desfavorables, tanto para el CC2420 como para el CC2520, con objeto de compensar la presencia de obstáculos que habrá en el caso real y que no están contemplados en la Ec. I.5.

La sensibilidad del módulo de radio representa la mínima potencia necesaria para activar sus circuitos y poder recibir un mensaje. En el caso del CC2420 estaba comprendida entre -90 y $-95 \mathrm{dBm}$. El caso más desfavorable es considerar el valor mayor ( $-90 \mathrm{dBm})$, el cual es equivalente a $0,001 \mathrm{nW}$. Aplicando el mismo razonamiento para el CC2520, el valor más desfavorable resulta, de 0,000316nW.

En el caso del CC2420, la potencia de transmisión $\left(\mathrm{P}_{\mathrm{r}}\right)$ se puede configurar entre $3,16 \mu \mathrm{W}$ y $1 \mathrm{~mW}$, y en la ecuación se utilizará en valor del centro de intervalo, $501,58 \mu \mathrm{W}$. Para el CC2520 resulta $1,5879 \mathrm{~mW}$.

La longitud de onda, $\lambda$, se obtiene dividiendo la velocidad de la luz $(300.000 \mathrm{~km} / \mathrm{s})$ entre la frecuencia de trabajo $(2,4 \mathrm{GHz})$, resultando una longitud de onda de $0,125 \mathrm{~m}$.

Los dispositivos basados en el CC2420 y en el CC2520 estarán separados, entre ellos, 100 y 400m, respectivamente. Con estos valores queda definido el alcance (R).

Todos los dispositivos dispondrán de la misma antena. Por tanto, el término $\mathrm{G}_{0 \mathrm{t}} \mathrm{G}_{0 \mathrm{r}}$ es equivalente a un término $\mathrm{G}^{2}$. La ganancia de una antena en dBi y la ganancia lineal (requerida en la Ec. I.5) se obtiene mediante el producto del término 10 por el logaritmo en base diez de la ganancia lineal. Así, las ganancias de $3 \mathrm{dBi}$ y $2 \mathrm{dBi}$ son equivalentes en términos lineales a 1,995 y 1,584, respectivamente. Estas son las ganancias que, a priori, se consideran más adecuadas para los dispositivos.

La Ec. 1.6 demuestra que una ganancia de $2 \mathrm{dBi}$, para un enlace de $100 \mathrm{~m}$ con el CC2420, es adecuada, ya que la potencia es superior a la requerida por el dispositivo.

$$
P_{r}=P_{t} \cdot\left(\frac{\lambda}{4 \pi \cdot R}\right)^{2} \cdot G^{2}=501,58 \cdot 10^{-6} \cdot\left(\frac{0,125}{4 \pi \cdot 100}\right)^{2} \cdot 1,584^{2}=0,0124 n W>0,001 n W
$$

En la misma línea, la Ec. I.7 demuestra que una antena con una ganancia de $3 \mathrm{dBi}$ para un enlace de $400 \mathrm{~m}$ basado en el CC2520 es adecuada, ya que la potencia es superior a la mínima requerida por el dispositivo, que se obtiene a partir de la sensibilidad del mismo.

$$
P_{r}=P_{t} \cdot\left(\frac{\lambda}{4 \pi \cdot R}\right)^{2} \cdot G^{2}=1,5879 \cdot 10^{-3} \cdot\left(\frac{0,125}{4 \pi \cdot 400}\right)^{2} \cdot 1,995^{2} \cong 0,004 n W>0,000316 n W \quad \text { Ec. }
$$

Por lo tanto, la estaciones se podrán comunicar utilizando antenas de $2 \mathrm{dBi}$ y $3 \mathrm{dBi}$ de ganancia en el caso del CC2420 y del 2520, respectivamente. En concreto, la Figura I.18 
muestra dos antenas con las características requeridas. Las dos son omnidireccionales y con conector SMA. La antena para el CC2420, que se puede adquirir en Solwise [Solwise] es de muy pequeño tamaño $(70 \mathrm{~mm})$ y se puede instalar con facilidad en cualquier carcasa debido a la tuerca y la junta tórica que incluye. La antena de la derecha, comercializada por Farnell [Farnell] es una antena de tipo rubber duck de 3dBi de ganancia. Esta antena complica el diseño de una carcasa porque no es de uso exterior y necesitaría un grado de protección adecuado.

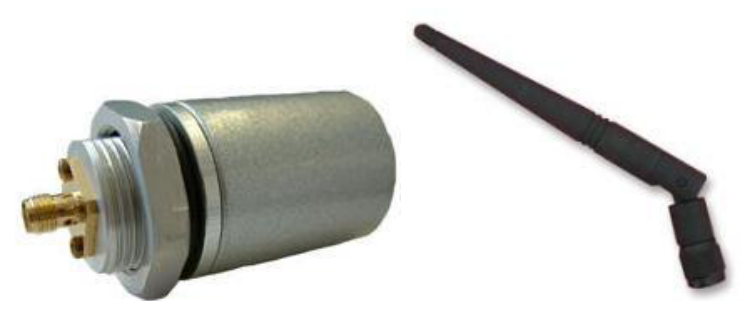

Figura I.18. Antenas seleccionadas para los módulos de radio.

\section{I.3.3 Micro-controladores.}

En relación con el micro-controlador, la mayoría de estos elementos incluyen las interfaces de E/S analógicas y digitales necesarias para que el nodo sensor o mote pueda interactuar con la instrumentación agrícola requerida en las especificaciones del diseño a implementar.

El módulo de radio que se especifica para una plataforma hardware es compatible con unas determinadas bibliotecas software. Sin embargo, para que la plataforma sea totalmente compatible con las bibliotecas, el micro-controlador también lo tiene que ser. De esta forma, una vez especificados el módulo de radio y las bibliotecas software, el micro-controlador queda especificado.

En el capítulo 4, se especificó que los nodos sensores de la arquitectura objeto de estudio e implementación tenían que ser compatibles con el sistema operativo para WSN TinyOS. Por ello, se especificó que le micro-controlador de los primeros nodos sensores a implementar fuese el MSP430F1611, que es el otro elemento principal del mote TelosB.

En un capítulo posterior al anterior, se especificó que la plataforma que se estaba diseñando tenía que ser compatible con la biblioteca Z-Stack, desarrollada por Texas Instruments. El primer micro-controlador compatible con la Z-Stack y con el CC2520 fue el MSP430F2618, que se describirá posteriormente, al igual que el MSP430F1611.

Cabe destacar que los dos micro-controladores de la familia MSP430 tienen los recursos necesarios (interfaces de entrada y salida analógicas y digitales) para poder conectarles toda la instrumentación agrícola a conectar con los nodos sensores. 
Al igual que el MSP430F2618, el MSP430F1611 es un micro-controlador RISC de 16 bits con un ultra-bajo consumo. Por ello, es indicado para un amplio rango de aplicaciones de bajo consumo y portátiles. La Tabla I.7 resume las principales características de este micro-controlador.

\begin{tabular}{|c|c|c|}
\hline \multicolumn{2}{|r|}{ Característica } & Descripción \\
\hline \multicolumn{2}{|c|}{ Frecuencia máxima de trabajo } & $8 \mathrm{MHz}$ \\
\hline \multicolumn{2}{|r|}{ Memoria Flash } & $48 \mathrm{~KB}$ \\
\hline \multicolumn{2}{|r|}{ Memoria RAM } & $10 \mathrm{~KB}$ \\
\hline \multicolumn{2}{|c|}{ Pines de entrada/salida } & 48 \\
\hline \multicolumn{2}{|c|}{ Interfaces de entrada/salida } & 10 ADCs SAR de 12 bits, 2 DACs de 12 bits, UART, SPI, I2C \\
\hline \multicolumn{2}{|r|}{ Tensión de trabajo } & $1,8-3,6 \mathrm{~V}$ \\
\hline \multirow{5}{*}{ Consumo } & Modo Activo & Típico: $330 \mu \mathrm{A}$; máximo: $600 \mu \mathrm{A}$ \\
\hline & Modo de bajo consumo 0 & Típico: $50 \mu \mathrm{A}$; máximo: $90 \mu \mathrm{A}$ \\
\hline & Modo de bajo consumo 2 & Típico: $11 \mu \mathrm{A}$; máximo: $22 \mu \mathrm{A}$ \\
\hline & Modo de bajo consumo 3 & Típico: $2,2 \mu \mathrm{A}$; máximo: $4,3 \mu \mathrm{A}$ \\
\hline & Modo de bajo consumo 4 & Típico: $0,1 \mu \mathrm{A}$; máximo: $2,5 \mu \mathrm{A}$ \\
\hline
\end{tabular}

Tabla I.7. Características principales del micro-controlador MSP430F1611 de Texas Instruments.

Posteriormente al MSP430F1611, Texas Instruments lanzo al mercado una versión con mejores prestaciones dentro de la familia de ultra-bajo consumo MSP430. La Tabla I.8 resume las principales características del MSP430F2618.

\begin{tabular}{|c|c|c|}
\hline \multicolumn{2}{|r|}{ Característica } & Descripción \\
\hline \multicolumn{2}{|c|}{ Frecuencia máxima de trabajo } & $16 \mathrm{MHz}$ \\
\hline \multicolumn{2}{|r|}{ Memoria Flash } & $116 \mathrm{~KB}$ \\
\hline \multicolumn{2}{|r|}{ Memoria RAM } & $8 \mathrm{~KB}$ \\
\hline \multicolumn{2}{|c|}{ Pines de entrada/salida } & 48 ó 64 \\
\hline \multicolumn{2}{|c|}{ Interfaces de entrada/salida } & 10 ADCs SAR de 12 bits, 2 DACs de 12 bits, UART, SPI, I2C \\
\hline \multicolumn{2}{|r|}{ Tensión de trabajo } & $1,8-3,6 \mathrm{~V}$ \\
\hline \multirow{5}{*}{ Consumo } & Modo Activo & Típico: $99 \mu \mathrm{A}$; máximo: $595 \mu \mathrm{A}$ \\
\hline & Modo de bajo consumo 0 & Típico: $37 \mu \mathrm{A}$; máximo: $125 \mu \mathrm{A}$ \\
\hline & Modo de bajo consumo 2 & Típico: $23 \mu \mathrm{A}$; máximo: $55 \mu \mathrm{A}$ \\
\hline & Modo de bajo consumo 3 & Típico: 5,5uA; máximo: $30 \mu \mathrm{A}$ \\
\hline & Modo de bajo consumo 4 & Típico: $0,1 \mu \mathrm{A}$; máximo: $6 \mu \mathrm{A}$ \\
\hline
\end{tabular}

Tabla I.8. Características principales del micro-controlador MSP430F2618 de Texas Instruments. 


\section{I.3.4 Baterías y módulos de alimentación seleccionados.}

Los elementos principales (micro-controlador y módulo de radio) de los nodos sensores, satisfacen la condición de poder operar con tensiones comprendidas entre 2,1 y 3,6V. Además, en el mercado electrónico hay disponibles multitud de reguladores lineales de tensión, con salida estabilizada de 2,5 y 3V. Por ello, una tensión nominal óptima para la batería estaría en torno a $3,7 \mathrm{~V}$, que se puede conseguir a partir de una única celda, o combinando 3 celdas recargables de 1,2V. Por otro lado, para determinar la capacidad de la batería, se podría hacer una estimación a partir de los consumos de los diferentes elementos, o bien, realizar un estudio de consumo de una primera versión hardware del dispositivo a diseñar e implementar. Esto último, que se detallará en otros capítulos, es lo que se realizó en esta tesis. Al final se seleccionaron las siguientes baterías: (1) celda de tipo AA recargable de NiMH (Níquel-Metal-Hidruro), de 1,2V y $2.700 \mathrm{mAh}$, (2) polímero de litio de 3,7V y $2.000 \mathrm{mAh}$ y (3) celda de Li-ION, de 3,7V y $4.000 \mathrm{mAh}$.

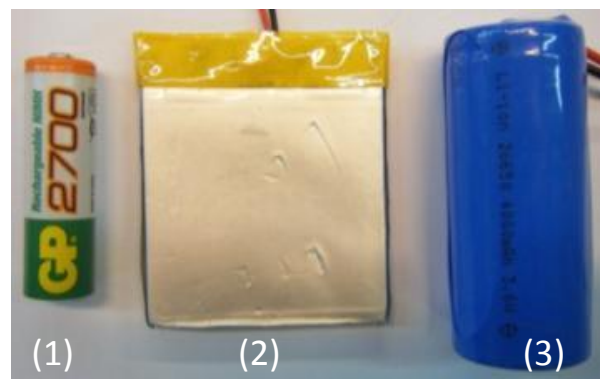

Figura I.19. Baterías seleccionadas para los nodos sensores objeto de estudio.

La batería de la izquierda ( $\mathrm{n}^{\mathrm{o}}$ 1) es una celda de $\mathrm{NiMH}$, de 1,2V de tensión nominal y $2.700 \mathrm{mAh}$ de capacidad, de tipo AA, y comercializada por GP Batteries [GPBatteries]. En concreto, la batería usada tiene la referencia GP270AAHC. Las baterías de níquel-metal hidruro presentan importantes ventajas sobre las baterías de tecnología níquel-cadmio $(\mathrm{NiCd})$, como que tienen una mayor densidad de energía, la capacidad de carga es mayor, menor efecto memoria y no utilizan cadmio, que es muy costoso y muy peligroso para el medio ambiente al ser tóxico. La batería de los nodos sensores se encuentra compuesta por 3 celdas, y la tensión es de unos 4,2V cuando se haya totalmente cargada. La batería central $\left(\mathrm{n}^{\mathrm{o}} 2\right)$ es de polímero de litio, con una tensión nominal de $3,7 \mathrm{~V}$ y $2.000 \mathrm{~m}$ Ah de capacidad. Las baterías de litio tienen un mayor precio que las de NiMH. Sin embargo, presentan una serie de ventajas, entre las que cabe mencionar las siguientes: no tienen efecto memoria, mayor densidad de energía, triplican el voltaje en una sola celda, muy ligeras y tienen una baja auto descarga. Esta batería, con referencia PRT-08483, fue adquirida a través de Sparkfun Electronics [Sparkfun]. Su principal desventaja fue que, si la batería se descargaba excesivamente, quedaba inutilizada. Por ello, en la última versión de nodo sensor se emplearon baterías como la mostrada en la imagen derecha de la Figura I.19 ( $\left.\mathrm{n}^{\circ} 3\right)$. Es una 
batería de tecnología Li-ION, de 3,7V de tensión nominal y 4.000mAh de capacidad. Su referencia es $\mathrm{LC} 26650+$ pc y son distribuidas por BPI [BPI]. Las principales diferencias con la anterior son el tipo de electrolítico (sólido en las de polímero de litio y una sal de litio en las de Li-ION), y que incluyen circuitos de protección de sobrecarga, sobredescarga, sobrevoltaje y voltaje mínimo.

Todas las baterías descritas anteriormente tienen, aproximadamente, una tensión de 4,2V cuando están totalmente cargadas. Este valor supera el máximo permitido por los micro-controladores y los módulos de radio. Además, es importante que los anteriores tengan una tensión estable ante posibles cambios en la tensión de la batería.

Para regular la tensión de las distintas baterías utilizadas se emplearon reguladores lineales de tensión con bajo dropout y con salidas de 2,5 y 3V. El regulador de tensión de 2,5V usado en los dispositivos es el LP38690DT-2.5 de National [National]. Este regulador tiene un dropout de $450 \mathrm{mV}$ y puede proporcionar hasta $1 \mathrm{~A}$ de corriente de salida con un consumo de $55 \mu \mathrm{A}$ (en el pin de masa) a carga completa. Además, la tensión de salida del regulador tiene una precisión del 2,5\% utilizando un condensador de salida con bajo ESR (Equivalent Serial Resistor). Por otro lado, el chip utilizado para estabilizar la tensión a 3V es el MCP1826S-3002E/DB de Microchip [Microchip], que proporciona una corriente de salida de hasta $1 \mathrm{~A}$, con una precisión de regulación en la salida del 0,5\%. El regulador consume una corriente de $120 \mu \mathrm{A}$ y tiene un dropout de $225 \mathrm{mV}$. El condensador de salida se puede utilizar de tipo cerámico, aluminio o de tántalo.

La mayoría de la instrumentación agronómica que se especificó en los capítulos anteriores, pueden funcionar correctamente con una tensión de alimentación de $12 \mathrm{~V}$ de corriente continua. La tensión de salida de los reguladores descritos anteriormente se puede elevar a un valor de tensión de $12 \mathrm{~V}$ usando convertidores continua/continua (DC/DC). En concreto, los nodos utilizan el MAX761ESA y el MAX1771, ambos de Maxim [Maxim]. El MAX761ESA es un convertidor DC/DC, de tipo elevador, que proporciona $12 \mathrm{~V}$ y una corriente de hasta $150 \mathrm{~mA}$ de salida. El chip incluye un pin de habilitación que permite activarlo sólo cuando es necesario. Cuando está habilitado consume $110 \mu \mathrm{A}$, frente a los $5 \mu \mathrm{A}$ en modo de bajo consumo. En el diseño final, debido a que el MAX761ESA dejó de estar disponible por el fabricante, para alimentar los sensores se utilizó el MAX1771. También se trata de un convertidor DC/DC, de tipo elevador, de alta eficiencia (90\%) con una tensión de $12 \mathrm{~V}$ y una corriente de hasta $2 \mathrm{~A}$ de salida. Al igual que el anterior, tiene un pin de habilitación y consumos de $110 \mu \mathrm{A}$ y $5 \mu \mathrm{A}$ en modo activo y modo de bajo consumo, respectivamente.

Por otro lado, hay instrumentación que requiere de una tensión de alimentación muy estable. Por este motivo, la solución más adecuada es utilizar un circuito de referencia de tensión. En concreto, se ha seleccionado el MAX6176 de Maxim. Este circuito de 
referencia proporciona una tensión de salida muy estable (10V), incluso antes cambios de temperatura de trabajo y una corriente de salida de hasta $30 \mathrm{~mA}$ a partir de una tensión de entrada de $12 \mathrm{~V}$.

\section{I.4 Herramientas.}

En esta sección se describirán las herramientas hardware y software utilizadas durante el estudio e implementación de los nodos sensores desarrollados en la tesis objeto de estudio, entendiéndose como tales el simulador ns-2, el sistema operativo TinyOS, la pila ZigBee (Z-Stack) de Texas Instruments y el entorno de compilación y depuración IAR Embedded Workbench para el micro-controlador MSP430, así como el kit de desarrollo CC2520DK y los módulos de inserción de micro-controladores y módulos de radio.

\section{I.4.1 Herramientas software.}

\section{I.4.1.1 Network Simulator 2.}

El simulador de redes Network Simulator 2 [Ramachandran, 2007; NS2], también conocido popularmente como ns-2 en referencia a la versión 2 del mismo, es un simulador de eventos discretos y orientado a objetos que tiene sus inicios en 1989, como una variante del simulador REAL Network Simulator, y ha evolucionado substancialmente durante los últimos años. Provee soporte para simulación de TCP, multicast y distintos algoritmos de enrutamiento sobre redes cableadas y redes inalámbricas de área local y redes usando enlaces vía satélite. El simulador fue ampliando sus capacidades mediante la incorporación de proyectos tales como SensorSim [SensorSim] y NRL [NRL], los cuales permiten modelar distintos tipos de WSNs. A partir de 1995, su desarrollo lo llevaba acabo la agencia de proyectos de investigación avanzada para la defensa de Estados Unidos (DARPA) a través del proyecto VINT (Virtual Inter Network Testbed), en el que intervienen Xerox PARC, UCB y USC/ISI. Posteriormente, ha sido mantenido por diversos proyectos de investigación de la Universidad del Sur de California. En julio de 2006, se inició el desarrollo de la versión 3 del simulador [NS3], de la que ya existen versiones muy estables, como la última versión 3.9 publicada el 20 de agosto de 2010. La versión 3 está desarrollada en $\mathrm{C}++$ con una interfaz opcional de Python. Surje con objeto de evitar algunos problemas de ns-2, entre los que cabe destacar los siguientes: interoperabilidad y acoplamiento entre modelos, pérdida de gestión de la memoria y la depuración de los objetos del lenguaje divididos.

Entre las principales características de ns-2, cabe destacar que está desarrollado en $\mathrm{C}++$ con una programación orientada a objetos, que es un simulador de código abierto, permite simular gran variedad de protocolos en diferentes niveles de red y dispone de 
herramientas de análisis y visualización. Cabe destacar que su programación orientada a objetos flexibiliza y simplifica la tarea de programación, tanto de su código fuente como de su interfaz, así como que, al ser un simulador de código abierto, deriva en que existan números grupos de investigación por todo el mundo que utilizan el simulador.

Como se ha descrito anteriormente, el núcleo de ns-2 está desarrollado en $\mathrm{C}++$. Sin embargo, proporciona una interfaz de simulación a través de OTcl [OTcl], un dialecto orientado a objetos de $\mathrm{Tcl}$ (Object Tool command language, lenguaje orientado a objetos de tipo intérprete). La razón principal de emplear dos lenguajes diferentes se debe principalmente a que ns-2 lleva a cabo dos tareas diferentes, cada una de ellas con distintos requisitos. En primer lugar, debe implementar todos los detalles de los protocolos de comunicaciones, para ello requiere un lenguaje que implemente algoritmos de manera eficiente y con relativa facilidad, además de ser capaz de manipular bytes, cabeceras de paquetes y en definitiva grandes volúmenes de datos. Por ello, el simulador emplea para este propósito el lenguaje $\mathrm{C}++$, pues la velocidad de procesamiento es un punto clave. Por otra parte, se requiere que el lenguaje aporte cierta flexibilidad y rapidez a la hora de realizar configuraciones y variaciones en los parámetros, para poder explorar los resultados de las simulaciones sin necesidad de tener que recompilar el código (lenguaje interpretado), por esta razón, ns-2 emplea el lenguaje OTcl. El uso de dos lenguajes se traduce en que el simulador debe soportar una jerarquía de clases en $\mathrm{C}++$, o jerarquía compilada, y una jerarquía de clases equivalente en OTcl, o jerarquía interpretada [Chung, 2009]. Ambas jerarquías se encuentran estrechamente relacionadas entre sí, de modo que, desde la perspectiva del usuario, hay una correspondencia uno a uno entre una clase de la jerarquía interpretada y otra de la compilada (Figura 1.20). La raíz de esta jerarquía es una clase que se llama TclObject. Cuando el usuario crea un objeto simulador desde el intérprete, cosa con la que generalmente se comienza un 'script', este objeto es instanciado dentro del intérprete y se crea una estrecha relación con otro objeto idéntico, pero dentro de la jerarquía compilada. La jerarquía de clases interpretada se establece automáticamente a través de métodos definidos dentro de la clase llamada TclClass. Además, es posible crear otras jerarquías personalizadas en los scripts, escritas en OTcl, y que no estén desdobladas en la jerarquía compilada.

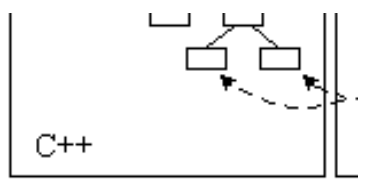

Figura I.20. Dualidad C++ y OTcl en ns-2. 
La Figura I.21 muestra la estructura simplificada de ns-2. El usuario percibe ns-2 como un intérprete de OTcl y que cuenta con: un planificador de eventos, bibliotecas de objetos con componentes de red (nodos, aplicaciones y protocolos) y librerías de configuración de red que permiten establecer los enlaces entre los componentes de la red.

Figura I.21. Estructura simplificada del simulador ns-2.

Para abordar un problema con ns-2, hay que llevar a cabo una serie de fases. En primer lugar, hay que implementar los protocolos o módulos necesarios para realizar el estudio mediante la incorporación de código $\mathrm{C}++$ y OTcl dentro del núcleo del simulador. Posteriormente, se describe la simulación mediante un script OTcl en el que se detalla el escenario a simular. La descripción incluye la iniciación del planificador de eventos, la configuración de la topología de la red usando las bibliotecas de componentes de red y de configuración de red y la especificación del comienzo y fin de transmisión de cada fuente de tráfico a través del planificador de eventos, entre otras. Nótese que este 'script' es codificado por el usuario y es la entrada que se proporciona al simulador. A medida que se está simulando la topología, el simulador genera una traza en la que se registran todos los eventos que se han generado durante la simulación. Básicamente, la salida de la simulación consta de dos archivos de texto (uno con extensión .tr y otro con extensión .nam). Ambos ficheros, aunque contienen la misma información, tienen distintos formatos. Finalmente, hay que analizar los resultados incluidos en los ficheros de salida. Para ello, el simulador dispone de una herramienta denominada NAM (Network AniMator) que permite la visualización del comportamiento de los terminales de la red. A partir de ella, es posible visualizar por ejemplo, cómo los distintos paquetes de datos se van encaminando a través de los distintos nodos, la pérdida de paquetes cuando la red entra en congestión, etc. Mientras que el archivo tr es necesario procesarlo con un lenguaje de scripts con reconocimiento de patrones y manejo de expresiones regulares, para obtener resultados cuantitativos del escenario simulado.

En resumen, este simulador (ver Figura I.22) es un entorno gratuito y muy potente dentro del campo de la simulación de redes. Es, a la vez, muy flexible dada la posibilidad de trabajar con scripts OTcl, que permite agregar toda la potencia de un lenguaje de programación a los elementos propios de la simulación. 


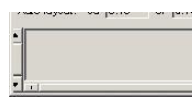

Figura I.22. Interfaz gráfica del simulador ns-2.

\section{I.4.1.2 TinyOS.}

TinyOS [TinyOS; Hill, 2000] es un sistema operativo, de código abierto, con una filosofía de diseño orientada a componentes y dirigida por eventos que está diseñado para redes inalámbricas embebidas de sensores. Está desarrollado por un consorcio, liderado por la Universidad de California en Berkeley, en cooperación con Intel Research. En el año 1999, la Universidad de Berkeley trabajaba en el desarrollo de la primera plataforma (WeC) para TinyOS y la implementación del sistema operativo. El año siguiente, se publica la versión 0.43 del sistema operativo, a nivel público. Actualmente, la última versión estable es la 2.1.1, que se publicó en abril de 2010 .

El sistema operativo está distribuido para ser usado en diferentes distribuciones Linux. Sin embargo, el desarrollo de software para WSN también se puede llevar a cabo en otras plataformas como Windows. Para ello es necesario utilizar el software Cygwin [Cygwin], que emula la consola o terminal de Linux bajo un sistema operativo Windows. Otra alternativa, sería utilizar un software de virtualización ejecutando una máquina virtual. Cabe destacar que, casi todo el flujo de diseño en TinyOS, se lleva a cabo desde la consola o terminal. La Figura 1.23 muestra la consola Cygwin con el comando ejecutado para compilar y descargar una aplicación en un mote TelosB, así como la salida generada en respuesta al comando.

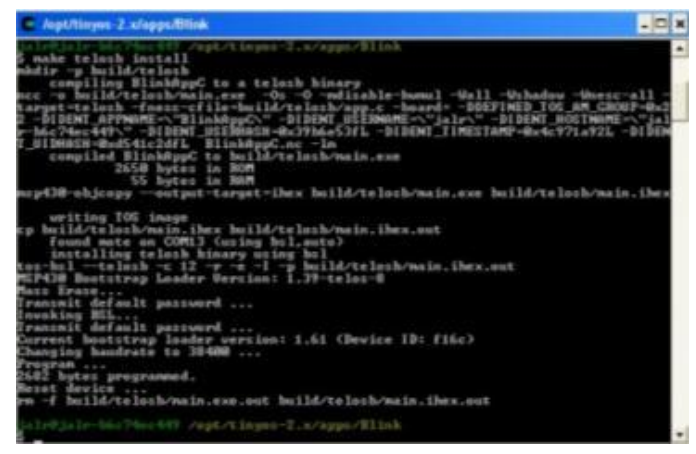

Figura I.23. Salida de Cygwin durante la compilación y descarga de una aplicación. 
TinyOS utiliza una arquitectura software única, que fue diseñada específicamente para nodos sensores con restricciones de recursos. Basado ante todo en el concepto de wiring (conexión de interfaces entre componentes) de componentes para crear una aplicación, el paradigma se esfuerza en usar la mínima memoria posible, al mismo tiempo que maneja múltiples funciones en una red de sensores. Inicialmente escrito en lenguaje $\mathrm{C}$, el proyecto ha sido escrito en un lenguaje propio, nesC [Gay, 2003]. Este "Network Embedded System C" usa una sintaxis similar a la del lenguaje C, pero añade nuevas características para soportar la estructura y el modelo de ejecución de una aplicación escrita en TinyOS [Stojmenovic, 2005].

Durante la ejecución de una típica tarea en cualquier sistema operativo, la pila de la memoria asigna espacio para el almacenamiento de los registros de activación y las variables locales. Esto, típicamente conlleva asignación de espacio de una pila separada para cada tarea en ejecución; sin embargo, debido a las limitaciones de memoria de los nodos sensores, los desarrolladores de TinyOS eligieron adoptar un nuevo modelo de ejecución, que es muy adecuado para dispositivos con poca memoria.

Para minimizar la memoria necesaria durante la ejecución, las aplicaciones de TinyOS consisten en múltiples tareas que comparten una única pila. Debido a este diseño, una tarea tiene que empezar y terminar la ejecución, antes de ceder el procesador y la pila a otra tarea. Las tareas pueden ser interrumpidas por manejadores de eventos hardware, que también se ejecutan hasta completar su ejecución. Naturalmente, antes de ejecutarse el evento, la tarea interrumpida almacena en memoria los estados necesarios para poder continuar su ejecución posteriormente.

Como se ha comentado anteriormente, otro aspecto único de TinyOS es su uso de componentes para crear aplicaciones, al contrario que desarrollar bibliotecas de funciones que pueden ser invocadas en el programa de usuario. Los componentes son bloques separados de código que tienen interfaces definidas de entrada y salida. Para proporcionar una interfaz, el componente debe implementar los comandos definidos en la interfaz. Para usar una interfaz, el componente implementa un conjunto diferentes de funciones, llamados eventos. Usando esta estructura, un componente que quiere utilizar los comandos de una interfaz específica, debe, también, implementar los eventos de la misma interfaz.

Cualquier aplicación desarrollada en TinyOS está constituida por componentes e interfaces. A su vez, los componentes pueden ser de tipo módulo o configuración, que, a su vez, pueden ser clasificados como generic o singleton. Los de tipo singleton son de los que sólo puede haber uno en todo el sistema operativo. Al contrario, pueden haber muchos tipo generic. Todo ellos tiene el mismo nombre, pero pueden coexistir varios en una misma aplicación porque cada uno tiene asociado un alias diferente. La diferencia entre componente de tipo módulo o configuración reside en el contenido del componente. Así, 
un componente de tipo módulo incluye código fuente en nesC para implementar el funcionamiento requerido por el componente. Por otro lado, los componentes de tipo configuración se utilizar para realizar el conexionado o 'wiring' de otros componentes. Cualquier aplicación desarrollada en TinyOS tendrá, al menos, dos componentes. Uno de tipo módulo, en el que el desarrollador escribirá el código fuente de la aplicación utilizando las interfaces de otros componentes, y otro de tipo configuración detallando el wiring entre el anterior y los componentes usados del sistema operativo o de tipo primitiva. Finalmente, cabe destacar que todos los componentes que conforman TinyOS se pueden clasificar en tres niveles de abstracción [Handziski, 2005; Levis, 2005]: Hardware Presentation Layer (HPL), Hardware Adaptation Layer (HAL) y Hardware Interface Layer (HIL). Los componentes del nivel de abstracción HPL están situados directamente sobre la interfaz hardware y software, y acceden al hardware, bien por memoria o mediante el mapeo de líneas de E/S. En un nivel superior se encuentran los de tipo HAL, que usan las interfaces de bajo nivel proporcionadas por los componentes de la HPL para construir interfaces de mayor nivel de abstracción que ocultan la complejidad de acceder directamente al hardware. El nivel final en la arquitectura está formado por los componentes de la HIL, que utilizan las abstracciones específicas de la plataforma proporcionada por la HAL y las convierte a interfaces independientes del hardware. Estas interfaces, proporcionan una plataforma de abstracción independiente del hardware sobre la que se simplifica el desarrollo del software de la aplicación al ocultar las diferencias del hardware. Al igual que con los componentes, las interfaces también están presentes en los 3 niveles de abstracción descritos anteriormente. Así, hay interfaces a nivel de abstracción HPL que permiten acceder directamente y a bajo nivel con el hardware y el software, las HAL con un mayor nivel de abstracción, y las HIL independientes del hardware.

Para ilustrar brevemente parte de lo anteriormente expuesto, se describirá la aplicación Blink (Blink A $\not p C$ ). Esta aplicación instancia 3 temporizadores, y cada uno de ellos cambia el estado de un LED del mote. La apariencia final es que los LEDs muestran una cuenta binaria entre 0 y 7. La Figura 1.24 muestra los componentes que incluye la configuración Blink $A p p C$. Esta figura, la genera TinyOS usando la herramienta gráfica Graphviz [Graphviz].

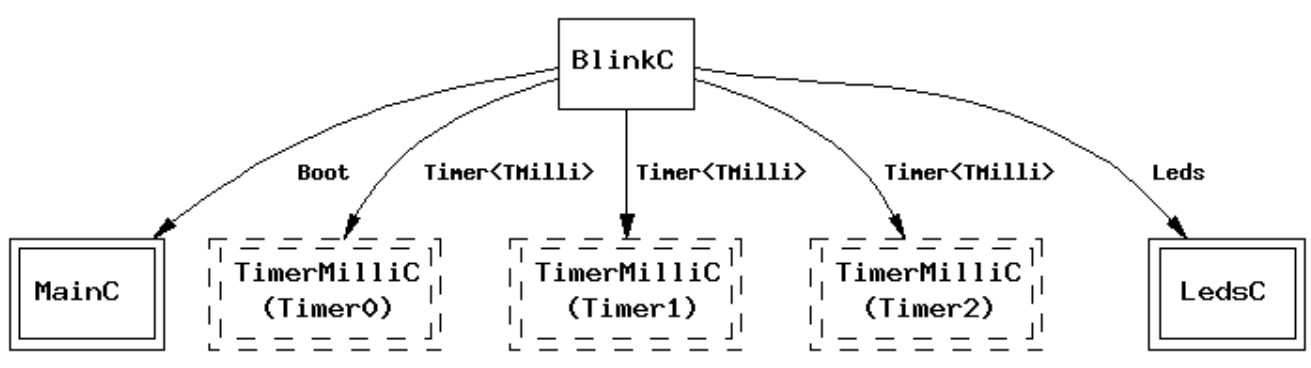

Figura I.24. Descripción gráfica de la aplicación BlinkAppC. 
En la citada figura, los componentes con línea simple son de tipo módulo y los de doble línea son de tipo configuración. A su vez, los de tipo generic se diferencias de los de tipo singleton por una línea discontinua. Como se puede observar en la figura, es lógico que el componente de tipo configuración MainC sea de tipo singleton, ya que sólo hay uno por aplicación. En cambio, se pueden necesitar diferentes temporizadores, y por ello el componente TimerMilliC es un componente de tipo configuración y generic. En el componente de tipo módulo Blink $C$ reside el código fuente nesC de la aplicación. El siguiente listado muestra un extracto del mismo:

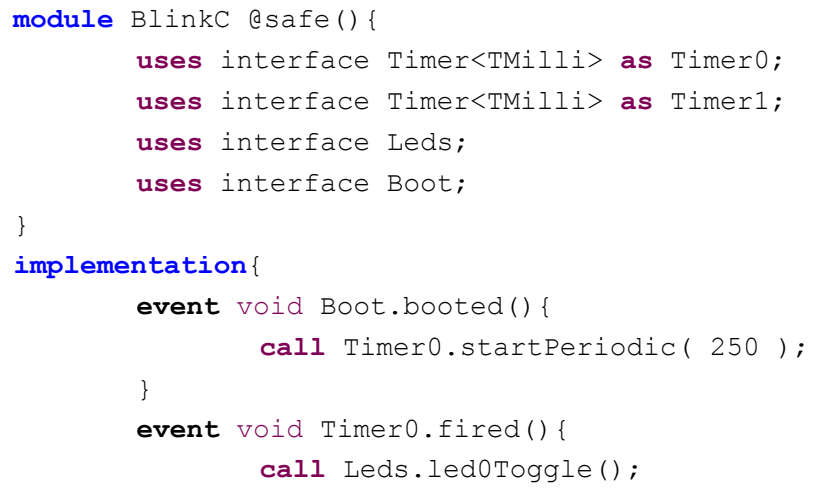

La primera línea define el nombre del módulo y las interfaces que usa, tanto propias como proporcionadas por otros componentes (MainC, TimerMilliC y LedsC). A continuación, dentro de la implementación aparecen los eventos. El primer evento es señalado cuando comienza la ejecución de la aplicación y es equivalente a la clásica función main() del lenguaje C. Dentro de este evento, se inicializan 3 temporizados (sólo se muestra uno) utilizando el comando startPeriodic de la interfaz Timer, proporcionada por el componente TimerMilliC. Finalmente, en respuesta al temporizador configurador, cada vez que transcurre el tiempo establecido la ejecución, llega al evento fired de la interfaz Timer. Conviene destacar que, como se usan 3 temporizadores, es necesario diferenciar la interfaz Timer con un alias, el cual se usa, posteriormente, para acceder a ella.

En último lugar, para definir el flujo de diseño se muestra un extracto del código fuente del componente de tipo configuración BlinkAppC:

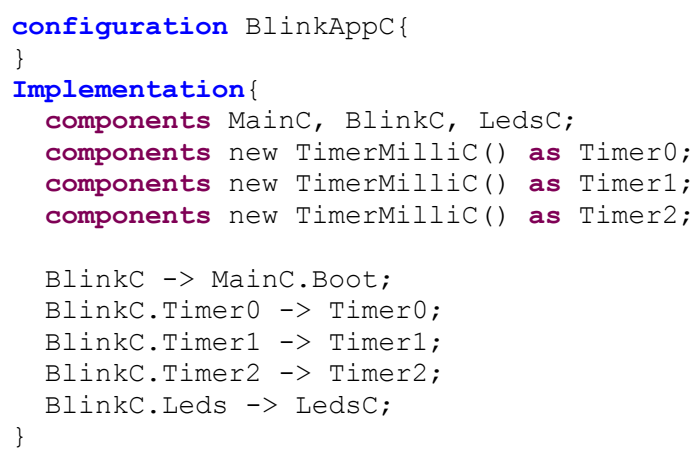


La primera línea define el componente de tipo configuración con su nombre. En este caso, el cuerpo está vacío (no se define que se usa ni se proporciona una interfaz) porque se trata de la configuración de nivel de abstracción superior de la aplicación y todas las aplicaciones deben tener una de este tipo. Como se puede observar, en primer lugar, se definen los componentes de la aplicación y que aparecen en el diagrama de la Figura I.24 y, posteriormente, se realiza el wiring entre ellos. Esto consiste en indicar que la interfaz que usa un componente es proporcionada por otro componente, utilizando, para ello, la siguiente sintaxis: componente_usa_interfaz:Interfaz_-> componente_proporciona_interfaz:Interfaz:

Finalmente, cabe destacar que TinyOS no es sólo una biblioteca de componentes e interfaces implementadas en lenguaje nesC. En realidad, es un entorno de desarrollo de software que incluye numerosas bibliotecas y herramientas para facilitar el diseño. En relación con las bibliotecas, están implementadas en lenguajes C, Java y Python, y permiten acceder a los mensajes que llegan al servidor a través del nodo sumidero de la WSN. Dentro de las herramientas, cabe destacar la aplicación Java Oscilloscope, la cual permite ver los datos recibidos de la red gráficamente, y el simulador TOSSIM [TOSSIM, 2003].

Además de TinyOS, existen otros sistemas operativos como Mantis [Mantis], SOS [SOS] y Contiki [Contiki]. Por describir brevemente alguno más, Contiki [Dunkels, 2004] En el diseño de un sistema operativo para redes de sensores, el reto consiste en encontrar mecanismos y abstracciones ligeros que proporcionen un entorno. Contiki [Dunkels, 2004] es un sistema operativo desarrollado para entornos de ejecución suficientemente ricos, dentro de las limitaciones de los dispositivos con restricciones. Contiki proporciona carga y liberación de programas individuales y servicios. El kernel es dirigido por eventos, pero el sistema soporta multi-threading anticipado, que puede ser aplicado sobre un proceso base. El multi-threading anticipado está implementado en una biblioteca que es enlazada sólo cuando los programas requieren explícitamente multi-threading.

Contiki está implementado en lenguaje $\mathrm{C}$ y ha sido portado a diferentes microcontroladores, incluyendo el MSP430 de Texas Instruments y el AVR de Atmel. Cabe destacar su uIPv6, que se trata de la implementación de código abierto, compatible con la pila IPv6 más pequeña que existe. uIPv6 pasa todas las pruebas requeridas por una pila IPv6, y por tanto es IPv6 Ready. Por este motivo, no es de extrañar que los desarrolladores de uIPv6 formen parte de la alianza IPSO [IPSO], que promueve el uso del protocolo IP para los denominados smart devices, y referido en otros lugares como el Internet de las cosas.

\section{I.4.1.3 Bibliotecas software.}

Para desarrollar el software del último mote estudiado e implementado en la presente tesis, se hizo uso de la biblioteca Z-Stack, desarrollada por Texas Instruments. La Z-Stack es la implementación de la pila ZigBee de Texas Instruments para diferentes dispositivos 
IEEE 802.15.4 y plataformas comercializados por el fabricante. Esta implementación se puede descargar gratuitamente en [Z-Stack]. Esta biblioteca es compatible con la especificación de ZigBee 2007 (ZigBee PRO y ZigBee), soportando tanto el conjunto de funciones de ZigBee como de ZigBee PRO en las plataformas formada por un microcontrolador de la familia MSP430 y el módulo de radio CC2520 y en el System-on-Chip (SoC) CC2530. La biblioteca, también soporta los perfiles Smart Energy y Home Automation. En cuanto a sus características, cabe destacar que incluye una batería de aplicaciones que reduce el tiempo de desarrollo de una nueva aplicación software, que soporta descarga Over the Air (los nodos se pueden programar inalámbricamente) y que es compatible con range extender CC2591, que se trata de un chip que permite ampliar el alcance inalámbrico y mejora la sensibilidad de recepción. Finalmente, cabe destacar que la biblioteca no implementa sólo la funcionalidad del protocolo de comunicaciones, sino que proporciona una serie de drivers para poder manejar el hardware del micro-controlador, como los convertidores analógico-digitales y de las placas de los kits de desarrollo, como un LCD, asociados con un transceptor determinado.

\section{I.4.1.4 IAR Embedded Workbench para MSP430.}

La biblioteca Z-Stack está desarrollada en lenguaje C con el entorno IAR Embedded Workbench, desarrollado y comercializado por IAR Systems [IAR]. Se trata de un entorno de desarrollo de software que permite edición, compilación, descarga y depuración del código desarrollado en lenguaje C. El entorno no incluye ningún simulador. Sin embargo, el código se puede verificar realizando una simulación real en el micro-controlador (depuración). El entorno está disponible para diferentes familias de micro-controladores. Existen diferentes versiones compatibles con el 8051, la familia Atmel AVR y la serie MSP430, entre otros. En este caso, es necesario usar la última versión mencionada. La Figura I.25 presenta la vista de la interfaz de este entorno de desarrollo de software. En ella, se puede observar en la parte superior izquierda el árbol de archivos que componen el proyecto, la ventana de edición a la derecha y la ventana de salida en la parte inferior.

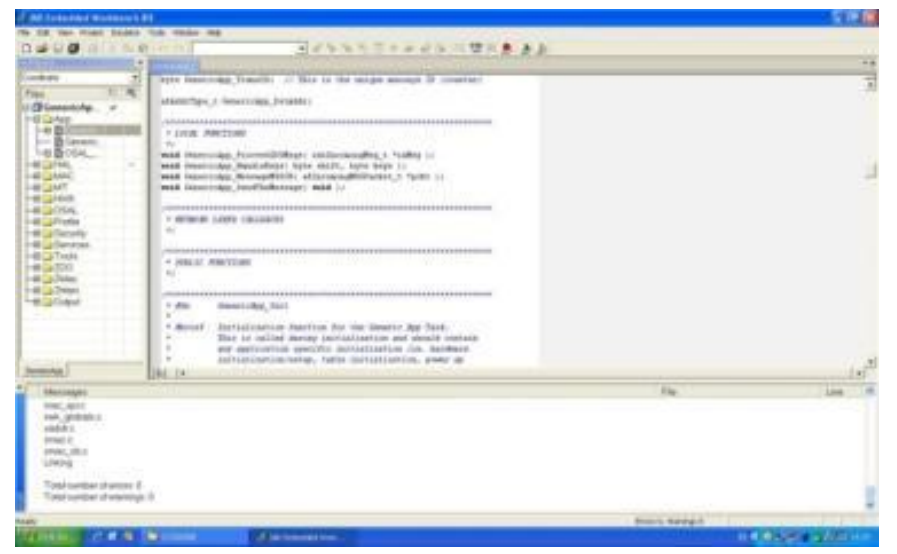

Figura I.25. Entorno de compilación y depuración IAR Embedded Workbench para MSP430. 


\section{I.4.2 Herramientas hardware.}

En este apartado se van a describir las herramientas hardware utilizada durante el transcurso de la tesis por orden cronológico. Estas son las siguientes: la plataforma TelosB, la placa de desarrollo MSP430-P1611, el cable de programación MSP430-JTAG, los módulos de inserción MSP430-H1611, CC2420EMK y CC2520-CC2591EMK, y el kit de desarrollo CC2520DK.

Para aprender a diseñar software para WSN con el sistema operativo TinyOS se utilizó la plataforma hardware TelosB [TelosB]. Se trata de un desarrollo de la Universidad de Berkeley, que es ampliamente utilizado a nivel de investigación. Como se puede observar en la Figura I.26, la plataforma está basada en el micro-controlador MSP430F1611 y el módulo de radio CC2420, ambos de Texas Instruments. Además, incluye sensores y otros elementos externos (memoria flash, LEDs, pulsadores, entre otros) que facilitan el desarrollo de aplicaciones. El dispositivo se puede alimentar desde el puerto USB o mediante dos baterías de tipo AA. La programación del micro-controlador se puede llevar a cabo por el puerto USB (que emula un puerto serie) o por interfaz JTAG, que también permite la depuración de la aplicación cargada.
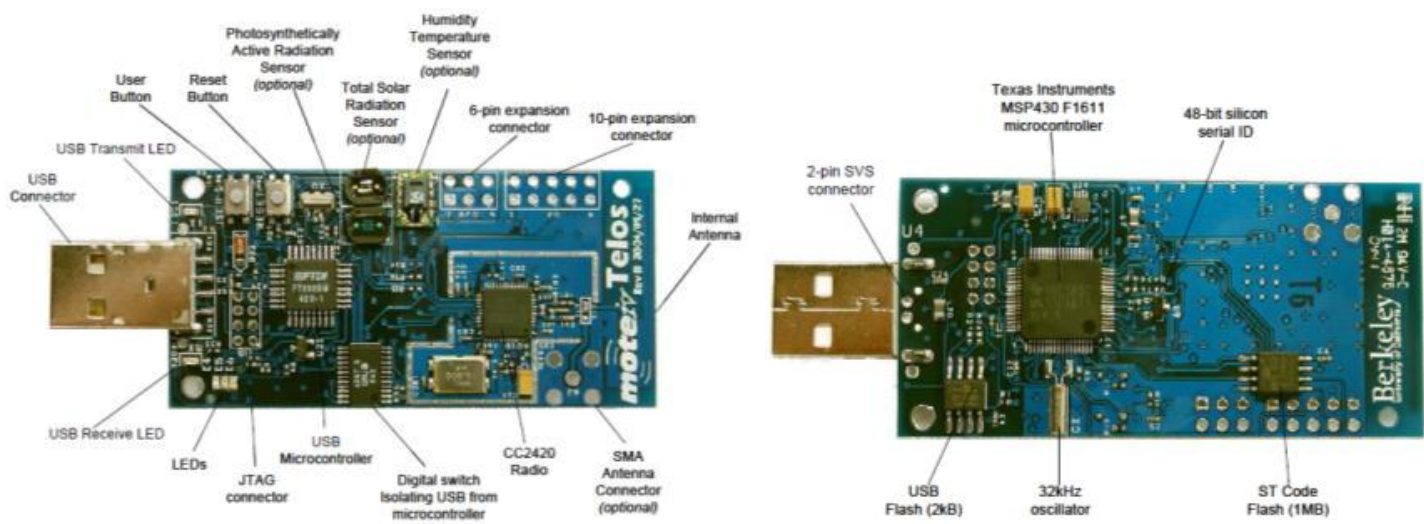

Figura I.26. Vistas y detalle de componentes del mote TelosB.

Con el propósito de que el primer prototipo de nodo sensor objeto de estudio en la tesis fuese compatible con el sistema operativo TinyOS, su diseño se basó en los elementos principales del mote TelosB: micro-controlador MSP430F1611 y módulo de radio CC2420. Por ello, para comenzar con el estudio e implementación del mismo era necesario adquirir placas de desarrollo y/o módulos de inserción de estos elementos.

El primer reto consistió en conseguir programar un micro-controlador con un firmware generando con TinyOS. Para ello se adquirieron placas de desarrollo MSP430P1611 y programadores mediante interfaz JTAG MSP430-JTAG, siendo ambos elementos diseñados y comercializados por Olimex [Olimex]. Como se puede obervar en la Figura 1.27, la placa de desarrollo básicamente hace accesibles los pines del micro y facilita la 
alimentación y descarga del firmware en el dispositivo utilizando el cable también mostrado en la figura. Cabe destacar que la programación de una aplicación generada con TinyOS en la placa de desarrollo se llevó a cabo con la utilidad msp430-jtag, perteneciente al conjunto de herramientas para la familia MSP430, de libre distribución, MSPGCC [Mspgcc].
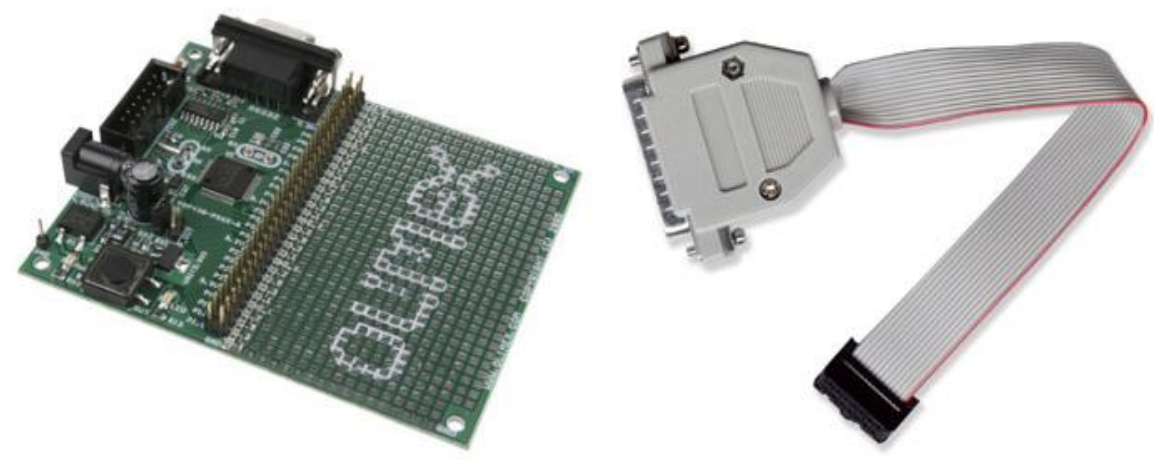

Figura I.27. Placa de desarrollo MSP430-P1611 y programador por interfaz JTAG MSP430-JTAG.

Posteriormente, con objeto de diseñar prototipos con el menor tamaño posible, se adquirieron módulos de inserción del micro-controlador MSP430F1611 y del transceptor de radio CC2420. Estos módulos de inserción se muestran en la Figura I.28. La imagen de la izquierda se corresponde con el módulo de inserción del micro, con referencia MSP430H1611 y comercializado por Olimex. La imagen de la derecha es el módulo de inserción del módulo de radio CC2420. Este elemento está desarrollado y lo comercializa Texas Instruments, con la referencia CC2420EMK, y se trata de un kit que incluye dos módulos de inserción del chip CC2420. Conectando adecuadamente estos dos elementos, se tendría la estructura básica del mote TelosB en un tamaño relativamente reducido.

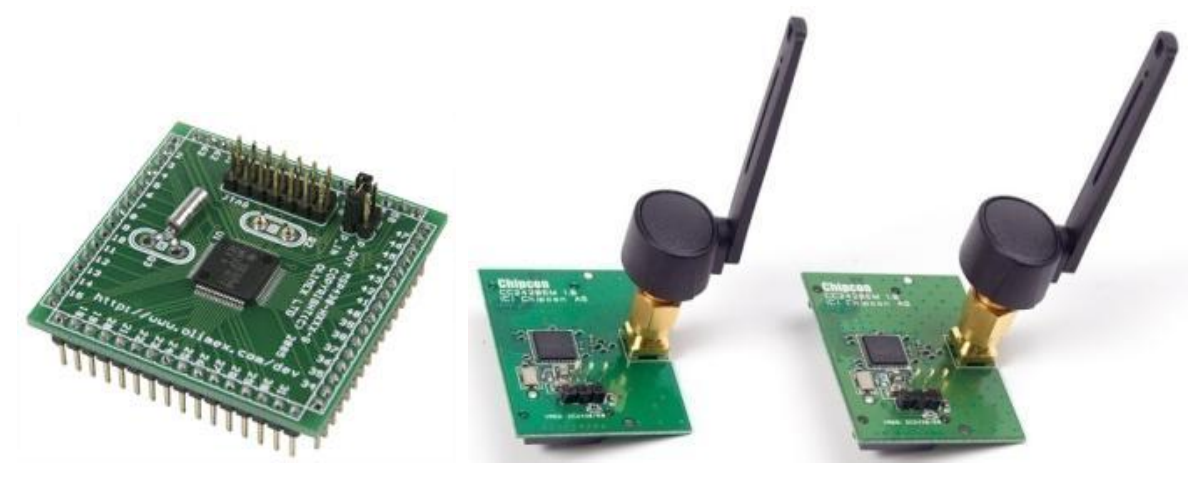

Figura I.28. Módulo de inserción MSP430-H1611 y kit de módulos de inserción CC2420EMK.

Por otro lado, el último nodo sensor objeto de estudio e implementación de la presente tesis está basado el chip de radio CC2520. Para agilizar el flujo de diseño se adquirió el kit de desarrollo CC2520DK, que facilita las tareas de diseño con el mencionado módulo y es comercializado por Texas Instruments. El kit CC2520DK (Figura I.29) está compuesto por los siguientes elementos: 3 placas de expansión SmartRF05EB, 3 módulos 
de evaluación del CC2520 (CC2520EM) con sus antenas, 2 placas de desarrollo del microcontrolador MSP430F2618 (CCMSP-EM430F2618) y una interfaz de programación y depuración mediante interfaz JTAG MSP-FET430UIF. La vista de la derecha muestra las 3 placas diferentes ensambladas y conectados al programador, que constituye un nodo sensor completo con el micro-controlador MSP430F2618 y el módulo de radio CC2520DK. La placa de desarrollo SmartRF05EB incluye un LCD, un puerto serie RS-232, 4 diodos LED, 2 botones, un potenciómetro y un joystick. Todos estos elementos facilitan en gran medida la tarea de depuración a la hora de desarrollar una nueva aplicación. Finalmente, cabe destacar que el kit incluye 3 CC2520EMs y 3 SmartRF05EBs porque con la conexión de las anteriores se obtiene un analizador de paquetes o 'packet sniffer', que permite ver el tráfico que hay en la red de sensores, así como inyectar paquetes en ella.
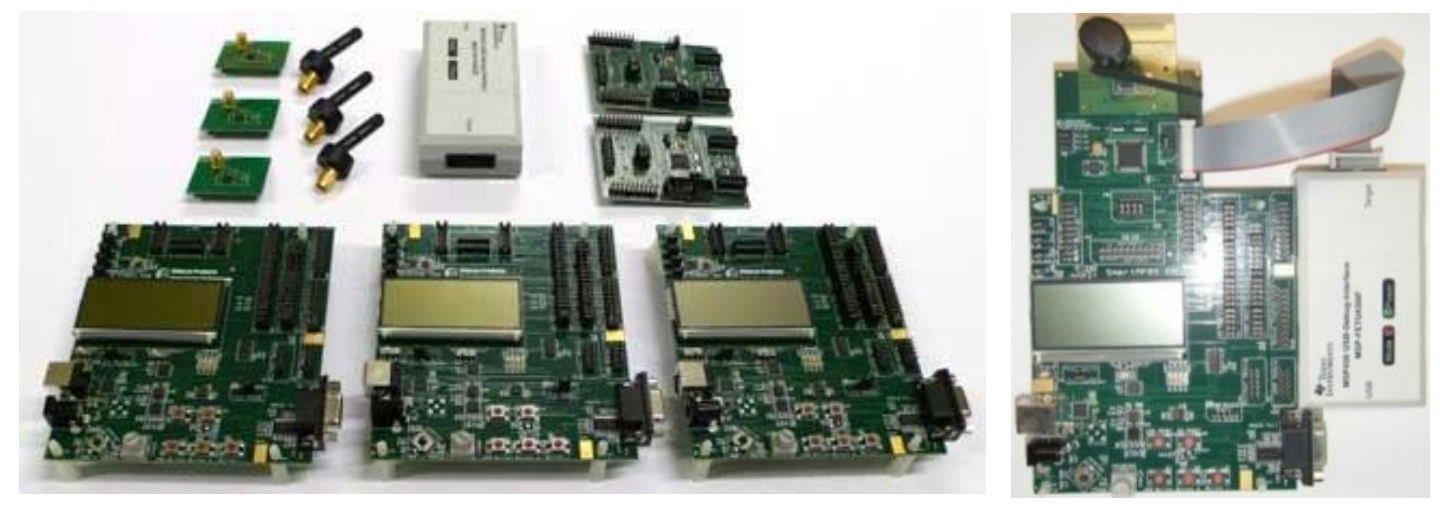

Figura I.29. Kit de desarrollo CC2520DK.

En el kit anterior se puede substituir la conexión del módulo de inserción CC2520EM por el CC2520-CC2591EM (Figura I.30), también comercializado por Texas Instruments. El módulo de inserción es la combinación del módulo de radio CC2520 y del CC2591, que se trata de un range extender. Como su nombre indica, permite incrementar el alcance, y por tanto la calidad del enlace inalámbrico. Utiliza, para ello, un amplificador de potencia (PA, Power Amplifier), el cual permite incrementar la potencia de salida hasta 20dBm. Dispone, también, de un amplificador de bajo ruido (LNA, Low Noise Amplifier) para mejorar la sensibilidad del receptor.

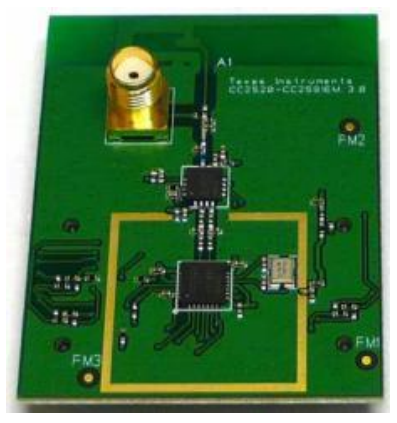

Figura I.30. Módulo de inserción CC2520-CC2591EM. 


\section{I.4.3 Herramientas para el despliegue.}

Una herramienta muy útil para el despliegue de WSN es el analizador de espectros. Las versiones portátiles del analizador de espectro se puede clasificar dentro del método heterodino y el de la transformada de Fourier. El método del heterodino también se llama el método barrido de frecuencia y permite medir hasta decenas de $\mathrm{GHz}$, solamente para la señal inmóvil. Por otra parte, el método de la transformada de Fourier puede manejar una señal no estacionaria desde bajas frecuencias hasta los MHz.

Los analizadores de espectros de gran tamaño y consumo eran aptos para trabajar en laboratorio. Pero han tenido que reducir su tamaño y hacerse portátiles para trabajar en el campo, debido a la reciente dispersión de los equipos de telecomunicaciones sin cables. La mayoría de analizadores de espectro portátiles permiten medir en campo la potencia del canal, el ancho de banda utilizado, así como ver la actividad de los canales de radio, con objeto de escoger el menos saturado para establecer los enlaces inalámbricos en al comunicación. Un equipo comercial es el MSA438 de Micronix [Micronix]. Este instrumento permite determinar la potencia en un canal, así como en el adyacente, el ancho de banda ocupado, la intensidad del campo eléctrico y magnético, y la frecuencia. El equipo permite analizar frecuencias desde los $50 \mathrm{KHz}$ hasta los $3,3 \mathrm{GHz}$, y por lo tanto, se puede emplear en aplicaciones de telefonía móvil, 2,4GHz LAN inalámbrica, WiMAX, RF-ID y redes de sensores.

Cuando se emplean protocolos de comunicación complejos, como, por ejemplo, ZigBee resulta muy útil disponer de un analizador de redes. Estas herramientas visualizan, de forma intuitiva mediante una interfaz gráfica, los paquetes del protocolo de comunicación seleccionado que están circulando por la red en el punto en el que está colocado el receptor del analizador. Está por tanto compuesto de una parte hardware y otra software. Daintree Networks [Daintree] ha desarrollado un analizador de redes. El sistema está compuesto (ver Figura I.31) del adaptador de red 2400E y el software SNA (Sensor Network Analyzer). La herramienta permite analizar paquetes del IEEE 802.15.4 y ZigBee, y recientemente ha ampliado su soporte para incluir otros protocolos de comunicación de red, basados en estándares y en sistemas propietarios como ZigBee RF4CE, 6LoWPAN, Jennet (de Jennic), SimpliciTI (de Texas Instruments) y Synkro (de Freescale Semiconductor). Las características de la SNA incluyen un decodificador de protocolos de gran alcance que permite navegar por los distintos paquetes, campos, y a nivel de byte; capacidades únicas de visualización que permiten ver todos los dispositivos de red y de las interacciones simultáneamente; opciones de configuración incluyendo filtrado, etiquetado y codificación por colores para que resulte fácil localizar los paquetes de interés; medidas de rendimiento de 802.15.4 y ZigBee, y herramientas intuitivas que facilitan para realizar 
funciones complejas tales como la captura de varios canales y la puesta en marcha (o 'commissioning') de ZigBee.
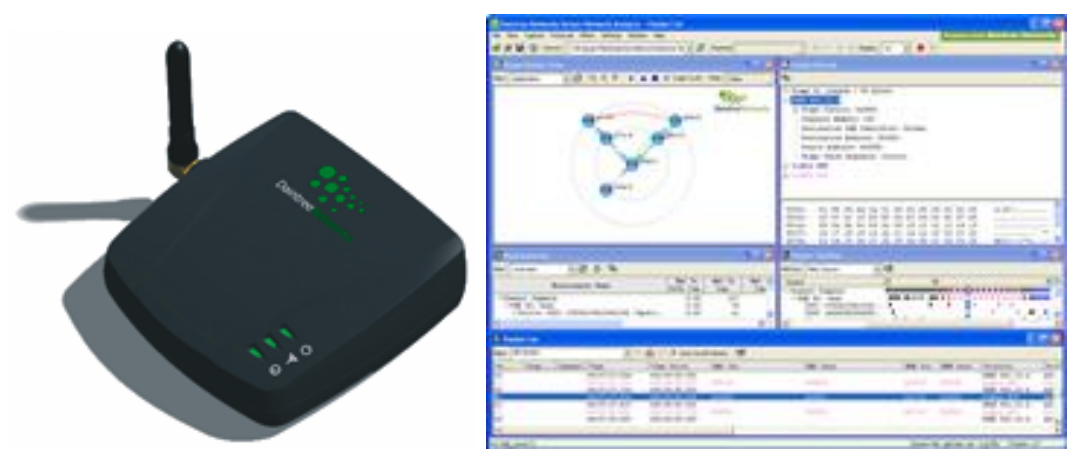

Figura I.31. Adaptador de red 2400E y software SNA de Daintree Networks.

Una alternativa, de bajo coste, a los analizadores de redes, son los denominados 'packet sniffer', que suelen ofrecen los desarrolladores de chips wireless junto con los kits de desarrollo que comercializan. Un ejemplo es el SmartRF Packet Sniffer [Sniffer], de Texas Instruments. Esta solución soporta ZigBee, RF4CE y SimpliciTI, así como muestra tramas de otros protocolos a nivel de byte. Esta solución no ofrece tanta funcionalidad como la ofrecida por Daintree Networks, pero muestra los paquetes del protocolo seleccionado de una manera intuitiva.

\section{I.5 Métodos.}

\section{I.5.1 Desarrollo de hardware para redes de sensores.}

El diseño e implementación de un nodo sensor se puede realizar por dos caminos diferentes: partir de un mote comercial, como el TelosB, y diseñar la electrónica de acondicionamiento con los sensores y/o actuadores, o bien, diseñar un dispositivo 'ad-boc' desde cero partiendo de un conjunto de elementos básicos. El último caso, conlleva un tiempo y gasto económico bastante considerable, y sólo se debería seguir este camino cuando se pretendan fabricar muchas unidades del dispositivo.

Para seleccionar un mote comercial para un caso de estudio concreto, es necesario tener en cuenta una serie de características, entre las que se pueden citar las siguientes: frecuencia de trabajo y potencia de transmisión del módulo de radio, interfaces de entrada/salida (SPI, I I C, UART, convertidores analógico-digitales, etc.) del microcontrolador o microprocesador, disponibles en el conector de expansión, tensión de alimentación y consumo de potencia requerido, etc. Atendiendo a estas características, se puede obtener una tabla comparativa de los dispositivos comerciales existentes y optar por el más adecuado. El siguiente paso a llevar a cabo, si fuese necesario, sería el desarrollo de 
la electrónica de acondicionamiento entre los periféricos y el mote seleccionado. A continuación, una vez conocidos los requerimientos energéticos del dispositivo con la electrónica de interfaz y los periféricos (sensores y actuadores), habría que definir el sistema de alimentación del dispositivo. En los casos más sencillos, bastará con usar un conjunto de baterías de tipo AAA o AA y, en los más complejos, serán necesarios convertidores DC/DC y elementos para obtener energía alternativa del entorno (paneles solares, aerogeneradores, etc.). Finalmente, los motes comerciales están preparados para usarse en laboratorio y, si se quieren desplegar en una aplicación real, será necesario que tengan asegurado un determinado grado de protección IP mediante una carcasa adecuada. Ésta se podría diseñar a medida, o bien usar una caja estanca comercial.

Por otro lado, el flujo de diseño para fabricar un nuevo dispositivo hardware resulta una tarea ardua y compleja en cualquier disciplina y, en el caso de una WSN, no iba a ser menos. El primer paso, consiste en determinar los requisitos que debe cumplir el dispositivo. Entre ellos, se pueden enumerar los siguientes: frecuencia de trabajo y potencia de transmisión (alcance) del módulo de radio, grado de protección IP, interfaces de entrada/salida necesarias, autonomía, topología de la red, etc. A partir de la topología requerida, se puede determinar el protocolo de comunicaciones necesario (IEEE 802.15.4, ZigBee, etc.), y con el anterior y los requisitos de frecuencia de trabajo y potencia de transmisión se escogería un transceptor o módulo de radio de los existentes en el mercado. Conocidos los periféricos (módulo de radio, sensores, actuadores, memorias externas, etc.) de la unidad de procesamiento (micro-controlador o microprocesador), se puede determinar un micro-controlador o microprocesador comercial para el mote. A continuación, habría que adquirir un kit de desarrollo o módulos de inserción del transceptor de radio y la unidad de procesamiento. Con ellos sería necesario construir un prototipo, basado en módulos de inserción, que asegure el funcionamiento del dispositivo. Naturalmente, en esta última compleja tarea es necesario un trabajo paralelo en desarrollo de software para verificar el correcto funcionamiento entre los periféricos y la unidad de procesamiento. Partiendo del diseño anterior y, como la placa del dispositivo final estará basada en componentes de montaje superficial (SMD, Surface Mounted Devices), es necesario construir una segunda versión del mote con la mayor parte de componentes SMD y la menor cantidad de componentes de inserción. Con el prototipo anterior se realizaría un estudio de consumo, con el fin de dimensionar la batería necesaria para asegurar la autonomía del dispositivo impuesta como requisito. En determinados casos, se puede reducir el tamaño de la batería usando una o varias fuentes de energía para recargarla. Por lo tanto, será necesario estudiar si es necesario emplear una o varias fuentes de energía alternativas y diseñar la electrónica necesaria entre el dispositivo, el sistema de energía alternativo y la batería, que normalmente estará constituida por reguladores lineales de tensión, cargadores, convertidores DC/DC, etc. Llegado este punto, el dispositivo a nivel electrónico está completamente definido y se puede realizar la llamada miniaturización del 
diseño, que consiste en definir una placa de circuito impreso completamente en SMD con un software de tipo CAD. A continuación, se fabricaría una pequeña pre-serie del dispositivo (10 ó menos) para verificar el correcto funcionamiento (pruebas de alcance, consumo, autonomía, etc.). Una vez verificado un correcto funcionamiento, y conocidos los tamaños de la placa de circuito impreso y de la batería, faltaría determinar una carcasa que asegure el grado de protección impuesto en los requisitos. Al igual que partiendo del mote comercial para el diseño, en este caso se puede optar por diseñar una carcasa a medida o usar una caja estanca comercial. El diagrama de la Figura 1.32 muestra gráficamente la selección de elementos que hay que llevar a cabo para diseñar un nodo sensor.

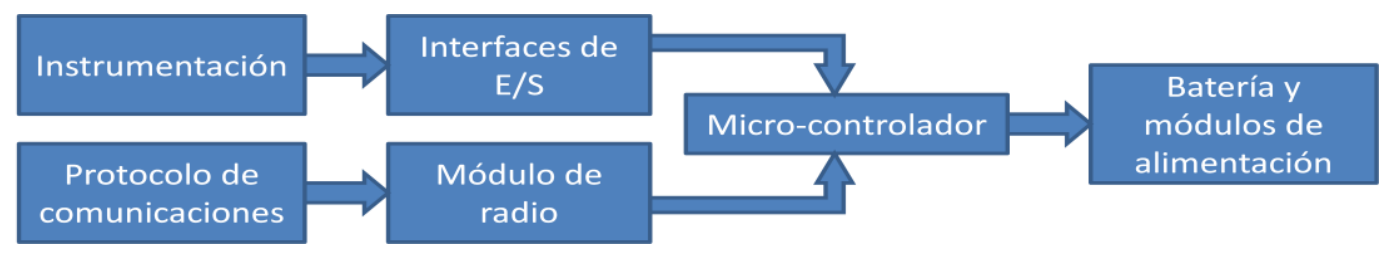

Figura I.32. Selección de elementos para implementar un nodo sensor.

Lo que se ha expuesto sería un breve resumen del proceso a seguir para diseñar e implementar un nuevo dispositivo. Conviene resaltar que un punto crítico de diseño es el consumo del mote en modo 'standby', que es en el que normalmente se encuentra y el cual debe ser mínimo. A modo de ejemplo, aunque parezca excesivo, es necesario reducir el consumo de 'standby' de $200 \mu \mathrm{A}$ a $150 \mu \mathrm{A}$. Esta reducción es necesaria porque permitiría ampliar la autonomía del mote en varias semanas.

\section{I.5.2 Desarrollo de software para redes de sensores.}

Tener que trabajar directamente con los recursos hardware de los motes convierte la programación de una aplicación en una dura tarea. Para simplificar esto se está recurriendo a sistemas operativos que aíslen al programador de las particularidades del hardware. Estos sistemas operativos constituyen el núcleo en torno al cual se van a desarrollar aplicaciones, ofreciendo unos servicios básicos que serán ampliados por otros servicios y herramientas adicionales. Entre estas ampliaciones podemos encontrar middleware distribuidos sobre los que implementar las aplicaciones software, como por ejemplo TinyDB [Madden, 2005], que trabaja con la red de sensores como si de una base de datos se tratara (usando sentencias SQL).

Puesto que los sistemas operativos constituyen la pieza sobre la que se va a construir el software para redes de sensores, conviene realizar una serie de consideraciones sobre su diseño. Principalmente, dadas las características y limitaciones de estas redes, los sistemas operativos que vayan a trabajar con ellas deben tener en cuenta dos aspectos muy importantes: la gestión de la potencia y la escasez de recursos hardware disponibles. 
Respecto al primero de éstos, cada sistema operativo tiene sus propias técnicas, pero todas coinciden en desactivar los subsistemas hardware que no están siendo utilizados, en concreto la radio y la CPU. En cuanto al segundo aspecto, se deben reducir las necesidades que tanto de memoria RAM, como de memoria de programa tenga el sistema desarrollado. De esta forma, se permite que, tanto sistemas operativos como aplicaciones, puedan ser usados en motes con capacidades muy limitadas.

La imposición de un bajo consumo por parte de los motes, ha popularizado el desarrollo de sistemas operativos dirigidos por eventos (aunque no todos siguen esta aproximación). Con esta filosofía, el procesado de los datos por la CPU comienza en el momento que se genera un evento, que es gestionado por su correspondiente manejador. El código generado mediante esta aproximación será asíncrono, lo que para redes de sensores permite lograr una mayor eficiencia energética. Gracias a esta asincronía, tras solicitar un dato, una aplicación no queda bloqueada a la espera de que el dato esté disponible. De esta forma es posible dormir la CPU mientras se está esperando el dato para despertarla una vez se obtenga. Como contrapartida, la programación de aplicaciones es algo más compleja.

Un buen sistema operativo para redes de sensores, además, debe contar con otras características. Dada la constante evolución de esta tecnología, un sistema operativo debe ser extensible y adaptable. La extensibilidad permite que las aplicaciones desarrolladas puedan ir contando con los últimos avances tecnológicos, a medida que se vayan incorporando al sistema operativo. La flexibilidad permite que las aplicaciones se adapten correctamente a los requisitos que se les exigen. De igual manera, el sistema operativo también debe adaptarse y manejar con facilidad los cambios en el hardware, ya que la variedad de dispositivos existentes es enorme y sigue creciendo. Además, como explica Hill [Hill, 2000], la aplicación debe poder controlar directamente todos estos recursos hardware, y permitir que exista interacción entre las distintas capas que la forman.

Otra característica deseable para un nodo sensor es la posibilidad de reprogramarlo sin tener que extraerlo de la red. La forma en que esto se hace difiere entre sistemas operativos. Algunos no permiten esta característica, otros como TinyOS pueden hacerlo pero se debe volcar el programa entero al mote para después reiniciarlo con el nuevo código. Por último, algunos sistemas operativos [Han, 2005][Bhatti, 2005][Dunkels, 2004] pueden cargar dinámicamente módulos aislados. Esto permite la adición de funcionalidades a los nodos sin interrumpir su funcionamiento.

Cada sistema operativo tomará decisiones que mejorarán unos aspectos del sistema en detrimento de otros. Generalmente, la adición de nuevos servicios a los nodos suele acarrear una mayor complejidad del sistema operativo que desborda las capacidades de muchos motes. Los principales ejemplos de esto son la gestión dinámica y protección de 
memoria. Por lo tanto, muchos sistemas operativos están optando por no contar con ellos para reducir los recursos que consumen. Otro caso parecido es la gestión de procesos. Puesto que el cambio de contexto entre dos procesos consume muchos ciclos del procesador se tiende a simplificar este aspecto lo más posible.

Cabe destacar que la Z-Stack no es sólo una biblioteca que contiene una batería de funciones que permiten trabajar con ZigBee. En realidad se trata de una compleja implantación orientada a eventos que informa en todo momento del estado del programa.

Por todo lo citado en los párrafos anteriores, las alternativas tradicionales usadas en otros sistemas empotrados no son válidas para las redes de sensores. Sistemas operativos como WinCE, VxWorks, PalmOS, QNX y pOSEK no cumplen con los requisitos anteriores. Se basan en micro-kernels que permiten la adición o substracción de funcionalidad según se requiera, por lo que no permiten comunicación directa con los recursos hardware. El entorno de ejecución es similar al de un PC, con cambios de contexto entre procesos excesivamente pesados. Algunos de estos sistemas operativos, además, incorporan mecanismos de protección de memoria que complican en exceso el sistema. Éstos son útiles para una PDA, un teléfono móvil o un PC empotrado pero no para un nodo sensor. Otras alternativas como plataformas middleware distribuidas existentes (CORBA, DCOM o RMI) tampoco ofrecen una buena solución por su consumo excesivo de memoria. Se están realizando implementaciones ligeras de CORBA para redes de sensores, sin embargo, tampoco resultan apropiadas por los problemas de bloqueo que ocasiona el mecanismo síncrono de pregunta y respuesta de CORBA. 
CONTRIBUCIÓN A LAS REDES DE SENSORES INALÁMBRICAS. ESTUDIO E IMPLEMENTACIÓN DE SOluCiones Hardware Para Agricultura de PreCisión. 


\section{Anexo II}

\section{Tramas de Datos Intercambiadas entre los Nodos Sensores Diseñados e Implementados durante la Fase de Validación de los Dispositivos.}

\footnotetext{
$n$ este anexo se describen los diferentes tipos de tramas intercambiadas por las redes inalámbricas
de sensores, desplegadas para validar los diferentes nodos sensores diseñados e implementados en la presente tesis doctoral. Se decidió recogerlo en un anexo para facilitar la lectura de los capitulos fundamentales del documento de tesis. La descripción comienza presentando las tramas intercambiadas por los dispositivos de la WSN diseñada e implementada con módulos de inserción, que son descritas en el Capitulo 4. Las tramas que se utilizaron para validar los dispositivos de los capitulos 5 y 6 no es necesario describirlas, ya que se utilizaron las mismas que las utilizadas en la WSN del Capitulo 4. Finalmente, se describirán las tramas utilizadas para validar el nodo sensor $M E W i N$, descrito en el Capitulo 7.
} 


\section{II.1 Introducción.}

En la presente tesis doctoral se ha llevado a cabo el diseño e implementación de diferentes nodos sensores, con los que poder realizar tareas de monitorización y control en cultivos agrícolas.

Todos los dispositivos diseñados se sometieron a un proceso de validación en cultivos reales. Para ello se realizó el despliegue de varias WSNs, en las que fue necesario definir los tipos de tramas necesarios para, tanto recibir y procesar, en la interfaz gráfica de usuario, los datos provenientes de los sensores y poder configurar, desde el software de monitorización de la WSN, la frecuencia de lectura de los sensores. Por ello, y para facilitar la lectura de los capítulos del documento, se decidió describir las tramas utilizadas en este anexo.

En primer lugar, se describirán las tramas intercambiadas por los dispositivos de la WSN diseñada e implementada con módulos de inserción, que son descritas en el Capítulo 4. Finalmente, se describirán las tramas utilizadas para validar el nodo sensor multi-entorno MEWiN, descrito en el Capítulo 7.

Las tramas que se utilizaron para validar el GAIA Soil-Mote y el GAIA 2 no es necesario describirlas, ya que se utilizaron las mismas que en los dispositivos diseñados e implementados con módulos de inserción del Capítulo 4.

\section{II.2Tramas de los nodos sensores basados en módulos de inserción.}

En el Capítulo 4 se describen un conjunto de dispositivos, que se diseñaron e implementaron con módulos de inserción, después de realizar los trabajos de simulación necesarios con la tecnología y el protocolo de comunicaciones seleccionado. Estos dispositivos conforman una arquitectura hardware, basada en WSN, para cultivos hortícolas. Para validar esta arquitectura, se definieron un conjunto de tramas que intercambiaban los dispositivos. Las tramas se pueden clasificar en dos grupos: las intercambiadas entre un Gateway con sus correspondientes nodos sensores, y las intercambiadas entre un Gateway y el Water-Mote con el software de monitorización.

Los Soil-Motes enviaban tramas de 26 bytes con las lecturas de cada sensor Hydra Probe II (HP2) al Gateway (ver Tabla II.1). Los dos primeros bytes (variable de tipo uint16_t) se utilizan para codificar la dirección de red o identificador del nodo sensor dentro de la red. El siguiente byte (variable de tipo uint8_t) es necesario para que el Gateway pueda diferenciar si la trama proviene del sensor HP2 0 ó 1, conectados con cada Soil-Mote. 
Finalmente, los 23 bytes restantes provienen de un array de 23 elementos de tipo vint8_t. En este array se almacena la lectura realizada de cada sensor HP2.

\begin{tabular}{|c|c|c|c|c|c|}
\hline Byte 0-Byte 1 & Byte 2 & \multicolumn{4}{|c|}{ Byte 3-Byte 25} \\
\hline \multirow{2}{*}{$\begin{array}{l}\text { Dirección de } \\
\text { red Variable de } \\
\text { tipo uint16_t }\end{array}$} & \multirow{2}{*}{$\begin{array}{l}\text { Identificador del } \\
\text { sensor Variable de } \\
\text { tipo uint8_t }\end{array}$} & \multicolumn{4}{|c|}{ Lectura del sensor HP2 Array de 23 elementos de tipo uint8_t } \\
\hline & & $\begin{array}{c}\text { Temperatura } \\
\text { del suelo en }{ }^{\circ} \mathrm{C}\end{array}$ & $\begin{array}{l}\text { Porcentaje volumétrico de } \\
\text { humedad de suelo en Water } \\
\text { Fraction Volume (WFV) }\end{array}$ & $\begin{array}{c}\text { Conductividad eléctrica (con } \\
\text { corrección de temperatura) } \\
\text { del suelo en } \mathrm{S} / \mathrm{m}\end{array}$ & $\begin{array}{c}\text { Salinidad del } \\
\text { suelo en } \mathrm{g} / \mathrm{l} \\
\text { de } \mathrm{ClNa}\end{array}$ \\
\hline $0-65535$ & $0-1$ & “ $\pm \mathrm{xx} \mathrm{x} "$ & “ $\pm \mathrm{x}$ xxx" & “ \pm x xxx" & “ $\pm \mathrm{x} x \mathrm{xx} "$ \\
\hline
\end{tabular}

Tabla II.1. Descripción de la trama enviada con la lectura de los sensores HP2 desde los Soil-Motes al Gateway.

Los Environmental-Motes enviaban tramas de 6 bytes con las lecturas del sensor de temperatura y humedad relativa del ambiente SHT71 al Gateway (ver Tabla II.2). Los dos primeros bytes (variable de tipo uint16_t) se utilizan para codificar la dirección de red o identificador del nodo sensor dentro de la red. Los dos bytes siguientes (variable de tipo uint16_t) almacenaban la lectura realizada de temperatura del ambiente en unidades raw. Los 2 bytes restantes almacenan la humedad relativa del ambiente en unidades 'raw'.

\begin{tabular}{|c|c|c|}
\hline Byte 0-Byte 1 & Byte 2-Byte 3 & Byte 4-Byte 5 \\
\hline $\begin{array}{l}\text { Dirección de red Variable } \\
\text { de tipo uint16_t }\end{array}$ & $\begin{array}{l}\text { Temperatura del ambiente } \\
\text { Variable de tipo uint16_t }\end{array}$ & $\begin{array}{l}\text { Humedad relativa del ambiente } \\
\text { Variable de tipo uint16_t }\end{array}$ \\
\hline $0-\left(2^{16}-1\right)$ & $0-\left(2^{14}-1\right)$ & $0-\left(2^{12}-1\right)$ \\
\hline
\end{tabular}

Tabla II.2. Descripción de la trama enviada con la lectura del sensor SHT71 desde los Environmental-Motes al Gateway.

Los Environmental-Motes y los Soil-Motes enviaban tramas de 4 bytes con la lectura del voltaje de la batería al Gateway (ver Tabla II.3). Los dos primeros bytes (variable de tipo uint16_t), se utilizan para codificar la dirección de red o identificador del nodo sensor dentro de la red. Los dos bytes siguientes (variable de tipo vint16_t), almacenaban la lectura del voltaje de la batería en unidades raw. La lectura se realizaba con un convertidor analógico-digital de 12 bits.

\begin{tabular}{|c|c|}
\hline Byte 0-Byte 1 & Byte 2-Byte 3 \\
\hline $\begin{array}{l}\text { Dirección de red Variable de } \\
\text { tipo uint16_t }\end{array}$ & $\begin{array}{l}\text { Lectura del voltaje de la batería } \\
\text { Variable de tipo uint16_t }\end{array}$ \\
\hline $0-\left(2^{16}-1\right)$ & $0-\left(2^{12}-1\right)$ \\
\hline
\end{tabular}

Tabla II.3. Descripción de la trama enviada con la lectura del voltaje de la batería desde los Environmental-Motes y los Soil-Motes al Gateway. 
El Gateway enviaba tramas de 4 bytes a los Environmental-Motes y los Soil-Motes para configurar la frecuencia de lectura de los sensores (ver Tabla II.4). La frecuencia de lectura se podía configurar entre un minuto y cero horas, y 255 minutos y 255 horas. Los dos primeros bytes (variable de tipo uint16_t) se utilizan para codificar la dirección de red o identificador del nodo sensor dentro de la red. El byte siguiente (variable de tipo uint8_t) almacenaba las horas de la nueva frecuencia de lectura a configurar. El último byte (variable de tipo vint8_t) indicaba los minutos de la nueva frecuencia de lectura a establecer en el dispositivo.

\begin{tabular}{|c|c|c|}
\hline \multicolumn{2}{|c|}{ Byte 0-Byte 1 } & Byte 2 \\
\hline $\begin{array}{c}\text { Dirección de red } \\
\text { Variable de tipo } \\
\text { uint16_t }\end{array}$ & $\begin{array}{c}\text { Horas de la frecuencia de } \\
\text { lectura de los sensores } \\
\text { Variable de tipo uint8_t }\end{array}$ & $\begin{array}{c}\text { Minutos de la frecuencia de } \\
\text { lectura de los sensores } \\
\text { Variable de tipo uint8_t }\end{array}$ \\
\hline 0-(216-1) & 0-255 horas & 0-255 minutos \\
\hline
\end{tabular}

Tabla II.4. Descripción de la trama enviada desde el Gateway a los Soil-Motes y a los Environmental-Motes para configurar la frecuencia de lectura de los sensores.

En las 4 tablas anteriores se han descrito las tramas intercambiadas entre los nodos sensores y el Gateway. Cuando el Gateway recibe una trama de un nodo sensor, la envía con otro formato al software de monitorización usando el radio módem XStream. De igual forma, para que el Gateway disemine en los nodos sensores una trama para configurar una nueva frecuencia de lectura de los sensores, es necesario que reciba previamente una trama desde la interfaz gráfica de usuario. Todas las tramas tienen una cabecera común, en la que los dos primeros bytes identifican la subred y a un nodo sensor específico dentro de la subred. El siguiente byte (longitud), almacena la cantidad de bytes que hay que leer desde el byte de longitud para completar la trama. El último byte de la cabecera almacena el tipo de de la trama. Estas tramas se describen en las siguientes tablas.

Cuando el Gateway recibe una trama de datos de un Soil-Mote, envía una trama de 30 bytes al software de monitorización (ver Tabla II.5). En esta trama el campo longitud tiene un valor de 27. El tipo puede ser 50 ó 51, en función de si el sensor es el HP2 0 o el 1.

\begin{tabular}{|c|c|c|c|c|}
\hline Byte 0 & Byte 1 & Byte 2 & Byte 3 & Byte 4-Byte 29 \\
\hline $\begin{array}{r}\text { Identificador } \\
\text { de la subred }\end{array}$ & $\begin{array}{c}\text { Identificador } \\
\text { del nodo dentro } \\
\text { de la subred }\end{array}$ & Longitud & $\begin{array}{l}\text { Tipo del } \\
\text { mensaje }\end{array}$ & Lectura realizada del sensor HP2 \\
\hline 1 & $0-255$ & 27 & $\begin{array}{l}50 \text { sensor } 0 \\
51 \text { sensor } 1\end{array}$ & $\begin{array}{c}“ \pm \mathrm{xx} x \pm \mathrm{x} \text { xxx } \pm \mathrm{x} \text { xxx } \pm \mathrm{x} \times x x " \\
\text { Ejemplo: }+25 \quad 5+0255+0 \quad 100+0099\end{array}$ \\
\hline
\end{tabular}

Tabla II.5. Descripción de la trama enviada desde el Gateway al software de monitorización cuando el Gateway recibe una trama de datos de un Soil-Mote. 


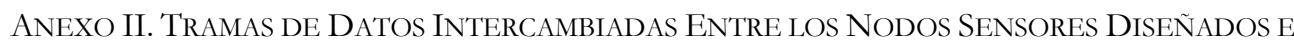
IMPLEMENTADOS DURANTE LA FASE DE VALIDACIÓN DE LOS DisPOSITIVOS.

Cuando el Gateway recibe una trama de datos de un Soil-Mote, envía una trama de 8 bytes al software de monitorización (ver Tabla II.6). En esta trama, el campo longitud tiene un valor de 5 y el tipo siempre tiene un valor de 20 .

\begin{tabular}{|c|c|c|c|c|c|c|c|}
\hline Byte 0 & Byte 1 & Byte 2 & Byte 3 & Byte 4 & Byte 5 & Byte 6 & Byte 7 \\
\hline $\begin{array}{l}\text { Identificador } \\
\text { de la subred }\end{array}$ & $\begin{array}{l}\text { Identificador } \\
\text { del nodo dentro } \\
\text { de la subred }\end{array}$ & Longitud & $\begin{array}{c}\text { Tipo } \\
\text { del } \\
\text { mensaje }\end{array}$ & $\begin{array}{c}\text { Byte más } \\
\text { significativo (MSB) } \\
\text { de la lectura de la } \\
\text { temperatura }\end{array}$ & $\begin{array}{c}\text { Byte menos } \\
\text { significativo (LSB) } \\
\text { de la lectura de la } \\
\text { temperatura }\end{array}$ & $\begin{array}{l}\text { Byte MSB de } \\
\text { la lectura de } \\
\text { la humedad } \\
\text { relativa }\end{array}$ & $\begin{array}{c}\text { Byte LSB de } \\
\text { la lectura de } \\
\text { la humedad } \\
\text { relativa }\end{array}$ \\
\hline 3 & $0-255$ & 5 & 20 & $0-255$ & $0-255$ & $0-255$ & $0-255$ \\
\hline
\end{tabular}

Tabla II.6. Descripción de la trama enviada desde el Gateway al software de monitorización cuando el Gateway recibe una trama de datos de un Environmental-Mote.

El Water-Mote envía tramas de datos de 8 bytes al software de monitorización (ver Tabla II.7). En esta trama, el campo longitud tiene un valor de 5 y el tipo siempre tiene un valor de 10.

\begin{tabular}{|c|c|c|c|c|c|c|c|}
\hline \multicolumn{2}{|c|}{ Byte 0 } & Byte 2 & Byte 3 & Byte 4 & Byte 5 & Byte 6 \\
\hline $\begin{array}{c}\text { Identificador } \\
\text { de la subred }\end{array}$ & $\begin{array}{c}\text { Identificador } \\
\text { del nodo dentro } \\
\text { de la subred }\end{array}$ & Longitud & $\begin{array}{c}\text { Tipo } \\
\text { del } \\
\text { mensaje }\end{array}$ & $\begin{array}{c}\text { Byte MSB de la } \\
\text { lectura de la } \\
\text { temperatura del } \\
\text { agua }\end{array}$ & $\begin{array}{c}\text { Byte LSB de la } \\
\text { lectura de la } \\
\text { temperatura del } \\
\text { agua }\end{array}$ & $\begin{array}{c}\text { Byte MSB de la } \\
\text { lectura de la } \\
\text { conductividad } \\
\text { eléctrica del agua }\end{array}$ & $\begin{array}{c}\text { Byte LSB de la } \\
\text { lectura de la } \\
\text { conductividad } \\
\text { eléctrica del agua }\end{array}$ \\
\hline 2 & $0-255$ & 5 & 10 & $0-255$ & $0-255$ & $0-255$ & $0-255$ \\
\hline
\end{tabular}

Tabla II.7. Descripción de la trama de datos del sensor EC250 enviada desde el Water-Mote al software de monitorización.

A cualquier nodo sensor de las tres subredes, o a todos los nodos sensores de una subred, se le podía modificar la frecuencia de lectura de los sensores desde el software de monitorización. Para ello, era necesario enviar una trama de 6 bytes (ver Tabla II.8).

\begin{tabular}{|c|c|c|c|c|c|}
\hline Byte 0 & Byte 1 & Byte 2 & Byte 3 & Byte 4 & Byte 5 \\
\hline $\begin{array}{c}\text { Identificador } \\
\text { de la subred }\end{array}$ & $\begin{array}{c}\text { Identificador } \\
\text { del nodo }\end{array}$ & Longitud & $\begin{array}{c}\text { Tipo } \\
\text { del } \\
\text { mensaje }\end{array}$ & $\begin{array}{c}\text { Horas } \\
\text { de } \\
\text { lectura }\end{array}$ & $\begin{array}{c}\text { Minutos } \\
\text { de } \\
\text { lectura }\end{array}$ \\
\hline 1,2 ó 3 & $0-255$ & 3 & 30 & $0-255$ & $1-255$ \\
\hline
\end{tabular}

Tabla II.8. Descripción de la trama enviada desde el software de monitorización a un Gateway o al Water-Mote para modificar la frecuencia de lectura de los sensores.

El byte 0 identifica la subred destinataria del mensaje, y puede ser la subred de SoilMotes, la subred de Environmental-Motes, y la subred 2 con un único nodo sensor, el WaterMote. El byte 1 indica el nodo o nodos que deben recibir el mensaje de modificación de la frecuencia de lectura. En el caso de la subred 2, como sólo hay un nodo sensor, el valor será siempre 1. Para las subredes de Soil-Motes y Environmental-Mote podrá ser el valor 
específico de un nodo sensor o un valor de 255, para modificar la frecuencia de lectura de todos los nodos sensores de la subred. El byte 2 siempre tiene un valor de 3 , que es la longitud del mensaje. El tipo del mensaje, byte 3, siempre tiene un valor de 30 . El cuarto byte representa el número de horas que tienen que pasar entre una lectura y la siguiente, que junto con los minutos (quinto byte) definen completamente la frecuencia de lectura de los sensores de un mote.

Las tablas anteriores han descrito todos los mensajes posibles intercambiados por los nodos sensores descritos en el Capítulo 4.

Para validar el GAIA Soil-Mote y el nodo sensor multifuncional GAIA 2 no fue necesario definir nuevas tramas, ya que se utilizaron las descritas en las tablas anteriores. Por ello, en la siguiente sección, se describen del último nodo sensor diseñado e implementado en la presente tesis.

\section{II.3Tramas utilizadas para validar el nodo sensor multi- entorno MEWiN.}

En esta sección del anexo se describirán las tramas que se utilizaron para validar en nodo sensor multi-entorno MEWiN en un cultivo real. En concreto, se describirán las tramas definidas que se utilizaron para validar el dispositivo en el cultivo de almendros de la Estación Experimental Agroalimentaria Tomás Ferro.

$\mathrm{Al}$ igual que en la red descrita en la sección anterior, hay dos tipos de tramas: las tramas que circulan por los dispositivos de la red y la que se intercambian bidireccionalmente entre el nodo coordinador y el software de monitorización.

Cada vez que un ZigBee End Device (ZED) realiza la lectura de un sensor HP2, envía una trama de 33 bytes al ZigBee Coordinator (ZC). En realidad la trama enviada tiene más bytes, debido a que incluye la cabecera definida por ZigBee. Esta cabecera, incluye entre otros campos la dirección de red del nodo que envía la trama. En la trama el primer byte (ver Tabla II.9) almacena la dirección del sensor (sensor 0 ó 1), los siguientes 17 bytes almacenan la lectura realizada del sensor HP2, los 7 bytes siguientes almacenan la hora y fecha actuales obtenidas del reloj de tiempo real (RTC, Real Time Clock) del nodo sensor, y los últimos 8 bytes almacenan la dirección MAC del nodo sensor que envía el mensaje.

Para transmitir la lectura del sensor, sólo se utilizan 17 bytes, en lugar de los 23 bytes descritos en la sección anterior, ya que en este caso sólo se leían tres variables en el sensor: temperatura, porcentaje volumétrico y conductividad eléctrica. 
Anexo II. Tramas de Datos Intercambiadas Entre los Nodos SENSORES Diseñados E IMPLEMENTADOS DURANTE LA FASE DE VALIDACIÓN DE LOS DisPOSITIVOS.

Los 7 bytes obtenidos del RTC incluyen los siguientes campos, todos ellos de 1 byte: segundos, minutos, horas, día de la semana, día del mes, mes y año.

\begin{tabular}{|c|c|c|c|c|c|c|c|c|c|c|c|}
\hline Byte 0 & \multicolumn{3}{|c|}{ Byte 1-Byte 17} & \multicolumn{7}{|c|}{ Byte 17-Byte 24} & $\begin{array}{l}\text { Byte 25- } \\
\text { Byte 32 }\end{array}$ \\
\hline \multirow{2}{*}{$\begin{array}{l}\text { Dirección } \\
\text { del sensor }\end{array}$} & \multicolumn{3}{|c|}{ Lectura del sensor HP2 } & \multicolumn{7}{|c|}{ Hora y fecha obtenidas del RTC } & \multirow{2}{*}{$\begin{array}{l}\text { Dirección } \\
\text { MAC del } \\
\text { nodo } \\
\text { sensor }\end{array}$} \\
\hline & Temperatura & $\begin{array}{l}\text { Porcentaje } \\
\text { volumétrico }\end{array}$ & $\begin{array}{l}\text { Conductividad } \\
\text { eléctrica }\end{array}$ & Segundos & Minutos & Horas & $\begin{array}{l}\text { Día de } \\
\text { la } \\
\text { semana }\end{array}$ & $\begin{array}{l}\text { Día } \\
\text { del } \\
\text { mes }\end{array}$ & Mes & Año & \\
\hline $\begin{array}{c}\text { 48: sensor } \\
049 \text { : } \\
\text { sensor } 1\end{array}$ & “土xx x" & $" \pm x x x "$ & $" \pm x$ xxx" & $\begin{array}{c}0 \times 00- \\
0 \times 59\end{array}$ & $\begin{array}{c}0 \times 00- \\
0 \times 59\end{array}$ & $\begin{array}{l}0 \times 00- \\
0 \times 23\end{array}$ & $\begin{array}{l}\text { 0x01- } \\
\text { 0x07 }\end{array}$ & $\begin{array}{l}0 \times 01- \\
0 \times 31\end{array}$ & $\begin{array}{l}0 \times 01- \\
0 \times 12\end{array}$ & $\begin{array}{r}0 \times 00- \\
0 \times 99\end{array}$ & $\begin{array}{c}\text { Entero sin } \\
\text { signo de } 64 \\
\text { bits }\end{array}$ \\
\hline
\end{tabular}

Tabla II.9. Descripción de la trama enviada de la lectura de un sensor HP2 desde un ZED al ZC.

Cada vez que un ZigBee Router (ZR) realiza la lectura del voltaje del panel solar con un convertidor analógico-digital (ADC) del micro-controlador, envía una trama de 17 bytes al ZC. Los dos primeros bytes (ver Tabla II.10) almacenan la lectura obtenida del ADC, los 7 bytes siguientes almacenan la hora y fecha actuales obtenidas del RTC, y los 8 bytes restantes almacenan la dirección MAC del nodo sensor.

\begin{tabular}{|c|c|c|c|c|c|c|c|c|c|}
\hline Byte 0 & Byte 1 & \multicolumn{7}{|c|}{ Byte 2-Byte 8} & Byte 9-Byte 16 \\
\hline \multirow{2}{*}{$\begin{array}{l}\text { Valor MSB de la } \\
\text { lectura del voltaje } \\
\text { del panel solar }\end{array}$} & \multirow{2}{*}{$\begin{array}{l}\text { Valor LSB de la } \\
\text { lectura del voltaje } \\
\text { del panel solar }\end{array}$} & \multicolumn{7}{|c|}{ Hora y fecha obtenidas del RTC } & \multirow{2}{*}{$\begin{array}{l}\text { Dirección MAC } \\
\text { del nodo sensor }\end{array}$} \\
\hline & & Segundos & Minutos & Horas & $\begin{array}{c}\text { Día de la } \\
\text { semana }\end{array}$ & $\begin{array}{l}\text { Día del } \\
\text { mes }\end{array}$ & Mes & Año & \\
\hline $0-255$ & $0-255$ & $\begin{array}{c}0 \times 00- \\
0 \times 59\end{array}$ & $\begin{array}{l}0 \times 00- \\
0 \times 59\end{array}$ & $\begin{array}{l}0 \times 00- \\
0 \times 23\end{array}$ & 0x01-0x07 & $\begin{array}{c}0 \times 01- \\
0 \times 31\end{array}$ & $\begin{array}{l}0 \times 01- \\
0 \times 12\end{array}$ & $\begin{array}{l}0 \times 00- \\
0 \times 99\end{array}$ & $\begin{array}{c}\text { Entero sin signo } \\
\text { de } 64 \text { bits }\end{array}$ \\
\hline
\end{tabular}

Tabla II.10. Descripción de la trama enviada con la lectura del voltaje del panel solar del ZR al ZC.

Tanto el ZED con el ZR envían tramas con la lectura realizada con un ADC del voltaje de la batería. Esta trama tiene el mismo formato que el de la trama de la Tabla II.10, siendo los dos primeros bytes la lectura obtenida con el ADC del voltaje de la batería.

Un ZED y un ZR pueden recibir una trama del ZC para configurar la frecuencia de lectura periódica de los elementos asociados al dispositivo. Este trama tiene un tamaño de 2 bytes (ver Tabla II.11), siendo el primer byte las horas de lectura y el segundo byte los minutos de lectura.

\begin{tabular}{|c|c|}
\hline Byte 0 & Byte 1 \\
\hline Horas de lectura & Minutos de lectura \\
\hline $0-255$ & $1-255$ \\
\hline
\end{tabular}

Tabla II.11. Descripción de la trama enviada desde el ZC a un nodo sensor (ZED ó ZR) para modificar la frecuencia de lectura de los sensores. 
Para facilitar el procesamiento de las tramas recibidas en el software de monitorización utilizado para validar el nodo sensor MEWiN, se decidió enviar tramas codificadas en ASCII delimitadas por el carácter de inicio ':’ y el carácter de fin retorno de carro o 'CR'.

Cuando se recibe una trama de datos de un ZED se prepara una trama ASCII de 55 (ver Tabla II.12) bytes que se envía por RS-232 a la interfaz gráfica de usuario. Después del carácter de inicio, se almacena entre los bytes 1 y 16 la dirección MAC del nodo que envió el mensaje en ASCII y en hexadecimal. Los dos bytes siguientes almacenan el tipo de la trama, siendo '10' en este caso. El siguiente byte identifica al sensor HP2 del mote del que se está recibiendo la trama, y puede ser ' 0 ' para el sensor 0 y el carácter ' 1 ' para el sensor 1. Entre los bytes 20 y el 36 se almacena la lectura recibida del sensor. La hora y la fecha está recogida entre los bytes 37 y el 49. Los siguientes 4 caracteres son el un CRC de 16 bits, que se calcula de la trama que se está preparando, y se almacena en la trama mediante cuatro caracteres ASCII en hexadecimal. El último byte recoge el fin de la trama.

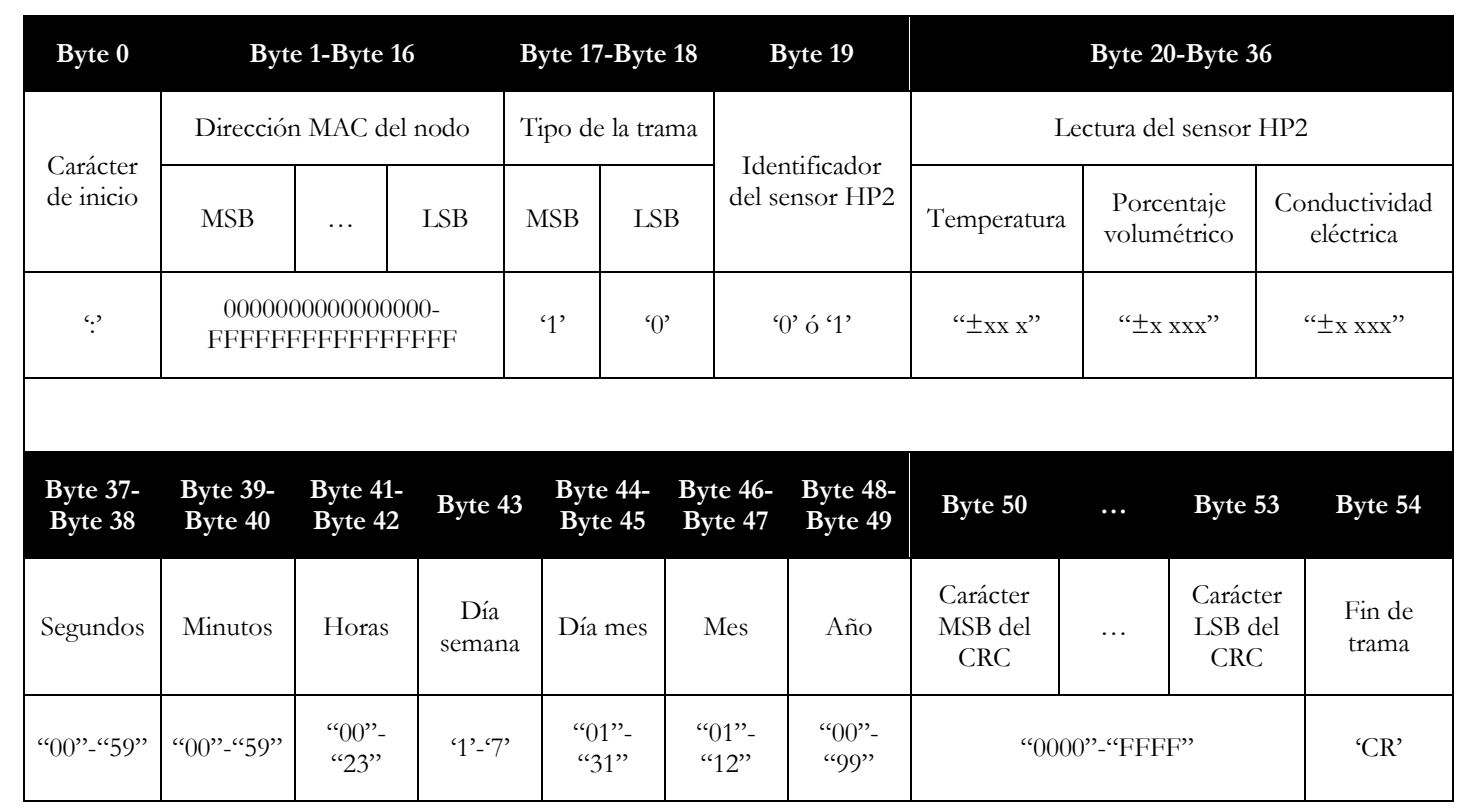

Tabla II.12. Trama que se envía a la interfaz gráfica de usuario al recibir una trama de datos de un ZED en el ZC.

Cuando se recibe en el ZC una trama de batería de un ZED o de un ZR, se prepara una trama ASCII de 40 (ver Tabla II.13) bytes que se envía por RS-232 a la interfaz gráfica de usuario. Después del carácter de inicio, se almacena entre los bytes 1 y 16 la dirección MAC del nodo que envió el mensaje en ASCII y en hexadecimal. Los dos bytes siguientes almacenan el tipo de la trama, siendo '20' en este caso. Los tres bytes siguientes almacenan la lectura del voltaje de la batería en ASCII y en hexadecimal. La hora y la fecha está recogida entre los bytes 22 y el 34. Los siguientes 4 caracteres son el un CRC de 16 bits, que se calcula de la trama que se está preparando y se almacena en la trama mediante cuatro caracteres ASCII en hexadecimal. El último byte recoge el fin de la trama. 
Anexo II. Tramas de Datos Intercambiadas Entre los Nodos SENSORES Diseñados E IMPLEMENTADOS DURANTE LA FASE DE VALIDACIÓN DE LOS DiSPOSITIVOS.

\begin{tabular}{|c|c|c|c|c|c|c|c|c|c|c|c|}
\hline Byte 0 & \multicolumn{3}{|c|}{ Byte 1-Byte 16} & \multicolumn{3}{|c|}{ Byte 17-Byte 18} & \multicolumn{2}{|l|}{ Byte 19} & yte 20 & \multicolumn{2}{|c|}{ Byte 21} \\
\hline \multirow{2}{*}{$\begin{array}{l}\text { Carácter } \\
\text { de inicio }\end{array}$} & \multicolumn{3}{|c|}{ Dirección MAC del nodo } & \multicolumn{3}{|c|}{ Tipo de la trama } & \multicolumn{5}{|c|}{ Lectura del voltaje de la batería en formato raw } \\
\hline & MSB & $\ldots$ & LSB & MSB & LSI & & arácter MSB & & $\ldots$ & & ter LSB \\
\hline$\because$ & \multicolumn{3}{|c|}{$\begin{array}{l}\text { 0000000000000000- } \\
\text { FFFFFFFFFFFFFFFF }\end{array}$} & '2' & '0’ & \multicolumn{2}{|c|}{${ }^{\prime} 0, \ldots,{ }^{\prime} \mathrm{O}^{\prime}, \mathrm{C}^{\prime}, \ldots,{ }^{\circ} \mathrm{F}$ ' } & \multicolumn{2}{|r|}{$\cdots$} & \multicolumn{2}{|c|}{${ }^{\prime} 0, \ldots,{ }^{\prime}, 9^{\prime}, \mathrm{A}^{\prime}, \ldots,{ }^{\circ} \mathrm{F}$} \\
\hline $\begin{array}{l}\text { Byte 22- } \\
\text { Byte 23 }\end{array}$ & $\begin{array}{l}\text { Byte 24- } \\
\text { Byte 25 }\end{array}$ & $\begin{array}{l}\text { Byte 26- } \\
\text { Byte 27 }\end{array}$ & Byte 28 & $\begin{array}{l}3 \text { Byt } \\
\text { Byt }\end{array}$ & & $\begin{array}{l}\text { Byte 31- } \\
\text { Byte 32 }\end{array}$ & $\begin{array}{l}\text { Byte 33- } \\
\text { Byte } 34\end{array}$ & Byte 35 & $\cdots$ & Byte 38 & Byte 39 \\
\hline Segundos & Minutos & Horas & $\begin{array}{c}\text { Día } \\
\text { semana }\end{array}$ & Día & & Mes & Año & $\begin{array}{c}\text { Carácter } \\
\text { MSB del } \\
\text { CRC }\end{array}$ & $\cdots$ & $\begin{array}{c}\text { Carácter } \\
\text { LSB del } \\
\text { CRC }\end{array}$ & $\begin{array}{l}\text { Fin de } \\
\text { trama }\end{array}$ \\
\hline “00”-“"59” & “00”-“59” & $\begin{array}{l}\text { “00”- } \\
\text { “23” }\end{array}$ & '1'-‘7' & "0 & & $\begin{array}{l}\text { “01"- } \\
\text { " } 12 "\end{array}$ & $\begin{array}{l}\text { “00"- } \\
\text { "99" }\end{array}$ & & $00 ”-“ F$ & & 'CR' \\
\hline
\end{tabular}

Tabla II.13. Trama que se envía a la interfaz gráfica de usuario al recibir una trama de batería de un ZED o del ZR en el ZC.

Cuando se recibe en el ZC una trama con el voltaje del panel solar del ZR, también se prepara una trama ASCII de 40 bytes que se envía por RS-232 a la interfaz gráfica de usuario. Esta trama es similar a la descrita en la Tabla II.13, salvo que en los 3 bytes que se almacena la lectura de la batería se almacena el voltaje medido del panel solar. Además, el tipo de la trama tiene el valor ' 30 '.

Finalmente, desde la interfaz gráfica de usuario se puede preparar una trama de 28 (ver Tabla II.14) bytes para modificar la frecuencia de lectura de los nodos sensores (ZED y/o ZR). Esta trama también empieza con el carácter de inicio y acaba con el CRC, y el carácter de fin de trama. Se ha definido que la trama tenga el tipo ‘40' y en ella se envían las horas y los minutos de lectura de los dispositivos asociados con los nodos sensores. Tanto las horas como los minutos se codifican con dos caracteres ASCII en hexadecimal. De esta manera, con los caracteres se puede codificar un valor entre 0 y 255.

\begin{tabular}{|c|c|c|c|c|c|c|c|c|c|c|c|}
\hline Byte 0 & \multicolumn{3}{|c|}{ Byte 1-Byte 16} & \multicolumn{2}{|c|}{ Byte 17-Byte 18} & $\begin{array}{l}\text { Byte 19- } \\
\text { Byte 20 }\end{array}$ & $\begin{array}{l}\text { Byte 21- } \\
\text { Byte 22 }\end{array}$ & Byte 23 & $\ldots$ & Byte 26 & $\begin{array}{c}\text { Byte } \\
27\end{array}$ \\
\hline \multirow{2}{*}{$\begin{array}{l}\text { Carácter } \\
\text { de inicio }\end{array}$} & \multicolumn{3}{|c|}{ Dirección MAC del nodo } & \multicolumn{2}{|c|}{ Tipo de la trama } & Horas de & Minutos & \multirow{2}{*}{$\begin{array}{c}\text { Carácter } \\
\text { MSB del } \\
\text { CRC }\end{array}$} & \multirow{2}{*}{$\ldots$} & \multirow{2}{*}{$\begin{array}{c}\text { Carácter } \\
\text { LSB del } \\
\text { CRC }\end{array}$} & \multirow{2}{*}{$\begin{array}{l}\text { Fin de } \\
\text { trama }\end{array}$} \\
\hline & MSB & $\ldots$ & LSB & MSB & LSB & \multirow{2}{*}{$\begin{array}{l}\text { "O0"- } \\
\text { "FF" }\end{array}$} & \multirow{2}{*}{ “01"-"FF" } & & & & \\
\hline ' & \multicolumn{3}{|c|}{$\begin{array}{l}\text { 0000000000000000- } \\
\text { FFFFFFFFFFFFFFFF }\end{array}$} & '2' & $0^{\prime}$ & & & \multicolumn{3}{|c|}{ "0000"-"FFFF" } & 'CR' \\
\hline
\end{tabular}

Tabla II.14. Trama que se envía desde la interfaz gráfica de usuario para modificar la frecuencia de lectura de un nodo sensor de la red.

Con la descripción de la trama anterior, quedan completamente definidas todas las tramas usadas para validar los nodos sensores diseñados en la presente tesis doctoral. 
CONTRIBUCIÓN A LAS REDES DE SENSORES INALÁMBRICAS. ESTUdiO E IMPLEMENTACIÓN DE SOLUCIONES Hardware PARA Agricultura de PRECISIÓN. 


\section{Anexo III}

\section{Descripción del Software desarrollado para el GAIA Soil-Mote.}

$\square$ neste anexo, se describe en profundidad el software desarrollado para el GALA Soil-Mote. Se tesis. Se optó por describir el software más complejo y extenso de todos los nodos sensores para que el lector interesado en este tipo de programación pudiese adquirir mayores conocimientos. La descripción comienza presentando la vista estática de componentes nes $C$ del nodo sensor. A continuación, se describen brevemente cada uno de los componentes y, finalmente, se describe en detalle el código fuente desarrollado del componente de tipo módulo que implementa la funcionalidad del nodo sensor. 


\section{III.1Introducción.}

Este anexo describe el software desarrollado para el GAIA Soil-Mote. Para ello, se presentan y describen todos los componentes instanciados del sistema operativo TinyOS, y posteriormente se describe en profundidad el código fuente del componente HP2C.

\section{III.2 Descripción del software.}

El software del dispositivo se ha desarrollado con la versión 2.0 del sistema operativo TinyOS [TinyOS] y el lenguaje de programación orientado a componentes asociado nesC [Gay, 2003]. Dicho software, ha sido programado de forma que ofrezca las funcionalidades principales de enviar el valor de la tensión de la batería con un periodo de una hora, y de adquirir y enviar las lecturas procedentes de hasta 10 sensores Hydra Probe II, con una frecuencia configurable remotamente con valores comprendidos entre 30 minutos y 10 días. La Figura III.1 muestra un diagrama con la vista estática de componentes nesC del software desarrollado. En ella se muestra el componente asociado al módulo HP2C desarrollado y los componentes instanciados del sistema operativo para conseguir esta funcionalidad. Los componentes se comunican usando interfaces. De esta forma, por ejemplo, el componente MainC proporciona la interfaz Boot y el componente HP2C la utiliza.

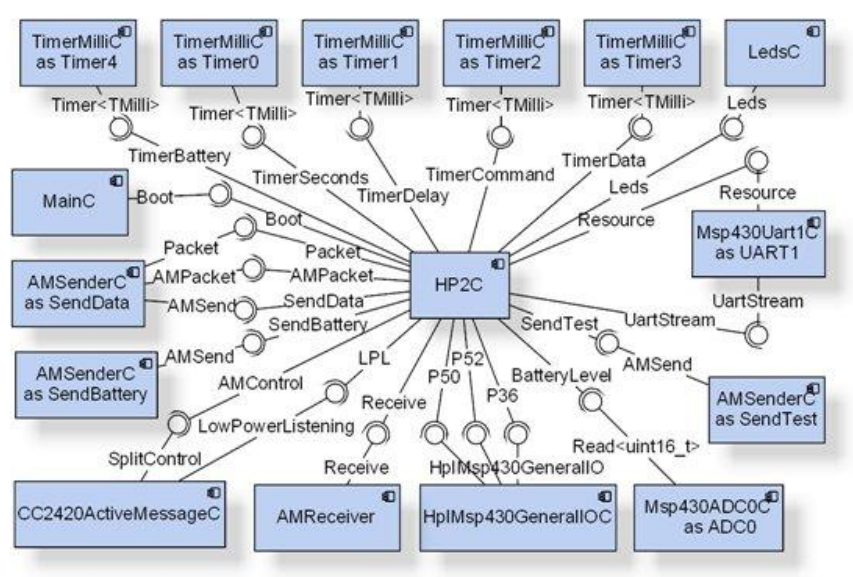

Figura III.1. Vista estática de componentes nesC del GSM.

El Listado III.1 representa el código nesC de la configuración de nivel jerárquico superior del software desarrollado. En ella se declaran y se establecen todas la relaciones de los componentes mostrados en la Figura III.1.

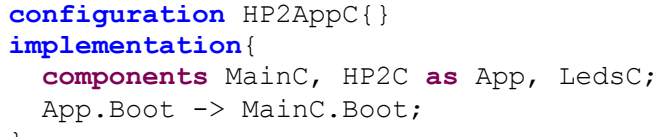

Listado III.1. Extracto de código del archivo de configuración de nivel jerárquico superior del GSM. 
Otro archivo importante del software es el fichero de cabecera "HP2.h", en el que se definen una serie de constantes necesarias y los campos asociados a cada mensaje que se envía inalámbricamente por la red. En el Listado III.2 se muestra un extracto del mismo.

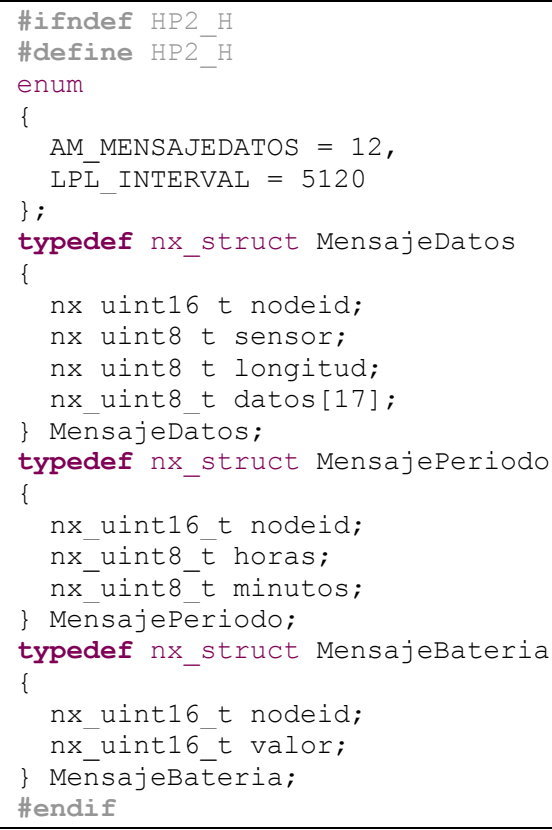

Listado III.2. Extracto del archivo de cabecera "HP2.h".

La línea 6 del Listado III.2, define el periodo de LowPowerListening de 5s. Cabe mencionar que, en TinyOS, para codificar el valor de $5000 \mathrm{~ms}$, es necesario utilizar el valor decímal 5120. De igual forma, para codificar 1000ms se utilizaría la constante decímal 1024. Entre las líneas 8 y 14 se define la estructura asociada al tipo de datos que se envían tras realizar la lectura de un sensor. La línea 10 identifica al nodo sensor que envía el mensaje. La línea 11 detalla el sensor leído y es necesaria porque es posible conectar hasta 10 sensores en el bus SDI-12. La lectura en formato ASCII de la temperatura, porcentaje volumétrico y conductividad del suelo se almacena en el array etiquetado como 'datos'. Además, como la cadena ASCII no tiene un formato fijo se utiliza el campo longitud (línea 12) para indicar los datos útiles del array. De igual forma, entre las líneas 15 y 20 se definen los mensajes para cambiar la frecuencia de lectura, que pueden recibir los GSMs del sumidero de la red. Combinando los 255 valores de los minutos y de las horas, la frecuencia se puede fijar hasta en aproximadamente 10 días. Los mensajes que envía el GSM de batería incluyen el identificador del nodo sensor (línea 23) y el valor entero de 12 bits (04095, línea 24) adquirido por el ADC al que está conectada la batería.

El punto de entrada del programa lo proporciona el componente MainC por medio de la interfaz Boot. En concreto, cuando el sistema operativo ha realizado todas las inicializaciones previas, señal el evento booted de la interfaz anterior, y es dentro de este evento donde se inicializa la ejecución de la aplicación del GSM. Esta inicialización implica configurar e iniciar el módulo de radio con un periodo de encendido (LowPowerListening) de 
5 s, establecer 3 pines del micro a nivel alto e inicializar un conjunto de variables. El Listado III.3 muestra parte del código contenido dentro de este evento.

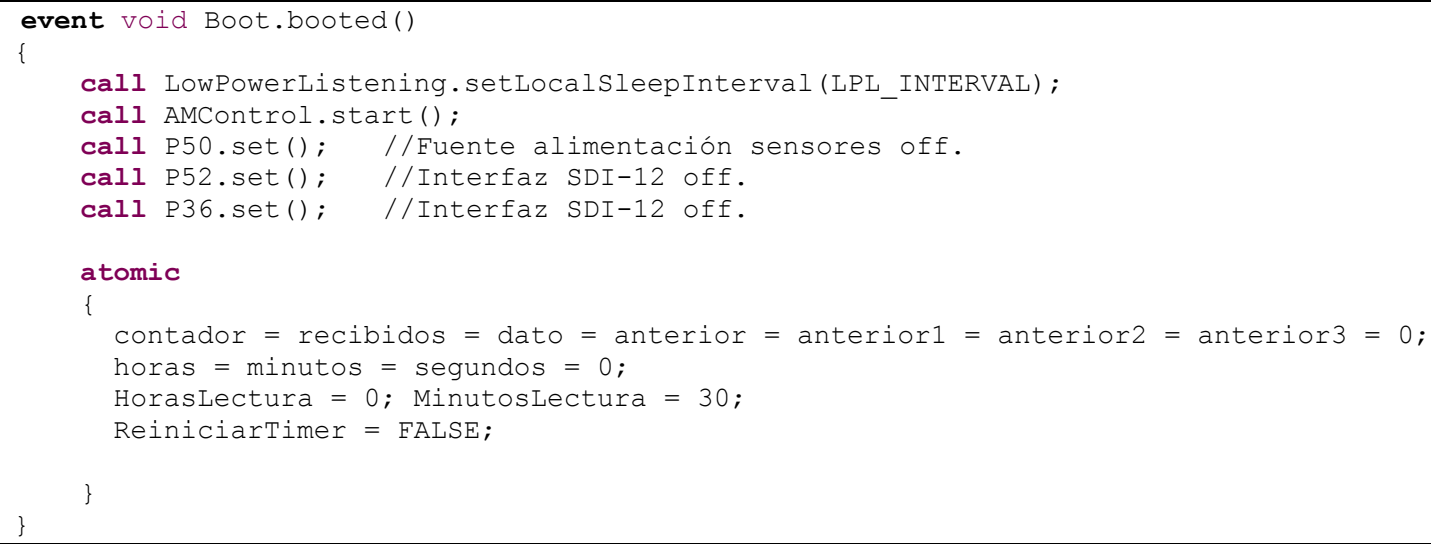

Listado III.3. Extracto de la inicialización de la aplicación del GSM.

En respuesta a la ejecución del comando start de la interfaz $A M C o n t r o l$ y tras el intento de inicializar el módulo de radio, el sistema operativo señala el evento startDone (Listado III.4) de la interfaz anterior. La inicialización puede haber sido correcta o incorrecta. En el primer caso, se inicia un temporizador periódico de 1s que gestiona la temporización del programa con objeto de saber cuándo hay que realizar una lectura (Componente Timer0) de los sensores. Además, se inicializa otro temporizador periódico de una hora (componente Timert) para gestionar las lecturas del nivel de tensión en la batería. Por otro lado, si la inicialización del módulo de radio ha sido errónea se realiza un reintento y se indica esta situación cambiando de estado el LED2 del mote.

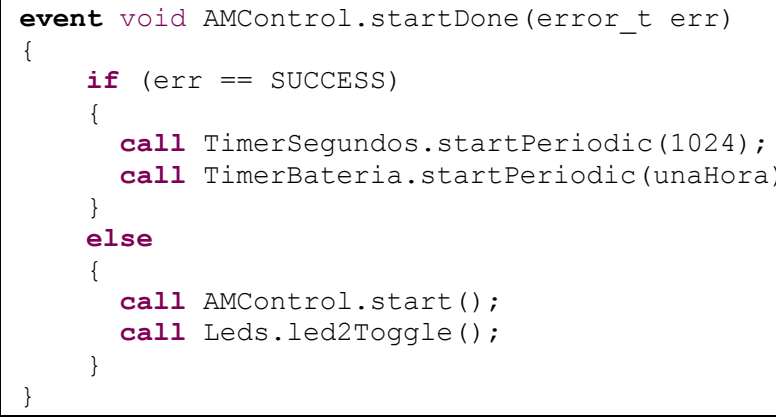

Listado III.4. Código del evento startDone de la interfaz AMControl.

En respuesta a la ejecución del comando de la línea 6 del Listado III.4, el sistema operativo señala el evento fired (Listado III.5) de la interfaz TimerBateria cada hora. En el cuerpo de este evento se ejecuta un comando (línea 3) para iniciar la lectura del ADC al que está conectada la batería a través de un divisor de tensión resistivo. Cuando TinyOS ha realizado la lectura del ADC solicitado, señala el evento readDone (línea 6) de la interfaz NivelBateria. A este evento se le pasan dos variables: el resultado de la operación y valor leído del ADC. Así, si el resultado de la operación ha sido correcto, se almacena la lectura 
realizada en una variable auxiliar y se ejecuta la tarea FuncionEnviarAlarma (línea 13), que se encarga de enviar la lectura realizada por radio hasta el Gateway de la subred.

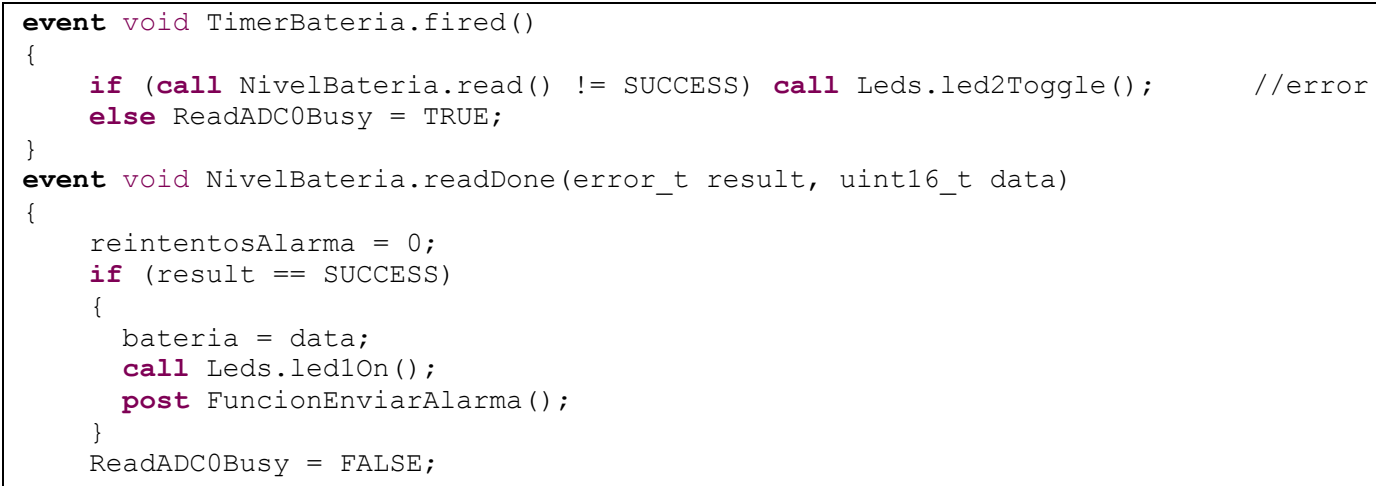

Listado III.5. Código nesC de los eventos asociados a la lectura del nivel de tensión de la batería.

Por otro lado, el componente Timer0 de tipo TimerMilliC permite mantener el contador de minutos y horas que se utiliza en el programa para decidir cuándo hay que realizar una lectura de los sensores HP2 conectados. El evento fired asociado a este temporizador se muestra en el Listado III.6.

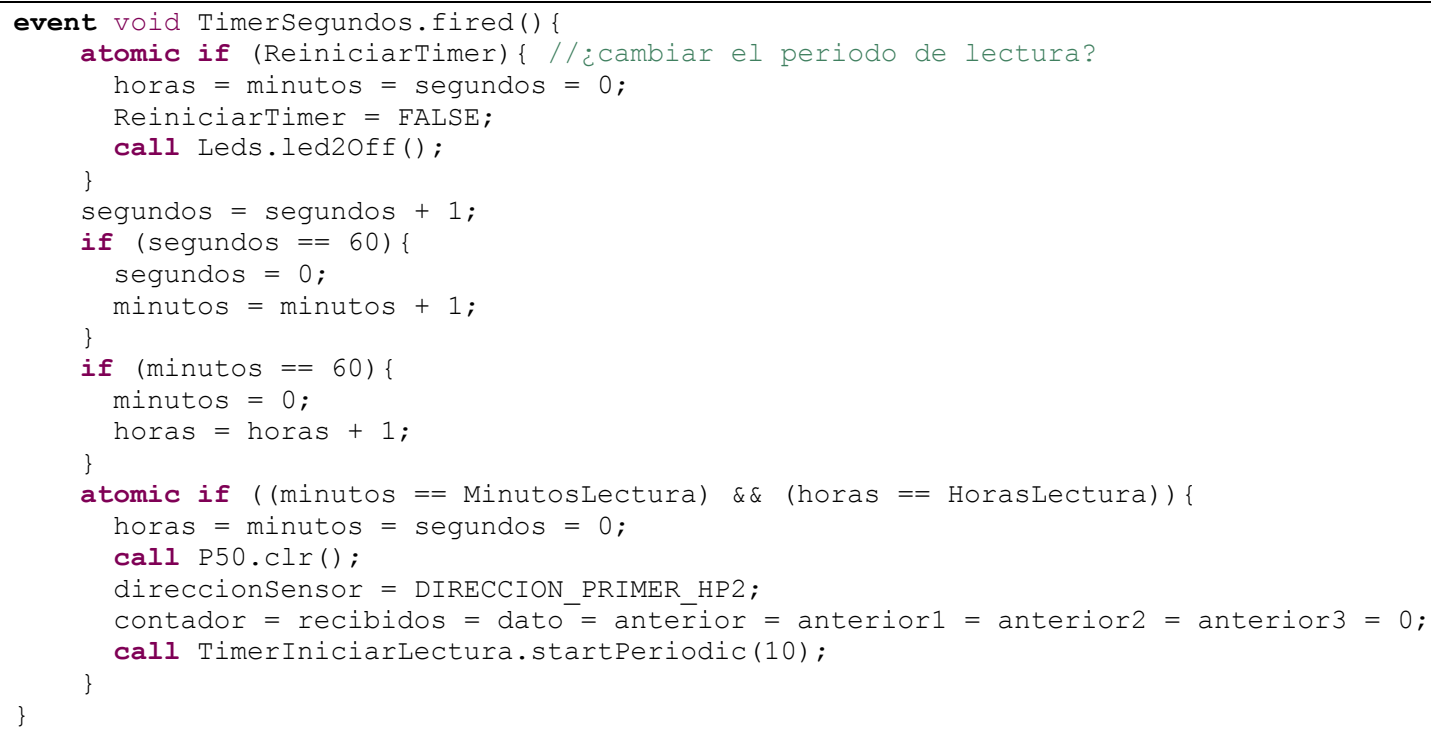

Listado III.6. Código nesC del evento fired del temporizador principal.

Primero se comprueba si se ha recibido un mensaje para modificar la frecuencia de lectura de los sensores (línea 2). Al recibir este mensaje, a la variable ReiniciarTimer se le asigna el valor TRUE y se reinician las variables del temporizador. A continuación se actualiza el temporizador. Posteriormente, se comprueba si es el momento de realizar una lectura de los sensores. En tal caso, se reinician los contadores (línea 17), se habilita el convertidor DC/DC que alimenta los sensores (línea 18), se configura la dirección del primer sensor conectado en el bus SDI-12, se inicializan las variables necesarias (línea 19) y se ejecuta el temporizador que inicia la lectura de los sensores (línea 21). 
A continuación, se presenta el código asociado a la tarea que envía los datos de batería y la recepción de mensajes para modificar la frecuencia de lectura de los sensores.

La tarea para enviar los datos de batería y el evento señalado cuando se envía un mensaje se muestran en el Listado III.7.

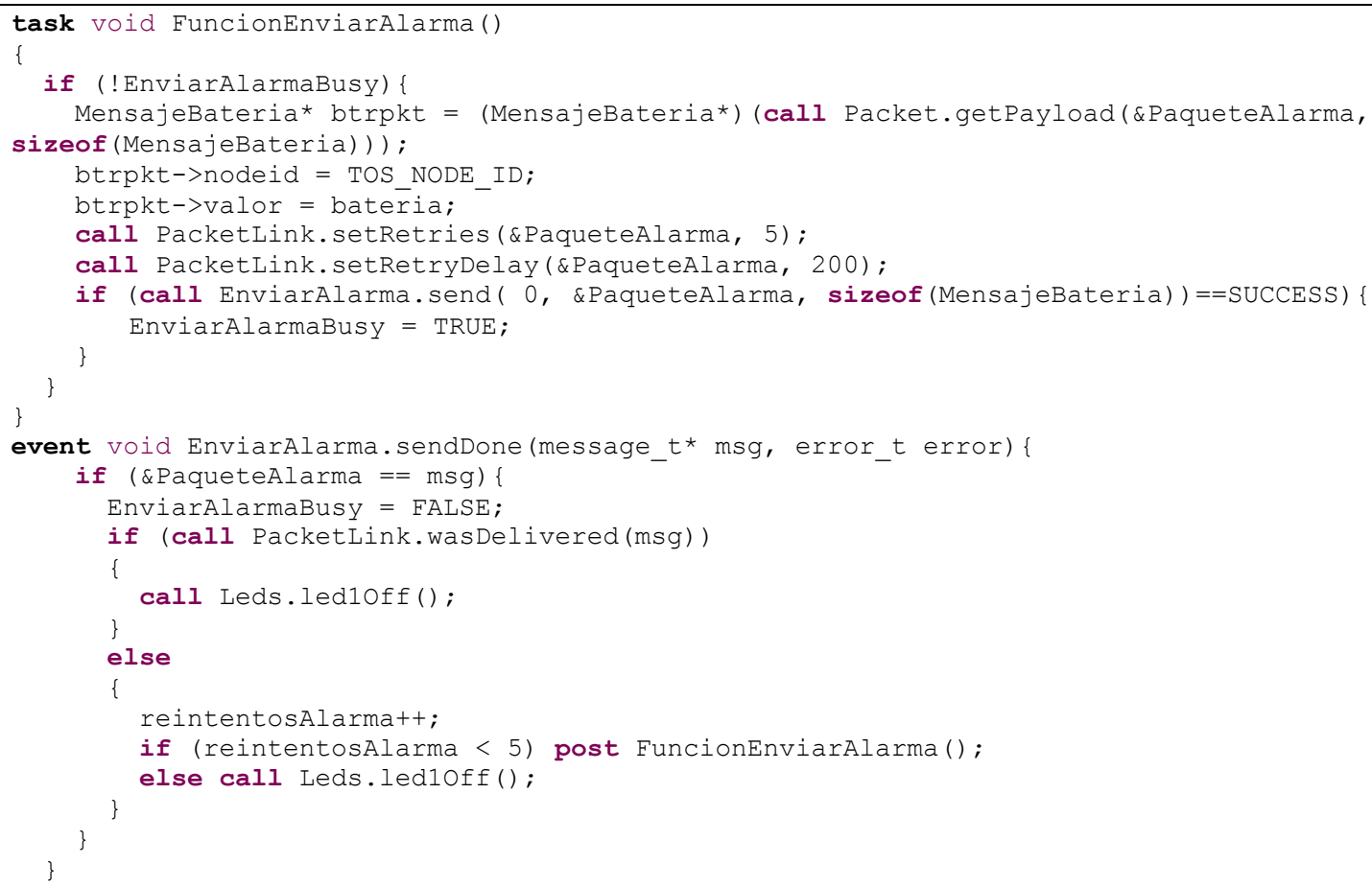

Listado III.7. Tarea para enviar datos de batería y evento asociado al envío.

En el caso de la tarea, en primer lugar se crea e inicializa una variable para enviar el mensaje del tipo definido el archivo de cabecera "HP2. $h$ ", en la que se define el nodo que envía el mensaje (línea 6) y el valor de la batería (línea 7). Posteriormente, para asegurar la recepción del mensaje se configuran 5 reintentos en el envío (línea 8) y se ejecuta el comando send de la interfaz EnviarAlarma para realizar el envío (línea 10). En respuesta al comando anterior, y después de que TinyOS realice el envío, éste señala el evento sendDone de la interfaz mencionada anteriormente. En primer lugar, se comprueba si el mensaje ha sido enviado correctamente (línea 18), en cuyo caso se apaga el LED (línea 20) que se encendió antes de comenzar el envío (línea 12 del Listado III.5). En caso contrario, se incrementa la variable que controla los reintentos realizados y si es menor que 5 se vuelve a ejecutar la tarea. Cabe destacar que también puede ocurrir que, tras completar los 5 reintentos, no se haya enviado el mensaje.

Cuando el módulo de radio recibe un mensaje para modificar la frecuencia de lectura de los sensores, TinyOS señala el evento receive de la interfaz Receive. Esta interfaz la proporciona el componente AMReceiver, mostrado en la Figura III.1. Las sentencias ejecutadas cuando se produce este evento se muestran en el Listado III.8. 


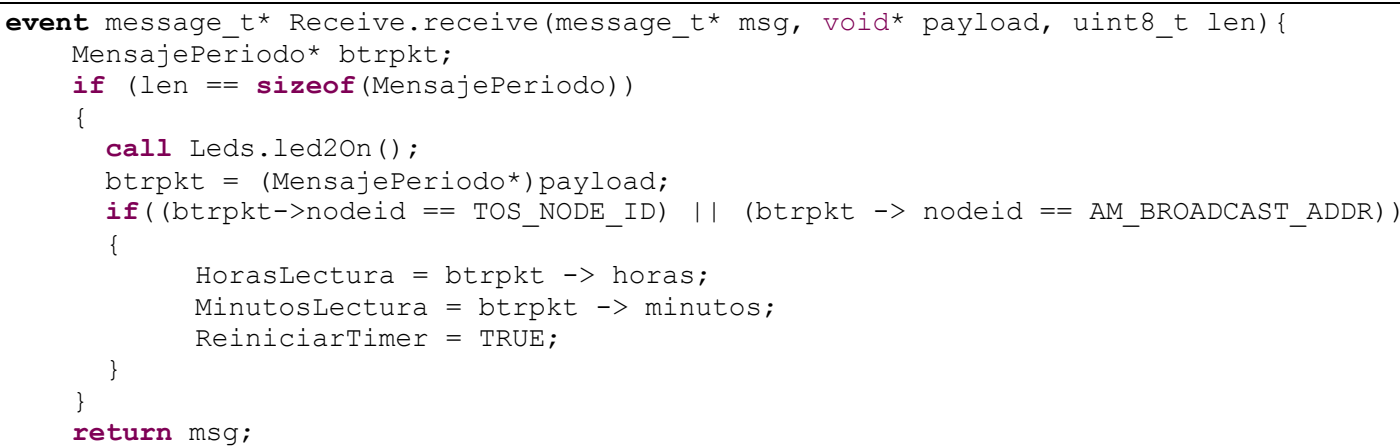

Listado III.8. Sentencias nesC ejecutadas en respuesta a la recepción de un mensaje.

En la línea 2 se declara un mensaje del tipo MensajePeriodo, que fue definido en el archivo de cabecera "HP2.h". En la siguiente línea se comprueba si el mensaje recibido tiene la longitud del mensaje esperado. En caso afirmativo, se enciende el LED2 (recordar su apagado al principio del Listado III.6) y se inicializa la variable declarada con el mensaje recibido. En la línea 8, se comprueba si el mensaje es para el nodo (TOS_NODE_ID es la dirección del nodo sensor actual) o si el mensaje iba direccionado a todos los nodos sensores (AM_BROADCAST_ADDR). Finalmente, si todo es correcto se actualizan las variables HorasLectura y MinutosLectura con los valores recibidos y se indica que hay que reiniciar el temporizador principal.

El proceso de lectura y envío de datos de los sensores HP2 se inicia (Figura III.2) enviando el comando aM! (a es la dirección del sensor) al sensor y analizar la respuesta que contiene el tiempo de espera (ttt) necesario antes de obtener los datos del sensor. A continuación, puede ocurrir que el sensor tarde el tiempo especificado en disponer de los datos, o que los disponga antes. Esta última situación se indica con el envío de una petición de servicio $(a<$ retorno de carro $><$ nueva línea $>$ ). Finalmente se envían uno o dos comandos, cuya respuesta incluye los datos solicitados al sensor.

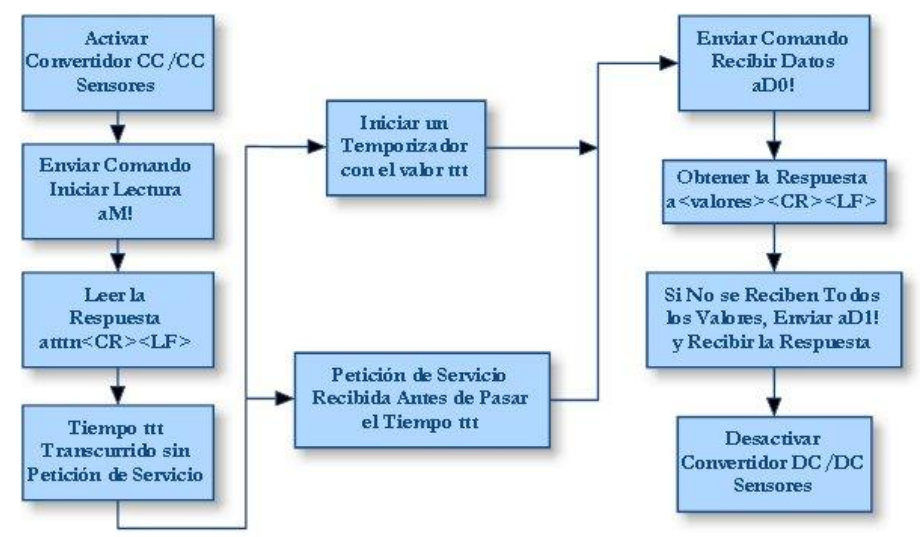

Figura III.2. Pasos para realizar la lectura de un sensor HP2.

El código nesC con el inicio de lectura del primer sensor HP2 especificado se mostró al final del Listado III.6, en el que hay, entre otras, sentencias para activar el 
convertidor DC/DC que alimenta los sensores, establecer la dirección del primer sensor y se iniciaba un temporizador periódico de $10 \mathrm{~ms}$, cuyo evento aparece en el Listado III.9.

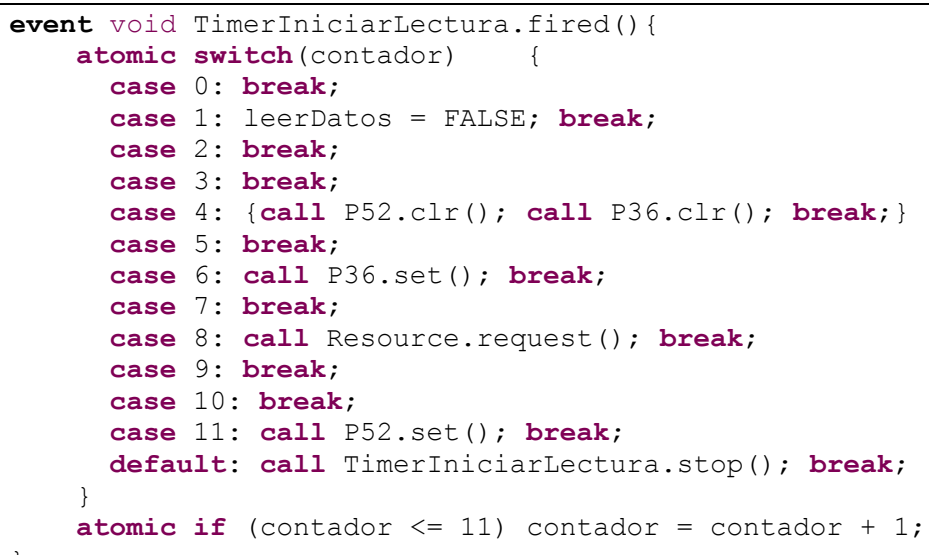

Listado III.9. Código nesC que envía el comando para iniciar la lectura al sensor.

La función principal del temporizador es enviar el comando para iniciar la lectura del sensor. Inicialmente, hay una espera de $50 \mathrm{~ms}$ (del case 0 al 4) para que se estabilice la tensión del convertidor DC/DC. En la línea 4 se inicia el proceso de enviar un break al sensor. Para ello, se habilita el buffer triestado (P52.clr) para escribir en el bus SDI-12 y se establece la línea de datos a nivel bajo (P36.clr) durante 20ms. A continuación se establece la línea de bus a nivel alto (línea 9) durante $20 \mathrm{~ms}$. Este proceso consigue que los sensores HP2 conectados en el bus pasen de modo sleep a modo activo y poder enviar el comando de iniciar la lectura, ejecutando el comando request de la interfaz Resource (línea 11) para solicitar el uso de la UART conectada con la interfaz SDI-12. El siguiente cambio de estado se realiza 30ms después (línea 14). Este tiempo es necesario, porque se está enviando un comando de 3 bytes a una velocidad de 1.200bps. A continuación, se deshabilita el buffer triestado configurando la interfaz SDI-12 en modo recepción a la espera de la respuesta del comando enviado. Finalmente, en la siguiente iteración del temporizador se detiene el mismo (línea 15).

Cuando el recurso solicitado (UART) por el temporizador con interfaz TimerIniciarLectura está disponible, TinyOS señala el evento granted de la misma interfaz (Listado III.10). En el cuerpo de este evento, en primer lugar se habilita la interrupción de recepción datos (línea 2) y se actualiza la primera posición de array a enviar con la dirección del sensor establecida previamente (recordar el dígito 'a' de los comandos SDI-12 del sensor asociado a la dirección del sensor). A continuación se comprueba el estado de la variable leerDatos (línea 4) con objeto de saber si hay que enviar un comando para iniciar la lectura o un comando de lectura de datos. En el primer caso se envía el comando correspondiente (array comandoIniciarLectura) para iniciar la lectura de un sensor (aM!). En otro caso, se trata del envío de un comando para obtener los datos del sensor y pueden darse dos casos diferentes: un primero en el que se realiza la primera petición de datos 
(aD0!) y un segundo en el que se realiza la segunda petición datos (aD1!), al no haberse recibido todas las variables solicitadas en la primera petición. La ejecución de estos dos casos se controla con la variable "cinco", que se describirá posteriormente.

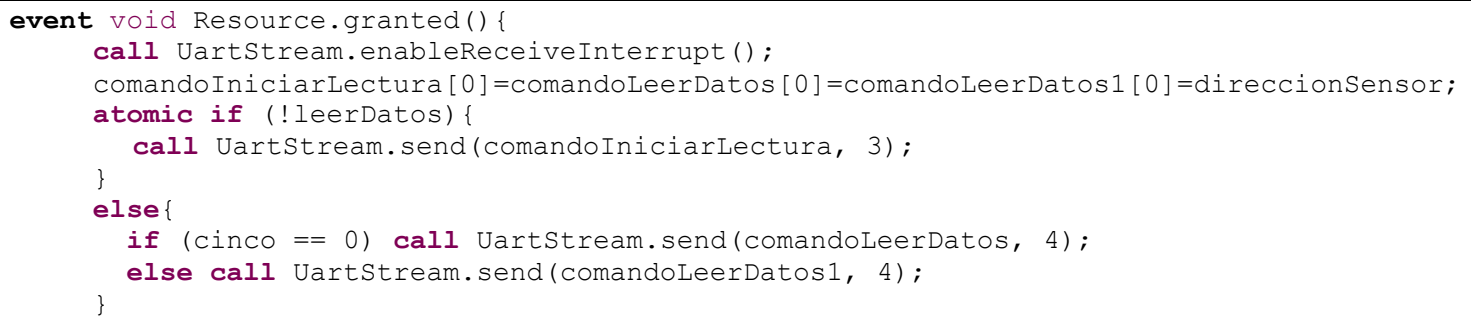

Listado III.10. Código nesC ejecutado cuando TinyOS cede el uso de la UART.

La ejecución continúa en el evento que señala TinyOS (Listado III.11) cada vez que se recibe un byte por la UART. Si no se están solicitando los datos al sensor, sino iniciando la lectura, se ejecutarán las sentencias de la sentencia 'if que comienza en la línea 3. En éste, se comprueba si se está recibiendo una respuesta, con o sin petición de servicio. Lo habitual es recibir una respuesta y la petición de servicio posteriormente. Todas las respuestas acaban con $<\mathrm{CR}><\mathrm{LF}>$ o 1310 en decimal. Así, para detectar la respuesta al comando de iniciar la lectura (línea 7) hay que recibir 10 bytes y que la respuesta acabe en 13 10. Son 10 bytes porque se recibe el comando enviado, aM!, así como la respuesta recibida, atttn $<C R><L F>$. " $t t t$ " es el tiempo (en segundos) que hay que esperar para el sensor disponga de los datos; siendo ' $n$ ' es el número de variables que devolverá el sensor y que está configurado con el valor 3, para así poder recibir las lecturas asociadas a los valores de temperatura, porcentaje volumétrico y conductividad del suelo adquiridos por el sensor. Cuando se detecta la respuesta (línea 7), se inicializa un temporizador de espera con el tiempo indicado en la respuesta. Si posteriormente se recibe una petición de servicio (dirección 13 10) se cancela el temporizador de espera (líneas 4-6). Además, entre las líneas 13-16 se actualizan las variables auxiliares necesarias para detectar las secuencias.

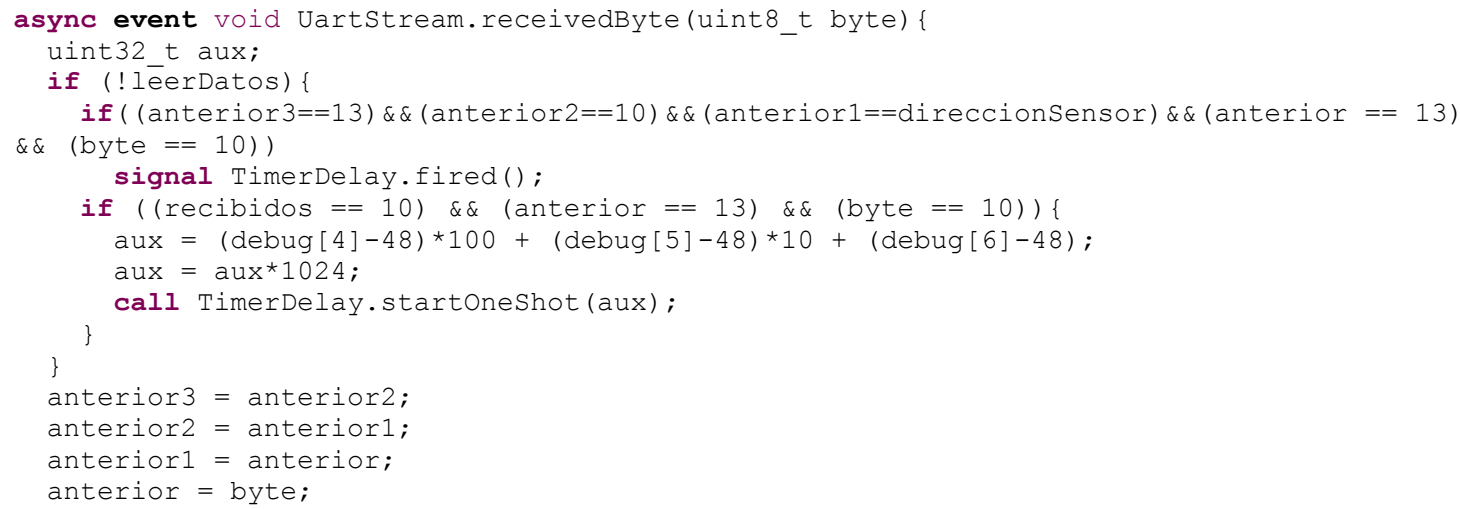

Listado III.11. Evento que recibe datos de la UART asociado al inicio de lectura de un HP2. 
En cualquier caso, tanto si se recibe una petición de servicio o no, se ejecutan las sentencias incluidas en el evento fired del temporizador de una sola iteración TimerDelay (Listado III.12). En la línea 2, se libera el uso de la UART y del propio temporizador por seguridad. Posteriormente, se inicializan las variables necesarias y se crea un temporizador periódico de 10ms para enviar el comando de petición de datos (línea 7) al sensor HP2.

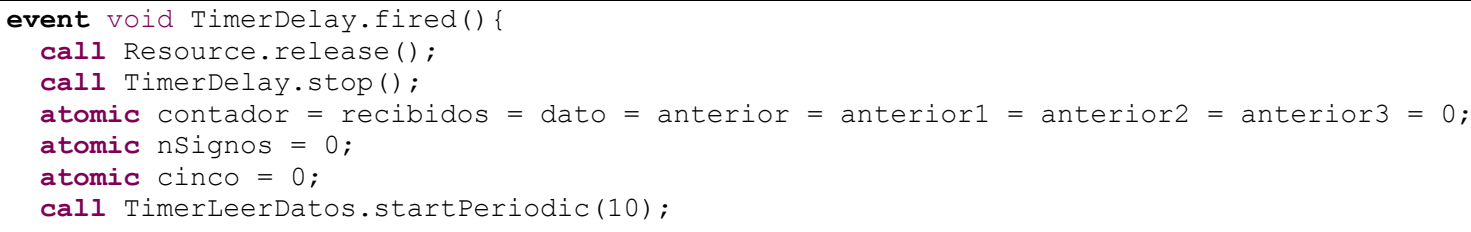

Listado III.12. Sentencias nesC del evento del temporizador de espera.

El Listado III.13 muestra el código nesC del temporizador mencionado anteriormente. Su estructura es similar a la del utilizado para iniciar la lectura. En este caso, se establece la variable leerDatos a verdadero (línea 4) para controlar el flujo de ejecución en los eventos de cuando la UART ha sido cedida por TinyOS (granted) y en la recepción de bytes (receiveByte). Otro cambio es que desde que se solicita la UART (línea 11) hasta que se configura la interfaz SDI-12 en modo recepción (línea 15) transcurren 40ms. Esto es así porque se envía un comando de 4 bytes (aD0!)

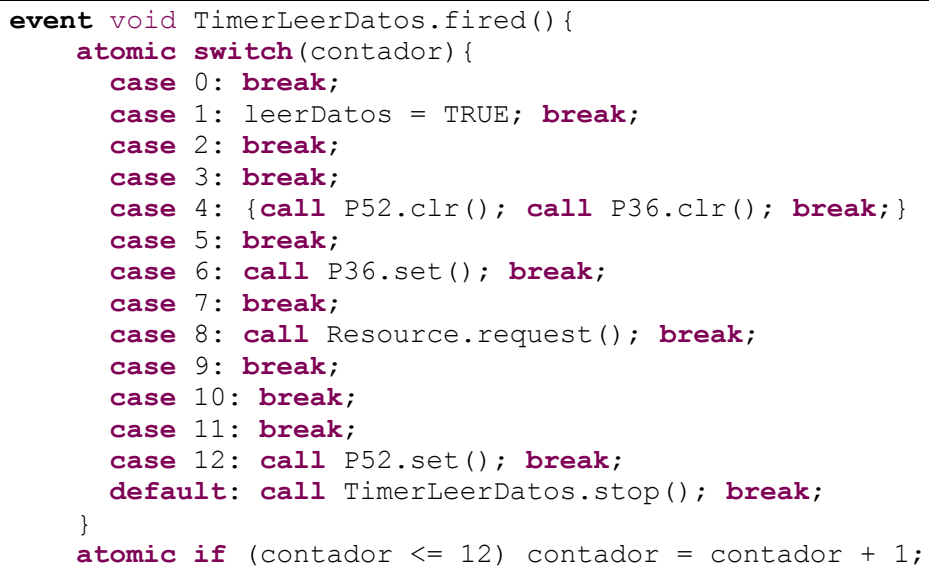

Listado III.13. Código del temporizador para realizar la petición de datos del sensor.

De nuevo, cuando el sensor reciba el comando, se recibirían bytes con la respuesta del comando por la UART (Listado III.14). En este caso la respuesta contiene los datos de las variables que monitoriza el sensor y tiene el formato a \pm tt.t \pm p.ppp \pm c.ccc $<\mathrm{CR}><\mathrm{LF}>$. El signo de la variable puede ser negativo o positivo. La temperatura con un decimal de precisión está representada por t. El porcentaje volumétrico y la conductividad con 3 decimales de precisión están representados por p y c, respectivamente. Al realizar la primera petición (aD0!) es posible que la respuesta no contenga las 3 variables esperadas. 
En este caso habrá que realizar una segunda petición con el envío del comando aD1!. Supóngase el siguiente ejemplo para el sensor con dirección 0:

$$
\text { “OD0!" "0+25.5+0.625<CR }><L F>\text { " "0D1!" "0-0.321<CR }><L F>\text { ” }
$$

Como se puede observar en la secuencia de ejemplo propuesta, para componer la trama con los datos obtenidos del sensor en el caso de realizar una segunda petición, es necesario desechar los 5 bytes marcados en negrita. Para este fin es la variable cinco mostrada en la línea 3 del Listado III.14. Antes de enviar el segundo comando de petición de datos esta variable se establece con el valor 5 (línea 20). Así, los 5 siguientes bytes recibidos no se almacenan en el array debug. En este caso el algoritmo detecta la recepción de un signo (ASCII 43 ó 45) e incrementa la variable nSignos, de manera que cuando se recibe fin de trama (13 10) se comprueba si nSignos es distinto de 3 (línea 19). En este caso, se enviará un segundo comando de petición de datos (línea 21). También puede ocurrir que al recibir fin de trama nSignos tenga en valor 3 (línea 23), en cuyo caso se han recibido todos los datos del sensor con un solo comando. Si ocurre esto, se desactiva el convertidor DC/DC (línea 24) que alimenta los sensores HP2 y se ejecuta la tarea encargada de enviar los datos (línea 25). Cabe destacar que en la línea 18 se restan dos bytes en la variable recibidos. Esto se realiza para no tener en cuenta en el array debug la secuencia de fin de trama 13 10. También hay que resaltar que cuando se detecta fin de trama se desactiva la interrupción y se libera el uso de la UART (líneas 15 y 16).

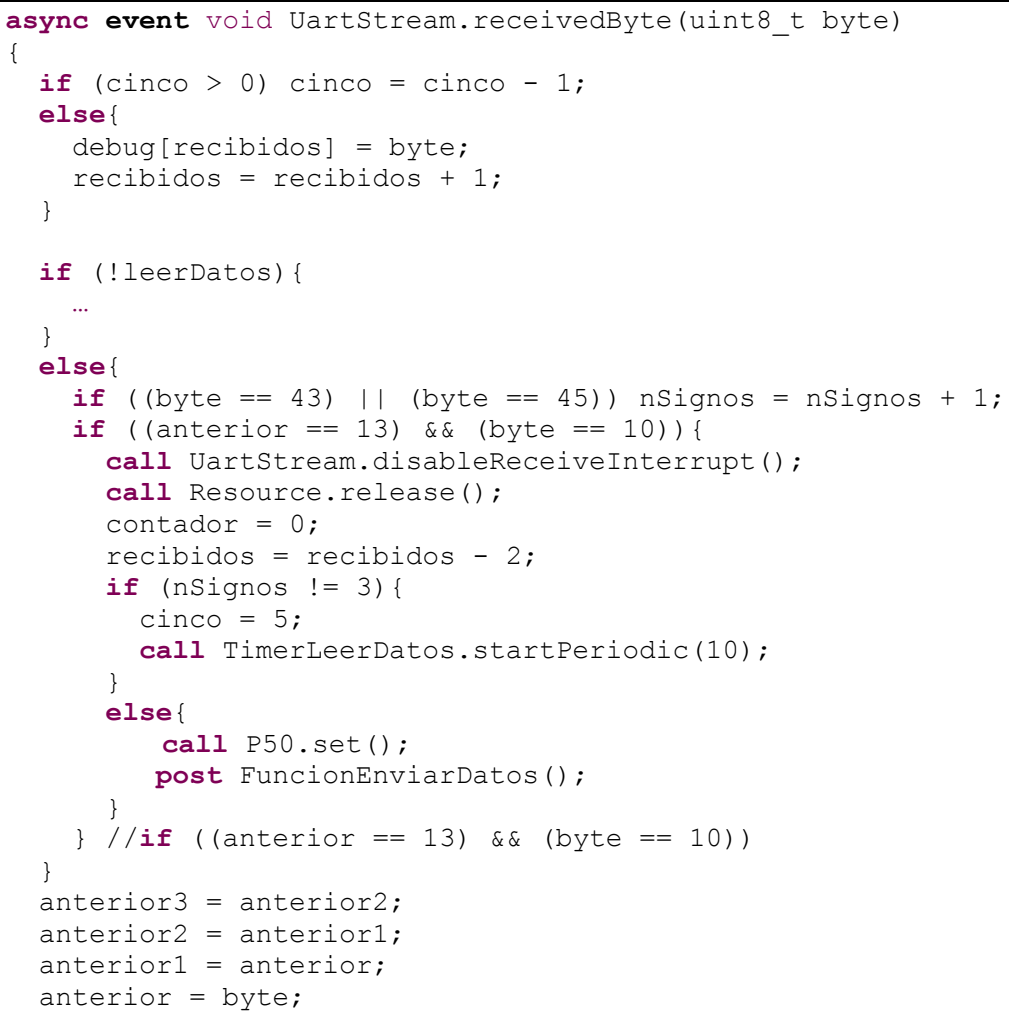

Listado III.14. Evento que recibe datos de la UART asociado con la lectura de datos de un HP2. 
En el Listado III.15 se muestran las sentencias ejecutadas en la tarea FuncionEnviarDatos, que tiene una estructura similar a la tarea FuncionEnviarAlarma presentada en el Listado III.7. En este caso, la principal diferencia es que hay que usar un bucle para insertar la lectura del sensor en el campo datos de la estructura MensajeDatos definida en el archivo de cabecera "HP2.h". Además, en este caso se utilizan 10 reintentos en las comunicaciones, en lugar de 5 , ya que la pérdida de un paquete de datos de los sensores es menos deseable que la del nivel de la batería. Finalmente, cabe destacar que el array debug contiene, en las primeras posiciones, el primer comando de petición de datos del sensor junto con el inicio de la respuesta. Por ello, la variable "len" se inicializa con "recibidos - 5" en la línea 7 y el paso de datos de un array al otro en el bucle 'for' tiene un offset de 5 (línea 12) para ignorar las 5 primeras posiciones del array.

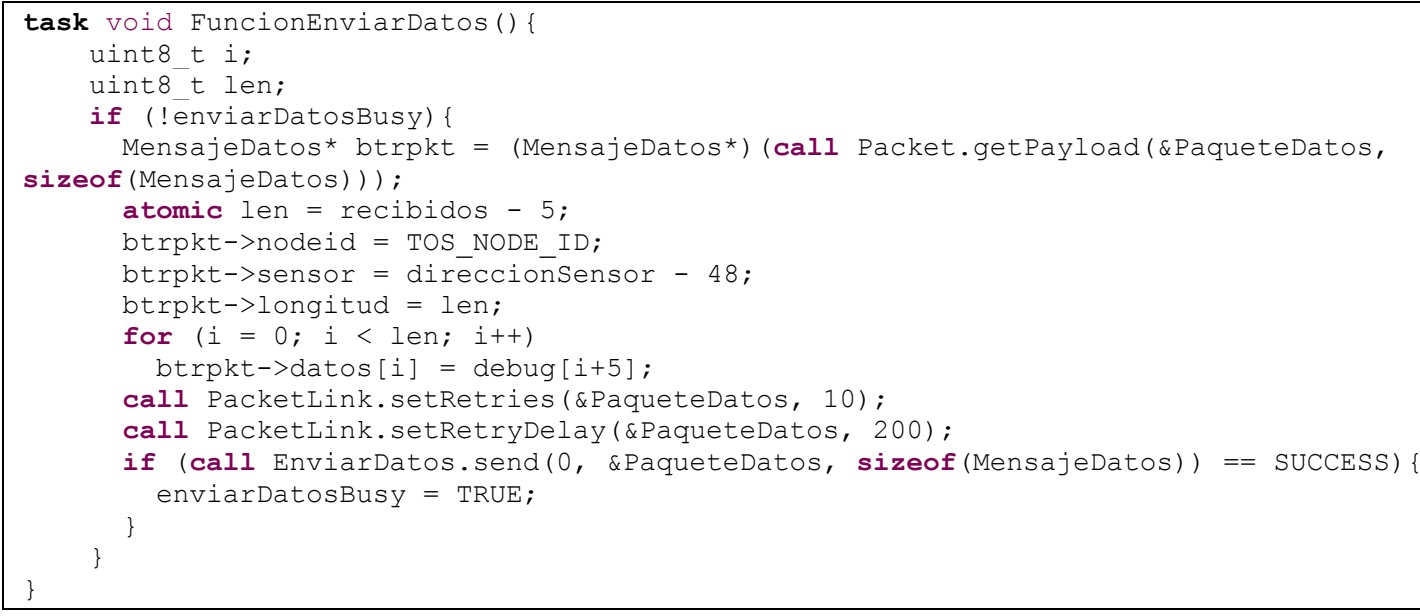

Listado III.15. Tarea para enviar los datos del sensor HP2.

Tal y como ocurría con el envío de mensajes del estado de la batería, la respuesta al comando send de la interfaz EnviarDatos es el evento sendDonde de la misma interfaz (Listado III.16). Este evento incluye como parámetros un puntero al mensaje enviado y la variable "error" para indicar el resultado de la operación. Antes de ejecutar cualquier otra sentencia, en la línea 3 se realiza una comprobación general de si el mensaje enviado y el indicado por el evento son el mismo. En caso afirmativo, puede ocurrir que el mensaje haya sido recibido por la estación receptora correctamente (líneas 5-18) o no (líneas 19-40). En este último caso, se incrementará la variable que cuenta los reintentos realizados y se volverá a enviar el mensaje (líneas 21 y 22). También puede ocurrir que tras realizar 10 reintentos el mensaje no se haya entregado correctamente en la estación receptora. En este caso, y al igual que si el mensaje ha sido entregado correctamente (línea 5), se ejecutan posteriormente las mismas sentencias. En concreto, como en el bus SDI-12 pueden haber hasta 10 sensores, se incrementa la dirección del último sensor leído y se comprueba si es menor o igual a la dirección del último sensor que hay que leer (líneas 6, 7, 25 y 26). Si hay que leer otro sensor HP2, se activa el convertidor DC/DC que alimenta los sensores (líneas 
8 y 28), se inicializan todas las variables necesarias y se crea un temporizador periódico de $10 \mathrm{~ms}$ para comenzar el proceso de lectura y que se ha descrito anteriormente en el Listado III.9. Finalmente, lo último que puede ocurrir es que se haya enviado el mensaje del último sensor del que había que realizar una lectura. En este caso se apaga el LED que se activo para indicar que comenzaba el proceso de lectura y se desactiva el convertidor DC/DC (líneas 15-16 y 36-37).

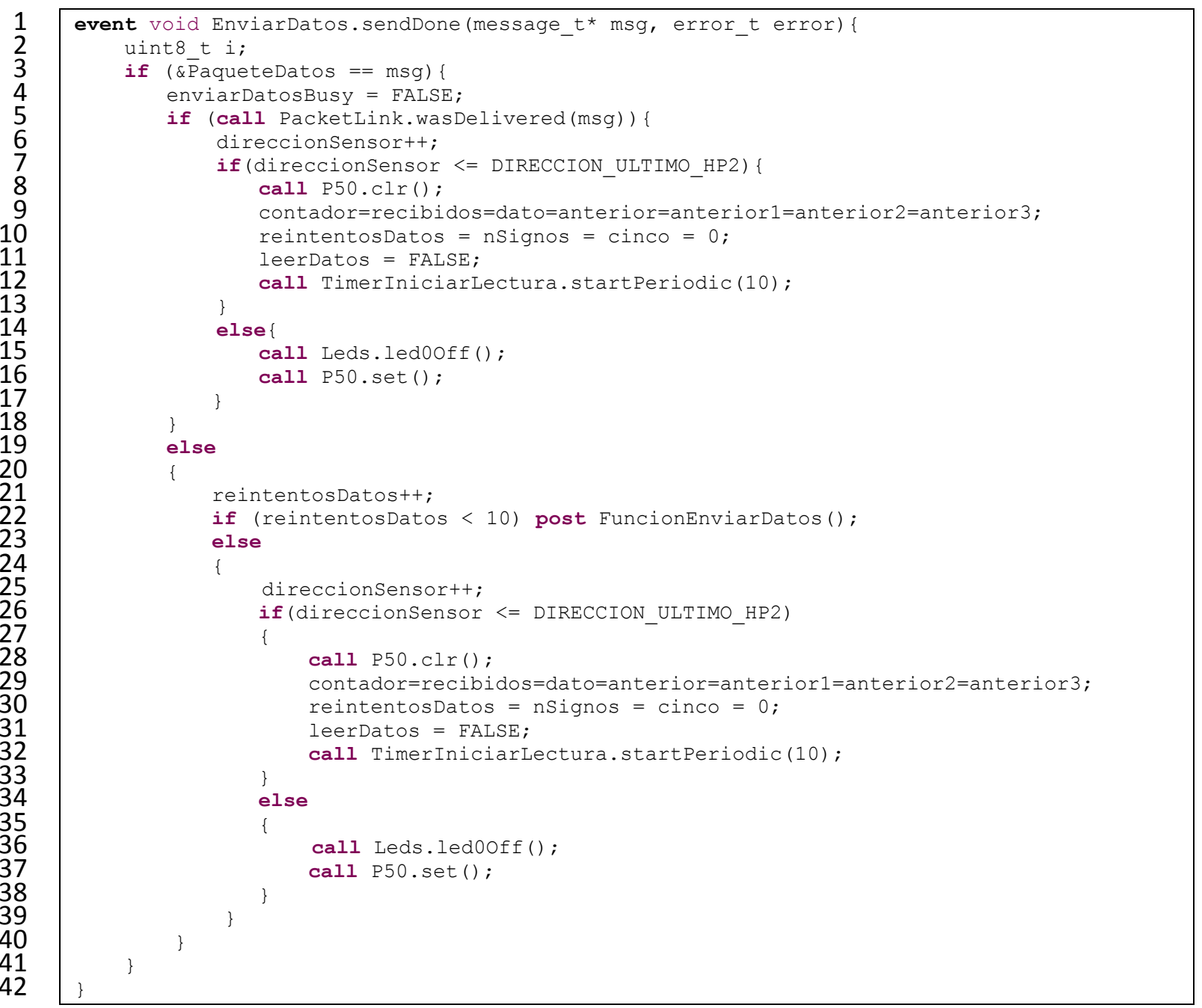

Listado III.16. Evento que señala TinyOS al enviar un mensaje de datos. 
CONTRIBUCIÓN A LAS REDES DE SENSORES INALÁMBRICAS. ESTUDIO E IMPLEMENTACIÓN DE SOluCiones Hardware Para Agricultura de PreCisión. 


\section{Anexo IV}

\section{Descripción del Software Desarrollado para un Nodo Sensor MEWiN con el Rol de ZigBee Router.}

\footnotetext{
$n$ este anexo, se describe en profundidad el software desarrollado para el nodo sensor multi-entorno
$M E W i N$ con el rol de ZigBee Router. Se decidió recogerlo en un anexo para facilitar la lectura de los capitulos fundamentales de la presente tesis. Leyendo este anexo, el lector interesado en el desarrollo de software con la biblioteca Z-Stack de Texas Instruments podrá adquirir mayores conocimientos con este tipo de programación. La descripción comienza presentando un diagrama que ilustra, a grandes rasgos, el funcionamiento del software del nodo sensor. Finalmente, se describe en detalle el código fuente desarrollado que implementa la funcionalidad del nodo sensor.
} 


\section{IV.1 Introducción.}

Para validar el nodo sensor multi-entorno MEWiN fue necesario desarrollar el software para los tres dispositivos que intervienen en una red ZigBee: Coordinator, Router y End Device. El software se implementó utilizando la biblioteca Z-Stack, que facilita Texas Instruments [TI]. Con esta biblioteca, se puede desarrollar software para los circuitos integrados que comercializa el fabricante para diseñar e implementar redes inalámbricas de bajo consumo.

En este anexo se describe en profundidad el software desarrollado para el nodo sensor multi-entorno MEWiN con el rol de ZigBee Router (ZR). Leyendo este anexo, el lector interesado en el desarrollo de software con la biblioteca Z-Stack, de Texas Instruments, podrá adquirir mayores conocimientos con este tipo de programación. La descripción comienza presentando un diagrama, que ilustra a grandes rasgos el funcionamiento del software desarrollado del nodo sensor. Finalmente, se describe en detalle el código fuente desarrollado que implementa la funcionalidad del nodo sensor con el rol de ZR.

\section{IV.2 Descripción del software.}

El desarrollo de aplicaciones con la biblioteca Z-Stack de Texas Instruments se realiza definiendo las tareas necesarias a nivel de aplicación. La ejecución de las tareas se lleva a cabo mediante un algoritmo de planificación de procesos Round Robin. Existen tareas de mayor prioridad, como la tarea de más bajo nivel encargada de la de gestión de control de acceso al medio (MAC, Medium Access Control) y la siguiente tarea con más prioridad, encargada de gestionar la red. A nivel de aplicación final debe haber al menos una tarea.

Cada tarea tiene asociadas dos funciones. La primera realiza la inicialización asociada a la tarea, y se realiza una sola vez al iniciarse la ejecución. La segunda función es un manejador de eventos, y se ejecuta periódicamente. El parámetro de este método es una máscara de 16 bits, la cual permite procesar hasta 16 eventos diferentes. En cada ejecución de esta última, se comprueba si está pendiente el procesamiento de un determinado evento. En caso afirmativo, se procesa el evento y se desactiva el bit de la máscara correspondiente al evento procesado.

El desarrollador puede definir los eventos necesarios asociados a cada tarea para implementar el funcionamiento del software necesario. Estos eventos se pueden configurar para que se ejecuten pasado un determinado tiempo, especificado en la planificación del evento. 
La Figura IV.1 muestra de forma visual el funcionamiento del software que se diseñó para el nodo sensor con el rol de ZR.

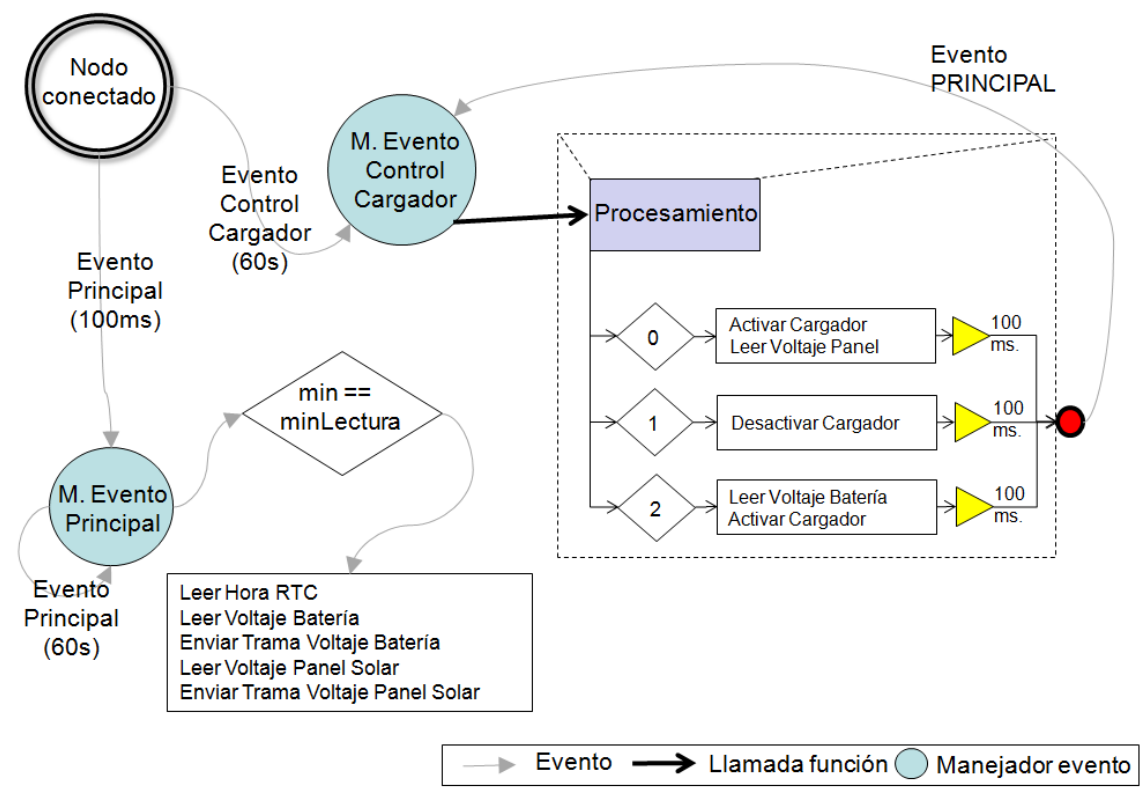

Figura IV.1. Diagrama simplificado del software diseñado para el nodo sensor con el rol de ZR.

Cuando el nodo se conecta con el coordinador de la red, se planifica la ejecución del evento principal a los $100 \mathrm{~ms}$ y la del evento para controlar el cargador al minuto.

El evento principal vuelve a planificar el mismo evento al minuto. La ejecución del mismo comprueba en primer lugar si hay que realizar una lectura del voltaje de la batería y del panel. En caso afirmativo, se realizan las lecturas y se envían las tramas con los datos leídos al coordinador.

El evento para controlar el cargador de la batería a través del panel solar fotovoltaico está implementado mediante una máquina de estados descrita con una sentencia de control Switch. En el 'case O' se comprueba si ha pasado el tiempo necesario para volver a ejecutar el algoritmo de control del cargador. En caso afirmativo, se activa el cargador y se realiza la lectura del voltaje del panel solar. En el 'case 1' se desactiva el cargador. Esto es necesario para realizar una lectura de la tensión de la batería real. Finalmente, en el 'case 2' se realiza la lectura del voltaje de la batería y si está por debajo del valor requerido, se vuelve a activar el cargador.

Los siguientes párrafos describen en mayor detalle el software que se ha implementado para el dispositivo. Esta descripción se realizará mediante la explicación de varios listados de código extraídos de la implementación real.

Como especifica ZigBee, los mensajes que envía y recibe un nodo se definen en un 'clúster'. El ZR envía mensajes del estado del panel y del estado de la batería, y puede recibir 
mensajes para modificar la frecuencia de lectura de la batería y del panel. Por ello, el clúster tiene 2 comandos de salida y 1 comando de entrada (ver Listado IV.1).

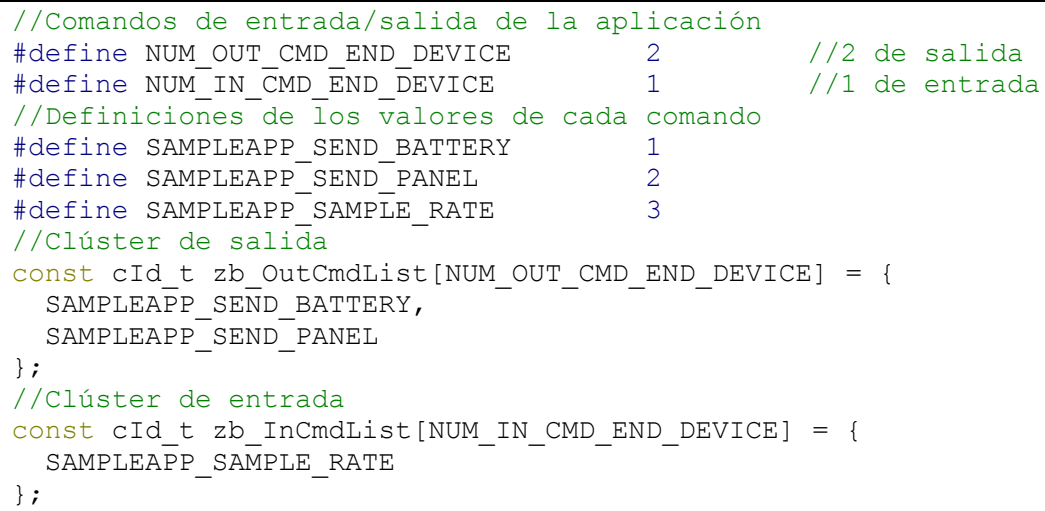

Listado IV.1. Comandos de entrada y salida que recibe y envía el ZR con el coordinador.

Continuado con el software del ZR, para conseguir un determinado funcionamiento con la biblioteca Z-Stack es necesario planificar la ejecución de un conjunto de eventos. Para este nodo ha sido necesario definir 5 eventos (ver Tabla IV.1). El evento "SYS_EVENT_MSG" es propio del sistema y permite gestionar eventos de la pila ZigBee y del hardware de bajo nivel. La tabla siguiente describe los 5 eventos que tiene definidos el ZR para su ejecución.

\begin{tabular}{|c|c|c|}
\hline Evento & Valor & Descripción \\
\hline SYS_EVENT_MSG & $0 \times 8000$ & $\begin{array}{l}\text { Evento del sistema. Eventos de la pila ZigBee y del hardware } \\
\text { de bajo nivel }\end{array}$ \\
\hline EVENTO_PRINCIPAL & $0 \times 0001$ & $\begin{array}{l}\text { Evento que gestiona el flujo principal del nodo sensor: lectura } \\
\text { y envío del voltaje de la batería y del panel solar }\end{array}$ \\
\hline CONTROL_PANEL_SOLAR & $0 \mathrm{x} 0002$ & Evento para controlar el estado del cargador de la batería \\
\hline LEER_PANEL_SOLAR & 0x0004 & Evento para realizar la lectura del voltaje del panel solar \\
\hline LEER_BATERIA & $0 \mathrm{x} 0008$ & Evento para realizar la lectura del voltaje de la batería \\
\hline LEER_HORA_RTC & $0 \times 0010$ & Evento para realizar la lectura del RTC \\
\hline
\end{tabular}

Tabla IV.1. Eventos definidos en el software del ZR.

Para la aplicación del nodo ZR sólo ha sido necesario implementar una tarea a nivel de aplicación. En la primera ejecución de la tarea se procesa la función SampleApp_Init. En esta función se configuran los pines de E/S del micro-controlador necesarios, se inicializan las variables, y se inicializan los convertidores analógico-digitales y los LEDs del dispositivo (ver Listado IV.2).

El método manejador de eventos de la tarea de la aplicación puede requerir el procesamiento del evento SYS_EVENT_MSG por diferentes motivos. Uno de ellos, es que el nodo haya establecido correctamente la conexión con el coordinador de red. En este caso, como se muestra en el Listado IV.3, se programa una secuencia en los LEDs, se 
inicializar las variables necesarias, se activa el cargador de la batería y se planifican la ejecución del EVENTO_PRINCIPAL y del evento CONTROL_PANEL_SOLAR a los $100 \mathrm{~ms}$ y a los $60 \mathrm{~s}$, respectivamente.

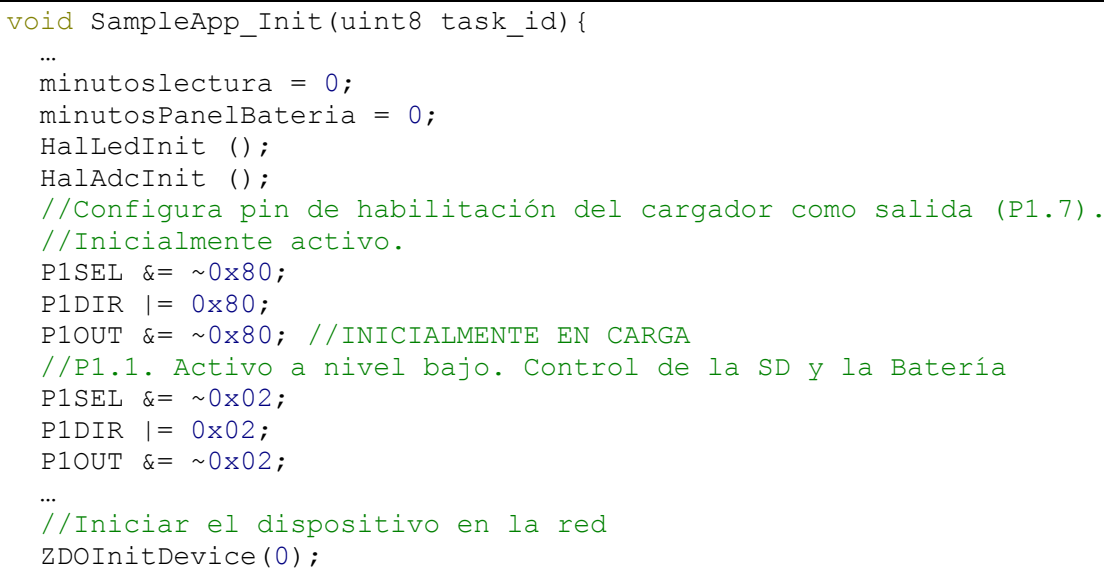

Listado IV.2. Extracto del método SampleApp_Init.

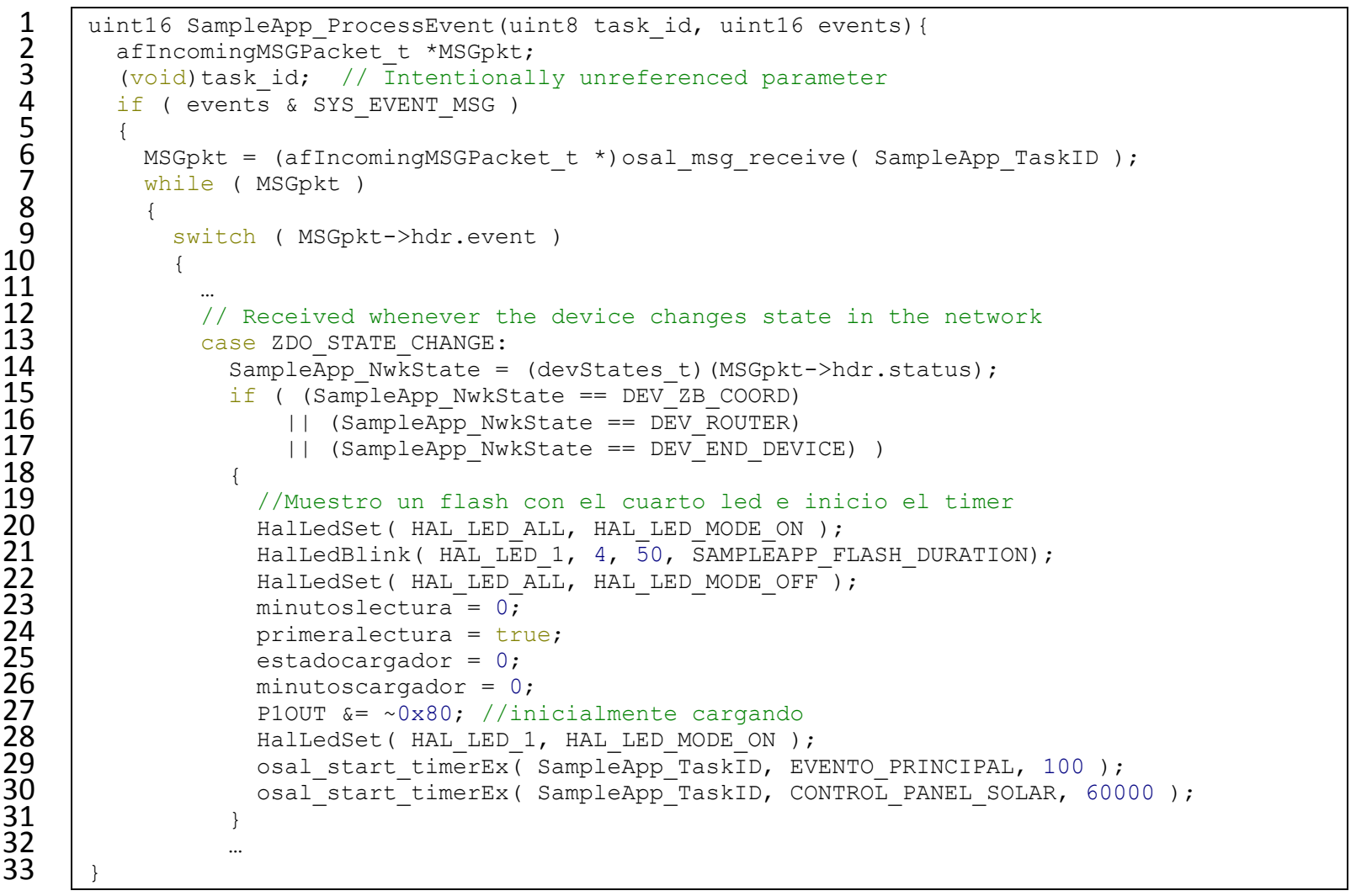

Listado IV.3. Sentencias ejecutadas cuando el ZR se conecta con el coordinador.

Cuando transcurran 100ms, se ejecutaran las sentencias del EVENTO_PRINCIPAL, que se muestran en el Listado IV.4. En primer lugar, se vuelve a configurar la ejecución del evento transcurridos $60 \mathrm{~s}$ y se incrementa la variable que almacena los minutos que han transcurrido. Posteriormente, se comprueba si han pasado los minutos que ha configurado el usuario (minutosPanelBateria, 30 en la inicialización) o si es 
la primera lectura que se realiza tras conectarse el nodo con el coordinador. Esta última variable booleana permite realizar una primera lectura a los $100 \mathrm{~ms}$ de una conexión establecida y se utiliza para verificar el correcto funcionamiento del nodo sensor. En caso afirmativo, se configura la ejecución de los tres eventos necesarios para realizar la lectura de la hora y la fecha del reloj de tiempo-real, leer y enviar la lectura del voltaje de la batería, así como leer y enviar la lectura del voltaje del panel solar.

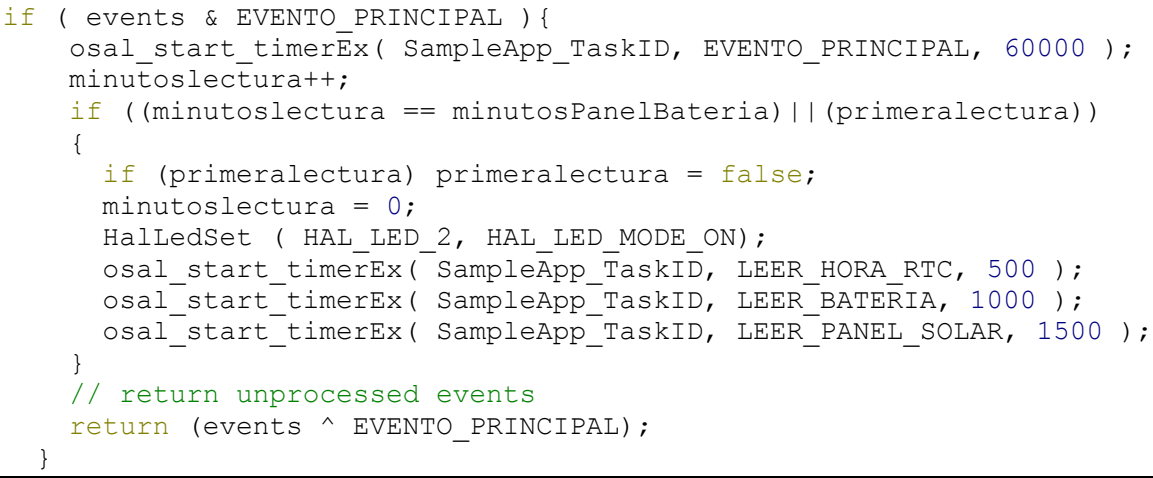

\section{Listado IV.4. Sentencias del evento principal.}

Como se puede observar en el Listado IV.5, la ejecución del evento LEER_HORA_RTC se realiza con la llamada a los métodos para iniciar el bus $\mathrm{I}^{2} \mathrm{C}$ (IniciarI2C) y realizar la lectura (LeerI2C) de los registros necesarios del componente. Cuando hayan transcurrido unos pocos milisegundos, la hora y la fecha estarán almacenas en el vector de 7 elementos $u 8 R \times B$ uffer. En dicho listado, también se puede observar que la ejecución de los eventos LEER_BATERIA y LEER_PANEL_SOLAR implica inicializar a cero una variable, realizar 8 lecturas del convertidor analógico-digital correspondiente, dividir el valor acumulado entre 8 y enviar la lectura realizada.

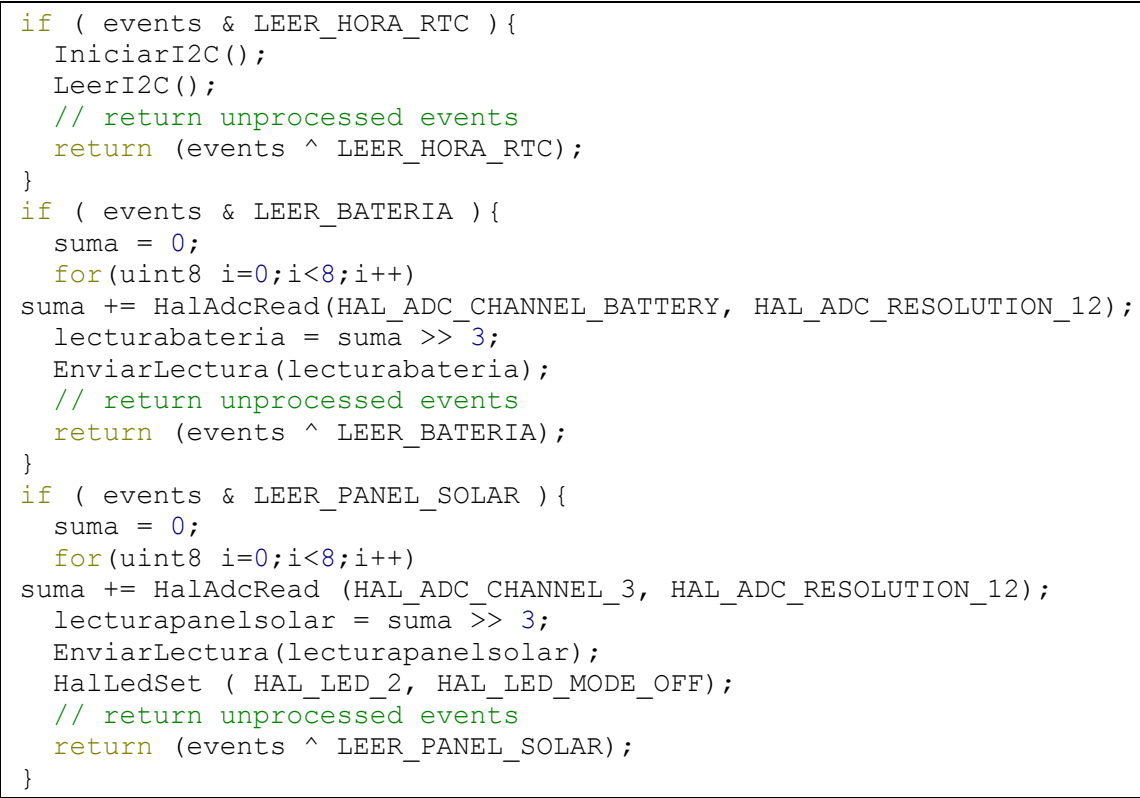

Listado IV.5. Sentencias de los eventos LEER_HORA_RTC, LEER_BATERIA y LEER_PANEL_SOLAR. 
El método EnviarLectura (ver Listado IV.6) es el encargado de enviar por radio la lectura realizada del voltaje del panel solar o de la batería. Para ello, en primer lugar la trama con la vector buffer, de 17 elementos. En las primeras dos posiciones se almacena la lectura a enviar, que como es de 16 bits y los elementos del vector son de un byte, es necesario escribir en el primer elemento del vector la parte menos significativa, y las más significativa en el segundo elemento del vector. En las siguientes 7 posiciones se asigna la lectura de la hora y la fecha del RTC, que se obtuvo en el vector $u 8 R \times B$ uffer. En las 8 posiciones restantes se almacena la MAC del nodo sensor, y finalmente se envía el mensaje por radio al coordinador de la red.

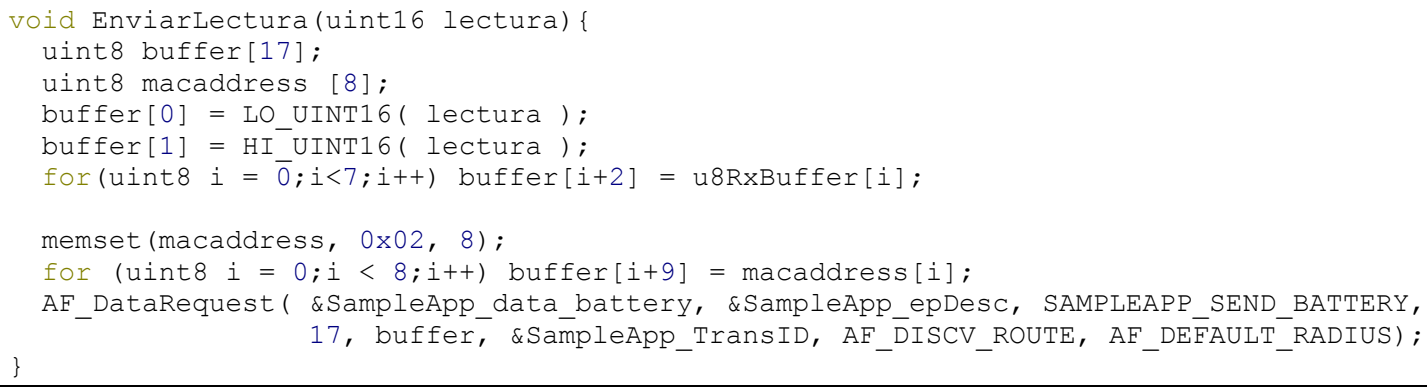

Listado IV.6. Sentencias del método para enviar los valores leídos de los ADCs.

Con lo expuesto anteriormente, queda definida la ejecución asociada al EVENTO_PRINCIPAL y queda por explicar el código fuente asociado al evento CONTROL_PANEL_SOLAR. La ejecución de este evento se ha implementado con una máquina de estados de tres estados. La máquina de estados se ha definido mediante una sentencia de control de tipo 'switch' (ver Listado IV.7).

En el primer estado de la máquina de estados (case 0), se incrementa la variable minutoscargador y se comprueba si dicha variable tiene el mismo valor que MINUTOS_COMPROBACION_CARGADOR, que se ha definido con un valor de 5. En caso de no coincidir, se vuelve a planificar la ejecución del evento transcurridos 60s (línea 18). En caso contrario, se activa el cargador antes de realizar la lectura del voltaje del panel solar para obtener un valor real. Esto es necesario hacerlo porque cuando está el cargador inactivo, el panel solar está en cortocircuito y se mediría un voltaje proporcional de la tensión emisor-colector del transistor. El panel solar está conectado con el ADC del microcontrolador a través de un divisor resistivo de valor 9,72, formado por tres resistencias. Por ello, en la línea 14 se utiliza el valor 9,72 para obtener el valor real de voltaje del panel solar en voltios, en la variable, de tipo float, flecturapanelsolar. Finalmente se incremente la variable estadocargador para entrar en el siguiente estado en la próxima ejecución y se vuelve a planificar la ejecución del evento a los 100ms.

El segundo estado (case 1) es necesario para establecer un retraso desde que se desactiva el cargador hasta que se realiza la lectura del voltaje de la batería. Este retraso es 
necesario para medir el voltaje real de la batería, ya que cuando el cargador está activo, el voltaje de la batería es superior al real.

En el último estado (case 2) se realiza la lectura de la batería en unidades sin procesar (líneas 27-30). En la línea 31 se obtiene el voltaje de la batería en voltios. El primer 2,5 de la expresión es necesario porque la batería está conectada con el ADC por medio de un divisor resistivo de valor 2,5. La fracción 2,5/4095 deriva del hecho que el ADC de 12 bits ofrece a la salida un valor de 4095 o fondo de escala para una tensión de entrada de 2,5V. Posteriormente, en la sentencia de selección 'if 'de la línea 32 se comprueba si el voltaje de la batería es menor de 4,1V y que el voltaje del panel sea superior al de la batería. En caso afirmativo, se activa el cargador para seguir cargando la batería. Tanto si la condición de la sentencia de selección 'if devuelve verdadero o falso, se asigna 0 a la variable estadocargador (para entrar en el estado 0 en la próxima ejecución) y se vuelve a planificar el evento a los 60 s.

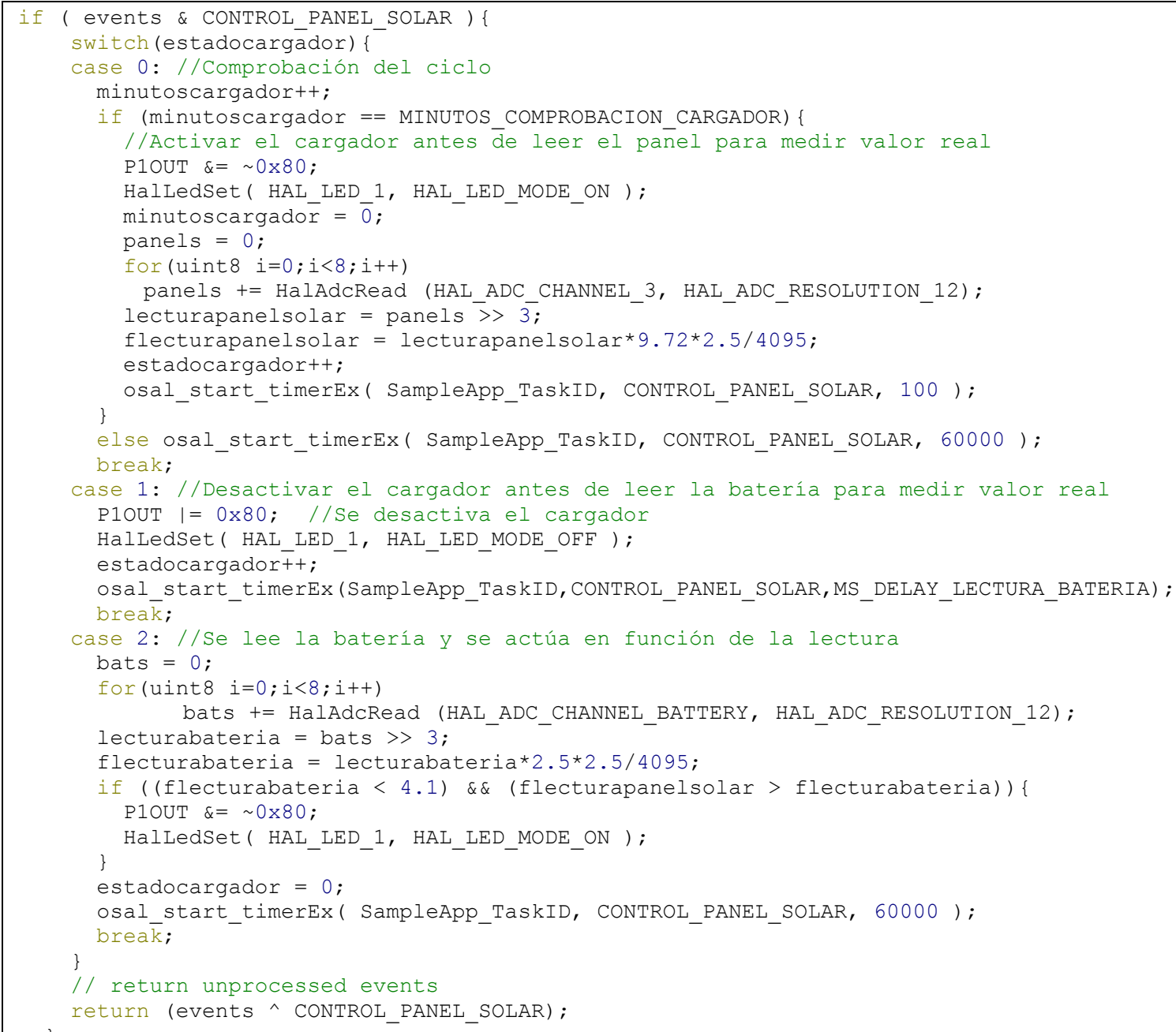

Listado IV.7. Sentencias de la máquina de estados implementada para la ejecución del evento CONTROL_PANEL_SOLAR. 


\section{Bibliografía}

[6LowWPAN]

[Acciona]

[Adrian, 2005]

[Agostinho, 2008]

[Ahn, 1999]

[AIT]

[Akyildiz, 2002]

[Akyildiz, 2004]

[Akyildiz, 2005]

[Allen, 1990]

[Allen, 2000]
6LoWPAN, <http:// Glowpan.net/>.

Acciona Infraestructuras. Disponible on-line en: www.accionainfraestructuras.es.

Adrian A.M., Norwood S.H., Mask P.L. "Producers' Perceptions and Attitudes Toward Precision Agriculture Technologies". Computers and Electronics in Agriculture 48 (2005). 256-271.

Feni Agostinho, Guaraci Diniz, Raúl Siche, Enrique Ortega. "The use of emergy assessment and the Geographical Information System in the diagnosis of small family farms in Brazil". Ecological Modelling, Volume 210, Issues 1-2, 10 January 2008, Pages 37-57.

Ahn, C.W., Baumgardner, M.F., Biehl, L.L., 1999. "Delineation of soil variability using geostatistics and fuzzy clustering analyses of hyperspectral data". Soil Sci. Soc. Am. J. 63, 142-150.

Grupo de investigación "Área de Ingeniería Telemática". Universidad Politécnica de Cartagena. Disponible on-line en: http://www.ait.upct.es.

Akyildiz, I.F., Su, W., Sankarasubramaniam, Y., Cayirci, E., 2002. Wireless sensor networks: a survey, Computer Networks 38, 393-422.

Akyildiz, I.F., Kasimoglu, I.H. "Wireless sensor and actor networks: Research and challenges". Ad Hoc Networks, 2(4):351-367, October 2004.

Akyildiz, I.F., Xudong Wang: "A Survey on Wireless Mesh Networks". IEEE Communications Magazine. 2005.

Allen, R.G., Fisher, D.K. 1990. "Low-cost electronic weighing lysimeters". Transactions of the Asae (33):1823-1833.

Allen, R.G., 2000. "Report on AM400 and HOBO loggers". University of Idaho, Kimberly, Idaho, www.kimberly.uidaho.edu/water/swm/. 
[Anurag, 2008]

[Apogee]

[Ascough, 1999]

[Auernhammer, 1994]

[Ayday, 2009]

[AZoNanotechnology]

[Baggio, 2005]

[Balanis, 2005]

[Balmart]

[Baronti, 2007]

[Basso, 2006]

[Beckwith, 2004]

[Beckwith, 2004]

[Beckwith, 2004a]

[Berkeley]
Anurag D, Siuli Roy and Somprakash Banyopadhyay. "Agro-sense: Precision agriculture using sensor-based wireless mesh networks. Innovations in NGN: Future Network and Services". Proceedings of the First ITU-T Kaleidoscope Academic Conference (K-INGN 2008), pp. 383-388.

Apogee Instruments Inc. Disponible on-line en: http://www.apogeeinst.com/

Ascough J.C., Hoag D.L., Frasier W.M., McMaster G.S. 1999. "Computer use in agriculture: an analysis of Great Plain producers”. Computers and Electronics in Agriculture, 23, 189-204.

Auernhammer, H. 1994. "Global positioning systems in agriculture (special issue)". Computers and Electronics in Agriculture 11.

Can Ayday, Saban Safak. "Application of Wireless Sensor Networks with GIS on the Soil Moisture Distribution Mapping”. Proceedings of GIS 2009. Ostrava, Czech Republic. January 2009.

AZoNanotechnology Article: "Precision Agriculture -Nanotech Methods Used, Such as 'Smart Dust', Smart Fields' and Nanosensors". http:/ /www.azonano.com/details.asp.ArticleID=1318. http:/ /www.azonano.com/details.asp.ArticleID=1318.

A. Baggio. "Wireless Sensor Networks in Precision Agriculture". ACM Workshop on Real-World Wireless Sensor Networks. Stockholm, Sweden, June 2005.

Constantine A. Balanis. “Antenna Theory: Analysis and Design”. John Wiley \& Sons, 2005.

Balmart. Sistemas Electrónicos y de Comunicaciones. Disponible on-line en: http://www.balmart.es/

Baronti, P., Pillai, P., Chook, V.W., Chessa, S., Gotta, A., Fu, Y.F., 2007. "Wireless Sensor Networks: a survey on the state of the art and the 802.15.4 and ZigBee standards". Comput. Commun. 30, 1655-1695.

Basso B., Sartori L., Bertocco M. "Manual de Agricultura de Precisión". Eumedia. 2006.

Richard Beckwith, Dan Teibel, Pat Bowen. "Report from the Field: Results from an Agricultural Wireless Sensor Network". Proceedings of the 29th Annual IEEE International Conference on Local Computer Networks, pp. 471-478, November 16-18, 2004.

R. Beckwith, D. Teibel, and P. Bowen. "Unwired wine: sensor networks in vineyards". 2004, pp. 561-564."

Beckwith, R., Teibel, D., Bowen, P., 2004. "Report from the field: results from an agricultural wireless sensor network". In: Proceedings of the 29th Annual IEEE International Conference on Local Computer Networks.

University of California, Berkeley, http://berkeley.edu. 
[Bertoni, 2000]

[Bhatti, 2005]

[Blackmore, 1994]

[Bogena, 2007]

[Bouwer, 2000]

[BPI]

[Camilli, 2007]

[CampbellScientific]

[Cano, 2007]

[Castia, 1992]

[CC2420]

$[\mathrm{CC} 2520]$

[CC2591]

[Cerpa, 2009]

[Chibuzor, 2006]
Bertoni, Henry L. "Radio propagation for modern wireless systems". Prentice Hall. 2000.

Shah Bhatti, James Carlson, Hui Dai, Jing Deng, Jeff Rose, Anmol Sheth, Brian Shucker, Charles Gruenwald, Adam Torgerson, Richard Han. "MANTIS OS: An Embedded Multithreaded Operating System for Wireless Micro Sensor Platforms". Mobile Networks and Applications, v.10 n.4, p.563-579, August 2005.

Blackmore, S. "Precision Farming: An Introduction. Outlook on Agriculture". 23(4) 4, 275-280.

Bogena, H.R., Huisman, J.A., Oberdörster, C., and H. Vereecken. 2007. "Evaluation of a low-cost soil water content sensor for wireless network applications". Journal of Hydrology 344, 32-42.

Bouwer, "Integrated water management: emerging issues and challenges", Agricultural Water Management, 45 (2000), pp. 217-228.

Baterías y Pilas Industriales. Disponible on-line en: http://www.bpi.com

Camilli, A., Cugnasca, C.E., Saraiva, A.M., Hirakawa, A.R., Corrêa, P.L.P., 2007. "From wireless sensors to field mapping: anatomy of an application for precision agriculture." Comp. Electron. Agric. 58, 25-36.

CampbellScientific Inc. Disponible on-line en: http:/ /www.campbellsci.com/

Cano, A.; Lopez-Baeza, E.; Anon, J.L.; Reig, C.; Millan-Scheiding, C. "Wireless Sensor Network for Soil Moisture Applications". Proceedings of the International Conference on Sensor Technologies and Applications 2007 (SensorComm 2007), 508-513."

Castia, T., Franco, M.A., Mattivi, F.,Muggiolu, G., Sferlazzo, G., Versini, G., 1992. Characterization of grapes cultivated in Sardinia: chemometric methods applied to the anthocyanic fraction. Sci. Aliments 12, 239-255.

Módulo de radio compatible con la versión 2003 del estándar IEEE 802.15.4 y ZigBee. Disponible on-line en: http://www.ti.com/product/cc2420.

Transceiver de segunda generación RF ZigBee/IEEE 802.15.4, de 2.4 GHz. Disponible on-line en: http://www.ti.com/product/cc2520.

Extensor de rango RF para circuitos integrados de baja potencia de 2.4 GHz. Disponible on-line en: http://www.ti.com/product/cc2591.

A. Cerpa, J. Elson, M. Hamilton, J. Zhao. "Habitat monitoring: application driver for wireless communications technology”. ACM SIGCOMM'2000, Costa Rica, April 2000.

Chibuzor Edordu and Lionel Sacks, "Self Organising Wireless Sensor Networks as a Land Management Tool in Developing Countries: A Preliminary Survey." Proceedings of the London Communications Symposium (LCS 2006), London, September 2006." 
[Cho, 2000]

[Chung, 2009]

[Cichota, 2006]

[Comax, 1990]

[Contiki]

[Cox, 2002]

[Cygwin]

[Daintree]

[Delenne, 2010]

[Digi]

[Downey, 2006]

[DSIE]

[Dunkels, 2004]

[Earl, 2000]

[EDOSOFT]

[EplusE]

[Embarcader]
S. Cho, A. Chandrakasan. "Energy-efficient protocols for low duty cycle wireless microsensor". Proceedings of the 33rd Annual Hawaii International Conference on System Sciences, Maui, HI Vol. 2 (2000), p. 10.

Jae Chung y Mark Claypool, "NS by Example", Home Page. Artículo de Página web, 2009. http://nile.wpi.edu/NS

Rogerio Cichota, Ana Lucia Berretta Hurtado, Quirijn de Jong van Lier. "Spatio-temporal variability of soil water tension in a tropical soil in Brazil". Geoderma, Volume 133, Issues 3-4, August 2006, Pages 231-243.

Hal Lemmon. "Comax: An Expert System for Cotton Crop Management". Springer Netherlands. 1990, 177-185.

Contiki Operating System. Disponible on-line en: http://www.sics.se/contiki/.

Sidney Cox. "Information technology: the global key to precision agriculture and sustainability". Computers and Electronics in Agriculture 36 (2002) 93111.

Emulación de la consola de Linux bajo Windows. Disponible on-line en: http://www.cygwin.com

Daintree Networks, disponible on-line en: http://www.daintree.net/

Carole Delenne, Sylvie Durrieu, Gilles Rabatel, Michel Deshayes. "From pixel to vine parcel: A complete methodology for vineyard delineation and characterization using remote-sensing data". Computers and Electronics in Agriculture, Volume 70, Issue 1, January 2010, Pages 78-83.

Digi Inc. Diponible on-line en: http:/ /www.digi.com/

Downey, M.O., Dokoozlian, N.K., Krstic, M.P., 2006. "Cultural practice and environmental impacts on the flavonoid composition of grapes and wine: a review of recent research". Am. J. Enol. Vitic. 57, 257-268.

Grupo de investigación "División de Sistemas e Ingeniería Electrónica" de la Universidad Politécnica de Cartagena. Diponible on-line en: http://www.dsie.upct.es/

A. Dunkels, B. Grönvall, and T. Voigt., Contiki - a Lightweight and Flexible Operating System for Tiny Networked Sensors, In Proceedings of the First IEEE Workshop on Embedded Networked Sensors 2004 (IEEE EmNetSI), Tampa, Florida, USA, November 2004.

R. Earl, G. Thomas, B. S. Blackmore. "The potential role of GIS in autonomous field operations". Computers and Electronics in Agriculture, Volume 25, Issues 1-2, January 2000, Pages 107-120.

Edosoft Factory S.L. Disponible on-line en: http://www.edosoftfactory.com/

E+E Inc. Disponible on-line en: http://www.epluse.com/

Embarcadero Inc. Disponible on-line en: http://www.embarcadero.com/ 
[ESEA]

[ESNA]

[Farnell]

[Fall, 2010]

[Favre, 1998]

[Fereres, 2000]

[Fleming, 2000]

[Fountas, 2006]

[Fraisse, 2001]

[Friis, 1946]

[Fukatsu, 2003]

[Gallardo, 2007]

[Gay, 2003]

[Ge, 2009]

[Gelb, 1999]
Estación Experimental Agroalimentaria 'Tomás Ferro'. Disponible on-line en: http://www.upct.es/eseatomasferro/

"European Sensor Network Architecture". Ref. ITEA 2006. Information Technology for European Advancement.

Distribuidor de components electronic Farnell. Diponible on-line en: http://es.farnell.com/

Fall, K., Varadhan, K. (2010). "The ns manual". http://www.isi.edu/nsnam/ns/.

P. Favre et al. "A 2V, 600 1A, $1 \mathrm{GHz}$ BiCMOS super regenerative receiver for ISM applications". IEEE Journal of Solid State Circuits 33 (1998) 21862196.

Fereres, E., Goldhamer, D.A. 2000. “Avances recientes en la programación de los riegos”. Ingeniería del Agua (7):47-54.

Fleming, K.L.,Westfall, D.G.,Wiens, D.W., Brodah, M.C., 2000. "Evaluating farmer developed management zone maps for variable rate fertilizer application”. Precision Agric. 2, 201-215.

S. Fountas, D. Wulfsohn, B.S. Blackmore, H.L. Jacobsen, S.M. Pedersen. “A model of decision-making and information flows for information-intensive agriculture”. Agricultural Systems 87 (2006) 192-210.

Fraisse, C.W., Sudduth, K.A., Kitchen, N.R., 2001. "Delineation of sitespecific management zones by unsupervised classification of topographic attributes and soil electrical conductivity". Trans. ASAE 44, 155-166.

H.T. Friis. "A note on a simple transmission formula". Proc. IRE, vol. 34, p. 254. 1946."

Fukatsu T, Hirafuji M. 2003. "Development of Field Servers for a field monitoring system". Agric. Info. Res. 12:1-12.

Antonio Gallardo, Rocio Paramá. "Spatial variability of soil elements in two plant communities of NW Spain”. Geoderma 139 (2007) 199-208.

Gay , D., Levis , P., von Behren , R., Welsh , M., Brewer , E., Culler, D., 2003. "The nesC Language: A Holistic Approach to Network Embedded Systems". ACM SIGPLAN 2003 Conference on Programming Language Design and Implementation, San Diego, California, USA, 1-11.

Yufeng Ge, J. Alex Thomasson, Ruixiu Sui, Cristine L.S. Morgan. “A module-specific post-processing calibration method to improve cotton yield mapping". Computers and Electronics in Agriculture, Volume 68, Issue 2, October 2009, Pages 161-167.

E. M. Gelb. "Adoption of IT by farmers--does reality reflect the potential benefit". In: Role and Potential of IT, Intranet and Internet for Advisory Services (Rickert U; Helbig R; Scheifer G eds). European Symposium as part of EFITA 99, Second European Conference of the European Federation for 
[GPBatteries]

[Graphviz]

[Gutierrez, 2001]

[Guzmán, 2009]

[Hamrita, 2005]

[Han, 2005]

[Handle, 2006]

[Handziski, 2005]

[HART, 2007]

[HART]

[Henao, 2006]

[Hill, 2000]

[Hirafuji, 2002]
Information Technology in Agriculture, Food and the Environment, 27-30 September, 1999, Bonn, Germany, pp. 13-23.

GP Batteries Incorporation. Disponible on-line en: http://www.gpbatteries.com

Graph Visualization Software. Disponible on-line en: http://www.graphviz.org/

Gutierrez, J.A.; Naeve, M.; Callaway, E.; Bourgeois, M.; Mitter, V.; Heile, B. "IEEE 802.15.4: a developing standard for low-power low-cost wireless personal area networks". IEEE Network. Vol. 15, 5. Pp. 12-19. Sep./Oct. 2001."

G.I. Guzmán Casado, M. González de Molina. "Preindustrial agriculture versus organic agriculture: The land cost of sustainability". Land Use Policy, Volume 26, Issue 2, April 2009, Pages 502-510.

Hamrita, T.K., Hoffacker, E.C., 2005. "Development of a "smart" wireless soil monitoring sensor prototype using RFID technology". Appl. Eng. Agric. 21 (1), 139-143.

C. Han, R. K. Rengaswamy, R. Shea, E. K. and M. Srivastava, SOS: A dynamic operating system for sensor networks, Proceedings of the Third International Conference on Mobile Systems, Applications, And Services (Mobisys), 2005.

Hande, A., Polk, T., Walker W., Bhatia, D. "Self-Powered Wireless Sensor Networks for remote Patient Monitoring in Hospitals". Sensors 2006, 6, pp. $1102,-1117$.

V. Handziski, J.Polastre, J.H.Hauer, C.Sharp, A.Wolisz and D.Culler. "Flexible Hardware Abstraction for Wireless Sensor Networks", in Proceedings of the 2nd European Workshop on Wireless Sensor Networks (EWSN 2005), Istanbul, Turkey, 2005."

Draft standard: What's in the April'07 WirelessHART specification, <http://www.controleng.com/article/CA6427951.html>.

HART. The Logical Wireless Solution, <http://www.hartcomm2.org/ hart_protocol/wireless_hart/hart_the_logical_solution.html $>$.

J. Henao and C. Baanante, "Agricultural Production and Soil Nutrient Mining in Africa: Implications for Resource Conservation and Policy Development." IFDC Technical Bulletin, Mar.2006."

Hill, J., Szewczyk, R., Woo, A., Hollar, S., Culler, D., Pister, K., 2000. "System Architecture Directions for Networked Sensors". ACM SIGPLAN notice. Vol. 35, No. 11., 93-104.

Hirafuji M, Fukatsu T. 2002. "Architecture of Field Monitoring Servers". Proceedings of the Third Asian Conference for Information Technology in Agriculture. p 405-409. 
[Hirafuji, 2004]

[Hoblos, 2000]

[Howitt, 2003]

[Huang, 2003]

[Huber, 1937]

[IAR]

[IEEE 802.15.3, 2003]

[IEEE, 2006]

[Intanagonwiwat, 2000]

[Intel]

[IPSO]

[Irrometer]

[ISA]

[Jensen, 2000]
Masayuki Hirafuji, Tokihiro Fukatsu, Hu Haoming, Takuji Kiura, Tominari Watanabe and Seishi Ninomiya. "A wireless sensor network with FieldMonitoring Servers and MetBroker in paddy fields". World Rice Rsearch Confrence 2004, 202.

G. Hoblos, M. Staroswiecki, A. Aitouche. "Optimal design of fault tolerant sensor networks". IEEE International Conference on Control Applications, Anchorage, AK, September 2000, pp. 467-472.

I. Howitt, J.A. Gutierrez. "IEEE802.15.4 low rate-wireless personal area network coexistence issues". Wireless Communications and Networking 3 (2003) 1481-1486.

G. Huang. "Casting the Wireless Sensor Net". July/August 2003. MIT Technology Review, pp. 51-56.

Huber, B., Schmidt, E. "Eine Kompensations-methode zur thermoelektrischen Messung Langsamer Saftstrome" Ber. deut. bot, Ges, 50,514-529. 1937.

IAR Systems. Disponible on-line en: http://www.iar.com/

IEEE Standard 802.15.3. "Wireless medium access control (MAC) and physical layer (PHY) specifications for high rate wireless person area networks (WPANs)". September 2003.

IEEE, Institute of Electrical and Electronics Engineers, 2006. "IEEE Standard for Information technology-Telecomunications and information exchange between systems - Local and metropolitan area networks Specific requirements Part 15.4: Wireless Medium Access Control (MAC) and Physical Layer (PHY) Specifications for Low-RateWireless Personal Area Networks (WPANs)". IEEE Standard 802.15.4-2006, Institute of Electrical and Electronics Engineers, New York.

C. Intanagonwiwat, R. Govindan, D. Estrin. "Directed diffusion: a scalable and robust communication paradigm for sensor networks". Proceedings of the ACM Mobi-Com'00, Boston, MA, 2000, pp. 56-67.

Intel Corporation, http:/ /www.intel.com

IPSO Alliance. Disponible on-line en: http://www.ipsoalliance.org/Pages/Overview.php

IRROMETER Company, Inc. Disponible on-line en: http://www.irrometer.com/

ISA100.11a,

$<$ http://www.isa.org/MSTemplate.cfm?MicrositeID=1134\&CommitteeID $=6891>$.

Jensen A.L., Boll P.S., Thysen I., Pathak B.K. 2000. “Pl@nteInfo--a webbased system for personalised decision support in crop management". Computers and Electronics in Agriculture, 25, 271-293. 
[Johnson, 2001]

[Jones, 2003]

[Kabashi, 2008]

[Kahn, 1999]

[Katsalis, 2007]

[King, 2000]

[KippZonen]

[Kitchen, 2005]

[Konstantinos, 2007]

[Kozlowski, 1967]

[Kuhlmann, 1999]

[Kwang, 2006]
Johnson, H., Robinson, J., 2001. The World Atlas of Wine, 5th ed. Mitchell Beasley, London.

J. W. Jones, G. Hoogenboom, C. H. Porter, K. J. Boote, W. D. Batchelor, L. A. Hunt, P. W. Wilkens, U. Singh, A. J. Gijsman and J. T. Ritchie. "The DSSAT cropping system model" European Journal of Agronomy 18 (2003), 235-265.

Amar H. Kabashi and J.M.H. Elmirghani. "A Technical Framework for Designing Wireless Sensor Networks for Agricultural Monitoring in Developing Regions". The Second International Conference on Next Generation Mobile Applications, Services and Technologies. IEEE Computer Society. 2008. Pp 395-401.

J.M. Kahn, R.H. Katz, K.S.J. Pister. "Next century challenges: mobile networking for smart dust". Proceedings of the ACM MobiCom'99, Washington, USA, 1999, pp. 271-278.

Katsalis Konstantinos, Xenakis Apostolos, Kikiras Panagiotis, Stamoulis George. "Topology Optimization in Wireless Sensor Networks for Precision Agriculture Applications". Proceedings of the 2007 International Conference on Sensor Technologies and Applications. Valencia, Spain. pp. 526-530, 2007.

King, B.A., Wall, R.W., Wall, L.R., 2000. "Supervisory Control and Data Acquisition System for Closed-Loop Center Pivot Irrigation”. ASAE Technical Paper No. 00-2020. The American Society of Agriculture Engineers, St. Joseph, Michigan, USA.

KippZonen Inc. Disponible on-line en: http://www.kippzonen.com/

N.R. Kitchen, K.A. Sudduth, D.B. Myers, S.T. Drummond, S.Y. Hong. "Delineating productivity zones on claypan soil fields using apparent soil electrical conductivity". Computers and Electronics in Agriculture 46 (2005) 285-308.

K. Konstantinos et al, "Topology Optimization in Wireless Sensor Networks for Precision Agriculture Applications", Proceedings of International Conference on Sensor Technologies and Applications 2007 (SensorComm 2007), 526-530.

Kozlowski, T.T.. "Diurnal variations in stem diameters of small trees". Botanical Gazette (128):60-68. 1967.

Kuhlmann F. 1999. "Farm-level-information-systems: developments and perspectives". In: Perspectives of Modern Information and Communication Systems in Agriculture, Food Production and Environmental Control (Scheifer G; Helbig R; Rickert U eds). Second European Conference of the European Federation for Information Technology in Agriculture, Food and the Environment, 27-30 September, 1999, Bonn, Germany, pp 7-17."

Kwang Koog Lee, Seong Hoon Kim, Yong Soon Choi, Hong Seong Park "A Mesh Routing Protocol using Cluster Label in the ZigBee Network". 
[Langendoen, 2006]

[Langmead]

[Levis, 2005]

[Libelium]

[López, 2008a]

[López, 2008b]

[López, 2009a]

[López, 2009b]

[López, 2009c]

[López, 2009d]

[López, 2009e]
IEEE International Conference on Mobile Adhoc and Sensor Systems. October 2006.

Langendoen, K.; Baggio, A.; Visser, O. "Murphy loves potatoes: experiences from a pilot sensor network deployment in precision agriculture". Parallel and Distributed Processing Symposium, 2006. IPDPS 2006. 20th International Digital Object Identifier: 10.1109/IPDPS.2006.1639412"

Langmead Farms Ltd. Disponible on-line en: http://www.langmeadfarms.co.uk.

P. Levis, D. Gay, V. Handziski, J.-H.Hauer, B.Greenstein, M.Turon, J.Hui, K.Klues, C.Sharp, R.Szewczyk, J.Polastre, P.Buonadonna, L.Nachman, G.Tolle, D.Culler, and A.Wolisz. "'T2: A Second Generation OS For Embedded Sensor Networks"". Technical Report TKN-05-007, Telecommunication Networks Group, Technische Universitaet Berlin, November 2005."

Libelium Company, disponible on-line en: http://www.libelium.com/

J. A. López Riquelme, F. Soto, J. Suardíaz, A. Iborra. "Red de Sensores Inalámbrica para Agricultura de Precisión”. Telecoforum 2008. ISSN: 16982924. Marzo 2009.

López Riquelme, J.A., Soto, F., Suardíaz, J., Iborra, A. "Desarrollo de una Red de Sensores para Agricultura de Precisión". Seminario Anual de Automática, Electrónica Industrial e Instrumentación (SAAEI 2008), Cartagena, Murcia.

J.A. López, F. Soto, J. Suardiaz, A. Iborra, J. Longares, J.A.Vera. "Wireless Sensor Networks for precision horticulture in Southerm Spain". Internacional Journal of Computer and Electronics in Agriculture, volumen 68, páginas 25-35. 2009.

Juan A. López, F. Soto, P. Sánchez, A. Iborra, J. Suardíaz, J.A.Vera. "Development of a Sensor Node for Precision Horticulture". Sensors, volumen 9, páginas 3240-3255. 2009.

Juan A. López, Fulgencio Soto, Andrés Iborra, Pedro Sánchez, Juan Suardíaz Muro. "Design and Implementation of a Wireless Sensor Network for Precision Horticulture". First International Conference on Sensor Applications, Experimentation, and Logistics (SENSAPPEAL 2009). Páginas 27-42. Atenas, Grecia.

J.A. López, F. Soto, J. Suardíaz, P. Sánchez, A. Iborra, J.A. Vera. "Development of a Mote for Horticulture based on the SDI-12 standard". 35th Annual Conference of the IEEE Industrial Electronics Society (IECON 2009), Porto, Portugal.

J.A. López Riquelme, F. Soto, J. Suardíaz, A. Iborra. "GAIA: un Mote para la Monitorización de Parámetros del Suelo Basado en el Estándar SDI-12”. Seminario Anual de Automática, Electrónica Industrial e Instrumentación (SAAEI 2009), Leganés, Madrid. 
[López, 2010]

[López, 2011a]

[López, 2011b]

[Losilla, 2009]

[Lowenberg-DeBoer, 2000]

[Madden, 2005]

[Mahgoub, 2006]

[Mantis]

[Mason, 1998]

[Matese, 2009]

[Maxim]

[Mckinion, 2001]

[Meguerdichian, 2001]

[Melly, 1999]
J.A. López Riquelme, F. Soto, C. Albaladejo, A. Iborra, J. Suardíaz. "GAIA 2: Un Mote Multifuncional para Agricultura de Precisión”. Seminario Anual de Automática, Electrónica Industrial e Instrumentación (SAAEI 2010), Bilbao, España.

J.A. López, A.J. García, F. Soto, A. Iborra, F. García, J. García. "Design and Validation of a Wireless Sensor Network Architecture for Precision Horticulture Applications". Precision Agriculture, volumen 12, páginas 280295. 2011.

J.A. López Riquelme, F. Soto Valles, R. Torres Sánchez, C. Albaladejo Pérez, J. Suardíaz Muro, H. Navarro Hellín. "MEWiN: un nodo sensor inalámbrico multi-entorno. Validación en Agricultura de Precisión". Seminario Anual de Automática, Electrónica Industrial e Instrumentación (SAAEI 2011), Badajoz, España.

Fernando Losilla López. Desarrollo de aplicaciones para redes de sensores inalámbricas: un enfoque dirigido por modelos. Tesis Doctoral. 2009.

Lowenberg-DeBoer, J. and K. Erickson. 2000. Precision Farming Profitability.Agricultural Research Programs, Purdue University.

S. Madden, et al., TinyDB: An Acquisitional Query Processing System for Sensor Networks, ACM Transactions on Database Systems, vol. 30, pp. 122173, No.1, March 2005.

Mahgoub, I., Ilyas, M., 2006. "Smart Dust: Sensor Network Applications, Architecture, and Design". CRC Press. ISBN-10: 0-8493-7037-X.

Mantis Operating System. Disponible on-line en: http://mantisos.org/index/tiki-index.php.html.

Mason, A. et al., 1998. "A Generic Multielement Microsystem for Portable Wireless Applications”. Proc. IEEE, 86(8), 1733-1746.

A. Matese, S.F. Di Gennaro, A. Zaldei, L. Genesio, F.P. Vaccari. “A wireless sensor network for precision viticulture: The NAV system". Computers and Electronics in Agriculture, Volume 69, Issue 1, November 2009, Pages 51 58.

Maxim Integrated Products. Disponible on-line en: http://www.maximic.com

J. M. McKinion, J. N. Jenkins, D. Akins, S. B. Turner, J. L. Willers, E. Jallas, F. D. Whisler. "Analysis of a precision agriculture approach to cotton production". Computers and Electronics in Agriculture, Volume 32, Issue 3, October 2001, Pages 213-228.

S. Meguerdichian, F. Koushanfar, G. Qu, M. Potkonjak. "Exposure in wireless ad-hoc sensor networks". Proceedings of ACM MobiCom'01, Rome, Italy, 2001, pp. 139-150.

T. Melly, A. Porret, C.C. Enz, E.A. Vittoz. “A 1.2 V, $430 \mathrm{MHz}, 4 \mathrm{dBm}$ power amplifier and a $250 \mathrm{lW}$ Frontend, using a standard digital CMOS 
[MEMSIC]

[Microchip]

[Min, 1995]

[Montenegro, 2007]

[Morais, 2008]

[MOTE-FRUT, 2008]

[MSP430F1611]

[Mspgcc]

[MTSP]

[Mulligan, 2007]

[National]

[Nortes, 2008]

[NRL]

[NS2]

[NS3]

[Odum, 1968] process". IEEE International Symposium on Low Power Electronics and Design Conference, San Diego, August 1999, pp. 233-237.

MEMS + IC, disponible on-line en: http://www.memsic.com.

Microchip Technology Incorporation. Disponible on-line en: http://www.microchip.com.

R. Min, T. Furrer, A. Chandrakasan. "Dynamic voltage scaling techniques for distributed microsensor networks". Proceedings of ACM MobiCom'95, August 1995.

G. Montenegro, N. Kushalnagar, J. Hui, D. Culler. "Transmission of IPv6 packets over IEEE 802.15.4 networks”. RFC 4944 (2007).

Morais, R.; Fernandes, M.A.; Matos, S.G.; Serodio, C.; Ferreira, P.J.S.G.; Reis, M.J.C.S. "A ZigBee multi-powered wireless acquisition device for remote sensing applications in precision viticulture". Comput. Electron. Agric. 2008, 62, 94-106."

"Sistema para la Monitorización y Seguimiento de los Indicadores de Estado Hídrico Basado en Redes de Sensores Inalámbricas (MOTE-FRUT)”. Ref. 08850/PI/08. Fundación Séneca. Región de Murcia.

Datasheet del microcontrolador MSP430F1611. Disponible on-line en: http:// focus.ti.com/lit/ds/symlink/msp430f1611.pdf

The GCC toolchain for the Texas Instruments MSP430 MCUs. Disponible on-line en: http://mspgcc.sourceforge.net/

Métodos y Tecnología de Sistemas y Procesos. Disponible on-line en: http://www.mtp.es.

G. Mulligan, L.W. Group. "The 6LoWPAN architecture". Proceedings of the EmNets, Cork, Ireland, 2007.

National Semiconductor Corporation. Disponible on-line en: http://www.national.com.

Pedro Antonio Nortes Tortosa. "Respuesta Agronómica y Fisiológica del Almendro al Riego Deficitario. Indicadores de Estrés Hídrico”. Tesis Doctoral. 2008.

Navy Research Laboratory sensor network extension. Disponible on-line en: http://pf.itd.nrl.navy.mil/projects/nrlsensorsim/.

Network Simulator 2. Página Web oficial. Disponible on-line en: http://www.isi.edu/nsnam/ns/

Network Simulator 3. Página Web oficial. Disponible on-line en: http://www.nsnam.org/

Odum P. Eugene. "Energy Flow in Ecosystems: A Historical Review". American Zoologist. 1968. Pp. 11-18. 
[Öhlmer, 1998]

[Olimex]

[OTcl]

[Panchard, 2006]

[Panchard, 2008]

[Parker, 1999]

[Pérez, 2004]

[Pering, 1998]

[Pierce, 2008]

[Polastre, 2004]

[Porret, 2000]

[Porter, 2005]

[Pottie, 2000]

[Rabaey, 2000]
O.G. Öhlmer B., Olson K., Brehmer B.. "Understanding farmers'decision making processes and improving managerial assistance”. Agricultural Economics, 18, 273-290. 1998.

Olimex Ltd. Disponible on-line en: http://www.olimex.com/

MIT Object Tcl: http://bmrc.berkeley.edu/research/cmt/cmtdoc/otcl

Jacques Panchard, Seshagiri Rao, Prabhakar T.V., H.S. Jamadagni and JeanPierre Hubaux. "COMMON-Sense Net: Improved water management for resource-poor farmers via sensor networks", Int'l Conf. on Information and Communication Technologies and Development, (ICTD 2006).

Panchard J., Rao S., Sheshshayee M., Papadimitratos P., Kumar S., Hubaux J. "Wireless sensor networking for rain-fed farming decision support". Proceedings of the second ACM SIGCOMM workshop on Networked systems for developing regions. Seattle, USA. pp. 31-36. 2008.

C. Parker. 1999. "Decision support systems: lessons from past Failures". Farm Management, 10, 273-289.

Pérez García M. A., Álvarez Antón J. C., Campo Rodríguez J. C., Ferrero Martín F. J., Grillo Ortega G. J. "Instrumentación Electrónica”. Interntional Thomson Editores Spain. Paraninfo, S.A. 2004.

T. Pering, T. Burd, R. Brodersen. "The simulation and evaluation of dynamic voltage scaling algorithms". Proceedings of International Symposium on Low Power Electronics and Design ISLPED'98, August 1998, pp. 76-81.

Pierce, F.J., Elliot, T.V., 2008. "Regional and on-farm wireless sensor networks for agricultural systems in Eastern Washington". Computers and Electronics in Agriculture 61, 32-43.

J. Polastre, J. Hill, and D. Culler. "Versatile low power media access for wireless sensor networks". In Proceedings of the 2nd ACM International Conference on Embedded Networked Sensor Systems (SenSys '04), pp. 95107, Baltimore, Md, USA, November 2004.

A. Porret, T. Melly, C.C. Enz, E.A. Vittoz. "A low-power low-voltage transceiver architecture suitable for wireless distributed sensors network". IEEE International Symposium on Circuits and Systems'00, Geneva, Vol. 1, 2000, pp. 56-59.

Porter, J., Arzberger, P., Braun, H., Bryant, W., Gage, P., Hansen, S., Hanson, T., Lin, P., Lin, C.C., Kratz, F.P., Michener, T., Shapiro, W., Williams, S.T., 2005. "Wireless sensor networks for ecology". BioScience 55 (7), 561-572.

G.J. Pottie, W.J. Kaiser. "Wireless integrated network sensors". Communications of the ACM 43 (5) (2000) 551-558.

J. Rabaey, J. Ammer, J.L. da Silva Jr., D. Patel. "Pico-Radio: ad-hoc wireless networking of ubiquitous lowenergy sensor/monitor nodes". Proceedings of 
[Rabaey, 2000a]

[Ramachandran, 2007]

[Ramos, 2007]

[Reyniers, 2006]

[RIMSI]

[Ryu, 2009]

[SDI12]

[SensorSim]

[Sentek]

[Shen, 2001]

[Shih, 2001]

[Shock, 1999]

[Sivilotti, 2005] the IEEE Computer Society Annual Workshop on VLSI (WVLSI'00), Orlanda, Florida, April 2000, pp. 9-12.

J.M. Rabaey, M.J. Ammer, J.L. da Silva Jr., D. Patel, S. Roundy. "PicoRadio supports ad hoc ultra-low power wireless networking”. IEEE Computer Magazine (2000) 42-48.

Ramachandran, I., Das, A.K. \& Roy, S. (2007). Analysis of the contention access period of IEEE 802.15.4 MAC. ACM Transactions on Sensor Networks, 3, 4-29. doi.acm.org/10.1145/1210669.1210673.

M.I. Ramos, A.J. Gil, F.R. Feito, A. García-Ferrer. "Using GPS and GIS tools to monitor olive tree movements". Computers and Electronics in Agriculture, Volume 57, Issue 2, July 2007, Pages 135-148.

M. Reyniers, K. Maertens, E. Vrindts, J. De Baerdemaeker. "Yield variability related to landscape properties of a loamy soil in central Belgium". Soil and Tillage Research, Volume 88, Issues 1-2, July 2006, Pages 262-273.

"Sistemas de Redes Inalámbricas de Micro Dispositivos para Monitorización Sensorial Inteligente". Ref. FIT-340000-2006-202. Ministerio de Industria, Comercio y Turismo. Fondo Europeo de Desarrollo Regional (FEDER).

Chanseok Ryu, Masahiko Suguri, Mikio Umeda. "Model for predicting the nitrogen content of rice at panicle initiation stage using data from airborne hyperspectral remote sensing". Biosystems Engineering, Volume 104, Issue 4, December 2009, Pages 465-475.

Interfaz digital de datos serie para sensores basados en micro-controladores. Versión 1.3. 18 de Julio, 2005. Realizado por el grupo de soporte SDI-12 (Comite Técnico). USA. Disponible on-line en: http://www.sdi-12.org.

SensorSim Project. Disponible on-line http://nesl.ee.ucla.edu/projects/sensorsim/

Sentek Technologies. Disponible on-line en: http://www.sentek.com/

C. Shen, C. Srisathapornphat, C. Jaikaeo. "Sensor information networking architecture and applications". IEEE Personal Communications. August 2001. pp. 52-59.

E. Shih, S. Cho, N. Ickes, R. Min, A. Sinha, A. Wang, A. Chandrakasan. "Physical layer driven protocol and algorithm design for energy-efficient wireless sensor networks". Proceedings of ACM MobiCom'01, Rome, Italy, July 2001, pp. 272-286.

Shock, C.C., Barnum, J.M., Seddigh, M. 1998. "Calibration of Watermark soil moisture sensors for irrigation management". In: Proceedings of the 1998 Irrigation Association Technical Conference, The Irrigation Association, Falls Church, VA, USA, pp. 123-129.

Sivilotti, P., Bonetto, C., Paladin, M., Peterlunger, E., 2005. Effect of soil moisture availability on vitis vinifera $\mathrm{cv}$ 'merlot': from leaf water potential to grape composition. Am. J. Enol. Vitic. 56 (1), 9-18. 
[Sniffer]

[Soilmoisture]

[SoilNet]

[Solartron]

[Solwise]

[Song, 2008]

[SOS]

[Sparkfun]

[Stafford, 1996]

[Stafford, 2000]

[Stevens]

[Stojmenovic, 2005]

[Sun, 2010]

[TelosB]

[TI]

[TinyOS]

[TOSSIM, 2003]

[Vaisala]

[VectorInstruments]
Manual del SmartRF Packet Sniffer de Texas Instruments. Disponible online en: http://focus.ti.com/lit/ug/swru187b/swru187b.pdf.

Soilmoisture Equipment Corp. Disponible on-line en: http://www.soilmoisture.com/

"SoilNet - A Zigbee based soil moisture sensor network" Project Group, Institute Of Chemistry And Dynamics Of The Geosphere (ICG), Agrosphere Institute, ICG 4, Forschungszentrum Jülich GmbH, 52425 Jülich, http://www.fz-juelich.de/icg/icg-4/index.php.index=739.

Solartron Metrology. Disponible on-line en: http://www.solartronmetrology.com/

Solwise Company. Disponible on-line en: http://www.solwise.co.uk

Song G., Zhou Y., Ding F., Song A. “A Mobile Sensor Network System for Monitoring of Unfriendly Environments". Sensors 2008, 8, pp. 7259-7274.

SOS Operating System. Disponible on-line en: https://projects.nesl.ucla.edu/public/sos-2x/doc/

Sparkfun Electronics. Disponible on-line en: http://www.sparkfun.com

Stafford J.V. "Special Issue: Spatially variable field operations". Computers and Electronics in Agriculture 14, 99-253.

Stafford, J.V. "Implementing Precision Agriculture in the 21st Century". J. Agric. Engng. Res. 76, 267-275.

Stevens Water Monitoring Systems, Inc. Disponible on-line en: http://www.stevenswater.com/

Stojmenovic I. "Handbook of Sensor Networks Algorithms and Architectures". Wiley-Intersciencie, 2005.

H. Sun, D.C.Slaughter, M. Pérez Ruiz, C. Gliever, S.K. Upadhyaya, R.F. Smith. "RTK GPS mapping of transplanted row crops". Computers and Electronics in Agriculture, Volume 71, Issue 1, April 2010, Pages 32-37.

Memsic Corporation. Disponible on-line en: http://www.memsic.com/

Texas Instruments Inc. . Disponible on-line en: http://www.ti.com/

TinyOS Operating System. Disponible on-line en: http://www.tinyos.net

P. Levis, N. Lee, M. Welsh, D. Culler. "TOSSIM: Accurate and Scalable Simulation of Entire TinyOS Applications”. In Proc. of the 1st international conference on Embedded networked sensor systems. Los Angeles, California, USA. Pp. 126 - 137, 2003.

Vaisala Inc. Disponible on-line en: http://www.vaisala.com/

Vector Instruments Inc. Disponible on-line en: http://www.windspeed.co.uk/ 
[Vellidis, 2008]

[Villalobos, 2002]

[Vrindts, 2003]

[Wall, 2004]

[Wang, 2001]

[Wang, 2006]

[Wang, 2007]

[Warneke, 2001]

[Webster, 1998]

[Wibree]

[Winkler, 1974]

[Wolfgang, 2000]

[Xia, 2007]

[Yick, 2008]

[Yu, 2010]
Vellidis, G., Tucker, M., Perry, C., Kvien, C., Bednarz, C., 2008. “A real-time wireless smart sensor array for scheduling irrigation". Comput. Electron. Agric. 61, 44-50.

Villalobos F., Mateos L., Orgaz F., Fereres E. "Fitotecnia: Bases y Tecnologías de la Producción Agrícola”. Mundi-Prensa. 2002.

E. Vrindts, M. Reyniers, P. Darius, J. De baerdemaeker, M. Gilot, Y. Sadaoui, M. Frankinet, B. Hanquet, M. -F. Destain. "Analysis of Soil and Crop Properties for Precision Agriculture for Winter Wheat". Biosystems Engineering, Volume 85, Issue 2, June 2003, Pages 141-152.

Wall, R.W., King, B.A., 2004. "Incorporating plug and play technology into measurement and control systems for irrigation management". ASAE Technical Paper No. 04-2189. The American Society of Agriculture Engineers, St. Joseph, Michigan, USA.

Wang Maohua. "Possible adoption of precision agriculture for developing countries at the threshold of the new millennium”. Millennium Special Issue. Computers and Electronics in Agriculture 30, 45, 50.

Ning Wang, Naiqian Zhang, Maohua Wang, "Wireless sensors in agriculture and food industry-Recent development and future perspective", published in Computers and Electronics in Agriculture 50 (2006) 1-14.

Wang, X., Wang, S., Bi, D., Ma, J. “Distributed Peer-to-Peer Target Tracking in Wireless Sensor Networks”. Sensors 2007, 7, 1001-1027.

B. Warneke, M. Last, B. Liebowitz and K. Pister. "Smart Dust: Communicating with a Cubic-Millimeter Computer". IEEE Computer, 34, pp. 44-51, 2001.

Webster, J. G. (Ed.). "The Measurement, Instrumentation and Sensors Handbook". CRC Press, 1998.

Wibree, <http://www.wibree.com/>.

Winkler, A.J., Cook, J.A., Kliewer,W.M., Lider, L.A., 1974. General Viticulture. University of California Press, Berkeley.

Wolfgang Lechner, Stefan Baumann. "Global navigation satellite systems". Computers and Electronics in Agriculture 25 (2000) 67-85.

Xia, F., Tian, Y., Sun, Y. "Wirelles sensor/actuator network design for mobile control applications”. Sensors 2007, 7, pp. 2157-2173.

Jennifer Yick, Biswanath Mukherjee, Dipak Ghosal. "Wireless sensor network survey”. Computer Networks, Volume 52, Issue 12, 22 August 2008, Pages 2292-2330.

Long Yu, Li Zhou, Wei Liu, Hua-Kun Zhou. "Using Remote Sensing and GIS Technologies to Estimate Grass Yield and Livestock Carrying Capacity of Alpine Grasslands in Golog Prefecture, China". Pedosphere, Volume 20, Issue 3, June 2010, Pages 342-351. 
[Zhang, 2002]

[Zheng, 2006]

[Zhao, 2004]

[ZigBee, 2007]

[ZigBee, 2008]

[Z-Stack]
Zhang, N.,Wang, M., Wang N. 2002. Precision agriculture-a worldwide overview, Computers and Electronics in Agriculture 36, 113-132.

Zheng, J., \& Lee, M. J. “A comprehensive performance study of IEEE 802.15.4". Sensor network operations (pp. 218-237). 2006.

Feng Zhao, Leonidas Guibas. "Wireless Sensor Networks. An Information Processing Approach”. Morgan Kaufmann Publishers. El Sevier.

ZigBee Alliance, especificación de la revisión ZigBee 2007. Disponible online en: http://www.zigbee.org.

Drew Gislason. “ZigBee Wireless Networking”. Newnes. 2008. ISBN-10: 9780750685979 .

ZigBee Protocol Stack. Disponible on-line en: http:/ / focus.ti.com/docs/toolsw/folders/print/z-stack.htm. 